\title{
Thermodynamics of atomic layer etching chemistry on copper and nickel surfaces from first principles
}

\author{
Yantao $\mathrm{Xia}^{*, \dagger}$ and Philippe Sautet ${ }^{*, \dagger, \ddagger}$ \\ $\dagger$ Department of Chemical and Biomolecular Engineering, University of California, Los \\ Angeles, CA 90095, USA \\ $\ddagger$ Department of Chemistry and Biochemistry, University of California, Los Angeles, CA \\ 90095, USA \\ E-mail: xyttyxy@ucla.edu; sautet@ucla.edu
}

\section{Estimation of reactant and product pressures}

The reactant and product pressures are estimated roughly based on the fluxes used in the experiments. Assuming complete vaporization and perfect mixing, the etchant pressure is calculated by:

$$
P_{\text {etchant }}=\frac{f_{\text {etchant }}}{f_{\text {etchant }}+f_{\text {carrier }}} P_{\text {total }}
$$

Where $f^{\prime}$ 's are the molar flow rates of etchant and the carrier gas. It is reported in Ref. ${ }^{1}$ that the flow rate of $\mathrm{N}_{2}$ carrier gas is $100 \mathrm{sccm}$, the flow rate of formic acid into the vaporizer is 0.1 $\mathrm{g} / \mathrm{min}$, and $P_{\text {total }}=350$ Torr. Thus $f_{\text {etchant }}=0.0021 \mathrm{~mol} / \mathrm{min}$, and $f_{\text {carrier }}=0.0035 \mathrm{~mol} / \mathrm{min}$. This gives $P_{\text {etchant }}=18025 \mathrm{~Pa}$. The estimation of the product pressure is difficult and we simply put it as $10 \%$ of the etchant pressure. The proposed processes using formamidine as the etchant are simulated at the same conditions. We expect that the pressure of the product 
will not significantly affect thermodynamics since at such low pressures, the deviation from ideal gas can be neglected. We verified that alternative estimates of product pressure to be $5 \%$ and $20 \%$ of the etchant pressure only changes the removal step energies by around $0.02 \mathrm{eV}$, and does not change our conclusions. 


\section{Validation of Computational Methods}

The relaxed crystal structure and their atomization energies are compared to experimental values in Table S1. The free energy is used throughout our study. The enthalpies are provided since the experimental free energy of formation of $\mathrm{Cu}_{3} \mathrm{~N}$ is not available. The results indicate that except for $\mathrm{NiO}$, the calculated energies agree very well with experimental energies. The calculated values for the oxides are shown but not used since they are known pathological cases for the PBE exchange-correlation functional. The functional is retained nevertheless due to the need to calculate and compare energies on diverse systems, that include surfaces, bulk structures, organic molecules, and organometallic complexes.

Table S1: Optimized bulk lattice and formation energy. All experimental values are obtained from CRC Handbook of Chemistry and Physics ${ }^{2}$ unless otherwise stated. The two numbers in $\mathrm{Ni}_{3} \mathrm{~N}$ lattice correspond to $a$ and $c$, respectively. The calculated values are obtained via self-consistent minimization in VASP with the PBE exchange-correlation functional. The values used are marked with boldface.

\begin{tabular}{|c|c|c|c|c|c|c|}
\hline \multirow[b]{2}{*}{ System } & \multicolumn{2}{|l|}{ Lattice $[\AA]$} & \multicolumn{2}{|c|}{ Cohesive enthalpy $[\mathrm{eV}]$} & \multicolumn{2}{|c|}{ Cohesive free energy $[\mathrm{eV}]$} \\
\hline & Exp. & Calc. & Exp. & Calc. & Exp. & Calc. \\
\hline $\mathrm{Ni}$ & 3.52 & 3.52 & 4.45 & 4.60 & 3.98 & 4.19 \\
\hline $\mathrm{Cu}$ & 3.62 & 3.64 & 3.50 & 3.44 & 3.09 & 3.05 \\
\hline $\mathrm{NiO}$ & 4.18 & 4.20 & $9.52^{3}$ & 8.82 & 8.58 & 7.78 \\
\hline $\mathrm{Cu}_{2} \mathrm{O}$ & 4.27 & 4.31 & 11.32 & 11.46 & 10.09 & 10.03 \\
\hline $\mathrm{Ni}_{3} \mathrm{~N}$ & $4.64 / 4.31^{4}$ & $4.62 / 4.31$ & $\mathrm{~N} / \mathrm{A}$ & 18.75 & N/A & 17.14 \\
\hline $\mathrm{Cu}_{3} \mathrm{~N}$ & 3.81 & 3.83 & $14.15^{5}$ & 14.25 & $\mathrm{~N} / \mathrm{A}$ & 12.71 \\
\hline
\end{tabular}

Van der Waals interaction could have a potentially important influence on our removal step energy results. The contribution of van der Waals interaction to the removal step energy is not quantified directly, since the $\mathrm{PBE}+\mathrm{dDs}^{6,7}$ formalism does not accurately reproduce the bulk formation energies, which is a significant term in Equation 5. Alternative methods, including the vdw-DF family of functionals, suffer similar problem. ${ }^{8}$. Instead, we chose to quantify the contribution by comparing the adsorption energies with and without this contribution. Figure S1 shows the adsorption energies (Eqn. S2) calculated with and without the $\mathrm{dDsC}$ contributions on the $\mathrm{Cu}(100)$ surface and $\mathrm{Ni}$ (111) surface, with $\mathrm{N}$ and $\mathrm{O}$ adsorbed. 
With the $\mathrm{dDsC}$ correction, the adsorption becomes more favorable by $\sim 0.1 \mathrm{eV}$ on both $\mathrm{Cu}$ and $\mathrm{Ni}$ systems. Notably, the magnitude of the correction does not change much with increasing coverage. This uniformity over the entire range of coverage investigated suggest that the deviation introduced by neglecting van der Waals interaction is approximately $0.05 \mathrm{eV}$ on the $\mathrm{Cu}$ systems and $0.1 \mathrm{eV}$ on the $\mathrm{Ni}$ systems. Errors of this magnitude will influence the quantitative thermochemical predictions, but they will not invalidate our results of whether given etching chemistry will lead to favorable reaction.
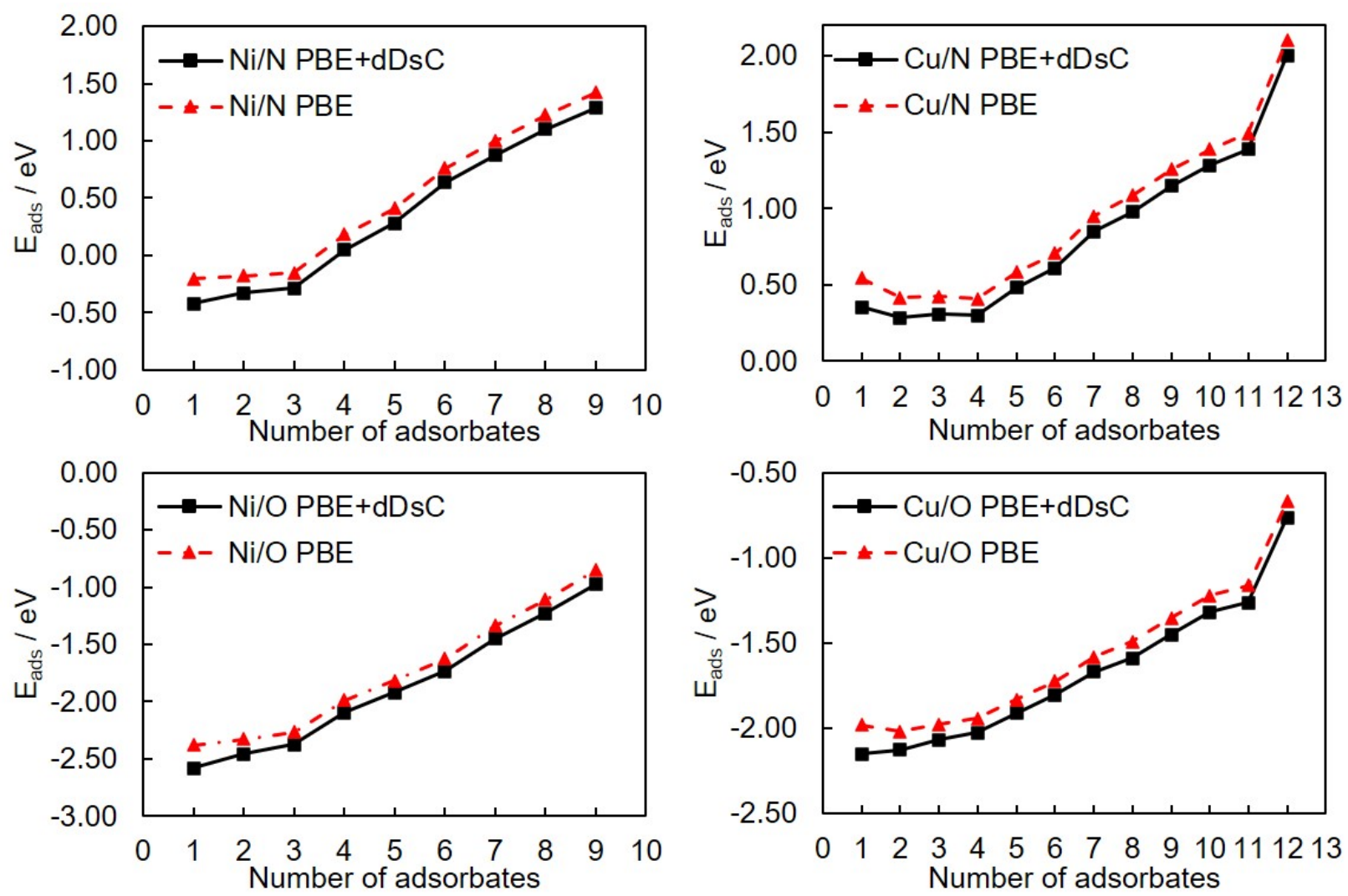

Figure S1: Adsorption energies with and without dDsC correction 


\section{Adsorption configuration search setup}

Figure S2 shows the supercell used in the configuration search and the "symmetry unique" sites. Except for the sites that merged into the same post-relaxation structure, the singleadsorbate adsorption energies of the four combinations of metals ( $\mathrm{Ni}$ and $\mathrm{Cu}$ ) and modifiers (N and $\mathrm{O}$ ) are shown in Table S2, calculated from Eqn. S2 (written for Ni/O system).

$$
\Delta G_{\mathrm{ads}}=\left(G_{\mathrm{Ni}_{\mathrm{s}} \mid \mathrm{O}_{\mathrm{y}}}-G_{\mathrm{Ni}_{\mathrm{s}} \mid}-y \mu_{\mathrm{O}}\right) \approx\left(E_{\mathrm{Ni}_{\mathrm{s}} \mid \mathrm{O}_{\mathrm{y}}}-E_{\mathrm{Ni}_{\mathrm{s}} \mid}-y \mu_{\mathrm{O}}\right) / y
$$

Table S2: Adsorption sites, labels, and adsorption energies (in $\mathrm{eV}$ ) referenced to $\mathrm{O}_{2}$ and $\mathrm{N}_{2}$ at $80^{\circ} \mathrm{C}$, 350 Torr. A blank cell means the site is not stable in relaxation and coalesced to other sites.

\begin{tabular}{|c|c|c|c|c|c|c|}
\hline \multirow[b]{2}{*}{ Termination } & \multirow[b]{2}{*}{ Site name } & \multicolumn{4}{|c|}{$\Delta E_{\text {ads }}[\mathrm{eV}]$} & \multirow[b]{2}{*}{$\mathrm{Cu} / \mathrm{O}$} \\
\hline & & Site label & $\mathrm{Ni} / \mathrm{N}$ & $\mathrm{Ni} / \mathrm{O}$ & $\mathrm{Cu} / \mathrm{N}$ & \\
\hline \multirow{3}{*}{$(100)$} & $\mathrm{t}$ & A1 & 2.43 & -0.43 & 3.58 & 0.20 \\
\hline & $\mathrm{b}$ & $\mathrm{A} 2$ & 0.78 & -1.63 & 2.15 & -1.01 \\
\hline & $4 \mathrm{~h}$ & A3 & -0.80 & -2.34 & 0.56 & -1.85 \\
\hline \multirow{5}{*}{$(110)$} & t-tr & B5 & 0.24 & -1.53 & 1.46 & -1.09 \\
\hline & t-pk & B4 & 2.42 & -0.58 & 3.96 & 0.43 \\
\hline & $\mathrm{sb}$ & $\mathrm{B} 2$ & 0.81 & -1.76 & 2.37 & -0.87 \\
\hline & $\mathrm{lb}$ & B3 & -0.05 & -1.54 & 1.52 & -1.14 \\
\hline & $3 \mathrm{~h}$ & B1 & 0.29 & -1.80 & & -1.21 \\
\hline \multirow{3}{*}{ (111) } & $\mathrm{t}$ & C3 & 2.58 & -0.22 & 3.98 & 0.70 \\
\hline & 3h-hcp & $\mathrm{C} 2$ & 0.11 & -1.92 & 1.93 & -1.00 \\
\hline & $3 \mathrm{~h}-\mathrm{fcc}$ & $\mathrm{C} 1$ & 0.08 & -2.04 & 1.85 & -1.09 \\
\hline \multirow{7}{*}{$(210)$} & t-tr & D6 & & & 2.23 & -0.81 \\
\hline & t-pk & D1 & 2.41 & -0.70 & & \\
\hline & b-tr & D2 & 0.31 & -1.59 & 1.88 & -0.81 \\
\hline & b-pk & D7 & -0.48 & -2.26 & 1.14 & -1.55 \\
\hline & $4 \mathrm{~h}$ & D3 & -0.06 & -1.66 & 1.42 & -1.20 \\
\hline & $3 h-1$ & D4 & 0.34 & -1.67 & 1.42 & -1.12 \\
\hline & $3 \mathrm{~h}-2$ & D5 & 0.34 & -1.67 & & \\
\hline
\end{tabular}


...continued

\begin{tabular}{|c|c|c|c|c|c|c|}
\hline \multirow[b]{2}{*}{ Termination } & \multirow[b]{2}{*}{ Site name } & \multicolumn{4}{|c|}{$\Delta E_{\text {ads }}[\mathrm{eV}]$} & \multirow[b]{2}{*}{$\mathrm{Cu} / \mathrm{O}$} \\
\hline & & Site label & $\mathrm{Ni} / \mathrm{N}$ & $\mathrm{Ni} / \mathrm{O}$ & $\mathrm{Cu} / \mathrm{N}$ & \\
\hline \multirow{9}{*}{$(211)$} & t-st & E9 & 2.70 & & & \\
\hline & t-pk & E1 & 2.46 & -0.59 & & \\
\hline & b-pk & E2 & & -1.86 & & \\
\hline & b-cs & E3 & 1.16 & -1.22 & 2.35 & -0.60 \\
\hline & $4 \mathrm{~h}$ & E4 & -0.24 & -1.82 & 0.98 & -1.39 \\
\hline & 3h-hcp-st-l & E6 & 0.28 & -1.68 & 1.85 & -1.07 \\
\hline & 3h-hcp-st-h & E8 & -0.04 & -2.14 & 1.48 & -1.45 \\
\hline & 3h-fcc-st-l & E5 & 0.29 & -1.62 & & -1.14 \\
\hline & 3h-fcc-st-h & E7 & 0.24 & -1.92 & 1.58 & -1.30 \\
\hline \multirow{10}{*}{$(221)$} & t-tr & F7 & 0.16 & -0.58 & 1.29 & \\
\hline & t-pk & $\mathrm{F} 1$ & 2.45 & & 3.80 & \\
\hline & $\mathrm{lb}$ & F6 & -0.09 & -1.56 & 1.40 & -1.07 \\
\hline & b-pk & $\mathrm{F} 2$ & & -1.82 & & \\
\hline & 3h-hcp-st-l & F5 & 0.20 & -1.83 & 1.74 & -1.16 \\
\hline & 3h-hcp-st-h & F3 & 0.13 & -1.84 & 1.67 & -1.17 \\
\hline & 3h-fcc-st-m & $\mathrm{F} 4$ & 0.14 & -1.91 & 1.61 & -1.34 \\
\hline & 3h-fcc-st-l & F10 & & -1.58 & & -0.98 \\
\hline & 3h-fcc-st-h & F8 & 0.00 & -2.18 & 1.39 & -1.60 \\
\hline & 3h-hcp-cs & F9 & 0.26 & -1.85 & & -1.38 \\
\hline \multirow{5}{*}{$(311)$} & b-tr & G2 & 1.17 & & & -0.59 \\
\hline & b-pk & G1 & & -1.88 & 2.23 & \\
\hline & $4 \mathrm{~h}$ & G3 & -0.20 & -1.80 & 1.12 & -1.36 \\
\hline & 3h-hcp & G4 & 0.08 & -1.99 & 1.61 & -1.33 \\
\hline & 3h-fcc & G5 & 0.41 & -1.61 & & -1.07 \\
\hline
\end{tabular}

For validation against experiments, the adsorption energies on selected experimentally observed low-coverage surface adsorption structures are also calculated and shown in Table S3. Experimental structures that do not allow a direct comparison(i.e., those with extra or missing metal atoms) to the ones found through the configuration search are marked with 

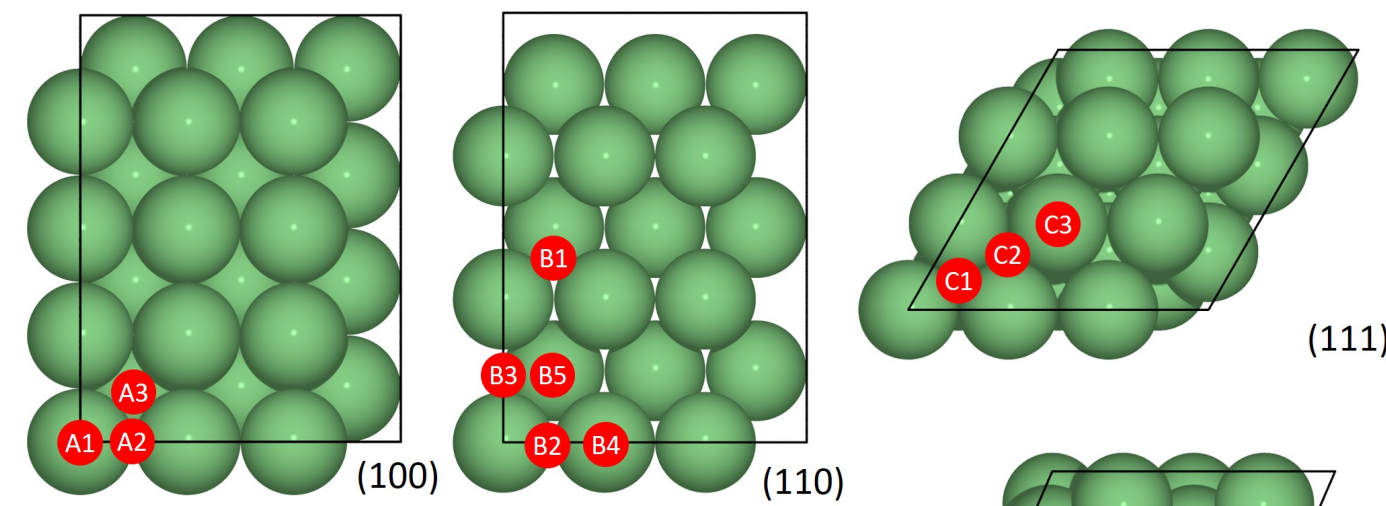

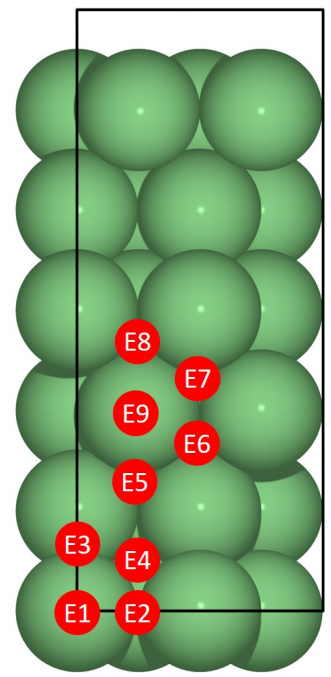

(211)

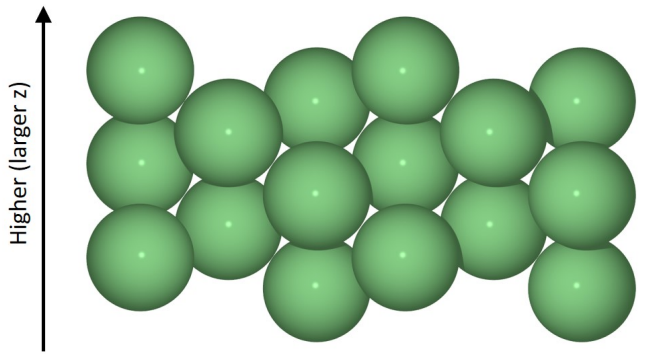

(211) - side view

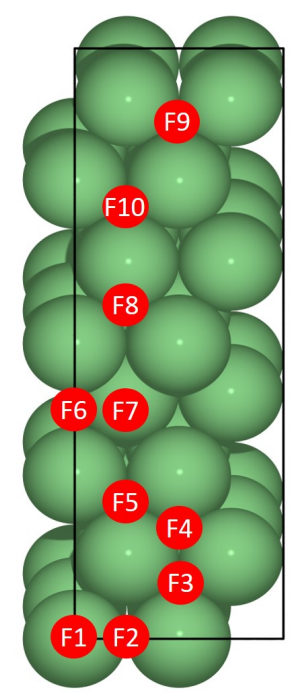

(221)
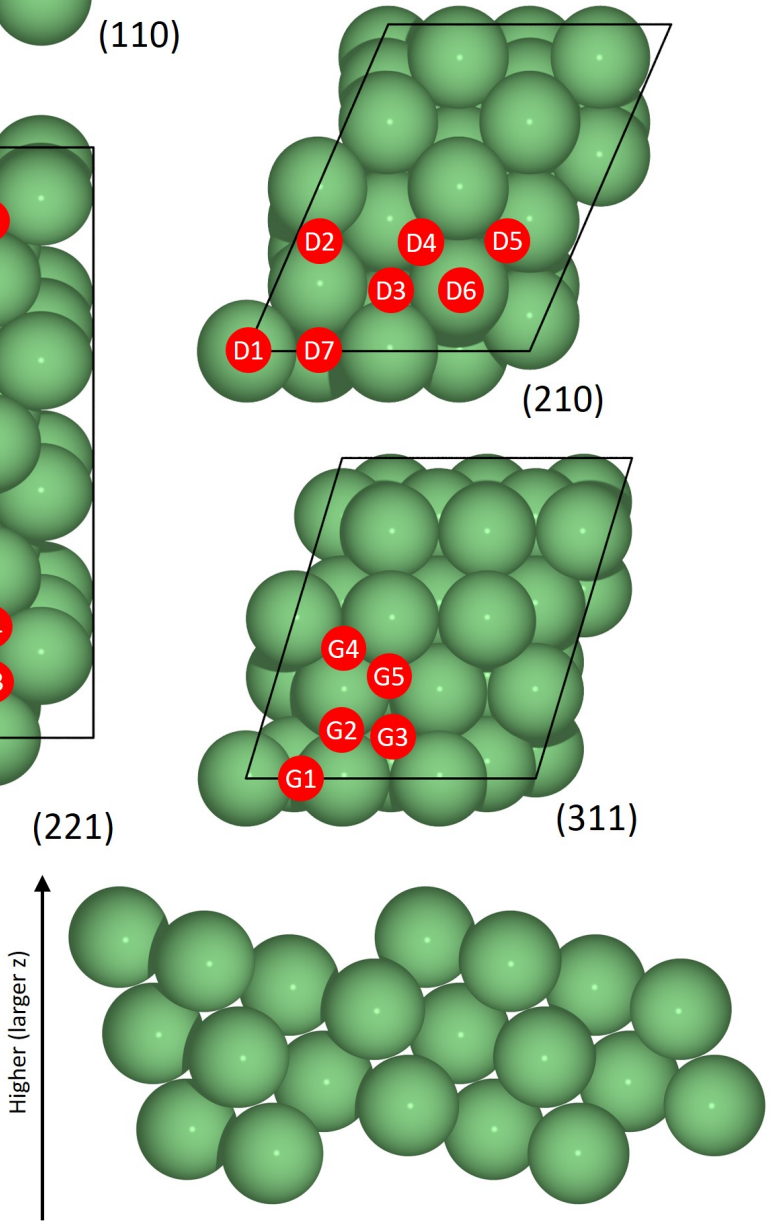

(221) - side view

Figure S2: Adsorption sites on (100), (111), (110), (210), (211), (221), and (311) terminations of $\mathrm{Ni}$ and $\mathrm{Cu}$. The supercells correspond to the actual slab model used in the configuration search. The (211) and (221) are shown in side view in addition to top view. Site labels are explained in Table $\mathrm{S} 2$ 
an asterisk.

Table S3: Experimentally observed surface reconstructions and adsorption superstructures for the $\mathrm{Ni} / \mathrm{O}$ and $\mathrm{Cu} / \mathrm{O}$ systems. The adsorption energy values marked with * indicate that these structures do not have a well-defined reference 'bare' surface, as metal atoms are added/removed to these surfaces. The 'bare' surface term in Eqn. S2 is approximately calculated from that of pristine surface, adjusted by the number of atoms. The Ni(110) missing row reconstruction and the $\mathrm{Cu}(110)$ pairing-row reconstruction had their bare surfaces recalculated, hence are not marked with *.

\begin{tabular}{lllllc} 
Substrate & Termination & $\theta_{\mathrm{O}}\left[\AA^{-2}\right]$ & Surface cell & Ref. & $\Delta G_{\text {ads }}[\mathrm{eV}]$ \\
\hline $\mathrm{Ni}$ & $(100)$ & 0.040 & $p(2 \times 2)$ & 9 & -1.92 \\
$\mathrm{Ni}$ & $(100)$ & 0.081 & $c(2 \times 2)$ & 9 & -2.27 \\
$\mathrm{Ni}$ & $(110)$ & 0.057 & $(2 \times 1)$ missing-row & 10 & -2.48 \\
$\mathrm{Ni}$ & $(110)$ & 0.076 & $(3 \times 1)$ missing-row & 10 & -2.13 \\
$\mathrm{Ni}$ & $(111)$ & 0.047 & $p(2 \times 2)$ & 11 & -1.52 \\
$\mathrm{Ni}$ & $(111)$ & 0.062 & $(\sqrt{3} \times \sqrt{3}) R 30^{\circ}$ & 11 & -1.60 \\
\hline $\mathrm{Cu}$ & $(100)$ & 0.076 & $c(2 \times 2)$ & 12 & -1.38 \\
$\mathrm{Cu}$ & $(100)$ & 0.076 & $(2 \sqrt{2} \times \sqrt{2}) R 45^{\circ}$ & 12 & -1.38 \\
$\mathrm{Cu}$ & $(110)$ & 0.054 & $(2 \times 1)$ pairing-row & 12 & -1.80 \\
$\mathrm{Cu}$ & $(110)$ & 0.071 & $c(6 \times 2)$ & 12 & $-3.35^{*}$ \\
$\mathrm{Cu}$ & $(111)$ & 0.087 & $p 4$ & 13 & $-2.66^{*}$ \\
$\mathrm{Cu}$ & $(111)$ & 0.098 & $p 4+\mathrm{O}_{F}$ & 13 & $-0.77^{*}$ \\
$\mathrm{Cu}$ & $(111)$ & 0.076 & $p 4+\mathrm{OCu}_{3}$ & 13 & $-1.02^{*}$
\end{tabular}

The supercells used in this study (see Figure S2) are selected and not thoroughly searched. Hence, it is possible that our configuration search cannot find more stable configurations that require a different repeating pattern. To quantify the magnitude of this error, Figure S3 compares the adsorption energies of structures from the configuration on different supercells. On (100), while the experimental adsorption structures of $p(2 \times 2), c(2 \times 2)$ and $\sqrt{2} \times \sqrt{2} R 45^{\circ}$ is not representable by the supercell used in the main results, the deviation errors introduced is on the order of $0.1 \mathrm{eV}$. On (111) and (211), the alternative supercells, through the same configuration search algorithm, finds less stable structures than the supercell used. The results here suggest that the structures used in this study are close to the true minima at the coverages investigated. An error of $0.1 \mathrm{eV}$ would not refute our conclusions. 

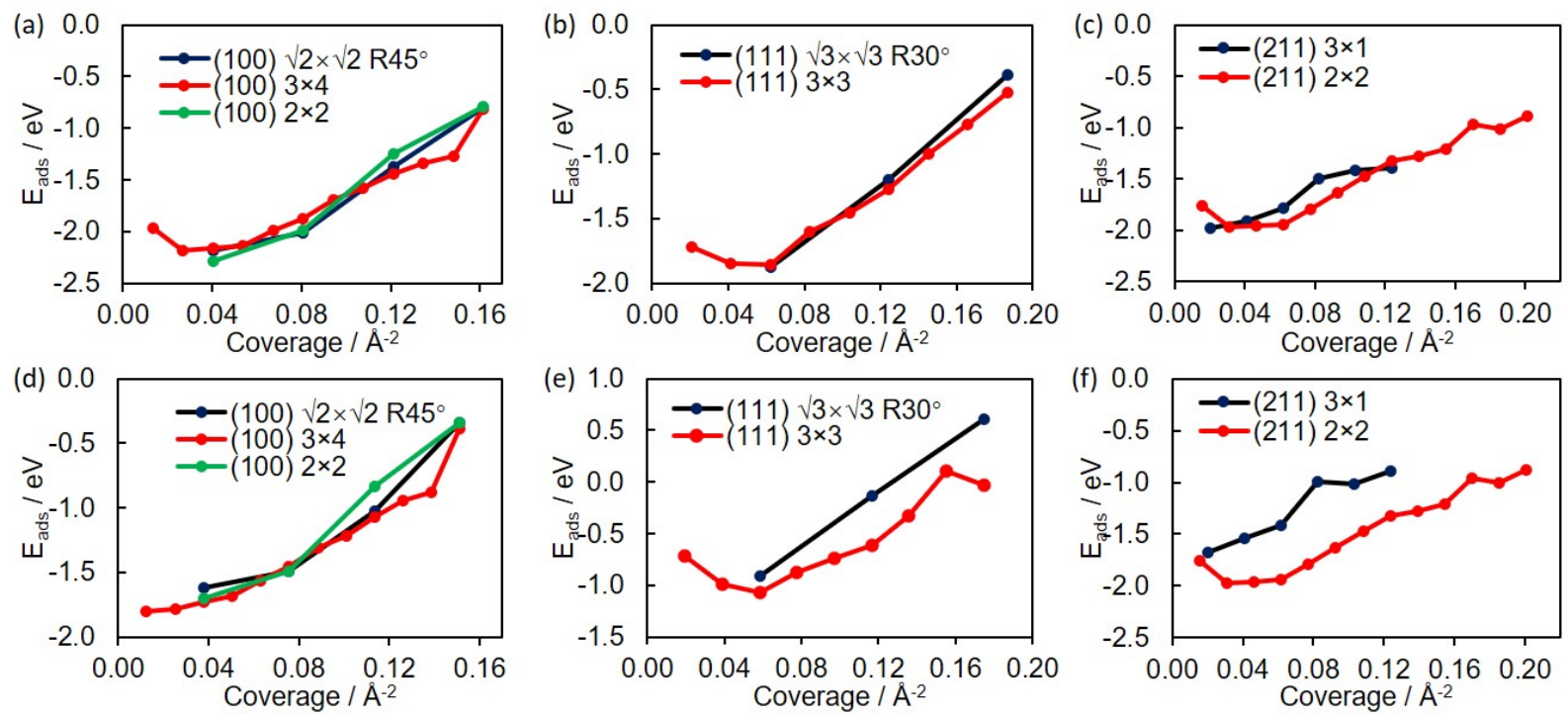

Figure S3: Adsorption energies of structures found by configuration search on the $\mathrm{Ni}$ (100)(a), $\mathrm{Ni}(111)(\mathrm{b}), \mathrm{Ni}(211)(\mathrm{c}), \mathrm{Cu}(100)(\mathrm{d}), \mathrm{Cu}(111)(\mathrm{e})$, and $\mathrm{Cu}(211)(\mathrm{f})$ surfaces. The results from alternative supercells are marked with green and black lines, whereas the original supercells are marked with red lines. The adsorption energies are calculated with Eqn. S2. 


\section{Averaging terminations using wulff construction}

The chemical potential used to obtain the surface formation energies are shown in Figure S4. Its definition is given in Eqn. 9. The surface energies resulting from the coverage-dependent chemical potential is shown in Figure S5. Its definition is given in Eqn. 10. The fraction of surfaces given by the Wulff construction is shown in Figure S6. The use of these quantifies are explained in Section 2.

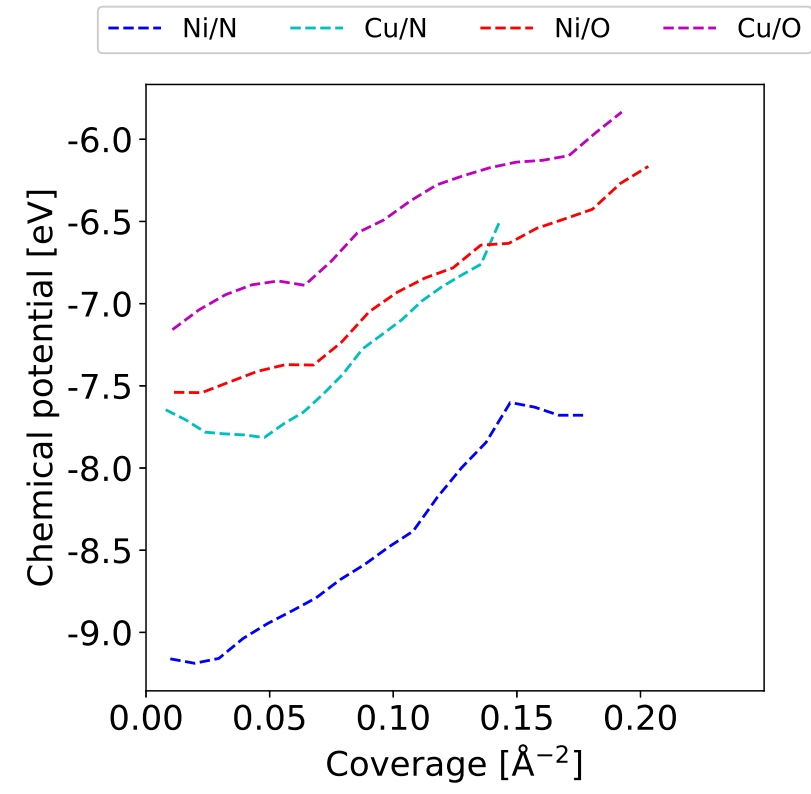

Figure S4: Relation of the chemical potential to the surface coverage(area density of modifier atoms), obtained from Eqn. 9. 
(a) $\mathrm{Ni} / \mathrm{N}$

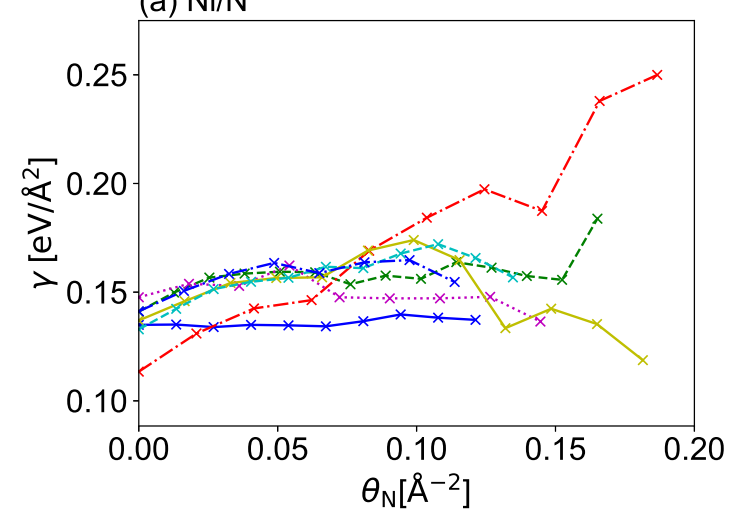

(c) $\mathrm{Ni} / \mathrm{O}$

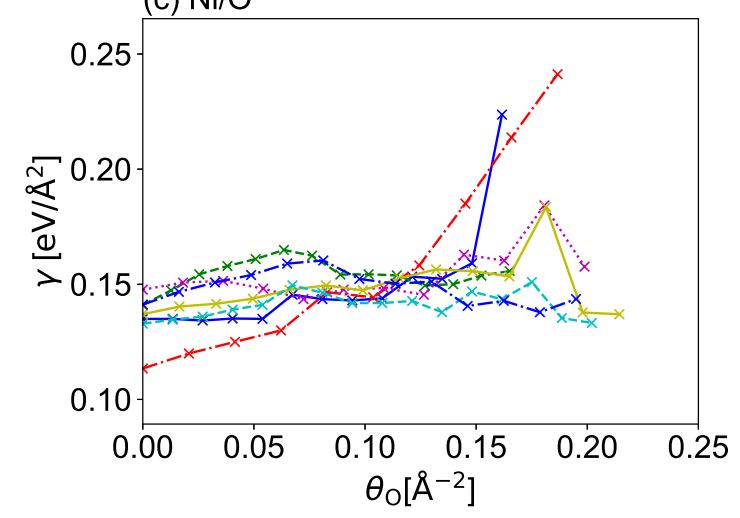

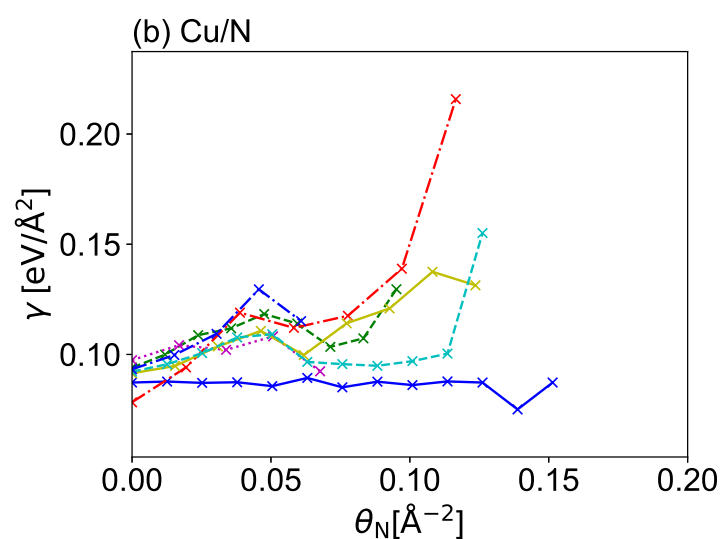

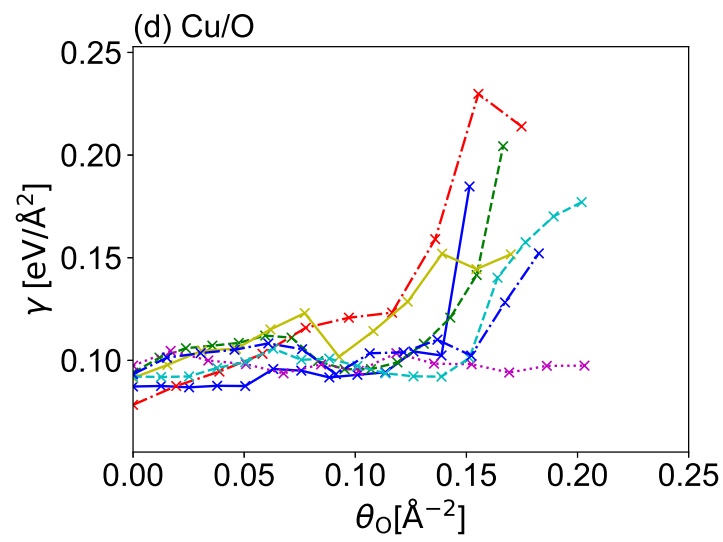

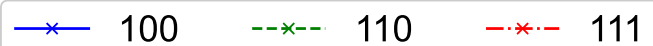

210

211

$221-* *-311$

Figure S5: Surface energies of terminations on the $\mathrm{Ni} / \mathrm{N}, \mathrm{Cu} / \mathrm{N}, \mathrm{Ni} / \mathrm{O}$ and $\mathrm{Cu} / \mathrm{O}$ systems as functions of coverage. 
(a) $\mathrm{Ni} / \mathrm{N}$

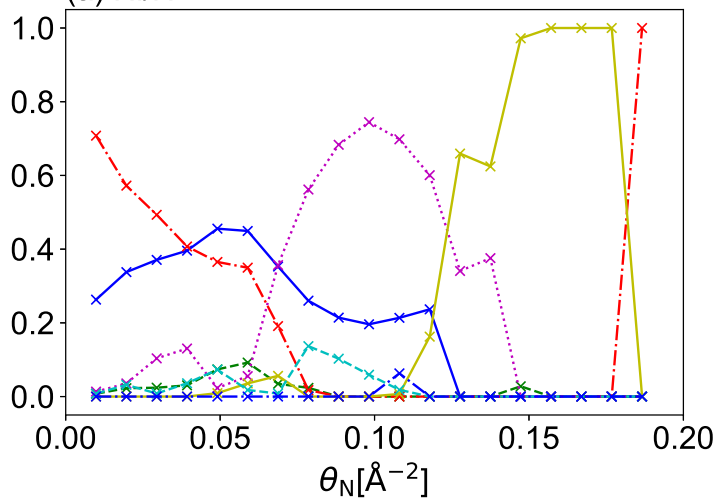

(c) $\mathrm{Ni} / \mathrm{O}$

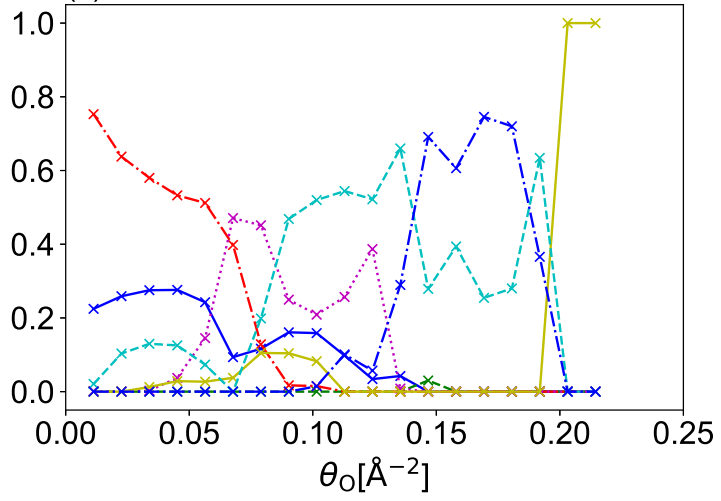

(b) $\mathrm{Cu} / \mathrm{N}$

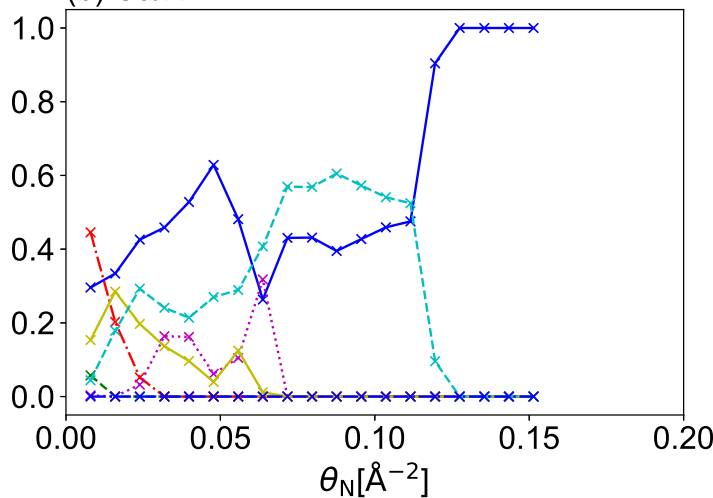

(d) $\mathrm{Cu} / \mathrm{O}$

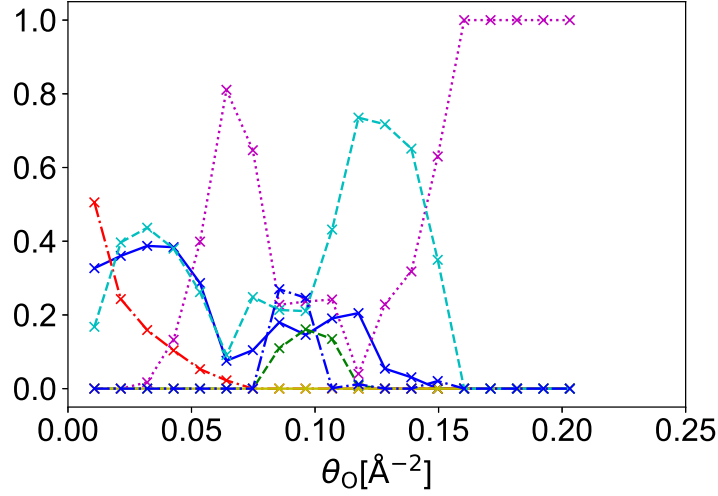

$\begin{array}{rlll}*-*--* & 110 & 111\end{array}$

210

211

$221-* \cdot *-311$

Figure S6: Fractions of terminations on the $\mathrm{Ni} / \mathrm{N}, \mathrm{Cu} / \mathrm{N}, \mathrm{Ni} / \mathrm{O}$ and $\mathrm{Cu} / \mathrm{O}$ systems as functions of coverage, determined by Wulff construction. 


\section{Trajectories resulting from the configuration search}

The relaxed structures at each step in the configuration search with the "greedy algorithm" are shown in Figure S7 - S10. The atomic coordinate files are provided as well. In the images, the green and brown substrate atoms represent $\mathrm{Ni}$ and $\mathrm{Cu}$, respectively. The blue and red modifier atoms represent $\mathrm{N}$ and $\mathrm{O}$ atoms, respectively. 


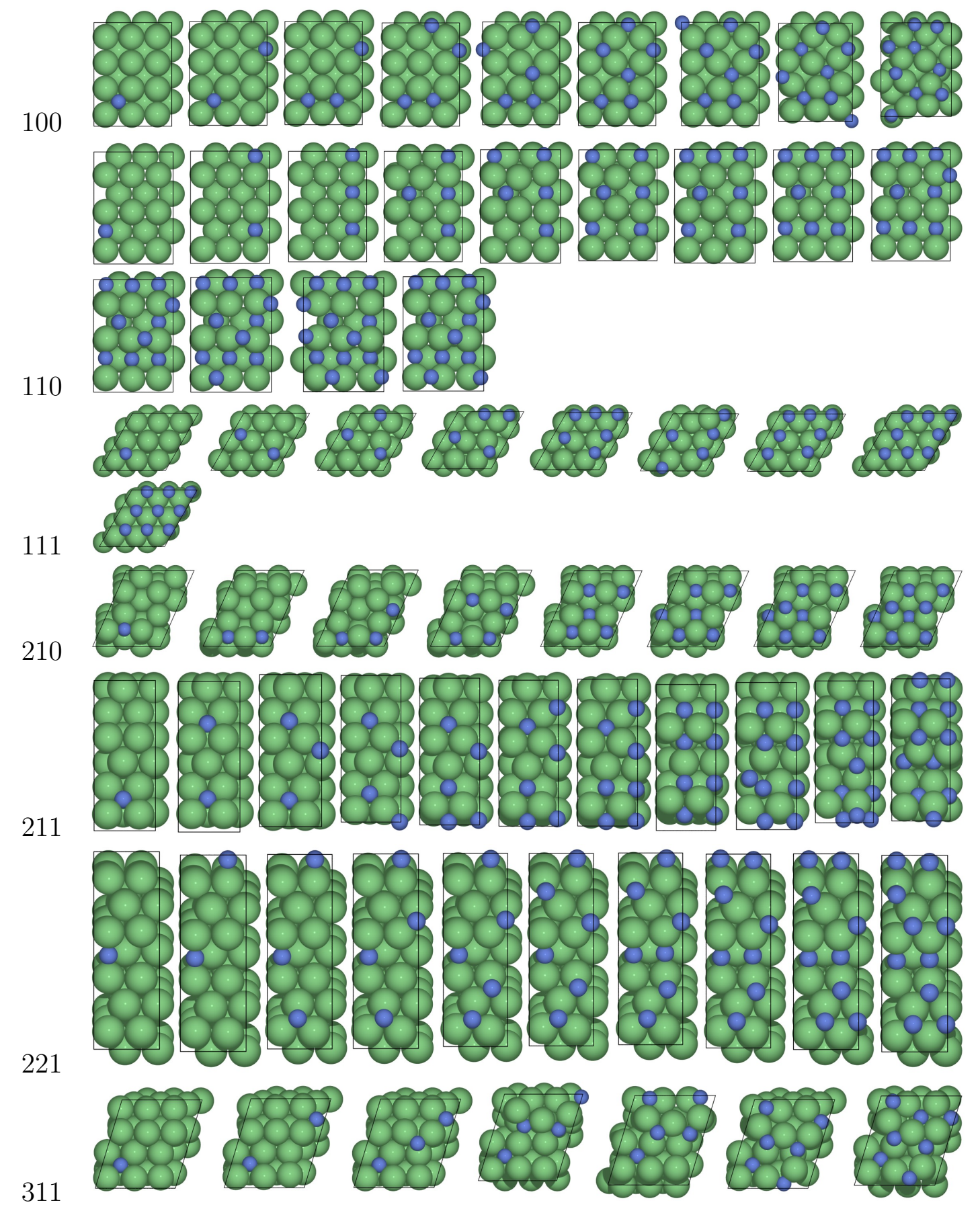

Figure S7: Configuration search iterations for $\mathrm{Ni} / \mathrm{N}$ system 


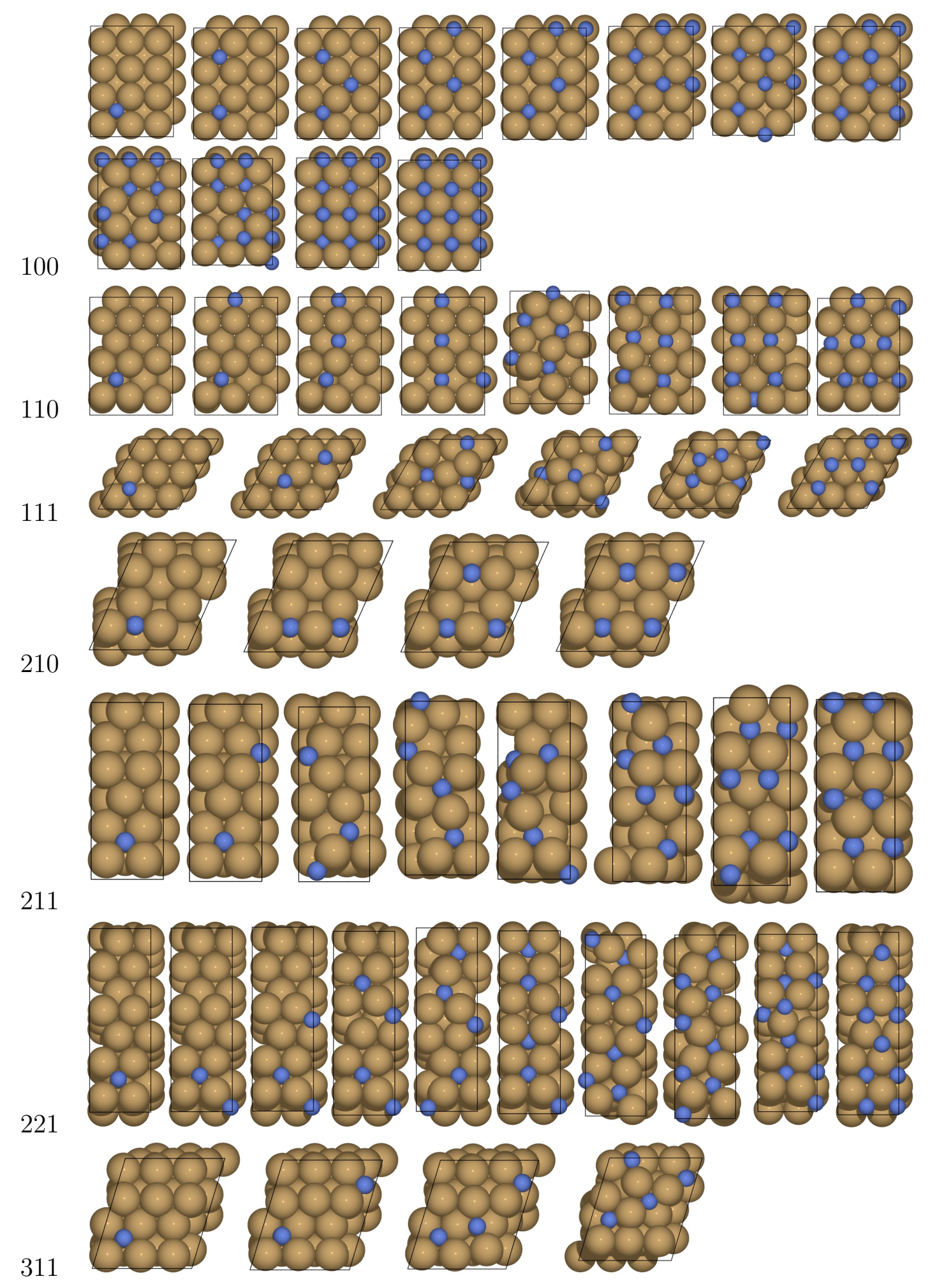

Figure S8: Configuration search iterations for $\mathrm{Cu} / \mathrm{N}$ system 


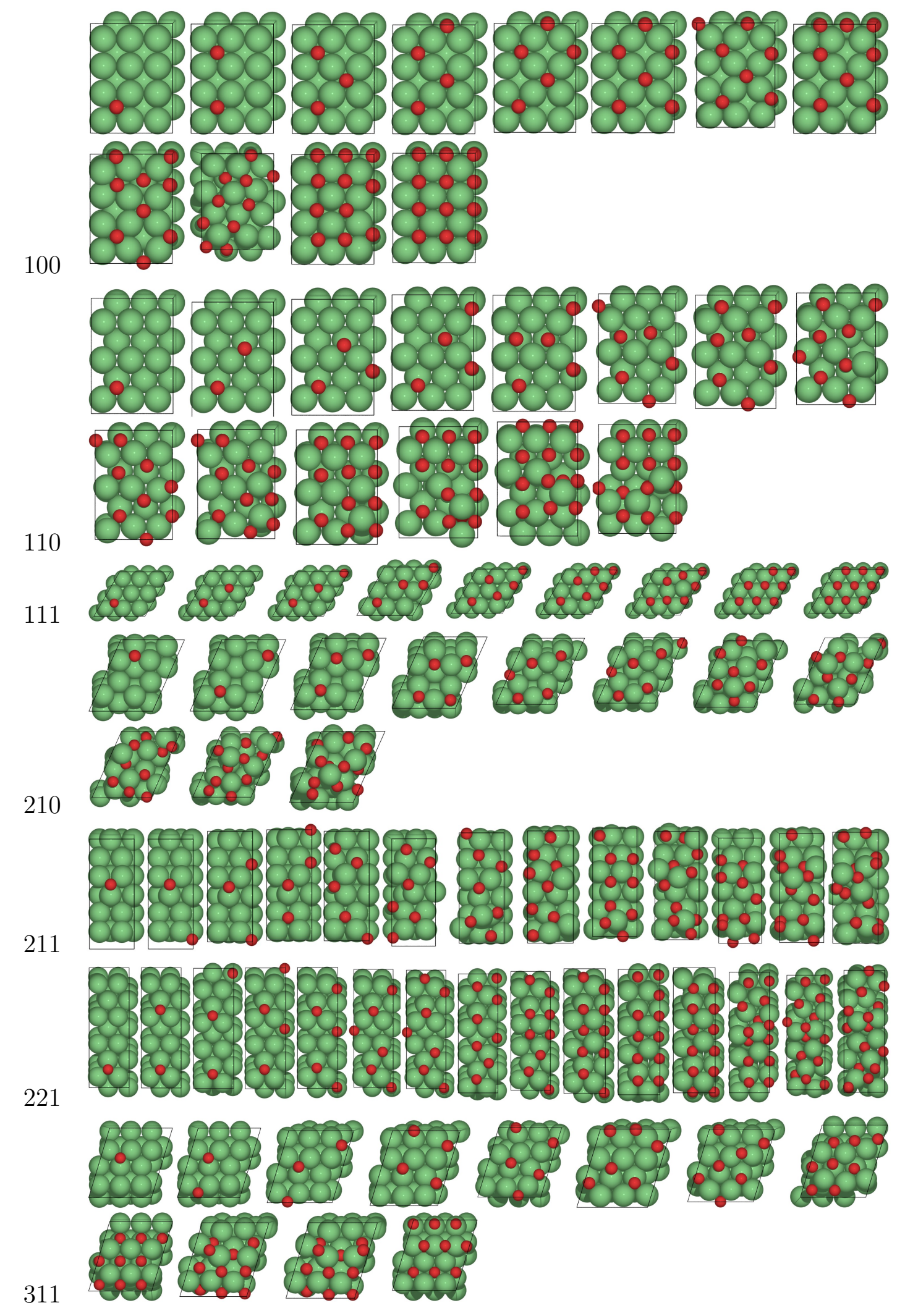

Figure S9: Configuration search iterations for Ni/O system 


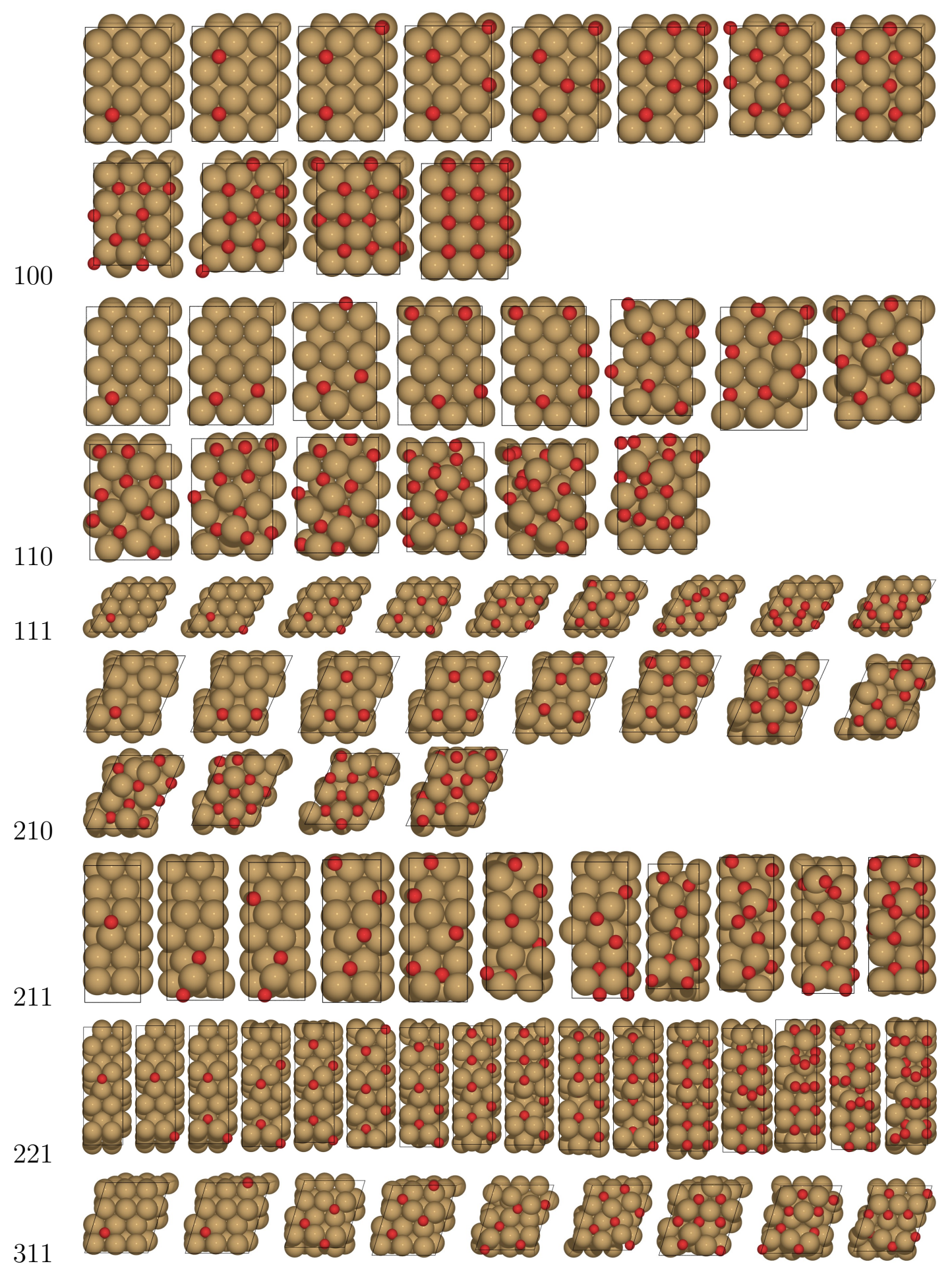

Figure S10: Configuration search iterations for $\mathrm{Cu} / \mathrm{O}$ system 


\section{Main results on $\mathrm{Ni}$ etching using monomer as product}

We noted that the thermodynamically most stable product may not be possible if kinetic barriers are significant. Hence we also provide the figures for the case where the nickel monomers are formed as opposed to the dimer and trimer. Changing the product only affects the absolute scale of the etching energy. Figure S11 reproduces Figure 5 when the nickel monomer complex is produced. Neither oxygen nor nitrogen can lead to a sufficiently activated overlayer adsorption structure that is favorable to etching on any of the terminations investigated.
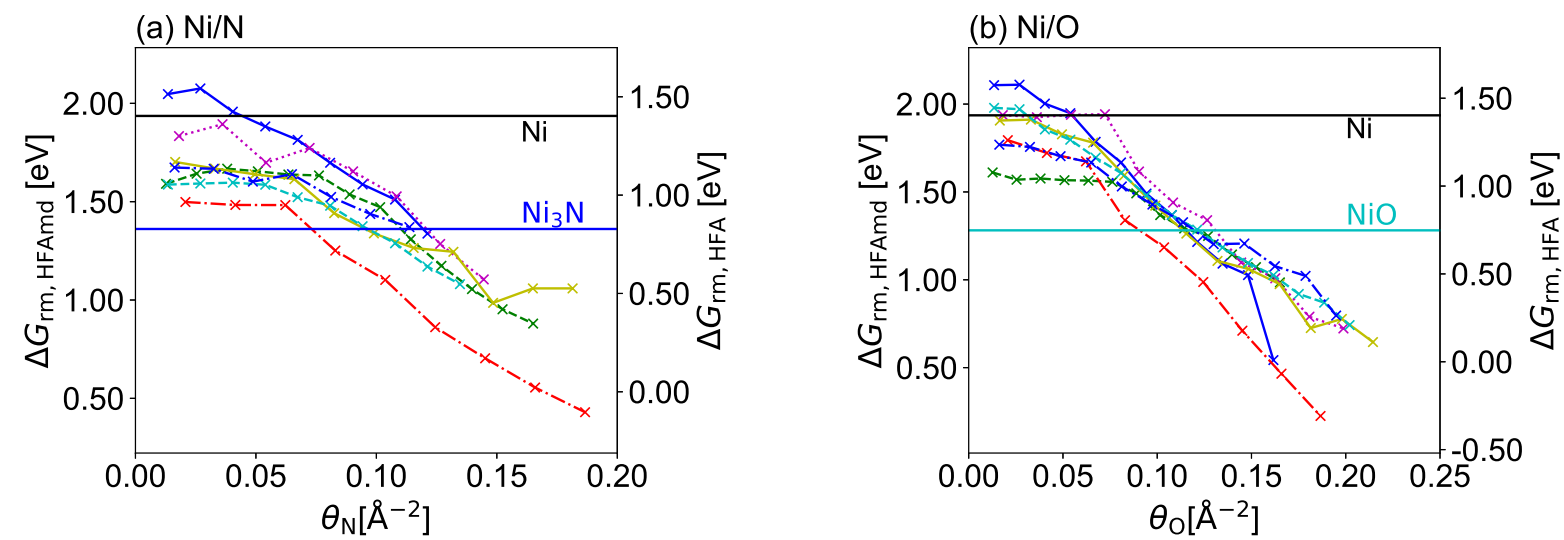

$\longrightarrow$ - $100 \quad--*--110 \quad-* \cdot-111$

210

211

$221-* *-311$

Figure S11: Removal step energies by terminations on the Ni/N, Ni/O systems as functions of coverage using nickel formate and formamidinate monomers as products. Two axis are shown. The y-axis on the left corresponds to formamidine chemistry. The y-axis on the right corresponds to formic acid chemistry. The solid and dashed horizontal lines represents the bulk model results on the pristine and activated substrates, respectively.

Similarly, Figure S12 reproduces Figure 6 with monomer complex as the product. Here, the dramatic effect of surface morphology is highlighted with crosses showing our previous results with metastable surface structures. It can be seen that the occupation of sub-surface sites are required for a favorable etch with the monomer complex as the product. 


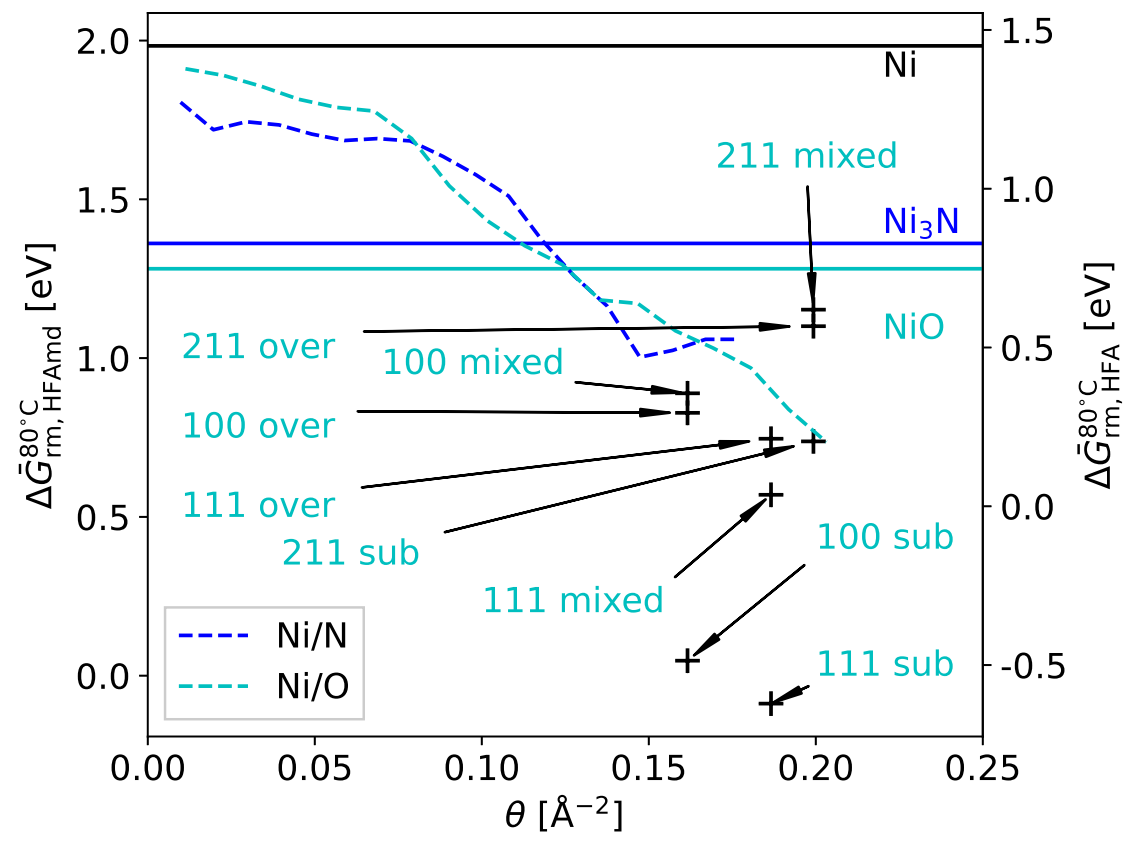

Figure S12: Wulff construction-averaged removal step energies as functions of coverage for nickel system using nickel formate and formamidinate monomers as products. 


\section{References}

(1) Sang, X.; Chang, J. P. Patterning nickel for extreme ultraviolet lithography mask application. II. Hybrid reactive ion etch and atomic layer etch processing. Journal of Vacuum Science 8 Technology A 2020, 38, 042604, Publisher: American Vacuum Society.

(2) Haynes, W. M. , CRC Handbook of Chemistry and Physics, 97th Edition, 97th ed.; CRC Press, 2016; pp 5-11, 5-29, 5-30.

(3) Boyle, B. J.; King, E. G.; Conway, K. C. Heats of Formation of Nickel and Cobalt Oxides ( $\mathrm{NiO}$ and $\mathrm{CoO}$ ) of Combustion Calorimetry. Journal of the American Chemical Society 1954, 76, 3835-3837.

(4) Vempaire, D.; Miraglia, S.; Sulpice, A.; Ortega, L.; Hlil, E.; Fruchart, D.; Pelletier, J. Structure and magnetic properties of nickel nitride thin film synthesized by plasmabased ion implantation. Journal of Magnetism and Magnetic Materials 2004, 272-276, E843 - E844, Proceedings of the International Conference on Magnetism (ICM 2003).

(5) Elder, S. H.; DiSalvo, F. J.; Topor, L.; Navrotsky, A. Thermodynamics of ternary nitride formation by ammonolysis: application to lithium molybdenum nitride (LiMoN2), sodium tungsten nitride (Na3WN3), and sodium tungsten oxide nitride (Na3WO3N). Chemistry of Materials 1993, 5, 1545-1553.

(6) Steinmann, S. N.; Corminboeuf, C. A generalized-gradient approximation exchange hole model for dispersion coefficients. The Journal of Chemical Physics 2011, 134, 044117.

(7) Steinmann, S. N.; Corminboeuf, C. Comprehensive Benchmarking of a DensityDependent Dispersion Correction. Journal of Chemical Theory and Computation 2011, 7, 3567-3577, PMID: 26598255.

(8) Silvestrelli, P. L.; Ambrosetti, A. Cohesive properties of noble metals by van der Waals- 
corrected density functional theory: $\mathrm{Au}, \mathrm{Ag}$, and $\mathrm{Cu}$ as case studies. Physical Review B 2016, 94, 045124, Publisher: American Physical Society.

(9) Baró, A.; Ollé, L. Adsorption site of oxygen chemisorption on Ni(110) at room temperature; an eels study. Surface Science 1983, 126, 170 - 176.

(10) Yagi-Watanabe, K.; Ikeda, Y.; Ishii, Y.; Inokuchi, T.; Fukutani, H. Reaction kinetics and mechanism of oxygen adsorption on the $\mathrm{Ni}(110)$ surface. Surface Science 2001, 482-485, $128-133$.

(11) Narusawa, T.; Gibson, W.; Törnqvist, E. Structure study of oxygen-adsorbed Ni(111) surface by high energy ion scattering. Surface Science 1982, 114, $331-348$.

(12) Duan, X.; Warschkow, O.; Soon, A.; Delley, B.; Stampfl, C. Density functional study of oxygen on $\mathrm{Cu}(100)$ and $\mathrm{Cu}(110)$ surfaces. Phys. Rev. B 2010, 81, 075430.

(13) Soon, A.; Todorova, M.; Delley, B.; Stampfl, C. Oxygen adsorption and stability of surface oxides on $\mathrm{Cu}(111)$ : A first-principles investigation. Phys. Rev. B 2006, 73, 165424 . 
. /all_structures.xyz ./bare/Cu/100/struct.xyz

48

Lattice $=" 7.710267940093008 \quad 0.0 \quad 0.0 \quad 0.0 \quad 10.280357253457344 \quad 0.0 \quad 0.0 \quad 0.021 .451982745204997 " \mathrm{P}$ roperties $=$ species:S:1:pos:R:3 pbc="T T T"

$\begin{array}{llll}\mathrm{Cu} & 1.28504466 & 1.28504466 & 8.00000000 \\ \mathrm{Cu} & 0.00000000 & 0.00000000 & 9.81732758\end{array}$

$\mathrm{Cu}$

$\mathrm{Cu} \quad 1.28504466 \quad 1.28504466$

$\begin{array}{lll}\mathrm{Cu} & 0.00000000 & 0.00000000\end{array}$

$\mathrm{Cu} \quad 1.28504466 \quad 3.85513397$

$\mathrm{Cu} \quad 0.00000000 \quad 2.57008931$

$\begin{array}{lll}\mathrm{Cu} & 1.28504466 & 3.85513397\end{array}$

$\mathrm{Cu} \quad 0.00000000$

3.85513397

1.28504466

6.42522328

0.00000000

5.14017863

1.28504466

5.14017863

0.00000000

5.14017863

1.28504466

8.99531260

0.00000000

8. 71026794

1.28504466

8.99531260

0.00000000

7.71026794

3. 85513397

1.28504466

2. 57008931

0.00000000

3. 85513397

1.28504466

2. 57008931

0.00000000

3. 85513397

3.85513397

2. 57008931

2. 57008931

3. 85513397

3. 85513397

2. 57008931

2. 57008931

3.85513397

6.42522328

2. 57008931

5.14017863

3. 85513397

6.42522328

2.57008931

5.14017863

3. 85513397

8.99531260

2. 57008931

7.71026794

3. 85513397

8.99531260

2. 57008931

7.71026794

6.42522328

1.28504466

5.14017863

0.00000000

6.42522328

1.28504466

5.14017863

0.00000000

6.42522328

3.85513397

2.57008931

5.14017863

3.85513397

2.57008931

5. 14017863

6.42522328

6.42522328

5.14017863

5.14017863

6.42522328

5.14017863

6.42522328

5.14017863

6.42522328

8.99531260

7.71026794

5.14017863

6.42522328

8.99531260

11.62798237

13.39654798

8.00000000

9.81732758

11.62798237

13.39654798

8.00000000

9.81732758

11.62798237

13.39654798

8.00000000

9.81732758

11.62798237

13.39654798

8.00000000

9.81732758

11.62798237

13.39654798

8.00000000

9.81732758

11.62798237

13.39654798

8.00000000

9.81732758

11.62798237

13.39654798

8.00000000

9.81732758

11.62798237

13.39654798

8.00000000

9.81732758

11.62798237

13.39654798

8.00000000

9.81732758

11.62798237

13.39654798

8.00000000

9.81732758

11.62798237

13.39654798

8.00000000

9.81732758

11.62798237

7.71026794

13.39654798

. /bare/Cu/110/struct. xyz

45

Lattice $=" 7.710267940093007 \quad 0.0 \quad 0.0 \quad 0.0 \quad 10.90396549041$ ties=species:S:1:pos:R:3 pbc="T T T"

0.00000000

$\mathrm{Cu} \quad 1.28504466 \quad 1.81732758$

$\mathrm{Cu} \quad 0.00000000 \quad 0.00000000$

$\mathrm{Cu} \quad 1.28504466 \quad 1.81732758$

$\mathrm{Cu} \quad 0.00000000 \quad 0.00000000$

$\mathrm{Cu} \quad 0.00000000 \quad 3.63465516$

$\mathrm{Cu} \quad 1.28504466 \quad 5.45198275$

$\mathrm{Cu} \quad 0.00000000 \quad 3.63465516$

$\mathrm{Cu} \quad 1.28504466 \quad 5.45198275$

$\mathrm{Cu} \quad 0.00000000 \quad 3.63465516$

$\mathrm{Cu} \quad 0.00000000$

3.63465516
7.26931033

8.00000000

9.28504466

10.57008931

11.90348877

13.06034034

8.00000000

9.28504466

10.57008931

11.90348877

13.06034034

8.00000000 


\section{$\mathrm{Cu}$}

$\mathrm{Cu}$

$\mathrm{Cu}$

$\mathrm{Cu}$

$\mathrm{Cu}$

$\mathrm{Cu}$

$\mathrm{Cu}$

$\mathrm{Cu}$

$\mathrm{Cu}$

$\mathrm{Cu}$

$\mathrm{Cu}$

$\mathrm{Cu}$

$\mathrm{Cu}$

$\mathrm{Cu}$

$\mathrm{Cu}$

$\mathrm{Cu}$

$\mathrm{Cu}$

$\mathrm{Cu}$

$\mathrm{Cu}$

$\mathrm{Cu}$

$\mathrm{Cu}$

$\mathrm{Cu}$

$\mathrm{Cu}$

$\mathrm{Cu}$

$\mathrm{Cu}$

$\mathrm{Cu}$

$\mathrm{Cu}$

$\mathrm{Cu}$

$\mathrm{Cu}$

$\mathrm{Cu}$

$\mathrm{Cu}$

$\mathrm{Cu}$

$\mathrm{Cu}$

$\mathrm{Cu}$

. 1

36

Lattice $=" 7.710267940093008$

$\mathrm{Cu}$

$\mathrm{Cu}$

$\mathrm{Cu}$

$\mathrm{Cu}$

$\mathrm{Cu}$

$\mathrm{Cu}$

$\mathrm{Cu}$

$\mathrm{Cu}$

$\mathrm{Cu}$

$\mathrm{Cu}$

$\mathrm{Cu}$

$\mathrm{Cu}$

$\mathrm{Cu}$

$\mathrm{Cu}$

$\mathrm{Cu}$

$\mathrm{Cu}$

$\mathrm{Cu}$

$\mathrm{Cu}$

$\mathrm{Cu}$

$\mathrm{Cu}$

$\mathrm{Cu}$

$\mathrm{Cu}$

$\mathrm{Cu}$

$\mathrm{Cu}$

$\mathrm{Cu}$

$\mathrm{Cu}$

$\mathrm{Cu}$

$\mathrm{Cu}$

$\mathrm{Cu}$

$\mathrm{Cu}$
1.28504466

0.00000000

1.28504466

0.00000000

2. 57008931

3. 85513397

2. 57008931

3. 85513397

2.57008931

2. 57008931

3. 85513397

2. 57008931

3. 85513397

2. 57008931

2. 57008931

3. 85513397

2. 57008931

3. 85513397

2. 57008931

5.14017863

6.42522328

5.14017863

6.42522328

5.14017863

5.14017863

6.42522328

5.14017863

6.42522328

5.14017863

5.14017863

6.42522328

5.14017863

6.42522328

5.14017863
0.00000000

1. 28504466

2.57008931

1. 28504466
Fri Apr 16 14:51:55 2021

9.08663791

7.26931033

9.08663791

7.26931033

0.00000000

1.81732758

0.00000000

1.81732758

0.00000000

3.63465516

5.45198275

3.63465516

5.45198275

3.63465516

7.26931033

9.08663791

7.26931033

9.08663791

7.26931033

0.00000000

1.81732758

0.00000000

1.81732758

0.00000000

3.63465516

5.45198275

3.63465516

5.45198275

3.63465516

7.26931033

9.08663791

7.26931033

9.08663791

7.26931033
9.28504466

10.57008931

11.90348877

13.06034034

8.00000000

9.28504466

10.57008931

11.90348877

13.06034034

8.00000000

9.28504466

10.57008931

11.90348877

13.06034034

8.00000000

9.28504466

10.57008931

11.90348877

13.06034034

8.00000000

9.28504466

10.57008931

11.90348877

13.06034034

8.00000000

9.28504466

10.57008931

11.90348877

13.06034034

8.00000000

9.28504466

10.57008931

11.90348877

13.06034034

$\begin{array}{llll}0.0 & 0.0 & 3.855133970046504\end{array}$

$6.67728790610526 \quad 0.0 \quad 0.0 \quad 0.0 \quad 22.295407$

$\mathrm{pbc}=" \mathrm{~T} \mathrm{~T} \mathrm{~T} "$

$0.00000000 \quad 8.00000000$

$0.74192088 \quad 10.09846914$

1.48384176

12.19345823

0.00000000

2.22576264

2.96768351

14.27510847

8.00000000

10.09846914

12.19345823

14.27510847

8.00000000

10.09846914

12.19345823

14.27510847

8.00000000

10.09846914

12.19345823

14.27510847

8.00000000

10.09846914

12.19345823

14.27510847

8.00000000

10.09846914

12.19345823

14.27510847

8.00000000

10.09846914

12.19345823

14.27510847

8.00000000

10.09846914 


\section{$\mathrm{Cu}$}

$\mathrm{Cu}$

$\mathrm{Cu}$

$\mathrm{Cu}$

$\mathrm{Cu}$

$\mathrm{Cu}$

$$
\begin{array}{r}
8.99531260 \\
6.42522328 \\
7.71026794 \\
8.99531260 \\
10.28035725 \\
7.71026794
\end{array}
$$

. $/$

32
Lattice $=" 7.26931032694000$
12.19345823

14.27510847

8.00000000

10.09846914

12.19345823

14.27510847

$35214202672 "$ Properties=species:S:1:pos:R:3 pbc="T T T"

$\mathrm{Cu} \quad 3.63465516 \quad 1.62546720$

$\begin{array}{lll}\mathrm{Cu} & 1.81732758 & 0.00000000\end{array}$

$\mathrm{Cu} \quad 1.81732758 \quad 2.43820081$

$\mathrm{Cu} \quad 3.63465516 \quad 0.82657142$

$\mathrm{Cu} \quad 3.63465516 \quad 3.24331062$

$\mathrm{Cu} \quad 1.81732758 \quad 1.63026287$

$\mathrm{Cu} \quad-0.00000000 \quad 0.01325132$

$\begin{array}{lll}\mathrm{Cu} & 3.63465516 & 7.31460242\end{array}$

$\mathrm{Cu} \quad 5.45198275$

$\mathrm{Cu} \quad 3.63465516$

5.68913521

6.50186882

5. 45198275
5.45198275

4.89023943

5.45198275

7.30697863

3.63465516

5.69393088

1.81732758

4.07691933

5.45198275

3.25093441

7.26931033

1.62546720

5.45198275

0.00000000

5.45198275

2.43820081

7.26931033

0.82657142

7.26931033

3.24331062

5.45198275

1. 63026287

3.63465516

0.01325132

7. 26931033

7.31460242

9.08663791

5.68913521

7.26931033

4.06366801

7.26931033

6.50186882

9.08663791

4.89023943

9.08663791

7.30697863

7. 26931033

5.69393088

5.45198275

4.07691933
8.00000000
8.81273360
9.62546720
10.43820081
11.25126707
12.10398449
12.88219960
13.56961510
8.00000000
8.81273360
9.62546720
10.43820081
11.25126707
12.10398449
12.88219960
13.56961510
8.00000000
8.81273360
9.62546720
10.43820081
11.25126707
12.10398449
12.88219960
13.56961510
8.00000000
8.81273360
9.62546720
10.43820081
11.25126707
12.10398449
12.88219960
13.56961510

. /bare/Cu/211/struct. xyz

32

Lattice $=" 5.140178626728673 \quad 0.0 \quad 0.0 \quad 0.0 \quad 12.590814822245207 \quad 0.0 \quad 0.0 \quad 0.021 .19344614919298 "$ Pr operties=species:S:1:pos:R:3 pbc="T T T"

Cis

$\mathrm{Cu}$

$\mathrm{Cu}$

$\mathrm{Cu}$

$\mathrm{Cu}$

$\mathrm{Cu}$

$\mathrm{Cu}$

$\mathrm{Cu}$

$\mathrm{Cu}$

$\mathrm{Cu}$

$\mathrm{Cu}$

$\mathrm{Cu}$

$\mathrm{Cu}$

$\mathrm{Cu}$

$\mathrm{Cu}$

$\mathrm{Cu}$

$\mathrm{Cu}$

$\mathrm{Cu}$

$\mathrm{Cu}$

$\mathrm{Cu}$

$\mathrm{Cu}$

$\mathrm{Cu}$
1.28504466

0.00000000

1. 28504466

0.00000000

1.28504466

0.00000000

1.28504466

0.00000000

1.28504466

0.00000000

1.28504466

0.00000000

1.28504466

0.00000000

1.28504466

0.00000000

3.85513397

2. 57008931

3. 85513397

2. 57008931

3. 85513397

2. 57008931
4.19693827

0.00000000

2.09846914

4.19693827

0.06083140

2.14558369

4.22508384

$-0.01509055$

10.49234569

6.29540741

8.39387655

10.49234569

6.35623881

8.44099110

10.52049125

6.28031686

4. 19693827

0.00000000

2.09846914

4.19693827

0.06083140

2.14558369
8.00000000

8.74192088

9.48384176

10.22576264

10.94225268

11.78936613

12.43009883

13.06621861

8.00000000

8.74192088

9.48384176

10.22576264

10.94225268

11.78936613

12.43009883

13.06621861

8.00000000

8.74192088

9.48384176

10.22576264

10.94225268

11.78936613

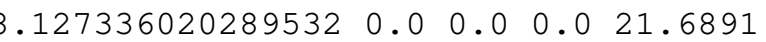
T"

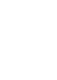

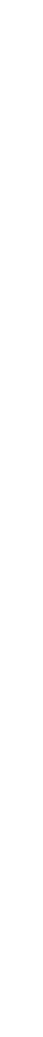




\section{$\mathrm{Cu}$}

$\mathrm{Cu}$

$\mathrm{Cu}$

$\mathrm{Cu}$

$\mathrm{Cu}$

$\mathrm{Cu}$

$\mathrm{Cu}$

$\mathrm{Cu}$

$\mathrm{Cu}$

$\mathrm{Cu}$
3. 85513397

2. 57008931

3.85513397

2. 57008931

3. 85513397

2. 57008931

3.85513397

2.57008931

3.85513397

2.57008931
Fri Apr 16 14:51:55 2021

4.22508384

$-0.01509055$

10.49234569

6.29540741

8.39387655

10.49234569

6.35623881

8.44099110

10.52049125

6.28031686
12.43009883

13.06621861

8.00000000

8.74192088

9.48384176

10.22576264

10.94225268

11.78936613

12.43009883

13.06621861

40

Lattice $=" 5.140178626728671 \quad 0.0 \quad 0.0 \quad 0.0 \quad 15.42053588018602 \quad 0.0 \quad 0.0 \quad 0.021 .451982745205 "$ Prope rties=species:S:1:pos:R:3 pbc="T T T"

$1.28504466 \quad 3.85513397$

1.28504466

$\mathrm{Cu} \quad 0.00000000$

1.28504466

3.85513397
5.99687506

0.42834822

0.00000000

2.57008931

1.28504466

0.00000000

4.71183041

1.28504466

6.88407719

0.00000000

1. 28504466

0.00000000

1.28504466

0.00000000

1.28504466

0.00000000

1. 28504466

0.00000000

1.28504466

0.00000000

1.28504466

0.00000000

3. 85513397

1. 37131568

3. 46951214

5.59227435

$-0.01861891$

11.56540191

13.70714300

8.13861616

10.28035725

12.42209835

14.59434514

9. 08158362

11.17978008

13.30254229

7.69164903

3.85513397

5.99687506

0.42834822

2. 57008931

4.71183041

6.88407719

1.37131568

3. 46951214

5.59227435

$-0.01861891$

11.56540191

13.70714300

8.13861616

10.28035725

12.42209835

14.59434514

9.08158362

11.17978008

13.30254229

7.69164903

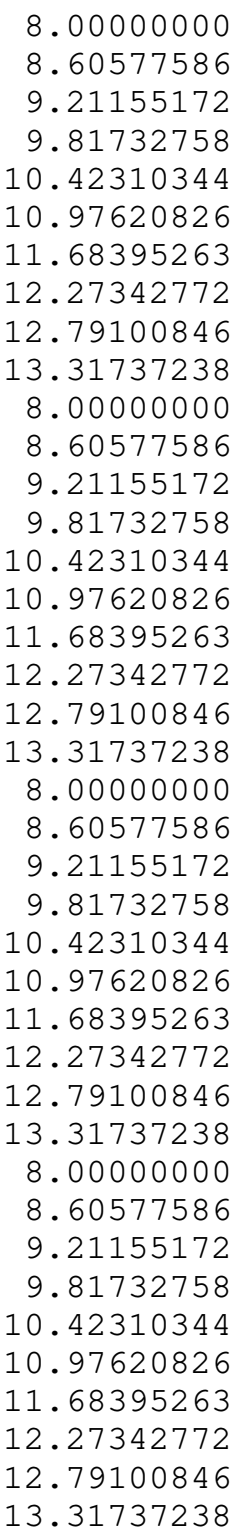

3.85513397

2. 57008931

. /bare/Cu/311/struct. xyz

36

Lattice $=" 7.71026794009301 \quad 0.0 \quad 0.0 \quad 2.5700893133643348 \quad 8.524021930131648 \quad 0.0 \quad 0.0 \quad 0.021 .47944$ $8827071757 "$ Properties=species:S:1:pos:R:3 pbc="T T T"

$\mathrm{Cu}$

.57008931

3.09964434

0.77491108

2.71218880

1. 28504466

0.40645163

1.28681939

2. 32782952

2. 57214039

0.00034527

3. 85513397

$-0.00436813$

7.36165530

5.03692205

6.97419976

2. 57008931

4.66846259

9.09588977

10.19177953

11.29310094

12.41944128

13.38087545

8.00000000

9.09588977

10.19177953

11.29310094 
$\mathrm{Cu}$

$\mathrm{Cu}$

$\mathrm{Cu}$

$\mathrm{Cu}$

$\mathrm{Cu}$

$\mathrm{Cu}$

$\mathrm{Cu}$

$\mathrm{Cu}$

$\mathrm{Cu}$

$\mathrm{Cu}$

$\mathrm{Cu}$

$\mathrm{Cu}$

$\mathrm{Cu}$

$\mathrm{Cu}$

$\mathrm{Cu}$

$\mathrm{Cu}$

$\mathrm{Cu}$

$\mathrm{Cu}$

$\mathrm{Cu}$

$\mathrm{Cu}$

$\mathrm{Cu}$

$\mathrm{Cu}$

$\mathrm{Cu}$

$\mathrm{Cu}$

$\mathrm{Cu}$

$\mathrm{Cu}$

.$/ 8$ 48
6.58984048

4.25764284

3.09964434

0.77491108

2.71218880

0.40645163

2.32782952

$-0.00436813$

7.36165530

5.03692205

6.97419976

4.66846259

6.58984048

4.25764284

3.09964434

0.77491108

2.71218880

0.40645163

2.32782952

$-0.00436813$

7.36165530

5.03692205

6.97419976

4.66846259

6.58984048

4.25764284
12.41944128

13.38087545

8.00000000

9.09588977

10.19177953

11.29310094

12.41944128

13.38087545

8.00000000

9.09588977

10.19177953

11.29310094

12.41944128

13.38087545

8.00000000

9.09588977

10.19177953

11.29310094

12.41944128

13.38087545

8.00000000

9.09588977

10.19177953

11.29310094

12.41944128

13.38087545

Lattice $=" 7.462281943147142 \quad 0.0 \quad 0.0 \quad 0.0 \quad 9.9497092575295230 .00 .0 \quad 0.021 .27663016512527 "$ Pro perties=species:S:1:pos:R:3 pbc="T T T"

$\begin{array}{lllr}\mathrm{Ni} & 1.24371366 & 1.24371366 & 8.00000000 \\ \mathrm{Ni} & 0.00000000 & 0.00000000 & 9.75887672 \\ \mathrm{Ni} & 1.24371366 & 1.24371366 & 11.53677529 \\ \mathrm{Ni} & 0.0000000 & 0.0000000 & 13.22964925 \\ \mathrm{Ni} & 1.24371366 & 3.73114097 & 8.0000000 \\ \mathrm{Ni} & 0.0000000 & 2.48742731 & 9.75887672 \\ \mathrm{Ni} & 1.24371366 & 3.73114097 & 11.53677529 \\ \mathrm{Ni} & 0.00000000 & 2.48742731 & 13.22964925 \\ \mathrm{Ni} & 1.24371366 & 6.21856829 & 8.00000000 \\ \mathrm{Ni} & 0.00000000 & 4.97485463 & 9.75887672 \\ \mathrm{Ni} & 1.24371366 & 6.21856829 & 11.53677529 \\ \mathrm{Ni} & 0.0000000 & 4.97485463 & 13.22964925 \\ \mathrm{Ni} & 1.24371366 & 8.70599560 & 8.0000000 \\ \mathrm{Ni} & 0.0000000 & 7.46228194 & 9.75887672 \\ \mathrm{Ni} & 1.24371366 & 8.70599560 & 11.53677529 \\ \mathrm{Ni} & 0.00000000 & 7.46228194 & 13.22964925 \\ \mathrm{Ni} & 3.73114097 & 1.24371366 & 8.00000000 \\ \mathrm{Ni} & 2.48742731 & 0.00000000 & 9.75887672 \\ \mathrm{Ni} & 3.73114097 & 1.24371366 & 11.53677529 \\ \mathrm{Ni} & 2.48742731 & 0.0000000 & 13.22964925 \\ \mathrm{Ni} & 3.73114097 & 3.73114097 & 8.0000000 \\ \mathrm{Ni} & 2.48742731 & 2.48742731 & 9.75887672 \\ \mathrm{Ni} & 3.73114097 & 3.73114097 & 11.53677529 \\ \mathrm{Ni} & 2.48742731 & 2.48742731 & 13.22964925 \\ \mathrm{Ni} & 3.73114097 & 6.21856829 & 8.00000000 \\ \mathrm{Ni} & 2.48742731 & 4.97485463 & 9.75887672 \\ \mathrm{Ni} & 3.73114097 & 6.21856829 & 11.53677529 \\ \mathrm{Ni} & 2.48742731 & 4.97485463 & 13.22964925 \\ \mathrm{Ni} & 3.73114097 & 8.70599560 & 8.0000000 \\ \mathrm{Ni} & 2.48742731 & 7.46228194 & 9.75887672 \\ \mathrm{Ni} & 3.73114097 & 8.70599560 & 11.53677529 \\ \mathrm{Ni} & 2.48742731 & 7.46228194 & 13.22964925 \\ \mathrm{Ni} & 6.21856829 & 1.24371366 & 8.00000000 \\ \mathrm{Ni} & 4.97485463 & 0.00000000 & 9.75887672 \\ \mathrm{Ni} & 6.21856829 & 1.24371366 & 11.53677529 \\ \mathrm{Ni} & 4.97485463 & 0.0000000 & 13.22964925 \\ \mathrm{Ni} & 6.21856829 & 3.73114097 & 8.00000000 \\ \mathrm{Ni} & 4.97485463 & 2.48742731 & 9.75887672 \\ & & & \end{array}$


$\mathrm{Ni}$

$\mathrm{Ni}$

$\mathrm{Ni}$

$\mathrm{Ni}$

$\mathrm{Ni}$

$\mathrm{Ni}$

$\mathrm{Ni}$

$\mathrm{Ni}$

$\mathrm{Ni}$

$\mathrm{Ni}$

.$/ \mathrm{b}$

6.21856829

4.97485463

6.21856829

4.97485463

6.21856829

4.97485463

6.21856829

4.97485463

6.21856829

4.97485463

45

/Ni/110/struct.xyz

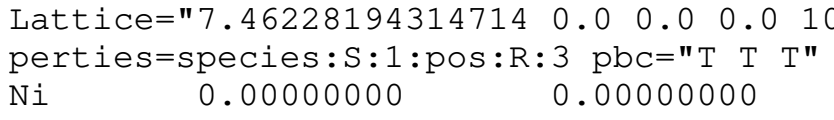

$\mathrm{Ni}$

$\mathrm{Ni}$

$\mathrm{Ni}$

$\mathrm{Ni}$

$\mathrm{Ni}$

$\mathrm{Ni}$

$\mathrm{Ni}$

$\mathrm{Ni}$

$\mathrm{Ni}$

$\mathrm{Ni}$

$\mathrm{Ni}$

$\mathrm{Ni}$

$\mathrm{Ni}$

$\mathrm{Ni}$

$\mathrm{Ni}$

$\mathrm{Ni}$

$\mathrm{Ni}$

$\mathrm{Ni}$

$\mathrm{Ni}$

$\mathrm{Ni}$

$\mathrm{Ni}$

$\mathrm{Ni}$

$\mathrm{Ni}$

$\mathrm{Ni}$

$\mathrm{Ni}$

$\mathrm{Ni}$

$\mathrm{Ni}$

$\mathrm{Ni}$

$\mathrm{Ni}$

$\mathrm{Ni}$

$\mathrm{Ni}$

$\mathrm{Ni}$

$\mathrm{Ni}$

$\mathrm{Ni}$

$\mathrm{Ni}$

$\mathrm{Ni}$

$\mathrm{Ni}$

$\mathrm{Ni}$

$\mathrm{Ni}$

$\mathrm{Ni}$

$\mathrm{Ni}$

$\mathrm{Ni}$

$\mathrm{Ni}$

$\mathrm{Ni}$

$\mathrm{Ni}$

36

0.00000000

1.24371366

0.00000000

1.24371366

0.00000000

0.00000000

1.24371366

0.00000000

1.24371366

0.00000000

0.00000000

1.24371366

0.00000000

1.24371366

0.00000000

2. 48742731

3.73114097

2. 48742731

3. 73114097

2. 48742731

2. 48742731

3. 73114097

2. 48742731

3. 73114097

2. 48742731

2. 48742731

3. 73114097

2. 48742731

3. 73114097

2. 48742731

4.97485463

6.21856829

4.97485463

6.21856829

4.97485463

4.97485463

6.21856829

4.97485463

6.21856829

4.97485463

4.97485463

6.21856829

4.97485463

6.21856829

4.97485463

1. 75887672

0.00000000

1. 75887672

0.00000000

3.51775344

5.27663017

3.51775344

5.27663017

3.51775344

7.03550689

8.79438361

7.03550689

8.79438361

7.03550689

0.00000000

1.75887672

0.00000000

1.75887672

0.00000000

3.51775344

5.27663017

3. 51775344

5.27663017

3.51775344

7.03550689

8.79438361

7.03550689

8.79438361

7.03550689

0.00000000

1.75887672

0.00000000

1.75887672

0.00000000

3.51775344

5.27663017

3.51775344

5.27663017

3. 51775344

7.03550689

8.79438361

7.03550689

8.79438361

7.03550689
11.53677529

13.22964925

8.00000000

9.75887672

11.53677529

13.22964925

8.00000000

9.75887672

11.53677529

13.22964925

\section{6}

$.5532603302505410 .0 \quad 0.0 \quad 0.020 .97485462876476 "$ Pro

8.00000000

10.48742731

11.78463603

12.87754996

8.00000000

9.24371366

10.48742731

11.78463603

12.87754996

8.00000000

9.24371366

10.48742731

11.78463603

12.87754996

8.00000000

9.24371366

10.48742731

11.78463603

12.87754996

8.00000000

9.24371366

10.48742731

11.78463603

12.87754996

8.00000000

9.24371366

10.48742731

11.78463603

12.87754996

8.00000000

9.24371366

10.48742731

11.78463603

12.87754996

8.00000000

9.24371366

10.48742731

11.78463603

12.87754996

8.00000000

9.24371366

10.48742731

11.78463603

12.87754996

Lattice $=" 7.4622819431471425 \quad 0.0 \quad 0.0 \quad 3.731140971573571 \quad 6.462525732967329 \quad 0.0 \quad 0.0 \quad 0.022 .0929$ $2769249835 "$ Properties=species:S:1:pos:R:3 pbc="T T T"

$\mathrm{Ni} \quad 0.00000000 \quad 0.00000000 \quad 8.00000000$

$\mathrm{Ni} \quad 1.24371366 \quad 0.71805841 \quad 10.03097590$

$\mathrm{Ni} \quad 2.48742731 \quad 1.43611683 \quad 12.04995298$

$\mathrm{Ni} \quad 0.00000000 \quad 0.00000000$

$\mathrm{Ni}$

1.24371366

2.15417524

14.04972536

8.00000000 
$\mathrm{Ni}$

$\mathrm{Ni}$

2.48742731

3.73114097

1.24371366

2.48742731

3.73114097

4.97485463

2.48742731

2.48742731

3.73114097

4.97485463

2.48742731

3.73114097

4.97485463

6.21856829

3.73114097

4.97485463

6.21856829

7.46228194

4.97485463

4.97485463

6.21856829

7.46228194

4.97485463

6.21856829

7.46228194

8.70599560

6.21856829

7.46228194

8.70599560

$\begin{array}{ll}\mathrm{Ni} & 9.94970926 \\ \mathrm{Ni} & 7.46228194\end{array}$

$\begin{array}{ll}\mathrm{Ni} & 9.94970926 \\ \mathrm{Ni} & 7.46228194\end{array}$

. /bare/Ni/210/struct. xyz

32

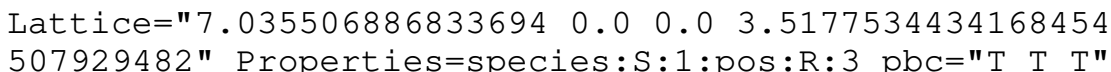

$\mathrm{Ni}$

$\mathrm{Ni}$

$\mathrm{Ni}$

$\mathrm{Ni}$

$\mathrm{Ni}$

$\mathrm{Ni}$

$\mathrm{Ni}$

$\mathrm{Ni}$

$\mathrm{Ni}$

$\mathrm{Ni}$

$\mathrm{Ni}$

$\mathrm{Ni}$

$\mathrm{Ni}$

$\mathrm{Ni}$

$\mathrm{Ni}$

$\mathrm{Ni}$

$\mathrm{Ni}$

$\mathrm{Ni}$

$\mathrm{Ni}$

$\mathrm{Ni}$

$\mathrm{Ni}$

$\mathrm{Ni}$

$\mathrm{Ni}$

$\mathrm{Ni}$

$\mathrm{Ni}$

$\mathrm{Ni}$

$\mathrm{Ni}$

$\mathrm{Ni}$

$\mathrm{Ni}$

$\mathrm{Ni}$

$\mathrm{Ni}$

$\mathrm{Ni}$

./bare/Ni/211/struct.xyz

\section{Fri Apr 16 14:51:55 2021}

\section{7}

3.59029207

2.15417524

5.02640890

5.74446732

4.30835049

0.00000000

0.71805841

1.43611683

0.00000000

2.15417524

2.87223366

3.59029207

2.15417524

4.30835049

5.02640890

5.74446732

4.30835049

0.00000000

0.71805841

1. 43611683

0.00000000

2.15417524

2. 87223366

3. 59029207

2.15417524

4.30835049

5.02640890

5.74446732

4.30835049

10.03097590

12.04995298

14.04972536

8.00000000

12.04995298

14.04972536

8.00000000

10.03097590

12.04995298

14.04972536

8.00000000

10.03097590

12.04995298

14.04972536

8.00000000

12.04995298

14.04972536

8.00000000

10.03097590

12.04995298

14.04972536

8.00000000

10.03097590

12.04995298

14.04972536

8.00000000

10.03097590

12.04995298

14.04972536
2. 87223366

4.30835049

10.03097590

10.03097590
1.75887672
3. 51775344
3.14637433
8.00000000
1.75887672
8.78659358
1. 57318717
9.57318717
1.75887672
0.00000000
2.35978075
10.35978075
3.51775344
0.78065983
11.14210658
3. 51775344
3.12139247
11.97682061
1. 75887672
1.56912209
0.01261651
3.51775344
12.69037217
13.38820109
7.07934224
5. 27663017
5.50615508
3.93296791
3. 51775344
6.29274866
5. 27663017
5.27663017
4.71362774
7.05436039
3. 51775344
5.50209000
3.94558443
3.14637433
5.27663017
7. 03550689
5.27663017
5.27663017
7. 03550689
7.03550689
5.27663017
3. 51775344
7.03550689
8.79438361
7.03550689
7.03550689
8.79438361
8.79438361
7.03550689
1. 57318717
0.00000000
2.35978075
0.78065983
3.12139247
1. 56912209
0.01261651
7.07934224
5.50615508
3.93296791
6.29274866
4.71362774
7.05436039
5.50209000
8.00000000
8.78659358
9.57318717
10.35978075
11.14210658
11.97682061
12.69037217
13.38820109
8.00000000
8.78659358
9.57318717
10.35978075
11.14210658
11.97682061
12.69037217
13.38820109
8.00000000
8.78659358
9.57318717
10.35978075
11.14210658
11.97682061
12.69037217
13.38820109
3.94558443

$\begin{array}{llllll}7.86593582756403 & 0.0 & 0.0 & 0.0 & 21.50615\end{array}$ 
32 Lattice $=" 4.974854628764761 \quad 0.0 \quad 0.0 \quad 0.0 \quad 12.1858553849967 \quad 0.0 \quad 0.0 \quad 0.021 .026408903419032 "$ Pro perties=species:S:1:pos:R:3 pbc="T T T"

$\begin{array}{lrrr}\mathrm{Ni} & 1.24371366 & 4.06195179 & 8.00000000 \\ \mathrm{Ni} & 0.00000000 & 0.00000000 & 8.71805841 \\ \mathrm{Ni} & 1.24371366 & 2.03097590 & 9.43611683 \\ \mathrm{Ni} & 0.00000000 & 4.06195179 & 10.15417524 \\ \mathrm{Ni} & 1.24371366 & 0.04366438 & 10.83715760 \\ \mathrm{Ni} & 0.00000000 & 2.05715392 & 11.62781921 \\ \mathrm{Ni} & 1.24371366 & 4.06953236 & 12.26445268 \\ \mathrm{Ni} & 0.00000000 & -0.04063967 & 12.88389803 \\ \mathrm{Ni} & 1.24371366 & 10.15487949 & 8.00000000 \\ \mathrm{Ni} & 0.00000000 & 6.09292769 & 8.71805841 \\ \mathrm{Ni} & 1.24371366 & 8.12390359 & 9.43611683 \\ \mathrm{Ni} & 0.00000000 & 10.15487949 & 10.15417524 \\ \mathrm{Ni} & 1.24371366 & 6.13659207 & 10.83715760 \\ \mathrm{Ni} & 0.00000000 & 8.15008161 & 11.62781921 \\ \mathrm{Ni} & 1.24371366 & 10.16246006 & 12.26445268 \\ \mathrm{Ni} & 0.00000000 & 6.05228803 & 12.88389803 \\ \mathrm{Ni} & 3.73114097 & 4.06195179 & 8.00000000 \\ \mathrm{Ni} & 2.48742731 & 0.00000000 & 8.71805841 \\ \mathrm{Ni} & 3.73114097 & 2.03097590 & 9.43611683 \\ \mathrm{Ni} & 2.48742731 & 4.06195179 & 10.15417524 \\ \mathrm{Ni} & 3.73114097 & 0.04366438 & 10.83715760 \\ \mathrm{Ni} & 2.48742731 & 2.05715392 & 11.62781921 \\ \mathrm{Ni} & 3.73114097 & 4.06953236 & 12.26445268 \\ \mathrm{Ni} & 2.48742731 & -0.04063967 & 12.88389803 \\ \mathrm{Ni} & 3.73114097 & 10.15487949 & 8.00000000 \\ \mathrm{Ni} & 2.48742731 & 6.09292769 & 8.71805841 \\ \mathrm{Ni} & 3.73114097 & 8.12390359 & 9.43611683 \\ \mathrm{Ni} & 2.48742731 & 10.15487949 & 10.15417524 \\ \mathrm{Ni} & 3.73114097 & 6.13659207 & 10.83715760 \\ \mathrm{Ni} & 2.48742731 & 8.15008161 & 11.62781921 \\ \mathrm{Ni} & 3.73114097 & 10.16246006 & 12.26445268 \\ \mathrm{Ni} & 2.48742731 & 6.05228803 & 12.88389803 \\ \mathrm{~N} & 1 / 221 / 56946 & & \end{array}$

. /bare/Ni/221/struct. xyz

40

Lattice $=" 4.974854628764762 \quad 0.0 \quad 0.0 \quad 0.0 \quad 14.9245638862942820 .0 \quad 0.0 \quad 0.021 .27663016512527 "$ Pr operties=species:S:1:pos:R:3 pbc="T T T"

$\begin{array}{lrrr}\mathrm{Ni} & 1.24371366 & 3.73114097 & 8.00000000 \\ \mathrm{Ni} & 0.00000000 & 1.65828488 & 8.58629224 \\ \mathrm{Ni} & 1.24371366 & 7.04771072 & 9.17258448 \\ \mathrm{Ni} & 0.00000000 & 4.97485463 & 9.75887672 \\ \mathrm{Ni} & 1.24371366 & 2.90199853 & 10.34516896 \\ \mathrm{Ni} & 0.00000000 & 0.78025784 & 10.89832514 \\ \mathrm{Ni} & 1.24371366 & 6.12811874 & 11.53987017 \\ \mathrm{Ni} & 0.00000000 & 4.08841631 & 12.10774236 \\ \mathrm{Ni} & 1.24371366 & 2.03232508 & 12.62942347 \\ \mathrm{Ni} & 0.00000000 & 0.00357981 & 13.14238110 \\ \mathrm{Ni} & 1.24371366 & 11.19342291 & 8.00000000 \\ \mathrm{Ni} & 0.00000000 & 9.12056682 & 8.58629224 \\ \mathrm{Ni} & 1.24371366 & 14.50999267 & 9.17258448 \\ \mathrm{Ni} & 0.00000000 & 12.43713657 & 9.75887672 \\ \mathrm{Ni} & 1.24371366 & 10.36428048 & 10.34516896 \\ \mathrm{Ni} & 0.00000000 & 8.24253978 & 10.89832514 \\ \mathrm{Ni} & 1.24371366 & 13.59040069 & 11.53987017 \\ \mathrm{Ni} & 0.00000000 & 11.55069826 & 12.10774236 \\ \mathrm{Ni} & 1.24371366 & 9.49460702 & 12.62942347 \\ \mathrm{Ni} & 0.00000000 & 7.46586175 & 13.14238110 \\ \mathrm{Ni} & 3.73114097 & 3.73114097 & 8.00000000 \\ \mathrm{Ni} & 2.48742731 & 1.65828488 & 8.58629224 \\ \mathrm{Ni} & 3.73114097 & 7.04771072 & 9.17258448 \\ \mathrm{Ni} & 2.48742731 & 4.97485463 & 9.75887672 \\ \mathrm{Ni} & 3.73114097 & 2.90199853 & 10.34516896 \\ \mathrm{Ni} & 2.48742731 & 0.78025784 & 10.89832514 \\ \mathrm{Ni} & 3.73114097 & 6.12811874 & 11.53987017 \\ \mathrm{Ni} & 2.48742731 & 4.08841631 & 12.10774236 \\ \mathrm{Ni} & 3.73114097 & 2.03232508 & 12.62942347\end{array}$


all_structures.xyz

$\mathrm{Ni}$

$\mathrm{Ni}$

$\mathrm{Ni}$

$\mathrm{Ni}$

$\mathrm{Ni}$

$\mathrm{Ni}$

$\mathrm{Ni}$

$\mathrm{Ni}$

$\mathrm{Ni}$

$\mathrm{Ni}$

$\mathrm{Ni}$

. /bare/Ni/311/struct.xyz

2.48742731

3.73114097

2.48742731

3. 73114097

2. 48742731

3.73114097

2. 48742731

3. 73114097

2. 48742731

3.73114097

2.48742731
Fri Apr 16 14:51:55 2021
0.00357981

11.19342291

9.12056682

14.50999267

12.43713657

10.36428048

8.24253978

13.59040069

11.55069826

9.49460702

7.46586175
13.14238110

8.00000000

8.58629224

9.17258448

9.75887672

10.34516896

10.89832514

11.53987017

12.10774236

12.62942347

13.14238110

Lattice $=" 7.462281943147142 \quad 0.0 \quad 0.0 \quad 2.4874273143823786 \quad 8.24986309508776 \quad 0.0 \quad 0.0 \quad 0.021 .30321$ 2853088358" Properties=species:S:1:pos:R:3 pbc="T T T"

$\begin{array}{lrrr}\mathrm{Ni} & 2.48742731 & 2.99995022 & 8.00000000 \\ \mathrm{Ni} & 2.48742731 & 0.74998755 & 9.06064257 \\ \mathrm{Ni} & 1.24371366 & 2.62495644 & 10.12128514 \\ \mathrm{Ni} & 1.24274907 & 0.39720605 & 11.16773022 \\ \mathrm{Ni} & 2.48703020 & 2.24780712 & 12.27945968 \\ \mathrm{Ni} & -0.00081923 & -0.00759730 & 13.17902073 \\ \mathrm{Ni} & 3.73114097 & 7.12488176 & 8.00000000 \\ \mathrm{Ni} & 3.73114097 & 4.87491910 & 9.06064257 \\ \mathrm{Ni} & 2.48742731 & 6.74988799 & 10.12128514 \\ \mathrm{Ni} & 2.48646273 & 4.52213760 & 11.16773022 \\ \mathrm{Ni} & 3.73074386 & 6.37273867 & 12.27945968 \\ \mathrm{Ni} & 1.24289443 & 4.11733425 & 13.17902073 \\ \mathrm{Ni} & 4.97485463 & 2.99995022 & 8.00000000 \\ \mathrm{Ni} & 4.97485463 & 0.74998755 & 9.06064257 \\ \mathrm{Ni} & 3.73114097 & 2.62495644 & 10.12128514 \\ \mathrm{Ni} & 3.73017638 & 0.39720605 & 11.16773022 \\ \mathrm{Ni} & 4.97445751 & 2.24780712 & 12.27945968 \\ \mathrm{Ni} & 2.48660809 & -0.00759730 & 13.17902073 \\ \mathrm{Ni} & 6.21856829 & 7.12488176 & 8.00000000 \\ \mathrm{Ni} & 6.21856829 & 4.87491910 & 9.06064257 \\ \mathrm{Ni} & 4.97485463 & 6.74988799 & 10.12128514 \\ \mathrm{Ni} & 4.97389004 & 4.52213760 & 11.16773022 \\ \mathrm{Ni} & 6.21817117 & 6.37273867 & 12.27945968 \\ \mathrm{Ni} & 3.73032175 & 4.11733425 & 13.17902073 \\ \mathrm{Ni} & 7.46228194 & 2.99995022 & 8.00000000 \\ \mathrm{Ni} & 7.46228194 & 0.74998755 & 9.06064257 \\ \mathrm{Ni} & 6.21856829 & 2.62495644 & 10.12128514 \\ \mathrm{Ni} & 6.21760370 & 0.39720605 & 11.16773022 \\ \mathrm{Ni} & 7.46188483 & 2.24780712 & 12.27945968 \\ \mathrm{Ni} & 4.97403540 & -0.00759730 & 13.17902073 \\ \mathrm{Ni} & 8.70599560 & 7.12488176 & 8.00000000 \\ \mathrm{Ni} & 8.70599560 & 4.87491910 & 9.06064257 \\ \mathrm{Ni} & 7.46228194 & 6.74988799 & 10.12128514 \\ \mathrm{Ni} & 7.46131735 & 4.52213760 & 11.16773022 \\ \mathrm{Ni} & 8.70559849 & 6.37273867 & 12.27945968 \\ \mathrm{Ni} & 6.21774906 & 4.11733425 & 13.17902073\end{array}$

./nitrogen/Cu/100/iter_1/struct.xyz

49

Lattice $=" 7.710267940093008 \quad 0.0 \quad 0.0 \quad 0.0 \quad 10.2803572534573440 .0 \quad 0.0 \quad 0.021 .451982745204997 " \mathrm{P}$ roperties $=$ species:S:1:pos:R:3 pbc="T T T"

cu

$\mathrm{Cu}$

$\mathrm{Cu}$

$\mathrm{Cu}$

$\mathrm{Cu}$

$\mathrm{Cu}$

$\mathrm{Cu}$

$\mathrm{Cu}$

$\mathrm{Cu}$

$\mathrm{Cu}$

$\mathrm{Cu}$

$\mathrm{Cu}$

$\mathrm{Cu}$
1.28504466

0.00000000

1.28504466

$-0.04337534$

1. 28504466

0.00000000

1. 28504466

$-0.04337534$

1.28504466

0.00000000

1.28504466

$-0.00886070$

1.28504466
1.28504466

0.00000000

1.28504466

$-0.05838098$

3. 85513397

2. 57008931

3.86796825

2. 62847029

6.42522328

5.14017863

6.42522328

5.15495360

8. 99531260
8.00000000

9.81732758

11.63245659

13.48914812

8.00000000

9.81732758

11.63342251

13.48914812

8.00000000

9.81732758

11.62827825

13.39259635

8.00000000 
$\mathrm{Cu}$

$\mathrm{Cu}$

$\mathrm{Cu}$

$\mathrm{Cu}$

$\mathrm{Cu}$

$\mathrm{Cu}$

$\mathrm{Cu}$

$\mathrm{Cu}$

$\mathrm{Cu}$

$\mathrm{Cu}$

$\mathrm{Cu}$

$\mathrm{Cu}$

$\mathrm{Cu}$

$\mathrm{Cu}$

$\mathrm{Cu}$

$\mathrm{Cu}$

$\mathrm{Cu}$

$\mathrm{Cu}$

$\mathrm{Cu}$

$\mathrm{Cu}$

$\mathrm{Cu}$

$\mathrm{Cu}$

$\mathrm{Cu}$

$\mathrm{Cu}$

$\mathrm{Cu}$

$\mathrm{Cu}$

$\mathrm{Cu}$

$\mathrm{Cu}$

$\mathrm{Cu}$

$\mathrm{Cu}$

$\mathrm{Cu}$

$\mathrm{Cu}$

$\mathrm{Cu}$

$\mathrm{Cu}$

$\mathrm{Cu}$

$\mathrm{N}$
0.00000000

1.28504466

$-0.00886070$

3.85513397

2.57008931

3.86049954

2. 61346466

3. 85513397

2.57008931

3.84424406

2.61346466

3.85513397

2.57008931

3.85939750

2.57895001

3.85513397

2. 57008931

3.84424406

2.57895001

6.42522328

5.14017863

6.41985771

5.14017863

6.42522328

5.14017863

6.43611319

5.14017863

6.42522328

5.14017863

6.42095976

5.14017863

6.42522328

5.14017863

6.43611319

5.14017863

1.28504466
Fri Apr 16 14:51:55 2021

7.71026794

8.98247831

7.69549297

1.28504466

0.00000000

1.28504466

$-0.05838098$

3.85513397

2.57008931

3. 84852941

2.62847029

6.42522328

5.14017863

6.42522328

5.15495360

8.99531260

7.71026794

9.00191716

7.69549297

1.28504466

0.00000000

1.28504466

$-0.01557490$

3.85513397

2.57008931

3.84852941

2.58566422

6.42522328

5.14017863

6.42522328

5.12974524

8.99531260

7.71026794

9.00191716

7.72070132

1.28504466
9. 81732758

11.63342251

13.39259635

8.00000000

9.81732758

11.63317354

13.48914812

8.00000000

9.81732758

11.63859865

13.48914812

8.00000000

9.81732758

11.62649102

13.39259635

8.00000000

9.81732758

11.63859865

13.39259635

8.00000000

9.81732758

11.63317354

13.39326846

8.00000000

9.81732758

11.63859865

13.39326846

8.00000000

9.81732758

11.62649102

13.39417814

8.00000000

9.81732758

11.63859865

13.39417814

13.81064087

58

Lattice $=" 7.710267940093008 \quad 0.0 \quad 0.0 \quad 0.0 \quad 10.280357253457344 \quad 0.0 \quad 0.0 \quad 0.021 .451982745204997 "$ P roperties=species:S:1:pos:R:3 pbc="T T T"

$\mathrm{Cu}$

$\mathrm{Cu}$

$\mathrm{Cu}$

$\mathrm{Cu}$

$\mathrm{Cu}$

$\mathrm{Cu}$

$\mathrm{Cu}$

$\mathrm{Cu}$

$\mathrm{Cu}$

$\mathrm{Cu}$

$\mathrm{Cu}$

$\mathrm{Cu}$

$\mathrm{Cu}$

$\mathrm{Cu}$

$\mathrm{Cu}$

$\mathrm{Cu}$

$\mathrm{Cu}$

$\mathrm{Cu}$

$\mathrm{Cu}$

$\mathrm{Cu}$

$\mathrm{Cu}$

$\mathrm{Cu}$

$\mathrm{Cu}$

$\mathrm{Cu}$

$\mathrm{Cu}$

$\mathrm{Cu}$

$\mathrm{Cu}$

$\mathrm{Cu}$
1.28504466

0.00000000

1.27182740

0.00000000

1.28504466

0.00000000

1.28597815

$-0.03767297$

1.28504466

0.00000000

1.26283697

0.00000000

1.28504466

0.00000000

1. 32205268

0.03767297

3.85513397

2. 57008931

3. 85524442

2.56108594

3. 85513397

2.57008931

3.86828319

2. 54219639

3. 85513397

2. 57008931

3.84198475

2.51462010
1.28504466

0.00000000

1.24317315

0.00000000

3.85513397

2.57008931

3.81561453

2. 33265674

6.42522328

5.14017863

6.45449224

5.14017863

8.99531260

7.71026794

9.01225556

7.94770052

1.28504466

0.00000000

1. 25318212

$-0.08329755$

3.85513397

2.57008931

3.80851981

2.35894110

6.42522328

5.14017863

6.47183744

5.06042997
8.00000000

9.81732758

11.62884520

14.86253519

8.00000000

9.81732758

11.69354332

13.68721554

8.00000000

9.81732758

11.65618237

13.77505831

8.00000000

9.81732758

11.59556772

13.68721554

8.00000000

9.81732758

11.60242155

14.63017323

8.00000000

9.81732758

11.62005523

13.67209941

8.00000000

9.81732758

11.62005523

13.70240950 


\section{$\mathrm{Cu}$}

$\mathrm{Cu}$

$\mathrm{Cu}$

$\mathrm{Cu}$

$\mathrm{Cu}$

$\mathrm{Cu}$

$\mathrm{Cu}$

$\mathrm{Cu}$

$\mathrm{Cu}$

$\mathrm{Cu}$

$\mathrm{Cu}$

$\mathrm{Cu}$

$\mathrm{Cu}$

$\mathrm{Cu}$

$\mathrm{Cu}$

$\mathrm{Cu}$

$\mathrm{Cu}$

$\mathrm{Cu}$

$\mathrm{Cu}$

$\mathrm{Cu}$

$\mathrm{N}$

$\mathrm{N}$

$\mathrm{N}$

$\mathrm{N}$

$\mathrm{N}$

$\mathrm{N}$

$\mathrm{N}$

$\mathrm{N}$

$\mathrm{N}$

$\mathrm{N}$

.$/$

59 rogen/Cu/100/iter_11/struct. xyz

3.85513397

2. 57008931

3. 85502352

2. 58144082

6.42522328

5.14017863

6.38821526

5.14918200

6.42522328

5.14017863

6.44743097

5.12882712

6.42522328

5.14017863

6.42428979

5.19564784

6.42522328

5.14017863

6.43844054

5.16807155

1.26556230

1.23080117

3. 95275797

6.44470564

6.47946677

3. 90712397

6.48814713

1.22212081

3.75750997
Fri Apr 16 14:51:55 2021

3.80314397
8.99531260

7.71026794

9.02717513

7.67689867

1.28504466

0.00000000

1.26810169

0.08329755

3.85513397

2.57008931

3.82586501

2.60345858

6.42522328

5.14017863

6.46474272

5.21992728

8.99531260

7.71026794

9.03718411

7.92141615

0.96988161

6.49132143

3.70579661

8.87900205

9.31047564

3.78903582

6.57456065

1. 42875131

8.85160594

1.40135521
8.00000000

9.81732758

11.60242155

14.41197968

8.00000000

9.81732758

11.59556772

14.63017323

8.00000000

9.81732758

11.65618237

14.41197968

8.00000000

9.81732758

11.69354332

13.70240950

8.00000000

9.81732758

11.62884520

13.67209941

13.52461769

13.62340952

13.55871601

15.03486763

13.52461769

13.62340952

13.55871601

15.09158210

15.09158210

15.03486763

Lattice $=" 7.710267940093008 \quad 0.0 \quad 0.0 \quad 0.0 \quad 10.280357253457344 \quad 0.0 \quad 0.0 \quad 0.021 .451982745204997 " \mathrm{P}$ roperties=species:S: $1:$ pos:R: 3 pbc="T T T"

$\mathrm{Cu}$

$\mathrm{Cu}$

$\mathrm{Cu}$

$\mathrm{Cu}$

$\mathrm{Cu}$

$\mathrm{Cu}$

$\mathrm{Cu}$

$\mathrm{Cu}$

$\mathrm{Cu}$

$\mathrm{Cu}$

$\mathrm{Cu}$

$\mathrm{Cu}$

$\mathrm{Cu}$

$\mathrm{Cu}$

$\mathrm{Cu}$

$\mathrm{Cu}$

$\mathrm{Cu}$

$\mathrm{Cu}$

$\mathrm{Cu}$

$\mathrm{Cu}$

$\mathrm{Cu}$

$\mathrm{Cu}$

$\mathrm{Cu}$

$\mathrm{Cu}$

$\mathrm{Cu}$

$\mathrm{Cu}$

$\mathrm{Cu}$

$\mathrm{Cu}$

$\mathrm{Cu}$

$\mathrm{Cu}$

$\mathrm{Cu}$

$\mathrm{Cu}$

$\mathrm{Cu}$
1.28504466

0.00000000

1.28737034

0.01329942

1.28504466

0.00000000

1.28346505

0.01329942

1.28504466

0.00000000

1.26762177

$-0.04874200$

1.28504466

0.00000000

1.28346505

$-0.04874200$

3.85513397

2. 57008931

3.85280828

2. 57008931

3.85513397

2. 57008931

3. 85671358

2. 57008931

3. 85513397

2. 57008931

3.87255685

2. 57008931

3. 85513397

2. 57008931

3. 85671358

2.57008931

6.42522328

5.14017863
1.28504466

0.00000000

1.28504466

$-0.05289626$

3.85513397

2.57008931

3.85173154

2. 62298558

6.42522328

5.14017863

6.42522328

5.11749779

8.99531260

7.71026794

8.99871502

7.73294878

1.28504466

0.00000000

1.28504466

$-0.01468346$

3.85513397

2.57008931

3. 85173154

2. 58477277

6.42522328

5.14017863

6.42522328

5.12658697

8.99531260

7.71026794

8.99871502

7.72385959

1.28504466

0.00000000
8.00000000

9.81732758

11.60972599

14.11982203

8.00000000

9.81732758

11.62414007

14.11982203

8.00000000

9.81732758

11.55812284

13.83295253

8.00000000

9.81732758

11.62414007

13.83295253

8.00000000

9.81732758

11.60972599

14.20633247

8.00000000

9.81732758

11.62414007

14.20633247

8.00000000

9.81732758

11.55812284

14.07645356

8.00000000

9.81732758

11.62414007

14.07645356

8.00000000

9.81732758 


\section{$\mathrm{Cu}$}

$\mathrm{Cu}$

$\mathrm{Cu}$

$\mathrm{Cu}$

$\mathrm{Cu}$

$\mathrm{Cu}$

$\mathrm{Cu}$

$\mathrm{Cu}$

$\mathrm{Cu}$

$\mathrm{Cu}$

$\mathrm{Cu}$

$\mathrm{Cu}$

$\mathrm{Cu}$

$\mathrm{Cu}$

$\mathrm{N}$

$\mathrm{N}$

$\mathrm{N}$

$\mathrm{N}$

$\mathrm{N}$

$\mathrm{N}$

$\mathrm{N}$

$\mathrm{N}$

$\mathrm{N}$

$\mathrm{N}$

$\mathrm{N}$

1. 31550036

Lattice $=" 7.7102679400930080 .0 \quad 0.00 .0$
roperties $=$ species:S:1:pos:R:3 pbc="T T T"

$\mathrm{Cu}$

$\mathrm{Cu}$

$\mathrm{Cu}$

$\mathrm{Cu}$

$\mathrm{Cu}$

$\mathrm{Cu}$

$\mathrm{Cu}$

$\mathrm{Cu}$

$\mathrm{Cu}$

$\mathrm{Cu}$

$\mathrm{Cu}$

$\mathrm{Cu}$

$\mathrm{Cu}$

$\mathrm{Cu}$

$\mathrm{Cu}$

$\mathrm{Cu}$

$\mathrm{Cu}$

$\mathrm{Cu}$

$\mathrm{Cu}$

$\mathrm{Cu}$

$\mathrm{Cu}$

$\mathrm{Cu}$

$\mathrm{Cu}$

$\mathrm{Cu}$

$\mathrm{Cu}$

$\mathrm{Cu}$

$\mathrm{Cu}$

$\mathrm{Cu}$

$\mathrm{Cu}$

$\mathrm{Cu}$

$\mathrm{Cu}$

$\mathrm{Cu}$

$\mathrm{Cu}$

$\mathrm{Cu}$

$\mathrm{Cu}$

$\mathrm{Cu}$

$\mathrm{Cu}$

$\mathrm{Cu}$

$\mathrm{Cu}$
6.42522328

5.12687920

6.42522328

5.14017863

6.42522328

5.12687920

6.42522328

5.14017863

6.42522328

5.18892062

6.42522328

5.14017863

6.42522328

5.18892062

1.28150652

1.25024163

3.82467827

3.82467827

6.42522328

6.42522328

3.88993699

6.42522328

1.31550036

3.85867211
$-0.05289626$

$1.28504466 \quad 1.28504466$

0.00000000

$1.28504466 \quad 1.28504466$

0.00000000

0.00000000

3.85513397

1.28504466

2.57008931

$1.28504466 \quad 3.85513397$

0.00000000

2.57008931

1.28504466

6.42522328

0.00000000

5.14017863

1.28504466

6.42522328

0.00000000

5.14017863

1. 28504466

0.00000000

8.99531260

7.71026794

1. 28504466

8.99531260

0.00000000

3. 85513397

7.71026794

1.28504466

2. 57008931

0.00000000

3. 85513397

1.28504466

0.00000000

2. 57008931

3. 85513397

3.85513397

2.57008931

3.85513397

3. 85513397

2.57008931

6.42522328

3. 85513397

2. 57008931

5.14017863

3. 85513397

6.42522328

5.14017863

8.99531260

3.85513397

7. 71026794

2. 57008931

3. 85513397

8.99531260

7.71026794

2. 57008931

1. 28504466

0.00000000

1.28504466

0.00000000

3.85513397

2. 57008931

3.85513397
Fri Apr 16 14:51:55 2021

1.28504466

3.85513397

2.57008931

3.89651705

2.62298558

6.42522328

5.14017863

6.42522328

5.11749779

8.99531260

7.71026794

8.95392952

7.73294878

1.28504466

6.42522328

3.86467440

8.98577217

8.91527843

3.93516814

6.42522328

1.28504466

8.98577217

1.28504466

3.86467440

struct. xyz
11.57810886

14.11982203

8.00000000

9.81732758

11.67012778

14.11982203

8.00000000

9.81732758

11.74859190

13.83295253

8.00000000

9.81732758

11.67012778

13.83295253

13.52806528

13.44899797

14.82670251

14.82670251

14.58197722

14.58197722

13.44899797

13.48388157

14.82670251

13.52806528

14.82670251

$.280357253457344 \quad 0.0 \quad 0.0 \quad 0.0 \quad 21.451982745204997 " \mathrm{P}$

8.00000000

9.81732758

11.61294956

13.65250616

8.00000000

9.81732758

11.61294956

13.65250616

8.00000000

9.81732758

11.61294956

13.65250616

8.00000000

9.81732758

11.61294956

13.65250616

8.00000000

9.81732758

11.61294956

13.65250616

8.00000000

9.81732758

11.61294956

13.65250616

8.00000000

9.81732758

11.61294956

13.65250616

8.00000000

9.81732758

11.61294956

13.65250616

8.00000000

9.81732758

11.61294956

13.65250616

8.00000000

9.81732758

11.61294956 
$\mathrm{Cu}$

$\mathrm{Cu}$

$\mathrm{Cu}$

$\mathrm{Cu}$

$\mathrm{Cu}$

$\mathrm{Cu}$

$\mathrm{Cu}$

$\mathrm{Cu}$

$\mathrm{Cu}$

$\mathrm{N}$

$\mathrm{N}$

$\mathrm{N}$

$\mathrm{N}$

$\mathrm{N}$

$\mathrm{N}$

$\mathrm{N}$

$\mathrm{N}$

$\mathrm{N}$

$\mathrm{N}$

$\mathrm{N}$

$\mathrm{N}$
5.14017863

6.42522328

5.14017863

6.42522328

5.14017863

6.42522328

5.14017863

6.42522328

5.14017863

1.28504466

1.28504466

3.85513397

3.85513397

6.42522328

6.42522328

3.85513397

6.42522328

1.28504466

3.85513397

6.42522328

1.28504466
Fri Apr 16 14:51:55 2021

2.57008931

6.42522328

5.14017863

6.42522328

5.14017863

8.99531260

7.71026794

8.99531260

7.71026794

1.28504466

6.42522328

3.85513397

8.99531260

8.99531260

3.85513397

6.42522328

1.28504466

8.99531260

1. 28504466

6.42522328

3. 85513397
13.65250616

8.00000000

9.81732758

11.61294956

13.65250616

8.00000000

9.81732758

11.61294956

13.65250616

14.21016099

14.21016099

14.21016099

14.21016099

14.21016099

14.21016099

14.21016099

14.21016099

14.21016099

14.21016099

14.21016099

14.21016099

50

Lattice $=" 7.710267940093008 \quad 0.0 \quad 0.0 \quad 0.0 \quad 10.280357253457344 \quad 0.0 \quad 0.0 \quad 0.021 .451982745204997 "$ P roperties=species:S: $1:$ pos:R: 3 pbc="T T T"

$\mathrm{Cu}$

$\mathrm{Cu}$

$\mathrm{Cu}$

$\mathrm{Cu}$

$\mathrm{Cu}$

$\mathrm{Cu}$

$\mathrm{Cu}$

$\mathrm{Cu}$

$\mathrm{Cu}$

$\mathrm{Cu}$

$\mathrm{Cu}$

$\mathrm{Cu}$

$\mathrm{Cu}$

$\mathrm{Cu}$

$\mathrm{Cu}$

$\mathrm{Cu}$

$\mathrm{Cu}$

$\mathrm{Cu}$

$\mathrm{Cu}$

$\mathrm{Cu}$

$\mathrm{Cu}$

$\mathrm{Cu}$

$\mathrm{Cu}$

$\mathrm{Cu}$

$\mathrm{Cu}$

$\mathrm{Cu}$

$\mathrm{Cu}$

$\mathrm{Cu}$

$\mathrm{Cu}$

$\mathrm{Cu}$

$\mathrm{Cu}$

$\mathrm{Cu}$

$\mathrm{Cu}$

$\mathrm{Cu}$

$\mathrm{Cu}$

$\mathrm{Cu}$

$\mathrm{Cu}$

$\mathrm{Cu}$

$\mathrm{Cu}$

$\mathrm{Cu}$

$\mathrm{Cu}$

$\mathrm{Cu}$
1.28504466

0.00000000

1.28504466

$-0.05210215$

1.28504466

0.00000000

1.28504466

$-0.05210215$

1.28504466

0.00000000

1.28504466

$-0.05210215$

1.28504466

0.00000000

1.28504466

$-0.05210215$

3.85513397

2.57008931

3.86637153

2. 62219147

3. 85513397

2.57008931

3.84408880

2. 62219147

3. 85513397

2.57008931

3. 86637153

2. 62219147

3.85513397

2. 57008931

3.84408880

2. 62219147

6.42522328

5.14017863

6.41398572

5.14017863

6.42522328

5.14017863

6.43626845

5.14017863

6.42522328

5.14017863

6.41398572
1.28504466

0.00000000

1.28504466

$-0.03572796$

3.85513397

2. 57008931

3. 85513397

2. 60581727

6.42522328

5.14017863

6.42522328

5.10445067

8.99531260

7.71026794

8.99531260

7.74599590

1.28504466

0.00000000

1.28504466

$-0.03572796$

3.85513397

2.57008931

3.85513397

2. 60581727

6.42522328

5.14017863

6.42522328

5.10445067

8.99531260

7.71026794

8.99531260

7.74599590

1.28504466

0.00000000

1.28504466

$-0.02136215$

3. 85513397

2.57008931

3.85513397

2.59145146

6.42522328

5.14017863

6.42522328
8.00000000

9.81732758

11.62557330

13.47748363

8.00000000

9.81732758

11.63011075

13.47748363

8.00000000

9.81732758

11.62557330

13.47748363

8.00000000

9.81732758

11.63011075

13.47748363

8.00000000

9.81732758

11.63402544

13.47748363

8.00000000

9.81732758

11.65116194

13.47748363

8.00000000

9.81732758

11.63402544

13.47748363

8.00000000

9.81732758

11.65116194

13.47748363

8.00000000

9.81732758

11.63402544

13.40278525

8.00000000

9.81732758

11.65116194

13.40278525

8.00000000

9.81732758

11.63402544 


\section{$\mathrm{Cu}$}

$\mathrm{Cu}$

$\mathrm{Cu}$

$\mathrm{Cu}$

$\mathrm{Cu}$

$\mathrm{N}$

$\mathrm{N}$
5.14017863

6.42522328

5.14017863

6.43626845

5.14017863

1.28504466

1.28504466
Fri Apr 16 14:51:55 2021

5.11881648

8.99531260

7.71026794

8.99531260

7.73163009

1.28504466

6.42522328
13.40278525

8.00000000

9.81732758

11.65116194

13.40278525

13.82213964

13.82213964

./nitrogen/Cu/100/iter_3/struct.xyz

51

Lattice $=" 7.710267940093008 \quad 0.0 \quad 0.0 \quad 0.0 \quad 10.280357253457344 \quad 0.0 \quad 0.0 \quad 0.021 .451982745204997 "$ P roperties=species:S:1:pos:R:3 pbc="T T T"

$\mathrm{Cu}$

$\mathrm{Cu}$

1.28504466

0.00000000

1.28504466

1.31499397

$-0.09278952$

1.28504466

0.00000000

1.29698329

$-0.01910979$

1.28504466

0.00000000

1.31499397

$-0.01910979$

1.28504466

0.00000000

1.26403256

$-0.09278952$

3.85513397

2. 57008931

0.00000000

1.28759788

$-0.04295341$

3.85513397

2. 57008931

3. 85513397

2. 60627062

6.42522328

5.14017863

6.42267006

5.10399732

8.99531260

7.71026794

8.99531260

7.75322135

1.28504466

0.00000000

3. 86173561

2. 62028508

1.28215920

3.85513397

$-0.06014453$

3.85513397

2. 57008931

2. 57008931

3. 82817938

3.85513397

2. 58453087

2. 54149348

3. 85513397

6.42522328

2. 57008931

5.14017863

3. 86173561

6.42810874

2. 58453087

3.85513397

5.16877446

8.99531260

7.71026794

2. 57008931

3. 82282375

2. 62028508

8.99531260

7.77041247

6.42522328

1.28504466

0.00000000

1.28156974

6.42219395

5.12564023

6.42522328

5.14017863

$-0.04252081$

3.85513397

2.57008931

3.85513397

2. 50546574

5.20269273

6.42522328

6.42522328

5.14017863

6.42869820

6.42219395

5.20269273

5.20480220

8.99531260

7.71026794

5.14017863

6.42411176

8.99531260

7.75278875

1.22902685

6.48124109

1.26174880

1.26174880

3.90938563

3.85513397

./nitrogen/Cu/100/iter_4/struct.xyz

52

Lattice $=" 7.710267940093008 \quad 0.0 \quad 0.0 \quad 0.0 \quad 10.280357253457344 \quad 0.0 \quad 0.0 \quad 0.021 .451982745204997 " \mathrm{P}$ roperties=species:S: $1:$ pos:R: 3 pbc="T T T"
$\mathrm{Cu}$
1.28504466
1.28504466
8.00000000
$\mathrm{Cu}$
0.00000000
0.00000000

8.00000000
9.81732758

11.63645819

13.47028476

8.00000000

9.81732758

11.63597751

13.42778221

8.00000000

9.81732758

11.63645819

13.42778221

8.00000000

9.81732758

11.63114151

13.47028476

8.00000000

9.81732758

11.63263826

13.48224734

8.00000000

9.81732758

11.65964591

13.68923432

8.00000000

9.81732758

11.63263826

13.68923432

8.00000000

9.81732758

11.63842186

13.48224734

8.00000000

9.81732758

11.62981909

13.38844738

8.00000000

9.81732758

11.64345663

13.43483068

8.00000000

9.81732758

11.62981909

13.43483068

8.00000000

9.81732758

11.63006536

13.38844738

13.82968819

13.82968819

13.83548738 
$\mathrm{Cu}$

$\mathrm{Cu}$

$\mathrm{Cu}$

$\mathrm{Cu}$

$\mathrm{Cu}$

$\mathrm{Cu}$

$\mathrm{Cu}$

$\mathrm{Cu}$

$\mathrm{Cu}$

$\mathrm{Cu}$

$\mathrm{Cu}$

$\mathrm{Cu}$

$\mathrm{Cu}$

$\mathrm{Cu}$

$\mathrm{Cu}$

$\mathrm{Cu}$

$\mathrm{Cu}$

$\mathrm{Cu}$

$\mathrm{Cu}$

$\mathrm{Cu}$

$\mathrm{Cu}$

$\mathrm{Cu}$

$\mathrm{Cu}$

$\mathrm{Cu}$

$\mathrm{Cu}$

$\mathrm{Cu}$

$\mathrm{Cu}$

$\mathrm{Cu}$

$\mathrm{Cu}$

$\mathrm{Cu}$

$\mathrm{Cu}$

$\mathrm{Cu}$

$\mathrm{Cu}$

$\mathrm{Cu}$

$\mathrm{Cu}$

$\mathrm{Cu}$

$\mathrm{Cu}$

$\mathrm{Cu}$

$\mathrm{Cu}$

$\mathrm{Cu}$

$\mathrm{Cu}$

$\mathrm{Cu}$

$\mathrm{Cu}$

$\mathrm{Cu}$

$\mathrm{Cu}$

$\mathrm{Cu}$

$\mathrm{N}$

$\mathrm{N}$

$\mathrm{N}$

$\mathrm{N}$
1.34097266

$-0.05231999$

1.28504466

0.00000000

1.27834681

$-0.05231999$

1.28504466

0.00000000

1.34097266

$-0.05231999$

1.28504466

0.00000000

1.27834681

$-0.05231999$

3.85513397

2.57008931

3.86183182

2.57008931

3. 85513397

2.57008931

3.79920596

2.57008931

3.85513397

2.57008931

3.86183182

2.57008931

3.85513397

2.57008931

3.79920596

2.57008931

6.42522328

5.14017863

6.42926474

5.19249862

6.42522328

5.14017863

6.42118183

5.19249862

6.42522328

5.14017863

6.42926474

5.19249862

6.42522328

5.14017863

6.42118183

5.19249862

1.23113867

1.23113867

3.90903995

3.90903995
Fri Apr 16 14:51:55 2021

1.28504466

$-0.04469425$

3.85513397

2.57008931

3.85513397

2. 61478356

6.42522328

5.14017863

6.42522328

5.09548438

8.99531260

7.71026794

8.99531260

7.75496219

1.28504466

0.00000000

1.28504466

0.00000000

3.85513397

2.57008931

3.85513397

2. 57008931

6.42522328

5.14017863

6.42522328

5.14017863

8.99531260

7.71026794

8.99531260

7.71026794

1.28504466

0.00000000

1.28504466

0.04469425

3.85513397

2. 57008931

3. 85513397

2. 52539507

6.42522328

5.14017863

6.42522328

5.18487287

8.99531260

7.71026794

8.99531260

7.66557369

1.28504466

6.42522328

3. 85513397

8.99531260
11.65314153

13.41957827

8.00000000

9.81732758

11.63627418

13.41957827

8.00000000

9.81732758

11.65314153

13.41957827

8.00000000

9.81732758

11.63627418

13.41957827

8.00000000

9.81732758

11.63627418

13.69894776

8.00000000

9.81732758

11.65314153

13.69894776

8.00000000

9.81732758

11.63627418

13.69894776

8.00000000

9.81732758

11.65314153

13.69894776

8.00000000

9.81732758

11.62796229

13.41957827

8.00000000

9.81732758

11.62796229

13.41957827

8.00000000

9.81732758

11.62796229

13.41957827

8.00000000

9.81732758

11.62796229

13.41957827

13.86594344

13.86594344

13.86594344

13.86594344

./nitrogen/Cu/100/iter_5/struct.xyz

53

Lattice $=" 7.710267940093008 \quad 0.0 \quad 0.0 \quad 0.0 \quad 10.280357253457344 \quad 0.0 \quad 0.0 \quad 0.021 .451982745204997 " \quad P$ roperties $=$ species: $\mathrm{S}: 1: \mathrm{pos}: \mathrm{R}: 3 \mathrm{pbc}=" \mathrm{~T} \mathrm{~T} \mathrm{~T}$ "

$\begin{array}{lrrr}\mathrm{Cu} & 1.28504466 & 1.28504466 & 8.00000000 \\ \mathrm{Cu} & 0.00000000 & 0.00000000 & 9.81732758 \\ \mathrm{Cu} & 1.31073599 & 1.28059659 & 11.64895581 \\ \mathrm{Cu} & -0.03560276 & 0.05465200 & 13.67855781 \\ \mathrm{Cu} & 1.28504466 & 3.85513397 & 8.00000000 \\ \mathrm{Cu} & 0.00000000 & 2.57008931 & 9.81732758 \\ \mathrm{Cu} & 1.29935203 & 3.85513397 & 11.62328385 \\ \mathrm{Cu} & -0.06033789 & 2.65233551 & 13.39386791 \\ \mathrm{Cu} & 1.28504466 & 6.42522328 & 8.00000000 \\ \mathrm{Cu} & 0.00000000 & 5.14017863 & 9.81732758 \\ \mathrm{Cu} & 1.31073599 & 6.42967135 & 11.64895581 \\ \mathrm{Cu} & -0.06033789 & 5.05793243 & 13.39386791 \\ \mathrm{Cu} & 1.28504466 & 8.99531260 & 8.00000000 \\ \mathrm{Cu} & 0.00000000 & 7.71026794 & 9.81732758\end{array}$


$\mathrm{Cu}$

$\mathrm{Cu}$

$\mathrm{Cu}$

$\mathrm{Cu}$

$\mathrm{Cu}$

$\mathrm{Cu}$

$\mathrm{Cu}$

$\mathrm{Cu}$

$\mathrm{Cu}$

$\mathrm{Cu}$

$\mathrm{Cu}$

$\mathrm{Cu}$

$\mathrm{Cu}$

$\mathrm{Cu}$

$\mathrm{Cu}$

$\mathrm{Cu}$

$\mathrm{Cu}$

$\mathrm{Cu}$

$\mathrm{Cu}$

$\mathrm{Cu}$

$\mathrm{Cu}$

$\mathrm{Cu}$

$\mathrm{Cu}$

$\mathrm{Cu}$

$\mathrm{Cu}$

$\mathrm{Cu}$

$\mathrm{Cu}$

$\mathrm{Cu}$

$\mathrm{Cu}$

$\mathrm{Cu}$

$\mathrm{Cu}$

$\mathrm{Cu}$

$\mathrm{Cu}$

$\mathrm{Cu}$

$\mathrm{N}$

$\mathrm{N}$

$\mathrm{N}$

N

$\mathrm{N}$
1.25786424

$-0.03560276$

3. 85513397

2.57008931

3.86471192

2. 54626862

3.85513397

2.57008931

3.81438787

2. 62593761

3. 85513397

2. 57008931

3. 86471192

2. 62593761

3. 85513397

2.57008931

3. 82414198

2.54626862

6.42522328

5.14017863

6.42697102

5.16582997

6.42522328

5.14017863

6.41013044

5.19396960

6.42522328

5.14017863

6.42697102

5.19396960

6.42522328

5.14017863

6.45172964

5.16582997

1.25858842

1.25858842

3.96242748

3. 77146821

6.49743896
Fri Apr 16 14:51:55 2021

8.99531260

7.65561594

1.28504466

0.00000000

1.29970562

0.05731936

3.85513397

2.57008931

3.85513397

2.60882468

6.42522328

5.14017863

6.41056232

5.10144326

8.99531260

7.71026794

8.99531260

7.65294858

1.28504466

0.00000000

1.27214699

0.04984451

3.85513397

2.57008931

3.85513397

2. 55445193

6.42522328

5.14017863

6.43812095

5.15581601

8.99531260

7.71026794

8.99531260

7.66042343

1. 37397658

6.33629136

3. 85513397

8.99531260

8.99531260
11.68599187

13.67855781

8.00000000

9.81732758

11.64885785

13.61873219

8.00000000

9.81732758

11.62347432

13.70113268

8.00000000

9.81732758

11.64885785

13.70113268

8.00000000

9.81732758

11.62437112

13.61873219

8.00000000

9.81732758

11.63885171

13.50345622

8.00000000

9.81732758

11.60981084

13.41617744

8.00000000

9.81732758

11.63885171

13.41617744

8.00000000

9.81732758

11.63561561

13.50345622

13.81124753

13.81124753

14.04695115

13.67190550

14.06906400

54

Lattice $=" 7.710267940093008 \quad 0.0 \quad 0.0 \quad 0.0 \quad 10.280357253457344 \quad 0.0 \quad 0.0 \quad 0.021 .451982745204997 "$ P roperties $=$ species: $\mathrm{S}: 1:$ pos $: \mathrm{R}: 3$ pbc $=" \mathrm{~T} T \mathrm{~T}$ "

$\begin{array}{lll}\mathrm{Cu} & 1.28504466 & 1.28504466\end{array}$

$\mathrm{Cu} \quad 0.00000000 \quad 0.00000000$

$\begin{array}{lll}\mathrm{Cu} & 1.28504466 & 1.28504466\end{array}$

$\begin{array}{lll}\mathrm{Cu} & -0.03838691 & 0.00860146\end{array}$

$\begin{array}{lll}\mathrm{Cu} & 1.28504466 & 3.85513397\end{array}$

$\begin{array}{lll}\mathrm{Cu} & 0.00000000 & 2.57008931\end{array}$

$\mathrm{Cu} \quad 1.28504466 \quad 3.85513397$

$\begin{array}{lll}\mathrm{Cu} & -0.03838691 & 2.56148785\end{array}$

$\mathrm{Cu} \quad 1.28504466$

0.00000000

1.28504466

$-0.03838691$

1.28504466

0.00000000

1.28504466

$-0.03838691$

3. 85513397

2. 57008931

6.42522328

5.14017863

6.42522328

5.14878009

8.99531260

7.71026794

8.99531260

7.70166648

1.28504466

0.00000000

3.87113308

2.60847622

1.28504466

0.00860146

3.85513397

3.85513397

2.57008931

3.85513397
2.57008931

3.83540649

3.85513397

2.60847622

2. 56148785

3. 85513397

6.42522328

8.00000000

9.81732758

11.61919339

13.60543580

8.00000000

9.81732758

11.65551445

13.60543580

8.00000000

9.81732758

11.61919339

13.60543580

8.00000000

9.81732758

11.65551445

13.60543580

8.00000000

9.81732758

11.65616669

13.60543580

8.00000000

9.81732758

11.61723345

13.60543580

8.00000000 


\section{$\mathrm{Cu}$}

$\mathrm{Cu}$

$\mathrm{Cu}$

$\mathrm{Cu}$

$\mathrm{Cu}$

$\mathrm{Cu}$

$\mathrm{Cu}$

$\mathrm{Cu}$

$\mathrm{Cu}$

$\mathrm{Cu}$

$\mathrm{Cu}$

$\mathrm{Cu}$

$\mathrm{Cu}$

$\mathrm{Cu}$

$\mathrm{Cu}$

$\mathrm{Cu}$

$\mathrm{Cu}$

$\mathrm{Cu}$

$\mathrm{Cu}$

$\mathrm{Cu}$

$\mathrm{Cu}$

$\mathrm{Cu}$

$\mathrm{Cu}$

$\mathrm{N}$

$\mathrm{N}$

$\mathrm{N}$

$\mathrm{N}$

$\mathrm{N}$

$\mathrm{N}$
2.57008931

3. 87113308

2. 60847622

3. 85513397

2. 57008931

3.83540649

2. 60847622

6.42522328

5.14017863

6.40922418

5.14017863

6.42522328

5.14017863

6.44495076

5.14017863

6.42522328

5.14017863

6.40922418

5.14017863

6.42522328

5.14017863

6.44495076

5.14017863

1.28504466

1.28504466

3. 80292889

3.80292889

6.47742836

6.47742836
Fri Apr 16 14:51:55 2021

5.14017863

6.42522328

5.14878009

8.99531260

7.71026794

8.99531260

7.70166648

1.28504466

0.00000000

1.28504466

0.01776510

3.85513397

2.57008931

3.85513397

2.55232421

6.42522328

5.14017863

6.42522328

5.15794373

8.99531260

7.71026794

8.99531260

7.69250284

1.28504466

6.42522328

3.85513397

8.99531260

8.99531260

3.85513397
9.81732758

11.65616669

13.60543580

8.00000000

9.81732758

11.61723345

13.60543580

8.00000000

9.81732758

11.65616669

13.50460424

8.00000000

9.81732758

11.61723345

13.50460424

8.00000000

9.81732758

11.65616669

13.50460424

8.00000000

9.81732758

11.61723345

13.50460424

13.67855836

13.67855836

14.06515088

14.06515088

14.06515088

14.06515088

./nitrogen/Cu/100/iter_7/struct.xyz

55

Lattice $=" 7.710267940093008 \quad 0.0 \quad 0.0 \quad 0.0 \quad 10.2803572534573440 .00 .00 .021 .451982745204997 "$ P roperties $=$ species:S: $1:$ pos: $\mathrm{R}: 3$ pbc="T T T"

$\mathrm{Cu} \quad 1.28504466 \quad 1.28504466$

$\mathrm{Cu} \quad 0.00000000 \quad 0.00000000$

$\mathrm{Cu} \quad 1.28175099 \quad 1.28504466$

$\mathrm{Cu} \quad-0.04349714 \quad 0.03368119$

$\begin{array}{lll}\mathrm{Cu} & 1.28504466 & 3.85513397\end{array}$

$\begin{array}{lll}\mathrm{Cu} & 0.00000000 & 2.57008931\end{array}$

$\begin{array}{lll}\mathrm{Cu} & 1.31412925 & 3.85564723\end{array}$

$\begin{array}{lll}\mathrm{Cu} & -0.04349714 & 2.53640812\end{array}$

$\begin{array}{lll}\mathrm{Cu} & 1.28504466 & 6.42522328\end{array}$

$\mathrm{Cu} \quad 0.00000000 \quad 5.14017863$

$\begin{array}{lll}\mathrm{Cu} & 1.33333807 & 6.42522328\end{array}$

$\begin{array}{lll}\mathrm{Cu} & 0.05837534 & 5.14965677\end{array}$

$\begin{array}{lll}\mathrm{Cu} & 1.28504466 & 8.99531260\end{array}$

$\begin{array}{lll}\mathrm{Cu} & 0.00000000 & 7.71026794\end{array}$

$\begin{array}{lll}\mathrm{Cu} & 1.31412925 & 8.99479934\end{array}$

$\begin{array}{lll}\mathrm{Cu} & 0.05837534 & 7.70078980\end{array}$

$\begin{array}{lll}\mathrm{Cu} & 3.85513397 & 1.28504466\end{array}$

$\begin{array}{lll}\mathrm{Cu} & 2.57008931 & 0.00000000\end{array}$

$\mathrm{Cu} \quad 3.83842667$

$\mathrm{Cu} \quad 2.57886880$

$\mathrm{Cu} \quad 3.85513397$

$\mathrm{Cu} \quad 2.57008931$

3.82240205

2.57886880

3.85513397

1.28504466

$-0.02628037$

3. 85513397

2.57008931

3.88172431

2. 59636969

6.42522328

2. 57008931

3. 89209392

5.14017863

6.42522328

2. 63821311

5.06791387

8.99531260

7.71026794

2. 57008931

3. 82240205

8.96872225

7.78253270

1.28504466

6.42522328

0.00000000

8.00000000

9.81732758

11.62557261

13.57321265

8.00000000

9.81732758

11.64992275

13.57321265

8.00000000

9.81732758

11.61574843

13.56257515

8.00000000

9.81732758

11.64992275

13.56257515

8.00000000

9.81732758

11.64152724

13.59709413

8.00000000

9.81732758

11.62468367

13.59709413

8.00000000

9.81732758

11.65408737

13.94206299

8.00000000

9.81732758

11.62468367

13.94206299

8.00000000

9.81732758

1.28504466

11.63778405 
$\mathrm{Cu}$

$\mathrm{Cu}$

$\mathrm{Cu}$

$\mathrm{Cu}$

$\mathrm{Cu}$

$\mathrm{Cu}$

$\mathrm{Cu}$

$\mathrm{Cu}$

$\mathrm{Cu}$

$\mathrm{Cu}$

$\mathrm{Cu}$

$\mathrm{Cu}$

$\mathrm{Cu}$

$\mathrm{N}$

$\mathrm{N}$

$\mathrm{N}$

$\mathrm{N}$

$\mathrm{N}$

$\mathrm{N}$

$\mathrm{N}$
5.06312621

6.42522328

5.14017863

6.41596413

5.06312621

6.42522328

5.14017863

6.39766730

5.12491487

6.42522328

5.14017863

6.41596413

5.12491487

1.28005252

1.38215740

3.79863729

3.79863729

6.45952681

6.45952681

3.99729461
Fri Apr 16 14:51:55 2021

0.03692105

3.85513397

2.57008931

3.88423456

2.53316826

6.42522328

5.14017863

6.42522328

5.15743077

8.99531260

7.71026794

8.96621200

7.69301579

1.28504466

6.42522328

3.68503868

9.16540789

8.96445506

3.88599151

6.42522328
13.47885068

8.00000000

9.81732758

11.60511213

13.47885068

8.00000000

9.81732758

11.67797472

13.89824037

8.00000000

9.81732758

11.60511213

13.89824037

13.72330495

13.59542527

14.42034463

14.42034463

13.60125819

13.60125819

14.61534253

./nitrogen/Cu/100/iter_8/struct.xyz 56

Lattice $=" 7.710267940093008 \quad 0.0 \quad 0.0 \quad 0.0 \quad 10.280357253457344 \quad 0.0 \quad 0.0 \quad 0.0 \quad 21.451982745204997 " \mathrm{P}$ roperties $=$ species: $\mathrm{S}: 1: \mathrm{pos}: \mathrm{R}: 3 \mathrm{pbc}=" \mathrm{~T} \mathrm{~T} \mathrm{~T} "$

$\mathrm{Cu} \quad 1.28504466 \quad 1.28504466$

$\mathrm{Cu} \quad 0.00000000 \quad 0.00000000$

$\mathrm{Cu} \quad 1.25485919 \quad 1.28504466$

$\begin{array}{lll}\mathrm{Cu} & -0.07436691 & 0.01178196\end{array}$

$\mathrm{Cu} \quad 1.28504466 \quad 3.85513397$

$\mathrm{Cu} \quad 0.00000000 \quad 2.57008931$

$\begin{array}{lll}\mathrm{Cu} & 1.28504466 & 3.85513397\end{array}$

$\mathrm{Cu} \quad-0.07436691 \quad 2.55830735$

$\mathrm{Cu} \quad 1.28504466 \quad 6.42522328$

$\mathrm{Cu} \quad 0.00000000 \quad 5.14017863$

$\mathrm{Cu} \quad 1.31523013 \quad 6.42522328$

$\mathrm{Cu} \quad 0.07086552 \quad 5.11161938$

$\mathrm{Cu} \quad 1.28504466 \quad 8.99531260$

$\begin{array}{lll}\mathrm{Cu} & 0.00000000 & 7.71026794\end{array}$

$\mathrm{Cu} \quad 1.28504466 \quad 8.99531260$

$\begin{array}{lll}\mathrm{Cu} & 0.07086552 & 7.73882719\end{array}$

$\begin{array}{lll}\mathrm{Cu} & 3.85513397 & 1.28504466\end{array}$

$\begin{array}{lll}\mathrm{Cu} & 2.57008931 & 0.00000000\end{array}$

$\begin{array}{llr}\mathrm{Cu} & 3.85968703 & 1.28504466\end{array}$

$\mathrm{Cu} \quad 2.49922379$

$\mathrm{Cu} \quad 3.85513397$

$\mathrm{Cu} \quad 2.57008931$

3. 84102890

2.49922379

3. 85513397

2. 57008931

3. 89117539

2. 64445622

3. 85513397

2. 57008931

3.84102890

2. 64445622

6. 42522328

5. 14017863

6.38918186

5.10842757

6.42522328

$-0.02855925$

3. 85513397

2.57008931

3. 84519896

2. 59864856

6.42522328

5.14017863

6.42522328

5.15196059

8.99531260

7.71026794

9.00524761

7.69848598

1. 28504466

0.00000000

1. 28504466

0.07391858

3.85513397

2. 57008931

3. 86506898

6.43932836

5.10842757

2.49617073

6.42522328

5.14017863

5. 14017863

6.42067022

6.42522328

8.00000000

9.81732758

11.69694176

13.78353820

8.00000000

9.81732758

11.66849070

13.78353820

8.00000000

9.81732758

11.69694176

13.69017176

8.00000000

9.81732758

11.66849070

13.69017176

8.00000000

9. 81732758

11.69396525

13.69017176

8.00000000

9.81732758

11.57440997

13.69017176

8.00000000

9.81732758

11.61754952

13.78353820

8.00000000

9.81732758

11.57440997

13.78353820

8.00000000

9.81732758

11.61754952

14.02581907

8.00000000

9.81732758

11.57440997

14.02581907

8.00000000

9.81732758

11.69396525

5.21409721

14.02581907 


\section{$\mathrm{Cu}$}

$\mathrm{Cu}$

$\mathrm{Cu}$

$\mathrm{Cu}$

$\mathrm{N}$

$\mathrm{N}$

$\mathrm{N}$

$\mathrm{N}$

$\mathrm{N}$

$\mathrm{N}$

$\mathrm{N}$

$\mathrm{N}$

.$/$

57

rogen/Cu/100/iter_9/struct.xyz
Fri Apr $16 \quad 14: 51: 552021$

19

8.99531260

7.71026794

8.98537758

7.63634936

1.28504466

6.42522328

3.78718932

9.06325725

8.92736795

3.92307862

6.42522328

1.28504466
8.00000000

9.81732758

11.57440997

13.81022623

13.81022623

13.50224519

13.50224519

13.50224519

13.50224519

14.74263109

14.74263109
14.02581907

Lattice="7.710267940093008 $0.0 \quad 0.0 \quad 0.0 \quad 10.280357253457344 \quad 0.0 \quad 0.0 \quad 0.021 .451982745204997 " \mathrm{P}$ roperties $=$ species:S:1:pos:R:3 pbc="T T T"

$\mathrm{Cu}$

$\mathrm{Cu}$

$\mathrm{Cu}$

$\mathrm{Cu}$

$\mathrm{Cu}$

$\mathrm{Cu}$

$\mathrm{Cu}$

$\mathrm{Cu}$

$\mathrm{Cu}$

$\mathrm{Cu}$

$\mathrm{Cu}$

$\mathrm{Cu}$

$\mathrm{Cu}$

$\mathrm{Cu}$

$\mathrm{Cu}$

$\mathrm{Cu}$

$\mathrm{Cu}$

$\mathrm{Cu}$

$\mathrm{Cu}$

$\mathrm{Cu}$

$\mathrm{Cu}$

$\mathrm{Cu}$

$\mathrm{Cu}$

$\mathrm{Cu}$

$\mathrm{Cu}$

$\mathrm{Cu}$

$\mathrm{Cu}$

$\mathrm{Cu}$

$\mathrm{Cu}$

$\mathrm{Cu}$

$\mathrm{Cu}$

$\mathrm{Cu}$

$\mathrm{Cu}$

$\mathrm{Cu}$

$\mathrm{Cu}$

$\mathrm{Cu}$

$\mathrm{Cu}$

$\mathrm{Cu}$

$\mathrm{Cu}$

$\mathrm{Cu}$

$\mathrm{Cu}$

$\mathrm{Cu}$

$\mathrm{Cu}$

$\mathrm{Cu}$

$\mathrm{Cu}$

$\mathrm{Cu}$

$\mathrm{Cu}$

$\mathrm{Cu}$

$\mathrm{N}$

$\mathrm{N}$

$\mathrm{N}$

$\mathrm{N}$

\subsection{6}

0.00000000

1.29854849

0.03643301

1.28504466

0.00000000

1. 28504466

0.07004582

1. 28504466

0.00000000

1.27154082

$-0.18760124$

1. 28504466

0.00000000

1.28504466

0.05506957

3.85513397

2. 57008931

3. 86243284

2. 51501974

3.85513397

2.57008931

3. 85121194

2.75769055

3. 85513397

2. 57008931

3. 86771417

2.50004350

3. 85513397

2. 57008931

3. 84608517

2.53365630

6.42522328

5.14017863

6.41264308

5.00846682

6.42522328

5.14017863

6.42914531

5.17236215

6.42522328

5.14017863

6.41792442

5.10799510

6.42522328

5.14017863

6.43427208

5.27189043

1. 30342400

1.26666531

3. 74418631

3.83744507
1. 28504466

0.00000000

1. 30977971

0.04380704

3. 85513397

2.57008931

3. 85513397

2. 77228737

6.42522328

5.14017863

6.40048823

5.18957406

8.99531260

7.71026794

8.99531260

7.70940528

1.28504466

0.00000000

1.31681131

0.00086266

3.85513397

2.57008931

3. 82929808

2.52069388

6.42522328

5.14017863

6.38795281

4.93798057

8.99531260

7.71026794

9.00987483

7.66646090

1.28504466

0.00000000

1. 32231513

0.14936384

3.85513397

2. 57008931

3.88096986

2.61464381

6.42522328

5.14017863

6.39345663

5.09562413

8.99531260

7.71026794

8.98075036

7.56090410

1.43480332

6.27546462

3.73250655

8.99267287
8.00000000

9.81732758

11.58742402

13.98156185

8.00000000

9.81732758

11.72567849

14.03252810

8.00000000

9.81732758

11.58742402

13.77209020

8.00000000

9.81732758

11.57906396

13.67371772

8.00000000

9.81732758

11.65622797

13.67371772

8.00000000

9.81732758

11.66531999

13.77209020

8.00000000

9.81732758

11.60515902

14.03252810

8.00000000

9.81732758

11.62723979

13.98156185

8.00000000

9.81732758

11.60515902

13.76915862

8.00000000

9.81732758

11.66531999

14.23957897

8.00000000

9.81732758

11.65622797

14.23957897

8.00000000

9.81732758

11.62723979

13.76915862

13.50742721

13.50742721

14.74373970

13.58759393 


\section{$\mathrm{N}$}

$\mathrm{N}$

$\mathrm{N}$

$\mathrm{N}$

$\mathrm{N}$
6.44291219

6.53617094

3.89535591

6.38500134

1.28504466
Fri Apr 16 14:51:55 2021

8.99795232

3.97776139

6.31868165

1.39158629

8.99531260
13.58759393

14.74373970

13.53363191

13.53363191

14.65047965

./nitrogen/Cu/110/iter_1/struct.xyz

46

Lattice $=" 7.710267940093007 \quad 0.0 \quad 0.0 \quad 0.0 \quad 10.90396549041$ ties=species:S:1:pos:R:3 pbc="T T T"

$\begin{array}{lll}\mathrm{Cu} & 0.00000000 & 0.00000000\end{array}$

$\mathrm{Cu} \quad 1.28504466 \quad 1.81732758$

$\mathrm{Cu} \quad 0.00000000 \quad 0.00000000$

$\begin{array}{lll}\mathrm{Cu} & 1.28010947 & 1.81816558\end{array}$

$\mathrm{Cu} \quad 0.00547619 \quad 0.31020711$

$\mathrm{Cu} \quad 0.00000000 \quad 3.63465516$

$\mathrm{Cu} \quad 1.28504466 \quad 5.45198275$

$\mathrm{Cu} \quad 0.00000000 \quad 3.63465516$

$\mathrm{Cu} \quad 1.26877131 \quad 5.35218936$

$\begin{array}{lll}\mathrm{Cu} & 0.00132913 & 3.34400297\end{array}$

$\mathrm{Cu} \quad 0.00000000 \quad 7.26931033$

$\mathrm{Cu} \quad 1.28504466 \quad 9.08663791$

$\mathrm{Cu} \quad 0.00000000 \quad 7.26931033$

$\mathrm{Cu} \quad 1.27150100$

$\mathrm{Cu} \quad-0.01731921$

2.57008931

3.85513397

2.57008931

3.89606214

2. 55125402

2.57008931

3. 85513397

2. 57008931

3.80729387

2. 55717300

2.57008931

3.85513397

.57008931

2.53691066

5.14017863

5.14017863

6.36560207

5.14017863

.42522328

./nitrogen/Cu/110/iter_2/struct.xyz

47

9.19472104

7.27173160

0.00000000

1. 81732758

0.00000000

1.81772072

0.29088706

3.63465516

5.45198275

3.63465516

5.38054834

3.36569470

7.26931033

9.08663791

7.26931033

9.17218641

7.27500576

0.00000000

1.81732758

0.00000000

1.81883240

0.04890629

3.63465516

5.45198275

3.63465516

5.37810265

3.59929710

7.26931033

9.08663791

7.26931033

9.16756665

7.27351528

1.83408537

8.00000000

9.28504466

10.57008931

11.66961778

13.21054164

8.00000000

9.28504466

10.57008931

11.97605333

13.20363824

8.00000000

9.28504466

10.57008931

11.98232177

13.01889602

8.00000000

9.28504466

10.57008931

11.81576172

13.20893758

8.00000000

9.28504466

10.57008931

11.95368775

13.19528735

8.00000000

9.28504466

10.57008931

11.95752554

13.01809186

8.00000000

9.28504466

10.57008931

11.81256056

13.08974958

8.00000000

9.28504466

10.57008931

11.94845091

13.08718790

8.00000000

9.28504466

10.57008931

11.95128747

13.00294110

13.62914649

$0.0 \quad 0.00 .021 .14017862672867 "$ Proper
Lattice $=" 7.710267940093007 \quad 0.0 \quad 0.0 \quad 0.0 \quad 10.90396549041$

ties=species:S:1:pos:R:3 pbc="T T T"

$\begin{array}{lll}\mathrm{Cu} & 0.00000000 & 0.00000000\end{array}$

$\mathrm{Cu} \quad 1.28504466 \quad 1.81732758$

$\mathrm{Cu} \quad 0.00000000 \quad 0.00000000$

$\mathrm{Cu} \quad 1.31077100 \quad 1.80010741$

$\mathrm{Cu} \quad-0.05679819 \quad 0.38091280$

$\mathrm{Cu} \quad 0.00000000$

0.38091280

$1.28504466 \quad 5.45198275$

$0.00000000 \quad 3.63465516$

8.00000000

9.28504466

10.57008931

11.68866973

13.24228061

8.00000000

9.28504466

10.57008931

11.98926620

5.36915673

$0.0 \quad 0.0 \quad 0.021 .14017862672867 "$ Proper

$\mathrm{Cu}$

1. 31298723 
$\mathrm{Cu}$

$\mathrm{Cu}$

$\mathrm{Cu}$

$\mathrm{Cu}$

$\mathrm{Cu}$

$\mathrm{Cu}$

$\mathrm{Cu}$

$\mathrm{Cu}$

$\mathrm{Cu}$

$\mathrm{Cu}$

$\mathrm{Cu}$

$\mathrm{Cu}$

$\mathrm{Cu}$

$\mathrm{Cu}$

$\mathrm{Cu}$

$\mathrm{Cu}$

$\mathrm{Cu}$

$\mathrm{Cu}$

$\mathrm{Cu}$

$\mathrm{Cu}$

$\mathrm{Cu}$

$\mathrm{Cu}$

$\mathrm{Cu}$

$\mathrm{Cu}$

$\mathrm{Cu}$

$\mathrm{Cu}$

$\mathrm{Cu}$

$\mathrm{Cu}$

$\mathrm{Cu}$

$\mathrm{Cu}$

$\mathrm{Cu}$

$\mathrm{Cu}$

$\mathrm{Cu}$

$\mathrm{Cu}$

$\mathrm{Cu}$

$\mathrm{Cu}$

$\mathrm{N}$

$\mathrm{N}$
2. 62880927

0.02537701

0.00000000

1.28504466

0.00000000

1.22998537

$-0.01799061$

2.57008931

3.85513397

2.57008931

3.87274567

2. 41228406

2.57008931

3.85513397

2.57008931

3.81443960

2.57891460

2.57008931

3.85513397

2.57008931

3.90062569

2.56885443

5.14017863

6.42522328

5.14017863

6.36376558

5.16277602

5.14017863

6.42522328

5.14017863

6.46813291

5.14300609

5.14017863

6.42522328

5.14017863

6.45203158

5.18518201

1.33906495
Fri Apr 16 14:51:55 2021

3.33852995

7.26931033

9.08663791

7.26931033

9.20455069

7.23452446

0.00000000

1.81732758

0.00000000

1.79593925

0.21185007

3.63465516

5.45198275

3.63465516

5.38745618

3.38996262

7.26931033

9.08663791

7.26931033

9.16649449

7.32939945

0.00000000

1.81732758

0.00000000

1.83205634

0.08740339

3.63465516

5.45198275

3.63465516

5.35424451

3.58628776

7.26931033

9.08663791

7.26931033

9.16974248

7.23938152

1.86521328

9.23475132
13.20309856

8.00000000

9.28504466

10.57008931

11.99248406

13.02488608

8.00000000

9.28504466

10.57008931

11.84122940

13.42755503

8.00000000

9.28504466

10.57008931

11.97135229

13.21868748

8.00000000

9.28504466

10.57008931

11.92737025

13.11411158

8.00000000

9.28504466

10.57008931

11.79213907

13.10770782

8.00000000

9.28504466

10.57008931

11.95775011

13.08923283

8.00000000

9.28504466

10.57008931

11.92840576

13.01188769

13.65541687

13.37536049

48

Lattice $=" 7.710267940093007 \quad 0.0 \quad 0.0 \quad 0.0 \quad 10.90396549041$

ties=species:S:1:pos:R:3 pbc="T T T"

$\mathrm{Cu} \quad 0.00000000$

$\mathrm{Cu} \quad 1.28504466 \quad 1.81732758$

$\mathrm{Cu} \quad 0.00000000 \quad 0.00000000$

$\begin{array}{lll}\mathrm{Cu} & 1.32337405 & 1.81732758\end{array}$

$\mathrm{Cu} \quad-0.04785218 \quad 0.39142557$

$\mathrm{Cu} \quad 0.00000000$

$\mathrm{Cu} \quad 1.28504466$

3. 63465516

5.45198275

0.00000000

3.63465516

$\begin{array}{lr}\mathrm{Cu} & 1.25217644 \\ \mathrm{Cu} & -0.04785218\end{array}$

5.37893780

3.24322960

0.00000000

1.28504466

0.00000000

7.26931033

9.08663791

7.26931033

1. 25217644

$-0.02699141$

2.57008931

9.15968285

7. 26931033

0.00000000

3. 85513397

1. 81732758

2. 57008931

0.00000000

3.82286355

1.81732758

2.40842269

0.11778775

2. 57008931

3. 85513397

3.63465516

5.45198275

3. 63465516

5.40695447

3. 89351295

2. 40842269

3.51686742

7. 26931033

\author{
8.00000000 \\ 9.28504466 \\ 10.57008931 \\ 11.69938692 \\ 13.22675177 \\ 8.00000000 \\ 9.28504466 \\ 10.57008931 \\ 11.97439874 \\ 13.22675177 \\ 8.00000000 \\ 9.28504466 \\ 10.57008931 \\ 11.97439874 \\ 13.04177948 \\ 8.00000000 \\ 9.28504466 \\ 10.57008931 \\ 11.88499441 \\ 13.44843578 \\ 8.00000000 \\ 9.28504466 \\ 10.57008931 \\ 11.93779012 \\ 13.44843578 \\ 8.00000000
}




\section{$\mathrm{Cu}$}

$\mathrm{Cu}$

$\mathrm{Cu}$

$\mathrm{Cu}$

$\mathrm{Cu}$

$\mathrm{Cu}$

$\mathrm{Cu}$

$\mathrm{Cu}$

$\mathrm{Cu}$

$\mathrm{Cu}$

$\mathrm{Cu}$

$\mathrm{Cu}$

$\mathrm{Cu}$

$\mathrm{Cu}$

$\mathrm{Cu}$

$\mathrm{Cu}$

$\mathrm{Cu}$

$\mathrm{Cu}$

$\mathrm{Cu}$

$\mathrm{N}$

$\mathrm{N}$

$\mathrm{N}$
3. 85513397

2. 57008931

3.89351295

2. 62395017

5.14017863

6.42522328

5.14017863

6.33273342

5.15985001

5.14017863

6.42522328

5.14017863

6.46004217

5.15985001

5.14017863

6.42522328

5.14017863

6.46004217

5.22597921

1. 46841960

2. 61359267

2. 61359267
Fri Apr 16 14:51:55 2021

9.08663791

7.26931033

9.13166619

7.26931033

0.00000000

1. 81732758

0.00000000

1.81732758

0.08854593

3.63465516

5.45198275

3.63465516

5.35785005

3.54610923

7.26931033

9.08663791

7.26931033

9.18077061

7.26931033

1.81732758

9.14060315

5.39801750
9.28504466

10.57008931

11.93779012

13.37641078

8.00000000

9.28504466

10.57008931

11.78028285

13.10496258

8.00000000

9.28504466

10.57008931

11.93377771

13.10496258

8.00000000

9.28504466

10.57008931

11.93377771

13.02859584

13.64465334

13.37945907

13.37945907

./nitrogen/Cu/110/iter_4/struct.xyz

49

Lattice $=" 7.710267940093007 \quad 0.0 \quad 0.0 \quad 0.0 \quad 10.90396549041$

ties $=$ species:S:1:pos:R:3 pbc="T T T"

$\mathrm{Cu} \quad 0.00000000$

1.28504466

0.00000000

0.00000000

1.81732758

$\mathrm{Cu}$

1. 31519306

0.00000000

$-0.05160787$

1.81732758

0.28532900

0.00000000

3.63465516

1.28504466

5.45198275

0.00000000

3.63465516

1.21872100

5. 36814290

$-0.05160787$

0.00000000

3. 34932617

7.26931033

1.28504466

9.08663791

0.00000000

1. 21872100

7.26931033

9.17047776

7.26931033

0.00000000

2. 57008931

3. 85513397

1. 81732758

2. 57008931

0.00000000

1.81732758

3. 82266829

2. 56530101

0.00149275

2. 57008931

3. 85513397

2. 57008931

3. 91624824

2. 56530101

2. 57008931

3. 85513397

2. 57008931

3. 91624824

2. 56691970

5. 14017863

6.42522328

5. 14017863

6.42278924

5.18431905

5.14017863

6.42522328

5.14017863

3.63465516

5.45198275

3.63465516

5. 37015105

3. 63316241

7.26931033

9.08663791

7.26931033

9.16846961

7.26931033

0.00000000

1.81732758

0.00000000

1.81732758

0.28021689

3.63465516

5. 45198275

3.63465516

5. 35266780

3. 35443827

7.26931033

8.00000000

9.28504466

10.57008931

11.83242118

13.21898760

8.00000000

9.28504466

10.57008931

11.98599375

13.21898760

8.00000000

9.28504466

10.57008931

11.98599375

13.03214399

8.00000000

9.28504466

10.57008931

11.83156320

13.41784592

8.00000000

9.28504466

10.57008931

11.98643576

13.41784592

8.00000000

9.28504466

10.57008931

11.98643576

13.35422714

8.00000000

9.28504466

10.57008931

11.65843794

13.21744574

8.00000000

9.28504466

10.57008931

11.92790207

13. 21744574

8.00000000

9.28504466

$0.00 .00 .021 .14017862672867 "$ Proper

5.18431905

5.14017863

9.08663791

6.42522328 


\section{$\mathrm{Cu}$}

$\mathrm{Cu}$

$\mathrm{Cu}$

$\mathrm{N}$

$\mathrm{N}$

$\mathrm{N}$

$\mathrm{N}$

6.41946618

.$/$

50

gen/cu/110/iter_5/struct.xyz

tice $=" 7.7102679400930$

ties=species:S:1:pos:R:3 pbc="T T T"

$\mathrm{Cu}$

$\mathrm{Cu}$

$\mathrm{Cu}$

$\mathrm{Cu}$

$\mathrm{Cu}$

$\mathrm{Cu}$

$\mathrm{Cu}$

$\mathrm{Cu}$

$\mathrm{Cu}$

$\mathrm{Cu}$

$\mathrm{Cu}$

$\mathrm{Cu}$

$\mathrm{Cu}$

$\mathrm{Cu}$

$\mathrm{Cu}$

$\mathrm{Cu}$

$\mathrm{Cu}$

$\mathrm{Cu}$

$\mathrm{Cu}$

$\mathrm{Cu}$

$\mathrm{Cu}$

$\mathrm{Cu}$

$\mathrm{Cu}$

$\mathrm{Cu}$

$\mathrm{Cu}$

$\mathrm{Cu}$

$\mathrm{Cu}$

$\mathrm{Cu}$

$\mathrm{Cu}$

$\mathrm{Cu}$

$\mathrm{Cu}$

$\mathrm{Cu}$

$\mathrm{Cu}$

$\mathrm{Cu}$

$\mathrm{Cu}$

$\mathrm{Cu}$

$\mathrm{Cu}$

$\mathrm{Cu}$

$\mathrm{Cu}$

$\mathrm{Cu}$

$\mathrm{Cu}$

$\mathrm{Cu}$

$\mathrm{Cu}$

$\mathrm{Cu}$

$\mathrm{Cu}$

$\mathrm{N}$

N

N

N

$\mathrm{N}$

. /

51

Lattice $=" 7.7102679400930070 .00 .00 .0$

ties=species:S:1:pos:R:3 pbc="T T T"

1. 28504466

$\mathrm{Cu} \quad 0.00000000$

1.81732758

0.00000000

\section{3}

10.57008931

11.92790207

13.03389382

13.36350003

13.39627408

13.39627408

13.63094145
0.90396549041

8.00000000
9.28504466

10.57008931

11.80105648

13.92374494

8.00000000

9.28504466

10.57008931

12.18983273

13.67436503

8.00000000

9.28504466

10.57008931

11.73347745

13.41657772

8.00000000

9.28504466

10.57008931

11.84547870

13.53660311

8.00000000

9.28504466

10.57008931

11.74849947

13.57779319

8.00000000

9.28504466

10.57008931

12.24931030

13.50126598

8.00000000

9.28504466

10.57008931

11.79785060

13.17357042

8.00000000

9.28504466

10.57008931

11.71233734

13.40659230

8.00000000

9.28504466

10.57008931

11.87386608

13.13890034

13.70701089

13.38515675

13.42503276

13.71346007

13.41859923

$0.00 .00 .021 .14017862672867 "$ Proper 
$\mathrm{Cu}$

$\mathrm{Cu}$

$\mathrm{Cu}$

$\mathrm{Cu}$

$\mathrm{Cu}$

$\mathrm{Cu}$

$\mathrm{Cu}$

$\mathrm{Cu}$

$\mathrm{Cu}$

$\mathrm{Cu}$

$\mathrm{Cu}$

$\mathrm{Cu}$

$\mathrm{Cu}$

$\mathrm{Cu}$

$\mathrm{Cu}$

$\mathrm{Cu}$

$\mathrm{Cu}$

$\mathrm{Cu}$

$\mathrm{Cu}$

$\mathrm{Cu}$

$\mathrm{Cu}$

$\mathrm{Cu}$

$\mathrm{Cu}$

$\mathrm{Cu}$

$\mathrm{Cu}$

$\mathrm{Cu}$

$\mathrm{Cu}$

$\mathrm{Cu}$

$\mathrm{Cu}$

$\mathrm{Cu}$

$\mathrm{Cu}$

$\mathrm{Cu}$

$\mathrm{Cu}$

$\mathrm{Cu}$

$\mathrm{Cu}$

$\mathrm{Cu}$

$\mathrm{Cu}$

$\mathrm{Cu}$

$\mathrm{Cu}$

$\mathrm{Cu}$

$\mathrm{Cu}$

$\mathrm{Cu}$

$\mathrm{N}$

$\mathrm{N}$

$\mathrm{N}$

$\mathrm{N}$

$\mathrm{N}$

$\mathrm{N}$
1.30700129

0.73387714

0.00000000

1.28504466

0.00000000

1.24466736

$-0.42286732$

0.00000000

1. 28504466

0.00000000

1.30998068

$-0.61282061$

2.57008931

3. 85513397

2.57008931

3. 81559588

2.64335465

2.57008931

3. 85513397

2. 57008931

3. 90486657

2. 62737574

2. 57008931

3. 85513397

2.57008931

3. 83436112

2.83215667

5.14017863

6.42522328

5.14017863

6.46257353

6.55394739

5. 14017863

6.42522328

5. 14017863

6.37296018

5. 06366101

5.14017863

6.42522328

5.14017863

6.43021536

4. 70807298

2. 52749096

2.83716902

2.76055351

6.58155102

$-0.21773837$

6.54948474
Fri Apr 16 14:51:55 2021

1.81213361

1.87852089

3. 63465516

5.45198275

3.63465516

5.52313386

3. 98917884

7.26931033

9.08663791

7.26931033

9.02507944

7. 54909168

0.00000000

1. 81732758

0.00000000

1. 74220192

$-0.04962061$

3.63465516

5.45198275

3.63465516

5.38283755

3. 60872478

7.26931033

9.08663791

7. 26931033

9. 09017288

7.22294148

0.00000000

1.81732758

0.00000000

1.80727553

0.24659578

3.63465516

5.45198275

3.63465516

5.46306520

3. 34234009

7.26931033

9.08663791

7.26931033

9.18991109

9.12820204

1.77176549

9.04062226

5.41647910

2.17922922

5.78647005

9.29925722
11.74637799

14.07071844

8.00000000

9.28504466

10.57008931

12.08724742

13.66988551

8.00000000

9.28504466

10.57008931

12.01128409

13.57549369

8.00000000

9.28504466

10.57008931

11.86048810

13.39613583

8.00000000

9.28504466

10.57008931

11.85608120

13.40321469

8.00000000

9.28504466

10.57008931

11.71264405

13.38030585

8.00000000

9.28504466

10.57008931

11.82454769

13.79467190

8.00000000

9.28504466

10.57008931

11.82961814

13.21253034

8.00000000

9.28504466

10.57008931

11.84923682

13.93216386

13.43659807

13.47771629

13.37308114

13.82260170

13.35126793

13.82235996

52

Lattice $=" 7.710267940093007 \quad 0.0 \quad 0.0 \quad 0.0 \quad 10.90396549041$ ties=species:S:1:pos:R:3 pbc="T T T"

$\mathrm{Cu} \quad 0.00000000 \quad 0.00000000$

$\mathrm{Cu} \quad 1.28504466 \quad 1.81732758$

$\mathrm{Cu} \quad 0.00000000 \quad 0.00000000$

$\mathrm{Cu} \quad 1.28289727 \quad 1.89888793$

$\mathrm{Cu} \quad-0.30180630$

$\mathrm{Cu} \quad 0.00000000$

$-0.00293475$

3.63465516

1.28504466

0.00000000

1.28286819

5.45198275

3.63465516

5.44914172

$-0.34150204$

0.00000000

1.28504466

3.61948262

7.26931033

9.08663791

0.00000000

1.28307659

7.26931033

9.00478379

7.27645902

$-0.33536910$

0.00000000

8.00000000

9.28504466

10.57008931

11.89478361

13.69639408

8.00000000

9.28504466

10.57008931

12.08273368

13.45003230

8.00000000

9.28504466

10.57008931

11.89724504

13.44435007

8.00000000

$0.00 .00 .021 .14017862672867 "$ Proper

2. 57008931 


\section{$\mathrm{Cu}$}

$\mathrm{Cu}$

$\mathrm{Cu}$

$\mathrm{Cu}$

$\mathrm{Cu}$

$\mathrm{Cu}$

$\mathrm{Cu}$

$\mathrm{Cu}$

$\mathrm{Cu}$

$\mathrm{Cu}$

$\mathrm{Cu}$

$\mathrm{Cu}$

$\mathrm{Cu}$

$\mathrm{Cu}$

$\mathrm{Cu}$

$\mathrm{Cu}$

$\mathrm{Cu}$

$\mathrm{Cu}$

$\mathrm{Cu}$

$\mathrm{Cu}$

$\mathrm{Cu}$

$\mathrm{Cu}$

$\mathrm{Cu}$

$\mathrm{Cu}$

$\mathrm{Cu}$

$\mathrm{Cu}$

$\mathrm{Cu}$

$\mathrm{Cu}$

$\mathrm{Cu}$

$\mathrm{N}$

$\mathrm{N}$

$\mathrm{N}$

$\mathrm{N}$

$\mathrm{N}$

$\mathrm{N}$

$\mathrm{N}$

.$/$

53

$\begin{array}{llll}\text { Lattice }=" 7.710267940093007 & 0.0 & 0.0 & 0.0 \\ \text { ties }=\text { species:S: } 1: \text { pos:R:3 } & \text { pbc="T T T" } \\ \mathrm{Cu} & 0.00000000 & 0.00000000\end{array}$

$\mathrm{Cu} \quad 1.28504466 \quad 1.81732758$

$\mathrm{Cu} \quad 0.00000000$

$\mathrm{Cu} \quad 1.29914594$

$\mathrm{Cu} \quad 0.00981405$

$\mathrm{Cu} \quad 0.00000000$

$\mathrm{Cu} \quad 1.28504466$

$\mathrm{Cu} \quad 0.00000000$

$\mathrm{Cu} \quad 1.29847799$

$\mathrm{Cu} \quad-0.03682062$

$\mathrm{Cu} \quad 0.00000000$

$\mathrm{Cu} \quad 1.28504466$

$\mathrm{Cu} \quad 0.00000000$

$\mathrm{Cu} \quad 1.24408546$

$\mathrm{Cu} \quad-0.08638118$

$\mathrm{Cu} \quad 2.57008931$

$\mathrm{Cu} \quad 3.85513397$

$\mathrm{Cu} \quad 2.57008931$

$\mathrm{Cu} \quad 3.84103269$

$\mathrm{Cu} \quad 2.57008931$

$\mathrm{Cu} \quad 2.57008931$

$\mathrm{Cu} \quad 3.85513397$

$\mathrm{Cu} \quad 2.57008931$

$\mathrm{Cu} \quad 3.84170064$

$\mathrm{Cu} \quad 2.57008931$

$\mathrm{Cu} \quad 2.57008931$

$\mathrm{Cu} \quad 3.85513397$

$\mathrm{Cu} \quad 2.57008931$
Fri Apr 16 14:51:55 2021

25

1.81732758

0.00000000

1.86021509

0.00198159

3.63465516

5.45198275

3.63465516

5.45057444

3.62797462

7.26931033

9.08663791

7.26931033

9.04841464

7.28337226

0.00000000

1.81732758

0.00000000

1.85520619

0.00234443

3.63465516

5.45198275

3.63465516

5.45069692

2.05572812

7.26931033

9.08663791

7.26931033

9.05020633

8.85050097

0.00146983

9.04742296

5.45618249

1.85832807

5.44832705

9.03771825

1.86462620

1.83290130

0.27025263

3.63465516

5.45198275

3.63465516

5. 37723318

3. 23298172

7.26931033

9.08663791

7.26931033

9.11669356

7.03725591

0.00000000

1.81732758

0.00000000

1.83290130

0.09664344

3.63465516

5.45198275

3.63465516

5.37723318

3. 39302739

7.26931033

9.08663791

7.26931033
9.28504466

10.57008931

11.83658952

8.00000000

9.28504466

10.57008931

11.80221767

13.45261389

8.00000000

9.28504466

10.57008931

13.44813517

8.00000000

9.28504466

10.57008931

11.83725063

12.89245368

8.00000000

9.28504466

10.57008931

11.80013842

14.03573006

8.00000000

9.28504466

10.57008931

11.83684902

14.03505406

12.42248756

13.80577241

13.33774492

13.81263507

13.33620541

13.81175712

13.80937062
11.83400686

0.90396549041

8.00000000

9.28504466

10.57008931

11.74541723

13.34375139

8.00000000

10.57008931

11.90192941

13.68455170

8.00000000

9.28504466

10.57008931

12.00471322

13.44041222

8.00000000

9.28504466

10.57008931

11.74541723

13.58052626

8.00000000

9.28504466

10.57008931

11.90192941

13.59843453

8.00000000

9.28504466

10.57008931
13.68986321

$0.00 .00 .021 .14017862672867 "$ Proper

9.28504466 


\section{$\mathrm{Cu}$}

$\mathrm{Cu}$

$\mathrm{Cu}$

$\mathrm{Cu}$

$\mathrm{Cu}$

$\mathrm{Cu}$

$\mathrm{Cu}$

$\mathrm{Cu}$

$\mathrm{Cu}$

$\mathrm{Cu}$

$\mathrm{Cu}$

$\mathrm{Cu}$

$\mathrm{Cu}$

$\mathrm{Cu}$

$\mathrm{Cu}$

$\mathrm{Cu}$

$\mathrm{Cu}$

$\mathrm{N}$

$\mathrm{N}$

$\mathrm{N}$

$\mathrm{N}$

$\mathrm{N}$

$\mathrm{N}$

$\mathrm{N}$

$\mathrm{N}$

37

Lattice $=" 7.710267940093008 \quad 0.0 \quad 0.0 \quad 3.855133970046504$

411122603" Properties=species:S:1:pos:R:3 pbc="T T T"

$\mathrm{Cu}$

$\mathrm{Cu}$

$\mathrm{Cu}$

$\mathrm{Cu}$

$\mathrm{Cu}$

$\mathrm{Cu}$

$\mathrm{Cu}$

$\mathrm{Cu}$

$\mathrm{Cu}$

$\mathrm{Cu}$

$\mathrm{Cu}$

$\mathrm{Cu}$

$\mathrm{Cu}$

$\mathrm{Cu}$

$\mathrm{Cu}$

$\mathrm{Cu}$

$\mathrm{Cu}$

$\mathrm{Cu}$

$\mathrm{Cu}$

$\mathrm{Cu}$

$\mathrm{Cu}$

$\mathrm{Cu}$

$\mathrm{Cu}$

$\mathrm{Cu}$

$\mathrm{Cu}$

$\mathrm{Cu}$

$\mathrm{Cu}$

$\mathrm{Cu}$

$\mathrm{Cu}$

$\mathrm{Cu}$

$\mathrm{Cu}$

$\mathrm{Cu}$

$\mathrm{Cu}$

$\mathrm{Cu}$

$\mathrm{Cu}$

$\mathrm{Cu}$

$\mathrm{N}$
5.06563863

3.89609317

2.57008931

5.14017863

6.42522328

6.42522328

5.13036458

5.14017863

6.42522328

5.14017863

6.42522328

5.17699925

5.14017863

6.42522328

5.14017863

6.42522328

5.22655981

1.38469568

2.57008931

2.57008931

6.42522328

0.07453999

6.42522328

3.75548295

0.00000000

1.28504466

$-0.06600523$

1.28504466

2. 57008931

3.85328211

1. 28504466

2.57008931

3.85513397

5.14017863

2.56109167

2.57008931

3.85513397

5.14017863

2.63609455

3. 85513397

5.14017863

6.42707514

3.87709673

5.14017863

6.42522328

7.69946539

5.14017863

5.14017863

6.42522328

7.71888910

5.14017863

6.42522328

7.71026794

8.99531260

6.40326052

7.71026794

8.99531260

10.29115981

7.71926558

1.28504466
5.14017863

2.56146815
9.11669356

7.23523140

0.00000000

1.81732758

0.00000000

1.85285582

0.27025263

3.63465516

5. 45198275

3.63465516

5.26252970

3.23298172

7.26931033

9.08663791

7.26931033

9.19599602

7.03725591

1.73537251

9.10159094

5.33878697

1.68878838

5.12047188

8.47674113

1.73537251

5.12047188

struct. $x y z$
Fri Apr 16 14:51:55 2021
12.00471322

13.42945538

8.00000000

9.28504466

10.57008931

11.77694286

13.34375139

9.28504466

10.57008931

11.96729145

13.68455170

8.00000000

9.28504466

10.57008931

11.93586781

13.44041222

13.66446913

13.40583388

13.36841195

13.77494694

13.35191160

13.63398727

13.66446913

13.35191160
8.00000000
$0.00000000 \quad 8.00000000$

0.74192088

1.47886433

$-0.03810814$

2.22576264

2.96768351

3.71067356

2.30197891

5.19344615

5.94532189

4.45672006

0.00000000

0.74192088

$-0.03810814$

2. 22576264

2. 96768351

3. 71067356

2.23844284

4.45152527

5.19344615

5.94160389

4.42616485

0.00000000

0.74192088

1. 47886433

$-0.01038958$

2. 22576264

2. 96768351

3. 69713068

2. 23844284

4. 45152527

5.19344615

5.94160389

4.45672006

0.74192088
10.09846914

12.21299594

14.38385857

8.00000000

10.09846914

12.19086959

14.38385857

8.00000000

10.09846914

12.21299594

14.26112543

8.00000000

10.09846914

12.19086959

14.38385857

8.00000000

10.09846914

12.19086959

14.26768342

8.00000000

10.09846914

12.19883722

14.26768342

8.00000000

10.09846914

12.21299594

14.26112543

8.00000000

10.09846914

12.19883722

14.26768342

8.00000000

10.09846914

12.19883722

14.26112543
4.45152527

1. 48170342

15.36855710 38 
Lattice $=" 7.710267940093008 \quad 0.0 \quad 0.0 \quad 3.855133970046504 \quad 6.67728790610526 \quad 0.0 \quad 0.0 \quad 0.0 \quad 22.295407$ 411122603" Properties=species:S:1:pos:R:3 pbc="T T T"

\begin{tabular}{|c|c|c|c|}
\hline $\mathrm{Cu}$ & 0.00000000 & 0.00000000 & 8.00000000 \\
\hline $\mathrm{Cu}$ & 1.28504466 & 0.74192088 & 10.09846914 \\
\hline $\mathrm{Cu}$ & 2.57008931 & 1.48384176 & 12.19654159 \\
\hline $\mathrm{Cu}$ & -0.02491361 & -0.01438388 & 14.22548956 \\
\hline $\mathrm{Cu}$ & 1.28504466 & 2.22576264 & 8.00000000 \\
\hline $\mathrm{Cu}$ & 2.57008931 & 2.96768351 & 10.09846914 \\
\hline $\mathrm{Cu}$ & 3.86446643 & 3.70746613 & 12.19200338 \\
\hline $\mathrm{Cu}$ & 1.21101939 & 2.26850114 & 14.38002096 \\
\hline $\mathrm{Cu}$ & 2.57008931 & 4.45152527 & 8.00000000 \\
\hline $\mathrm{Cu}$ & 3.85513397 & 5.19344615 & 10.09846914 \\
\hline $\mathrm{Cu}$ & 5.14669665 & 5.92835401 & 12.19200338 \\
\hline $\mathrm{Cu}$ & 2.57008931 & 4.48029303 & 14.22548956 \\
\hline $\mathrm{Cu}$ & 2.57008931 & 0.00000000 & 8.00000000 \\
\hline $\mathrm{Cu}$ & 3.85513397 & 0.74192088 & 10.09846914 \\
\hline $\mathrm{Cu}$ & 5.14299306 & 1.49299303 & 12.19200338 \\
\hline $\mathrm{Cu}$ & 2.57008931 & -0.08547701 & 14.38002096 \\
\hline $\mathrm{Cu}$ & 3.85513397 & 2.22576264 & 8.00000000 \\
\hline $\mathrm{Cu}$ & 5.14017863 & 2.96768351 & 10.09846914 \\
\hline $\mathrm{Cu}$ & 6.42522328 & 3.70960439 & 12.17749534 \\
\hline $\mathrm{Cu}$ & 3.92915923 & 2.26850114 & 14.38002096 \\
\hline $\mathrm{Cu}$ & 5.14017863 & 4.45152527 & 8.00000000 \\
\hline $\mathrm{Cu}$ & 6.42522328 & 5.19344615 & 10.09846914 \\
\hline $\mathrm{Cu}$ & 7.70374992 & 5.92835401 & 12.19200338 \\
\hline $\mathrm{Cu}$ & 5.08794968 & 4.48167967 & 14.32031981 \\
\hline $\mathrm{Cu}$ & 5.14017863 & 0.00000000 & 8.00000000 \\
\hline $\mathrm{Cu}$ & 6.42522328 & 0.74192088 & 10.09846914 \\
\hline $\mathrm{Cu}$ & 7.70745350 & 1.49299303 & 12.19200338 \\
\hline $\mathrm{Cu}$ & 5.16509224 & -0.01438388 & 14.22548956 \\
\hline $\mathrm{Cu}$ & 6.42522328 & 2.22576264 & 8.00000000 \\
\hline $\mathrm{Cu}$ & 7.71026794 & 2.96768351 & 10.09846914 \\
\hline $\mathrm{Cu}$ & 8.98598014 & 3.70746613 & 12.19200338 \\
\hline $\mathrm{Cu}$ & 6.42522328 & 2.16545385 & 14.32031981 \\
\hline $\mathrm{Cu}$ & 7.71026794 & 4.45152527 & 8.00000000 \\
\hline $\mathrm{Cu}$ & 8.99531260 & 5.19344615 & 10.09846914 \\
\hline $\mathrm{Cu}$ & 10.28035725 & 5.93536703 & 12.1853137 \\
\hline $\mathrm{Cu}$ & 7.76249688 & 4.48167967 & 14.3203198 \\
\hline $\mathrm{N}$ & 2.57008931 & 1.48384176 & 15.3167055 \\
\hline $\mathrm{N}$ & 6.42522328 & 3.70960439 & 15.3385328 \\
\hline
\end{tabular}

./nitrogen/Cu/111/iter_3/struct.xyz

39

Lattice $=" 7.710267940093008 \quad 0.0 \quad 0.0 \quad 3.855133970046504 \quad 6.67728790610526 \quad 0.0 \quad 0.0 \quad 0.0 \quad 22.295407$

411122603" Properties=species:S:1:pos:R:3 pbc="T T T"

$\mathrm{Cu} \quad 0.00000000$

$\mathrm{Cu} \quad 1.28504466$

$\mathrm{Cu} \quad 2.57079522$

$\mathrm{Cu} \quad-0.11114249$

$\mathrm{Cu} \quad 1.28504466$

$\mathrm{Cu} \quad 2.57008931$

$\mathrm{Cu} \quad 3.91936644$

$\mathrm{Cu} \quad 0.67494739$

$\mathrm{Cu} \quad 2.57008931$

$\mathrm{Cu} \quad 3.85513397$

$\mathrm{Cu} \quad 5.14716177$

$\mathrm{Cu} \quad 2.57448435$

$\mathrm{Cu} \quad 2.57008931$

$\mathrm{Cu} \quad 3.85513397$

$\mathrm{Cu} \quad 5.16184946$

$\mathrm{Cu} \quad 2.57831370$

$\mathrm{Cu} \quad 3.85513397$

$\mathrm{Cu} \quad 5.14017863$

$\mathrm{Cu} \quad 6.42718468$

$\mathrm{Cu} \quad 4.47911603$

$\mathrm{Cu} \quad 5.14017863$

$\mathrm{Cu} \quad 6.42522328$

$\mathrm{Cu} \quad 7.70323329$

$\mathrm{Cu} \quad 5.05283818$
$0.00000000 \quad 8.00000000$

$0.74192088 \quad 10.09846914$

1.52767104

$-0.10335140$

2.22576264

2.96768351

3. 69720212

2.14159802

4. 45152527

5.19344615

5.96293185

4.04001354

0.00000000

0.74192088

1.44683747

0.13142737

2.22576264

2.96768351

3. 68727224

2.14594503

4.45152527

5.19344615

5.96154701

4.61225301
12.38297789

14.30756428

8.00000000

10.09846914

12.15656097

14.45135130

8.00000000

10.09846914

12.17742596

14.23459430

8.00000000

10.09846914

12.13174906

14.76673135

8.00000000

10.09846914

12.22509404

14.44300258

8.00000000

10.09846914

12.18145402

14.21909811 


\section{$\mathrm{Cu}$}

$\mathrm{Cu}$

$\mathrm{Cu}$

$\mathrm{Cu}$

$\mathrm{Cu}$

$\mathrm{Cu}$

$\mathrm{Cu}$

$\mathrm{Cu}$

$\mathrm{Cu}$

$\mathrm{Cu}$

$\mathrm{Cu}$

$\mathrm{Cu}$

$\mathrm{N}$

$\mathrm{N}$

$\mathrm{N}$

.$/$

40

/rogen/Cu/111/iter_4/struct. $x y z$
5.14017863

6.42522328

7.68808364

5.26789723

6.42522328

7.71026794

8.93595044

6.43086400

7.71026794

8.99531260

10.28146461

7.81152642

2.57739353

6.42804302

6.43596758
Fri Apr 16 14:51:55 2021

0.00000000

0.74192088

1. 45098056

$-0.09824307$

2.22576264

2. 96768351

3. 69712949

3.15889080

4.45152527

5.19344615

5.83078192

4.61586475

2.01649993

5.05237725

1.41958391
8.00000000

10.09846914

12.13721009

14.30990837

8.00000000

10.09846914

12.15835033

15.59349164

8.00000000

10.09846914

12.20900043

14.22444703

14.36877475

15.48073811

14.88161788

$411122603 "$ Properties=species: $:$ : $1:$ pos:R:3 pbc="T T"

$\mathrm{Cu}$

$\mathrm{Cu}$

$\mathrm{Cu}$

$\mathrm{Cu}$

$\mathrm{Cu}$

$\mathrm{Cu}$

$\mathrm{Cu}$

$\mathrm{Cu}$

$\mathrm{Cu}$

$\mathrm{Cu}$

$\mathrm{Cu}$

$\mathrm{Cu}$

$\mathrm{Cu}$

$\mathrm{Cu}$

$\mathrm{Cu}$

$\mathrm{Cu}$

$\mathrm{Cu}$

$\mathrm{Cu}$

$\mathrm{Cu}$

$\mathrm{Cu}$

$\mathrm{Cu}$

$\mathrm{Cu}$

$\mathrm{Cu}$

$\mathrm{Cu}$

$\mathrm{Cu}$

$\mathrm{Cu}$

$\mathrm{Cu}$

$\mathrm{Cu}$

$\mathrm{Cu}$

$\mathrm{Cu}$

$\mathrm{Cu}$

$\mathrm{Cu}$

$\mathrm{Cu}$

$\mathrm{Cu}$

$\mathrm{Cu}$

$\mathrm{Cu}$

$\mathrm{N}$

N

$\mathrm{N}$

$\mathrm{N}$

9.76798793

. $/$

0.00000000

1.28504466

2. 61482720

$-0.57124011$

1.28504466

2. 57008931

3. 85513397

1. 67949037

2. 57008931

3. 85513397

5.15620821

2.49987071

2. 57008931

3. 85513397

5. 16671541

2. 45071207

3. 85513397

5.14017863

6.38630265

4. 12181663

5.14017863

6.42522328

7. 71026794

4.94371457

5. 14017863

6.42522328

7.71026794

4. 84449946

6.42522328

7.71026794

8. 95274624

6.60201768

7. 71026794

8.99531260

10.27454000

8.12532435

3. 25769749

6.24998443

7.71026794

41

gen/cu/111/iter_5/struct.xyz

Lattice $=" 7.710267940093008 \quad 0.0 \quad 0.0 \quad 3.855133970046504$

411122603" Properties=species:S:1:pos:R:3 pbc="T T T"

$\mathrm{Cu}$

$\mathrm{Cu}$

$\mathrm{Cu}$

$\mathrm{Cu}$

0.00000000

1.28504466

2. 56014193

0.35251794

1.28504466

0.00000000

0.74192088

1.50157072

0.50168180

2. 22576264
8.00000000

10.09846914

12.21498165

14.64461727

8.00000000

10.09846914

12.13730307

14.64461727

8.00000000

10.09846914

12.15685883

14.18788688

8.00000000

10.09846914

12.15685883

15.45457996

8.00000000

10.09846914

14.18788688

8.00000000

10.09846914

12.26515841

14.18788688

8.00000000

10.09846914

12.11239924

15.45457996

8.00000000

10.09846914

12.15685883

14.64461727

8.00000000

10.09846914

12.21498165

15.45457996

15.50602074

15.50602074

13.97477815
12.21498165

15.50602074

$\mathrm{Cu}$

2. 22576264

2.29865048

5.19344615

1.28919939

4.40241173

1.48384176

$6.67728790610526 \quad 0.0 \quad 0.0 \quad 0.0 \quad 22.295407$

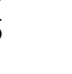




\section{$\mathrm{Cu}$}

$\mathrm{Cu}$

$\mathrm{Cu}$

$\mathrm{Cu}$

$\mathrm{Cu}$

$\mathrm{Cu}$

$\mathrm{Cu}$

$\mathrm{Cu}$

$\mathrm{Cu}$

$\mathrm{Cu}$

$\mathrm{Cu}$

$\mathrm{Cu}$

$\mathrm{Cu}$

$\mathrm{Cu}$

$\mathrm{Cu}$

$\mathrm{Cu}$

$\mathrm{Cu}$

$\mathrm{Cu}$

$\mathrm{Cu}$

$\mathrm{Cu}$

$\mathrm{Cu}$

$\mathrm{Cu}$

$\mathrm{Cu}$

$\mathrm{Cu}$

$\mathrm{Cu}$

$\mathrm{Cu}$

$\mathrm{Cu}$

$\mathrm{Cu}$

$\mathrm{Cu}$

$\mathrm{Cu}$

$\mathrm{Cu}$

$\mathrm{N}$

$\mathrm{N}$

$\mathrm{N}$

$\mathrm{N}$

$\mathrm{N}$

. /

42

/nitrogen/Cu/111/iter_6/struct.xyz

2. 57008931

3. 84475393

1.10467069

2. 57008931

3.85513397

5.18361678

3.03858474

2. 57008931

3. 85513397

5.07275780

2. 80597122

3. 85513397

5.14017863

6.35377575

3. 56701251

5. 14017863

6.42522328

7. 73827732

5.05960851

5.14017863

6.42522328

7. 70131634

5.39838885

6.42522328

7. 71026794

9.01564002

5.81449513

7.71026794

10.35672969

7.55495615

1.87442026

4.70774118

6.42522328

8. 83837444

2. 57008931
Fri Apr 16 14:51:55 2021

8.99531260
2.96768351

3. 69212523

2. 66259560

4. 45152527

5.19344615

5.99278850

4. 38931723

0.00000000

0.74192088

1.43457990

2. 22576264

2. 96768351

3. 71851220

1.85113772

4.45152527

5.19344615

5.86903775

4.67738106

0.00000000

0.74192088

1.56686084

$-0.55613039$

2. 22576264

2. 96768351

3. 70935459

2. 28021122

4.45152527

5.19344615

5.90160980

4.26882161

0.90098047

3.39856797

0.74192088

4.60350211

2. 96768351
$-0.04315212$
10.09846914

12.18291498

15.67740966

8.00000000

10.09846914

12.17457496

15.67740966

8.00000000

10.09846914

12.21202609

14.21112306

8.00000000

10.09846914

12.17457496

15.67740966

8.00000000

10.09846914

12.17457496

14.21112306

8.00000000

10.09846914

12.21202609

14.52240458

8.00000000

10.09846914

12.18291498

14.52240458

8.00000000

10.09846914

12.21202609

14.21112306

15.53972848

15.53972848

13.58367567

15.53972848

16.85574221

Lattice $=" 7.710267940093008 \quad 0.0 \quad 0.0 \quad 3.855133970046504 \quad 6.67728790610526 \quad 0.0 \quad 0.0 \quad 0.0 \quad 22.295407$

411122603" Properties=species:S:1:pos:R:3 pbc="T T T"

$\begin{array}{llll}\mathrm{Cu} & 0.00000000 & 0.00000000 & 8.00000000\end{array}$

$\mathrm{Cu} \quad 1.28504466 \quad 0.74192088 \quad 10.09846914$

$\begin{array}{lll}\mathrm{Cu} & 2.57486330 & 1.48108549\end{array}$

$\mathrm{Cu} \quad 0.00000000 \quad-0.04873510$

$\mathrm{Cu} \quad 1.28504466 \quad 2.22576264$

$\begin{array}{lll}\mathrm{Cu} & 2.57008931 & 2.96768351\end{array}$

$\mathrm{Cu} \quad 3.85513397$

3.71511692

1.24283882

2.25013019

4.45152527

2.57008931

5.19344615

5.93261077

5.13540464

2.61229515

4.47589282

0.00000000

0.74192088

3. 85513397

1.48108549

0.02436755

2. 22576264

2.96768351

3.70684813

2. 17702753

4.45152527

5.19344615

5.94087955

12.20318181

14.34746655

8.00000000

10.09846914

12.20318181

14.34746655

8.00000000

10.09846914

12.20318181

14.34746655

8.00000000

10.09846914

12.20318181

14.34746655

8.00000000

10.09846914

12.20318181

14.34746655

8.00000000

10.09846914

12.20318181

14.34746655

8.00000000

10.09846914

12.20318181

0.74192088

1.48935428

0.02436755

14.34746655 


\section{$\mathrm{Cu}$}

$\mathrm{Cu}$

$\mathrm{Cu}$

$\mathrm{Cu}$

$\mathrm{Cu}$

$\mathrm{Cu}$

$\mathrm{Cu}$

$\mathrm{Cu}$

$\mathrm{N}$

$\mathrm{N}$

$\mathrm{N}$

$\mathrm{N}$

$\mathrm{N}$

$\mathrm{N}$
6.42522328

7.71026794

8.99053861

6.46742912

7.71026794

8.99531260

10.28513124

7.71026794

1.28504466

5.14017863

6.42522328

8.99531260

2.57008931

6.42522328
Fri Apr 16 14:51:55 2021

2.22576264

2. 96768351

3.70684813

2.25013019

4.45152527

5.19344615

5.93261077

4.40279017

0.74192088

2.96768351

0.74192088

5.19344615

2.96768351

5.19344615
8.00000000

10.09846914

12.20318181

14.34746655

8.00000000

10.09846914

12.20318181

14.34746655

15.47957842

15.47957842

15.47957842

15.47957842

15.47957842

15.47957842

./nitrogen/Cu/210/iter_1/struct.xyz 33

Lattice $=" 7.269310326940001 \quad 0.0 \quad 0.0 \quad 3.6346551634699993 \quad 8.127336020289532 \quad 0.0 \quad 0.0 \quad 0.021 .6891$ 35214202672" Properties=species:S:1:pos:R:3 pbc="T T T"

$\begin{array}{lrlr}\mathrm{Cu} & 1.81732758 & 3.25093441 & 8.00000000 \\ \mathrm{Cu} & 3.63465516 & 1.62546720 & 8.81273360 \\ \mathrm{Cu} & 1.81732758 & 0.00000000 & 9.62546720 \\ \mathrm{Cu} & 1.81732758 & 2.43820081 & 10.43820081 \\ \mathrm{Cu} & 3.64251887 & 0.82055295 & 11.25958643 \\ \mathrm{Cu} & 3.63857661 & 3.25474461 & 12.08393908 \\ \mathrm{Cu} & 1.81732758 & 1.71883392 & 12.92177560 \\ \mathrm{Cu} & -0.05086980 & 0.01616005 & 13.69038294 \\ \mathrm{Cu} & 3.63465516 & 7.31460242 & 8.00000000 \\ \mathrm{Cu} & 5.45198275 & 5.68913521 & 8.81273360 \\ \mathrm{Cu} & 3.63465516 & 4.06366801 & 9.62546720 \\ \mathrm{Cu} & 3.63465516 & 6.50186882 & 10.43820081 \\ \mathrm{Cu} & 5.45198275 & 4.88929053 & 11.23883062 \\ \mathrm{Cu} & 5.45198275 & 7.28668953 & 12.16112543 \\ \mathrm{Cu} & 3.62891612 & 5.68264313 & 12.85728652 \\ \mathrm{Cu} & 1.81732758 & 4.14813409 & 13.55326406 \\ \mathrm{Cu} & 5.45198275 & 3.25093441 & 8.00000000 \\ \mathrm{Cu} & 7.26931033 & 1.62546720 & 8.81273360 \\ \mathrm{Cu} & 5.45198275 & 0.00000000 & 9.62546720 \\ \mathrm{Cu} & 5.45198275 & 2.43820081 & 10.43820081 \\ \mathrm{Cu} & 7.26144662 & 0.82055295 & 11.25958643 \\ \mathrm{Cu} & 7.26538888 & 3.25474461 & 12.08393908 \\ \mathrm{Cu} & 5.45198275 & 1.61671061 & 12.87225697 \\ \mathrm{Cu} & 3.68552496 & 0.01616005 & 13.69038294 \\ \mathrm{Cu} & 7.26931033 & 7.31460242 & 8.00000000 \\ \mathrm{Cu} & 9.08663791 & 5.68913521 & 8.81273360 \\ \mathrm{Cu} & 7.26931033 & 4.06366801 & 9.62546720 \\ \mathrm{Cu} & 7.26931033 & 6.50186882 & 10.43820081 \\ \mathrm{Cu} & 9.08663791 & 4.89272796 & 11.23068940 \\ \mathrm{Cu} & 9.08663791 & 7.29927186 & 12.15034621 \\ \mathrm{Cu} & 7.27504937 & 5.68264313 & 12.85728652 \\ \mathrm{Cu} & 5.45198275 & 4.06094329 & 13.55088114 \\ \mathrm{~N} & 1.81732758 & 0.10622754 & 13.92318077\end{array}$

./nitrogen/Cu/210/iter_2/struct.xyz 34

Lattice $=" 7.269310326940001 \quad 0.0 \quad 0.0 \quad 3.6346551634699993 \quad 8.127336020289532 \quad 0.0 \quad 0.0 \quad 0.021 .6891$ 35214202672" Properties=species:S:1:pos:R:3 pbc="T T T"

$\begin{array}{lllr}\mathrm{Cu} & 1.81732758 & 3.25093441 & 8.00000000 \\ \mathrm{Cu} & 3.63465516 & 1.62546720 & 8.81273360 \\ \mathrm{Cu} & 1.81732758 & 0.00000000 & 9.62546720 \\ \mathrm{Cu} & 1.81732758 & 2.43820081 & 10.43820081 \\ \mathrm{Cu} & 3.63465516 & 0.82580764 & 11.26545540 \\ \mathrm{Cu} & 3.63465516 & 3.28015942 & 12.07451332 \\ \mathrm{Cu} & 1.81732758 & 1.70403018 & 12.89316848 \\ \mathrm{Cu} & 0.00000000 & -0.00079488 & 13.86858821 \\ \mathrm{Cu} & 3.63465516 & 7.31460242 & 8.00000000 \\ \mathrm{Cu} & 5.45198275 & 5.68913521 & 8.81273360 \\ \mathrm{Cu} & 3.63465516 & 4.06366801 & 9.62546720 \\ \mathrm{Cu} & 3.63465516 & 6.50186882 & 10.43820081 \\ \mathrm{Cu} & 5.45198275 & 4.89345406 & 11.23238829\end{array}$




\section{$\mathrm{Cu}$}

$\mathrm{Cu}$

$\mathrm{Cu}$

$\mathrm{Cu}$

$\mathrm{Cu}$

$\mathrm{Cu}$

$\mathrm{Cu}$

$\mathrm{Cu}$

$\mathrm{Cu}$

$\mathrm{Cu}$

$\mathrm{Cu}$

$\mathrm{Cu}$

$\mathrm{Cu}$

$\mathrm{Cu}$

$\mathrm{Cu}$

$\mathrm{Cu}$

$\mathrm{Cu}$

$\mathrm{Cu}$

$\mathrm{Cu}$

$\mathrm{N}$

$\mathrm{N}$

.$/$

. /nitrogen/Cu/210/iter_3/struct. xyz

5.45198275

3.63465516

1.81732758

5.45198275

7.26931033

5.45198275

5.45198275

7.26931033

7.26931033

5.45198275

3.63465516

7.26931033

9.08663791

7.26931033

7.26931033

9.08663791

9.08663791

7.26931033

5.45198275

1. 81732758

5.45198275
Fri Apr 16 14:51:55 2021
7.29351166

5.68155916

4.11011957

3.25093441

1. 62546720

0.00000000

2. 43820081

0.82580764

3.28015942

1. 70403018

7.31460242

5.68913521

4.06366801

6.50186882

4.89345406

7.29351166

5.68155916

4.11011957

0.07453166

0.07453166
$-0.00079488$
12.14396473

12.86015731

13.55359171

8.00000000

8.81273360

9.62546720

10.43820081

11.26545540

12.07451332

12.89316848

13.86858821

8.00000000

8.81273360

9.62546720

10.43820081

11.23238829

12.14396473

12.86015731

13.55359171

13.90370437

13.90370437

35214202672" Properties=species:S:1:pos:R:3 pbc="T T T"

$\begin{array}{llll}\mathrm{Cu} & 1.81732758 & 3.25093441 & 8.00000000\end{array}$

$\begin{array}{llll}\mathrm{Cu} & 3.63465516 & 1.62546720 & 8.81273360\end{array}$

$\begin{array}{llll}\mathrm{Cu} & 1.81732758 & 0.00000000 & 9.62546720\end{array}$

$\mathrm{Cu} \quad 1.81732758 \quad 2.43820081$

$\mathrm{Cu} \quad 3.63465516 \quad 0.83059494$

$\mathrm{Cu} \quad 3.63465516 \quad 3.25717883$

$\mathrm{Cu} \quad 1.78835138 \quad 1.70689784$

$\mathrm{Cu} \quad-0.00000000 \quad 0.00580949$

$\begin{array}{lll}\mathrm{Cu} & 3.63465516 & 7.31460242\end{array}$

$\mathrm{Cu} \quad 5.45198275 \quad 5.68913521$

$\mathrm{Cu} \quad 3.63465516 \quad 4.06366801$

$\mathrm{Cu} \quad 3.63465516 \quad 6.50186882$

$\mathrm{Cu} \quad 5.45747191 \quad 4.89854939$

$\begin{array}{lll}\mathrm{Cu} & 5.45109154 & 7.31319027\end{array}$

$\mathrm{Cu} \quad 3.63465516 \quad 5.76141699$

$\mathrm{Cu} \quad 1.76652416 \quad 4.10633588$

$\begin{array}{lll}\mathrm{Cu} & 5.45198275 & 3.25093441\end{array}$

$\begin{array}{lll}\mathrm{Cu} & 7.26931033 & 1.62546720\end{array}$

$\mathrm{Cu} \quad 5.45198275 \quad 0.00000000$

$\begin{array}{lll}\mathrm{Cu} & 5.45198275 & 2.43820081\end{array}$

$\begin{array}{lll}\mathrm{Cu} & 7.26931033 & 0.83578839\end{array}$

$\begin{array}{lll}\mathrm{Cu} & 7.26931033 & 3.29730094\end{array}$

$\mathrm{Cu} \quad 5.48095895 \quad 1.70689784$

$\mathrm{Cu} \quad 3.63465516 \quad-0.02618746$

$\begin{array}{lll}\mathrm{Cu} & 7.26931033 & 7.31460242\end{array}$

$\begin{array}{lll}\mathrm{Cu} & 9.08663791 & 5.68913521\end{array}$

$\mathrm{Cu} \quad 7.26931033 \quad 4.06366801$

$\mathrm{Cu} \quad 7.26931033 \quad 6.50186882$

$\begin{array}{lll}\mathrm{Cu} & 9.08114874 & 4.89854939\end{array}$

$\begin{array}{lll}\mathrm{Cu} & 9.08752911 & 7.31319027\end{array}$

$\begin{array}{lll}\mathrm{Cu} & 7.26931033 & 5.67261196\end{array}$

$\mathrm{Cu} \quad 5.50278617 \quad 4.10633588$

$\begin{array}{lll}\mathrm{N} & 1.81939851 & 0.06086948\end{array}$

$\mathrm{N} \quad 5.44991182 \quad 0.06086948$

$\mathrm{N} \quad 3.63465516 \quad 4.11350903$

10.43820081

11.24893543

12.08898772

12.90851502

13.86763660

8.00000000

8.81273360

9.62546720

10.43820081

11.22668314

12.10750851

12.90410735

13.65521860

8.00000000

8.81273360

9.62546720

10.43820081

11.25596937

12.08689253

12.90851502

13.82702136

8.00000000

8.81273360

9.62546720

10.43820081

11.22668314

12.10750851

12.86745127

13.65521860

13.87384133

13.87384133

13.87204529

./nitrogen/Cu/210/iter_4/struct. xyz

36

Lattice $=" 7.269310326940001 \quad 0.0 \quad 0.0 \quad 3.6346551634699993 \quad 8.127336020289532 \quad 0.0 \quad 0.0 \quad 0.021 .6891$ 35214202672 " Properties=species:S:1:pos:R:3 pbc="T T T"

$\begin{array}{llll}\mathrm{Cu} & 1.81732758 & 3.25093441 & 8.00000000 \\ \mathrm{Cu} & 3.63465516 & 1.62546720 & 8.81273360 \\ \mathrm{Cu} & 1.81732758 & 0.00000000 & 9.62546720\end{array}$

$\mathrm{Cu}$

1. 81732758

2.43820081

10.43820081 


\section{$\mathrm{Cu}$}

$\mathrm{Cu}$

$\mathrm{Cu}$

$\mathrm{Cu}$

$\mathrm{Cu}$

$\mathrm{Cu}$

$\mathrm{Cu}$

$\mathrm{Cu}$

$\mathrm{Cu}$

$\mathrm{Cu}$

$\mathrm{Cu}$

$\mathrm{Cu}$

$\mathrm{Cu}$

$\mathrm{Cu}$

$\mathrm{Cu}$

$\mathrm{Cu}$

$\mathrm{Cu}$

$\mathrm{Cu}$

$\mathrm{Cu}$

$\mathrm{Cu}$

$\mathrm{Cu}$

$\mathrm{Cu}$

$\mathrm{Cu}$

$\mathrm{Cu}$

$\mathrm{Cu}$

$\mathrm{Cu}$

$\mathrm{Cu}$

$\mathrm{Cu}$

$\mathrm{N}$

$\mathrm{N}$

$\mathrm{N}$

$\mathrm{N}$
3.63465516

3.63465516

1. 81732758

0.00000000

3. 63465516

5.45198275

3.63465516

3. 63465516

5.45198275

5.45198275

3. 63465516

1.81732758

5.45198275

7.26931033

5.45198275

5.45198275

7.26931033

7.26931033

5.45198275

3.63465516

7.26931033

9.08663791

7.26931033

7.26931033

9.08663791

9.08663791

7.26931033

5.45198275

1.81732758

5.45198275

3.63465516

7.26931033
Fri Apr 16 14:51:55 2021

32

0.84281181

3.27718406

1.70164999

$-0.01840580$

7.31460242

5.68913521

4.06366801

6.50186882

4.90647982

7.34085207

5.76531800

4.04526221

3.25093441

1.62546720

0.00000000

2.43820081

0.84281181

3.27718406

1.70164999

$-0.01840580$

7.31460242

5.68913521

4.06366801

6.50186882

4.90647982

7.34085207

5.76531800

4.04526221

0.04781916

0.04781916

4.11148717

4.11148717
11.24258858

12.08340371

12.90320537

13.83811117

8.00000000

8.81273360

9. 62546720

10.43820081

11.24258858

12.08340371

12.90320537

13.83811117

8.00000000

8.81273360

9.62546720

10.43820081

11.24258858

12.08340371

12.90320537

13.83811117

8.00000000

8.81273360

9.62546720

10.43820081

12.08340371

12.90320537

13.83811117

13.86454297

13.86454297

13.86454297

13.86454297
11.24258858

. /nitrogen/Cu/211/iter_1/struct.xyz

33

Lattice $=" 5.140178626728673 \quad 0.0 \quad 0.0 \quad 0.0 \quad 12.5908148222452070 .0 \quad 0.0 \quad 0.021 .19344614919298 "$ Pr operties=species:S:1:pos:R:3 pbc="T T T"

$\begin{array}{lrrr}\mathrm{Cu} & 1.28504466 & 4.19693827 & 8.00000000 \\ \mathrm{Cu} & 0.00000000 & 0.00000000 & 8.74192088 \\ \mathrm{Cu} & 1.28504466 & 2.09846914 & 9.48384176 \\ \mathrm{Cu} & 0.00000000 & 4.19693827 & 10.22576264 \\ \mathrm{Cu} & 1.28504466 & 0.07185651 & 10.94922801 \\ \mathrm{Cu} & -0.03417336 & 2.22727724 & 11.85632891 \\ \mathrm{Cu} & 1.28504466 & 4.33629510 & 12.44359416 \\ \mathrm{Cu} & -0.06590879 & -0.09813885 & 13.14937805 \\ \mathrm{Cu} & 1.28504466 & 10.49234569 & 8.00000000 \\ \mathrm{Cu} & 0.00000000 & 6.29540741 & 8.74192088 \\ \mathrm{Cu} & 1.28504466 & 8.39387655 & 9.48384176 \\ \mathrm{Cu} & 0.00000000 & 10.49234569 & 10.22576264 \\ \mathrm{Cu} & 1.28504466 & 6.35458572 & 10.93279773 \\ \mathrm{Cu} & 0.00419570 & 8.44665178 & 11.73971302 \\ \mathrm{Cu} & 1.28504466 & 10.47046384 & 12.43610685 \\ \mathrm{Cu} & -0.00087580 & 6.36267407 & 13.07456533 \\ \mathrm{Cu} & 3.85513397 & 4.19693827 & 8.00000000 \\ \mathrm{Cu} & 2.57008931 & 0.00000000 & 8.74192088 \\ \mathrm{Cu} & 3.85513397 & 2.09846914 & 9.48384176 \\ \mathrm{Cu} & 2.57008931 & 4.19693827 & 10.22576264 \\ \mathrm{Cu} & 3.85513397 & 0.05920954 & 10.95727399 \\ \mathrm{Cu} & 2.60426268 & 2.22727724 & 11.85632891 \\ \mathrm{Cu} & 3.85513397 & 4.33470432 & 12.41738332 \\ \mathrm{Cu} & 2.63599810 & -0.09813885 & 13.14937805 \\ \mathrm{Cu} & 3.85513397 & 10.49234569 & 8.00000000 \\ \mathrm{Cu} & 2.57008931 & 6.29540741 & 8.74192088 \\ \mathrm{Cu} & 3.85513397 & 8.39387655 & 9.48384176 \\ \mathrm{Cu} & 2.57008931 & 10.49234569 & 10.22576264 \\ \mathrm{Cu} & 3.85513397 & 6.38488377 & 10.93054185 \\ \mathrm{Cu} & 2.56589361 & 8.44665178 & 11.73971302 \\ \mathrm{Cu} & 3.85513397 & 10.48318666 & 12.44472778 \\ \mathrm{Cu} & 2.57096511 & 6.36267407 & 13.07456533\end{array}$




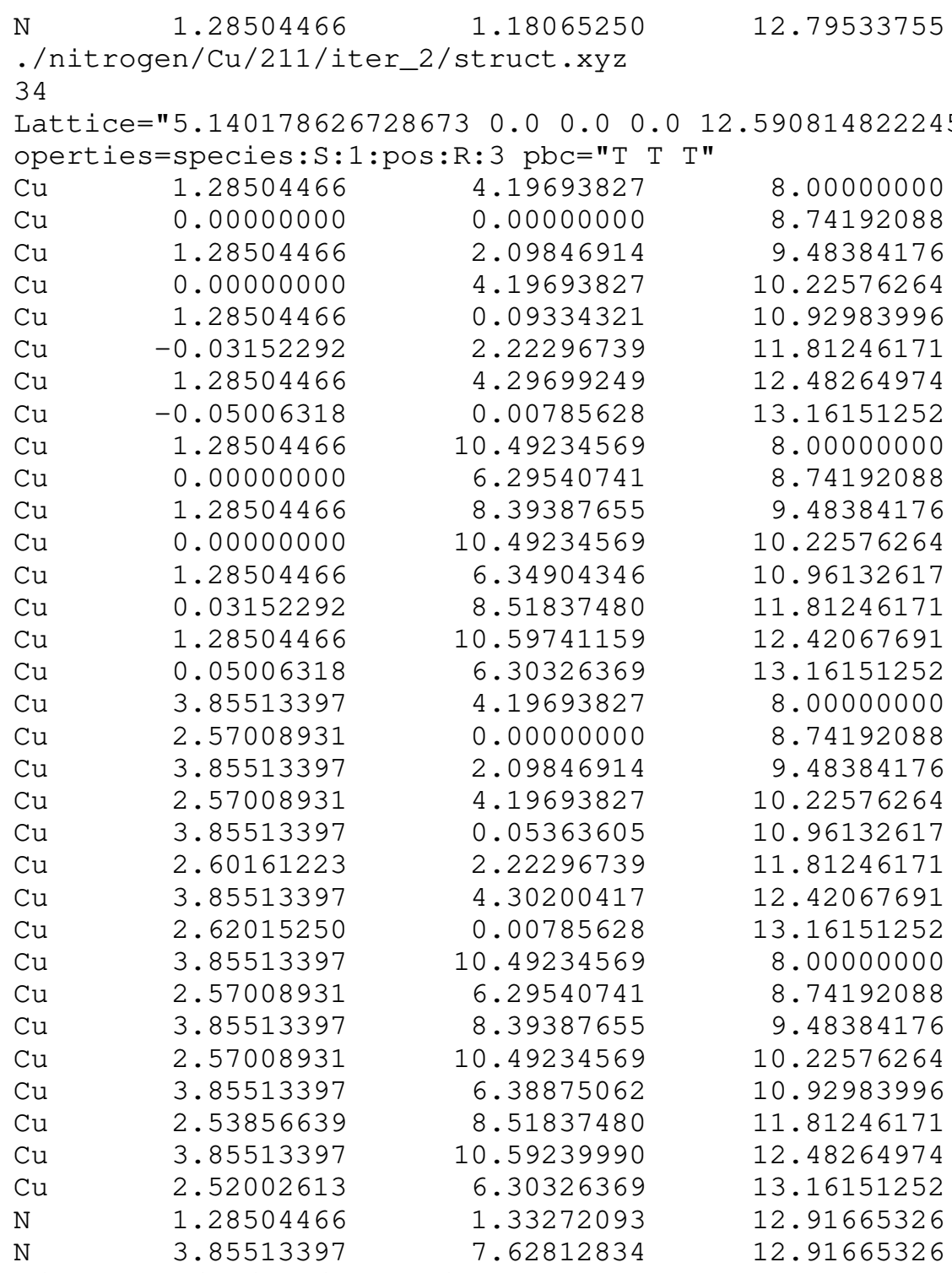

./nitrogen/Cu/211/iter_3/struct. xyz 35

Lattice $=" 5.140178626728673 \quad 0.0 \quad 0.0 \quad 0.0 \quad 12.590814822245207 \quad 0.0 \quad 0.0 \quad 0.021 .19344614919298 "$ Pr operties=species:S:1:pos:R:3 pbc="T T T"

$\begin{array}{lrrr}\mathrm{Cu} & 1.28504466 & 4.19693827 & 8.00000000 \\ \mathrm{Cu} & 0.00000000 & 0.00000000 & 8.74192088 \\ \mathrm{Cu} & 1.28504466 & 2.09846914 & 9.48384176 \\ \mathrm{Cu} & 0.00000000 & 4.19693827 & 10.22576264 \\ \mathrm{Cu} & 1.44027533 & 0.03893078 & 11.00225196 \\ \mathrm{Cu} & 0.11259214 & 2.20594157 & 11.71020512 \\ \mathrm{Cu} & 1.36751255 & 3.96781260 & 13.02714611 \\ \mathrm{Cu} & 0.45845181 & 0.56863537 & 13.46504857 \\ \mathrm{Cu} & 1.28504466 & 10.49234569 & 8.00000000 \\ \mathrm{Cu} & 0.00000000 & 6.29540741 & 8.74192088 \\ \mathrm{Cu} & 1.28504466 & 8.39387655 & 9.48384176 \\ \mathrm{Cu} & 0.00000000 & 10.49234569 & 10.22576264 \\ \mathrm{Cu} & 1.24416294 & 6.30972456 & 10.95271136 \\ \mathrm{Cu} & -0.10866629 & 8.55990534 & 11.82152463 \\ \mathrm{Cu} & 0.78269399 & 10.79203455 & 12.59244914 \\ \mathrm{Cu} & 0.06343782 & 6.20786335 & 13.13122947 \\ \mathrm{Cu} & 3.85513397 & 4.19693827 & 8.00000000 \\ \mathrm{Cu} & 2.57008931 & 0.00000000 & 8.74192088 \\ \mathrm{Cu} & 3.85513397 & 2.09846914 & 9.48384176 \\ \mathrm{Cu} & 2.57008931 & 4.19693827 & 10.22576264 \\ \mathrm{Cu} & 4.01107966 & 0.04907897 & 11.01884299 \\ \mathrm{Cu} & 2.73622680 & 2.22195402 & 11.64183020 \\ \mathrm{Cu} & 3.93686843 & 4.24817879 & 12.39708816 \\ \mathrm{Cu} & 2.98551440 & 0.49637786 & 13.36823004 \\ \mathrm{Cu} & 3.85513397 & 10.49234569 & 8.00000000\end{array}$




\section{$\mathrm{Cu}$}

$\mathrm{Cu}$

$\mathrm{Cu}$

$\mathrm{Cu}$

$\mathrm{Cu}$

$\mathrm{Cu}$

$\mathrm{Cu}$

$\mathrm{N}$

$\mathrm{N}$

$\mathrm{N}$
2. 57008931

3. 85513397

2. 57008931

3. 81443904

2. 37903214

3. 31967762

2.47057643

1. 62453596

3.78344136

$-0.72341796$
Fri Apr 16 14:51:55 2021

6.29540741

8.39387655

10.49234569

6.38244136

8.50075894

10.65439748

6.14870902

1.99141585

7.45667087

11.77153845
8.74192088

9.48384176

10.22576264

10.93871359

11.88196392

12.51238094

13.12824327

13.19078652

12.77683174

13.50210382

./nitrogen/Cu/211/iter_4/struct.xyz

36

Lattice $=" 5.140178626728673 \quad 0.0 \quad 0.0 \quad 0.0 \quad 12.590814822245207 \quad 0.0 \quad 0.0 \quad 0.0 \quad 21.19344614919298 "$ Pr operties=species:S:1:pos:R:3 pbc="T T T"

\begin{tabular}{|c|c|c|c|}
\hline $\mathrm{Cu}$ & 1.28504466 & 4.19693827 & 8.00000000 \\
\hline $\mathrm{Cu}$ & 0.00000000 & 0.00000000 & 8.74192088 \\
\hline $\mathrm{Cu}$ & 1.28504466 & 2.09846914 & 9.48384176 \\
\hline $\mathrm{Cu}$ & 0.00000000 & 4.19693827 & 10.22576264 \\
\hline $\mathrm{Cu}$ & 1.44170673 & 0.05094611 & 10.97234789 \\
\hline $\mathrm{Cu}$ & 0.19285158 & 2.20285783 & 11.70517258 \\
\hline $\mathrm{Cu}$ & 1.44603138 & 3.57977931 & 13.39325967 \\
\hline $\mathrm{Cu}$ & 0.36110205 & 0.41777193 & 13.41194423 \\
\hline $\mathrm{Cu}$ & 1.28504466 & 10.49234569 & 8.00000000 \\
\hline $\mathrm{Cu}$ & 0.00000000 & 6.29540741 & 8.74192088 \\
\hline $\mathrm{Cu}$ & 1.28504466 & 8.39387655 & 9.48384176 \\
\hline $\mathrm{Cu}$ & 0.00000000 & 10.49234569 & 10.22576264 \\
\hline $\mathrm{Cu}$ & 1.12378446 & 6.30622179 & 11.03452702 \\
\hline $\mathrm{Cu}$ & -0.19285158 & 8.49826525 & 11.70517258 \\
\hline $\mathrm{Cu}$ & 0.80614491 & 10.73613705 & 12.48896176 \\
\hline $\mathrm{Cu}$ & -0.36110205 & 6.71317934 & 13.41194423 \\
\hline $\mathrm{Cu}$ & 3.85513397 & 4.19693827 & 8.00000000 \\
\hline $\mathrm{Cu}$ & 2.57008931 & 0.00000000 & 8.74192088 \\
\hline $\mathrm{Cu}$ & 3.85513397 & 2.09846914 & 9.48384176 \\
\hline $\mathrm{Cu}$ & 2.57008931 & 4.19693827 & 10.22576264 \\
\hline $\mathrm{Cu}$ & 4.01639416 & 0.01081438 & 11.03452702 \\
\hline $\mathrm{Cu}$ & 2.85315681 & 2.26994001 & 11.74105810 \\
\hline $\mathrm{Cu}$ & 4.33403372 & 4.44072964 & 12.48896176 \\
\hline $\mathrm{Cu}$ & 2.91748304 & 0.21337482 & 13.28242575 \\
\hline $\mathrm{Cu}$ & 3.85513397 & 10.49234569 & 8.00000000 \\
\hline $\mathrm{Cu}$ & 2.57008931 & 6.29540741 & 8.74192088 \\
\hline $\mathrm{Cu}$ & 3.85513397 & 8.39387655 & 9.48384176 \\
\hline $\mathrm{Cu}$ & 2.57008931 & 10.49234569 & 10.22576264 \\
\hline $\mathrm{Cu}$ & 3.69847190 & 6.34635352 & 10.97234789 \\
\hline $\mathrm{Cu}$ & 2.28702182 & 8.56534743 & 11.74105810 \\
\hline $\mathrm{Cu}$ & 3.69414725 & 9.87518672 & 13.39325967 \\
\hline $\mathrm{Cu}$ & 2.22269558 & 6.50878223 & 13.2824257 \\
\hline $\mathrm{N}$ & 1.68208873 & 1.71256419 & 13.1936030 \\
\hline $\mathrm{N}$ & 3.45808990 & 8.00797160 & 13.19360305 \\
\hline $\mathrm{N}$ & -0.79766085 & 11.58438855 & 13.48372741 \\
\hline $\mathrm{N}$ & 0.79766085 & 5.28898114 & 13.4837274 \\
\hline
\end{tabular}

./nitrogen/Cu/211/iter_5/struct. xyz

37

Lattice $=" 5.140178626728673 \quad 0.0 \quad 0.0 \quad 0.0 \quad 12.590814822245207 \quad 0.0 \quad 0.0 \quad 0.021 .19344614919298 "$ Pr operties=species:S:1:pos:R:3 pbc="T T T"

$\begin{array}{llrr}\mathrm{Cu} & 1.28504466 & 4.19693827 & 8.00000000 \\ \mathrm{Cu} & 0.00000000 & 0.00000000 & 8.74192088 \\ \mathrm{Cu} & 1.28504466 & 2.09846914 & 9.48384176 \\ \mathrm{Cu} & 0.00000000 & 4.19693827 & 10.22576264 \\ \mathrm{Cu} & 1.26034668 & 0.01729239 & 11.05870064 \\ \mathrm{Cu} & 5.08290534 & 2.26245046 & 11.70860002 \\ \mathrm{Cu} & 0.77053097 & 3.76450213 & 13.60772798 \\ \mathrm{Cu} & 0.06093037 & 0.65312575 & 13.52437279 \\ \mathrm{Cu} & 1.28504466 & 10.49234569 & 8.00000000 \\ \mathrm{Cu} & 0.00000000 & 6.29540741 & 8.74192088 \\ \mathrm{Cu} & 1.28504466 & 8.39387655 & 9.48384176 \\ \mathrm{Cu} & 0.00000000 & 10.49234569 & 10.22576264 \\ \mathrm{Cu} & 1.33870961 & 6.29513955 & 10.88913611 \\ \mathrm{Cu} & 1.29007253 & 8.93941394 & 11.98651161\end{array}$




$\begin{array}{ll}\mathrm{Cu} & 2.55037276 \\ \mathrm{Cu} & 1.01087600 \\ \mathrm{Cu} & 3.85513397 \\ \mathrm{Cu} & 2.57008931 \\ \mathrm{Cu} & 3.85513397 \\ \mathrm{Cu} & 2.57008931 \\ \mathrm{Cu} & 3.82437945 \\ \mathrm{Cu} & 2.51872909 \\ \mathrm{Cu} & 3.79691783 \\ \mathrm{Cu} & 2.63316213 \\ \mathrm{Cu} & 3.85513397 \\ \mathrm{Cu} & 2.57008931 \\ \mathrm{Cu} & 3.85513397 \\ \mathrm{Cu} & 2.57008931 \\ \mathrm{Cu} & 3.82962707 \\ \mathrm{Cu} & 3.78188942 \\ \mathrm{Cu} & 0.00794539 \\ \mathrm{Cu} & 3.50283714 \\ \mathrm{~N} & 1.33838823 \\ \mathrm{~N} & 0.05478457 \\ \mathrm{~N} & 3.88558506 \\ \mathrm{~N} & 4.90409725 \\ \mathrm{~N} & 2.43593662\end{array}$

Fri Apr 16 14:51:55 2021

10.93537542

6.64883042

4.19693827

0.00000000

2.09846914

4.19693827

0.08551220

2.31588268

4.35968269

0.69255998

10.49234569

6.29540741

8.39387655

10.49234569

6.31941616

8.77110977

10.92913811

6.48391611

2.03021734

7.44966949

11.92075218

5.28621217

7.90781574
12.66178292

13.38547341

8.00000000

8.74192088

9.48384176

10.22576264

11.05905475

11.72633475

12.44392319

13.52707871

8.00000000

8.74192088

9.48384176

10.22576264

10.95265974

12.13223713

12.62373346

13.71688001

13.29545354

11.77650678

13.61997033

13.80584806

13.28338063

./nitrogen/Cu/211/iter_6/struct.xyz 38

Lattice $=" 5.140178626728673 \quad 0.0 \quad 0.0 \quad 0.0 \quad 12.5908148222452070 .0 \quad 0.0 \quad 0.021 .19344614919298 "$ Pr operties=species:S:1:pos:R:3 pbc="T T T"

$\begin{array}{lrrr}\mathrm{Cu} & 1.28504466 & 4.19693827 & 8.00000000 \\ \mathrm{Cu} & 0.00000000 & 0.00000000 & 8.74192088 \\ \mathrm{Cu} & 1.28504466 & 2.09846914 & 9.48384176 \\ \mathrm{Cu} & 0.00000000 & 4.19693827 & 10.22576264 \\ \mathrm{Cu} & 1.34195188 & 0.00833281 & 11.00304332 \\ \mathrm{Cu} & 0.05118861 & 2.17059668 & 11.79256623 \\ \mathrm{Cu} & 1.24944779 & 4.30431632 & 12.59344826 \\ \mathrm{Cu} & 0.15907661 & -0.03119557 & 13.36122670 \\ \mathrm{Cu} & 1.28504466 & 10.49234569 & 8.00000000 \\ \mathrm{Cu} & 0.00000000 & 6.29540741 & 8.74192088 \\ \mathrm{Cu} & 1.28504466 & 8.39387655 & 9.48384176 \\ \mathrm{Cu} & 0.00000000 & 10.49234569 & 10.22576264 \\ \mathrm{Cu} & 1.25722748 & 6.33581123 & 10.95562203 \\ \mathrm{Cu} & -0.06105360 & 8.57809427 & 11.77961321 \\ \mathrm{Cu} & 0.24720457 & 9.97472033 & 14.00463480 \\ \mathrm{Cu} & 0.05144069 & 6.76978166 & 13.58112384 \\ \mathrm{Cu} & 3.85513397 & 4.19693827 & 8.00000000 \\ \mathrm{Cu} & 2.57008931 & 0.00000000 & 8.74192088 \\ \mathrm{Cu} & 3.85513397 & 2.09846914 & 9.48384176 \\ \mathrm{Cu} & 2.57008931 & 4.19693827 & 10.22576264 \\ \mathrm{Cu} & 3.90929180 & 0.08319409 & 10.96728685 \\ \mathrm{Cu} & 2.64974237 & 2.19971584 & 11.73239975 \\ \mathrm{Cu} & 3.81713178 & 4.20065683 & 12.55024891 \\ \mathrm{Cu} & 2.74983586 & -0.04153586 & 13.43453194 \\ \mathrm{Cu} & 3.85513397 & 10.49234569 & 8.00000000 \\ \mathrm{Cu} & 2.57008931 & 6.29540741 & 8.74192088 \\ \mathrm{Cu} & 3.85513397 & 8.39387655 & 9.48384176 \\ \mathrm{Cu} & 2.57008931 & 10.49234569 & 10.22576264 \\ \mathrm{Cu} & 3.82653112 & 6.30530070 & 10.92411476 \\ \mathrm{Cu} & 2.35557992 & 8.67845059 & 11.94689617 \\ \mathrm{Cu} & 3.78804638 & 10.64074038 & 12.29945276 \\ \mathrm{Cu} & 2.31170716 & 6.81380569 & 13.65504069 \\ \mathrm{~N} & 1.44642913 & 1.12069065 & 12.72068750 \\ \mathrm{~N} & 3.71538741 & 7.38817348 & 12.49668846 \\ \mathrm{~N} & -1.05440181 & 11.35556751 & 14.05070087 \\ \mathrm{~N} & -0.10447380 & 4.94474057 & 13.71314856 \\ \mathrm{~N} & 1.10929408 & 8.38967306 & 13.55280037 \\ \mathrm{~N} & 2.57839495 & 4.98477873 & 13.70596765\end{array}$

. /nitrogen/Cu/211/iter_7/struct. xyz 39

Lattice $=" 5.140178626728673 \quad 0.0 \quad 0.0 \quad 0.0 \quad 12.590814822245207 \quad 0.0 \quad 0.0 \quad 0.0 \quad 21.19344614919298 "$ Pr 


$\begin{array}{llrl}\text { operties } & \text { species:S:1 : pos : } \mathrm{R}: 3 \text { pbC="T T T } & \\ \mathrm{Cu} & 1.28504466 & 4.19693827 & 8.00000000 \\ \mathrm{Cu} & 0.00000000 & 0.00000000 & 8.74192088 \\ \mathrm{Cu} & 1.28504466 & 2.09846914 & 9.48384176 \\ \mathrm{Cu} & 0.00000000 & 4.19693827 & 10.22576264 \\ \mathrm{Cu} & 1.28114891 & -0.04622170 & 10.90380469 \\ \mathrm{Cu} & 0.00000000 & 2.12001419 & 11.88341213 \\ \mathrm{Cu} & 1.27589200 & 4.26364561 & 12.51180445 \\ \mathrm{Cu} & 0.00000000 & -0.09748230 & 13.74382352 \\ \mathrm{Cu} & 1.28504466 & 10.49234569 & 8.00000000 \\ \mathrm{Cu} & 0.00000000 & 6.29540741 & 8.74192088 \\ \mathrm{Cu} & 1.28504466 & 8.39387655 & 9.48384176 \\ \mathrm{Cu} & 0.00000000 & 10.49234569 & 10.22576264 \\ \mathrm{Cu} & 1.28306181 & 6.37359091 & 10.97767791 \\ \mathrm{Cu} & 0.00000000 & 8.63434804 & 11.75988398 \\ \mathrm{Cu} & 1.22103188 & 9.97269567 & 14.14648604 \\ \mathrm{Cu} & 0.00000000 & 6.82819068 & 13.43417095 \\ \mathrm{Cu} & 3.85513397 & 4.19693827 & 8.00000000 \\ \mathrm{Cu} & 2.57008931 & 0.00000000 & 8.74192088 \\ \mathrm{Cu} & 3.85513397 & 2.09846914 & 9.48384176 \\ \mathrm{Cu} & 2.57008931 & 4.19693827 & 10.22576264 \\ \mathrm{Cu} & 3.85902971 & -0.04622170 & 10.90380469 \\ \mathrm{Cu} & 2.57008931 & 2.15674226 & 11.93930112 \\ \mathrm{Cu} & 3.86428663 & 4.26364561 & 12.51180445 \\ \mathrm{Cu} & 2.57008931 & -0.45668013 & 13.42722124 \\ \mathrm{Cu} & 3.85513397 & 10.49234569 & 8.00000000 \\ \mathrm{Cu} & 2.57008931 & 6.29540741 & 8.74192088 \\ \mathrm{Cu} & 3.85513397 & 8.39387655 & 9.48384176 \\ \mathrm{Cu} & 2.57008931 & 10.49234569 & 10.22576264 \\ \mathrm{Cu} & 3.85711682 & 6.37359091 & 10.97767791 \\ \mathrm{Cu} & 2.57008931 & 8.64532218 & 11.74412140 \\ \mathrm{Cu} & 3.91914675 & 9.97269567 & 14.14648604 \\ \mathrm{Cu} & 2.57008931 & 6.79883311 & 13.45264172 \\ \mathrm{~N} & 1.30266981 & 0.79793533 & 12.62668944 \\ \mathrm{~N} & 3.80595913 & 8.31485439 & 13.24789341 \\ \mathrm{~N} & 0.00000000 & 11.10377886 & 14.96018857 \\ \mathrm{~N} & 0.00000000 & 4.99577712 & 13.64868143 \\ \mathrm{~N} & 1.33421949 & 8.31485439 & 13.24789341 \\ \mathrm{~N} & 2.57008931 & 4.95151802 & 13.63988119 \\ \mathrm{~N} & 3.83750882 & 0.79793533 & 12.62668944\end{array}$

./nitrogen/Cu/211/iter_8/struct.xyz

40

Lattice $=" 5.1401786267286730 .0 \quad 0.0 \quad 0.0 \quad 12.5908148222452070 .0 \quad 0.0 \quad 0.021 .19344614919298 "$ Pr operties =species:S:1:pos:R:3 pbc="T T T"

$\begin{array}{lrrr}\mathrm{Cu} & 1.28504466 & 4.19693827 & 8.00000000 \\ \mathrm{Cu} & 0.00000000 & 0.00000000 & 8.74192088 \\ \mathrm{Cu} & 1.28504466 & 2.09846914 & 9.48384176 \\ \mathrm{Cu} & 0.00000000 & 4.19693827 & 10.22576264 \\ \mathrm{Cu} & 1.28504466 & -0.04548736 & 10.98813454 \\ \mathrm{Cu} & 0.00000000 & 2.17109853 & 11.65780538 \\ \mathrm{Cu} & 1.28504466 & 3.58545017 & 13.62584486 \\ \mathrm{Cu} & 0.00000000 & 0.25098123 & 13.37425590 \\ \mathrm{Cu} & 1.28504466 & 10.49234569 & 8.00000000 \\ \mathrm{Cu} & 0.00000000 & 6.29540741 & 8.74192088 \\ \mathrm{Cu} & 1.28504466 & 8.39387655 & 9.48384176 \\ \mathrm{Cu} & 0.00000000 & 10.49234569 & 10.22576264 \\ \mathrm{Cu} & 1.28504466 & 6.24992005 & 10.98813454 \\ \mathrm{Cu} & 0.00000000 & 8.46650594 & 11.65780538 \\ \mathrm{Cu} & 1.28504466 & 9.88085758 & 13.62584486 \\ \mathrm{Cu} & 0.00000000 & 6.54638864 & 13.37425590 \\ \mathrm{Cu} & 3.85513397 & 4.19693827 & 8.00000000 \\ \mathrm{Cu} & 2.57008931 & 0.00000000 & 8.74192088 \\ \mathrm{Cu} & 3.85513397 & 2.09846914 & 9.48384176 \\ \mathrm{Cu} & 2.57008931 & 4.19693827 & 10.22576264 \\ \mathrm{Cu} & 3.85513397 & -0.04548736 & 10.98813454 \\ \mathrm{Cu} & 2.57008931 & 2.17109853 & 11.65780538 \\ \mathrm{Cu} & 3.85513397 & 3.58545017 & 13.62584486 \\ \mathrm{Cu} & 2.57008931 & 0.25098123 & 13.37425590\end{array}$




\section{$\mathrm{Cu}$}

$\mathrm{Cu}$

$\mathrm{Cu}$

$\mathrm{Cu}$

$\mathrm{Cu}$

$\mathrm{Cu}$

$\mathrm{Cu}$

$\mathrm{Cu}$

$\mathrm{N}$

$\mathrm{N}$

$\mathrm{N}$

$\mathrm{N}$

$\mathrm{N}$

$\mathrm{N}$

$\mathrm{N}$

$\mathrm{N}$
3.85513397

2.57008931

3.85513397

2.57008931

3.85513397

2.57008931

3.85513397

2.57008931

1.28504466

3.85513397

0.00000000

0.00000000

1.28504466

2.57008931

3.85513397

2.57008931
Fri Apr 16 14:51:55 2021

10.49234569

6.29540741

8.39387655

10.49234569

6.24992005

8.46650594

9.88085758

6.54638864

1.70747127

8.00287868

11.12936040

4.83395299

8.00287868

4.83395299

1.70747127

11.12936040
8.00000000

8.74192088

9.48384176

10.22576264

10.98813454

11.65780538

13.62584486

13.37425590

13.11290299

13.11290299

14.02431670

14.02431670

13.11290299

14.02431670

13.11290299

14.02431670

./nitrogen/Cu/221/iter_1/struct.xyz

41

Lattice="5.140178626728671 $0.00 .00 .015 .420535880186020 .00 .00 .021 .451982745205 "$ Prope rties=species:S:1:pos:R: 3 pbc="T T T"

$1.28504466 \quad 3.85513397$

$\mathrm{Cu} \quad 1.28504466$

0.00000000

3.85513397

1.28504466

5.99687506

0.00000000

0.42834822

$\mathrm{Cu}$

1.28504466

2.57008931

0.00737244

4.71183041

1.28031715

6.88303236

0.00276438

1.29739259

3.21608078

1.29188234

5.48464475

$-0.00735612$

0.28718604

1.28504466

0.00000000

1.28504466

0.00000000

1.28504466

$-0.01111300$

1.29906190

$-0.00481267$

1.27629207

0.01884975

3.85513397

2. 57008931

3. 85513397

11.56540191

13.70714300

8.13861616

10.28035725

12.42209835

14.55984371

9. 12173704

11.28110897

13.42808947

7.59821879

3.85513397

5.99687506

0.42834822

2.57008931

4.71183041

6.88270926

1. 32911561

3.22672067

5. 42331415

0.27976928

11.56540191

13.70714300

8.13861616

10.28035725

12.42209835

14.55769868

9.11813251

11.28116797

13.49281717

7.59538365

$\begin{array}{lrr}\mathrm{N} & 1.28444858 & 1.80447740 \\ . / \text { nitrogen/Cu/221/iter_10/struct.xyz }\end{array}$
8.00000000
8.60577586
9.21155172
9.81732758
10.42310344
10.98230712
11.44089596
12.63676737
12.76624849
13.36414747
8.00000000
8.60577586
9.21155172
9.81732758
10.42310344
11.00941600
11.73234600
12.29213577
12.85395002
13.29764507
8.00000000
8.60577586
9.21155172
9.81732758
10.42310344
10.98229829
11.53318923
12.61463098
12.76823527
13.36143815
8.00000000
8.60577586
9.21155172
9.81732758
10.42310344
11.00873048
11.73597732
12.29160190
12.89291057
13.29835930
13.32113108

50

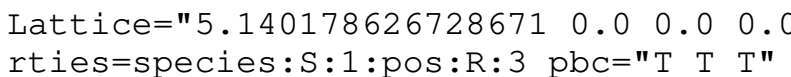

$\begin{array}{lll}\mathrm{Cu} & 1.28504466 & 3.85513397\end{array}$

$\mathrm{Cu} \quad 0.00000000 \quad 5.99687506$

$\mathrm{Cu} \quad 1.28504466 \quad 0.42834822$

8.00000000

8.60577586

9.21155172 
$\mathrm{Cu}$

$\mathrm{Cu}$

$\mathrm{Cu}$

$\mathrm{Cu}$

$\mathrm{Cu}$

$\mathrm{Cu}$

$\mathrm{Cu}$

$\mathrm{Cu}$

$\mathrm{Cu}$

$\mathrm{Cu}$

$\mathrm{Cu}$

$\mathrm{Cu}$

$\mathrm{Cu}$

$\mathrm{Cu}$

$\mathrm{Cu}$

$\mathrm{Cu}$

$\mathrm{Cu}$

$\mathrm{Cu}$

$\mathrm{Cu}$

$\mathrm{Cu}$

$\mathrm{Cu}$

$\mathrm{Cu}$

$\mathrm{Cu}$

$\mathrm{Cu}$

$\mathrm{Cu}$

$\mathrm{Cu}$

$\mathrm{Cu}$

$\mathrm{Cu}$

$\mathrm{Cu}$

$\mathrm{Cu}$

$\mathrm{Cu}$

$\mathrm{Cu}$

$\mathrm{Cu}$

$\mathrm{Cu}$

$\mathrm{Cu}$

$\mathrm{Cu}$

$\mathrm{Cu}$

$\mathrm{N}$

$\mathrm{N}$

$\mathrm{N}$

$\mathrm{N}$

$\mathrm{N}$

$\mathrm{N}$

$\mathrm{N}$

$\mathrm{N}$

$\mathrm{N}$

$\mathrm{N}$
1.26263246

0.00000000

1.28504466

0.00000000

1.28062671

0.00000000

1.23429473

0.00000000

1.28504466

0.00000000

1.28504466

0.00000000

1.28504466

0.00000000

1.28062671

0.00000000

1.23429473

0.00000000

3.85513397

2. 57008931

3. 85513397

2. 57008931

3. 85513397

2. 57008931

3. 85955192

2. 57008931

3. 90588390

2. 57008931

3.85513397

2. 57008931

3. 85513397

2. 57008931

2. 57008931

3. 85955192

2.57008931

3.90588390

2.57008931

1.25449280

3.87754617

3.87754617

1.25449280

2.57008931

2.57008931

3.88568582

3.88568582

1. 26263246
3.85513397

Fri Apr 16 14:51:55 2021

2.57008931

4.71183041

6.82907748

1.26509517

3.55002356

5.80960410

0.87655877

11.56540191

13.70714300

8.13861616

10.28035725

12.42209835

14.53934542

8.97536311

11.26029150

13.51987204

8.58682671

3.85513397

5.99687506

0.42834822

2.57008931

4.71183041

6.84651446

1.26509517

3. 52427761

5.80960410

0.82985441

11.56540191

13.70714300

8.13861616

10.28035725

12.42209835

14.55678240

8.97536311

11.23454555

13.51987204

8.54012235

2.21237121

14.98192630

7.27165836

9.92263915

12.53961795

4.82935001

2. 21237121

9.92263915

7.27165836

14.98192630

42

Lattice $=" 5.1401786267286710 .00 .00 .0$ rties=species:S:1:pos:R:3 pbc="T T T"

Cu

$\mathrm{Cu}$

$\mathrm{Cu} \quad 0.00000000$

$\mathrm{Cu} \quad 1.28504466$

$\mathrm{Cu} \quad 0.00000000$

$\mathrm{Cu} \quad 1.28504466$

$\mathrm{Cu} \quad-0.00280760$

$\mathrm{Cu} \quad 1.28504466$

$\mathrm{Cu} \quad-0.00164022$

$\mathrm{Cu} \quad 1.28504466$

$\mathrm{Cu} \quad 0.00469148$

$\mathrm{Cu} \quad 1.28504466$

$\mathrm{Cu} \quad 0.00000000$

$\mathrm{Cu} \quad 1.28504466$

$\mathrm{Cu} \quad 0.00000000$

$\mathrm{Cu} \quad 1.28504466$

$\mathrm{Cu} \quad-0.01193489$

$\mathrm{Cu}$

-0.01193489
1.28504466
3.85513397

5.99687506

0.42834822

2.57008931

4.71183041

6.89746638

1.27276859

3. 37085505

5.59524675

0.69229420

11.56540191

13.70714300

8.13861616

10.28035725

12.42209835

14.56531446

9.12946343
9.81732758

10.42310344

11.01679881

11.51870960

12.37622484

12.99422907

13.90445986

8.00000000

8.60577586

9.21155172

9.81732758

10.42310344

11.01679881

11.51870960

12.37622484

12.99422907

13.90445986

8.00000000

8.60577586

9.21155172

9.81732758

10.42310344

10.99069121

11.51870960

12.50717801

12.99422907

13.79278786

8.00000000

8.60577586

9.21155172

9.81732758

10.42310344

10.99069121

11.51870960

12.50717801

12.99422907

13.79278786

13.19893380

14.16269190

14.16269190

13.19893380

13.79558751

13.79558751

13.19893380

13.19893380

14.16269190

14.16269190 
$\mathrm{Cu}$

$\mathrm{Cu}$

$\mathrm{Cu}$

$\mathrm{Cu}$

$\mathrm{Cu}$

$\mathrm{Cu}$

$\mathrm{Cu}$

$\mathrm{Cu}$

$\mathrm{Cu}$

$\mathrm{Cu}$

$\mathrm{Cu}$

$\mathrm{Cu}$

$\mathrm{Cu}$

$\mathrm{Cu}$

$\mathrm{Cu}$

$\mathrm{Cu}$

$\mathrm{Cu}$

$\mathrm{Cu}$

$\mathrm{Cu}$

$\mathrm{Cu}$

$\mathrm{Cu}$

$\mathrm{Cu}$

$\mathrm{Cu}$

$\mathrm{N}$

$\mathrm{N}$
$-0.00222063$

1.28504466

0.00058543

3.85513397

2.57008931

3.85513397

2.57008931

3.85513397

2.57289692

3.85513397

2. 57172953

3.85513397

2.56539784

3.85513397

2.57008931

3.85513397

2.57008931

3.85513397

2.58202420

3.85513397

2.57230994

3.85513397

2.56950388

1.28504466

3.85513397
Fri Apr 16 14:51:55 2021

11.24511927

13.40949738

7.68154055

3.85513397

5.99687506

0.42834822

2.57008931

4.71183041

6.89746638

1. 32362234

3.37085505

5.55061027

0.69229420

11.56540191

13.70714300

8.13861616

10.28035725

12.42209835

14.56531446

9.11655424

11.24511927

13.38153905

7.68154055

2.10226019

14.87137789
12.28112379

12.80679023

13.30497548

8.00000000

8.60577586

9.21155172

9.81732758

10.42310344

10.97179745

11.54661837

12.42913911

12.75052035

13.59211090

8.00000000

8.60577586

9.21155172

9.81732758

10.42310344

11.01145118

11.71231281

12.28112379

12.90298763

13.30497548

13.27714851

13.98892318

43

Lattice $=" 5.1401786267286710 .0 \quad 0.0 \quad 0.015 .420535880186020 .00 .00 .021 .451982745205 "$ Prope rties=species:S:1:pos:R:3 pbc="T T T"

$1.28504466-3.85513397$

$\mathrm{Cu} \quad 0.00000000 \quad 5.99687506$

$\mathrm{Cu} \quad 1.28504466 \quad 0.42834822$

$\mathrm{Cu} \quad 0.00000000 \quad 2.57008931$

$\mathrm{Cu} \quad 1.28504466$

$\mathrm{Cu} \quad-0.00317210$

$\mathrm{Cu} \quad 1.28504466$

$\mathrm{Cu} \quad 0.00384189$

$\mathrm{Cu} \quad 1.28504466$

$\mathrm{Cu} \quad 0.00424423$

$\mathrm{Cu} \quad 1.28504466$

$\mathrm{Cu} \quad 0.00000000$

$\mathrm{Cu} \quad 1.28504466$

$\mathrm{Cu} \quad 0.00000000$

$\mathrm{Cu} \quad 1.28504466$

$\mathrm{Cu} \quad-0.01212967$

$\mathrm{Cu} \quad 1.28504466$

$\mathrm{Cu} \quad 0.00155517$

$\mathrm{Cu} \quad 1.28504466$

$\mathrm{Cu} \quad 0.04825461$

$\mathrm{Cu} \quad 3.85513397$

$\mathrm{Cu} \quad 2.57008931$

$\mathrm{Cu} \quad 3.85513397$

$\mathrm{Cu} \quad 2.57008931$

$\mathrm{Cu} \quad 3.85513397$

$\mathrm{Cu} \quad 2.57326141$

$\mathrm{Cu} \quad 3.85513397$

$\mathrm{Cu} \quad 2.56624743$

$\mathrm{Cu} \quad 3.85513397$

$\mathrm{Cu} \quad 2.56584508$

$\mathrm{Cu} \quad 3.85513397$

$\mathrm{Cu} \quad 2.57008931$

$\mathrm{Cu} \quad 3.85513397$

$\mathrm{Cu} \quad 2.57008931$

$\mathrm{Cu} \quad 3.85513397$

$\mathrm{Cu} \quad 2.58221898$

$\mathrm{Cu} \quad 3.85513397$

$\mathrm{Cu} \quad 2.56853414$

$\mathrm{Cu} \quad 3.85513397$

4.71183041

6.90187856

1.24422789

3.34849133

5.59201248

0.70056711

11.56540191

13.70714300

8.13861616

10.28035725

12.42209835

14.55357642

9.11399422

11.24022030

13.40005774

7.76650095

3.85513397

5.99687506

0.42834822

2.57008931

4.71183041

6.90187856

1. 30667358

3. 34849133

5.46109625

0.70056711

11.56540191

13.70714300

8.13861616

10.28035725

12.42209835

14.55357642

9.12363722

11.24022030

13.35723190

8.00000000

8.60577586

9. 21155172

9.81732758

10.42310344

10.99695034

11.47934450

12.38902086

12.73058958

13.59538299

8.00000000

8.60577586

9.21155172

9.81732758

10.42310344

11.00958052

11.66421371

12.27457791

12.81003185

13.35495700

8.00000000

8.60577586

9. 21155172

9.81732758

10.42310344

10.99695034

11.54172834

12.38902086

12.83844302

13.59538299

8.00000000

8.60577586

9. 21155172

9.81732758

10.42310344

11.00958052

11.67451925

12.27457791

12.90838063 
all_structures.xyz

$\mathrm{Cu}$

$\mathrm{N}$

$\mathrm{N}$

$\mathrm{N}$
2. 52183470

1.28504466

3.85513397

3.85513397
Fri Apr 16 14:51:55 2021

7.76650095

2.10222706

14.86907057

6.77929347

./nitrogen/Cu/221/iter_4/struct.xyz

44

Lattice $=" 5.140178626728671 \quad 0.0 \quad 0.0 \quad 0.0 \quad 15.420535880186020 .0 \quad 0.0 \quad 0.021 .451982745205 "$ Prope rties $=$ species:S:1:pos:R:3 pbc="T T T"

$\mathrm{Cu} \quad 0.00000000 \quad 5.99687506$

$\mathrm{Cu} \quad 1.28504466 \quad 0.42834822$

$\begin{array}{lll}\mathrm{Cu} & 0.00000000 & 2.57008931\end{array}$

$\mathrm{Cu} \quad 1.28504466$

2. 57008931

$-0.00893569$

6.87520302

1.28504466

1.29744514

0.00210441

1.28504466

3. 42471373

0.00630586

5.70747121

0.73773851

1.28504466

11.56540191

0.00000000

1.28504466

13.70714300

0.00000000

8.13861616

1. 28504466

10.28035725

12.42209835

$-0.00893569$

1. 28504466

14.58547096

9.00771308

0.00210441

11.13498167

1.28504466

0.00630586

13.41773915

8.44800645

3.85513397

5.99687506

2. 57008931

0.42834822

2.57008931

2.57008931

4.71183041

6.87520302

3.85513397

1. 34506015

3.85513397

3. 42471373

5.63091599

0.73773851

3.85513397

2. 56378345

11.56540191

3. 85513397

13.70714300

8.13861616

3.85513397

10.28035725

2. 57008931

3. 85513397

12.42209835

2. 57902500

14.58547096

9.05532809

3. 85513397

11.13498167

. 56798490

13.34118393

8.44800645

2.15164313

2. 56378345

1. 28504466

14.87269072

7.16242278

3. 85513397

9.86191107

./nitrogen/Cu/221/iter_5/struct.xyz

45

Lattice $=" 5.1401786267286710 .0 \quad 0.0 \quad 0.0$

rties=species:S:1:pos:R:3 pbc="T T T"

$\mathrm{Cu}$

1.28504466

3. 85513397

0.00000000

5.99687506

1.28504466

0.42834822

0.00000000

2. 57008931

1.28504466

4.71183041

$-0.01988552$

1.24247920

6.85301696

1. 21206232

3. 29766885

5.39153976

0.71383645

1.21046987

$-0.10027779$

1.28504466

11.56540191

$\mathrm{Cu}$

0.00000000

13.70714300

13.35495700

13.25736776

13.95855202

14.13359372
40
8.00000000
8.60577586
9. 21155172
9.81732758
10.42310344
10.99147625
11.48659981
12.40984916
12.74708073
13.59294900
8.00000000
8.60577586
9.21155172
9.81732758
10.42310344
10.99147625
11.48659981
12.40984916
12.74708073
13.59294900
8.00000000
8.60577586
9.21155172
9.81732758
10.42310344
10.99147625
11.54348527
12.40984916
12.82768689
13.59294900
8.00000000
8.60577586
9.21155172
9. 81732758
10.42310344
10.99147625
11.54348527
12.40984916
12.82768689
13.59294900
13.26491315
13.85547856
13.85547856
13.26491315

$15.420535880186020 .00 .00 .021 .451982745205 "$ Prope 8.00000000

8.60577586

9.21155172

9.81732758

10.42310344

11.09344970

11.49290119

12.36657668

12.82087833

13.62563861

8.00000000

8.60577586 
$\mathrm{Cu}$

$\mathrm{Cu}$

$\mathrm{Cu}$

$\mathrm{Cu}$

$\mathrm{Cu}$

$\mathrm{Cu}$

$\mathrm{Cu}$

$\mathrm{Cu}$

$\mathrm{Cu}$

$\mathrm{Cu}$

$\mathrm{Cu}$

$\mathrm{Cu}$

$\mathrm{Cu}$

$\mathrm{Cu}$

$\mathrm{Cu}$

$\mathrm{Cu}$

$\mathrm{Cu}$

$\mathrm{Cu}$

$\mathrm{Cu}$

$\mathrm{Cu}$

$\mathrm{Cu}$

$\mathrm{Cu}$

$\mathrm{Cu}$

$\mathrm{Cu}$

$\mathrm{Cu}$

$\mathrm{Cu}$

$\mathrm{Cu}$

$\mathrm{Cu}$

$\mathrm{N}$

$\mathrm{N}$

$\mathrm{N} \quad-0.04662810$

$\mathrm{N} \quad 1.13372482$

$-0.00671519$
1.28504466

0.00000000

1.28504466

1.32728894

0.18307994

$-0.25727746$

$-1.31597650$

3.85513397

2.57008931

3.85513397

2.57008931

3.85513397

2.55434358

3.82117786

2.52278722

3.80246383

2.50537572

3.85513397

3.85513397

2.57008931

3.85513397

2.60209488

3.85082081

2.69012275

2.37332418

1.25121161

1.18068972

3.71920709
2.57008931
Fri Apr 16 14:51:55 2021

8.13861616

10.28035725

12.42209835

14.55544803

9.09963123

10.88728074

13.52587726

3.85513397

5.99687506

0.42834822

2.57008931

4.71183041

6.90710659

1.26641212

3.24446776

5.33014686

0.78926119

11.56540191

13.70714300

8.13861616

10.28035725

12.42209835

14.55679290

9.15836752

11.27069166

13.66778157

7.73221077

2.09430298

14.81501466

6.38382901

9.03811122

12.46451219
7.69928437
9.21155172

9.81732758

10.42310344

10.96646861

12.89470937

13.20559239

13.54209635

8.00000000

8.60577586

9.21155172

9.81732758

10.42310344

11.08311897

11.52835752

12.36732417

12.81628912

13.63816396

8.00000000

8.60577586

9.21155172

9.81732758

10.96546314

11.59869780

12.24222361

13.27466078

13.58775638

13.28809217

13.93689727

13.77687091

13.15733927

12.49647173
11.58709670

10.42310344

46

Lattice $=" 5.140178626728671$
rties=species:S:1:pos:R:3 pbc="T T T"

$\mathrm{Cu} \quad 1.28504466 \quad 3.85513397$

$\mathrm{Cu} \quad 0.00000000 \quad 5.99687506$

0.42834822

1. 28504466

0.00000000

2.57008931

1.28504466

$-0.02177252$

4.71183041

6.87799365

1. 30218324

0.01409866

0.01531371

0.01374722

1. 28504466

0.00000000

1. 28504466

0.00000000

1. 28504466

$-0.02177252$

1.30218324

0.01409866

0.01531371

0.01374722

3. 85513397

2. 57008931

3. 85513397

2. 57008931

3. 85513397

1.26368346

3. 37058841

5.94795307

0.80337393

11.56540191

13.70714300

8.13861616

10.28035725

12.42209835

14.58826159

8.97395140

11.08085635

13.65822101

8.51364187

3.85513397

5.99687506

0.42834822

2.57008931

4.71183041

6.87404441

1. 32856515

3. 87467385

2. 62150436

1.32856515
3.36547360

5. 92595529

0.78931787

2. 61712741

3. 85513397

11.56540191
8.00000000
8.60577586
9.21155172
9.81732758
10.42310344
10.97540899
11.53293463
12.38621567
13.27132851
13.69776050
8.00000000
8.60577586
9.21155172
9.81732758
10.42310344
10.97540899
11.53293463
12.38621567
13.27132851
13.69776050
8.00000000
8.60577586
9.21155172
9.81732758
10.42310344
10.97819053
11.57623571
12.38139472
13.26791248
13.69309682
8.00000000 


\section{$\mathrm{Cu}$}

$\mathrm{Cu}$

$\mathrm{Cu}$

$\mathrm{Cu}$

$\mathrm{Cu}$

$\mathrm{Cu}$

$\mathrm{Cu}$

$\mathrm{Cu}$

$\mathrm{Cu}$

$\mathrm{N}$

$\mathrm{N}$

$\mathrm{N}$

$\mathrm{N}$

$\mathrm{N}$

$\mathrm{N}$
2.57008931

3.85513397

2.57008931

3.85513397

2.59137213

3.87467385

2.62150436

2.65584333

2. 61712741

1.31953274

3. 89284619

3.89284619

1.31953274

1.32539662

1.32539662
Fri Apr 16 14:51:55 2021

13.70714300

8.13861616

10.28035725

12.42209835

14.58431235

9.03883309

11.07574154

13.63622323

8.49958581

2.11117557

14.85288949

7.14262155

9.82144351

12.64306544

4.93279749
8.60577586

9.21155172

9.81732758

10.42310344

10.97819053

11.57623571

12.38139472

13.26791248

13.69309682

13.33306174

13.98727099

13.98727099

13.33306174

12.39325386

12.39325386

47

Lattice $=" 5.140178626728671$
rties

$\mathrm{Cu} \quad 1.28504466$

3. 85513397

0.00000000

5.99687506

1. 28504466

0.42834822

0.00000000

2.57008931

1.28504466

4.71183041

5.08792622

6.85298576

1.35201199

1.33695348

0.05880909

3. 61266959

0.29420861

6.02822957

0.11549880

0.87324224

1. 28504466

11.56540191

0.00000000

1. 28504466

0.00000000

1. 28504466

5.11980389

1. 31378653

0.05113204

1.10916267

0.07496301

3. 85513397

13.70714300

8.13861616

10.28035725

12.42209835

14.61974184

8.95140377

11.02680552

13.74326948

8.52439628

3. 85513397

5.99687506

0.42834822

3. 85513397

2. 57008931

3. 85513397

2.57008931

4.71183041

6.86810931

1. 41343781

3. 20977768

5.84658055

3. 91133008

2. 56214029

2. 90976070

3. 07285415

3. 85513397

15.39294602

11.56540191

2. 57008931

3. 85513397

13.70714300

8.13861616

2. 57008931

3. 85513397

2. 60105037

10.28035725

12.42209835

14.64627810

9.00564133

3. 89720381

2. 61818211

3. 88709095

10.99439023

13.22841254

2. 66801576

1. 89985793

4.76519639

8.42760683

1.38938680

14.44914387

4. 02945012

1. 36520371

2. 35182099

1.53909789

4. 27830347

7.12380154

9.81735666

12.85876243

4.77357682

2. 44102581

15.42053588018602 $0.0 \quad 0.00 .021 .451982745205 "$ Prope

8.00000000
8.60577586
9.21155172
9.81732758
10.42310344
10.96633047

11.61666736

12.30761302

13.26772054

13.88758798

8.00000000

8.60577586

9.21155172

9.81732758

10.42310344

11.00706900

11.52831131

12.39638073

13.14011195

13.69814706

8.00000000

8.60577586

9.21155172

9.81732758

10.42310344

10.96324880

11.68827016

13.15102901

13.18009240

13.75342458

8.00000000

8.60577586

9.21155172

9.81732758

10.42310344

11.05401847

11.55871058

12.49245149

12.97900592

13.64702913

13.48373927

14.08421756

13.94712728

13.35991451

11.97328374

12.50626753

13.37208116

/nitrogen/Cu/221/iter_8/struct.xyz 48 
Lattice $=" 5.140178626728671 \quad 0.0 \quad 0.0 \quad 0.0 \quad 15.420535880186020 .00 .0 \quad 0.021 .451982745205 "$ Prope rties=species:S:1:pos:R:3 pbc="T T T"

\begin{tabular}{|c|c|c|c|}
\hline $\mathrm{Cu}$ & 1.28504466 & 3.85513397 & 8.00000000 \\
\hline $\mathrm{Cu}$ & 0.00000000 & 5.99687506 & 8.60577586 \\
\hline $\mathrm{Cu}$ & 1.28504466 & 0.42834822 & 9.21155172 \\
\hline $\mathrm{Cu}$ & 0.00000000 & 2.57008931 & 9.81732758 \\
\hline $\mathrm{Cu}$ & 1.28504466 & 4.71183041 & 10.42310344 \\
\hline $\mathrm{Cu}$ & -0.06800500 & 6.85476921 & 11.00199019 \\
\hline $\mathrm{Cu}$ & 1.27207931 & 1.25083602 & 11.61071967 \\
\hline $\mathrm{Cu}$ & 0.04904522 & 3.48585959 & 12.29219707 \\
\hline $\mathrm{Cu}$ & 0.58705143 & 5.84349316 & 13.23447721 \\
\hline $\mathrm{Cu}$ & -0.13931309 & 0.58642386 & 13.80385657 \\
\hline $\mathrm{Cu}$ & 1.28504466 & 11.56540191 & 8.00000000 \\
\hline $\mathrm{Cu}$ & 0.00000000 & 13.70714300 & 8.60577586 \\
\hline $\mathrm{Cu}$ & 1.28504466 & 8.13861616 & 9.21155172 \\
\hline $\mathrm{Cu}$ & 0.00000000 & 10.28035725 & 9.81732758 \\
\hline $\mathrm{Cu}$ & 1.28504466 & 12.42209835 & 10.42310344 \\
\hline $\mathrm{Cu}$ & -0.06800500 & 14.56503715 & 11.00199019 \\
\hline $\mathrm{Cu}$ & 1.27207931 & 8.96110396 & 11.61071967 \\
\hline $\mathrm{Cu}$ & 0.04904522 & 11.19612753 & 12.29219707 \\
\hline $\mathrm{Cu}$ & 0.58705143 & 13.55376110 & 13.23447721 \\
\hline $\mathrm{Cu}$ & -0.13931309 & 8.29669180 & 13.80385657 \\
\hline $\mathrm{Cu}$ & 3.85513397 & 3.85513397 & 8.00000000 \\
\hline $\mathrm{Cu}$ & 2.57008931 & 5.99687506 & 8.60577586 \\
\hline $\mathrm{Cu}$ & 3.85513397 & 0.42834822 & 9.21155172 \\
\hline $\mathrm{Cu}$ & 2.57008931 & 2.57008931 & 9.81732758 \\
\hline $\mathrm{Cu}$ & 3.85513397 & 4.71183041 & 10.42310344 \\
\hline $\mathrm{Cu}$ & 2.53893832 & 6.86827110 & 11.02973582 \\
\hline $\mathrm{Cu}$ & 3.82335697 & 1.31995824 & 11.61347014 \\
\hline $\mathrm{Cu}$ & 2.40475146 & 2.94823930 & 13.20410707 \\
\hline $\mathrm{Cu}$ & 3.27783856 & 5.31065668 & 12.95085096 \\
\hline $\mathrm{Cu}$ & 2.63928654 & -0.17944570 & 13.68917850 \\
\hline $\mathrm{Cu}$ & 3.85513397 & 11.56540191 & 8.00000000 \\
\hline $\mathrm{Cu}$ & 2.57008931 & 13.70714300 & 8.60577586 \\
\hline $\mathrm{Cu}$ & 3.85513397 & 8.13861616 & 9.21155172 \\
\hline $\mathrm{Cu}$ & 2.57008931 & 10.28035725 & 9.81732758 \\
\hline $\mathrm{Cu}$ & 3.85513397 & 12.42209835 & 10.42310344 \\
\hline $\mathrm{Cu}$ & 2.53893832 & 14.57853904 & 11.02973582 \\
\hline $\mathrm{Cu}$ & 3.82335697 & 9.03022618 & 11.61347014 \\
\hline $\mathrm{Cu}$ & 2.40475146 & 10.65850724 & 13.20410707 \\
\hline $\mathrm{Cu}$ & 3.27783856 & 13.02092462 & 12.95085096 \\
\hline $\mathrm{Cu}$ & 2.63928654 & 7.53082224 & 13.68917850 \\
\hline $\mathrm{N}$ & 1.57242023 & 1.28466364 & 13.54803871 \\
\hline $\mathrm{N}$ & 4.20747478 & 14.26912019 & 14.03110261 \\
\hline $\mathrm{N}$ & 4.20747478 & 6.55885225 & 14.03110261 \\
\hline $\mathrm{N}$ & 1.57242023 & 8.99493158 & 13.54803871 \\
\hline $\mathrm{N}$ & 1.66157136 & 12.26671944 & 12.35399710 \\
\hline $\mathrm{N}$ & 1.66157136 & 4.55645150 & 12.35399710 \\
\hline $\mathrm{N}$ & 4.18413549 & 2.27869300 & 13.27696801 \\
\hline $\mathrm{N}$ & 4.18413549 & 9.98896094 & 13.27696801 \\
\hline
\end{tabular}

./nitrogen/Cu/221/iter_9/struct.xyz 49

Lattice $=" 5.140178626728671 \quad 0.0 \quad 0.0 \quad 0.0 \quad 15.420535880186020 .0 \quad 0.0 \quad 0.021 .451982745205 "$ Prope rties=species:S:1:pos:R:3 pbc="T T T"

$\begin{array}{lrrr}\mathrm{Cu} & 1.28504466 & 3.85513397 & 8.00000000 \\ \mathrm{Cu} & 0.00000000 & 5.99687506 & 8.60577586 \\ \mathrm{Cu} & 1.28504466 & 0.42834822 & 9.21155172 \\ \mathrm{Cu} & 0.00000000 & 2.57008931 & 9.81732758 \\ \mathrm{Cu} & 1.28504466 & 4.71183041 & 10.42310344 \\ \mathrm{Cu} & 5.08262806 & 6.90166720 & 10.95285681 \\ \mathrm{Cu} & 1.32345584 & 1.32221310 & 11.62462085 \\ \mathrm{Cu} & 0.21170314 & 3.87532385 & 12.73272671 \\ \mathrm{Cu} & 1.23346831 & 6.79971116 & 13.44623632 \\ \mathrm{Cu} & 0.18637970 & 1.13377706 & 14.06866335 \\ \mathrm{Cu} & 1.28504466 & 11.56540191 & 8.00000000 \\ \mathrm{Cu} & 0.00000000 & 13.70714300 & 8.60577586 \\ \mathrm{Cu} & 1.28504466 & 8.13861616 & 9.21155172 \\ \mathrm{Cu} & 0.00000000 & 10.28035725 & 9.81732758\end{array}$


$\mathrm{Cu}$

$\mathrm{Cu}$

$\mathrm{Cu}$

$\mathrm{Cu}$

$\mathrm{Cu}$

$\mathrm{Cu}$

$\mathrm{Cu}$

$\mathrm{Cu}$

$\mathrm{Cu}$

$\mathrm{Cu}$

$\mathrm{Cu}$

$\mathrm{Cu}$

$\mathrm{Cu}$

$\mathrm{Cu}$

$\mathrm{Cu}$

$\mathrm{Cu}$

$\mathrm{Cu}$

$\mathrm{Cu}$

$\mathrm{Cu}$

$\mathrm{Cu}$

$\mathrm{Cu}$

$\mathrm{Cu}$

$\mathrm{Cu}$

$\mathrm{Cu}$

$\mathrm{Cu}$

$\mathrm{Cu}$

$\mathrm{N}$

$\mathrm{N}$

$\mathrm{N}$

$\mathrm{N}$

$\mathrm{N}$

$\mathrm{N}$

$\mathrm{N}$

$\mathrm{N}$

$\mathrm{N}$
1.28504466

5.09671921

1.31855516

0.06052403

0.21161787

0.04043764

3. 85513397

2. 57008931

3.85513397

2. 57008931

3.85513397

2.47478703

3.89356393

2.79490762

3.53634027

2. 74836698

3.85513397

2.57008931

3.85513397

2.57008931

3. 85513397

2. 53877608

3. 88614704

2. 67121482

2.83332629

2.69696255

1.46895264

4.06057926

4.62385642

1.35665866

1.46794355

1.68858884

4.14129873

3. 94918954

1.35372773
Fri Apr 16 14:51:55 2021

12.42209835

14.56039844

9.02603615

11.68257362

14.14076386

9.28935241

3.85513397

5.99687506

0.42834822

2.57008931

4.71183041

6.88883918

1.24507815

3.66783333

5.94960413

1.08271014

11.56540191

13.70714300

8.13861616

10.28035725

12.42209835

14.55539443

9.07049821

11.64679447

14.06893990

9.19989735

2.28018759

15.14659314

7.44793441

10.17983837

13.15056413

5.21477160

2.43740823

10.35266120

8.10451353
10.42310344

10.97438000

11.57812497

12.74659464

13.42748656

14.32381751

8.00000000

8.60577586

9.21155172

9.81732758

10.42310344

10.91947946

11.57478609

12.82611233

12.83533273

14.00760725

8.00000000

8.60577586

9.21155172

9.81732758

10.42310344

10.99653346

11.72626448

12.83714755

13.42550253

14.20081350

13.33258002

14.33385420

12.74687455

13.08257738

12.37403561

12.38935853

13.05869134

13.33275229

14.77568542

./nitrogen/Cu/311/iter_1/struct.xyz

37

Lattice $=" 7.71026794009301 \quad 0.0 \quad 0.0 \quad 2.5700893133643348 \quad 8.524021930131648 \quad 0.0 \quad 0.0 \quad 0.0 \quad 21.47944$ 8827071757 " Properties=species:S:1:pos:R:3 pbc="T T T"

$\mathrm{Cu} \quad 2.57008931$

$\mathrm{Cu} \quad 2.57008931$

$\mathrm{Cu} \quad 1.28504466$

$\mathrm{Cu} \quad 1.29470286$

$\mathrm{Cu} \quad 2.61154210$

$\mathrm{Cu} \quad-0.03745775$

$\mathrm{Cu} \quad 3.85513397$

$\mathrm{Cu} \quad 3.85513397$

$\mathrm{Cu} \quad 2.57008931$

$\mathrm{Cu} \quad 2.58539686$

$\mathrm{Cu} \quad 3.86915273$

$\mathrm{Cu} \quad 1.30362093$

$\mathrm{Cu} \quad 5.14017863$

$\mathrm{Cu} \quad 5.14017863$

$\mathrm{Cu} \quad 3.85513397$

$\mathrm{Cu} \quad 3.86636550$

$\mathrm{Cu} \quad 5.16476914$

$\mathrm{Cu} \quad 2.63379767$

$\mathrm{Cu} \quad 6.42522328$

$\mathrm{Cu} \quad 6.42522328$

$\mathrm{Cu} \quad 5.14017863$

$\mathrm{Cu} \quad 5.15227340$

$\mathrm{Cu} \quad 6.44162442$

$\mathrm{Cu} \quad 3.89449276$

$\mathrm{Cu} \quad 7.71026794$

$\mathrm{Cu} \quad 7.71026794$

$\mathrm{Cu} \quad 6.42522328$

$\mathrm{Cu} \quad 6.43363789$

$\mathrm{Cu}$
3.09964434

0.77491108

2.71218880

0.40516835

2. 34594989

$-0.03901992$

7.36165530

5.03692205

6.97419976

4.65623725

6.57511241

4. 35320464

3.09964434

0.77491108

2.71218880

0.40647297

2. 34803383

$-0.06015810$

7.36165530

5.03692205

6.97419976

4.67125498

6.57930019

4.29625381

3. 09964434

0.77491108

2.71218880

0.41424444

2.36953966
8.00000000

9.09588977

10.19177953

11.26869680

12.49014582

13.42044778

8.00000000

9.09588977

10.19177953

11.29328633

12.40414452

13.39811979

8.00000000

9.09588977

10.19177953

11.28330830

12.41324407

13.42289581

8.00000000

9.09588977

10.19177953

11.28463047

12.41829838

13.39045040

8.00000000

9.09588977

10.19177953

11.28530534

12.49299794 


\section{$\mathrm{Cu}$}

$\mathrm{Cu}$

$\mathrm{Cu}$

$\mathrm{Cu}$

$\mathrm{Cu}$

$\mathrm{Cu}$

$\mathrm{Cu}$

$\mathrm{N}$

\subsection{7}

8.99531260

8.99531260

7.71026794

7.71940413

9.01128647

6.42634776

1.31041365
Fri Apr 16 14:51:55 2021

$-0.03623905$

7.36165530

5.03692205

6.97419976

4.65812771

6.59476635

4.30573067

1.29217235
13.37312445

8.00000000

9.09588977

10.19177953

11.28594229

12.40383082

13.39107140

13.48453770

38

Lattice $=" 7.71026794009301 \quad 0.0 \quad 0.0 \quad 2.5700893133643348 \quad 8.524021930131648 \quad 0.0 \quad 0.0 \quad 0.0 \quad 21.47944$ 8827071757 " Properties=species:S:1:pos:R:3 pbc="T T T"

$\mathrm{Cu}$

$\mathrm{Cu}$

2.57008931

3.09964434

2.57008931

0.77491108

1.28504466

2.71218880

1.28779228

0.40274234

2.60709742

2. 34426177

0.00894524

0.06812492

3.85513397

7.36165530

3. 85513397

5.03692205

2.57008931

6.97419976

2.56783203

4.67201691

3. 87442126

6.63148582

1.32015537

4.10329754

5.14017863

3.09964434

5.14017863

0.77491108

3.85513397

2.71218880

3.85658370

0.38242763

5.11385332

2.33414269

2. 58448244

0.06329513

6.42522328

7.36165530

6.42522328

5.03692205

5.14017863

6.97419976

5.13226090

4.67928941

6.40747502

6.64669428

3. 84199015

4.29700164

7.71026794

3.09964434

7.71026794

0.77491108

6.42522328

2.71218880

6.43263130

0.37935121

8.00000000

9.09588977

10.19177953

11.23039435

12.40979739

13.42918305

8.00000000

9.09588977

10.19177953

11.29649930

12.40821259

13.52964490

8.00000000

9.09588977

10.19177953

11.28599533

12.39016143

13.41886234

8.00000000

9.09588977

10.19177953

11.28369729

12.52655753

13.38597921

8.00000000

9.09588977

10.19177953

11.29611202

7.65780145

2.33367538

5.15688383

0.06718448

7.36165530

8.99531260

5.03692205

7.71026794

6.97419976

7.71329804

4.69078291

9.06663453

6.59835971

6.29275166

4.21796250

1.30217275

1.49324012

7.72171638

5.44654531

./nitrogen/Cu/311/iter_3/struct.xyz

12.41245838

13.39978947

8.00000000

9.09588977

10.19177953

11.31458842

12.56670847

13.44699614

13.59221494

13.35648050

39

Lattice $=" 7.71026794009301 \quad 0.0 \quad 0.0 \quad 2.5700893133643348 \quad 8.524021930131648 \quad 0.0 \quad 0.0 \quad 0.0 \quad 21.47944$ 8827071757 " Properties=species:S:1:pos:R:3 pbc="T T T"

$\begin{array}{lrlr}\mathrm{Cu} & 2.57008931 & 3.09964434 & 8.00000000 \\ \mathrm{Cu} & 2.57008931 & 0.77491108 & 9.09588977 \\ \mathrm{Cu} & 1.28504466 & 2.71218880 & 10.19177953 \\ \mathrm{Cu} & 1.27069342 & 0.36589734 & 11.26103109 \\ \mathrm{Cu} & 2.57823536 & 2.27750924 & 12.53318734 \\ \mathrm{Cu} & -0.16885293 & 0.03695367 & 13.42709874 \\ \mathrm{Cu} & 3.85513397 & 7.36165530 & 8.0000000 \\ \mathrm{Cu} & 3.85513397 & 5.03692205 & 9.09588977 \\ \mathrm{Cu} & 2.57008931 & 6.97419976 & 10.19177953 \\ \mathrm{Cu} & 2.57358850 & 4.64492793 & 11.32124333 \\ \mathrm{Cu} & 3.79238779 & 6.52243630 & 12.48326312 \\ \mathrm{Cu} & 1.38803880 & 4.31819920 & 13.48268421 \\ \mathrm{Cu} & 5.14017863 & 3.09964434 & 8.0000000 \\ \mathrm{Cu} & 5.14017863 & 0.77491108 & 9.09588977\end{array}$




\section{$\mathrm{Cu}$}

$\mathrm{Cu}$

$\mathrm{Cu}$

$\mathrm{Cu}$

$\mathrm{Cu}$

$\mathrm{Cu}$

$\mathrm{Cu}$

$\mathrm{Cu}$

$\mathrm{Cu}$

$\mathrm{Cu}$

$\mathrm{Cu}$

$\mathrm{Cu}$

$\mathrm{Cu}$

$\mathrm{Cu}$

$\mathrm{Cu}$

$\mathrm{Cu}$

$\mathrm{Cu}$

$\mathrm{Cu}$

$\mathrm{Cu}$

$\mathrm{Cu}$

$\mathrm{Cu}$

$\mathrm{Cu}$

$\mathrm{N}$

$\mathrm{N}$

$\mathrm{N}$

4.16465549

40

trogen/Cu/311/iter_4/struct.xyz

3.85513397

3.82186802

5.29745124

2.42024044

6.42522328

6.42522328

5.14017863

5.13054391

6.38327988

3.91829853

7.71026794

7.71026794

6.42522328

6.40215676

7.76885007

4.91802746

8.99531260

8.99531260

7.71026794

7.70203944

8.93198389

6.38371451

1.13957124

7.70710335

/n 0
Fri Apr 16 14:51:55 2021

$\begin{array}{llll}8827071757 " & \text { Properties=species:S:1:pos:R:3 pbc="T T T" } \\ \mathrm{Cu} & 2.57008931 & 3.09964434 & 8.00000000\end{array}$

$\mathrm{Cu} \quad 2.57008931$

1.28504466

1.30211984

2.87824954

0.34146517

3.85513397

3.85513397

2.57008931

2.56585483

3.93929317

0.81544553

5.14017863

5.14017863

3.85513397

3.89310333

5.03375035

2.72253503

6.42522328

6.42522328

5.14017863

5.10359134

6.50509772

3.47340170

7.71026794

7.71026794

6.42522328

6.43350870

7.62131284

5.26384658

8.99531260

8.99531260

7.71026794

7.71482640

9.02621136

5.96962236

1.11168600

7.51629845

4.44297279
2.71218880

0.37433468

2.40746639

$-0.04147206$

5.03692205

6.97419976

4.72980800

6.68527803

4.10556598

3.09964434

0.77491108

2.71218880

0.38565212

2.37897581

0.36796830

7.36165530

5.03692205

6.97419976

4.67348218

6.68525835

4.27141641

1.35500489

5.58514392

2.17902025
7.36165530
10.19177953

11.27205079

12.27417656

13.47001199

8.00000000

9.09588977

10.19177953

11.30266350

12.54876524

13.47918385

8.00000000

9.09588977

10.19177953

11.28417910

12.40615573

13.53527210

8.00000000

9.09588977

10.19177953

11.28950365

12.48221980

13.46836537

13.62004711

13.48394390

13.76274122
9.09588977

$2.71218880 \quad 10.19177953$

$0.42312354 \quad 11.22295287$

$2.47905844 \quad 13.85664076$

0.16666856

7.36165530

5.03692205

6.97419976

4.66098832

6.74412863

3.81394416

3.09964434

0.77491108

2.71218880

0.42843061

2. 37708821

0.15000391

7. 36165530

5.03692205

6.97419976

4.69763676

6.71640281

5.02619043

3.09964434

0.77491108

2.71218880

0.33699698

2.16719912

0.11051648

7.36165530

5.03692205

6.97419976

4. 63924737

6.40767127

4.39950169

1. 89577059

5.33345822

13.86499331

8.00000000

9.09588977

10.19177953

11.39288286

12.32913721

13.77586026

8.00000000

9.09588977

10.19177953

11.23820002

12.40278147

13.32068693

8.00000000

9.09588977

10.19177953

11.27452386

12.42403345

13.99266017

8.00000000

9.09588977

10.19177953

11.26971840

12.32044531

13.38704393

8.00000000

9.09588977

10.19177953

11.37758229

13.45480795

13.66611024

13.85476122

13.28590009

13.93514569 


\begin{tabular}{|c|c|c|c|}
\hline $\mathrm{N}$ & 3.00622906 & 6.85404773 & 14.02676029 \\
\hline \multicolumn{4}{|c|}{$\begin{array}{l}\text {./nitrogen/Ni/100/iter_1/struct.xyz } \\
49\end{array}$} \\
\hline \multicolumn{4}{|c|}{ Lattice $=" 7.462281943147142 \quad 0.0 \quad 0.0 \quad 0.0 \quad 9.9497092575295$} \\
\hline $\mathrm{Ni}$ & 1.24371366 & 1.24371366 & 8.00000000 \\
\hline $\mathrm{Ni}$ & 0.00000000 & 0.00000000 & 9.75887672 \\
\hline $\mathrm{Ni}$ & 1.24371366 & 1.24371366 & 11.50918693 \\
\hline $\mathrm{Ni}$ & -0.02589532 & -0.02628328 & 13.31333276 \\
\hline $\mathrm{Ni}$ & 1.24371366 & 3.73114097 & 8.00000000 \\
\hline $\mathrm{Ni}$ & 0.00000000 & 2.48742731 & 9.75887672 \\
\hline $\mathrm{Ni}$ & 1.24371366 & 3.75187394 & 11.51796394 \\
\hline $\mathrm{Ni}$ & -0.02589532 & 2.51371059 & 13.31333276 \\
\hline $\mathrm{Ni}$ & 1.24371366 & 6.21856829 & 8.00000000 \\
\hline $\mathrm{Ni}$ & 0.00000000 & 4.97485463 & 9.75887672 \\
\hline $\mathrm{Ni}$ & 1.24371366 & 6.21856829 & 11.52741694 \\
\hline $\mathrm{Ni}$ & 0.00404056 & 4.98231815 & 13.22885258 \\
\hline $\mathrm{Ni}$ & 1.24371366 & 8.70599560 & 8.00000000 \\
\hline $\mathrm{Ni}$ & 0.00000000 & 7.46228194 & 9.75887672 \\
\hline $\mathrm{Ni}$ & 1.24371366 & 8.68526264 & 11.51796394 \\
\hline $\mathrm{Ni}$ & 0.00404056 & 7.45481842 & 13.22885258 \\
\hline $\mathrm{Ni}$ & 3.73114097 & 1.24371366 & 8.00000000 \\
\hline $\mathrm{Ni}$ & 2.48742731 & 0.00000000 & 9.75887672 \\
\hline $\mathrm{Ni}$ & 3.74870757 & 1.24371366 & 11.51632091 \\
\hline $\mathrm{Ni}$ & 2.51332263 & -0.02628328 & 13.31333276 \\
\hline $\mathrm{Ni}$ & 3.73114097 & 3.73114097 & 8.00000000 \\
\hline $\mathrm{Ni}$ & 2.48742731 & 2.48742731 & 9.75887672 \\
\hline $\mathrm{Ni}$ & 3.72927823 & 3.72374127 & 11.53891648 \\
\hline $\mathrm{Ni}$ & 2.51332263 & 2.51371059 & 13.31333276 \\
\hline $\mathrm{Ni}$ & 3.73114097 & 6.21856829 & 8.00000000 \\
\hline $\mathrm{Ni}$ & 2.48742731 & 4.97485463 & 9.75887672 \\
\hline $\mathrm{Ni}$ & 3.73364632 & 6.21856829 & 11.53764526 \\
\hline $\mathrm{Ni}$ & 2.48338675 & 4.98231815 & 13.22885258 \\
\hline $\mathrm{Ni}$ & 3.73114097 & 8.70599560 & 8.00000000 \\
\hline $\mathrm{Ni}$ & 2.48742731 & 7.46228194 & 9.75887672 \\
\hline $\mathrm{Ni}$ & 3.72927823 & 8.71339530 & 11.53891648 \\
\hline $\mathrm{Ni}$ & 2.48338675 & 7.45481842 & 13.22885258 \\
\hline $\mathrm{Ni}$ & 6.21856829 & 1.24371366 & 8.00000000 \\
\hline $\mathrm{Ni}$ & 4.97485463 & 0.00000000 & 9.75887672 \\
\hline $\mathrm{Ni}$ & 6.20100169 & 1.24371366 & 11.51632091 \\
\hline $\mathrm{Ni}$ & 4.97485463 & 0.00811283 & 13.23379606 \\
\hline $\mathrm{Ni}$ & 6.21856829 & 3.73114097 & 8.00000000 \\
\hline $\mathrm{Ni}$ & 4.97485463 & 2.48742731 & 9.75887672 \\
\hline $\mathrm{Ni}$ & 6.22043102 & 3.72374127 & 11.53891648 \\
\hline $\mathrm{Ni}$ & 4.97485463 & 2.47931449 & 13.23379606 \\
\hline $\mathrm{Ni}$ & 6.21856829 & 6.21856829 & 8.00000000 \\
\hline $\mathrm{Ni}$ & 4.97485463 & 4.97485463 & 9.75887672 \\
\hline $\mathrm{Ni}$ & 6.21606294 & 6.21856829 & 11.53764526 \\
\hline $\mathrm{Ni}$ & 4.97485463 & 4.96373965 & 13.22835385 \\
\hline $\mathrm{Ni}$ & 6.21856829 & 8.70599560 & 8.00000000 \\
\hline $\mathrm{Ni}$ & 4.97485463 & 7.46228194 & 9.75887672 \\
\hline $\mathrm{Ni}$ & 6.22043102 & 8.71339530 & 11.53891648 \\
\hline $\mathrm{Ni}$ & 4.97485463 & 7.47339693 & 13.22835385 \\
\hline $\mathrm{N}$ & 1.24371366 & 1.24371366 & 13.66630909 \\
\hline
\end{tabular}

. /nitrogen/Ni/100/iter_2/struct.xyz 50

Lattice $=" 7.462281943147142 \quad 0.0 \quad 0.0 \quad 0.0 \quad 9.9497092575295230 .0 \quad 0.0 \quad 0.021 .27663016512527 "$ Pro perties=species:S:1:pos:R:3 pbc="T T T"

$\begin{array}{lllr}\mathrm{Ni} & 1.24371366 & 1.24371366 & 8.00000000 \\ \mathrm{Ni} & 0.00000000 & 0.00000000 & 9.75887672 \\ \mathrm{Ni} & 1.24874102 & 1.24371366 & 11.52396164 \\ \mathrm{Ni} & 7.43343611 & 9.92349871 & 13.31585178 \\ \mathrm{Ni} & 1.24371366 & 3.73114097 & 8.00000000 \\ \mathrm{Ni} & 0.00000000 & 2.48742731 & 9.75887672 \\ \mathrm{Ni} & 1.24251548 & 3.76173783 & 11.51825138 \\ \mathrm{Ni} & 7.43343611 & 2.51363786 & 13.31585178 \\ \mathrm{Ni} & 1.24371366 & 6.21856829 & 8.00000000 \\ \mathrm{Ni} & 0.00000000 & 4.97485463 & 9.75887672\end{array}$


$\mathrm{Ni}$

$\mathrm{Ni}$

$\mathrm{Ni}$

$\mathrm{Ni}$

$\mathrm{Ni}$

$\mathrm{Ni}$

$\mathrm{Ni}$

$\mathrm{Ni}$

$\mathrm{Ni}$

$\mathrm{Ni}$

$\mathrm{Ni}$

$\mathrm{Ni}$

$\mathrm{Ni}$

$\mathrm{Ni}$

$\mathrm{Ni}$

$\mathrm{Ni}$

$\mathrm{Ni}$

$\mathrm{Ni}$

$\mathrm{Ni}$

$\mathrm{Ni}$

$\mathrm{Ni}$

$\mathrm{Ni}$

$\mathrm{Ni}$

$\mathrm{Ni}$

$\mathrm{Ni}$

$\mathrm{Ni}$

$\mathrm{Ni}$

$\mathrm{Ni}$

$\mathrm{Ni}$

$\mathrm{Ni}$

$\mathrm{Ni}$

$\mathrm{Ni}$

$\mathrm{Ni}$

$\mathrm{Ni}$

$\mathrm{Ni}$

$\mathrm{Ni}$

$\mathrm{Ni}$

$\mathrm{Ni}$

$\mathrm{N}$

$\mathrm{N}$
1.26237968

0.03251365

1.24371366

0.00000000

1.24251548

0.03251365

3.73114097

2. 48742731

3. 74852849

2. 52420794

3. 73114097

2. 48742731

3.73133338

2. 52420794

3. 73114097

2. 48742731

3.71347287

2.48326524

3. 73114097

2. 48742731

3. 73133338

2. 48326524

6.21856829

4.97485463

6.20191957

4.98092493

6.21856829

4.97485463

6.22164579

4.98092493

6.21856829

4.97485463

6.21496654

4.93823218

6.21856829

4.97485463

6.22164579

4.93823218

1.25149944

6.21137107
Fri Apr 16 14:51:55 2021

6.21856829

4.94566038

8.70599560

7.46228194

8.67539874

7.49147620

1.24371366

0.00000000

1.24371366

9.90765024

3.73114097

2.48742731

3.73099243

2.52948633

6.21856829

4.97485463

6.21856829

4.99236900

8.70599560

7.46228194

8.70614414

7.44476757

1.24371366

0.00000000

1.24371366

0.01842050

3.73114097

2. 48742731

3.69991645

2.46900682

6.21856829

4.97485463

6.21856829

4.92958333

8.70599560

7.46228194

8.73722012

7.50755324

1.24371366

6.21856829
11.50627490

13.31527183

8.00000000

9.75887672

11.51825138

13.31527183

8.00000000

9.75887672

11.51411180

13.31905814

8.00000000

9.75887672

11.53790579

13.31905814

8.00000000

9.75887672

11.51418863

13.23054241

8.00000000

9.75887672

11.53790579

13.23054241

8.00000000

9.75887672

11.50633403

13.23024312

8.00000000

9.75887672

11.51774568

13.23024312

8.00000000

9.75887672

11.52621463

13.31737235

8.00000000

9.75887672

11.51774568

13.31737235

13.61714828

13.59505698

51

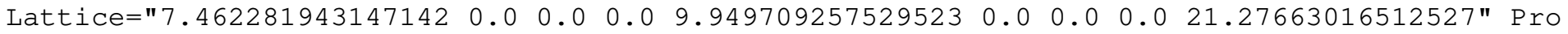
perties=species:S:1:pos:R:3 pbc="T T T"

$\mathrm{Ni} \quad 1.24371366 \quad 1.24371366$

$\mathrm{Ni} \quad 0.00000000 \quad 0.00000000$

$\mathrm{Ni} \quad 1.23390578 \quad 1.24371366$

$\mathrm{Ni} \quad 7.40389619 \quad 9.86214676$

$\mathrm{Ni} \quad 1.24371366$

0.00000000

2.48742731

1.25123166

3.75788627

7.40389619

2.57498981

1. 24371366

6.21856829

0.00000000

4.97485463

1. 26536749

6.21856829

0.02614913

4.96263073

1. 24371366

8.70599560

0.00000000

7.46228194

1. 25123166

0.02614913

3. 73114097

8.67925031

7.47450584

1.24371366

2. 48742731

3. 74316446

2. 48562661

3. 73114097

0.00000000

1. 24371366

$-0.01576460$

3.73114097

2. 48742731

3. 75773023

8.00000000

9.75887672

11.53967227

13.30743853

8.00000000

9.75887672

11.52543245

13.30743853

8.00000000

9.75887672

11.50898306

13.31346081

8.00000000

9.75887672

11.52543245

13.31346081

8.00000000

9.75887672

11.54028453

13.42289401

8.00000000

9.75887672

3. 72716793

2.50319191

11.52680825

13.42289401 
$\mathrm{Ni}$

$\mathrm{Ni}$

$\mathrm{Ni}$

$\mathrm{Ni}$

$\mathrm{Ni}$

$\mathrm{Ni}$

$\mathrm{Ni}$

$\mathrm{Ni}$

$\mathrm{Ni}$

$\mathrm{Ni}$

$\mathrm{Ni}$

$\mathrm{Ni}$

$\mathrm{Ni}$

$\mathrm{Ni}$

$\mathrm{Ni}$

$\mathrm{Ni}$

$\mathrm{Ni}$

$\mathrm{Ni}$

$\mathrm{Ni}$

$\mathrm{Ni}$

$\mathrm{Ni}$

$\mathrm{Ni}$

$\mathrm{Ni}$

$\mathrm{Ni}$

$\mathrm{N}$

$\mathrm{N}$

$\mathrm{N}$

.$/$

./nitrogen/Ni/100/iter_4/struct.xyz

3.73114097

3.71519189

2.48705070

3.73114097

2.48742731

3. 72716793

2.48705070

6.21856829

4.97485463

6.21858048

5.02870856

6.21856829

4.97485463

6.21678063

5.02870856

6.21856829

4.97485463

6.21785835

4.95155130

6.21856829

4.97485463

6.21678063

4.95155130

1.09448168

6.22114273

3.87746733
Fri Apr 16 14:51:55 2021

6.21856829

4.97485463

6.21856829
4.99277529

8.70599560

7.46228194

8.67940634

7.44436128

1.24371366

0.00000000

1.24371366

$-0.08581317$

3.73114097

2.48742731

3. 70860886

2.57324049

6.21856829

4.97485463

6.21856829

4.96241719

8.70599560

7.46228194

7.47471938

1.24371366

6.21856829

1.24371366
8.72852771
8.00000000

9.75887672

11.51255660

13.23840188

8.00000000

9.75887672

11.52680825

13.23840188

8.00000000

9.75887672

11.47475110

13.31031247

8.00000000

9.75887672

11.50089519

13.31031247

8.00000000

9.75887672

11.50007305

13.31873528

8.00000000

9.75887672

11.50089519

13.31873528

13.52464547

13.68184153

13.53570007

perties $=$ species:S:1:pos:R:3 pbc="T T T"

$\begin{array}{lllr}\mathrm{Ni} & 1.24371366 & 1.24371366 & 8.00000000 \\ \mathrm{Ni} & 0.00000000 & 0.00000000 & 9.75887672 \\ \mathrm{Ni} & 1.22831927 & 1.21737753 & 11.57235910 \\ \mathrm{Ni} & 7.34780101 & 9.87181721 & 13.33654540 \\ \mathrm{Ni} & 1.24371366 & 3.73114097 & 8.00000000 \\ \mathrm{Ni} & 0.00000000 & 2.48742731 & 9.75887672 \\ \mathrm{Ni} & 1.26127175 & 3.75286668 & 11.51072266 \\ \mathrm{Ni} & 7.39581651 & 2.56791169 & 13.30027196 \\ \mathrm{Ni} & 1.24371366 & 6.21856829 & 8.00000000 \\ \mathrm{Ni} & 0.00000000 & 4.97485463 & 9.75887672 \\ \mathrm{Ni} & 1.27947784 & 6.22126660 & 11.50502719 \\ \mathrm{Ni} & 0.10086894 & 4.92995925 & 13.31936308 \\ \mathrm{Ni} & 1.24371366 & 8.70599560 & 8.00000000 \\ \mathrm{Ni} & 0.00000000 & 7.46228194 & 9.75887672 \\ \mathrm{Ni} & 1.22906947 & 8.67770257 & 11.52275311 \\ \mathrm{Ni} & 0.05098362 & 7.46876550 & 13.31813597 \\ \mathrm{Ni} & 3.73114097 & 1.24371366 & 8.00000000 \\ \mathrm{Ni} & 2.48742731 & 0.00000000 & 9.75887672 \\ \mathrm{Ni} & 3.72682571 & 1.25177413 & 11.56325536 \\ \mathrm{Ni} & 2.49349587 & 0.02161626 & 13.61595073 \\ \mathrm{Ni} & 3.73114097 & 3.73114097 & 8.00000000 \\ \mathrm{Ni} & 2.48742731 & 2.48742731 & 9.75887672 \\ \mathrm{Ni} & 3.73594411 & 3.76041017 & 11.50478552 \\ \mathrm{Ni} & 2.43671179 & 2.54794586 & 13.42814830 \\ \mathrm{Ni} & 3.73114097 & 6.21856829 & 8.00000000 \\ \mathrm{Ni} & 2.48742731 & 4.97485463 & 9.75887672 \\ \mathrm{Ni} & 3.73069990 & 6.21604216 & 11.49580181 \\ \mathrm{Ni} & 2.53656519 & 4.99347073 & 13.22439127 \\ \mathrm{Ni} & 3.73114097 & 8.70599560 & 8.00000000 \\ \mathrm{Ni} & 2.48742731 & 7.46228194 & 9.75887672 \\ \mathrm{Ni} & 3.72060648 & 8.69226704 & 11.49563932 \\ \mathrm{Ni} & 2.45379659 & 7.42460947 & 13.30889436 \\ \mathrm{Ni} & 6.21856829 & 1.24371366 & 8.00000000 \\ \mathrm{Ni} & 4.97485463 & 0.00000000 & 9.75887672 \\ \mathrm{Ni} & 6.19748618 & 1.23306117 & 11.49212545 \\ \mathrm{Ni} & 4.92399289 & 9.87754870 & 13.43725181 \\ \mathrm{Ni} & 6.21856829 & 3.73114097 & 8.00000000\end{array}$


$\mathrm{Ni}$

$\mathrm{Ni}$

$\mathrm{Ni}$

$\mathrm{Ni}$

$\mathrm{Ni}$

$\mathrm{Ni}$

$\mathrm{Ni}$

$\mathrm{Ni}$

$\mathrm{Ni}$

$\mathrm{Ni}$

$\mathrm{Ni}$

$\mathrm{N}$

$\mathrm{N}$

$\mathrm{N}$

$\mathrm{N}$

. $/$

. /nitrogen/Ni/100/iter_5/struct.xyz perties $=$ species:S:1:pos:R:3 pbc="T T T"

$\mathrm{N}$

$\mathrm{Ni}$

1.24371366

0.00000000

1.22984776

7. 35517162

1.24371366

0.00000000

1.24346760

7.35689549

1.24371366

0.00000000

1.28891107

0.11557196

1.24371366

0.00000000

1.24534793

0.11657911

3.73114097

2.48742731

3. 71276199

2. 47659557

3.73114097

2.48742731

3. 73488017

2.47659383

3.73114097

2. 48742731

3. 73505153

2. 48625148

3. 73114097

2.48742731

3.73536993

2. 48752349

6.21856829

4.97485463

6.18138716

4.95706133

6.21856829

4.97485463

6.22096909

4.95650626

6.21856829

4.97485463

6.23163340

5.01789541

6.21856829

4.97485463

6.22135700

5.01866129

1.06540709

4.96316723

1.24371366

0.00000000

1.24556858

9.87855795

3.73114097

2. 48742731

3. 75052381

2. 55655455

6.21856829

4.97485463

6.22011404

4.92893157

8.70599560

7.46228194

8.68838457

7.50704485

1.24371366

0.00000000

1. 24390508

$-0.04251478$

3.73114097

2. 48742731

3. 75102959

2.52830805

6.21856829

4. 97485463

6.21875287

5.00716495

8.70599560

7.46228194

8.68463307

7. 42882187

1.24371366

0.00000000

1.24527560

9.82497338

3. 73114097

2. 48742731

2. 61265485

6.21856829

4.97485463

6.21876488

4.99219336

8.70599560

7.46228194

8.73106203

7.44803558

1.24204048
Fri Apr 16 14:51:55 2021

50

2.48742731

3.71154694

2.63085744

6.21856829

4.97485463

6.22956519

8.70599560

7.46228194

8.73084804

7.43997010

1.23337319

6.16536728

1.39630354

8.55087819
9.75887672
11.48135676
13.30998554
8.00000000
9.75887672
11.51615951
13.28011623
8.00000000
9.75887672
11.51905149
13.42814803
13.55532356
13.65536304
13.45976586
13.93320245
$.9497092575295230 .0 \quad 0.0 \quad 0.021 .27663016512527$ " Pro 8.00000000

3.70674248
9.75887672

11.60118788

13.33345862

9.75887672

11.50895023

13.33515858

8.00000000

9.75887672

11.49350089

13.31692963

8.00000000

9.75887672

11.50830119

13.31549976

8.00000000

9.75887672

11.53902802

13.60396490

8.00000000

9.75887672

11.47276471

13.60538707

8.00000000

9.75887672

11.48655849

13.29301295

8.00000000

9.75887672

11.47216433

13.29368606

8.00000000

9.75887672

11.51174383

13.43198476

8.00000000

9.75887672

11.50592435

13.43461889

9.75887672

11.54591780

13.41995746

8.00000000

9.75887672

11.50387064

13.41583323

13.55893023
8.00000000

8.00000000 
$\mathrm{N}$

$\mathrm{N}$

$\mathrm{N}$

$\mathrm{N}$

\section{69581101 \\ 6.37197149 \\ 3. 81898828 \\ 3.69579550}

. / n
Fri Apr 16 14:51:55 2021

$\begin{array}{ll}6.21897685 & 13.57924107 \\ 1.24394300 & 13.35297832 \\ 8.50740673 & 14.00225402 \\ 3.93004806 & 14.00163185\end{array}$

51

perties=species:S:1:pos:R:3 pbc="T T T"

$\mathrm{Ni}$

$\mathrm{Ni}$

$\mathrm{Ni}$

1.24371366

0.00000000

1.27959631

$-0.03583333$

1. 24371366

0.00000000

1. 21315060

$-0.03583333$

1. 24371366

0.00000000

1.23916922

$-0.00719484$

1.24371366

0.00000000

1. 21315060

$-0.00719484$

3.73114097

2.48742731

3. 74050606

2.59641568

3. 73114097

2. 48742731

3.72153251

2.59641568

3. 73114097

2.48742731

3.72904343

2. 43679660

3. 73114097

2.48742731

3. 72153251

2.43679660

6.21856829

4.97485463

6.19087657

5.02038781

6.21856829

4.97485463

6.24547143

5.02038781

6.21856829

4.97485463

6.22178378

5.02386928

6.21856829

4.97485463

6.24547143

5.02386928

1.12540192

6.13919703

4.00361422

3.73770217

3. 73770217

1. 31769110

.$/$

55
1.24371366

0.00000000

$-0.08866654$

3.73114097

2. 48742731

3. 74908151

2.57609385

6.21856829

4.97485463

6.21856829

5.01749009

8.70599560

7.46228194

8.68805507

7.41964648

1.24371366

0.00000000

1. 24371366

0.09288458

3.73114097

2. 48742731

3.72012636

2.39454273

6.21856829

4.97485463

6.21856829

5.00827192

8.70599560

7.46228194

8.71701021

7.42886465

1.24371366

0.00000000

1.24371366

0.03118548

3. 73114097

2.48742731

3.72205466

2. 45624184

6.21856829

4.97485463

6.21856829

5.01835087

8.70599560

7.46228194

8.71508191

7.41878570

1. 24371366

6.21856829

1. 24371366

8.63887374

3.79826283

6.21856829
1. 24371366
8.00000000

9.75887672

11.59279226

13.31287087

8.00000000

9.75887672

11.50479952

13.31287087

8.00000000

9.75887672

11.47404041

13.44987459

8.00000000

9.75887672

11.50479952

13.44987459

8.00000000

9.75887672

11.46097598

13.69898067

9.75887672

11.55073810

13.69898067

8.00000000

9.75887672

11.45194146

13.31760141

8.00000000

9.75887672

11.55073810

13.31760141

9.75887672

11.51795619

13.45146474

8.00000000

9.75887672

11.51937035

13.45146474

8.00000000

11.46711053

13.31273803

9.75887672

11.51937035

13.31273803

13.52082162

14.04819350

14.34123347

13.46077659

13.46077659

14.03786825
8.00000000

8.00000000

9.75887672

8.00000000

perties $=$ species:S:1:pos:R:3 pbc="T T T"
$\mathrm{Ni}$
1.24371366
1.24371366

$30.0 \quad 0.0 \quad 0.021 .27663016512527 "$ Pro 
$\mathrm{Ni}$

$\mathrm{Ni}$

$\mathrm{Ni}$

$\mathrm{Ni}$

$\mathrm{Ni}$

$\mathrm{Ni}$

$\mathrm{Ni}$

$\mathrm{Ni}$

$\mathrm{Ni}$

$\mathrm{Ni}$

$\mathrm{Ni}$

$\mathrm{Ni}$

$\mathrm{Ni}$

$\mathrm{Ni}$

$\mathrm{Ni}$

$\mathrm{Ni}$

$\mathrm{Ni}$

$\mathrm{Ni}$

$\mathrm{Ni}$

$\mathrm{Ni}$

$\mathrm{Ni}$

$\mathrm{Ni}$

$\mathrm{Ni}$

$\mathrm{Ni}$

$\mathrm{Ni}$

$\mathrm{Ni}$

$\mathrm{Ni}$

$\mathrm{Ni}$

$\mathrm{Ni}$

$\mathrm{Ni}$

$\mathrm{Ni}$

$\mathrm{Ni}$

$\mathrm{Ni}$

$\mathrm{Ni}$

$\mathrm{Ni}$

$\mathrm{Ni}$

$\mathrm{Ni}$

$\mathrm{Ni}$

$\mathrm{Ni}$

$\mathrm{Ni}$

$\mathrm{Ni}$

$\mathrm{Ni}$

$\mathrm{Ni}$

$\mathrm{Ni}$

$\mathrm{Ni}$

$\mathrm{Ni}$

$\mathrm{N}$

$\mathrm{N}$

$\mathrm{N}$

$\mathrm{N}$

$\mathrm{N}$

$\mathrm{N}$

$\mathrm{N}$
6.44921031

1.28640816

0.04220538

1.24371366

0.00000000

1.20991115

$-0.02831486$

1.24371366

0.00000000

1.21323987

$-0.01683427$

1.24371366

0.00000000

1.23545399

0.00204086

3.73114097

2.48742731

3.75562573

2. 64785910

3. 73114097

2. 48742731

3. 71951788

2.61848047

2. 48742731

3.73357429

2.41586589

3.73114097

2. 48742731

3.75392920

2.40349600

6.21856829

4.97485463

6.19180936

5.04631715

6.21856829

4.97485463

6.24291980

6.21856829

4.97485463

6.21715115

5.01725308

6.21856829

4.97485463

6.24503042

5.13524640

1.19312431

6.10069238

3. 99214412

3. 68432637

3. 76234454

1. 34899294
3.73114097

5.01132040
Fri Apr 16 14:51:55 2021

1.24779893

$-0.04775465$

3.73114097

2.48742731

3.75786966

2.60609167

6.21856829

4.97485463

6.22130051

5.02218369

8.70599560

7.46228194

8.68950181

7.38786860

1.24371366

0.00000000

1.22333016

0.11238417

3.73114097

2.48742731

3.70363091

2.41828353

6.21856829

4.97485463

6.23128141

4.95384080

8.70599560

7.46228194

8.70188192

7.37740753

1.24371366

0.00000000

1.24585027

$-0.07100874$

3.73114097

2. 48742731

3.72758642

2.40880295

6.21856829

4.97485463

6.24321751

5.02232030

8.70599560

7.46228194

8.70681634

7.47987537

1. 32518708

6.02139198

1.24758772

8.57941499

3.78698867

6.14985998

8.79938824
11.57592900

13.34688748

8.00000000

9.75887672

11.48573619

13.28390194

8.00000000

9.75887672

11.48225460

13.46518016

8.00000000

9.75887672

11.50331344

13.66318778

8.00000000

9.75887672

11.47868687

13.64773545

8.00000000

9.75887672

11.54318668

13.79253876

8.00000000

9.75887672

11.47105766

13.29844748

8.00000000

9.75887672

11.56143757

13.30013980

8.00000000

9.75887672

11.53182800

13.70268846

8.00000000

9.75887672

11.51001621

13.45228758

8.00000000

9.75887672

11.48530390

13.25505845

8.00000000

9.75887672

11.51491411

13.61654676

13.47013233

14.21862870

14.40614003

13.40352737

13.43406818

14.10124410

14.23776968

56

Lattice $=" 7.462281943147142 \quad 0.0 \quad 0.0 \quad 0.0 \quad 9.949709257529523 \quad 0.0 \quad 0.0 \quad 0.021 .27663016512527 "$ Pro perties=species:S:1:pos:R:3 pbc="T T T"

$\begin{array}{lllr}\mathrm{Ni} & 1.24371366 & 1.24371366 & 8.00000000 \\ \mathrm{Ni} & 0.00000000 & 0.00000000 & 9.75887672 \\ \mathrm{Ni} & 1.32762947 & 1.21970690 & 11.57089281 \\ \mathrm{Ni} & 0.21667228 & 9.77043623 & 13.34403925 \\ \mathrm{Ni} & 1.24371366 & 3.73114097 & 8.00000000 \\ \mathrm{Ni} & 0.00000000 & 2.48742731 & 9.75887672 \\ \mathrm{Ni} & 1.21601294 & 3.69839774 & 11.43681216 \\ \mathrm{Ni} & 0.20547354 & 2.53769095 & 13.37596984 \\ \mathrm{Ni} & 1.24371366 & 6.21856829 & 8.00000000 \\ \mathrm{Ni} & 0.00000000 & 4.97485463 & 9.75887672 \\ \mathrm{Ni} & 1.17309163 & 6.18366995 & 11.57425199\end{array}$


$\mathrm{Ni}$

$\mathrm{Ni} \quad 1.24371366$

$\mathrm{Ni} \quad 0.00000000$

$\mathrm{Ni} \quad 1.27278664$

$\mathrm{Ni} \quad 7.14588995$

$\mathrm{Ni} \quad 3.73114097$

$\mathrm{Ni} \quad 2.48742731$

$\mathrm{Ni} \quad 3.79014454$

$\mathrm{Ni} \quad 2.74363047$

$\mathrm{Ni} \quad 3.73114097$

$\mathrm{Ni} \quad 2.48742731$

$\mathrm{Ni} \quad 3.73359560$

$\mathrm{Ni} \quad 2.81713849$

$\mathrm{Ni} \quad 3.73114097$

$\mathrm{Ni} \quad 2.48742731$

$\mathrm{Ni} \quad 3.69448818$

$\mathrm{Ni} \quad 2.24400539$

$\mathrm{Ni} \quad 3.73114097$

$\mathrm{Ni} \quad 2.48742731$

$\mathrm{Ni} \quad 3.70243108$

$\mathrm{Ni} \quad 2.31346331$

$\mathrm{Ni} \quad 6.21856829$

$\mathrm{Ni}$

$\mathrm{Ni}$

$\mathrm{Ni}$

$\mathrm{Ni}$

$\mathrm{Ni}$

$\mathrm{Ni}$

$\mathrm{Ni}$

$\mathrm{Ni}$

$\mathrm{Ni}$

$\mathrm{Ni}$

$\mathrm{Ni}$

$\mathrm{Ni}$

$\mathrm{Ni}$

$\mathrm{Ni}$

$\mathrm{Ni}$

$\mathrm{N}$

$\mathrm{N}$

$\mathrm{N}$

$\mathrm{N}$

$\mathrm{N}$

$\mathrm{N}$

$\mathrm{N}$

/nitrogen/Ni/100/iter 9/struct.xyz
Fri Apr 16 14:51:55 2021

4.90671039

8.70599560

7.46228194

8.66795007

7.22955529

1. 24371366

0.00000000

1.24126285

9.87811593

3.73114097

2.48742731

3. 72590850

2. 25403468

6.21856829

4.97485463

6.28157659

4.74892667

8.70599560

7.46228194

8.72346325

7.48139623

1.24371366

0.00000000

1. 31128965

0.19087781

3.73114097

2. 48742731

3. 75236074

2.61716302

6.21856829

4.97485463

6.21355634

5.17642383

8.70599560

7.46228194

8.69810155

7.60397312

1.14356811

6.15314851

1.17324916

8.26881771

3.79436677

6.11072190

8.76933103

3.29735948
14.11166240

8.00000000

9.75887672

11.44657360

13.63569209

8.00000000

9.75887672

11.46391293

14.10997547

8.00000000

9.75887672

11.55594297

13.65071836

8.00000000

9.75887672

11.50398411

13.37850958

8.00000000

9.75887672

11.56495583

13.37066963

8.00000000

9.75887672

11.51164676

13.30149393

8.00000000

9.75887672

11.56253230

13.84838971

8.00000000

9.75887672

11.46066432

13.30566828

8.00000000

9.75887672

11.55186088

13.86512593

13.45639319

14.44910124

14.45330979

14.60984822

13.42284012

13.45554791

13.41570158

14.60994546

Lattice $=" 7.462281943147142 \quad 0.0 \quad 0.0 \quad 0.0 \quad 9.949709257529523 \quad 0.0 \quad 0.0 \quad 0.021 .27663016512527 "$ Pro perties=species:S:1:pos:R:3 pbc="T T T"

$\mathrm{N}$

$\mathrm{Ni}$

$\mathrm{Ni}$

$\mathrm{Ni}$

$\mathrm{Ni}$

$\mathrm{Ni}$

$\mathrm{Ni}$

$\mathrm{Ni}$

$\mathrm{Ni}$

$\mathrm{Ni}$

$\mathrm{Ni}$

$\mathrm{Ni}$

$\mathrm{Ni}$

$\mathrm{Ni}$

$\mathrm{Ni}$

$\mathrm{Ni}$

$\mathrm{Ni}$

$\mathrm{Ni}$

$\mathrm{Ni}$
1.24371366

0.00000000

1.28945624

0.31168427

1. 24371366

0.00000000

1. 22545449

0.14350357

1. 24371366

0.00000000

1. 19817533

$-0.17339818$

1. 24371366

0.00000000

1.26298526

$-0.19788379$

3.73114097

2. 48742731

3. 75345393
1. 24371366

0.00000000

1. 21134556

$-0.25247675$

3.73114097

2.48742731

3. 70667746

2.56125707

6.21856829

4.97485463

6.20506208

4.92448047

8.70599560

7.46228194

8.67689534

7. 33245725

1. 24371366

0.00000000

1.22682694
8.00000000

9.75887672

11.57716828

13.56710775

8.00000000

9.75887672

11.44153121

13.35836303

8.00000000

9.75887672

11.57591175

14.10954552

8.00000000

9.75887672

11.45537882

13.79773633

8.00000000

9.75887672

11.44005719 
$\mathrm{Ni}$

$\mathrm{Ni}$

$\mathrm{Ni}$

$\mathrm{Ni}$

$\mathrm{Ni}$

$\mathrm{Ni}$

$\mathrm{Ni}$

$\mathrm{Ni}$

$\mathrm{Ni}$

$\mathrm{Ni}$

$\mathrm{Ni}$

$\mathrm{Ni}$

$\mathrm{Ni}$

$\mathrm{Ni}$

$\mathrm{Ni}$

$\mathrm{Ni}$

$\mathrm{Ni}$

$\mathrm{Ni}$

$\mathrm{Ni}$

$\mathrm{Ni}$

$\mathrm{Ni}$

$\mathrm{Ni}$

$\mathrm{Ni}$

$\mathrm{Ni}$

$\mathrm{Ni}$

$\mathrm{Ni}$

$\mathrm{Ni}$

$\mathrm{Ni}$

$\mathrm{Ni}$

$\mathrm{N}$

$\mathrm{N}$

N

$\mathrm{N}$

$\mathrm{N}$

N

$\mathrm{N}$

$\mathrm{N}$

$\mathrm{N}$
2.59059449

3. 73114097

2. 48742731

3. 74633081

2.83499534

3.73114097

2. 48742731

3.71035083

2. 31435110

3. 73114097

2. 48742731

3. 69286222

2. 38544036

6.21856829

4.97485463

6.22445154

4.91107013

6.21856829

4.97485463

6.25848753

5.15450036

6.21856829

4.97485463

6.18869254

4.96269998

6.21856829

4.97485463

6.22114177

4.87086242

1. 34162553

5.91198462

4.06713990

3.54044564

3. 79124473

1.15014227

6.18960980

$-0.82390942$

1.19693753
Fri Apr 16 14:51:55 2021

$-0.09911125$

3.73114097

2.48742731

3.69972447

2.19278541

6.21856829

4.97485463

6.25781662

4.72469766

8.70599560

7.46228194

8.69791553

7.46113521

1.24371366

0.00000000

1.30522858

0.12976541

3.73114097

2.48742731

3.75221927

2.54385295

6.21856829

4.97485463

6.22891168

5.07823161

8.70599560

7.46228194

8.71358817

7.52176123

1.21579398

6.15915414

1.09567253

8.26968902

3.72180339

6.07042740

8.82799448

3. 35198912

8.50397178
14.33737464

8.00000000

9.75887672

11.54179741

13.66180757

8.00000000

9.75887672

11.52135511

13.39848253

8.00000000

9.75887672

11.52730201

13.48472352

8.00000000

9.75887672

11.54280059

13.27764182

8.00000000

9.75887672

11.54019050

13.98087651

8.00000000

9.75887672

11.49145874

13.31918411

8.00000000

9.75887672

11.52427017

13.63904077

13.42642113

14.38454006

14.51834578

14.57605571

13.39984448

13.41104079

13.34250167

14.59956633

14.63375940

46

Lattice $=" 7.46228194314714 \quad 0.0 \quad 0.0 \quad 0.0 \quad 10.553260330250541 \quad 0.0 \quad 0.0 \quad 0.020 .97485462876476 "$ Pro perties $=$ species: $:: 1:$ pos:R:3 pbc="T T T"

$\begin{array}{llrr}\mathrm{Ni} & 0.00000000 & 0.00000000 & 8.00000000 \\ \mathrm{Ni} & 1.24371366 & 1.75887672 & 9.24371366 \\ \mathrm{Ni} & 0.00000000 & 0.00000000 & 10.48742731 \\ \mathrm{Ni} & 1.28654318 & 1.75887672 & 11.82828732 \\ \mathrm{Ni} & 0.00000000 & -0.03313743 & 13.01465131 \\ \mathrm{Ni} & 0.00000000 & 3.51775344 & 8.00000000 \\ \mathrm{Ni} & 1.24371366 & 5.27663017 & 9.24371366 \\ \mathrm{Ni} & 0.00000000 & 3.51775344 & 10.48742731 \\ \mathrm{Ni} & 1.23726557 & 5.27219389 & 11.77494915 \\ \mathrm{Ni} & 0.00000000 & 3.55089087 & 13.01465131 \\ \mathrm{Ni} & 0.00000000 & 7.03550689 & 8.00000000 \\ \mathrm{Ni} & 1.24371366 & 8.79438361 & 9.24371366 \\ \mathrm{Ni} & 0.00000000 & 7.03550689 & 10.48742731 \\ \mathrm{Ni} & 1.23726557 & 8.79881988 & 11.77494915 \\ \mathrm{Ni} & 0.00000000 & 7.03550689 & 12.88093129 \\ \mathrm{Ni} & 2.48742731 & 0.00000000 & 8.00000000 \\ \mathrm{Ni} & 3.73114097 & 1.75887672 & 9.24371366 \\ \mathrm{Ni} & 2.48742731 & 0.00000000 & 10.48742731 \\ \mathrm{Ni} & 3.73114097 & 1.75887672 & 11.79666404 \\ \mathrm{Ni} & 2.52707335 & -0.06670626 & 12.88869399 \\ \mathrm{Ni} & 2.48742731 & 3.51775344 & 8.00000000 \\ \mathrm{Ni} & 3.73114097 & 5.27663017 & 9.24371366 \\ \mathrm{Ni} & 2.48742731 & 3.51775344 & 10.48742731 \\ \mathrm{Ni} & 3.73114097 & 5.30057780 & 11.76759764 \\ \mathrm{Ni} & 2.52707335 & 3.58445970 & 12.88869399 \\ \mathrm{Ni} & 2.48742731 & 7.03550689 & 8.00000000\end{array}$


$\mathrm{Ni}$

$\mathrm{Ni}$

$\mathrm{Ni}$

$\mathrm{Ni}$

$\mathrm{Ni}$

$\mathrm{Ni}$

$\mathrm{Ni}$

$\mathrm{Ni}$

$\mathrm{Ni}$

$\mathrm{Ni}$

$\mathrm{Ni}$

$\mathrm{Ni}$

$\mathrm{Ni}$

$\mathrm{Ni}$

$\mathrm{Ni}$

$\mathrm{Ni}$

$\mathrm{Ni}$

$\mathrm{Ni}$

$\mathrm{Ni}$

$\mathrm{N}$
Fri Apr 16 14:51:55 2021

3.73114097

2.48742731

3.73114097

2.47962912

4.97485463

6.21856829

4.97485463

6.17573876

4.93520860

4.97485463

6.21856829

4.97485463

6.22501637

4.93520860

4.97485463

6.21856829

4.97485463

6.22501637

4. 98265282

0.00000000
8.79438361

7.03550689

8.77043598

7.03550689

0.00000000

1.75887672

0.00000000

1.75887672

$-0.06670626$

3.51775344

5.27663017

3.51775344

5.27219389

3.58445970

7.03550689

8.79438361

7.03550689

8.79881988

7.03550689

1.75887672
9.24371366

10.48742731

11.76759764

12.88835234

8.00000000

9.24371366

10.48742731

11.82828732

12.88869399

8.00000000

9.24371366

10.48742731

11.77494915

12.88869399

8.00000000

9.24371366

10.48742731

11.77494915

12.88835234

13.13521242

55

Lattice $=" 7.462281943147140 .0 \quad 0.0 \quad 0.0 \quad 10.5532603302505410 .00 .0 \quad 0.020 .97485462876476 "$ Pro perties=species:S:1:pos:R:3 pbc="T T T"

$\mathrm{Ni}$

$\mathrm{Ni}$

0.00000000

1.00000000

$1.24371366 \quad 1.75887672$

$0.00000000 \quad 0.00000000$

1.23728064

1.81469676

$-0.00889356$

0.04193497

0.00000000

3.51775344

1.24371366

5.27663017

0.00000000

3.51775344

1.24371366

5.27663017

0.04494322

3.70039441

0.00000000

7.03550689

1.24371366

8.79438361

0.00000000

7.03550689

1.25014667

8.73856357

0.13125377

6.92539719

2. 48742731

0.00000000

3.73114097

1.75887672

2.48742731

0.00000000

3.70883292

1.82964057

2.49632088

2.48742731

3. 73114097

$-0.04193497$

3.51775344

5.27663017

2.48742731

3.72906978

2. 35617355

3.51775344

5.24093184

3.62786314

2. 48742731

3.73114097

2.48742731

7.03550689

8.79438361

7.03550689

3.74929978

2.44248409

4.97485463

8.70922798

6.85286592

0.00000000

6.21856829

1.75887672

4.97485463

0.00000000

6.20040948

1. 84403235

4.97485463

0.84403235

4.97485463

3.51775344

6.21856829

5.27663017

4.97485463

6.22063948

3.51775344

5. 31232849

3.53990774

4.93053734

7.03550689

8.79438361

6.21856829

4.97485463

7.03550689

8.00000000

9.24371366

10.48742731

11.84706097

13.19039016

8.00000000

9.24371366

10.48742731

11.69859932

13.17821058

8.00000000

9.24371366

10.48742731

11.84706097

13.67109607

8.00000000

9.24371366

10.48742731

11.86524768

13.19039016

8.00000000

9.24371366

10.48742731

11.78003361

13.67109607

8.00000000

9.24371366

10.48742731

11.84528584

13.17821058

8.00000000

9.24371366

10.48742731

11.84528584

13.19795513

8.00000000

9.24371366

10.48742731

11.78003361

13.59423081

8.00000000

9.24371366

10.48742731

8.72361976

11.86524768 
$\mathrm{Ni}$

$\mathrm{N} \quad 4.97236726$

$\mathrm{N} \quad 4.97734200$

4.97485463

1.24371366

0.03311418

$-0.02318468$

2. 51061199

2.45431314

6.27641721

3.67329205
Fri Apr 16 14:51:55 2021

7.01335259

1. 76001566

8.79324467

5.27663017

5.27663017

8.77160944

1.87250082

8.68075951

1.78165090

6.86148924

3. 69177109
13.59423081

13.21234467

13.21234467

13.06460878

13.51878487

13.21217570

13.22194514

13.22194514

13.21217570

14.75219307

14.75219307

. /nitrogen/Ni/110/iter_11/struct.xyz 56

Lattice $=" 7.46228194314714 \quad 0.0 \quad 0.0 \quad 0.0 \quad 10.5532603302505410 .0 \quad 0.0 \quad 0.020 .97485462876476 "$ Pro perties=species:S:1:pos:R:3 pbc="T T T"

\begin{tabular}{|c|c|c|c|}
\hline $\mathrm{Ni}$ & 0.00000000 & 0.00000000 & 8.00000000 \\
\hline $\mathrm{Ni}$ & 1.24371366 & 1.75887672 & 9.24371366 \\
\hline $\mathrm{Ni}$ & 0.00000000 & 0.00000000 & 10.48742731 \\
\hline $\mathrm{Ni}$ & 1.22488760 & 1.76951969 & 11.86585543 \\
\hline $\mathrm{Ni}$ & 0.00259537 & 0.01083540 & 13.54286259 \\
\hline $\mathrm{Ni}$ & 0.00000000 & 3.51775344 & 8.00000000 \\
\hline $\mathrm{Ni}$ & 1.24371366 & 5.27663017 & 9.24371366 \\
\hline $\mathrm{Ni}$ & 0.00000000 & 3.51775344 & 10.48742731 \\
\hline $\mathrm{Ni}$ & 1.24371366 & 5.27663017 & 11.69294266 \\
\hline $\mathrm{Ni}$ & 0.05342619 & 3.70173583 & 13.17582071 \\
\hline $\mathrm{Ni}$ & 0.00000000 & 7.03550689 & 8.00000000 \\
\hline $\mathrm{Ni}$ & 1.24371366 & 8.79438361 & 9.24371366 \\
\hline $\mathrm{Ni}$ & 0.00000000 & 7.03550689 & 10.48742731 \\
\hline $\mathrm{Ni}$ & 1.26253971 & 8.78374064 & 11.86585543 \\
\hline $\mathrm{Ni}$ & 0.12093234 & 6.89656289 & 13.67409072 \\
\hline $\mathrm{Ni}$ & 2.48742731 & 0.00000000 & 8.00000000 \\
\hline $\mathrm{Ni}$ & 3.73114097 & 1.75887672 & 9.24371366 \\
\hline $\mathrm{Ni}$ & 2.48742731 & 0.00000000 & 10.48742731 \\
\hline $\mathrm{Ni}$ & 3.71690899 & 1.78353549 & 11.83841371 \\
\hline $\mathrm{Ni}$ & 2.48483194 & -0.01083540 & 13.54286259 \\
\hline $\mathrm{Ni}$ & 2.48742731 & 3.51775344 & 8.00000000 \\
\hline $\mathrm{Ni}$ & 3.73114097 & 5.27663017 & 9.24371366 \\
\hline $\mathrm{Ni}$ & 2.48742731 & 3.51775344 & 10.48742731 \\
\hline $\mathrm{Ni}$ & 3.72783527 & 5.24093496 & 11.78224423 \\
\hline $\mathrm{Ni}$ & 2.36649497 & 3.65669744 & 13.67409072 \\
\hline $\mathrm{Ni}$ & 2.48742731 & 7.03550689 & 8.00000000 \\
\hline $\mathrm{Ni}$ & 3.73114097 & 8.79438361 & 9.24371366 \\
\hline $\mathrm{Ni}$ & 2.48742731 & 7.03550689 & 10.48742731 \\
\hline $\mathrm{Ni}$ & 3.74675660 & 8.75129133 & 11.84843975 \\
\hline $\mathrm{Ni}$ & 2.43400112 & 6.85152450 & 13.17582071 \\
\hline $\mathrm{Ni}$ & 4.97485463 & 0.00000000 & 8.00000000 \\
\hline $\mathrm{Ni}$ & 6.21856829 & 1.75887672 & 9.24371366 \\
\hline $\mathrm{Ni}$ & 4.97485463 & 0.00000000 & 10.48742731 \\
\hline $\mathrm{Ni}$ & 6.20295266 & 1.80196900 & 11.84843975 \\
\hline $\mathrm{Ni}$ & 4.97485463 & 0.00000000 & 13.18924120 \\
\hline $\mathrm{Ni}$ & 4.97485463 & 3.51775344 & 8.00000000 \\
\hline $\mathrm{Ni}$ & 6.21856829 & 5.27663017 & 9.24371366 \\
\hline $\mathrm{Ni}$ & 4.97485463 & 3.51775344 & 10.48742731 \\
\hline $\mathrm{Ni}$ & 6.22187399 & 5.31232537 & 11.78224423 \\
\hline $\mathrm{Ni}$ & 4.93754089 & 3.54067405 & 13.6002799 \\
\hline $\mathrm{Ni}$ & 4.97485463 & 7.03550689 & 8.00000000 \\
\hline $\mathrm{Ni}$ & 6.21856829 & 8.79438361 & 9.24371366 \\
\hline $\mathrm{Ni}$ & 4.97485463 & 7.03550689 & 10.48742731 \\
\hline $\mathrm{Ni}$ & 6.23280027 & 8.76972484 & 11.8384137 \\
\hline $\mathrm{Ni}$ & 5.01216837 & 7.01258628 & 13.6002799 \\
\hline $\mathrm{N}$ & 4.96217529 & 1.76431842 & 13.21206092 \\
\hline $\mathrm{N}$ & 4.98753397 & 8.78894191 & 13.21206092 \\
\hline $\mathrm{N}$ & 4.97485463 & 5.27663017 & 13.06211482 \\
\hline $\mathrm{N}$ & 1.24371366 & 5.27663017 & 13.51744168 \\
\hline $\mathrm{N}$ & 0.00603994 & 8.71060869 & 13.17241069 \\
\hline $\mathrm{N}$ & -0.03935558 & 1.88761221 & 13.20233793 \\
\hline $\mathrm{N}$ & 2.52678289 & 8.66564812 & 13.20233793 \\
\hline $\mathrm{N}$ & 2.48138737 & 1.84265164 & 13.1724106 \\
\hline
\end{tabular}




$\begin{array}{llll}\mathrm{N} & 6.26817189 & 6.83499507 & 14.76062033 \\ \mathrm{~N} & 3.68153737 & 3.71826526 & 14.76062033 \\ \mathrm{~N} & 1.24371366 & 0.00000000 & 14.76577384\end{array}$

./nitrogen/Ni/110/iter_12/struct. xyz

57

Lattice $=" 7.46228194314714 \quad 0.0 \quad 0.0 \quad 0.0 \quad 10.5532603302505410 .0 \quad 0.0 \quad 0.020 .97485462876476 "$ Pro perties=species:S:1:pos:R:3 pbc="T T T"

$\mathrm{Ni} \quad 1.24371366 \quad 1.75887672$

$\mathrm{Ni} \quad 0.00000000 \quad 0.00000000$

$\mathrm{Ni} \quad 1.20801242 \quad 1.74394634$

$\mathrm{Ni} \quad 0.00649219 \quad 0.02118765$

$\mathrm{Ni} \quad 0.00000000 \quad 3.51775344$

$\mathrm{Ni} \quad 1.24371366 \quad 5.27663017$

$\mathrm{Ni} \quad 0.00000000 \quad 3.51775344$

$\mathrm{Ni} \quad 1.24692945 \quad 5.27274987$

$\mathrm{Ni} \quad 0.02474896 \quad 3.71011273$

$\begin{array}{lll}\mathrm{Ni} & 0.00000000 & 7.03550689\end{array}$

$\begin{array}{lll}\mathrm{Ni} & 1.24371366 & 8.79438361\end{array}$

$\mathrm{Ni} \quad 0.00000000 \quad 7.03550689$

$\mathrm{Ni} \quad 1.25243463 \quad 8.80432692$

$\mathrm{Ni} \quad 0.11883454 \quad 6.88407356$

$\mathrm{Ni} \quad 2.48742731 \quad 0.00000000$

$\mathrm{Ni} \quad 3.73114097 \quad 1.75887672$

$\begin{array}{lll}\mathrm{Ni} & 2.48742731 & 0.00000000\end{array}$

$\mathrm{Ni} \quad 3.71838872 \quad 1.72980956$

$\mathrm{Ni} \quad 2.48826447$

$\mathrm{Ni} \quad 2.48742731$

$\mathrm{Ni} \quad 3.73114097$

10.54835206

3.51775344

5.27663017

2.48742731

3.51775344

3.73291017

5.23883077

2. 35423616

3.66384511

2. 48742731

3. 73114097

7.03550689

8.79438361

2. 48742731

7.03550689

3. 74353098

8.79374103

2. 43409894

6.86413215

4.97485463

0.00000000

6.21856829

1.75887672

4.97485463

0.00000000

6.21216425

1.76948018

4. 98010239

0.01494197

4.97485463

3.51775344

6.21856829

5.27663017

4. 97485463

3. 51775344

6.22789540

5. 31532210

4. 91915559

3.54822004

4. 97485463

7.03550689

6.21856829

8.79438361

7.03550689

4.97485463

8.82136386

8.00000000

9.24371366

10.48742731

11.86835923

13.93612669

8.00000000

9.24371366

10.48742731

11.70667820

13.20323876

8.00000000

9.24371366

10.48742731

11.85747593

13.69089518

8.00000000

9.24371366

10.48742731

11.82306317

13.52273508

8.00000000

9.24371366

10.48742731

11.78975625

13.66462535

8.00000000

9. 24371366

10.48742731

11.81036771

13.17117660

8.00000000

9.24371366

10.48742731

11.87313331

13.59416749

8.00000000

9.24371366

10.48742731

11.78028837

13.62994772

8.00000000

9.24371366

10.48742731

11.84952246

13.56344716

5.01858670

7.01338443

1.77978599

8.79917677

4.96923140

5.27345866

4.98922739

5.28510596

1. 24847197

8.71895711

0.01955832

1.89868927

7.44039302

1.89868927
8.67678067

1. 84541162

2. 48445680

6.92837697

13.18005520

13.15185719

13.06571408

13.53049719

13.18156087

13.21924632

13.17301737

13.15116454

14.76317342

14.77120440

14.90376503

0.00225849

1.46013585

10.48938076

./nitrogen/Ni/110/iter_13/struct.xyz

14.95882169

58

Lattice $=" 7.46228194314714 \quad 0.0 \quad 0.0 \quad 0.0 \quad 10.5532603302505410 .0 \quad 0.0 \quad 0.020 .97485462876476 "$ Pro perties=species:S:1:pos:R:3 pbc="T T T" 
$\mathrm{Ni}$

$\mathrm{Ni}$

$\mathrm{Ni}$

$\mathrm{Ni}$

$\mathrm{Ni}$

$\mathrm{Ni}$

$\mathrm{Ni}$

$\mathrm{Ni}$

$\mathrm{Ni}$

$\mathrm{Ni}$

$\mathrm{Ni}$

$\mathrm{Ni}$

$\mathrm{Ni}$

$\mathrm{Ni}$

$\mathrm{Ni}$

$\mathrm{Ni}$

$\mathrm{Ni}$

$\mathrm{Ni}$

$\mathrm{Ni}$

$\mathrm{Ni}$

$\mathrm{Ni}$

$\mathrm{Ni}$

$\mathrm{Ni}$

$\mathrm{Ni}$

$\mathrm{Ni}$

$\mathrm{N}$

$\mathrm{Ni}$

$\mathrm{Ni}$

$\mathrm{N}$

$\mathrm{Ni}$

$\mathrm{Ni}$

$\mathrm{N}$

$\mathrm{Ni}$

$\mathrm{Ni}$

$\mathrm{Ni}$

$\mathrm{Ni}$

$\mathrm{Ni}$

$\mathrm{Ni}$

$\mathrm{Ni}$

$\mathrm{Ni}$

$\mathrm{Ni}$

$\mathrm{Ni}$

$\mathrm{Ni}$

$\mathrm{Ni}$

$\mathrm{Ni}$

$\mathrm{N}$

$\mathrm{N}$

$\mathrm{N}$

$\mathrm{N}$

$\mathrm{N}$

$\mathrm{N}$

$\mathrm{N}$

$\mathrm{N}$

$\mathrm{N}$

$\mathrm{N}$

$\mathrm{N}$

$\mathrm{N}$

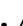

47

\subsection{0 \\ 1.24371366 \\ 0.00000000 \\ 1.23892271 \\ 0.01007870 \\ 0.00000000 \\ 1.24371366 \\ 0.00000000 \\ 1.22463601 \\ 0.08867457 \\ 0.00000000 \\ 1.24371366 \\ 0.00000000 \\ 1.24512498 \\ 0.14111956 \\ 2. 48742731 \\ 3. 73114097 \\ 2.48742731 \\ 3.75107993 \\ 2. 49303972 \\ 2.48742731 \\ 3.73114097 \\ 2. 48742731 \\ 3. 71191668 \\ 2. 42989887 \\ 2.48742731 \\ 3.73114097 \\ 2. 48742731 \\ 3.74988696 \\ 2.46516119 \\ 4.97485463 \\ 6.21856829 \\ 4.97485463 \\ 6.23477412 \\ 4.98483953 \\ 4.97485463 \\ 6.21856829 \\ 4.97485463 \\ 6.21441747 \\ 4.96399560 \\ 4.97485463 \\ 6.21856829 \\ 4.97485463 \\ 6.24177275 \\ 5.03020461 \\ 4.97009489 \\ 4.97036432 \\ 4.89554669 \\ 1. 34393278 \\ 0.01511181 \\ 0.00221180 \\ 2. 52932005 \\ 2. 52312221 \\ 6.26273132 \\ 3. 47654374 \\ 1.45657833 \\ 6.02042538 \\ 6.46954513}

Fri Apr 16 14:51:55 2021

0.00000000

1.75887672

0.00000000

1.78276309

0.14831044

3.51775344

5.27663017

3.51775344

5.25529298

3.82225188

7.03550689

8.79438361

7.03550689

8.82013348

6.99305014

0.00000000

1.75887672

0.00000000

1.75554359

0.05838387

3.51775344

5.27663017

3.51775344

5.24379877

3.72903283

7.03550689

8.79438361

7.03550689

8.80559167

6.93717950

0.00000000

1.75887672

0.00000000

1. 75731408

0.15445725

3.51775344

5.27663017

3.51775344

5.26291892

3. 68882665

7.03550689

8.79438361

7.03550689

8.82572749

7.07136109

1.97897821

8.89768182

5.28916172

5.40369131

8.83065653

1.99429646

8.75646870

1.91140973

6.89297782

3.75607227

0.13691564

0.16640378

3. 92012109
8.00000000

9.24371366

10.48742731

11.84724182

13.90508638

8.00000000

9.24371366

10.48742731

11.73231837

13.61209972

8.00000000

9.24371366

10.48742731

11.84486256

13.62852164

8.00000000

9.24371366

10.48742731

11.79937740

13.50209566

8.00000000

9.24371366

10.48742731

11.75802179

13.61302340

8.00000000

9.24371366

10.48742731

11.81092374

13.13087612

8.00000000

9.24371366

10.48742731

11.84840349

13.52955813

8.00000000

9.24371366

10.48742731

11.84740544

14.11701634

8.00000000

9.24371366

10.48742731

11.84829441

13.49565630

13.13867978

13.16150259

13.06593967

13.53744355

13.17910142

13.15542428

13.15981720

13.11305938

14.68942972

14.95926209

14.87732403

14.91633491

14.92539930

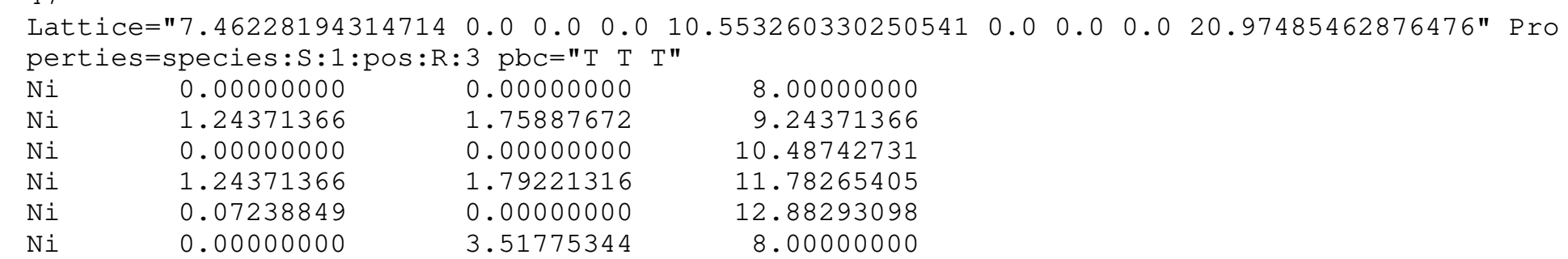


$\mathrm{Ni} \quad 0.00000000$

$\mathrm{Ni} \quad 1.24371366$

$\mathrm{Ni} \quad 0.03183453$

$\mathrm{Ni} \quad 0.00000000$

$\mathrm{Ni} \quad 1.24371366$

$\mathrm{Ni} \quad 0.00000000$

$\mathrm{Ni} \quad 1.24371366$

$\mathrm{Ni} \quad 0.03183453$

$\mathrm{Ni} \quad 2.48742731$

$\mathrm{Ni} \quad 3.73114097$

$\mathrm{Ni} \quad 2.48742731$

$\mathrm{Ni} \quad 3.70324592$

$\mathrm{Ni} \quad 2.41503882$

$\mathrm{Ni} \quad 2.48742731$

$\mathrm{Ni} \quad 3.73114097$

$\mathrm{Ni} \quad 2.48742731$

$\mathrm{Ni} \quad 3.74164002$

$\mathrm{Ni} \quad 2.45559278$

$\mathrm{Ni} \quad 2.48742731$

$\mathrm{Ni} \quad 3.73114097$

$\mathrm{Ni} \quad 2.48742731$

$\mathrm{Ni}$

$\mathrm{Ni}$

$\mathrm{Ni}$

$\mathrm{Ni}$

$\mathrm{Ni}$

$\mathrm{Ni}$

$\mathrm{Ni}$

$\mathrm{Ni}$

$\mathrm{Ni}$

$\mathrm{Ni}$

$\mathrm{Ni}$

$\mathrm{Ni}$

$\mathrm{Ni}$

$\mathrm{Ni}$

$\mathrm{Ni}$

$\mathrm{Ni}$

$\mathrm{Ni}$

$\mathrm{N}$

$\mathrm{N}$

3. 70324592

2. 45559278

4.97485463

6.21856829

4.97485463

6.24646334

4.97485463

4.97485463

6.21856829

4.97485463

6.20806923

4.97485463

4.97485463

6.21856829

4. 97485463

6.24646334

4.97485463

4.97485463

4.97485463
Fri Apr 16 14:51:55 2021

5.27663017

3.51775344

5.27663017

3.59292212

7.03550689

8.79438361

7.03550689

8.76104717

6.96033821

0.00000000

1.75887672

0.00000000

1.75983378

0.00000000

3.51775344

5.27663017

3.51775344

5.27663017

3.59292212

7.03550689

8.79438361

7.03550689

8.79342655

6.96033821

0.00000000

1.75887672

0.00000000

1.75983378

0.00000000

3.51775344

5.27663017

3.51775344

5.27663017

3.58920383

7.03550689

8.79438361

7.03550689

8.79342655

6.96405650

1.80897637

8.74428396
9.24371366

10.48742731

11.73450762

12.89123330

8.00000000

9.24371366

10.48742731

11.78265405

12.89123330

8.00000000

9.24371366

10.48742731

11.82412599

12.88293098

8.00000000

9.24371366

10.48742731

11.75968672

12.89123330

8.00000000

9.24371366

10.48742731

11.82412599

12.89123330

8.00000000

9.24371366

10.48742731

11.82412599

13.13856334

8.00000000

9.24371366

10.48742731

11.75968672

13.00503415

8.00000000

9.24371366

10.48742731

11.82412599

13.00503415

13.16055952

13.16055952

48

Lattice $=" 7.462281943147140 .0 \quad 0.0 \quad 0.0 \quad 10.5532603302505410 .0 \quad 0.0 \quad 0.020 .97485462876476 "$ Pro perties $=$ species:S:1:pos:R:3 pbc="T T T"

$\begin{array}{lllr}\mathrm{Ni} & 0.00000000 & 0.00000000 & 8.00000000 \\ \mathrm{Ni} & 1.24371366 & 1.75887672 & 9.24371366 \\ \mathrm{Ni} & 0.00000000 & 0.00000000 & 10.48742731 \\ \mathrm{Ni} & 1.24371366 & 1.75887672 & 11.75304183 \\ \mathrm{Ni} & 0.06391617 & 0.00000000 & 12.89304494 \\ \mathrm{Ni} & 0.00000000 & 3.51775344 & 8.00000000 \\ \mathrm{Ni} & 1.24371366 & 5.27663017 & 9.24371366 \\ \mathrm{Ni} & 0.00000000 & 3.51775344 & 10.48742731 \\ \mathrm{Ni} & 1.24371366 & 5.27663017 & 11.75304183 \\ \mathrm{Ni} & 0.06391617 & 3.51775344 & 12.89304494 \\ \mathrm{Ni} & 0.00000000 & 7.03550689 & 8.00000000 \\ \mathrm{Ni} & 1.24371366 & 8.79438361 & 9.24371366 \\ \mathrm{Ni} & 0.00000000 & 7.03550689 & 10.48742731 \\ \mathrm{Ni} & 1.24371366 & 8.79438361 & 11.75304183 \\ \mathrm{Ni} & 0.06391617 & 7.03550689 & 12.89304494 \\ \mathrm{Ni} & 2.48742731 & 0.00000000 & 8.00000000 \\ \mathrm{Ni} & 3.73114097 & 1.75887672 & 9.24371366 \\ \mathrm{Ni} & 2.48742731 & 0.00000000 & 10.48742731 \\ \mathrm{Ni} & 3.71651001 & 1.75887672 & 11.80950798 \\ \mathrm{Ni} & 2.42351114 & 0.00000000 & 12.89304494 \\ \mathrm{Ni} & 2.48742731 & 3.51775344 & 8.00000000 \\ \mathrm{Ni} & 3.73114097 & 5.27663017 & 9.24371366 \\ \mathrm{Ni} & 2.48742731 & 3.51775344 & 10.48742731\end{array}$


$\mathrm{Ni}$

$\mathrm{Ni}$

3.71651001

2.42351114

2. 48742731

3.73114097

2.48742731

3.71651001

2.42351114

4.97485463

6.21856829

4.97485463

6.23319925

4.97485463

4.97485463

6.21856829

4.97485463

6.23319925

4.97485463

4.97485463

6.21856829

4.97485463

6.23319925

4.97485463

4.97485463

4.97485463

4.97485463
Fri Apr 16 14:51:55 2021

5.27663017

3.51775344

7.03550689

8.79438361

7.03550689

8.79438361

7.03550689

0.00000000

1.75887672

0.00000000

1.75887672

0.00000000

3.51775344

5.27663017

3.51775344

5.27663017

3.51775344

7.03550689

8.79438361

7.03550689

8.79438361

7.03550689

1.75887672

8.79438361

5.27663017
11.80950798

12.89304494

8.00000000

9.24371366

10.48742731

11.80950798

12.89304494

8.00000000

9.24371366

10.48742731

11.80950798

13.18595424

8.00000000

9.24371366

10.48742731

11.80950798

13.18595424

8.00000000

9.24371366

10.48742731

11.80950798

13.18595424

13.18442989

13.18442989

13.18442989

./nitrogen/Ni/110/iter_4/struct.xyz

49

Lattice $=" 7.46228194314714 \quad 0.0 \quad 0.0 \quad 0.0 \quad 10.5532603302505410 .0 \quad 0.0 \quad 0.020 .97485462876476 "$ Pro perties=species:S:1:pos:R:3 pbc="T T T"

$\mathrm{Ni}$

$\mathrm{Ni}$

$\mathrm{Ni}$

$\mathrm{Ni}$

$\mathrm{Ni}$

$\mathrm{Ni}$

$\mathrm{Ni}$

$\mathrm{Ni}$

$\mathrm{Ni}$

$\mathrm{Ni}$

$\mathrm{Ni}$

$\mathrm{Ni}$

$\mathrm{Ni}$

$\mathrm{Ni}$

$\mathrm{Ni}$

$\mathrm{Ni}$

$\mathrm{Ni}$

$\mathrm{Ni}$

$\mathrm{Ni}$

$\mathrm{Ni}$

$\mathrm{Ni}$

$\mathrm{Ni}$

$\mathrm{Ni}$

$\mathrm{Ni}$

$\mathrm{Ni}$

$\mathrm{Ni}$

$\mathrm{Ni}$

$\mathrm{Ni}$

$\mathrm{Ni}$

$\mathrm{Ni}$

$\mathrm{Ni}$

$\mathrm{Ni}$

$\mathrm{Ni}$

$\mathrm{Ni}$

$\mathrm{Ni}$

$\mathrm{Ni}$

$\mathrm{Ni}$

$\mathrm{Ni}$

$\mathrm{Ni}$
0.00000000

1. 24371366

0.00000000

1.24371366

0.06739201

0.00000000

1.24371366

0.00000000

1. 24371366

0.06952583

0.00000000

1. 24371366

0.00000000

1. 24371366

0.06952583

2. 48742731

3. 73114097

2.48742731

3. 68508172

2. 42003530

2.48742731

3. 73114097

2. 48742731

3.77053461

2. 41790149

2. 48742731

3. 73114097

2. 48742731

3. 68508172

2. 41790149

4. 97485463

6.21856829

4. 97485463

6.26462754

4.97485463

4. 97485463

6.21856829

4.97485463

6.17917465
0.00000000

1.75887672

0.00000000

1.83871764

0.00000000

3.51775344

5.27663017

3.51775344

5.27663017

3.76637235

7.03550689

8.79438361

7.03550689

8.71454269

6.78688798

0.00000000

1.75887672

0.00000000

1.82059577

0.00000000

3.51775344

5. 27663017

3.51775344

5.27663017

3.76637235

7.03550689

8.79438361

7.03550689

8.73266456

6.78688798

0.00000000

1.75887672

0.00000000

1.82059577

0.00000000

3.51775344

5.27663017

3.51775344

5.27663017
8.00000000

9.24371366

10.48742731

11.82715025

12.86646569

8.00000000

9.24371366

10.48742731

11.57047185

13.03571221

8.00000000

9.24371366

10.48742731

11.82715025

13.03571221

8.00000000

9.24371366

10.48742731

11.85147411

12.86646569

8.00000000

9.24371366

10.48742731

11.73341485

13.03571221

8.00000000

9.24371366

10.48742731

11.85147411

13.03571221

8.00000000

9.24371366

10.48742731

11.85147411

13.14053499

8.00000000

9.24371366

10.48742731

11.73341485 
$\mathrm{Ni}$

$\mathrm{Ni}$

$\mathrm{Ni}$

$\mathrm{Ni}$

$\mathrm{Ni}$

$\mathrm{Ni}$

$\mathrm{N}$

$\mathrm{N}$

$\mathrm{N}$

$\mathrm{N}$

1.24371366

6.21856829

4.97485463

4.97485463

4.97485463

4.97485463
Fri Apr 16 14:51:55 2021

4.97485463

4.97485463

6.26462754

4.97485463
3.52043243

7.03550689

8.79438361

7.03550689

8.73266456

7.03282790

1.76193002

8.79133031

5.27663017

5.27663017
13.20505707

8.00000000

9.24371366

10.48742731

11.85147411

13.20505707

13.17739551

13.17739551

13.15618145

13.41015326

50

Lattice $=" 7.46228194314714 \quad 0.0 \quad 0.0 \quad 0.0 \quad 10.553260330250541 \quad 0.0 \quad 0.0 \quad 0.020 .97485462876476 "$ Pro perties=species:S:1:pos:R:3 pbc="T T T"

$\mathrm{Ni}$

0.00000000

1.24371366

0.00000000

1.75887672

0.00000000

0.00000000

1.25250025

1.82554733

0.08233849

0.00000000

$-0.07560616$

1.24371366

3.51775344

5.27663017

0.00000000

3. 51775344

1.21472680

5.30213206

0.07770221

3. 74943269

0.00000000

7.03550689

1.24371366

8.79438361

7.03550689

0.00000000

8.69783849

0.00062704

6.86627075

2. 48742731

0.00000000

3.73114097

1.75887672

2.48742731

0.00000000

3.70255405

1.83213124

2. 46124948

0.03944561

2.48742731

3.51775344

3.73114097

5.27663017

2. 48742731

3. 51775344

3.77988014

5.27698161

3.75100843

7.03550689

2. 48742731

8.79438361

7.03550689

3.73114

8.73896700

3.68763376

6.68796811

0.00000000

4.97485463

1.75887672

6.21856829

0.00000000

1.77844197

6.97485463

$-0.00097530$

4. 92657906

3. 51775344

5.27663017

6.21856829

3.51775344

4.97485463

5.32669561

3.52408773

7.03550689

4.99874110

4.97485463

8.79438361

7.03550689

4.97485463

8.69893304

6.20435655

7.03541022

1.76260256

8.79570777

4.99521962

5.28400746

4.78273250
4.97016487
1.24875679

5.22508901

1. 24875679

8.68268826

8.00000000

9.24371366

10.48742731

11.82969365

12.98711055

8.00000000

9.24371366

10.48742731

11.59476603

13.02877996

8.00000000

9.24371366

10.48742731

11.79168748

13.19933010

8.00000000

9.24371366

10.48742731

11.83787909

12.86627314

8.00000000

9.24371366

10.48742731

11.72382714

13.02452403

8.00000000

9.24371366

10.48742731

11.79872807

13.11709484

8.00000000

9.24371366

10.48742731

11.87075830

13.16131217

8.00000000

9.24371366

10.48742731

11.76707774

13.17854130

8.00000000

9.24371366

10.48742731

11.94947760

13.27604322

13.16849666

13.25119423

13.16492426

13.43467774

13.18703284

./nitrogen/Ni/110/iter_6/struct.xyz

51

Lattice $=" 7.462281943147140 .0 \quad 0.0 \quad 0.0 \quad 10.5532603302505410 .0 \quad 0.0 \quad 0.020 .97485462876476 "$ Pro perties=species:S:1:pos:R:3 pbc="T T T" 
$\mathrm{Ni}$

$\mathrm{Ni}$

$\mathrm{Ni}$

$\mathrm{Ni}$

$\mathrm{Ni}$

$\mathrm{Ni}$

$\mathrm{Ni}$

$\mathrm{Ni}$

$\mathrm{Ni}$

$\mathrm{Ni}$

$\mathrm{Ni}$

$\mathrm{Ni}$

$\mathrm{Ni}$

$\mathrm{Ni}$

$\mathrm{Ni}$

$\mathrm{Ni}$

$\mathrm{Ni}$

$\mathrm{Ni}$

$\mathrm{Ni}$

$\mathrm{Ni}$

$\mathrm{Ni}$

$\mathrm{Ni}$

$\mathrm{Ni}$

$\mathrm{Ni}$

$\mathrm{Ni}$

$\mathrm{N}$

$\mathrm{Ni}$

$\mathrm{Ni}$

$\mathrm{Ni}$

$\mathrm{Ni}$

$\mathrm{Ni}$

$\mathrm{Ni}$

$\mathrm{Ni}$

$\mathrm{Ni}$

$\mathrm{Ni}$

$\mathrm{Ni}$

$\mathrm{Ni}$

$\mathrm{Ni}$

$\mathrm{Ni}$

$\mathrm{Ni}$

$\mathrm{Ni}$

$\mathrm{Ni}$

$\mathrm{Ni}$

$\mathrm{Ni}$

$\mathrm{Ni}$

$\mathrm{N}$

$\mathrm{N}$

$\mathrm{N}$

$\mathrm{N}$

N

$\mathrm{N}$
0.00000000

1.24371366

0.00000000

1.28120881

0.11366614

0.00000000

1.24371366

0.00000000

1.18166862

0.08798580

0.00000000

1.24371366

0.00000000

1.28120881

0.08798580

2.48742731

3.73114097

2. 48742731

3.69684735

2.50608856

2. 48742731

3. 73114097

2. 48742731

3. 78893857

2.45037722

2. 48742731

3.73114097

2.48742731

3.69684735

2.45037722

4.97485463

6.21856829

4.97485463

6.23698449

4.90099354

4.97485463

6.21856829

4.97485463

6.20558259

4.92099345

4.97485463

6.21856829

4.97485463

6.23698449

4.92099345

4.80347648

4.80347648

4.97253990

1.25587100

0.16874045

0.16874045
Fri Apr 16 14:51:55 2021

0.00000000

1.75887672

0.00000000

1.82238086

0.00000000

3.51775344

5.27663017

3. 51775344

5.27663017

3.66332267

7.03550689

8.79438361

7.03550689

8.73087947

6.88993766

0.00000000

1.75887672

0.00000000

1. 82560227

0.00000000

3. 51775344

5.27663017

3.51775344

5.27663017

3.83411477

7.03550689

8.79438361

7.03550689

8.72765806

6.71914556

0.00000000

1.75887672

0.00000000

1.78632246

0.00000000

3.51775344

5.27663017

3.51775344

5.27663017

3.51925167

7.03550689

8.79438361

7.03550689

8.76693787

7.03400866

1.75984410

8.79341624

5.27663017

5.27663017

8.71323117

1.84002916
8.00000000

9.24371366

10.48742731

11.79670992

13.18186865

8.00000000

9.24371366

10.48742731

11.61682119

13.19030778

8.00000000

9.24371366

10.48742731

11.79670992

13.19030778

8.00000000

9.24371366

10.48742731

11.79805739

12.86972190

8.00000000

9.24371366

10.48742731

11.71790591

13.08091160

8.00000000

9.24371366

10.48742731

11.79805739

13.08091160

8.00000000

9.24371366

10.48742731

11.96233046

13.22210681

8.00000000

9.24371366

10.48742731

11.78725397

13.24146004

8.00000000

9.24371366

10.48742731

11.96233046

13.24146004

13.23798937

13.23798937

13.16304080

13.44600810

13.21665730

13.21665730

. /nitrogen/Ni/110/iter_7/struct.xyz

52

Lattice $=" 7.46228194314714 \quad 0.0 \quad 0.0 \quad 0.0 \quad 10.5532603302505410 .0 \quad 0.0 \quad 0.020 .97485462876476 "$ Pro perties $=$ species: $S: 1:$ pos:R: 3 pbc $=" T$ T T"

$\mathrm{Ni} \quad 0.00000000 \quad 0.00000000$

$\mathrm{Ni} \quad 1.24371366 \quad 1.75887672$

0.00000000

0.00000000

1.28316415

0.04308276

0.00000000

$-0.00780988$

3.51775344

1.24371366

5.27663017

3.51775344

5.31083575

1.20813103

3.65158385

7.03550689

8.79438361

0.00000000

8.79438361

8.00000000

9.24371366

10.48742731

11.82159002

13.20399134

8.00000000

9.24371366

10.48742731

11.64279122

13.19233817

8.00000000

9.24371366

10.48742731 
$\mathrm{Ni}$

$\mathrm{Ni}$

$\mathrm{Ni}$

$\mathrm{Ni}$

$\mathrm{Ni}$

$\mathrm{Ni}$

$\mathrm{Ni}$

$\mathrm{Ni}$

$\mathrm{Ni}$

$\mathrm{Ni}$

$\mathrm{Ni}$

$\mathrm{Ni}$

$\mathrm{Ni}$

$\mathrm{Ni}$

$\mathrm{Ni}$

$\mathrm{Ni}$

$\mathrm{Ni}$

$\mathrm{Ni}$

$\mathrm{Ni}$

$\mathrm{Ni}$

$\mathrm{Ni}$

$\mathrm{Ni}$

$\mathrm{Ni}$

$\mathrm{Ni}$

$\mathrm{Ni}$

$\mathrm{Ni}$

$\mathrm{Ni}$

$\mathrm{Ni}$

$\mathrm{Ni}$

$\mathrm{Ni}$

$\mathrm{Ni}$

$\mathrm{Ni}$

$\mathrm{N}$

$\mathrm{N}$

$\mathrm{N}$

$\mathrm{N}$

$\mathrm{N}$

$\mathrm{N}$

$\mathrm{N}$
1.22898571

0.07409651

2. 48742731

3.73114097

2.48742731

3.67889261

2. 49664165

2.48742731

3.73114097

2. 48742731

3. 77145658

2. 45253022

2. 48742731

3. 73114097

2. 48742731

3.75050646

2.42824788

4.97485463

6.21856829

4.97485463

6.22878669

4.93048072

4.97485463

6.21856829

4.97485463

6.20788606

4.91674231

4.97485463

6.21856829

4.97485463

6.22318070

5.00083273

4.78559703

4.98007974

4.97654289

1.23855852

$-0.00891745$

0.15075961

2.50333995
Fri Apr 16 14:51:55 2021

8.72979678

6.88016459

0.00000000

1.75887672

0.00000000

1.78633075

$-0.10138352$

3.51775344

5.27663017

3.51775344

5.32410902

3.81941069

7.03550689

8.79438361

7.03550689

8.70891281

6.84586905

0.00000000

1.75887672

0.00000000

1.79481415

0.00329415

3.51775344

5.27663017

3.51775344

5.27184310

3.52257602

7.03550689

8.79438361

7.03550689

8.77602574

7.03561039

1.76291701

8.80281062

5.28061635

5.23802365

8.70000395

1.82426201

8.65319195
11.82558180

13.23925040

8.00000000

9.24371366

10.48742731

11.81069627

13.02643657

8.00000000

9.24371366

10.48742731

11.74955168

13.06362027

8.00000000

9.24371366

10.48742731

11.82245247

13.22687479

8.00000000

9.24371366

10.48742731

11.95372656

13.25201291

8.00000000

9.24371366

10.48742731

11.78049783

13.20559596

8.00000000

9.24371366

10.48742731

11.84421816

13.27095537

13.24221958

13.26936161

13.16374324

13.46780918

13.22432410

13.21930348

13.17980935

53

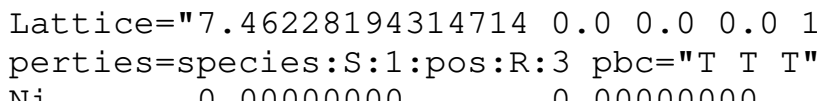

$\mathrm{Ni} \quad 0.00000000 \quad 0.00000000$

$\mathrm{Ni} \quad 1.24371366 \quad 1.75887672$

0.00000000

0.00000000

1.76718068

1.24371366

$-0.00599598$

0.00000000

0.00000000

3.51775344

1. 24371366

5.27663017

0.00000000

3. 51775344

1. 24371366

5.27663017

0.07137081

0.00000000

3.66887141

7.03550689

8.79438361

1.24371366

7.03550689

8.78607965

1.24371366

0.07137081

6.88438892

0.00000000

1. 75887672

3. 73114097

0.00000000

2. 48742731

1.79148541

0.00000000

3.51775344

5.27663017

3.51775344

5.27663017

3. 66887141
8.00000000
9.24371366
10.48742731
11.85291738
13.22652077
8.00000000
9.24371366
10.48742731
11.66835228
13.22357879
8.00000000
9.24371366
10.48742731
11.85291738
13.22357879
8.00000000
9.24371366
10.48742731
11.84127807
13.22652077
8.00000000
9.24371366
10.48742731
11.77640897
13.22357879 
$\mathrm{Ni}$

$\mathrm{Ni}$

$\mathrm{Ni}$

$\mathrm{Ni}$

$\mathrm{Ni}$

$\mathrm{Ni}$

$\mathrm{Ni}$

$\mathrm{Ni}$

$\mathrm{Ni}$

$\mathrm{Ni}$

$\mathrm{Ni}$

$\mathrm{Ni}$

$\mathrm{Ni}$

$\mathrm{Ni}$

$\mathrm{Ni}$

$\mathrm{Ni}$

$\mathrm{Ni}$

$\mathrm{Ni}$

$\mathrm{Ni}$

$\mathrm{Ni}$

$\mathrm{N}$

$\mathrm{N}$

$\mathrm{N}$

$\mathrm{N}$

$\mathrm{N}$

$\mathrm{N}$

$\mathrm{N}$

$\mathrm{N}$
2.48742731

3.73114097

2.48742731

3.72574805

2.41605650

4.97485463

6.21856829

4.97485463

6.22396121

4.97485463

4.97485463

6.21856829

4.97485463

6.20542598

4.97485463

4.97485463

6.21856829

4.97485463

6.22396121

4.97485463

4.97485463

4.97485463

4.97485463

1.24371366

$-0.01215673$

$-0.01215673$

2.49958404

2.49958404
Fri Apr 16 14:51:55 2021

7.03550689

8.79438361

7.03550689

8.76177492

6.88438892

0.00000000

1.75887672

0.00000000

1.79148541

0.00000000

3.51775344

5.27663017

3.51775344

5.27663017

3.52252708

7.03550689

8.79438361

7.03550689

8.76177492

7.03073325

1.75620365

8.79705668

5.27663017

5.27663017

8.70980771

1.84345262

8.70980771

1.84345262

. /nitrogen/Ni/110/iter_9/struct.xyz
8.00000000

9.24371366

10.48742731

11.84127807

13.22357879

8.00000000

9.24371366

10.48742731

11.84127807

13.27000013

8.00000000

9.24371366

10.48742731

11.77640897

13.23286392

8.00000000

9.24371366

10.48742731

11.84127807

13.23286392

13.25381658

13.25381658

13.15888607

13.48420291

13.22473909

13.22473909

13.22473909

13.22473909

54

Lattice $=" 7.46228194314714 \quad 0.0 \quad 0.0 \quad 0.0 \quad 10.553260330250541 \quad 0.0 \quad 0.0 \quad 0.020 .97485462876476 "$ Pro perties=species:S:1:pos:R:3 pbc="T T T"

$\mathrm{Ni} \quad 0.00000000 \quad 0.00000000$

$\mathrm{Ni} \quad 1.24371366 \quad 1.75887672$

$\mathrm{Ni} \quad 0.00000000 \quad 0.00000000$

$\mathrm{Ni} \quad 1.25258159 \quad 1.77092359$

$\mathrm{Ni} \quad-0.00099216$

0.00000000

0.02691347

3.51775344

1.24371366

5.27663017

0.00000000

3.51775344

1. 20742370

5.30591398

0.10624916

0.00000000

3. 68157648

7.03550689

1. 24371366

8.79438361

0.00000000

1.25854609

7.03550689

8.74478822

0.12318808

6.90222348

2. 48742731

3. 73114097

0.00000000

1. 75887672

2. 48742731

0.00000000

1.77702680

3. 73225413

2. 50261188

2. 48742731

3. 73114097

2. 48742731

3. 72323426

2. 44548568

2. 48742731

3. 73114097

2. 48742731

3. 75531383

$-0.01361823$

3.51775344

5. 27663017

3.51775344

5.30007234

3. 65394273

7.03550689

8.79438361

7.03550689

8.70992671

2. 44687381

4. 97485463

6.87121874

0.00000000

1. 75887672

6.21856829

1.75887672
0.00000000

1.79591250

6. 23804294

4. 99453716

$-0.00537734$

4. 97485463

3.51775344 
$\mathrm{Ni}$

$\mathrm{Ni}$

$\mathrm{Ni}$

$\mathrm{Ni}$

$\mathrm{Ni}$

$\mathrm{Ni}$

$\mathrm{Ni}$

$\mathrm{Ni}$

$\mathrm{Ni}$

$\mathrm{N}$

$\mathrm{N}$

$\mathrm{N}$

$\mathrm{N}$

$\mathrm{N}$

$\mathrm{N}$

$\mathrm{N}$

$\mathrm{N}$

$\mathrm{N}$

.$/$

6.21856829

4.97485463

6.19012167

4.98493482

4.97485463

6.21856829

4.97485463

6.24047155

5.00456451

4.98779387

4.98478129

4.86740986

1.31019914

0.04101297

$-0.00661888$

2.51239412

2.51141912

6.29255952
Fri Apr 16 14:51:55 2021

5.27663017

3.51775344

5.34463209

3.51839354

7.03550689

8.79438361

7.03550689

8.71669924

7.00679022

1.75155782

8.78712339

5.27278673

5.26628570

8.75747595

1.86305299

8.70047439

1.82703593

6.82423179
9.24371366

10.48742731

11.84695941

13.19294789

8.00000000

9.24371366

10.48742731

11.87354351

13.63497234

13.22489570

13.23181341

13.12973130

13.50085166

13.21162920

13.21513532

13.22988307

13.20960453

14.75547970

37

Lattice $=" 7.4622819431471425 \quad 0.0 \quad 0.0 \quad 3.731140971573571 \quad 6.462525732967329 \quad 0.0 \quad 0.0 \quad 0.022 .0929$

$2769249835 "$ Properties=species:S:1:pos:R:3 pbc="T T T"

$0.00000000 \quad 0.00000000$

1.24371366

0.71805841

2.48251645

$-0.02514897$

1.24371366

1.43328154

$\mathrm{Ni}$

$-0.01451976$

2. 48742731

2.15417524

2. 87223366

3. 72510700

3. 59377579

1. 24371366

2. 18321477

2. 48742731

4.30835049

3. 73114097

5.02640890

4. 97485463

5.75013790

2. 48309897

4.31084946

2. 48742731

0.00000000

3. 73114097

0.71805841

4.97485463

2. 51257628

1.42914940

3. 73114097

$-0.01451976$

2.15417524

2. 87223366

4. 97485463

3. 59377579

2. 17273582

3. 76328884

4. 97485463

4. 30835049

5.02640890

6.21856829

5.74741371

7. 45717864

4. 97485463

4.27122933

0.00000000

4.97485463

0.71805841

6.21856829

1.43328154

7. 46719281

$-0.00499795$

2.15417524

2. 87223366

6.21856829

7. 46228194

3. 58439928

8.00000000

10.03097590

12.06532098

14.18482263

8.00000000

10.03097590

12.05277257

14.18482263

8.00000000

10.03097590

12.06532098

14.05795655

8.00000000

10.03097590

12.05277257

14.18482263

8.00000000

10.03097590

12.05277257

14.05108111

8.00000000

10.03097590

12.06488793

14.05108111

8.00000000

10.03097590

12.06532098

14.05795655

8.00000000

10.03097590

12.06488793

14.05108111

8.00000000

2.17273582

4.30835049

5.02640890

5.74741371

10.03097590

12.06488793

14.05795655

4.31084946

15.16155991

/nitrogen/Ni/111/iter_2/struct.xyz

38

Lattice $=" 7.4622819431471425 \quad 0.0 \quad 0.0 \quad 3.731140971573571 \quad 6.462525732967329 \quad 0.0 \quad 0.0 \quad 0.022 .0929$ $2769249835 "$ Properties=species:S:1:pos:R:3 pbc="T T T"

8.00000000

$\mathrm{Ni} \quad 1.24371366 \quad 0.71805841$

10.03097590

2. $48742731 \quad 1.43663555$

12.05344183

6.45011247

14.15578312

3. 75264137

2.15417524

8.00000000

$\mathrm{Ni}$

1. 24371366 
$\mathrm{Ni}$

$\mathrm{Ni}$

2.48742731

3.73069175

8.70563785

2.48742731

3. 73114097

4.96452951

9.94970926

2.48742731

3.73114097

4.97324624

6.21856829

3. 73114097

4.97485463

6.21856829

3.73149873

4.97485463

6.21856829

7.47260706

5.01439296

4.97485463

6.21856829

7.46389033

8.68449520

6.21856829

7.46228194

8.70644483

6.21856829

7.46228194

8.70599560

9.94970926

7.42274361

6.21856829

2. 48742731
Fri Apr 16 14:51:55 2021

2.87223366

3. 59003271

2.15396870

4.30835049

5.02640890

5.75042853

4.30876359

0.00000000

0.71805841

1.43704543

6.41687080

2.15417524

2.87223366

3.57836966

2.15396870

4.30835049

5.02640890

5.75042853

4.33117796

0.00000000

0.71805841

1.43704543

6.45011247

2.15417524

2.87223366

3.59003271

2.17900176

4.30835049

5.02640890

5.74261012

4.33117796

0.71805841

2.87223366
10.03097590

12.05344183

14.16003412

8.00000000

10.03097590

12.04460149

14.16003412

8.00000000

10.03097590

12.06141921

14.02501570

8.00000000

10.03097590

12.04460149

14.16003412

8.00000000

10.03097590

12.04460149

14.02501570

8.00000000

10.03097590

12.06141921

14.15578312

8.00000000

10.03097590

12.05344183

14.15578312

8.00000000

10.03097590

12.06141921

14.02501570

15.13977486

15.16334742

./nitrogen/Ni/111/iter_3/struct.xyz

39

Lattice $=" 7.4622819431471425 \quad 0.0 \quad 0.0 \quad 3.731140971573571 \quad 6.462525732967329 \quad 0.0 \quad 0.0 \quad 0.0 \quad 22.0929$

$2769249835 "$ Properties=species:S:1:pos:R:3 pbc="T T T"

$\mathrm{Ni} \quad 0.00000000 \quad 0.00000000 \quad 8.00000000$

$\mathrm{Ni} \quad 1.24371366 \quad 0.71805841$

1.42810085

2.48742731

0.00390205

7.45552339

2.15417524

1.24371366

2.48742731

2.87223366

3. 73808301

3. 59430006

1.25047221

2.15807730

4.30835049

5.02640890

3.73114097

5.74847531

4.30054638

4.96791259

0.00000000

0.71805841

1.44012482

6.45472163

2.15417524

2.87223366

3. 58227610

2.15807730

4.30835049

5.02640890

10.03097590

12.05067420

14.13552018

8.00000000

10.03097590

12.05067420

14.13552018

8.00000000

10.03097590

12.05067420

14.13552018

8.00000000

10.03097590

12.05067420

14.13552018

8.00000000

10.03097590

12.05067420

14.13552018

8.00000000

10.03097590

12.05067420

14.13552018

8.00000000

10.03097590

12.05067420

14.13552018

8.00000000

10.03097590

12.05067420 
$\mathrm{Ni}$

$\mathrm{Ni}$

$\mathrm{Ni}$

$\mathrm{Ni}$

$\mathrm{Ni}$

$\mathrm{N}$

$\mathrm{N}$

$\mathrm{N}$

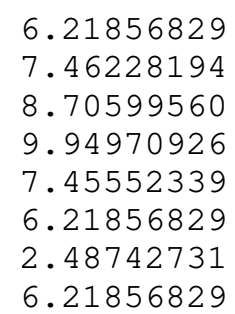

Fri Apr 16 14:51:55 2021

14.13552018

8.00000000

10.03097590

12.05067420

14.13552018

15.15096636

15.15096636

15.15096636

./nitrogen/Ni/111/iter_4/struct.xyz 40

Lattice $=" 7.4622819431471425 \quad 0.0 \quad 0.0 \quad 3.731140971573571 \quad 6.462525732967329 \quad 0.0 \quad 0.0 \quad 0.0 \quad 22.0929$ $2769249835 "$ Properties=species:S:1:pos:R:3 pbc="T T T"

$\begin{array}{lllr}\mathrm{Ni} & 0.00000000 & 0.00000000 & 8.00000000 \\ \mathrm{Ni} & 1.24371366 & 0.71805841 & 10.03097590 \\ \mathrm{Ni} & 2.48405598 & 1.43465538 & 12.02447378 \\ \mathrm{Ni} & 3.77857641 & 6.43463075 & 14.12222450 \\ \mathrm{Ni} & 1.24371366 & 2.15417524 & 8.00000000 \\ \mathrm{Ni} & 2.48742731 & 2.87223366 & 10.03097590 \\ \mathrm{Ni} & 3.71365984 & 3.58753772 & 12.04027051 \\ \mathrm{Ni} & 8.65812011 & 2.12704244 & 14.12222450 \\ \mathrm{Ni} & 2.48742731 & 4.30835049 & 8.00000000 \\ \mathrm{Ni} & 3.73114097 & 5.02640890 & 10.03097590 \\ \mathrm{Ni} & 4.97527465 & 5.74811771 & 12.02447378 \\ \mathrm{Ni} & 2.46981465 & 4.20361218 & 14.36814491 \\ \mathrm{Ni} & 2.48742731 & 0.00000000 & 8.00000000 \\ \mathrm{Ni} & 3.73114097 & 0.71805841 & 10.03097590 \\ \mathrm{Ni} & 4.98598054 & 1.42235490 & 12.04027051 \\ \mathrm{Ni} & 2.48786736 & 0.05502779 & 14.12222450 \\ \mathrm{Ni} & 3.73114097 & 2.15417524 & 8.00000000 \\ \mathrm{Ni} & 4.97485463 & 2.87223366 & 10.03097590 \\ \mathrm{Ni} & 6.22492351 & 3.60680836 & 12.04027051 \\ \mathrm{Ni} & 3.78643468 & 2.12511345 & 14.03497645 \\ \mathrm{Ni} & 4.97485463 & 4.30835049 & 8.00000000 \\ \mathrm{Ni} & 6.21856829 & 5.02640890 & 10.03097590 \\ \mathrm{Ni} & 7.47666403 & 5.75359403 & 12.09383539 \\ \mathrm{Ni} & 4.92203952 & 4.27499563 & 14.03497645 \\ \mathrm{Ni} & 4.97485463 & 0.00000000 & 8.00000000 \\ \mathrm{Ni} & 6.21856829 & 0.71805841 & 10.03097590 \\ \mathrm{Ni} & 7.46523326 & 1.43392789 & 12.02447378 \\ \mathrm{Ni} & 5.07436700 & 0.03711613 & 14.36814491 \\ \mathrm{Ni} & 6.21856829 & 2.15417524 & 8.00000000 \\ \mathrm{Ni} & 7.46228194 & 2.87223366 & 10.03097590 \\ \mathrm{Ni} & 8.69090059 & 3.59818397 & 12.09383539 \\ \mathrm{Ni} & 6.21608969 & 2.21659189 & 14.03497645 \\ \mathrm{Ni} & 7.46228194 & 4.30835049 & 8.00000000 \\ \mathrm{Ni} & 8.70599560 & 5.02640890 & 10.03097590 \\ \mathrm{Ni} & 9.95042218 & 5.72744870 & 12.09383539 \\ \mathrm{Ni} & 7.38038224 & 4.37597266 & 14.36814491 \\ \mathrm{~N} & 6.46787941 & 0.92256960 & 15.14891733 \\ \mathrm{~N} & 2.53988363 & 2.55406830 & 15.14891733 \\ \mathrm{~N} & 5.91680084 & 5.14006308 & 15.14891733 \\ \mathrm{~N} & 8.70599560 & 5.02640890 & 15.33395694\end{array}$

./nitrogen/Ni/111/iter_5/struct.xyz

41

Lattice $=" 7.4622819431471425 \quad 0.0 \quad 0.0 \quad 3.731140971573571 \quad 6.462525732967329 \quad 0.0 \quad 0.0 \quad 0.0 \quad 22.0929$ 2769249835 " Properties=species:S:1:pos:R:3 pbc="T T T"

1.24371366

$\begin{array}{ll}1.24371366 & 0.71805841 \\ 2.47431886 & 1.44639945\end{array}$

8.00000000

$\mathrm{Ni} \quad 2.47431886$

3. 81698734

10.03097590

$\mathrm{Ni}$

6.41296231

1. 24371366

2.15417524

2.48742731

2.87223366

3. 71568174

3.59650302

8.69284538

2.19382769

2.48742731

4. 30835049

3. 73114097

5.02640890

12.02675125

14.41993362

8.00000000

10.03097590

12.02675125

14.37077578

8.00000000

10.03097590

4.96714400

5.74891905

12.00233719

$\mathrm{Ni}$

4.29991270

14.37077578

\section{7}


$\mathrm{Ni}$

$\mathrm{Ni}$

$\mathrm{Ni}$

$\mathrm{Ni}$

$\mathrm{Ni}$

$\mathrm{Ni}$

$\mathrm{Ni}$

$\mathrm{Ni}$

$\mathrm{Ni}$

$\mathrm{Ni}$

$\mathrm{Ni}$

$\mathrm{Ni}$

$\mathrm{Ni}$

$\mathrm{Ni}$

$\mathrm{Ni}$

$\mathrm{Ni}$

$\mathrm{Ni}$

$\mathrm{Ni}$

$\mathrm{Ni}$

$\mathrm{Ni}$

$\mathrm{Ni}$

$\mathrm{Ni}$

$\mathrm{Ni}$

$\mathrm{Ni}$

$\mathrm{N}$

$\mathrm{N}$

$\mathrm{N}$

$\mathrm{N}$

$\mathrm{N}$
2. 48742731

3. 73114097

4.99560584

2. 50572211

3. 73114097

4.97485463

6.23648865

3. 75124830

4.97485463

6.21856829

7.47769465

4. 94875640

4.97485463

6.21856829

7. 47684740

4.92994761

6.21856829

7.46228194

8.70947943

6.20188929

7. 46228194

8.70599560

9.96189196

6.88623033

6.18374483

2. 70776098

5.29036801

8.58178918

7.37479347
Fri Apr 16 14:51:55 2021

0.00000000

0.71805841

1. 42413611

0.04069815

2.15417524

2. 87223366

3. 58284135

2. 14256627

4.30835049

5.02640890

5.73267319

4.27215766

0.00000000

0.71805841

1. 43045882

$-0.00666783$

2.15417524

2.87223366

3.58828068

2.19639978

4.30835049

5.02640890

5.73468227

4.64093404

0.73816375

2.74502396

5.56230559

5.05572128

2. 96514339
8.00000000

10.03097590

12.03729393

13.97455688

8.00000000

10.03097590

12.04195472

13.99377776

8.00000000

10.03097590

12.04195472

13.97455688

8.00000000

10.03097590

12.10342790

14.15918157

8.00000000

10.03097590

12.10319718

14.15918157

8.00000000

10.03097590

12.10342790

15.44091917

15.19111762

15.21993077

15.16220233

15.24743339

15.24743339

42

Lattice $=" 7.4622819431471425 \quad 0.0 \quad 0.0 \quad 3.731140971573571 \quad 6.4625257329673290 .0 \quad 0.0 \quad 0.022 .0929$ $2769249835 "$ Properties=species:S:1:pos:R:3 pbc="T T T"

$\begin{array}{lllr}\mathrm{Ni} & 0.00000000 & 0.00000000 & 8.00000000 \\ \mathrm{Ni} & 1.24371366 & 0.71805841 & 10.03097590 \\ \mathrm{Ni} & 2.48318658 & 1.45210384 & 12.01424320 \\ \mathrm{Ni} & 7.40808184 & 0.00893816 & 14.31135241 \\ \mathrm{Ni} & 1.24371366 & 2.15417524 & 8.00000000 \\ \mathrm{Ni} & 2.48742731 & 2.87223366 & 10.03097590 \\ \mathrm{Ni} & 3.73393936 & 3.60126547 & 12.06089905 \\ \mathrm{Ni} & 1.22539142 & 2.11360863 & 14.23816900 \\ \mathrm{Ni} & 2.48742731 & 4.30835049 & 8.00000000 \\ \mathrm{Ni} & 3.73114097 & 5.02640890 & 10.03097590 \\ \mathrm{Ni} & 4.96412442 & 5.75573065 & 12.04339371 \\ \mathrm{Ni} & 2.52225511 & 4.17932930 & 14.60290654 \\ \mathrm{Ni} & 2.48742731 & 0.00000000 & 8.00000000 \\ \mathrm{Ni} & 3.73114097 & 0.71805841 & 10.03097590 \\ \mathrm{Ni} & 4.98809413 & 1.42570617 & 12.00973629 \\ \mathrm{Ni} & 2.48123216 & 0.06615797 & 14.02609031 \\ \mathrm{Ni} & 3.73114097 & 2.15417524 & 8.00000000 \\ \mathrm{Ni} & 4.97485463 & 2.87223366 & 10.03097590 \\ \mathrm{Ni} & 6.22028175 & 3.60313796 & 12.05304530 \\ \mathrm{Ni} & 3.77911372 & 2.12271254 & 13.97971908 \\ \mathrm{Ni} & 4.97485463 & 4.30835049 & 8.00000000 \\ \mathrm{Ni} & 6.21856829 & 5.02640890 & 10.03097590 \\ \mathrm{Ni} & 7.46371052 & 5.74165835 & 12.05026978 \\ \mathrm{Ni} & 4.97379215 & 4.33273733 & 14.14660516 \\ \mathrm{Ni} & 4.97485463 & 0.00000000 & 8.00000000 \\ \mathrm{Ni} & 6.21856829 & 0.71805841 & 10.03097590 \\ \mathrm{Ni} & 7.47220392 & 1.43684073 & 12.06585287 \\ \mathrm{Ni} & 8.67928258 & 6.42826741 & 14.14823183 \\ \mathrm{Ni} & 6.21856829 & 2.15417524 & 8.00000000 \\ \mathrm{Ni} & 7.46228194 & 2.87223366 & 10.03097590 \\ \mathrm{Ni} & 8.70889094 & 3.59744711 & 12.11286910 \\ \mathrm{Ni} & 6.18003916 & 2.17723368 & 14.06592678 \\ \mathrm{Ni} & 7.46228194 & 4.30835049 & 8.00000000 \\ \mathrm{Ni} & 8.70599560 & 5.02640890 & 10.03097590 \\ \mathrm{Ni} & 9.95491423 & 5.73414040 & 12.08308132 \\ & & & \end{array}$


7.43338181

$\mathrm{N} \quad 6.08486729$

$\mathrm{N} \quad 2.65560344$

6.14918174

8.67059363

7.39875631

3.87231113

$\mathrm{N}$

.$/$

43
Fri Apr $16 \quad 14: 51: 552021$

69

$\begin{array}{ll}4.30716072 & 14.36064625 \\ 0.82271006 & 15.21757192 \\ 2.47873466 & 15.23872899 \\ 5.26431593 & 15.18873258 \\ 5.11858373 & 15.38089203 \\ 2.76313306 & 15.25983044 \\ 5.05411484 & 15.36173605\end{array}$

4.30716072

14.36064625

15.18873258

15.25983044

15.36173605

Lattice $=" 7.4622819431471425 \quad 0.0 \quad 0.0 \quad 3.731140971573571 \quad 6.462525732967329 \quad 0.0 \quad 0.0 \quad 0.0 \quad 22.0929$ $2769249835 "$ Properties=species:S:1:pos:R:3 pbc="T T T"

$\begin{array}{lllr}\mathrm{Ni} & 0.00000000 & 0.00000000 & 8.00000000 \\ \mathrm{Ni} & 1.24371366 & 0.71805841 & 10.03097590 \\ \mathrm{Ni} & 2.48445962 & 1.43273440 & 12.04379762 \\ \mathrm{Ni} & 7.37785283 & 0.04874517 & 14.62143613 \\ \mathrm{Ni} & 1.24371366 & 2.15417524 & 8.00000000 \\ \mathrm{Ni} & 2.48742731 & 2.87223366 & 10.03097590 \\ \mathrm{Ni} & 3.73258639 & 3.59455339 & 12.04379762 \\ \mathrm{Ni} & 1.25830735 & 2.15679298 & 14.35946216 \\ \mathrm{Ni} & 2.48742731 & 4.30835049 & 8.00000000 \\ \mathrm{Ni} & 3.73114097 & 5.02640890 & 10.03097590 \\ \mathrm{Ni} & 4.96628879 & 5.74941281 & 12.05764216 \\ \mathrm{Ni} & 2.49245714 & 4.29440312 & 14.35946216 \\ \mathrm{Ni} & 2.48742731 & 0.00000000 & 8.00000000 \\ \mathrm{Ni} & 3.73114097 & 0.71805841 & 10.03097590 \\ \mathrm{Ni} & 4.98909106 & 1.42789742 & 12.00728779 \\ \mathrm{Ni} & 2.46291793 & 0.01356095 & 14.14790987 \\ \mathrm{Ni} & 3.73114097 & 2.15417524 & 8.00000000 \\ \mathrm{Ni} & 4.97485463 & 2.87223366 & 10.03097590 \\ \mathrm{Ni} & 6.21652030 & 3.59283042 & 12.03713942 \\ \mathrm{Ni} & 3.77466803 & 2.12904489 & 13.98283735 \\ \mathrm{Ni} & 4.97485463 & 4.30835049 & 8.00000000 \\ \mathrm{Ni} & 6.21856829 & 5.02640890 & 10.03097590 \\ \mathrm{Ni} & 7.45905968 & 5.74497175 & 12.03713942 \\ \mathrm{Ni} & 4.95085581 & 4.32279577 & 14.14790987 \\ \mathrm{Ni} & 4.97485463 & 0.00000000 & 8.00000000 \\ \mathrm{Ni} & 6.21856829 & 0.71805841 & 10.03097590 \\ \mathrm{Ni} & 7.47473026 & 1.43101253 & 12.09123456 \\ \mathrm{Ni} & 4.93805437 & 0.00849354 & 14.09797647 \\ \mathrm{Ni} & 6.21856829 & 2.15417524 & 8.00000000 \\ \mathrm{Ni} & 7.46228194 & 2.87223366 & 10.03097590 \\ \mathrm{Ni} & 8.71532378 & 3.58490645 & 12.08276900 \\ \mathrm{Ni} & 6.19281253 & 2.18179843 & 14.09797647 \\ \mathrm{Ni} & 7.46228194 & 4.30835049 & 8.00000000 \\ \mathrm{Ni} & 8.70599560 & 5.02640890 & 10.03097590 \\ \mathrm{Ni} & 9.96035387 & 5.73623891 & 12.09123456 \\ \mathrm{Ni} & 7.45260701 & 4.31393631 & 14.14655954 \\ \mathrm{~N} & 5.92563075 & 0.88718598 & 15.29385749 \\ \mathrm{~N} & 2.75467590 & 2.71793761 & 15.23194286 \\ \mathrm{~N} & 6.19024226 & 5.04276294 & 15.23157773 \\ \mathrm{~N} & 8.64162329 & 5.11281429 & 15.26401760 \\ \mathrm{~N} & 7.35526653 & 2.88477902 & 15.26401760 \\ \mathrm{~N} & 3.81960860 & 4.91405249 & 15.41800355 \\ \mathrm{~N} & 1.38525098 & 0.69762141 & 15.41800355 \\ \mathrm{~N} & \mathrm{H} & & \end{array}$

./nitrogen/Ni/111/iter_8/struct.xyz 44

Lattice $=" 7.4622819431471425 \quad 0.0 \quad 0.0 \quad 3.731140971573571 \quad 6.462525732967329 \quad 0.0 \quad 0.0 \quad 0.0 \quad 22.0929$ 2769249835" Properties=species:S:1:pos:R:3 pbc="T T T" $\mathrm{Ni} \quad 0.00000000 \quad 0.00000000 \quad 8.00000000$

$\mathrm{Ni} \quad 1.24371366 \quad 0.71805841$

$2.47555112 \quad 1.42926011$

$-0.00923265 \quad-0.00533047$

$1.24371366 \quad 2.15417524$

$2.48742731 \quad 2.87223366$

$3.73302190 \quad 3.58920612$

$1.24371366 \quad 2.16483619$

$2.48742731 \quad 4.30835049$

10.03097590

12.07605295

14.39711199

8.00000000

10.03097590

12.02543690

14.39711199

8.00000000

$\mathrm{Ni}$

5.02640890

10.03097590 
$\mathrm{Ni}$

$\mathrm{Ni}$

$\mathrm{Ni}$

$\mathrm{Ni}$

$\mathrm{Ni}$

$\mathrm{Ni}$

$\mathrm{Ni}$

$\mathrm{Ni}$

$\mathrm{Ni}$

$\mathrm{Ni}$

$\mathrm{Ni}$

$\mathrm{Ni}$

$\mathrm{Ni}$

$\mathrm{Ni}$

$\mathrm{Ni}$

$\mathrm{Ni}$

$\mathrm{Ni}$

$\mathrm{Ni}$

$\mathrm{Ni}$

$\mathrm{Ni}$

$\mathrm{Ni}$

$\mathrm{Ni}$

$\mathrm{Ni}$

$\mathrm{Ni}$

$\mathrm{Ni}$

$\mathrm{Ni}$

$\mathrm{N}$

$\mathrm{N}$

$\mathrm{N}$

$\mathrm{N}$

$\mathrm{N}$

$\mathrm{N}$

$\mathrm{N}$
4.97485463

2.49374686

2. 48742731

3.73114097

4.97485463

2. 49665996

3.73114097

4.97485463

6.21668736

3. 74138880

4.97485463

6.21856829

7.45672599

4.97485463

4.97485463

6.21856829

7.47415814

4.97485463

6.21856829

7.46228194

8.70599560

6.20832046

7.46228194

8.70599560

9.95526521

7.45596240

6.13649910

2. 63296347

6.15510131

8.70599560

7.31674579

3.79460794

1.24371366

3. 81321015
Fri Apr 16 14:51:55 2021

5.75818077

4.30470190

0.00000000

0.71805841

1.43828874

$-0.00533047$

2.15417524

2.87223366

3.58920612

2.16009183

4.30835049

5.02640890

5.74767505

4.29651731

0.00000000

0.71805841

1.42926011

0.00729718

2.15417524

2.87223366

3.58387661

2.16009183

4.30835049

5.02640890

5.74767505

4.30470190

0.83872643

2.88297365

4.89500090

5.02640890

2.88297365

4.89500090

0.71805841

0.83872643
12.07605295

14.14757728

8.00000000

10.03097590

12.02543690

14.39711199

8.00000000

10.03097590

12.02543690

14.07966763

8.00000000

10.03097590

12.06242021

14.07966763

8.00000000

10.03097590

12.07605295

14.14757728

8.00000000

10.03097590

12.06242021

14.07966763

8.00000000

10.03097590

12.06242021

14.14757728

15.28705626

15.28705626

15.28705626

15.23390228

15.28705626

15.28705626

15.47696046

15.28705626

45

Lattice $=" 7.4622819431471425 \quad 0.0 \quad 0.0 \quad 3.731140971573571 \quad 6.462525732967329 \quad 0.0 \quad 0.0 \quad 0.0 \quad 22.0929$

$2769249835 "$ Properties $=$ species:S:1:pos:R:3 pbc="T T T"

0.00000000

1.24371366

0.00000000

8.00000000

2.48742731

0.71805841

0.00000000

1.43611683

0.00000000

1.24371366

2.15417524

2.48742731

2.87223366

10.03097590

12.06326442

14.20174683

8.00000000

3. 73114097

3.59029207

1. 24371366

2.15417524

2. 48742731

4.30835049

5.02640890

3. 73114097

4.97485463

5.74446732

2. 48742731

4.30835049

2. 48742731

0.00000000

3. 73114097

0.71805841

4.97485463

1. 43611683

0.00000000

2. 48742731

3. 73114097

2.15417524

4. 97485463

2.87223366

3. 59029207

2.15417524

3.73114097

4.30835049

5.02640890

6.21856829

5.74446732

7. 46228194

5.74446732
4.30835049

0.00000000

0.71805841

1.43611683

0.00000000

2.15417524

10.03097590

12.06326442

14.20174683

8.00000000

10.03097590

12.06326442

14.20174683

8.00000000

10.03097590

12.06326442

14.20174683

8.00000000

10.03097590

12.06326442

14.20174683

8.00000000

10.03097590

12.06326442

14.20174683

8.00000000

10.03097590

12.06326442

14.20174683

8.00000000

6.21856829

2.87223366

10.03097590 
$\mathrm{Ni}$

$\mathrm{Ni}$

$\mathrm{Ni}$

$\mathrm{Ni}$

$\mathrm{Ni}$

$\mathrm{Ni}$

$\mathrm{N}$

$\mathrm{N}$

$\mathrm{N}$

$\mathrm{N}$

$\mathrm{N}$

$\mathrm{N}$

$\mathrm{N}$

$\mathrm{N}$

$\mathrm{N}$

8.70599560

6.21856829

7.46228194

8.70599560

9.94970926

7.46228194

6.21856829

2. 48742731

6.21856829

8.70599560

7.46228194

3.73114097

1.24371366

3.73114097

4.97485463
Fri Apr 16 14:51:55 2021

3.59029207

2.15417524

4.30835049

5.02640890

5.74446732

4.30835049

0.71805841

2.87223366

5.02640890

5.02640890

2.87223366

5.02640890

0.71805841

0.71805841

2.87223366
12.06326442

14.20174683

8.00000000

10.03097590

12.06326442

14.20174683

15.31991696

15.31991696

15.31991696

15.31991696

15.31991696

15.31991696

15.31991696

15.31991696

15.31991696

33

Lattice $=" 7.035506886833694 \quad 0.0 \quad 0.0 \quad 3.5177534434168454$

$\begin{array}{llll}\mathrm{Ni} & 1.75887672 & 3.14637433 & 8.00000000\end{array}$

$\mathrm{Ni} \quad 3.51775344 \quad 1.57318717$

$\begin{array}{ll}1.75887672 & 0.00000000\end{array}$

$\begin{array}{ll}1.75887672 & 2.35978075 \\ 3.53788628 & 0.78917250\end{array}$

$3.53788628 \quad 0.78917250$

$3.52399289 \quad 3.12574101$

$1.75887672 \quad 1.67288357$

$\begin{array}{ll}-0.02445705 & -0.01175747\end{array}$

$\begin{array}{ll}3.51775344 & 7.07934224\end{array}$

$\begin{array}{ll}5.27663017 & 5.50615508\end{array}$

$3.51775344 \quad 3.93296791$

3.51775344

6.29274866

5.27663017

4.71184685

5.27663017

7.04171056

3.49481735

5.47580067

1.75887672

3.99549832

5.27663017

3.14637433

7.03550689

1.57318717

5.27663017

0.00000000

5.27663017

2.35978075

7.01537405

0.78917250

7.02926744

3.12574101

5.27663017

1.56602634

3.54221050

$-0.01175747$

7.03550689

7.07934224

8.79438361

5.50615508

3.93296791

7.03550689

6.29274866

$\begin{array}{ll}8.79438361 & 4.70922621\end{array}$

$\begin{array}{ll}8.79438361 & 7.05544522\end{array}$

$\begin{array}{ll}7.05844298 & 5.47580067\end{array}$

8.78659358

9.57318717

10.35978075

11.12439325

11.96338792

12.76669664

13.48030491

8.00000000

8.78659358

9.57318717

10.35978075

11.14210690

11.99401528

12.67885639

13.39592464

8.00000000

8.78659358

9.57318717

10.35978075

11.12439325

11.96338792

12.69041298

13.48030491

8.00000000

8.78659358

9.57318717

10.35978075

11.12829015

11.99768169

12.67885639

3.92755735

13.38077777

13.63026845

1.75887672

0.09805511

./nitrogen/Ni/210/iter_2/struct.xyz

34

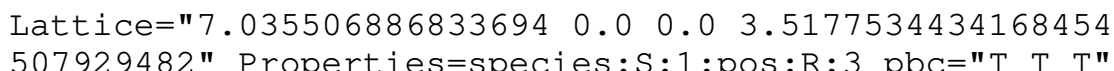

$\begin{array}{llll}\mathrm{Ni} & 1.75887672 & 3.14637433 & 8.00000000\end{array}$

3.5177534

1.57318717

1.75887672

0.00000000

8.78659358

$\mathrm{Ni} \quad 1.75887672$

2.35978075

9.57318717

0.79556943

3.51775344

3.11908914

10.35978075

11.10300522

1.75887672

1.63834118

11.95751819

12.75521045

7.84289334

3.51775344

7.07934224

13.58535416

8.00000000

8.78659358

9.57318717

5.27663017

5.50615508

3.93296791

10.35978075

$7.86593582756403 \quad 0.0 \quad 0.0 \quad 0.0 \quad 21.50615$

3.51775344

6.29274866

$\begin{array}{llllll}7.86593582756403 & 0.0 & 0.0 & 0.0 & 21.50615\end{array}$

3.51775344 
$\mathrm{Ni}$

$\mathrm{Ni}$

$\mathrm{Ni}$

$\mathrm{Ni}$

$\mathrm{Ni}$

$\mathrm{Ni}$

$\mathrm{Ni}$

$\mathrm{Ni}$

$\mathrm{Ni}$

$\mathrm{Ni}$

$\mathrm{Ni}$

$\mathrm{Ni}$

$\mathrm{Ni}$

$\mathrm{Ni}$

$\mathrm{Ni}$

$\mathrm{Ni}$

$\mathrm{Ni}$

$\mathrm{Ni}$

$\mathrm{Ni}$

$\mathrm{Ni}$

$\mathrm{N}$

$\mathrm{N}$

5.27663017

35

Lattice $=" 7.035506886833694 \quad 0.0 \quad 0.0 \quad 3.5177534434168454$ $507929482 "$ Properties=species:S:1:pos:R:3 pbc="T T T" $\mathrm{Ni}$

$\mathrm{Ni}$

$\mathrm{Ni}$

$\mathrm{Ni}$

$\mathrm{Ni}$

$\mathrm{Ni}$

$\mathrm{Ni}$

$\mathrm{Ni}$

$\mathrm{Ni}$

$\mathrm{Ni}$

$\mathrm{Ni}$

$\mathrm{Ni}$

$\mathrm{Ni}$

$\mathrm{Ni}$

$\mathrm{Ni}$

$\mathrm{Ni}$

$\mathrm{Ni}$

$\mathrm{Ni}$

$\mathrm{Ni}$

$\mathrm{Ni}$

$\mathrm{Ni}$

$\mathrm{Ni}$

$\mathrm{Ni}$

$\mathrm{Ni}$

$\mathrm{Ni}$

$\mathrm{Ni}$

$\mathrm{Ni}$

$\mathrm{Ni}$

$\mathrm{Ni}$

$\mathrm{Ni}$

$\mathrm{Ni}$

$\mathrm{Ni}$

$\mathrm{N}$

$\mathrm{N}$

$\mathrm{N}$

1.75887672

3.51775344

1.75887672

1. 75887672

3. 51775344

3.51775344

1.39189291

3. 51775344

3.51775344

5.27663017

3. 51775344

3.51775344

5.23180206

5.23771047

3.51775344

8.62434348

5.27663017

7.03550689

5.27663017

5.27663017

7.03550689

7.03550689

5.64361398

3.51775344

7.03550689

8.79438361

7.03550689

7.03550689

8.83921171

8.83330330

7.03550689

5.44667030

5.27593269

8.79508108

7.03550689
Fri Apr 16 14:51:55 2021

4.70592099

7.04002523

5.44199883

3.97301435

3.14637433

1.57318717

0.00000000

0.79556943

3.11908914

1.63834118

7.84289334

7.07934224

5.50615508

3.93296791

6.29274866

4.70592099

7.04002523

5.44199883

3.97301435

0.06636084

0.06636084
2.35978075
11.12922662

12.00992872

12.66655403

13.38299889

8.00000000

8.78659358

9.57318717

10.35978075

11.10300522

11.95751819

12.75521045

13.58535416

8.00000000

9.57318717

10.35978075

12.00992872

12.66655403

13.69099156

13.69099156
8.78659358

11.12922662

13.38299889

8.00000000

8.78659358

9.57318717

10.35978075

11.11334513

12.11241592

13.02582083

13.78955060

8.00000000

8.78659358

9.57318717

10.35978075

11.13095463

12.00535625

12.69995900

13.44365374

8.00000000

8.78659358

9.57318717

10.35978075

11.06253595

11.77991762

13.02582083

13.51567739

8.00000000

8.78659358

9.57318717

10.35978075

11.13095463

12.00535625

12.62644415

13.44365374

13.72648254

13.72648254

13.57903775

$\begin{array}{llllll}7.86593582756403 & 0.0 & 0.0 & 0.0 & 21.50615\end{array}$

36

Lattice $=" 7.035506886833694 \quad 0.0 \quad 0.0 \quad 3.5177534434168454$

$507929482 "$ Properties=species:S:1:pos:R:3 pbc="T T T"

$\begin{array}{llll}\mathrm{Ni} & 1.75887672 & 3.14637433 & 8.00000000\end{array}$

$\begin{array}{llll}\mathrm{Ni} & 3.51775344 & 1.57318717 & 8.78659358\end{array}$

$\begin{array}{llll}\mathrm{Ni} & 1.75887672 & 0.00000000 & 9.57318717\end{array}$ 
$\mathrm{Ni}$

$\mathrm{Ni}$

$\mathrm{Ni}$

$\mathrm{Ni}$

$\mathrm{Ni}$

$\mathrm{Ni}$

$\mathrm{Ni}$

$\mathrm{Ni}$

$\mathrm{Ni}$

$\mathrm{Ni}$

$\mathrm{Ni}$

$\mathrm{Ni}$

$\mathrm{Ni}$

$\mathrm{Ni}$

$\mathrm{Ni}$

$\mathrm{Ni}$

$\mathrm{Ni}$

$\mathrm{Ni}$

$\mathrm{Ni}$

$\mathrm{Ni}$

$\mathrm{Ni}$

$\mathrm{Ni}$

$\mathrm{Ni}$

$\mathrm{Ni}$

$\mathrm{Ni}$

$\mathrm{Ni}$

$\mathrm{Ni}$

$\mathrm{Ni}$

$\mathrm{Ni}$

$\mathrm{N}$

$\mathrm{N}$

$\mathrm{N}$

$\mathrm{N}$

. $/$

37 . nitrogen/Ni/210/iter_5/struct.xyz

Lat

$507929482 "$ Properties=species:S:1:pos:R:3 pbc="T T T"

$\mathrm{Ni}$

$\mathrm{Ni}$

$\mathrm{Ni}$

$\mathrm{Ni}$

$\mathrm{Ni}$

$\mathrm{Ni}$

$\mathrm{Ni}$

$\mathrm{Ni}$

$\mathrm{Ni}$

$\mathrm{Ni}$

$\mathrm{Ni}$

$\mathrm{Ni}$

$\mathrm{Ni}$

$\mathrm{Ni}$

$\mathrm{Ni}$

$\mathrm{Ni}$

$\mathrm{Ni}$

$\mathrm{Ni}$

$\mathrm{Ni}$

$\mathrm{Ni}$

$\mathrm{Ni}$

$\mathrm{Ni}$

$\mathrm{Ni}$

$\mathrm{Ni}$

$\mathrm{Ni}$

$\mathrm{Ni}$

$\mathrm{Ni}$

$\mathrm{Ni}$

$\mathrm{Ni}$

$\mathrm{Ni}$

$\mathrm{Ni}$
1. 75887672

3. 51775344

3. 51775344

1. 32859984

3. 51775344

3. 51775344

5. 27663017

3. 51775344

3. 51775344

5. 23626311

3. 51775344

8. 71651883

5.27663017

7. 03550689

5.27663017

5. 27663017

7.03550689

7.03550689

5.70690705

3.51775344

7.03550689

7.03550689

7.03550689

8.81279644

8.83475066

7.03550689

5. 35449494

5.27781626

8.79319751

3. 51775344

7.03550689
5. 25821733

8.79438361
Fri Apr 16 14:51:55 2021

2.35978075

0.77395320

2. 96804612

1.55439049

7.49650881

7.07934224

5.50615508

3.93296791

4.73537635

7.08330328

5.40856604

3.87501397

3.14637433

1. 57318717

0.00000000

2.35978075

0.77351293

3. 24280343

1. 55439049

0.10314933

7.07934224

5.50615508

3.93296791

6.29274866

4.73537635

7.08330328

5.53763551

3.87501397

7.79550130

7.79550130

3.92602135

2. 92438534
6.29274866
10.35978075

11.10002951

12.08846339

13.04204586

13.89923632

8.00000000

8.78659358

9.57318717

10.35978075

11.10218010

12.01977443

12.71032717

13.55688324

8.00000000

8.78659358

9.57318717

10.35978075

11.06070529

11.81507071

13.04204586

13.49136793

8.00000000

8.78659358

9.57318717

10.35978075

11.10218010

12.01977443

12.65988960

13.55688324

13.74671738

13.74671738

13.60784139
13.68436791

$\begin{array}{lll}1.75887672 & 3.14637433 & 8.00000000\end{array}$

$3.51775344 \quad 1.57318717$

8.78659358

9.57318717

1. 75887672

0.00000000

2.35978075

0.76113094

3.51775344

3. 51775344

3.22457782

1.52434443

1.71141995

0.00000000

3.51775344

5. 27663017

3. 51775344

3. 51775344

5. 28906566

5.27311093

3. 51775344

1. 76657868

5. 27663017

7.03550689

$-0.13316521$

7.07934224

5.50615508

3. 93296791

6.29274866

4.72451961

7.03703599

5.53377221

3.96237290

3.14637433

1.57318717

0.00000000

2.35978075

5. 27663017

7.03550689

0.78547441

3.12267233

1.52434443

5. 32408693

3. 51775344

7.03550689

$-0.03664460$

7.07934224

5.50615508

3.93296791

7.03550689

3.93296791
6.29274866

4.72451961

7.03703599

10.35978075

11.13365933

12.01265480

12.93025767

13.71665948

8.00000000

8.78659358

9.57318717

10.35978075

11.09478492

12.00459762

12.71122777

13.66358695

8.00000000

8.78659358

9.57318717

10.35978075

11.14810205

12.02395821

12.93025767

13.76833479

8.00000000

8.78659358

9.57318717

10.35978075

11.09478492

12.00459762

8.79790285

5.51711709

12.73323453

7.03550689

$\begin{array}{llllll}7.86593582756403 & 0.0 & 0.0 & 0.0 & 21.50615\end{array}$ 
$\mathrm{Ni}$

$\mathrm{N}$

$\mathrm{N}$

$\mathrm{N}$

$\mathrm{N}$

$\mathrm{N}$

. $/$

38

rogen/Ni/210/iter_6/struct.xyz

$507929482 "$ Properties=species:S: $1:$ pos:R:3 pbc="T T T"

$\mathrm{Ni}$

$\mathrm{Ni}$

1.75887672

3.51775344

1.75887672

1.75887672

3.51775344

3. 51775344

1.75887672

0.00000000

3.51775344

5.27663017

3.51775344

3.51775344

5.27663017

5.27663017

3.51775344

1.75887672

5.27663017

7.03550689

5.27663017

5.27663017

7.03550689

7.03550689

5.27663017

3.51775344

7.03550689

8.79438361

7.03550689

7.03550689

8.79438361

8.79438361

7.03550689

5.27663017

1.75887672

5.27663017

7.03550689

7.03550689

3.51775344

3.51775344
Fri Apr 16 14:51:55 2021

3. 96237290

$-0.18462406$

$-0.18462406$

3.97076600

4.12697613

1.58453227
13.66358695

13.77232200

13.77232200

13.70564072

13.82849644

12.73470624
$-0.44409144$

$-0.44409144$
5.5756807

4.01773217

4.14555522

1.57805049

4.14555522

1. 57805049

3.14637433

0.00000000

0.78179799

199135

$-0.04630836$

(2)

3.93296791

274866

7.06910547

568070

3.14637433

57318717

2.35978075

78179799

1. 47725858

5.50615508

.93296791

6.29274866

4.73653329

7.06910547

$\mathrm{pc}=" \mathrm{~T} T \mathrm{~T} "$
8.00000000

8.78659358

9.57318717

10.35978075

11.19126390

12.07596909

13.26738701

13.83254428

8.00000000

8.78659358

9.57318717

10.35978075

11.09846393

12.01726683

12.72471100

13.72097664

8.00000000

8.78659358

10.35978075

11.19126390

12.07596909

13.83254428

8.00000000

8.78659358

9.57318717

11.09846393

12.01726683

12.72471100

13.72097664

13.83754674

13.83754674

13.83283231

12.81974372

13.83283231

12.81974372
7.07934224

9.57318717

13.26738701

10.35978075

/nitrogen/Ni/210/iter_7/struct.xyz

39

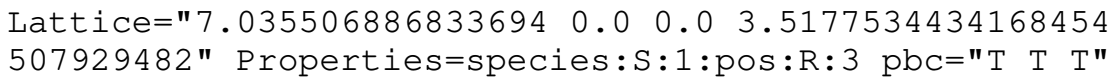

$\mathrm{Ni}$

1.75887672

3.14637433

1.57318717

8.00000000

3.51775344

0.00000000

1.75887672
1.75887672

2.35978075

3.48820480

0.79215862

3.42436049

3.16058204

1.75887672

$-0.00756098$

3.51775344

1.38522380

$-0.20734080$

7.07934224

5.27663017

3. 51775344

3. 51775344

5.50615508

3. 93296791

6.29274866

4.69211568

7.04112506

5.27663017

3.48309705

5.51625733

8.78659358

9.57318717

10.35978075

11.22471634

12.16695450

13.60105012

13.81756864

8.00000000

8.78659358

9.57318717

10.35978075

11.09511142

11.99812400

12.66178517

3.88998555

$7.86593582756403 \quad 0.0 \quad 0.0 \quad 0.0 \quad 21.50615$ .

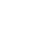


$\mathrm{Ni}$

$\mathrm{Ni}$

$\mathrm{Ni}$

$\mathrm{Ni}$

$\mathrm{Ni}$

$\mathrm{Ni}$

$\mathrm{Ni}$

$\mathrm{Ni}$

$\mathrm{Ni}$

$\mathrm{Ni}$

$\mathrm{Ni}$

$\mathrm{Ni}$

$\mathrm{Ni}$

$\mathrm{Ni}$

$\mathrm{Ni}$

$\mathrm{Ni}$

$\mathrm{N}$

$\mathrm{N}$

$\mathrm{N}$

$\mathrm{N}$

$\mathrm{N}$

$\mathrm{N}$

$\mathrm{N}$
5.27663017

7.03550689

5.27663017

5.27663017

7.06505553

7.12889984

5.27663017

3. 52531442

7.03550689

8.79438361

7.03550689

7.03550689

8.79438361

8.79438361

7.07016328

5.27663017

1.75887672

5.27663017

7.02967890

7.04655581

3.52358143

3.50670452

1.75887672
Fri Apr 16 14:51:55 2021

3.14637433

1.57318717

0.00000000

2.35978075

0.79215862

3.16058204

1.38118236

$-0.20734080$

7.07934224

5.50615508

3.93296791

6.29274866

4.71076098

7.05470162

5.51625733

3.93819107

$-0.60905181$

$-0.57843855$

4.17605351

1.49938151

4.17605351

1.49938151

2.41579513
8.00000000

8.78659358

9.57318717

10.35978075

11.22471634

12.16695450

13. 31349484

13.81756864

8.00000000

8.78659358

9.57318717

10.35978075

11.08038579

11.95140164

12.66178517

13.62863117

13.82674934

13.78689047

13.82571425

12.89416731

13.82571425

12.89416731

15.04779411

40

Lattice $=" 7.035506886833694 \quad 0.0 \quad 0.0 \quad 3.5177534434168454 \quad 7.86593582756403 \quad 0.0 \quad 0.0 \quad 0.0 \quad 21.50615$

$507929482 "$ Properties=species:S:1:pos:R:3 pbc="T T T" .

$\mathrm{Ni}$

1.75887672

3.51775344

3.14637433

1.57318717

0.00000000

1.75887672

2.35978075

1.75887672

0.80488885

.5.51775344

3.15105327

1.75887672

1. 34921557

$-0.00000000$

$-0.27119572$

3.51775344

7.07934224

5.27663017

5.50615508

3. 51775344

3.93296791

3. 51775344

6.29274866

5.27663017

4.68088318

5.27663017

7.06121058

3. 51775344

5.51103662

1. 75887672

3. 89331227

5.27663017

3.14637433

7.03550689

1. 57318717

0.00000000

5.27663017

2. 35978075

.27663017

0.80488885

3.15105327

7.03550689

1. 34921557

5.27663017

3. 51775344

7.03550689

$-0.27119572$

7.07934224

8.79438361

7.03550689

7.03550689

5.50615508

8.00000000

8.78659358

9.57318717

10.35978075

11.24488507

12.21117180

13.58803937

13.80276021

8.00000000

8.78659358

9.57318717

10.35978075

11.09710335

11.95590926

12.61516471

14.07073470

8.00000000

8.78659358

9.57318717

10.35978075

11.24488507

12.21117180

13.58803937

13.80276021

8.00000000

8.78659358

9.57318717

3.93296791

6.29274866

4.68088318

7.06121058

8.79438361

5.51103662

3.89331227

7.03550689
5.27663017
1.75887672

$-0.65768151$

1.75887672

$-0.65768151$

4.23035574

1.47087271

4.23035574

1.47087271

2.45403384

2.45403384

./nitrogen/Ni/211/iter_1/struct.xyz

10.35978075

11.09710335

11.95590926

12.61516471

14.07073470

13.79390093

13.79390093

13.82296377

12.92449945

13.82296377

12.92449945

14.98619637

14.98619637 
33

Lattice $=" 4.974854628764761 \quad 0.0 \quad 0.0 \quad 0.0 \quad 12.1858553849967 \quad 0.0 \quad 0.0 \quad 0.021 .026408903419032 "$ Pro perties=species:S:1:pos:R:3 pbc="T T T"

$\begin{array}{lrrr}\mathrm{Ni} & 1.24371366 & 4.06195179 & 8.00000000 \\ \mathrm{Ni} & 0.00000000 & 0.00000000 & 8.71805841 \\ \mathrm{Ni} & 1.24371366 & 2.03097590 & 9.43611683 \\ \mathrm{Ni} & 0.00000000 & 4.06195179 & 10.15417524 \\ \mathrm{Ni} & 1.24371366 & 0.03519080 & 10.84645810 \\ \mathrm{Ni} & -0.01566228 & 2.09695812 & 11.69517071 \\ \mathrm{Ni} & 1.24371366 & 4.15158715 & 12.27872973 \\ \mathrm{Ni} & -0.04739661 & -0.12958768 & 12.96748134 \\ \mathrm{Ni} & 1.24371366 & 10.15487949 & 8.00000000 \\ \mathrm{Ni} & 0.00000000 & 6.09292769 & 8.71805841 \\ \mathrm{Ni} & 1.24371366 & 8.12390359 & 9.43611683 \\ \mathrm{Ni} & 0.00000000 & 10.15487949 & 10.15417524 \\ \mathrm{Ni} & 1.24371366 & 6.13512274 & 10.83554489 \\ \mathrm{Ni} & 0.00020672 & 8.12265803 & 11.60845053 \\ \mathrm{Ni} & 1.24371366 & 10.07317735 & 12.26725093 \\ \mathrm{Ni} & -0.00215525 & 6.09938483 & 12.90572298 \\ \mathrm{Ni} & 3.73114097 & 4.06195179 & 8.00000000 \\ \mathrm{Ni} & 2.48742731 & 0.00000000 & 8.71805841 \\ \mathrm{Ni} & 3.73114097 & 2.03097590 & 9.43611683 \\ \mathrm{Ni} & 2.48742731 & 4.06195179 & 10.15417524 \\ \mathrm{Ni} & 3.73114097 & 0.01315690 & 10.81750234 \\ \mathrm{Ni} & 2.50308960 & 2.09695812 & 11.69517071 \\ \mathrm{Ni} & 3.73114097 & 4.13098031 & 12.28105100 \\ \mathrm{Ni} & 2.53482393 & -0.12958768 & 12.96748134 \\ \mathrm{Ni} & 3.73114097 & 10.15487949 & 8.00000000 \\ \mathrm{Ni} & 2.48742731 & 6.09292769 & 8.71805841 \\ \mathrm{Ni} & 3.73114097 & 8.12390359 & 9.43611683 \\ \mathrm{Ni} & 2.48742731 & 10.15487949 & 10.15417524 \\ \mathrm{Ni} & 3.73114097 & 6.13148335 & 10.83615394 \\ \mathrm{Ni} & 2.48722059 & 8.12265803 & 11.60845053 \\ \mathrm{Ni} & 3.73114097 & 10.09928828 & 12.24566943 \\ \mathrm{Ni} & 2.48958256 & 6.09938483 & 12.90572298 \\ \mathrm{~N} & 1.24371366 & 1.08606755 & 12.58994687\end{array}$

. /nitrogen/Ni/211/iter_10/struct.xyz 42

Lattice $=" 4.974854628764761 \quad 0.0 \quad 0.0 \quad 0.0 \quad 12.1858553849967 \quad 0.0 \quad 0.0 \quad 0.021 .026408903419032 "$ Pro perties=species:S:1:pos:R:3 pbc="T T T"

$\mathrm{N}$

$\mathrm{Ni}$

$\mathrm{Ni}$

$\mathrm{Ni}$

$\mathrm{Ni}$

$\mathrm{Ni}$

$\mathrm{Ni}$

$\mathrm{Ni}$

$\mathrm{Ni}$

$\mathrm{Ni}$

$\mathrm{Ni}$

$\mathrm{Ni}$

$\mathrm{Ni}$

$\mathrm{Ni}$

$\mathrm{Ni}$

$\mathrm{Ni}$

$\mathrm{Ni}$

$\mathrm{Ni}$

$\mathrm{Ni}$

$\mathrm{Ni}$

$\mathrm{Ni}$

$\mathrm{Ni}$

$\mathrm{Ni}$

$\mathrm{Ni}$

$\mathrm{Ni}$

$\mathrm{Ni}$

$\mathrm{Ni}$

$\mathrm{Ni}$

\subsection{6}

0.00000000

1.24371366

0.00000000

1.24878106

0.00000000

1. 21764101

0.00000000

1.24371366

0.00000000

1. 24371366

0.00000000

1. 24092952

0.00000000

1.24391389

0.00000000

3. 73114097

2. 48742731

3. 73114097

2. 48742731

3. 72607357

2. 48742731

3. 75721361

2. 48742731

3. 73114097

2. 48742731

3. 73114097

2. 48742731
4.06195179

0.00000000

2.03097590

4.06195179

$-0.01716536$

2. 21241462

4.35819839

0.01400454

10.15487949

6.09292769

8.12390359

10.15487949

6.06973360

8.12378110

10.09101602

6.92162195

4.06195179

0.00000000

2.03097590

4.06195179

$-0.01716536$

2.25996392

4.35819839

0.10807373

10.15487949

6.09292769

8.12390359

10.15487949
8.00000000

8.71805841

9.43611683

10.15417524

10.88368566

11.70313680

12.60670376

13.51230450

8.00000000

8.71805841

9.43611683

10.15417524

10.81399955

11.54168811

12.60266015

13.63494707

8.00000000

8.71805841

9.43611683

10.15417524

10.88368566

11.85534988

12.60670376

13.32336057

8.00000000

8.71805841

9.43611683

10.15417524 
$\mathrm{Ni}$

$\mathrm{Ni}$

$\mathrm{Ni}$

$\mathrm{Ni}$

$\mathrm{N}$

$\mathrm{N}$

$\mathrm{N}$

$\mathrm{N}$

$\mathrm{N}$

$\mathrm{N}$

$\mathrm{N}$

$\mathrm{N}$

$\mathrm{N}$

$\mathrm{N}$
3.73392510

2.48742731

3.73094074

2.48742731

1.17947573

1.25082537

3.74185296

3. 64778573

1.32706890

3.72402926

3.79537890

1.23300167

2. 48742731

2.48742731
Fri Apr 16 14:51:55 2021

6.06973360

8.09184575

10.09101602

6.91289566

0.96425090

8.30824481

5.67240384

10.88718196

10.88718196

8.30824481

0.96425090

5.67240384

11.23171415

3.32811334
10.81399955

11.54098607

12.60266015

13.67218404

12.39822797

12.90986846

13.81220102

14.30841501

14.30841501

12.90986846

12.39822797

13.81220102

14.94436246

13.27731803

. /nitrogen/Ni/211/iter_11/struct.xyz 43

Lattice $=" 4.974854628764761 \quad 0.0 \quad 0.0 \quad 0.0 \quad 12.1858553849967 \quad 0.0 \quad 0.0 \quad 0.021 .026408903419032 "$ Pro perties=species:S:1:pos:R:3 pbc="T T T"

\begin{tabular}{|c|c|c|c|}
\hline $\mathrm{Ni}$ & 1.24371366 & 4.06195179 & 8.00000000 \\
\hline $\mathrm{Ni}$ & 0.00000000 & 0.00000000 & 8.71805841 \\
\hline $\mathrm{Ni}$ & 1.24371366 & 2.03097590 & 9.43611683 \\
\hline $\mathrm{Ni}$ & 0.00000000 & 4.06195179 & 10.15417524 \\
\hline $\mathrm{Ni}$ & 1.24368600 & -0.04661253 & 10.95432715 \\
\hline $\mathrm{Ni}$ & 0.00000000 & 2.11862169 & 11.64335692 \\
\hline $\mathrm{Ni}$ & 1.24522326 & 4.81591601 & 12.97035262 \\
\hline $\mathrm{Ni}$ & 0.00000000 & 0.00223200 & 13.56870937 \\
\hline $\mathrm{Ni}$ & 1.24371366 & 10.15487949 & 8.00000000 \\
\hline $\mathrm{Ni}$ & 0.00000000 & 6.09292769 & 8.71805841 \\
\hline $\mathrm{Ni}$ & 1.24371366 & 8.12390359 & 9.43611683 \\
\hline $\mathrm{Ni}$ & 0.00000000 & 10.15487949 & 10.15417524 \\
\hline $\mathrm{Ni}$ & 1.24097084 & 6.11667242 & 10.78824484 \\
\hline $\mathrm{Ni}$ & 0.00000000 & 8.10787460 & 11.61093816 \\
\hline $\mathrm{Ni}$ & 1.24732257 & 10.13602402 & 12.58282684 \\
\hline $\mathrm{Ni}$ & 0.00000000 & 7.20422024 & 13.97435437 \\
\hline $\mathrm{Ni}$ & 3.73114097 & 4.06195179 & 8.00000000 \\
\hline $\mathrm{Ni}$ & 2.48742731 & 0.00000000 & 8.71805841 \\
\hline $\mathrm{Ni}$ & 3.73114097 & 2.03097590 & 9.43611683 \\
\hline $\mathrm{Ni}$ & 2.48742731 & 4.06195179 & 10.15417524 \\
\hline $\mathrm{Ni}$ & 3.73116863 & -0.04661253 & 10.95432715 \\
\hline $\mathrm{Ni}$ & 2.48742731 & 2.09526160 & 11.66868070 \\
\hline $\mathrm{Ni}$ & 3.72963137 & 4.81591601 & 12.97035262 \\
\hline $\mathrm{Ni}$ & 2.48742731 & 0.17623174 & 13.48610341 \\
\hline $\mathrm{Ni}$ & 3.73114097 & 10.15487949 & 8.00000000 \\
\hline $\mathrm{Ni}$ & 2.48742731 & 6.09292769 & 8.7180584 \\
\hline $\mathrm{Ni}$ & 3.73114097 & 8.12390359 & 9.43611683 \\
\hline $\mathrm{Ni}$ & 2.48742731 & 10.15487949 & 10.15417524 \\
\hline $\mathrm{Ni}$ & 3.73388379 & 6.11667242 & 10.78824484 \\
\hline $\mathrm{Ni}$ & 2.48742731 & 8.08666275 & 11.60437241 \\
\hline $\mathrm{Ni}$ & 3.72753206 & 10.13602402 & 12.58282684 \\
\hline $\mathrm{Ni}$ & 2.48742731 & 7.19370201 & 13.96731042 \\
\hline $\mathrm{N}$ & 1.19517415 & 0.96856469 & 12.48330090 \\
\hline $\mathrm{N}$ & 1.24926041 & 8.37044919 & 12.9679540 \\
\hline $\mathrm{N}$ & 3.73392200 & 5.98500591 & 14.35903953 \\
\hline $\mathrm{N}$ & 3.64399599 & 10.87478754 & 14.32578408 \\
\hline $\mathrm{N}$ & 1.33085864 & 10.87478754 & 14.32578408 \\
\hline $\mathrm{N}$ & 3.72559422 & 8.37044919 & 12.96795405 \\
\hline $\mathrm{N}$ & 3.77968048 & 0.96856469 & 12.4833009 \\
\hline $\mathrm{N}$ & 1.24093262 & 5.98500591 & 14.35903953 \\
\hline $\mathrm{N}$ & 2.48742731 & 11.15481930 & 15.00173274 \\
\hline $\mathrm{N}$ & 2.48742731 & 3.90213548 & 11.99756671 \\
\hline $\mathrm{N}$ & 4.97485463 & 3.91425463 & 11.9958599 \\
\hline
\end{tabular}

./nitrogen/Ni/211/iter_2/struct.xyz

34

Lattice $=" 4.974854628764761 \quad 0.0 \quad 0.0 \quad 0.0 \quad 12.1858553849967 \quad 0.0 \quad 0.0 \quad 0.021 .026408903419032 "$ Pro perties=species:S:1:pos:R:3 pbc="T T T"

$\mathrm{Ni}$

1.24371366

4.06195179

8.00000000

$\mathrm{Ni}$

0.00000000

0.00000000

8.71805841

$\mathrm{Ni}$

1. 24371366

2.03097590

9.43611683 
$\mathrm{Ni}$

$\mathrm{Ni}$

$\mathrm{Ni}$

$\mathrm{Ni}$

$\mathrm{Ni}$

$\mathrm{Ni}$

$\mathrm{Ni}$

$\mathrm{Ni}$

$\mathrm{Ni}$

$\mathrm{Ni}$

$\mathrm{Ni}$

$\mathrm{Ni}$

$\mathrm{Ni}$

$\mathrm{Ni}$

$\mathrm{Ni}$

$\mathrm{Ni}$

$\mathrm{Ni}$

$\mathrm{Ni}$

$\mathrm{Ni}$

$\mathrm{Ni}$

$\mathrm{Ni}$

$\mathrm{Ni}$

$\mathrm{Ni}$

$\mathrm{Ni}$

$\mathrm{Ni}$

$\mathrm{Ni}$

$\mathrm{Ni}$

$\mathrm{Ni}$

$\mathrm{Ni}$

$\mathrm{N}$

$\mathrm{N}$

35

itrogen/Ni/211/iter_3/struct.xyz
Fri Apr 16
4.06195179

0.02217179

2.06870874

4.05827734

12.10371028

10.15487949

6.09292769

8.12390359

10.15487949

6.11509948

8.16163643

10.15120503

6.01078259

4.06195179

0.00000000

2.03097590

4.06195179

0.01233321

2.06870874

4.07247135

12.10371028

10.15487949

6.09292769

8.12390359

10.15487949

6.10526091

8.16163643

10.16539905

6.01078259

1.12491348

7.21784117
10.15417524

10.83818658

11.66435519

12.27758358

12.97994200

8.00000000

8.71805841

9.43611683

10.15417524

10.83818658

11.66435519

12.27758358

12.97994200

8.00000000

8.71805841

9.43611683

10.15417524

10.81738414

11.66435519

12.26002262

12.97994200

8.00000000

8.71805841

9.43611683

10.15417524

10.81738414

11.66435519

12.26002262

12.97994200

12.61145245

12.61145245

Lattice $=" 4.974854628764761 \quad 0.0 \quad 0.0 \quad 0.0 \quad 12.1858553849967 \quad 0.0 \quad 0.0 \quad 0.021 .026408903419032 " \quad$ Pro perties=species:S:1:pos:R:3 pbc="T T T"

$\mathrm{Ni} \quad 1.24371366 \quad 4.06195179$

$\mathrm{Ni} \quad 0.00000000 \quad 0.00000000$

$\mathrm{Ni} \quad 1.24371366 \quad 2.03097590$

$\mathrm{Ni} \quad 0.00000000 \quad 4.06195179$

0.02714471

1.24371366

2.08696457

4. 95411434

4.09634002

1.24371366

4. 92634063

1.24371366

12.10034140

10.15487949

0.00000000

6.09292769

8.12390359

1.24371366

0.00000000

1. 24371366

10.15487949

6.11419510

8.15202822

4.95762788

1. 24371366

0.02432553

3. 73114097

10.13256742

6.10077110

4.06195179

0.00000000

2. 48742731

3. 73114097

2.03097590

4.06195179

2. 48742731

3. 73114097

0.01530271

2.08696457

4.08951309

3. 73114097

2. 53594131

3. 73114097

2. 48742731

3. 73114097

2. 48742731

3. 73114097

2. 50465407

3. 73114097

2. 46310179

12.10034140

10.15487949

6.09292769

8.12390359

10.15487949

6.11862696

8.15202822

10.16572904

6.10077110

8.00000000

8.71805841

9.43611683

10.15417524

10.84964734

11.66680886

12.23456275

12.98121598

8.00000000

8.71805841

9.43611683

10.15417524

10.83383605

11.64706144

12.29418188

13.07168048

8.00000000

8.71805841

9.43611683

10.15417524

10.81551216

11.66680886

12.36770779

12.98121598

8.00000000

8.71805841

9.43611683

10.15417524

10.80211906

11.64706144

12.25462012

13.07168048

12.57935923 
$\mathrm{N} \quad 1.24371366$

3. 73114097
Fri Apr 16 14:51:55 2021

12.80573199

13.80708801

./nitrogen/Ni/211/iter_4/struct.xyz 36

Lattice $=" 4.974854628764761 \quad 0.0 \quad 0.0 \quad 0.0 \quad 12.1858553849967 \quad 0.0 \quad 0.0 \quad 0.021 .026408903419032 "$ Pro perties=species:S:1:pos:R:3 pbc="T T T"

$\begin{array}{lrrr}\mathrm{Ni} & 1.24371366 & 4.06195179 & 8.00000000 \\ \mathrm{Ni} & 0.00000000 & 0.00000000 & 8.71805841 \\ \mathrm{Ni} & 1.24371366 & 2.03097590 & 9.43611683 \\ \mathrm{Ni} & 0.00000000 & 4.06195179 & 10.15417524 \\ \mathrm{Ni} & 1.24371366 & 0.02823059 & 10.84880147 \\ \mathrm{Ni} & 4.95016365 & 2.07662489 & 11.64459714 \\ \mathrm{Ni} & 1.24371366 & 4.07444169 & 12.24213320 \\ \mathrm{Ni} & 0.02115498 & 12.17368070 & 13.07615288 \\ \mathrm{Ni} & 1.24371366 & 10.15487949 & 8.00000000 \\ \mathrm{Ni} & 0.00000000 & 6.09292769 & 8.71805841 \\ \mathrm{Ni} & 1.24371366 & 8.12390359 & 9.43611683 \\ \mathrm{Ni} & 0.00000000 & 10.15487949 & 10.15417524 \\ \mathrm{Ni} & 1.24371366 & 6.12115829 & 10.84880147 \\ \mathrm{Ni} & 4.95016365 & 8.16955258 & 11.64459714 \\ \mathrm{Ni} & 1.24371366 & 10.16736938 & 12.24213320 \\ \mathrm{Ni} & 0.02115498 & 6.08075301 & 13.07615288 \\ \mathrm{Ni} & 3.73114097 & 4.06195179 & 8.00000000 \\ \mathrm{Ni} & 2.48742731 & 0.00000000 & 8.71805841 \\ \mathrm{Ni} & 3.73114097 & 2.03097590 & 9.43611683 \\ \mathrm{Ni} & 2.48742731 & 4.06195179 & 10.15417524 \\ \mathrm{Ni} & 3.73114097 & 0.02775823 & 10.79693957 \\ \mathrm{Ni} & 2.51211829 & 2.07662489 & 11.64459714 \\ \mathrm{Ni} & 3.73114097 & 4.08580099 & 12.35629064 \\ \mathrm{Ni} & 2.46627233 & 12.17368070 & 13.07615288 \\ \mathrm{Ni} & 3.73114097 & 10.15487949 & 8.00000000 \\ \mathrm{Ni} & 2.48742731 & 6.09292769 & 8.71805841 \\ \mathrm{Ni} & 3.73114097 & 8.12390359 & 9.43611683 \\ \mathrm{Ni} & 2.48742731 & 10.15487949 & 10.15417524 \\ \mathrm{Ni} & 3.73114097 & 6.12068592 & 10.79693957 \\ \mathrm{Ni} & 2.51211829 & 8.16955258 & 11.64459714 \\ \mathrm{Ni} & 3.73114097 & 10.17872868 & 12.35629064 \\ \mathrm{Ni} & 2.46627233 & 6.08075301 & 13.07615288 \\ \mathrm{~N} & 1.24371366 & 1.33288671 & 12.74240707 \\ \mathrm{~N} & 1.24371366 & 7.42581440 & 12.74240707 \\ \mathrm{~N} & 3.73114097 & 5.09772828 & 13.80860491 \\ \mathrm{~N} & 3.73114097 & 11.19065597 & 13.80860491 \\ . / n i t & 0 & & \end{array}$

./nitrogen/Ni/211/iter_5/struct. xyz

37

Lattice $=" 4.974854628764761 \quad 0.0 \quad 0.0 \quad 0.0 \quad 12.1858553849967 \quad 0.0 \quad 0.0 \quad 0.021 .026408903419032 "$ Pro perties=species:S:1:pos:R:3 pbc="T T T"

$\begin{array}{llrr}\mathrm{Ni} & 1.24371366 & 4.06195179 & 8.00000000 \\ \mathrm{Ni} & 0.00000000 & 0.00000000 & 8.71805841 \\ \mathrm{Ni} & 1.24371366 & 2.03097590 & 9.43611683 \\ \mathrm{Ni} & 0.00000000 & 4.06195179 & 10.15417524 \\ \mathrm{Ni} & 1.24371366 & 12.16651044 & 10.80202833 \\ \mathrm{Ni} & 4.94278337 & 2.01328903 & 11.55288480 \\ \mathrm{Ni} & 1.24371366 & 3.81793089 & 12.46570538 \\ \mathrm{Ni} & 0.06594466 & 0.44286316 & 13.39217194 \\ \mathrm{Ni} & 1.24371366 & 10.15487949 & 8.00000000 \\ \mathrm{Ni} & 0.00000000 & 6.09292769 & 8.71805841 \\ \mathrm{Ni} & 1.24371366 & 8.12390359 & 9.43611683 \\ \mathrm{Ni} & 0.00000000 & 10.15487949 & 10.15417524 \\ \mathrm{Ni} & 1.24371366 & 6.09998596 & 10.90035894 \\ \mathrm{Ni} & 4.95087530 & 8.20075047 & 11.65357113 \\ \mathrm{Ni} & 1.24371366 & 10.29383879 & 12.37076385 \\ \mathrm{Ni} & 0.02606111 & 5.96019306 & 13.08218324 \\ \mathrm{Ni} & 3.73114097 & 4.06195179 & 8.00000000 \\ \mathrm{Ni} & 2.48742731 & 0.00000000 & 8.71805841 \\ \mathrm{Ni} & 3.73114097 & 2.03097590 & 9.43611683 \\ \mathrm{Ni} & 2.48742731 & 4.06195179 & 10.15417524 \\ \mathrm{Ni} & 3.73114097 & 0.01484130 & 10.84543486 \\ \mathrm{Ni} & 2.51949858 & 2.01328903 & 11.55288480\end{array}$


$\mathrm{Ni}$

$\mathrm{Ni}$

3.73114097

2.42148265

3.73114097

2.48742731

3.73114097

2.48742731

3.73114097

2.51140665

3.73114097

2. 46136621

1.24371366

1.24371366

3.73114097

3.73114097

1.24371366
Fri Apr 16 14:51:55 2021

12.35336080

13.39217194

8.00000000

8.71805841

9.43611683

10.15417524

10.79746680

11.65357113

12.31144812

13.08218324

12.88108570

12.52442390

13.81955238

13.57484741

13.78016808

5.00722126

11.45330544

11.32526152

./nitrogen/Ni/211/iter_6/struct.xyz

80

38

Lattice $=" 4.974854628764761 \quad 0.0 \quad 0.0 \quad 0.0 \quad 12.1858553849967 \quad 0.0 \quad 0.0 \quad 0.021 .026408903419032 "$ Pro perties=species:S:1:pos:R:3 pbc="T T T"

$\mathrm{Ni}$

$\mathrm{Ni}$

$\mathrm{Ni}$

$\mathrm{Ni}$

$\mathrm{Ni}$

$\mathrm{Ni}$

$\mathrm{Ni}$

$\mathrm{Ni}$

$\mathrm{Ni}$

$\mathrm{Ni}$

$\mathrm{Ni}$

$\mathrm{Ni}$

$\mathrm{Ni}$

$\mathrm{Ni}$

$\mathrm{Ni}$

$\mathrm{Ni}$

$\mathrm{Ni}$

$\mathrm{Ni}$

$\mathrm{Ni}$

$\mathrm{Ni}$

$\mathrm{Ni}$

$\mathrm{Ni}$

$\mathrm{Ni}$

$\mathrm{Ni}$

$\mathrm{Ni}$

$\mathrm{Ni}$

$\mathrm{Ni}$

$\mathrm{Ni}$

$\mathrm{Ni}$

$\mathrm{Ni}$

$\mathrm{Ni}$

$\mathrm{Ni}$

$\mathrm{N}$

N

N

$\mathrm{N}$

N

$\mathrm{N}$

.

39

/ itrogen/Ni/211/iter_t/struct.xyz

Lat perties=species:S:1:pos:R: 3 pbc="T T T"

$\mathrm{Ni}$

$\mathrm{Ni}$

$\mathrm{Ni}$

$\mathrm{Ni}$

$\mathrm{Ni}$

$\mathrm{Ni}$

$\mathrm{Ni}$
1.24371366

0.00000000

1.24371366

0.00000000

1.24371366

$-0.03328262$

1.24371366

0.05940857

1.24371366

0.00000000

1.24371366

0.00000000

1.24371366

$-0.02050835$

1. 24371366

0.02593445

3.73114097

2.48742731

3. 73114097

2.48742731

3.73114097

2.52070993

3. 73114097

2. 42801875

3.73114097

2.48742731

3.73114097

2.48742731

3. 73114097

2.50793566

3.73114097

2. 46149286

1. 24371366

1. 24371366

3.73114097

3. 73114097

1. 24371366

3. 73114097
2.03097590

4.06195179

$-0.01271241$

1.99439947

3.79896226

0.49695452

10.15487949

6.09292769

8.12390359

10.15487949

6.12894023

8.24563640

10.39411700

5.86443493

4.06195179

0.00000000

2.03097590

4.06195179

0.00687160

1.99439947

3.94642647

0.49695452

10.15487949

6.09292769

8.12390359

10.15487949

8.24563640

10.43684268

1.99322153

7.11958300

4.93356194

11.60493690

11.38066137

8.73075696
4.06195179

0.00000000

6.10650929

5.86443493
8.00000000

8.71805841

9.43611683

10.15417524

10.78100239

11.53630000

12.42333262

13.42732077

8.00000000

8.71805841

9.43611683

10.15417524

10.87630466

11.78620274

12.37585198

13.06431322

8.00000000

8.71805841

9.43611683

10.15417524

10.83682107

11.53630000

12.35232152

13.42732077

8.00000000

8.71805841

9.43611683

10.15417524

10.80207469

11.78620274

12.58484141

13.06431322

12.86045036

12.50427581

13.82937065

13.92750072

13.80144453

12.98728701

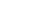


$\mathrm{Ni}$

$\mathrm{Ni}$

$\mathrm{Ni}$

$\mathrm{Ni}$

$\mathrm{Ni}$

$\mathrm{Ni}$

$\mathrm{Ni}$

$\mathrm{N}$

$\mathrm{Ni}$

$\mathrm{Ni}$

$\mathrm{Ni}$

$\mathrm{Ni}$

$\mathrm{Ni}$

$\mathrm{Ni}$

$\mathrm{Ni}$

$\mathrm{Ni}$

$\mathrm{Ni}$

$\mathrm{Ni}$

$\mathrm{Ni}$

$\mathrm{N}$

$\mathrm{Ni}$

$\mathrm{Ni}$

$\mathrm{Ni}$

$\mathrm{Ni}$

$\mathrm{Ni}$

$\mathrm{N}$

$\mathrm{N}$

$\mathrm{N}$

$\mathrm{N}$

$\mathrm{N}$

$\mathrm{N}$

$\mathrm{N}$
$-0.01712239$

1.24371366

0.00000000

1.24371366

0.00000000

1.24371366

$-0.01840307$

1.24371366

$-0.03408005$

3.73114097

2.48742731

3. 73114097

2. 48742731

3.73114097

2. 50069556

3. 73114097

2.50454971

3. 73114097

2. 48742731

3.73114097

2. 48742731

3. 73114097

2.50583038

3. 73114097

2. 52150737

1.24371366

1.24371366

3. 73114097

3.73114097

1.24371366

3.73114097

3.73114097
Fri Apr 16 14:51:55 2021

0.59232950

10.15487949

6.09292769

8.12390359

10.15487949

6.12044748

8.24846720

10.40783851

5.93639152

4.06195179

0.00000000

2.03097590

4.06195179

$-0.01539434$

1. 98143501

3.82738061

0.59232950

10.15487949

6.09292769

8.12390359

10.15487949

6.08004533

8.24846720

10.44604779

5.93639152

2.15089337

7.11292932

5.17800319

11.54817905

11.52301151

8.73322243

2.02578759
13.58724640

8.00000000

8.71805841

9.43611683

10.15417524

10.86655478

11.76430739

12.35088497

13.02354268

8.00000000

8.71805841

9.43611683

10.15417524

10.80348736

11.56081924

12.92765549

13.58724640

8.00000000

8.71805841

9.43611683

10.15417524

10.80234533

11.76430739

12.58187645

13.02354268

12.89641497

12.48453225

14.06076568

13.97371741

13.68795672

12.95579358

12.98221350

40

Lattice $=" 4.974854628764761 \quad 0.0 \quad 0.0 \quad 0.0 \quad 12.1858553849967 \quad 0.0 \quad 0.0 \quad 0.021 .026408903419032 "$ Pro perties=species:S:1:pos:R:3 pbc="T T T"

$\mathrm{Ni} \quad 1.24371366 \quad 4.06195179$

$\mathrm{Ni} \quad 0.00000000$

06195179

1.24371366

0.00000000

2.03097590

4.06195179

0.00000000

$-0.05958679$

.24371366

2.08954100

3. 98354795

0.80471982

1. 24371366

0.00000000

1.24371366

0.00000000

10.15487949

6.09292769

8.12390359

24371366

0.00000000

1.24371366

10.15487949

6.03334090

8.18246869

10.07647565

6.89764752

0.00000000

4.06195179

0.00000000

2.03097590

4.06195179

$-0.05958679$

2.08954100

3.98354795

0.80471982

10.15487949

6.09292769

8.12390359

10.15487949

6.03334090

8.18246869

10.07647565

6.89764752

8.00000000

8.71805841

9.43611683

10.15417524

11.08004256

11.51917202

12.48836408

13.48086005

8.00000000

8.71805841

9.43611683

10.15417524

11.08004256

11.51917202

12.48836408

13.48086005

8.00000000

8.71805841

9.43611683

10.15417524

11.08004256

11.51917202

12.48836408

13.48086005

8.00000000

8.71805841

9.43611683

10.15417524

11.08004256

11.51917202

12.48836408

13.48086005

$\mathrm{Ni}$

3.73114097
2.48742731 


$\begin{array}{ll}\mathrm{N} & 1.24371366 \\ \mathrm{~N} & 1.24371366 \\ \mathrm{~N} & 3.73114097 \\ \mathrm{~N} & 3.73114097 \\ \mathrm{~N} & 1.24371366 \\ \mathrm{~N} & 3.73114097 \\ \mathrm{~N} & 3.73114097 \\ \mathrm{~N} & 1.24371366\end{array}$

./nitrogen/Ni/211/iter_9/struct.xyz

2. 24307811

8.33600580

5.67914049

11.77206819

11.77206819

8.33600580

2.24307811

5.67914049
12.98805921

12.98805921

12.98304652

12.98304652

12.98304652

12.98805921

12.98805921

12.98304652 41

Lattice $=" 4.974854628764761 \quad 0.0 \quad 0.0 \quad 0.0 \quad 12.1858553849967 \quad 0.0 \quad 0.0 \quad 0.021 .026408903419032 "$ Pro perties=species:S:1:pos:R:3 pbc="T T T"

$\begin{array}{lrrr}\mathrm{Ni} & 1.24371366 & 4.06195179 & 8.00000000 \\ \mathrm{Ni} & 0.00000000 & 0.00000000 & 8.71805841 \\ \mathrm{Ni} & 1.24371366 & 2.03097590 & 9.43611683 \\ \mathrm{Ni} & 0.00000000 & 4.06195179 & 10.15417524 \\ \mathrm{Ni} & 1.26414453 & -0.03086499 & 10.84396923 \\ \mathrm{Ni} & 0.0000000 & 2.04521805 & 11.51917236 \\ \mathrm{Ni} & 1.24265456 & 4.31804386 & 12.39128968 \\ \mathrm{Ni} & 0.00000000 & 0.03470426 & 13.13637255 \\ \mathrm{Ni} & 1.24371366 & 10.15487949 & 8.00000000 \\ \mathrm{Ni} & 0.00000000 & 6.09292769 & 8.71805841 \\ \mathrm{Ni} & 1.24371366 & 8.12390359 & 9.43611683 \\ \mathrm{Ni} & 0.00000000 & 10.15487949 & 10.15417524 \\ \mathrm{Ni} & 1.24344724 & 6.08237255 & 10.78553245 \\ \mathrm{Ni} & 0.00000000 & 8.10526477 & 11.54765744 \\ \mathrm{Ni} & 1.25536683 & 10.06944564 & 12.57823578 \\ \mathrm{Ni} & 0.00000000 & 6.78321691 & 13.57528854 \\ \mathrm{Ni} & 3.73114097 & 4.06195179 & 8.00000000 \\ \mathrm{Ni} & 2.48742731 & 0.00000000 & 8.71805841 \\ \mathrm{Ni} & 3.73114097 & 2.03097590 & 9.43611683 \\ \mathrm{Ni} & 2.48742731 & 4.06195179 & 10.15417524 \\ \mathrm{Ni} & 3.71071010 & -0.03086499 & 10.84396923 \\ \mathrm{Ni} & 2.48742731 & 2.19045992 & 11.84409332 \\ \mathrm{Ni} & 3.73220006 & 4.31804386 & 12.39128968 \\ \mathrm{Ni} & 2.48742731 & 0.22123925 & 13.53974432 \\ \mathrm{Ni} & 3.73114097 & 10.15487949 & 8.00000000 \\ \mathrm{Ni} & 2.48742731 & 6.09292769 & 8.71805841 \\ \mathrm{Ni} & 3.73114097 & 8.12390359 & 9.43611683 \\ \mathrm{Ni} & 2.48742731 & 10.15487949 & 10.15417524 \\ \mathrm{Ni} & 3.73140739 & 6.08237255 & 10.78553245 \\ \mathrm{Ni} & 2.48742731 & 8.10483170 & 11.53140189 \\ \mathrm{Ni} & 3.71948780 & 10.06944564 & 12.57823578 \\ \mathrm{Ni} & 2.48742731 & 6.82884552 & 13.58854223 \\ \mathrm{~N} & 1.11751981 & 1.77449911 & 13.16761942 \\ \mathrm{~N} & 1.24920265 & 8.25033437 & 12.91734067 \\ \mathrm{~N} & 3.71333374 & 5.53466513 & 13.65682492 \\ \mathrm{~N} & 3.72358556 & 11.19143949 & 13.92761940 \\ \mathrm{~N} & 1.25126906 & 11.19143949 & 13.92761940 \\ \mathrm{~N} & 3.72565198 & 8.25033437 & 12.91734067 \\ \mathrm{~N} & 3.85733481 & 1.77449911 & 13.16761942 \\ \mathrm{~N} & 1.26152089 & 5.53466513 & 13.65682492 \\ \mathrm{~N} & 4.97485463 & 2.58997833 & 13.48917651\end{array}$

./nitrogen/Ni/221/iter_1/struct.xyz

41

Lattice $=" 4.974854628764762 \quad 0.0 \quad 0.0 \quad 0.0 \quad 14.9245638862942820 .0 \quad 0.0 \quad 0.0 \quad 21.27663016512527 "$ Pr operties=species:S:1:pos:R:3 pbc="T T T"

$\begin{array}{lrrr}\mathrm{Ni} & 1.24371366 & 3.73114097 & 8.00000000 \\ \mathrm{Ni} & 0.00000000 & 1.65828488 & 8.58629224 \\ \mathrm{Ni} & 1.24371366 & 7.04771072 & 9.17258448 \\ \mathrm{Ni} & 0.00000000 & 4.97485463 & 9.75887672 \\ \mathrm{Ni} & 1.24371366 & 2.90199853 & 10.34516896 \\ \mathrm{Ni} & 0.00000000 & 0.77319518 & 10.89378219 \\ \mathrm{Ni} & 1.26639014 & 6.14604956 & 11.58677655 \\ \mathrm{Ni} & 0.00000000 & 4.06145648 & 12.22983270 \\ \mathrm{Ni} & 1.24165899 & 1.98731947 & 12.63637619 \\ \mathrm{Ni} & 0.00000000 & -0.02575610 & 13.15469662 \\ \mathrm{Ni} & 1.24371366 & 11.19342291 & 8.00000000\end{array}$


$\mathrm{Ni}$

$\mathrm{Ni}$

$\mathrm{Ni}$

$\mathrm{Ni}$

$\mathrm{Ni}$

$\mathrm{Ni}$

$\mathrm{Ni}$

$\mathrm{Ni}$

$\mathrm{Ni}$

$\mathrm{Ni}$

$\mathrm{Ni}$

$\mathrm{Ni}$

$\mathrm{Ni}$

$\mathrm{Ni}$

$\mathrm{Ni}$

$\mathrm{Ni}$

$\mathrm{Ni}$

$\mathrm{Ni}$

$\mathrm{Ni}$

$\mathrm{Ni}$

$\mathrm{Ni}$

$\mathrm{Ni}$

$\mathrm{Ni}$

$\mathrm{Ni}$

$\mathrm{Ni}$

$\mathrm{Ni}$

$\mathrm{Ni}$

$\mathrm{Ni}$

$\mathrm{Ni}$

$\mathrm{N}$
0.00000000

1.24371366

0.00000000

1.24371366

0.00000000

1.24256262

0.00000000

1.24265437

0.00000000

3.73114097

2. 48742731

3.73114097

2. 48742731

3.73114097

2. 48742731

3.70846449

2.48742731

3.73319564

2. 48742731

3.73114097

2. 48742731

3. 73114097

2.48742731

3. 73114097

2. 48742731

3.73229201

2. 48742731

3. 73220026

2.48742731

0.00000000
Fri Apr 16 14:51:55 2021

83

9.12056682

14.50999267

12.43713657

10.36428048

8.27111974

13.59138497

11.55808116

9.52740412

7.48958298

3.73114097

1.65828488

7.04771072

4.97485463

2.90199853

0.77706888

6.14604956

3.99830633

1.98731947

$-0.02594584$

11.19342291

9.12056682

14.50999267

12.43713657

10.36428048

8.24004981

13.59138497

11.57453291

9.52740412

7.59019805

5.74970248
8.58629224

9.17258448

9.75887672

10.34516896

10.88777446

11.53062480

12.10630958

12.62631123

13.26262279

8.00000000

8.58629224

9.17258448

10.34516896

10.89074095

11.58677655

12.09027545

12.63637619

13.14774338

8.00000000

8.58629224

9.17258448

9.75887672

10.34516896

10.90379333

11.53062480

12.10438560

12.62631123

13.20600869

12.86053991
9.75887672

50

Lattice $=" 4.974854628764762 \quad 0.0 \quad 0.0 \quad 0.0 \quad 14.9245638862942820 .0 \quad 0.0 \quad 0.021 .27663016512527 "$ Pr operties=species:S:1:pos:R:3 pbc="T T T"

$\mathrm{Ni}$

$\mathrm{Ni}$

1.24371366

3.73114097

0.00000000

1. 65828488

7.04771072

1. 24371366

4.97485463

2. 90199853

0.00000000

0.90292798

6.22404443

3. 96294679

2.02036594

0.00000000

1.24697635

0.00000000

$-0.11583314$

1.24371366

0.00000000

11.19342291

9.12056682

1. 24371366

0.00000000

1.24371366

0.00000000

1.25676924

0.00000000

1.24045096

0.00000000

3.73114097

2. 48742731

3. 73114097

2. 48742731

3. 73114097

14.50999267

12.43713657

10.36428048

8.35237903

13.68632637

11.49286134

9.48264788

7.30974444

3.73114097

1. 65828488

7.04771072

4.97485463

2.90199853

0.89009709

6.22404443

4.03057939

2.02036594

$-0.15253750$

11.19342291

9.12056682

14.50999267

12.43713657
8.00000000
8.58629224
9.17258448
9.75887672
10.34516896
10.97306369
11.51449771
12.16088323
13.11419910
13.48958146
8.00000000
8.58629224
9.17258448
9.75887672
10.34516896
10.93425888
11.51449771
12.17895011
13.11419910
13.45702081
8.00000000
8.58629224
9.17258448
9.75887672
10.34516896
10.93425888
11.51449771
12.17895011
13.11419910
13.45702081
8.00000000
8.58629224
9.17258448
9.75887672 
$\mathrm{Ni}$

$\mathrm{Ni}$

$\mathrm{Ni}$

$\mathrm{Ni}$

$\mathrm{Ni}$

$\mathrm{Ni}$

$\mathrm{N}$

$\mathrm{N}$

$\mathrm{N}$

$\mathrm{N}$

$\mathrm{N}$

$\mathrm{N}$

$\mathrm{N}$

$\mathrm{N}$

$\mathrm{N}$

$\mathrm{N}$
3.73114097

2.48742731

3.71808539

2.48742731

3.73440367

2.48742731

0.00000000

2.48742731

1.24766746

3.73509477

2.48742731

0.00000000

2.48742731

0.00000000

3.72718717

1.23975986
Fri Apr 16 14:51:55 2021

10.36428048

8.36520992

13.68632637

11.42522873

9.48264788

7.34644881

5.61008163

13.07236357

0.76413185

8.22641379

3.15474081

10.61702275

5.71759036

13.17987231

0.76413185

8.22641379
10.34516896

10.97306369

11.51449771

12.16088323

13.11419910

13.48958146

12.76314770

12.76314770

14.39746037

14.39746037

13.69749487

13.69749487

12.73099131

12.73099131

14.39746037

14.39746037

42

Lattice $=" 4.974854628764762 \quad 0.0 \quad 0.0 \quad 0.0 \quad 14.9245638862942820 .0 \quad 0.0 \quad 0.0 \quad 21.27663016512527 "$ Pr operties=species:S:1:pos:R:3 pbc="T T T"

$\begin{array}{lrrr}\mathrm{Ni} & 1.24371366 & 3.73114097 & 8.00000000 \\ \mathrm{Ni} & 0.00000000 & 1.65828488 & 8.58629224 \\ \mathrm{Ni} & 1.24371366 & 7.04771072 & 9.17258448 \\ \mathrm{Ni} & 0.00000000 & 4.97485463 & 9.75887672 \\ \mathrm{Ni} & 1.24371366 & 2.90199853 & 10.34516896 \\ \mathrm{Ni} & 0.00000000 & 0.77168233 & 10.90262693 \\ \mathrm{Ni} & 1.26650847 & 6.14759390 & 11.57938646 \\ \mathrm{Ni} & 0.00000000 & 4.07580404 & 12.22032364 \\ \mathrm{Ni} & 1.24744724 & 2.01715878 & 12.63478945 \\ \mathrm{Ni} & 0.00000000 & 0.09597592 & 13.21571670 \\ \mathrm{Ni} & 1.24371366 & 11.19342291 & 8.00000000 \\ \mathrm{Ni} & 0.00000000 & 9.12056682 & 8.58629224 \\ \mathrm{Ni} & 1.24371366 & 14.50999267 & 9.17258448 \\ \mathrm{Ni} & 0.00000000 & 12.43713657 & 9.75887672 \\ \mathrm{Ni} & 1.24371366 & 10.36428048 & 10.34516896 \\ \mathrm{Ni} & 0.00000000 & 8.26867263 & 10.88482444 \\ \mathrm{Ni} & 1.22091885 & 13.60987585 & 11.57938646 \\ \mathrm{Ni} & 0.00000000 & 11.47286806 & 12.08418442 \\ \mathrm{Ni} & 1.23998008 & 9.47944072 & 12.63478945 \\ \mathrm{Ni} & 0.00000000 & 7.47013533 & 13.28101656 \\ \mathrm{Ni} & 3.73114097 & 3.73114097 & 8.00000000 \\ \mathrm{Ni} & 2.48742731 & 1.65828488 & 8.58629224 \\ \mathrm{Ni} & 3.73114097 & 7.04771072 & 9.17258448 \\ \mathrm{Ni} & 2.48742731 & 4.97485463 & 9.75887672 \\ \mathrm{Ni} & 3.73114097 & 2.90199853 & 10.34516896 \\ \mathrm{Ni} & 2.48742731 & 0.80639069 & 10.88482444 \\ \mathrm{Ni} & 3.70834616 & 6.14759390 & 11.57938646 \\ \mathrm{Ni} & 2.48742731 & 4.01058612 & 12.08418442 \\ \mathrm{Ni} & 3.72740739 & 2.01715878 & 12.63478945 \\ \mathrm{Ni} & 2.48742731 & 0.00785338 & 13.28101656 \\ \mathrm{Ni} & 3.73114097 & 11.19342291 & 8.00000000 \\ \mathrm{Ni} & 2.48742731 & 9.12056682 & 8.58629224 \\ \mathrm{Ni} & 3.73114097 & 14.50999267 & 9.17258448 \\ \mathrm{Ni} & 2.48742731 & 12.43713657 & 9.75887672 \\ \mathrm{Ni} & 3.73114097 & 10.36428048 & 10.34516896 \\ \mathrm{Ni} & 2.48742731 & 8.23396427 & 10.90262693 \\ \mathrm{Ni} & 3.75393578 & 13.60987585 & 11.57938646 \\ \mathrm{Ni} & 2.48742731 & 11.53808598 & 12.22032364 \\ \mathrm{Ni} & 3.73487455 & 9.47944072 & 12.63478945 \\ \mathrm{Ni} & 2.48742731 & 7.55825786 & 13.21571670 \\ \mathrm{~N} & 0.00000000 & 5.74503099 & 12.85576684 \\ \mathrm{~N} & 2.48742731 & 13.20731293 & 12.85576684 \\ \mathrm{~N} & \mathrm{~N} 1 \mathrm{n} & & \end{array}$

. /nitrogen/Ni/221/iter_3/struct.xyz

43

Lattice $=" 4.974854628764762 \quad 0.0 \quad 0.0 \quad 0.0 \quad 14.924563886294282 \quad 0.0 \quad 0.0 \quad 0.021 .27663016512527 "$ Pr operties=species:S:1:pos:R:3 pbc="T T T"
$\mathrm{Ni}$
1.24371366
3.73114097
8.00000000
$\mathrm{Ni}$
0.00000000
1. 65828488
8.58629224 
$\mathrm{Ni}$

$\mathrm{Ni} \quad 0.00000000$

$\mathrm{Ni} \quad 1.24371366$

$\mathrm{Ni} \quad 0.00373342$

$\mathrm{Ni} \quad 1.26585699$

$\mathrm{Ni} \quad 0.00712822$

$\mathrm{Ni} \quad 1.25569118$

$\mathrm{Ni} \quad 4.91952145$

$\mathrm{Ni} \quad 1.24371366$

$\mathrm{Ni} \quad 0.00000000$

$\mathrm{Ni} \quad 1.24371366$

$\mathrm{Ni} \quad 0.00000000$

$\mathrm{Ni} \quad 1.24371366$

$\mathrm{Ni} \quad 0.00093519$

$\mathrm{Ni} \quad 1.23850441$

$\mathrm{Ni} \quad 0.00780926$

$\mathrm{Ni} \quad 1.23912509$

$\mathrm{Ni} \quad 0.00102340$

$\mathrm{Ni} \quad 3.73114097$

$\mathrm{Ni} \quad 2.48742731$

$\mathrm{Ni} \quad 3.73114097$

$\mathrm{Ni} \quad 2.48742731$

$\mathrm{Ni}$

$\mathrm{Ni}$

$\mathrm{Ni}$

$\mathrm{Ni}$

$\mathrm{Ni}$

$\mathrm{Ni}$

$\mathrm{Ni}$

$\mathrm{Ni}$

$\mathrm{Ni}$

$\mathrm{Ni}$

$\mathrm{Ni}$

$\mathrm{Ni}$

$\mathrm{Ni}$

$\mathrm{Ni}$

$\mathrm{Ni}$

$\mathrm{Ni}$

$\mathrm{N}$

$\mathrm{N}$

$\mathrm{N}$

3.73114097

2. 48055179

3. 70938549

2.49184491

3. 71885795

2. 46710130

3.73114097

2. 48742731

3. 73114097

2.48742731

3. 73114097

2. 48803296

3.76244061

2.49513214

3. 74098131

2.48854124

4.97302096

2.53597473

1.20740788
Fri Apr 16 14:51:55 2021

7.04771072

4.97485463

2.90199853

0.78549913

6.16066925

4.07983245

2.07816409

0.06678418

11.19342291

9.12056682

14.50999267

12.43713657

10.36428048

8.27131549

13.62240674

11.48428909

9.48004522

7.46992723

3.73114097

1.65828488

7.04771072

4.97485463

2.90199853

0.81121640

6.15703197

4.04141422

2.04996454

14.89645156

11.19342291

9.12056682

14.50999267

12.43713657

10.36428048

8.24092715

13.63463667

11.52084359

9.47837581

7.55840192

5.74640444

13.17870917

0.97272161
9.17258448

9.75887672

10.34516896

10.90271140

11.57204843

12.20582906

12.74819045

13.31445388

8.00000000

8.58629224

9.17258448

9.75887672

10.34516896

10.88379538

11.58431178

12.08089066

12.63533489

13.28488639

8.00000000

8.58629224

9.17258448

9.75887672

10.34516896

10.88449036

11.57175852

12.07103771

12.60782894

13.37876752

8.00000000

8.58629224

9.17258448

9.75887672

10.34516896

10.90243034

11.57127097

12.20343846

12.63449555

13.21417688

12.84710029

12.84333332

14.12397444

44

Lattice $=" 4.974854628764762 \quad 0.0 \quad 0.0 \quad 0.0 \quad 14.9245638862942820 .0 \quad 0.0 \quad 0.021 .27663016512527 "$ Pr operties=species:S:1:pos:R:3 pbc="T T T"

$\begin{array}{lrrr}\mathrm{Ni} & 1.24371366 & 3.73114097 & 8.00000000 \\ \mathrm{Ni} & 0.00000000 & 1.65828488 & 8.58629224 \\ \mathrm{Ni} & 1.24371366 & 7.04771072 & 9.17258448 \\ \mathrm{Ni} & 0.00000000 & 4.97485463 & 9.75887672 \\ \mathrm{Ni} & 1.24371366 & 2.90199853 & 10.34516896 \\ \mathrm{Ni} & 0.00309752 & 0.78844038 & 10.89992893 \\ \mathrm{Ni} & 1.27313951 & 6.17986058 & 11.56764965 \\ \mathrm{Ni} & 0.01475787 & 4.06332346 & 12.19372239 \\ \mathrm{Ni} & 1.26107251 & 2.07303197 & 12.74446726 \\ \mathrm{Ni} & -0.05340549 & 0.06651828 & 13.31702262 \\ \mathrm{Ni} & 1.24371366 & 11.19342291 & 8.00000000 \\ \mathrm{Ni} & 0.00000000 & 9.12056682 & 8.58629224 \\ \mathrm{Ni} & 1.24371366 & 14.50999267 & 9.17258448 \\ \mathrm{Ni} & 0.00000000 & 12.43713657 & 9.75887672 \\ \mathrm{Ni} & 1.24371366 & 10.36428048 & 10.34516896 \\ \mathrm{Ni} & -0.00667262 & 8.27407093 & 10.88388516 \\ \mathrm{Ni} & 1.23827075 & 13.62916057 & 11.57931726 \\ \mathrm{Ni} & 0.01203139 & 11.50832514 & 12.07059136 \\ \mathrm{Ni} & 1.23083732 & 9.50891776 & 12.60894144 \\ \mathrm{Ni} & -0.01814226 & 7.43054465 & 13.38257571 \\ \mathrm{Ni} & 3.73114097 & 3.73114097 & 8.00000000 \\ \mathrm{Ni} & 2.48742731 & 1.65828488 & 8.58629224 \\ \mathrm{Ni} & 3.73114097 & 7.04771072 & 9.17258448\end{array}$


$\mathrm{Ni}$

$\mathrm{Ni} \quad 3.73114097$

$\mathrm{Ni} \quad 2.48075469$

$\mathrm{Ni} \quad 3.72569806$

$\mathrm{Ni} \quad 2.49945870$

$\mathrm{Ni} \quad 3.71826464$

$\mathrm{Ni} \quad 2.46928505$

$\mathrm{Ni} \quad 3.73114097$

$\mathrm{Ni} \quad 2.48742731$

$\mathrm{Ni} \quad 3.73114097$

$\mathrm{Ni} \quad 2.48742731$

$\mathrm{Ni} \quad 3.73114097$

$\mathrm{Ni} \quad 2.49052483$

$\mathrm{Ni} \quad 3.76056682$

$\mathrm{Ni} \quad 2.50218518$

$\mathrm{Ni} \quad 3.74849982$

$\mathrm{Ni} \quad 2.43402182$

$\mathrm{N} \quad 0.04263137$

$\mathrm{N} \quad 2.53005868$

$\begin{array}{ll}\mathrm{N} & 1.20955987\end{array}$

$\mathrm{N} \quad 3.69698718$
Fri Apr 16 14:51:55 2021

86

4.97485463

2.90199853

0.81178899

6.16687863

4.04604320

2. 04663581

$-0.03173729$

11.19342291

9.12056682

14.50999267

12.43713657

10.36428048

8.25072232

13.64214252

11.52560541

9.53531391

7.52880022

5.71669076

13.17897270

0.97449261

8.43677455
9.75887672

10.34516896

10.88388516

11.57931726

12.07059136

12.60894144

13.38257571

8.00000000

8.58629224

9.17258448

9.75887672

10.34516896

10.89992893

11.56764965

12.19372239

12.74446726

13.31702262

12.83748468

12.83748468

14.12175876

14.12175876

45

Lattice $=" 4.974854628764762 \quad 0.0 \quad 0.0 \quad 0.0 \quad 14.9245638862942820 .0 \quad 0.0 \quad 0.0 \quad 21.27663016512527 "$ Pr operties=species:S:1:pos:R:3 pbc="T T T"

$\mathrm{Ni}$

$\mathrm{Ni}$

$\mathrm{Ni}$

$\mathrm{Ni}$

$\mathrm{Ni}$

$\mathrm{Ni}$

$\mathrm{Ni}$

$\mathrm{Ni}$

$\mathrm{Ni}$

$\mathrm{Ni}$

$\mathrm{Ni}$

$\mathrm{Ni}$

$\mathrm{Ni}$

$\mathrm{Ni}$

$\mathrm{Ni}$

$\mathrm{Ni}$

$\mathrm{Ni}$

$\mathrm{Ni}$

$\mathrm{Ni}$

$\mathrm{Ni}$

$\mathrm{Ni}$

$\mathrm{Ni}$

$\mathrm{Ni}$

$\mathrm{Ni}$

$\mathrm{Ni}$

$\mathrm{Ni}$

$\mathrm{Ni}$

$\mathrm{Ni}$

$\mathrm{Ni}$

$\mathrm{Ni}$

$\mathrm{Ni}$

$\mathrm{Ni}$

$\mathrm{Ni}$

$\mathrm{Ni}$

$\mathrm{Ni}$

$\mathrm{Ni}$

$\mathrm{Ni}$

$\mathrm{Ni}$

$\mathrm{Ni}$

$\mathrm{Ni}$

$\mathrm{N}$

$\mathrm{N}$

$\mathrm{N}$

\subsection{6}

0.00000000

1. 24371366

0.00000000

1.24371366

0.01389891

1.27943383

0.01810748

1. 28457648

$-0.12475584$

1. 24371366

0.00000000

1.24371366

0.00000000

1. 24371366

$-0.00634000$

1.22670512

0.00935369

1.23026122

$-0.02312009$

3.73114097

2.48742731

3. 73114097

2. 48742731

3.73114097

2.48745501

3.73312447

2.48905519

3. 74286065

2.40199416

3.73114097

2. 48742731

3.73114097

2. 48742731

3. 73114097

2. 49150088

3.74370433

2.49955090

3. 74642161

2.43063342

0.04743948

2.53415585

1.09679591
3.73114097

1. 65828488

7.04771072

4.97485463

2.90199853

0.81376000

6.20845042

4.10531230

2.09313775

0.02118497

11.19342291

9.12056682

14.50999267

12.43713657

10.36428048

8.29041550

13.62602081

11.50931060

9.50731357

7.45911037

3.73114097

1. 65828488

7.04771072

4.97485463

2.90199853

0.82874779

6.20754371

4.13187641

2.07293683

$-0.08156785$

11.19342291

9.12056682

14.50999267

12.43713657

10.36428048

8.26470451

13.63795159

11.50705286

9.53512736

7.52998269

5.76354101

13.15521203

0.76104428
8.00000000

8.58629224

9.17258448

9.75887672

10.34516896

10.92513921

11.55919070

12.16357431

13.02711658

13.26261014

8.00000000

8.58629224

9.17258448

9.75887672

10.34516896

10.88053340

11.55757629

12.07071176

12.61096686

13.39824608

8.00000000

8.58629224

9.17258448

9.75887672

10.34516896

10.90500200

11.56536618

12.09501823

12.74070779

13.38068669

8.00000000

8.58629224

9.17258448

9.75887672

10.34516896

10.89788752

11.53302425

12.18050315

12.74925569

13.32802879

12.81749978

12.81075441

14.23251310 
$\mathrm{N}$

3.67982480

2.62664714
Fri Apr 16 14:51:55 2021

87

./nitrogen/Ni/221/iter_6/struct.xyz 46

Lattice $=" 4.974854628764762 \quad 0.0 \quad 0.0 \quad 0.0 \quad 14.9245638862942820 .0 \quad 0.0 \quad 0.0 \quad 21.27663016512527 "$ Pr operties=species:S:1:pos:R:3 pbc="T T T"

$\mathrm{Ni}$

$\mathrm{Ni}$

$\mathrm{Ni}$

$\mathrm{Ni}$

$\mathrm{Ni}$

$\mathrm{Ni}$

$\mathrm{Ni}$

$\mathrm{Ni}$

$\mathrm{Ni}$

$\mathrm{Ni}$

$\mathrm{Ni}$

$\mathrm{Ni}$

$\mathrm{Ni}$

$\mathrm{Ni}$

$\mathrm{Ni}$

$\mathrm{Ni}$

$\mathrm{Ni}$

$\mathrm{Ni}$

$\mathrm{Ni}$

$\mathrm{Ni}$

$\mathrm{Ni}$

$\mathrm{Ni}$

$\mathrm{Ni}$

$\mathrm{Ni}$

$\mathrm{Ni}$

$\mathrm{Ni}$

$\mathrm{Ni}$

$\mathrm{Ni}$

$\mathrm{Ni}$

$\mathrm{Ni}$

$\mathrm{Ni}$

$\mathrm{Ni}$

$\mathrm{Ni}$

$\mathrm{Ni}$

$\mathrm{Ni}$

$\mathrm{Ni}$

$\mathrm{Ni}$

$\mathrm{Ni}$

$\mathrm{Ni}$

$\mathrm{N}$

$\mathrm{N}$

$\mathrm{N}$

$\mathrm{N}$

$\mathrm{N}$

$\mathrm{N}$

.$/$

47

gen/Ni/221/iter_7/struct.xyz
3.73114097

1. 65828488

7.04771072

4.97485463

2.90199853

0.82729856

6.20178105

4.08054246

2.10458986

0.02671289

11.19342291

9.12056682

14.50999267

12.43713657

10.36428048

8.30785563

13.65840244

11.59549448

9.53819515

7.40538786

3.73114097

1.65828488

7.04771072

4.97485463

2.90199853

0.84557368

6.19612049

4.13321254

2.07591321

$-0.05689408$

11.19342291

9.12056682

14.50999267

12.43713657

10.36428048

8.28958050

13.66406299

11.54282440

9.56687180

7.48899484

5.72969553

13.19197748

0.76506948

8.22735142

3.17426992

10.63655186
14.13779693

13.52936570
8.00000000

8.58629224

9.17258448

9.75887672

10.34516896

10.92797235

11.52027832

12.14956124

13.04756406

13.26684708

8.00000000

8.58629224

9.17258448

9.75887672

10.34516896

10.90375514

11.54370590

12.09739131

12.74847784

13.37960146

8.00000000

8.58629224

9.17258448

9.75887672

10.34516896

10.90375514

11.54370590

12.09739131

12.74847784

13.37960146

8.00000000

8.58629224

9.17258448

9.75887672

10.34516896

10.92797235

11.52027832

12.14956124

13.04756406

13.26684708

12.78936902

12.78936902

14.24402351

14.24402351

13.54050973

13.54050973

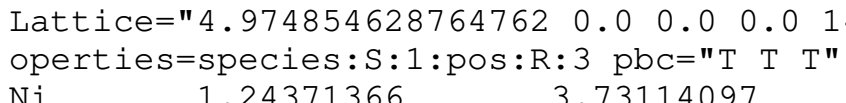

$\begin{array}{lrrr}\mathrm{Ni} & 1.24371366 & 3.73114097 & 8.00000000 \\ \mathrm{Ni} & 0.00000000 & 1.65828488 & 8.58629224 \\ \mathrm{Ni} & 1.24371366 & 7.04771072 & 9.17258448 \\ \mathrm{Ni} & 0.00000000 & 4.97485463 & 9.75887672 \\ \mathrm{Ni} & 1.24371366 & 2.90199853 & 10.34516896 \\ \mathrm{Ni} & 0.01252141 & 0.81473589 & 10.92550087 \\ \mathrm{Ni} & 1.21125777 & 6.24036865 & 11.52120117 \\ \mathrm{Ni} & -0.00288319 & 4.05148191 & 12.17008947 \\ \mathrm{Ni} & 1.23861573 & 2.03678503 & 13.05646058 \\ \mathrm{Ni} & -0.13605534 & -0.05277891 & 13.27401329 \\ \mathrm{Ni} & 1.24371366 & 11.19342291 & 8.00000000 \\ \mathrm{Ni} & 0.00000000 & 9.12056682 & 8.58629224\end{array}$


$\mathrm{Ni}$

$\mathrm{Ni}$

$\mathrm{Ni}$

$\mathrm{Ni}$

$\mathrm{Ni}$

$\mathrm{Ni}$

$\mathrm{Ni}$

$\mathrm{Ni}$

$\mathrm{Ni}$

$\mathrm{Ni}$

$\mathrm{Ni}$

$\mathrm{Ni}$

$\mathrm{Ni}$

$\mathrm{Ni}$

$\mathrm{Ni}$

$\mathrm{Ni}$

$\mathrm{Ni}$

$\mathrm{Ni}$

$\mathrm{Ni}$

$\mathrm{Ni}$

$\mathrm{Ni}$

$\mathrm{Ni}$

$\mathrm{Ni}$

$\mathrm{Ni}$

$\mathrm{Ni}$

$\mathrm{Ni}$

$\mathrm{Ni}$

$\mathrm{Ni}$

$\mathrm{N}$

$\mathrm{N}$

$\mathrm{N}$

$\mathrm{N}$

$\mathrm{N}$

$\mathrm{N}$

$\mathrm{N}$
1.24371366

0.00000000

1.24371366

$-0.00541313$

1.23284593

$-0.01186698$

1. 25065510

$-0.03524312$

3.73114097

2. 48742731

3. 73114097

2.48742731

3.73114097

2.47379667

3. 70609274

2.47823270

3. 72265676

2.39621822

3. 73114097

2.48742731

3. 73114097

2.48742731

3. 73114097

2.50306572

3. 73774545

2. 50440281

3. 76834127

2.40379215

$-0.00831686$

2. 54571530

1.06382267

3.68628697

2.62933273

0.16873380

2. 43188092
Fri Apr 16 14:51:55 2021

88

14.50999267

12.43713657

10.36428048

8.32655028

13.64721919

11.59999443

9.53913688

7.42346330

3.73114097

1. 65828488

7.04771072

4.97485463

2.90199853

0.83945780

6.21391584

4.10879236

1.99558935

$-0.09894888$

11.19342291

9.12056682

14.50999267

12.43713657

10.36428048

8.33629452

13.66150791

11.53022046

9.58942264

7.51039033

5.69808992

13.17029045

0.69619326

8.25700570

2.98909401

10.65262642

5.82046982
9.17258448

9.75887672

10.34516896

10.89674455

11.54187093

12.10065355

12.74547668

13.37076063

8.00000000

8.58629224

9.17258448

9.75887672

10.34516896

10.89460087

11.54259337

12.27736743

12.71581868

13.40514802

8.00000000

8.58629224

9.17258448

9.75887672

10.34516896

10.92479576

11.50142053

12.13912529

13.11378536

13.39034508

12.81737013

12.78574224

14.25533699

14.31281476

13.63403043

13.54603414

12.81988907

48

Lattice $=" 4.974854628764762 \quad 0.0 \quad 0.0 \quad 0.0 \quad 14.9245638862942820 .0 \quad 0.0 \quad 0.0 \quad 21.27663016512527 "$ Pr operties=species:S:1:pos:R:3 pbc="T T T"

$\begin{array}{lrrr}\mathrm{Ni} & 1.24371366 & 3.73114097 & 8.00000000 \\ \mathrm{Ni} & 0.00000000 & 1.65828488 & 8.58629224 \\ \mathrm{Ni} & 1.24371366 & 7.04771072 & 9.17258448 \\ \mathrm{Ni} & 0.00000000 & 4.97485463 & 9.75887672 \\ \mathrm{Ni} & 1.24371366 & 2.90199853 & 10.34516896 \\ \mathrm{Ni} & 0.02389063 & 0.86084077 & 10.91790575 \\ \mathrm{Ni} & 1.20356616 & 6.23647874 & 11.52155364 \\ \mathrm{Ni} & -0.00058056 & 4.03524432 & 12.16384659 \\ \mathrm{Ni} & 1.25211390 & 2.06637009 & 13.14389911 \\ \mathrm{Ni} & -0.10066690 & 0.00093685 & 13.41760015 \\ \mathrm{Ni} & 1.24371366 & 11.19342291 & 8.00000000 \\ \mathrm{Ni} & 0.00000000 & 9.12056682 & 8.58629224 \\ \mathrm{Ni} & 1.24371366 & 14.50999267 & 9.17258448 \\ \mathrm{Ni} & 0.00000000 & 12.43713657 & 9.75887672 \\ \mathrm{Ni} & 1.24371366 & 10.36428048 & 10.34516896 \\ \mathrm{Ni} & -0.01028772 & 8.31791540 & 10.89207792 \\ \mathrm{Ni} & 1.21301216 & 13.66543060 & 11.54086742 \\ \mathrm{Ni} & -0.01689931 & 11.55669082 & 12.26109390 \\ \mathrm{Ni} & 1.24819278 & 9.46039557 & 12.71066494 \\ \mathrm{Ni} & -0.04692944 & 7.38016299 & 13.39253907 \\ \mathrm{Ni} & 3.73114097 & 3.73114097 & 8.00000000 \\ \mathrm{Ni} & 2.48742731 & 1.65828488 & 8.58629224 \\ \mathrm{Ni} & 3.73114097 & 7.04771072 & 9.17258448 \\ \mathrm{Ni} & 2.48742731 & 4.97485463 & 9.75887672 \\ \mathrm{Ni} & 3.73114097 & 2.90199853 & 10.34516896 \\ \mathrm{Ni} & 2.47713959 & 0.85563346 & 10.89207792 \\ \mathrm{Ni} & 3.70043947 & 6.20314865 & 11.54086742 \\ \mathrm{Ni} & 2.47052801 & 4.09440887 & 12.26109390 \\ \mathrm{Ni} & 3.73562010 & 1.99811363 & 12.71066494\end{array}$


$\mathrm{Ni}$

$\mathrm{Ni}$

2.44049787

3. 73114097

2.48742731

3. 73114097

2. 48742731

3. 73114097

2. 51131794

3. 69099347

2. 48684676

3. 73954122

2. 38676041

$-0.01237675$

2. 47505057

1.18264523

3.67007254

2. 67941486

0.19198755

2.42733629

$-0.06009103$

49

Lattice $=" 4.974854628764762 \quad 0.0 \quad 0.0 \quad 0.0 \quad 14.9245638862942820 .0 \quad 0.0 \quad 0.0 \quad 21.27663016512527 "$ Pr operties=species:S:1:pos:R:3 pbc="T T T"

$\mathrm{Ni}$

1.24371366

0.00000000

1. 24371366

3.73114097

1.65828488

0.00000000

7.04771072

1.24371366

0.00005367

1.19612938

$-0.01594209$

1. 24180785

$-0.00832000$

1. 24371366

0.00000000

1.24371366

0.00000000

1.24371366

$-0.01222956$

1.24738822

$-0.00248007$

1.25000164

$-0.04162085$

3.73114097

2. 48742731

3.73114097

2. 48742731

3. 73114097

2.48585972

3. 70716341

2. 46810597

3. 71624722

2.47859170

3. 73114097

2. 48742731

3.73114097

2.48742731

3. 73114097

2.51208643

3. 72045504

2.50535345

3.73991098

2. 38999728

$-0.01612399$

2.48654636

1.23606994

3. 67873412

2.48477213

Fri Apr 16 14:51:55 2021

89

$-0.08211896$

11.19342291

9.12056682

14.50999267

12.43713657

10.36428048

8.32312271

13.69876068

11.49752626

9.52865203

7.46321880

5.67380267

13.13608461

0.72962803

8.19190998

3.01087199

10.47315394

5.79702674

13.25930868

13.39253907

8.00000000

8.58629224

9.17258448

9.75887672

10.34516896

10.91790575

11.52155364

12.16384659

13.14389911

13.41760015

12.81414258

12.81414258

14.34388009

14.34388009

13.64280791

13.64280791

12.80908137

12.80908137
4.97485463

2. 90199853

0.88810544

6.25578593

4.02838223

2.06017135

$-0.08980101$

11.19342291

9.12056682

14.50999267

12.43713657

10.36428048

8.32785686

13.66469687

11.47799409

9.41905185

7.34868279

3.73114097

1. 65828488

7.04771072

4.97485463

2.90199853

0.87450613

6.22244800

4.11044379

2.05532895

$-0.12589850$

11.19342291

9.12056682

14.50999267

12.43713657

10.36428048

8.32844146

13.66866272

11.43570120

9.48603039

7.43398508

5.66563673

13.07915077

0.80782485

8.15371075

3.18317718
8.00000000

8.58629224

9.17258448

9.75887672

10.34516896

10.95600099

11.51737967

12.15350828

13.06730633

13.45737947

8.00000000

8.58629224

9.17258448

9.75887672

10.34516896

10.89618546

11.52809073

12.25145376

12.74093122

13.43257095

8.00000000

8.58629224

9.17258448

9.75887672

10.34516896

10.92085141

11.53289715

12.18199142

13.05048594

13.43438306

8.00000000

8.58629224

9. 17258448

9.75887672

10.34516896

10.92552138

11.52343948

12.17016582

13.18417647

13.46571604

12.80519073

12.78253689

14.35384645

14.38923616

13.66402059 
$\mathrm{N}$

$\mathrm{N}$

0.19532219

2.42837022

$-0.00572587$

3.72124131
Fri Apr 16 14:51:55 2021

10.43830283

5.80475343

13.17128136

0.81281030

./nitrogen/Ni/311/iter_1/struct.xyz

37

Lattice $=" 7.462281943147142 \quad 0.0 \quad 0.0 \quad 2.4874273143823786 \quad 8.24986309508776 \quad 0.0 \quad 0.0 \quad 0.021 .30321$ 2853088358" Properties=species:S:1:pos:R:3 pbc="T T T"

$\begin{array}{lrrr}\mathrm{Ni} & 2.48742731 & 2.99995022 & 8.00000000 \\ \mathrm{Ni} & 2.48742731 & 0.74998755 & 9.06064257 \\ \mathrm{Ni} & 1.24371366 & 2.62495644 & 10.12128514 \\ \mathrm{Ni} & 1.24228550 & 0.39368028 & 11.19202475 \\ \mathrm{Ni} & 2.50904525 & 2.29687080 & 12.36643213 \\ \mathrm{Ni} & -0.07452415 & -0.08366504 & 13.27934506 \\ \mathrm{Ni} & 3.73114097 & 7.12488176 & 8.00000000 \\ \mathrm{Ni} & 3.73114097 & 4.87491910 & 9.06064257 \\ \mathrm{Ni} & 2.48742731 & 6.74988799 & 10.12128514 \\ \mathrm{Ni} & 2.48293589 & 4.50368871 & 11.17475197 \\ \mathrm{Ni} & 3.72860906 & 6.31614743 & 12.24797074 \\ \mathrm{Ni} & 1.24770289 & 4.26149314 & 13.22734976 \\ \mathrm{Ni} & 4.97485463 & 2.99995022 & 8.00000000 \\ \mathrm{Ni} & 4.97485463 & 0.74998755 & 9.06064257 \\ \mathrm{Ni} & 3.73114097 & 2.62495644 & 10.12128514 \\ \mathrm{Ni} & 3.74939838 & 0.39409824 & 11.15983953 \\ \mathrm{Ni} & 4.98058279 & 2.26487263 & 12.29613115 \\ \mathrm{Ni} & 2.54937637 & -0.09577922 & 13.27565277 \\ \mathrm{Ni} & 6.21856829 & 7.12488176 & 8.00000000 \\ \mathrm{Ni} & 6.21856829 & 4.87491910 & 9.06064257 \\ \mathrm{Ni} & 4.97485463 & 6.74988799 & 10.12128514 \\ \mathrm{Ni} & 4.97820224 & 4.50427057 & 11.16661769 \\ \mathrm{Ni} & 6.22240758 & 6.36263340 & 12.24375542 \\ \mathrm{Ni} & 3.74860749 & 4.15352476 & 13.22221272 \\ \mathrm{Ni} & 7.46228194 & 2.99995022 & 8.00000000 \\ \mathrm{Ni} & 7.46228194 & 0.74998755 & 9.06064257 \\ \mathrm{Ni} & 6.21856829 & 2.62495644 & 10.12128514 \\ \mathrm{Ni} & 6.19971808 & 0.39922525 & 11.16058375 \\ \mathrm{Ni} & 7.44361558 & 2.30927203 & 12.37965063 \\ \mathrm{Ni} & 4.96601345 & -0.01299319 & 13.18281547 \\ \mathrm{Ni} & 8.70599560 & 7.12488176 & 8.00000000 \\ \mathrm{Ni} & 8.70599560 & 4.87491910 & 9.06064257 \\ \mathrm{Ni} & 7.46228194 & 6.74988799 & 10.12128514 \\ \mathrm{Ni} & 7.46359652 & 4.50075043 & 11.17113739 \\ \mathrm{Ni} & 8.70033344 & 6.38410594 & 12.22779795 \\ \mathrm{Ni} & 6.20372780 & 4.18347815 & 13.21720088 \\ \mathrm{~N} & 1.24149476 & 1.11922136 & 13.00094571 \\ . & 1.251 & \end{array}$

./nitrogen/Ni/311/iter_2/struct.xyz 38

Lattice $=" 7.462281943147142 \quad 0.0 \quad 0.0 \quad 2.4874273143823786 \quad 8.24986309508776 \quad 0.0 \quad 0.0 \quad 0.021 .30321$ $2853088358 "$ Properties=species:S:1:pos:R:3 pbc="T T T"

$\mathrm{Ni}$

$\mathrm{Ni}$

$\mathrm{Ni}$

$\mathrm{Ni}$

$\mathrm{Ni}$

$\mathrm{Ni}$

$\mathrm{Ni}$

$\mathrm{Ni}$

$\mathrm{Ni}$

$\mathrm{Ni}$

$\mathrm{Ni}$

$\mathrm{Ni}$

$\mathrm{Ni}$

$\mathrm{Ni}$

$\mathrm{Ni}$

$\mathrm{Ni}$

$\mathrm{Ni}$

$\mathrm{Ni}$

$\mathrm{Ni}$
2. 48742731

2. 48742731

1. 24371366

1. 24701736

2. 52573373

9.89552224

3.73114097

3.73114097

2. 48742731

2.50666223

3. 73157817

1. 36028677

4.97485463

4.97485463

3.73114097

3.75104664

4.96735785

5.04599897

6.21856829
2.99995022

0.74998755

2.62495644

0.36972965

2. 27074077

8.18629780

7.12488176

4.87491910

6.74988799

4.49660586

6.32249046

4.14466129

2. 99995022

0.74998755

2.62495644

0.37106356

2. 26653375

8.19521124

7.12488176
13.67028327

12.78084573

12.76425032

14.34825684
90

8.00000000

10.12128514

9202475

13.27934506

8.00000000

9.06064257

12.24797074

9.06064257

10.12128514

12.29613115

13.27565277

8.00000000

.06064257

12.24375542

3.22221272

8.00000000

06064257

12.37965063

9.06064257

10.12128514

13.00094571 
$\mathrm{Ni}$

$\mathrm{Ni}$

$\mathrm{Ni}$

$\mathrm{Ni}$

$\mathrm{Ni}$

$\mathrm{Ni}$

$\mathrm{Ni}$

$\mathrm{Ni}$

$\mathrm{Ni}$

$\mathrm{Ni}$

$\mathrm{Ni}$

$\mathrm{Ni}$

$\mathrm{Ni}$

$\mathrm{Ni}$

$\mathrm{Ni}$

$\mathrm{Ni}$

$\mathrm{Ni}$

$\mathrm{N}$

$\mathrm{N}$

39

itrogen/Ni/311/iter_3/struct.xyz

6.21856829

4.97485463

4.96208241

6.19790562

3.76365255

7.46228194

7.46228194

6.21856829

6.19821517

7.42265275

4.97905688

8.70599560

8.70599560

7.46228194

7.46933811

8.72449310

6.13706157

1.25062288

7.46114711
Fri Apr 16 14:51:55 2021

4.87491910

6.74988799

4.50378521

6.41079218

4.14274824

2.99995022

0.74998755

2.62495644

0.38084794

2.23804892

0.13176479

7.12488176

4.87491910

6.74988799

4.49590970

6.42520807

4.08320149

1.13700387

5.26677538
truct.xyz

\section{5}

$\mathrm{Ni}$

$\mathrm{Ni}$

$\mathrm{Ni}$

$\mathrm{Ni}$

$\mathrm{Ni}$

$\mathrm{Ni}$

$\mathrm{Ni}$

$\mathrm{Ni}$

$\mathrm{Ni}$

$\mathrm{Ni}$

$\mathrm{Ni}$

$\mathrm{Ni}$

$\mathrm{Ni}$

$\mathrm{Ni}$

$\mathrm{Ni}$

$\mathrm{Ni}$

$\mathrm{Ni}$

$\mathrm{Ni}$

$\mathrm{Ni}$

$\mathrm{Ni}$

$\mathrm{Ni}$

$\mathrm{Ni}$

$\mathrm{Ni}$

$\mathrm{Ni}$

$\mathrm{Ni}$

$\mathrm{Ni}$

$\mathrm{Ni}$

$\mathrm{Ni}$

$\mathrm{Ni}$

$\mathrm{Ni}$

$\mathrm{Ni}$

$\mathrm{Ni}$

$\mathrm{Ni}$

$\mathrm{Ni}$

$\mathrm{Ni}$

$\mathrm{N}$

$\mathrm{N}$

$\mathrm{N}$

4.83673162

2. 48742731

2. 48742731

1. 24371366

1.24330743

2. 48200631

2. 44644972

3.73114097

3.73114097

2. 48742731

2. 49735784

3.74775095

1.25635639

4.97485463

4.97485463

3. 73114097

3. 76046547

4.94630175

5.03788858

6.21856829

6.21856829

4.97485463

4.95622326

6.21526291

3. 61892750

7. 46228194

7. 46228194

6.21856829

6.19134400

7.42639443

4.98486194

8.70599560

8.70599560

7. 46228194

7.44896535

8.74113106

6.18362242

1.24572860

7.48728532

.$/ 1$

40

Lattice $=" 7.462281943147142 \quad 0.0 \quad 0.0 \quad 2.4874273143823786 \quad 8.24986309508776 \quad 0.0 \quad 0.0 \quad 0.0 \quad 21.30321$ 2853088358" Properties=species:S:1:pos:R:3 pbc="T T T"

$\mathrm{Ni}$

2.48742731

2.99995022

8.00000000

$\mathrm{Ni}$

2.48742731

0.74998755

9.06064257
91

10.12128514

11.15295443

12.32888035

13.21659828

8.00000000

11.16210664

12.33016940

13.22520129

8.00000000

9.06064257

10.12128514

11.20075981

12.31797608

13.01316113

13.00582697
$73143823786 \quad 8.24986309508776 \quad 0.0 \quad 0.0 \quad 0.0 \quad 21.30321$

8.00000000

9.06064257

10.12128514

11.17480448

12.30055620

13.28800184

9.06064257

10.12128514

11.16410311

12.23648996

13.29130296

8.00000000

9.06064257

10.12128514

11.15969418

12.42990893

13.27355714

8.00000000

9.06064257

10.12128514

11.14351835

12.30750962

13.31892239

9.06064257

10.12128514

11.15866165

12.30866689

13.21883320

8.00000000

9.06064257

10.12128514

11.16717918

12.30580261

13.36469390

13.01416918

13.23557831

13.94082606 $\mathrm{pbc}=" \mathrm{~T} \mathrm{~T}$ T"

8.00000000

8.00000000 
$\mathrm{Ni}$

$\mathrm{Ni}$

$\mathrm{Ni}$

$\mathrm{Ni}$

$\mathrm{Ni}$

$\mathrm{Ni}$

$\mathrm{Ni}$

$\mathrm{Ni}$

$\mathrm{Ni}$

$\mathrm{Ni}$

$\mathrm{Ni}$

$\mathrm{Ni}$

$\mathrm{Ni}$

$\mathrm{Ni}$

$\mathrm{Ni}$

$\mathrm{Ni}$

$\mathrm{Ni}$

$\mathrm{Ni}$

$\mathrm{Ni}$

$\mathrm{Ni}$

$\mathrm{Ni}$

$\mathrm{Ni}$

$\mathrm{Ni}$

$\mathrm{Ni}$

$\mathrm{Ni}$

$\mathrm{Ni}$

$\mathrm{Ni}$

$\mathrm{Ni}$

$\mathrm{Ni}$

$\mathrm{Ni}$

$\mathrm{Ni}$

$\mathrm{Ni}$

$\mathrm{Ni}$

$\mathrm{Ni}$

$\mathrm{N}$

N

N

$\mathrm{N}$
1.24371366

1.23695406

2. 57523545

2. 44074376

3. 73114097

3. 73114097

2. 48742731

2. 51870258

3. 76907915

2. 40001225

4.97485463

4.97485463

3. 73114097

3. 77955941

5.08257738

2. 56016618

6.21856829

6.21856829

4.97485463

4.98560896

6.14314863

4.77041008

7.46228194

7.46228194

6.21856829

6.20707578

7.48286353

7.44053649

8.70599560

8.70599560

7.46228194

7.45000506

8.66899298

7. 35651665

1. 22468465

8. 51255970

6.34473811

3.07985403
Fri Apr 16 14:51:55 2021

2.62495644

0.44726980

2.22536685

7. 96189023

7.12488176

4.87491910

6.74988799

4. 36762891

6.34000570

5. 35148350

2.99995022

0.74998755

2. 62495644

0.42019261

2. 28131817

$-0.18072972$

7. 12488176

4.87491910

6.74988799

4.49477813

6.34620699

4.43593830

2.99995022

0.74998755

2.62495644

0.41755462

2. 30539045

8.01026746

7.12488176

4.87491910

6.74988799

4.43108305

6.32000432

5.01037624

0.99569310

6.62239055

3.54274608

3.86980951
10.12128514

11.26178045

12.61007024

13.46731962

8.00000000

9.06064257

10.12128514

11.41592923

12.13681761

13.91679575

8.00000000

9.06064257

10.12128514

11.22672921

12.38116272

13.35437205

8.00000000

9.06064257

10.12128514

11.06703539

12.12725340

13.47452808

8.00000000

9.06064257

10.12128514

11.20074327

12.58012882

13.32315803

8.00000000

9.06064257

10.12128514

11.20519709

12.06947087

13.69549674

13.09839003

13.94945310

13.30436527

13.19077475

41

Lattice $=" 7.462281943147142 \quad 0.0 \quad 0.0 \quad 2.4874273143823786 \quad 8.24986309508776 \quad 0.0 \quad 0.0 \quad 0.021 .30321$ 2853088358" Properties=species:S:1:pos:R:3 pbc="T T T"

$\begin{array}{lrrr}\mathrm{Ni} & 2.48742731 & 2.99995022 & 8.00000000 \\ \mathrm{Ni} & 2.48742731 & 0.74998755 & 9.06064257 \\ \mathrm{Ni} & 1.24371366 & 2.62495644 & 10.12128514 \\ \mathrm{Ni} & 1.27235768 & 0.45592017 & 11.23617221 \\ \mathrm{Ni} & 2.75847842 & 2.37111997 & 12.70338888 \\ \mathrm{Ni} & -0.00894658 & -0.03175395 & 13.37116640 \\ \mathrm{Ni} & 3.73114097 & 7.12488176 & 8.00000000 \\ \mathrm{Ni} & 3.73114097 & 4.87491910 & 9.06064257 \\ \mathrm{Ni} & 2.48742731 & 6.74988799 & 10.12128514 \\ \mathrm{Ni} & 2.45383322 & 4.44884141 & 11.15472213 \\ \mathrm{Ni} & 3.70650321 & 6.26709542 & 12.29829373 \\ \mathrm{Ni} & 2.24213078 & 4.65221528 & 13.65036750 \\ \mathrm{Ni} & 4.97485463 & 2.99995022 & 8.00000000 \\ \mathrm{Ni} & 4.97485463 & 0.74998755 & 9.06064257 \\ \mathrm{Ni} & 3.73114097 & 2.62495644 & 10.12128514 \\ \mathrm{Ni} & 3.77504744 & 0.42943307 & 11.16196427 \\ \mathrm{Ni} & 5.20943063 & 2.20697878 & 12.30445214 \\ \mathrm{Ni} & 2.56325698 & -0.08591737 & 13.32450061 \\ \mathrm{Ni} & 6.21856829 & 7.12488176 & 8.00000000 \\ \mathrm{Ni} & 6.21856829 & 4.87491910 & 9.06064257 \\ \mathrm{Ni} & 4.97485463 & 6.74988799 & 10.12128514 \\ \mathrm{Ni} & 4.94622704 & 4.48526917 & 11.12281776 \\ \mathrm{Ni} & 6.10507230 & 6.35213830 & 12.18871889 \\ \mathrm{Ni} & 4.65775654 & 4.18100420 & 13.57218335 \\ \mathrm{Ni} & 7.46228194 & 2.99995022 & 8.00000000 \\ \mathrm{Ni} & 7.46228194 & 0.74998755 & 9.06064257\end{array}$


$\mathrm{Ni}$

$\mathrm{Ni}$

$\mathrm{Ni}$

$\mathrm{Ni}$

$\mathrm{Ni}$

$\mathrm{Ni}$

$\mathrm{Ni}$

$\mathrm{Ni}$

$\mathrm{Ni}$

$\mathrm{Ni}$

$\mathrm{N}$

$\mathrm{N}$

$\mathrm{N}$

$\mathrm{N}$

$\mathrm{N}$

6.21856829

6.23424206

7.62237056

4.94791136

8.70599560

8.70599560

7.46228194

7.46205767

8.60459090

7.13888846

1.35079983

7.17734833

6.20506336

3.23279739

2.49849782
Fri Apr 16 14:51:55 2021

2.62495644

0.36674588

2.44994178

0.02277694

7.12488176

4.87491910

6.74988799

4.49304675

6.36198728

4.72205806

1.15490124

6.54312469

3. 19913249

3.30840836

6.44761893
10.12128514

11.15895564

12.54259518

13.30673549

8.00000000

9.06064257

10.12128514

11.12059332

12.33444403

13.62081769

13.00134774

13.63644932

13.47602409

14.15700433

13.63352255

42

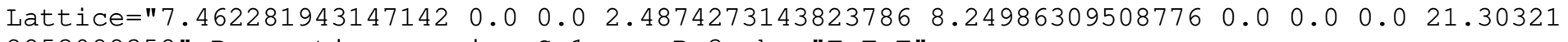

2853088358" Properties=species:S:1:pos:R:3 pbc="T T T"

.

Nit

$2.48742731 \quad 2.99995022$

8.00000000

2.48742731

0.74998755

9.06064257

$\mathrm{Ni}$

1.24371366

2.62495644

1.26585195

0.34856190

10.12128514

2.44391103

2. 46321937

2.41608722

8.22541922

3. 73114097

7.12488176

3.73114097

4.87491910

2. 48742731

6.74988799

2. 52689204

4.47571103

3.73224131

6.24593572

1.77936958

4.74584383

4.97485463

2.99995022

4.97485463

0.74998755

3. 73114097

2.62495644

3. 74960672

0.38428976

4.97877719

2. 31584039

2. 48570795

$-0.01352933$

6.21856829

7.12488176

6.21856829

4.87491910

4.97485463

6.74988799

4.97892212

4.53062560

6.21413632

6.35692346

4.10039206

3.92850850

7. 46228194

2.99995022

7.46228194

0.74998755

2.62495644

6.21856829

0.38384370

7.41644106

2.22165311

5.09872284

0.79342187

7.12488176

$8.70599560 \quad 4.87491910$

7.46228194

6.74988799

11.21500415

12.65772335

13.47538382

8.00000000

9.06064257

10.12128514

11.15958376

12.41690248

13.69678381

8.00000000

9.06064257

10.12128514

11.20668459

12.15550237

13.38840270

8.00000000

9.06064257

10.12128514

11.17865581

12.55183819

13.72991433

8.00000000

9.06064257

10.12128514

11.14736799

12.18324092

13.94808337

8.00000000

9.06064257

10.12128514

11.19705745

12.26136767

8.67855054

4.50695312

6.46571094

6.52726321

3.95380597

1.15855618

5.16118482

13.53156708

12.93130020

12.90512284

13.96319168

2.59988938

5.38244596

3. 28453266

14.26694467

13.68311878

2.46725694

6.46275021

13.73566257

./nitrogen/Ni/311/iter_7/struct. xyz

43

Lattice $=" 7.462281943147142 \quad 0.0 \quad 0.0 \quad 2.4874273143823786 \quad 8.24986309508776 \quad 0.0 \quad 0.0 \quad 0.021 .30321$

2853088358" Properties=species:S:1:pos:R:3 pbc="T T T"

$\begin{array}{rrrr}\mathrm{Ni} & 2.48742731 & 2.99995022 & 8.00000000 \\ \mathrm{Ni} & 2.48742731 & 0.74998755 & 9.06064257 \\ \mathrm{Ni} & 1.24371366 & 2.62495644 & 10.12128514\end{array}$

$\mathrm{Ni}$

1.24371366

2.62495644

10.12128514 
$\mathrm{Ni}$

$\mathrm{Ni}$

$\mathrm{Ni}$

$\mathrm{Ni}$

$\mathrm{Ni}$

$\mathrm{Ni}$

$\mathrm{Ni}$

$\mathrm{Ni}$

$\mathrm{Ni}$

$\mathrm{Ni}$

$\mathrm{Ni}$

$\mathrm{Ni}$

$\mathrm{Ni}$

$\mathrm{Ni}$

$\mathrm{Ni}$

$\mathrm{Ni}$

$\mathrm{Ni}$

$\mathrm{Ni}$

$\mathrm{Ni}$

$\mathrm{Ni}$

$\mathrm{Ni}$

$\mathrm{Ni}$

$\mathrm{Ni}$

$\mathrm{Ni}$

$\mathrm{Ni}$

$\mathrm{Ni}$

$\mathrm{Ni}$

$\mathrm{Ni}$

$\mathrm{Ni}$

$\mathrm{Ni}$

$\mathrm{Ni}$

$\mathrm{Ni}$

$\mathrm{Ni}$

$\mathrm{N}$

$\mathrm{N}$

$\mathrm{N}$

$\mathrm{N}$

$\mathrm{N}$

$\mathrm{N}$

$\mathrm{N}$

. / oxygen/Cu/100/2r2xr2R45/struct. xyz

17

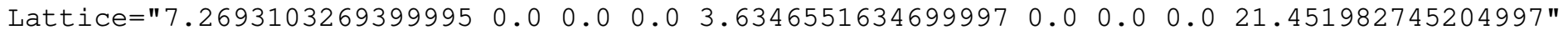
Properties=species:S:1:pos:R: 3 pbc="T T T"

$\mathrm{Cu}$

$\mathrm{Cu}$

$\mathrm{Cu}$

$\mathrm{Cu}$

$\mathrm{Cu}$

$\mathrm{Cu}$

$\mathrm{Cu}$

$\mathrm{Cu}$

$\mathrm{Cu}$

$\mathrm{Cu}$

$\mathrm{Cu}$

$\mathrm{Cu}$

$\mathrm{Cu}$

$\mathrm{Cu}$

0

0

1.81732758

0.00000000

0.00000000

0.00000000

0.00000000

0.00000000

1.81732758

1.81732758

1.81732758

0.00000000

0.00000000

0.00000000

0.00000000

1.81732758

1.81732758

1.81732758

1.81732758

1.81732758

1.81732758
11.20734980

12.55704276

13.47601790

8.00000000

9.06064257

10.12128514

11.13397924

12.50418549

13.74535678

8.00000000

9.06064257

10.12128514

11.22280098

12.13313092

13.42925759

8.00000000

9.06064257

10.12128514

11.30185795

13.17885718

14.01343489

8.00000000

9.06064257

10.12128514

11.13442456

12.17729964

13.69345644

8.00000000

9.06064257

10.12128514

11.24100997

12.31066538

13.60718594

12.88270959

12.99450460

13.90299678

14.26445380

13.77830029

14.34516555

13.07749877
8.00000000

9.81732758

11.54336030

13.53735607

8.00000000

9.81732758

11.63300738

8.00000000

9.81732758

11.64814958

13.53735607

8.00000000

9.81732758

11.63300738

13.65884309

13.87373054

13.87373054

. /oxygen/Cu/100/c2x2/struct. $x y z$

18

Lattice $=" 5.1401786267286720 .0 \quad 0.0 \quad 0.0 \quad 5.1401786267286720 .0 \quad 0.0 \quad 0.021 .451982745204997 "$ Pr operties $=$ species: $\mathrm{S}: 1:$ pos $: \mathrm{R}: 3 \mathrm{pbc}=" \mathrm{~T} \mathrm{~T} \mathrm{~T}$ "

$\begin{array}{lllr}\mathrm{Cu} & 1.28504466 & 1.28504466 & 8.00000000 \\ \mathrm{Cu} & 0.00000000 & 0.00000000 & 9.81732758 \\ \mathrm{Cu} & 1.28504466 & 1.28504466 & 11.59845140\end{array}$

$\mathrm{Cu}$

1.28504466

1.28504466 


\section{$\mathrm{Cu}$}

$\mathrm{Cu}$

$\mathrm{Cu}$

$\mathrm{Cu}$

$\mathrm{Cu}$

$\mathrm{Cu}$

$\mathrm{Cu}$

$\mathrm{Cu}$

$\mathrm{Cu}$

$\mathrm{Cu}$

$\mathrm{Cu}$

$\mathrm{Cu}$

$\mathrm{Cu}$

o

0

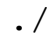

49

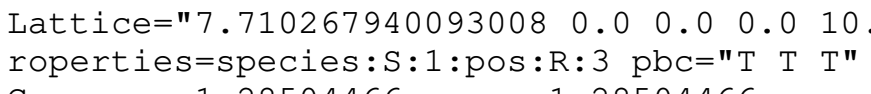

$\mathrm{Cu}$

$\mathrm{Cu}$

$\mathrm{Cu}$

$\mathrm{Cu}$

$\mathrm{Cu}$

$\mathrm{Cu}$

$\mathrm{Cu}$

$\mathrm{Cu}$

$\mathrm{Cu}$

$\mathrm{Cu}$

$\mathrm{Cu}$

$\mathrm{Cu}$

$\mathrm{Cu}$

$\mathrm{Cu}$

$\mathrm{Cu}$

$\mathrm{Cu}$

$\mathrm{Cu}$

$\mathrm{Cu}$

$\mathrm{Cu}$

$\mathrm{Cu}$

$\mathrm{Cu}$

$\mathrm{Cu}$

$\mathrm{Cu}$

$\mathrm{Cu}$

$\mathrm{Cu}$

$\mathrm{Cu}$

$\mathrm{Cu}$

$\mathrm{Cu}$

$\mathrm{Cu}$

$\mathrm{Cu}$

$\mathrm{Cu}$

$\mathrm{Cu}$

$\mathrm{Cu}$

$\mathrm{Cu}$

$\mathrm{Cu}$

$\mathrm{Cu}$

$\mathrm{Cu}$

$\mathrm{Cu}$

$\mathrm{Cu}$

$\mathrm{Cu}$

$\mathrm{Cu}$

$\mathrm{Cu}$

$\mathrm{Cu}$

$\mathrm{Cu}$

$\mathrm{Cu}$

$\mathrm{Cu}$

$\mathrm{Cu}$

$\mathrm{Cu}$

o
0.00000000

1.28504466

0.00000000

1.28504466

0.00000000

3.85513397

2. 57008931

3. 85513397

2.57008931

3. 85513397

2.57008931

3. 85513397

2. 57008931

1.28504466

3.85513397
1.28504466

0.00000000

1.28504466

1.28504466

0.00000000

1.28504466

$-0.02280669$

1.28504466

0.00000000

1.28504466

$-0.00762925$

1.28504466

0.00000000

1.28504466

$-0.00762925$

3. 85513397

2. 57008931

3. 84018714

2. 59289601

3.85513397

2. 57008931

3. 83750048

2. 59289601

3. 85513397

2. 57008931

3. 86062646

2. 57771856

3.85513397

2. 57008931

3.83750048

2. 57771856

6.42522328

5.14017863

6.44017011

5.14017863

6.42522328

5.14017863

6.44285678

5.14017863

6.42522328

5.14017863

6.41973079

5.14017863

6.42522328

5.14017863

6.44285678

5.14017863

1.28504466
$-0.02280669$
1.28504466

0.00000000

1.28504466

$-0.03015739$

3.85513397

2.57008931

3.84352120

2.60024671

6.42522328

5.14017863

6.42522328

5.15450127

8.99531260

7.71026794

9.00692537

7.69594529

1.28504466

0.00000000

1.28504466

$-0.03015739$

3.85513397

2.57008931

3. 84123984

2.60024671

6.42522328

5.14017863

6. 42522328

5.15450127

8.99531260

7.71026794

9.00920673

7.69594529

1.28504466

0.00000000

1.28504466

$-0.00332518$

3.85513397

2.57008931

3. 84123984

2.57341449

6.42522328

5.14017863

6.42522328

5.14159903

8.99531260

7.71026794

9.00920673

7.70884754

1.28504466
Fri Apr 16 14:51:55 2021

0.00000000

3.85513397

2.57008931

3.85513397

2.57008931

1.28504466

0.00000000

1.28504466

0.00000000

3.85513397

2.57008931

3.85513397

2.57008931

1.28504466

3.85513397
13.54793534

8.00000000

9.81732758

11.65570317

13.54793534

8.00000000

9.81732758

11.65570317

13.54793534

8.00000000

9.81732758

11.59845140

13.54793534

14.22269073

14.22269073
8.00000000

9.81732758

11.57440670

13.47547038

8.00000000

9.81732758

11.64164395

13.47547038

8.00000000

9.81732758

11.62315246

13.38291892

8.00000000

9.81732758

11.64164395

13.38291892

8.00000000

9.81732758

11.63845698

13.47547038

8.00000000

9.81732758

11.64847417

13.47547038

8.00000000

9.81732758

11.62125078

13.38291892

8.00000000

9.81732758

11.64847417

13.38291892

8.00000000

9.81732758

11.63845698

13.37733524

8.00000000

9.81732758

11.64847417

13.37733524

8.00000000

9.81732758

11.62125078

13.39108621

8.00000000

9.81732758

11.64847417

13.39108621

14.23628391

$44 \quad 0.0 \quad 0.0 \quad 0.021 .451982745204997 " \mathrm{P}$ ( 
. /oxygen/Cu/100/iter_10/struct.xyz

58

Lattice $=" 7.710267940093008 \quad 0.0 \quad 0.0 \quad 0.0 \quad 10.280357253457344 \quad 0.0 \quad 0.0 \quad 0.021 .451982745204997 " \mathrm{P}$ roperties=species:S:1:pos:R:3 pbc="T T T" 1.28504466

1. 28504466

0.00000000

0.00000000

8.00000000

$\mathrm{Cu}$

1.31889498

1.31303239

0.00000000

0.00000000

1.28504466

3.85513397

0.00000000

2.57008931

1.28473077

3.90095169

0.15501624

2. 63024745

1. 28504466

0.00000000

6.42522328

1.24716283

5.14017863

0.00000000

6.46037394

1. 28504466

5.14017863

0.00000000

8.99531260

7.71026794

1. 22280848

9.02435586

7. 55525170

7.65010980

3.85513397

1.28504466

2. 57008931

0.00000000

3. 91192029

1.27551044

2. 56587923

0.06832180

3. 85513397

3.85513397

2. 57008931

2. 57008931

3.87523765

3.81573320

2. 64653683

2.47285357

3. 85513397

6.42522328

2. 57008931

5.14017863

3. 83503029

6.46462405

5.28169297

2. 47470040

8.99531260

7.71026794

2. 57008931

9.00484681

7.91050985

3.79834765

1.28504466

0.00000000

1.25600139

. 42522328

5.14017863

6.48745946

5. 14438871

10.21203546

3.85513397

2. 57008931

5.14017863

3.81998331

6.46310511

2. 36984741

6.42522328

5.14017863

6.42522328

5.14017863

6.37940556

4. 99866428

5.23556754

6.42522328

8.99531260

7.71026794

8.96732486

6.39137296

7.80750369

1.16092102

6.56685250

9.11943623

3.71350476

3. 87772930

9.81732758

11.60829616

13.60579647

8.00000000

9.81732758

11.57900721

13.42250588

8.00000000

9.81732758

11.63562631

14.87925786

8.00000000

9.81732758

11.65885745

13.42250588

8.00000000

9.81732758

11.61106817

13.43496197

8.00000000

9.81732758

11.60548648

14.43649852

8.00000000

9.81732758

11.60548648

13.95967407

8.00000000

9.81732758

11.61106817

13.65907384

8.00000000

9.81732758

11.65885745

13.43496197

8.00000000

9.81732758

11.63562631

13.65907384

8.00000000

9.81732758

11.57900721

13.95967407

8.00000000

9.81732758

11.60829616

14.43649852

14.44560197

14.65408895

14.44560197

14.65408895

13.57661544

14.40364633

14.40364633

13.57661544

14.56857824

1.30460754

6.40262795

6.41405689

14.56857824

. /oxygen/Cu/100/iter_11/struct.xyz

59

Lattice $=" 7.710267940093008 \quad 0.0 \quad 0.0 \quad 0.0 \quad 10.280357253457344 \quad 0.0 \quad 0.0 \quad 0.021 .451982745204997 " \quad \mathrm{P}$ roperties=species:S:1:pos:R:3 pbc="T T T"
$\mathrm{Cu}$
1.28504466
1.28504466
8.00000000
$\mathrm{Cu}$
0.00000000
0.00000000
9.81732758 
$\mathrm{Cu}$

$\mathrm{Cu}$

$\mathrm{Cu}$

$\mathrm{Cu}$

$\mathrm{Cu}$

$\mathrm{Cu}$

$\mathrm{Cu}$

$\mathrm{Cu}$

$\mathrm{Cu}$

$\mathrm{Cu}$

$\mathrm{Cu}$

$\mathrm{Cu}$

$\mathrm{Cu}$

$\mathrm{Cu}$

$\mathrm{Cu}$

$\mathrm{Cu}$

$\mathrm{Cu}$

$\mathrm{Cu}$

$\mathrm{Cu}$

$\mathrm{Cu}$

$\mathrm{Cu}$

$\mathrm{Cu}$

$\mathrm{Cu}$

$\mathrm{Cu}$

$\mathrm{Cu}$

$\mathrm{Cu}$

$\mathrm{Cu}$

$\mathrm{Cu}$

$\mathrm{Cu}$

$\mathrm{Cu}$

$\mathrm{Cu}$

$\mathrm{Cu}$

$\mathrm{Cu}$

$\mathrm{Cu}$

$\mathrm{Cu}$

$\mathrm{Cu}$

$\mathrm{Cu}$

$\mathrm{Cu}$

$\mathrm{Cu}$

$\mathrm{Cu}$

$\mathrm{Cu}$

$\mathrm{Cu}$

$\mathrm{Cu}$

$\mathrm{Cu}$

$\mathrm{Cu}$

$\mathrm{Cu}$

0

0

0

o

0

0

0

o

0

o

0
1.28504466

0.11589265

1.28504466

0.00000000

1.28504466

0.05944302

1.28504466

0.00000000

1.28504466

0.05944302

1.28504466

0.00000000

1.28504466

0.11589265

3.85513397

2. 57008931

3. 82236820

2. 45419666

3.85513397

2.57008931

3. 76943992

2. 51064629

3. 85513397

2. 57008931

3. 82236820

2. 51064629

3.85513397

2. 57008931

3. 84661715

2. 45419666

6.42522328

5.14017863

6.45798905

5.14017863

6.42522328

5.14017863

6.51091733

5.14017863

6.42522328

5.14017863

6.45798905

5.14017863

6.42522328

5.14017863

6.43374011

5.14017863

1.28504466

1.28504466

6.52113797

6.69378934

3.58656791

3.75921929

3.85655577

3.85655577

6.42380149

1.28504466

6.42380149

Fri Apr 16 14:51:55 2021

97

1.28398669

$-0.10989420$

3.85513397

2.57008931

3.85513397

2.43831057

6.42522328

5.14017863

6.42628125

5.27195737

8.99531260

7.71026794

8.99531260

7.82016214

1.28504466

0.00000000

1.24243215

$-0.10989420$

3.85513397

2.57008931

3.85513397

2.43831057

6.42522328

5.14017863

6.46783579

5.27195737

8.99531260

7.71026794

8.99531260

7.82016214

1.28504466

0.00000000

1.24243215

$-0.02357404$

3.85513397

2. 57008931

3. 85513397

2.64061653

6.42522328

5.14017863

6.46783579

5.06965141

8.99531260

7.71026794

8.99531260

7.73384198

1.00705635

6.70321159

8.99531260

3.85513397

3. 85513397

8.99531260

1.24744695

6.46282099

6.46282099

3.85513397

1.24744695
11.62102465

13.50240549

8.00000000

9.81732758

11.58054008

14.49446831

8.00000000

9.81732758

11.62102465

14.49446831

8.00000000

9.81732758

11.59912560

13.50240549

8.00000000

9.81732758

11.63350418

13.50240549

8.00000000

9.81732758

11.60129746

14.49446831

8.00000000

9.81732758

11.63350418

14.49446831

8.00000000

9.81732758

11.61755712

13.50240549

8.00000000

9.81732758

11.63350418

14.38439084

8.00000000

9.81732758

11.60129746

13.36358723

8.00000000

9.81732758

11.63350418

13.36358723

8.00000000

9.81732758

11.61755712

14.38439084

14.85312765

14.85312765

14.71354862

13.73829223

13.73829223

14.71354862

13.78822017

13.78822017

13.78822017

15.08850972

13.78822017

. /oxygen/Cu/100/iter_12/struct.xyz 60

Lattice $=" 7.710267940093008 \quad 0.0 \quad 0.0 \quad 0.0 \quad 10.280357253457344 \quad 0.0 \quad 0.0 \quad 0.0 \quad 21.451982745204997 " \quad P$ roperties=species:S:1:pos:R:3 pbc="T T T" $\mathrm{Cu} \quad 1.28504466 \quad 1.28504466$

$\mathrm{Cu} \quad 0.00000000 \quad 0.00000000$

$\mathrm{Cu} \quad 1.28504466 \quad 1.28504466$

$\mathrm{Cu} \quad 0.00000000 \quad 0.00000000$

$\mathrm{Cu} \quad 1.28504466 \quad 3.85513397$

$\mathrm{Cu} \quad 0.00000000 \quad 2.57008931$

$\mathrm{Cu} \quad 1.28504466$

3.85513397

8.00000000

9.81732758

11.59266803

13.69785888

8.00000000

9.81732758

11.59266803 


\section{$\mathrm{Cu}$}

$\mathrm{Cu}$

$\mathrm{Cu}$

$\mathrm{Cu}$

$\mathrm{Cu}$

$\mathrm{Cu}$

$\mathrm{Cu}$

$\mathrm{Cu}$

$\mathrm{Cu}$

$\mathrm{Cu}$

$\mathrm{Cu}$

$\mathrm{Cu}$

$\mathrm{Cu}$

$\mathrm{Cu}$

$\mathrm{Cu}$

$\mathrm{Cu}$

$\mathrm{Cu}$

$\mathrm{Cu}$

$\mathrm{Cu}$

$\mathrm{Cu}$

$\mathrm{Cu}$

$\mathrm{Cu}$

$\mathrm{Cu}$

$\mathrm{Cu}$

$\mathrm{Cu}$

$\mathrm{Cu}$

$\mathrm{Cu}$

$\mathrm{Cu}$

$\mathrm{Cu}$

$\mathrm{Cu}$

$\mathrm{Cu}$

$\mathrm{Cu}$

$\mathrm{Cu}$

$\mathrm{Cu}$

$\mathrm{Cu}$

$\mathrm{Cu}$

$\mathrm{Cu}$

$\mathrm{Cu}$

$\mathrm{Cu}$

$\mathrm{Cu}$

$\mathrm{Cu}$

0

O

0

0

0

o

0

0

o

o

0

0
0.00000000

1.28504466

0.00000000

1.28504466

0.00000000

1.28504466

0.00000000

1.28504466

0.00000000

3. 85513397

2. 57008931

3. 85513397

2. 57008931

3. 85513397

2. 57008931

3. 85513397

2. 57008931

3. 85513397

2. 57008931

3. 85513397

2. 57008931

3. 85513397

2. 57008931

3. 85513397

2.57008931

6.42522328

5.14017863

6.42522328

5.14017863

6.42522328

5.14017863

6.42522328

5.14017863

6.42522328

5.14017863

6.42522328

5.14017863

6.42522328

5.14017863

6.42522328

5.14017863

1.28504466

1.28504466

6.42522328

6.42522328

3.85513397

3. 85513397

3.85513397

3. 85513397

6.42522328

1.28504466

6.42522328

1.28504466
Fri Apr 16 14:51:55 2021

2.57008931

6.42522328

5.14017863

6.42522328

5.14017863

8.99531260

7.71026794

8.99531260

7.71026794

1.28504466

0.00000000

1.28504466

0.00000000

3.85513397

2.57008931

3.85513397

2.57008931

6.42522328

5.14017863

6.42522328

5.14017863

8.99531260

7.71026794

8.99531260

7.71026794

1.28504466

0.00000000

1.28504466

0.00000000

3.85513397

2.57008931

3. 85513397

2. 57008931

6.42522328

5.14017863

6.42522328

5.14017863

8.99531260

7.71026794

8.99531260

7.71026794

1.28504466

6.42522328

8.99531260

3.85513397

3. 85513397

8.99531260

1.28504466

6.42522328

6.42522328

3.85513397

1.28504466

8.99531260
13.69785888

8.00000000

9.81732758

11.59266803

13.69785888

8.00000000

9.81732758

11.59266803

13.69785888

8.00000000

9.81732758

11.59266803

13.69785888

8.00000000

9.81732758

11.59266803

13.69785888

8.00000000

9.81732758

11.59266803

13.69785888

8.00000000

9.81732758

11.59266803

13.69785888

8.00000000

9.81732758

11.59266803

13.69785888

8.00000000

9.81732758

11.59266803

13.69785888

8.00000000

9.81732758

11.59266803

13.69785888

8.00000000

9.81732758

11.59266803

13.69785888

14.18402533

14.18402533

14.18402533

14.18402533

14.18402533

14.18402533

14.18402533

14.18402533

14.18402533

14.18402533

14.18402533

14.18402533

. /oxygen/Cu/100/iter_2/struct.xyz

50

Lattice $=" 7.710267940093008 \quad 0.0 \quad 0.0 \quad 0.0 \quad 10.2803572534573440 .0 \quad 0.0 \quad 0.021 .451982745204997 " \mathrm{P}$ roperties $=$ species: $\mathrm{S}: 1: \mathrm{pos}: \mathrm{R}: 3 \mathrm{pbc}=" \mathrm{~T} \mathrm{~T} \mathrm{~T} "$

$\begin{array}{lrrr}\mathrm{Cu} & 1.28504466 & 1.28504466 & 8.00000000 \\ \mathrm{Cu} & 0.00000000 & 0.00000000 & 9.81732758 \\ \mathrm{Cu} & 1.28504466 & 1.28504466 & 11.56339925 \\ \mathrm{Cu} & 7.68389678 & 10.26222879 & 13.46445722 \\ \mathrm{Cu} & 1.28504466 & 3.85513397 & 8.00000000 \\ \mathrm{Cu} & 0.00000000 & 2.57008931 & 9.81732758 \\ \mathrm{Cu} & 1.28504466 & 3.85513397 & 11.64409294 \\ \mathrm{Cu} & 7.68389678 & 2.58821778 & 13.46445722 \\ \mathrm{Cu} & 1.28504466 & 6.42522328 & 8.00000000 \\ \mathrm{Cu} & 0.00000000 & 5.14017863 & 9.81732758 \\ \mathrm{Cu} & 1.28504466 & 6.42522328 & 11.56339925\end{array}$


$\mathrm{Cu}$

$\mathrm{Cu}$

$\mathrm{Cu}$

$\mathrm{Cu}$

$\mathrm{Cu}$

$\mathrm{Cu}$

$\mathrm{Cu}$

$\mathrm{Cu}$

$\mathrm{Cu}$

$\mathrm{Cu}$

$\mathrm{Cu}$

$\mathrm{Cu}$

$\mathrm{Cu}$

$\mathrm{Cu}$

$\mathrm{Cu}$

$\mathrm{Cu}$

$\mathrm{Cu}$

$\mathrm{Cu}$

$\mathrm{Cu}$

$\mathrm{Cu}$

$\mathrm{Cu}$

$\mathrm{Cu}$

$\mathrm{Cu}$

$\mathrm{Cu}$

$\mathrm{Cu}$

$\mathrm{Cu}$

$\mathrm{Cu}$

$\mathrm{Cu}$

$\mathrm{Cu}$

$\mathrm{Cu}$

$\mathrm{Cu}$

$\mathrm{Cu}$

$\mathrm{Cu}$

$\mathrm{Cu}$

$\mathrm{Cu}$

$\mathrm{Cu}$

$\mathrm{Cu}$

0

0
7.68389678

1.28504466

0.00000000

1.28504466

7.68389678

3.85513397

2. 57008931

3. 84945364

2.59646047

3. 85513397

2.57008931

3. 82666861

2. 59646047

3. 85513397

2. 57008931

3. 84945364

2. 59646047

3. 85513397

2. 57008931

3. 82666861

2. 59646047

6.42522328

5.14017863

6.43090361

5.14017863

6.42522328

5.14017863

6.45368865

5.14017863

6.42522328

5.14017863

6.43090361

5.14017863

6.42522328

5.14017863

6.45368865

5.14017863

1.28504466

1.28504466
Fri Apr 16 14:51:55 2021

5.12205016

8.99531260

7.71026794

8.99531260

7.72839640

1.28504466

0.00000000

1.28504466

10.26222879

3.85513397

2.57008931

3.85513397

2.58821778

6.42522328

5.14017863

6.42522328

5.12205016

8.99531260

7.71026794

8.99531260

7.72839640

1.28504466

0.00000000

1.28504466

10.27790534

3.85513397

2.57008931

3.85513397

2.57254122

6.42522328

5.14017863

6.42522328

5.13772672

8.99531260

7.71026794

8.99531260

7.71271985

1.28504466

6.42522328
13.46445722

8.00000000

9.81732758

11.64409294

13.46445722

8.00000000

9.81732758

11.63321729

13.46445722

8.00000000

9.81732758

11.67233443

13.46445722

8.00000000

9.81732758

11.63321729

13.46445722

8.00000000

9.81732758

11.67233443

13.46445722

8.00000000

9.81732758

11.63321729

13.38573654

8.00000000

9.81732758

11.67233443

13.38573654

8.00000000

9.81732758

11.63321729

13.38573654

8.00000000

9.81732758

11.67233443

13.38573654

14.22427808

14.22427808

51

Lattice $=" 7.710267940093008 \quad 0.0 \quad 0.0 \quad 0.0 \quad 10.280357253457344 \quad 0.0 \quad 0.0 \quad 0.021 .451982745204997 " \mathrm{P}$ roperties $=$ species: $\mathrm{S}: 1:$ pos $: \mathrm{R}: 3$ pbc $=" \mathrm{~T} T \mathrm{~T}$ "

$\begin{array}{lll}\mathrm{Cu} & 0.00000000 & 0.00000000\end{array}$

$\mathrm{Cu} \quad 1.27216358 \quad 1.26915930$

$\begin{array}{lll}\mathrm{Cu} & -0.02301791 & 0.02623007\end{array}$

$\begin{array}{lll}\mathrm{Cu} & 1.28504466 & 3.85513397\end{array}$

$\mathrm{Cu} \quad 0.00000000 \quad 2.57008931$

$\begin{array}{lll}\mathrm{Cu} & 1.30067853 & 3.85513397\end{array}$

$\begin{array}{lll}\mathrm{Cu} & 0.00278380 & 2.60156546\end{array}$

$\mathrm{Cu} \quad 1.28504466$

6.42522328

$0.00000000 \quad 5.14017863$

$1.27216358 \quad 6.44110864$

$0.00278380 \quad 5.10870248$

$1.28504466 \quad 8.99531260$

$0.00000000 \quad 7.71026794$

$1.22818601 \quad 8.99531260$

$\begin{array}{rl}-0.02301791 & 7.68403787\end{array}$

$3.85513397 \quad 1.28504466$

$2.57008931 \quad 0.00000000$

$\begin{array}{ll}3.86573717 & 1.27250125\end{array}$

$2.56941550 \quad-0.00803234$

$\begin{array}{ll}3.85513397 & 3.85513397\end{array}$

$\begin{array}{ll}2.57008931 & 2.57008931\end{array}$

$\begin{array}{ll}3.84129440 & 3.85513397\end{array}$

$2.62368902 \quad 2.59931276$

$\begin{array}{ll}3.85513397 & 6.42522328\end{array}$

8.00000000

9.81732758

11.56224205

13.73587227

8.00000000

9.81732758

11.62426075

13.43085862

8.00000000

9.81732758

11.56224205

13.43085862

8.00000000

9.81732758

11.67374790

13.73587227

8.00000000

9.81732758

11.63756907

13.41931468

8.00000000

9.81732758

11.64028440

13.43271060

8.00000000 


\section{$\mathrm{Cu}$}

$\mathrm{Cu}$

$\mathrm{Cu}$

$\mathrm{Cu}$

$\mathrm{Cu}$

$\mathrm{Cu}$

$\mathrm{Cu}$

$\mathrm{Cu}$

$\mathrm{Cu}$

$\mathrm{Cu}$

$\mathrm{Cu}$

$\mathrm{Cu}$

$\mathrm{Cu}$

$\mathrm{Cu}$

$\mathrm{Cu}$

$\mathrm{Cu}$

$\mathrm{Cu}$

$\mathrm{Cu}$

$\mathrm{Cu}$

$\mathrm{Cu}$

$\mathrm{Cu}$

$\mathrm{Cu}$

$\mathrm{Cu}$

O

O

0
2.57008931

3. 86573717

2. 62368902

3. 85513397

2. 57008931

3.81800884

2. 56941550

6.42522328

5.14017863

6.44967463

5.09434408

6.42522328

5.14017863

6.46988611

5.15617755

6.42522328

5.14017863

6.44967463

5.15617755

6.42522328

5.14017863

6.43551619

5.09434408

1. 32028622

1. 32028622

6.35617986
Fri Apr 16 14:51:55 2021

100

5.14017863

6.43776669

5.11095518

8.99531260

7.71026794

8.99531260

7.71830028

1.28504466

0.00000000

1.26289051

0.03408604

3. 85513397

2.57008931

3.85513397

2. 58919282

6.42522328

5.14017863

6.44737743

5.12107512

8.99531260

7.71026794

8.99531260

7.67618190

1.32360547

6.38666247

8.99531260
9.81732758

11.63756907

13.43271060

8.00000000

9.81732758

11.66469191

13.41931468

8.00000000

9.81732758

11.64778803

13.41481396

8.00000000

9.81732758

11.65253295

13.36292609

8.00000000

9.81732758

11.64778803

13.36292609

8.00000000

9.81732758

11.58914258

13.41481396

14.23485142

14.23485142

14.24414510

. /oxygen/Cu/100/iter_4/struct.xyz

52

Lattice $=" 7.710267940093008 \quad 0.0 \quad 0.0 \quad 0.0 \quad 10.280357253457344 \quad 0.0 \quad 0.0 \quad 0.021 .451982745204997 " \mathrm{P}$ roperties $=$ species: $\mathrm{S}: 1: \mathrm{pos}: \mathrm{R}: 3 \mathrm{pbc}=" \mathrm{~T} \mathrm{~T} \mathrm{~T} "$

$\begin{array}{llrr}\mathrm{Cu} & 1.28504466 & 1.28504466 & 8.00000000 \\ \mathrm{Cu} & 0.00000000 & 0.00000000 & 9.81732758 \\ \mathrm{Cu} & 1.25470217 & 1.28504466 & 11.57236781 \\ \mathrm{Cu} & 0.00000000 & 0.00000000 & 13.64850694 \\ \mathrm{Cu} & 1.28504466 & 3.85513397 & 8.00000000 \\ \mathrm{Cu} & 0.00000000 & 2.57008931 & 9.81732758 \\ \mathrm{Cu} & 1.25175909 & 3.85513397 & 11.65053172 \\ \mathrm{Cu} & 0.00000000 & 2.57008931 & 13.64850694 \\ \mathrm{Cu} & 1.28504466 & 6.42522328 & 8.00000000 \\ \mathrm{Cu} & 0.00000000 & 5.14017863 & 9.81732758 \\ \mathrm{Cu} & 1.25470217 & 6.42522328 & 11.57236781 \\ \mathrm{Cu} & 0.00000000 & 5.14017863 & 13.64850694 \\ \mathrm{Cu} & 1.28504466 & 8.99531260 & 8.00000000 \\ \mathrm{Cu} & 0.00000000 & 7.71026794 & 9.81732758 \\ \mathrm{Cu} & 1.25175909 & 8.99531260 & 11.65053172 \\ \mathrm{Cu} & 0.00000000 & 7.71026794 & 13.64850694 \\ \mathrm{Cu} & 3.85513397 & 1.28504466 & 8.00000000 \\ \mathrm{Cu} & 2.57008931 & 0.00000000 & 9.81732758 \\ \mathrm{Cu} & 3.87473396 & 1.28504466 & 11.63947870 \\ \mathrm{Cu} & 2.59904725 & -0.02333566 & 13.39600795 \\ \mathrm{Cu} & 3.85513397 & 3.85513397 & 8.00000000 \\ \mathrm{Cu} & 2.57008931 & 2.57008931 & 9.81732758 \\ \mathrm{Cu} & 3.83553398 & 3.85513397 & 11.63947870 \\ \mathrm{Cu} & 2.59904725 & 2.59342498 & 13.39600795 \\ \mathrm{Cu} & 3.85513397 & 6.42522328 & 8.00000000 \\ \mathrm{Cu} & 2.57008931 & 5.14017863 & 9.81732758 \\ \mathrm{Cu} & 3.87473396 & 6.42522328 & 11.63947870 \\ \mathrm{Cu} & 2.59904725 & 5.11684296 & 13.39600795 \\ \mathrm{Cu} & 3.85513397 & 8.99531260 & 8.00000000 \\ \mathrm{Cu} & 2.57008931 & 7.71026794 & 9.81732758 \\ \mathrm{Cu} & 3.83553398 & 8.99531260 & 11.63947870 \\ \mathrm{Cu} & 2.59904725 & 7.73360360 & 13.39600795 \\ \mathrm{Cu} & 6.42522328 & 1.28504466 & 8.00000000 \\ \mathrm{Cu} & 5.14017863 & 0.00000000 & 9.81732758 \\ \mathrm{Cu} & 6.45850885 & 1.28504466 & 11.65053172 \\ \mathrm{Cu} & 5.11122069 & 0.02333566 & 13.39600795 \\ \mathrm{Cu} & 6.42522328 & 3.85513397 & 8.00000000 \\ \mathrm{Cu} & 5.14017863 & 2.57008931 & 9.81732758\end{array}$




\section{$\mathrm{Cu}$}

$\mathrm{Cu}$

$\mathrm{Cu}$

$\mathrm{Cu}$

$\mathrm{Cu}$

$\mathrm{Cu}$

$\mathrm{Cu}$

$\mathrm{Cu}$

$\mathrm{Cu}$

$\mathrm{Cu}$

O

0

○

0
6.45556577

5.11122069

6.42522328

5.14017863

6.45850885

5.11122069

6.42522328

5.14017863

6.45556577

5.11122069

1.35118056

1.35118056

6.35908738

6.35908738
Fri Apr 16 14:51:55 2021

3.85513397

2.54675365

6.42522328

5.14017863

6.42522328

5.16351429

8.99531260

7.71026794

8.99531260

7.68693228

1.28504466

6.42522328

8.99531260

3.85513397
11.57236781

13.39600795

8.00000000

9.81732758

11.65053172

13.39600795

8.00000000

9.81732758

11.57236781

13.39600795

14.22701104

14.22701104

14.22701104

14.22701104

53

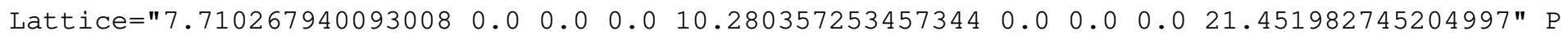
roperties=species:S:1:pos:R:3 pbc="T T T"

$\mathrm{Cu} \quad 1.28504466 \quad 1.28504466$

$\mathrm{Cu} \quad 0.00000000 \quad 0.00000000$

$\begin{array}{lll}\mathrm{Cu} & 1.26939007 & 1.30781935\end{array}$

$\begin{array}{lll}\mathrm{Cu} & -0.04439746 & -0.02565918\end{array}$

$\begin{array}{lll}\mathrm{Cu} & 1.28504466 & 3.85513397\end{array}$

$\mathrm{Cu} \quad 0.00000000 \quad 2.57008931$

$\begin{array}{lll}\mathrm{Cu} & 1.29620802 & 3.85513397\end{array}$

$\begin{array}{lll}\mathrm{Cu} & 0.03824998 & 2.52968147\end{array}$

$\begin{array}{lll}\mathrm{Cu} & 1.28504466 & 6.42522328\end{array}$

$\begin{array}{lll}\mathrm{Cu} & 0.00000000 & 5.14017863\end{array}$

$\begin{array}{lll}\mathrm{Cu} & 1.26939007 & 6.40244859\end{array}$

$\begin{array}{lll}\mathrm{Cu} & 0.03824998 & 5.18058647\end{array}$

$\begin{array}{lll}\mathrm{Cu} & 1.28504466 & 8.99531260\end{array}$

$\begin{array}{lll}\mathrm{Cu} & 0.00000000 & 7.71026794\end{array}$

$\begin{array}{lll}\mathrm{Cu} & 1.23580288 & 8.99531260\end{array}$

$\begin{array}{lll}\mathrm{Cu} & -0.04439746 & 7.73592712\end{array}$

$\begin{array}{lll}\mathrm{Cu} & 3.85513397 & 1.28504466\end{array}$

$\begin{array}{lll}\mathrm{Cu} & 2.57008931 & 0.00000000\end{array}$

$\mathrm{Cu} \quad 3.85944323 \quad 1.29495088$

$\mathrm{Cu} \quad 2.58078954$

$\mathrm{Cu} \quad 3.85513397$

$-0.04631658$

3.85513397

$\begin{array}{ll}2.57008931 & 2.57008931\end{array}$

$\begin{array}{ll}3.83637101 & 3.85513397\end{array}$

$2.60096180 \quad 2.51448063$

$\begin{array}{ll}3.85513397 & 6.42522328\end{array}$

$\begin{array}{ll}2.57008931 & 5.14017863\end{array}$

$3.85944323 \quad 6.41531706$

2.60096180 $\quad 5.19578731$

$3.85513397 \quad 8.99531260$

$\begin{array}{ll}2.57008931 & 7.71026794\end{array}$

$3.82697210 \quad 8.99531260$

$\begin{array}{ll}2.58078954 & 7.75658452\end{array}$

$6.42522328 \quad 1.28504466$

$\begin{array}{ll}5.14017863 & 0.00000000\end{array}$

$6.45322181 \quad 1.28732093$

$\begin{array}{ll}5.09089888 & 0.00900297\end{array}$

$\begin{array}{ll}6.42522328 & 3.85513397\end{array}$

$\begin{array}{ll}5.14017863 & 2.57008931\end{array}$

$\begin{array}{ll}6.46272636 & 3.85513397\end{array}$

$\begin{array}{ll}5.15739631 & 2.50876587\end{array}$

$6.42522328 \quad 6.42522328$

$5.14017863 \quad 5.14017863$

$\begin{array}{ll}6.45322181 & 6.42294701\end{array}$

$\begin{array}{ll}5.15739631 & 5.20150207\end{array}$

$6.42522328 \quad 8.99531260$

$\begin{array}{ll}5.14017863 & 7.71026794\end{array}$

$6.44330922 \quad 8.99531260$

$\begin{array}{ll}5.09089888 & 7.70126497\end{array}$

8.00000000

9.81732758

11.58285306

13.62790590

8.00000000

9.81732758

11.68176271

13.64144751

8.00000000

9.81732758

11.58285306

13.64144751

8.00000000

9.81732758

11.63479508

13.62790590

8.00000000

9.81732758

11.64746379

13.36990659

8.00000000

9.81732758

11.59973123

13.63825071

8.00000000

9.81732758

11.64746379

13.63825071

8.00000000

9.81732758

11.62640038

13.36990659

8.00000000

9.81732758

11.65664238

13.38541955

8.00000000

9.81732758

11.59552949

13.40723521

8.00000000

9.81732758

11.65664238

13.40723521

8.00000000

9.81732758

11.56543757

13.38541955

14.24559581

1.31835469

1.20233792

14.24559581 


\section{0}

O

0

\subsection{6 \\ 6.51111720 \\ 3.80435154}

Fri Apr 16 14:51:55 2021

102

oxygen/Cu/100/iter_6/struct.xyz

$\begin{array}{ll}8.99531260 & 14.25114737 \\ 3.85513397 & 14.16677414 \\ 3.85513397 & 14.19503223\end{array}$

54

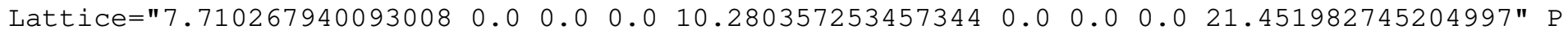
roperties=species:S:1:pos:R:3 pbc="T T T"

$\mathrm{Cu}$

1.28504466

1.28504466

0.00000000

8.00000000

0.00000000

1.28504466

1.28504466

0.00484147

1. 28504466

3.85513397

0.00000000

2.57008931

1.28504466

3.85513397

$-0.01789944$

2.56524785

1.28504466

6.42522328

0.00000000

5.14017863

1.28504466

6.42522328

$-0.01789944$

5.14502009

1.28504466

8.99531260

7.71026794

0.00000000

8.99531260

1.28504466

7.70542647

1.28504466

3.85513397

0.00000000

57008931

1.28504466

2. 58798876

0.00484147

3.85513397

3.85513397

2. 57008931

2.57008931

3. 83211395

3.85513397

2. 58798876

2.56524785

3.85513397

6.42522328

2. 57008931

5.14017863

3.84491995

6.42522328

2. 58798876

5.14502009

3. 85513397

8.99531260

7.71026794

57008931

8.99531260

7.70542647

2. 58798876

1. 28504466

6.42522328

0.00000000

1.28504466

0.04456484

3.85513397

2. 57008931

3. 85513397

2.52552447

6.42522328

5.14017863

6.42522328

5.18474347

8.99531260

7.71026794

8.99531260

7.66570310

1.28504466

6.42522328

8.99531260

3.85513397

3. 85513397

9.81732758

11.59163221

13.57400594

8.00000000

9.81732758

11.64894134

13.57400594

8.00000000

9.81732758

11.59163221

13.57400594

8.00000000

9.81732758

11.64894134

13.57400594

8.00000000

9.81732758

11.65109642

13.57400594

8.00000000

9.81732758

11.58120785

13.57400594

8.00000000

9.81732758

11.65109642

13.57400594

8.00000000

9.81732758

11.58120785

13.57400594

8.00000000

9.81732758

11.65109642

13.39649080

8.00000000

9.81732758

11.58120785

13.39649080

8.00000000

9.81732758

11.65109642

13.39649080

8.00000000

9.81732758

11.58120785

13.39649080

14.17161818

14.17161818

14.21470029

14.21470029

14.21470029

14.21470029

. /oxygen/Cu/100/iter_7/struct.xyz

55

Lattice $=" 7.710267940093008 \quad 0.0 \quad 0.0 \quad 0.0 \quad 10.280357253457344 \quad 0.0 \quad 0.0 \quad 0.021 .451982745204997 " \mathrm{P}$ roperties=species:S:1:pos:R:3 pbc="T T T"

$\mathrm{Cu}$

1.28504466

0.00000000

1. 28504466

8.00000000

$\mathrm{Cu}$

1.27129015

0.00000000

9.81732758

1.28504466

11.60269780 


\section{$\mathrm{Cu}$}

$\mathrm{Cu}$

$\mathrm{Cu}$

$\mathrm{Cu}$

$\mathrm{Cu}$

$\mathrm{Cu}$

$\mathrm{Cu}$

$\mathrm{Cu}$

$\mathrm{Cu}$

$\mathrm{Cu}$

$\mathrm{Cu}$

$\mathrm{Cu}$

$\mathrm{Cu}$

$\mathrm{Cu}$

$\mathrm{Cu}$

$\mathrm{Cu}$

$\mathrm{Cu}$

$\mathrm{Cu}$

$\mathrm{Cu}$

$\mathrm{Cu}$

$\mathrm{Cu}$

$\mathrm{Cu}$

$\mathrm{Cu}$

$\mathrm{Cu}$

$\mathrm{Cu}$

$\mathrm{Cu}$

$\mathrm{Cu}$

$\mathrm{Cu}$

$\mathrm{Cu}$

$\mathrm{Cu}$

$\mathrm{Cu}$

$\mathrm{Cu}$

$\mathrm{Cu}$

$\mathrm{Cu}$

$\mathrm{Cu}$

$\mathrm{Cu}$

$\mathrm{Cu}$

$\mathrm{Cu}$

$\mathrm{Cu}$

$\mathrm{Cu}$

$\mathrm{Cu}$

$\mathrm{Cu}$

$\mathrm{Cu}$

$\mathrm{Cu}$

$\mathrm{Cu}$

O

0

O

0

o

o
0.04020146

1.28504466

0.00000000

1.31739316

0.04020146

1.28504466

0.00000000

1.29106704

$-0.00152280$

1.28504466

0.00000000

1.31739316

$-0.00152280$

3.85513397

2. 57008931

3. 82214584

2. 41049326

3.85513397

2. 57008931

3. 86096520

2. 41049326

3.85513397

2. 57008931

3. 86314943

2. 59117762

3. 85513397

2.57008931

3.86096520

2. 59117762

6.42522328

5.14017863

6.40718257

5.21618107

6.42522328

5.14017863

6.44440215

5.21618107

6.42522328

5.14017863

6.41825489

5.12169282

6.42522328

5.14017863

6.44440215

5.12169282

1.17540763

1.27604784

6.53541689

6.53541689

3.74438665

3. 74438665

3.93732566
Fri Apr 16 14:51:55 2021

0.03225496

3.85513397

2.57008931

3.83604163

2.53783435

6.42522328

5.14017863

6.42522328

5.12974443

8.99531260

7.71026794

9.01440494

7.72070214

1.28504466

0.00000000

1.28504466

10.15645761

3.85513397

2. 57008931

3.86428164

2.69398895

6.42522328

5.14017863

6.42522328

5.12968915

8.99531260

7.71026794

8.98616492

7.72075742

1.28504466

0.00000000

1.28504466

10.21453593

3.85513397

2.57008931

3.84065190

2. 63591064

6.42522328

5.14017863

6.42522328

5.17615869

8.99531260

7.71026794

9.00979466

7.67428788

1. 28504466

6.42522328

8.99724915

3. 85319742

3.98754800

8.86289856

1.28504466
13.44353262

8.00000000

9.81732758

11.65365855

13.44353262

8.00000000

9.81732758

11.59281683

13.52708043

8.00000000

9.81732758

11.65365855

13.52708043

8.00000000

9.81732758

11.57240297

14.28771967

8.00000000

9.81732758

11.60164188

14.28771967

8.00000000

9.81732758

11.65366821

13.48972684

8.00000000

9.81732758

11.60164188

13.48972684

8.00000000

9.81732758

11.66945374

13.71516440

8.00000000

9.81732758

11.60062586

13.71516440

8.00000000

9.81732758

11.66346702

13.43998185

8.00000000

9.81732758

11.60062586

13.43998185

14.53379406

14.14321920

14.28260187

14.28260187

14.45660271

14.45660271

13.52037333

. /oxygen/Cu/100/iter_8/struct.xyz

56

Lattice $=" 7.710267940093008 \quad 0.0 \quad 0.0 \quad 0.0 \quad 10.280357253457344 \quad 0.0 \quad 0.0 \quad 0.021 .451982745204997 " \mathrm{P}$ roperties=species:S:1:pos:R:3 pbc="T T T"

$\begin{array}{lllr}\mathrm{Cu} & 1.28504466 & 1.28504466 & 8.00000000 \\ \mathrm{Cu} & 0.00000000 & 0.00000000 & 9.81732758 \\ \mathrm{Cu} & 1.28693790 & 1.28504466 & 11.61129154 \\ \mathrm{Cu} & 0.00000000 & 0.00000000 & 13.37972717 \\ \mathrm{Cu} & 1.28504466 & 3.85513397 & 8.00000000 \\ \mathrm{Cu} & 0.00000000 & 2.57008931 & 9.81732758 \\ \mathrm{Cu} & 1.30608165 & 3.85513397 & 11.63383379 \\ \mathrm{Cu} & 0.00000000 & 2.57008931 & 13.37972717 \\ \mathrm{Cu} & 1.28504466 & 6.42522328 & 8.00000000 \\ \mathrm{Cu} & 0.00000000 & 5.14017863 & 9.81732758 \\ \mathrm{Cu} & 1.28693790 & 6.42522328 & 11.61129154 \\ \mathrm{Cu} & 0.00000000 & 5.14017863 & 13.37972717\end{array}$


$\mathrm{Cu}$

$\mathrm{Cu}$

$\mathrm{Cu}$

$\mathrm{Cu}$

$\mathrm{Cu}$

$\mathrm{Cu}$

$\mathrm{Cu}$

$\mathrm{Cu}$

$\mathrm{Cu}$

$\mathrm{Cu}$

$\mathrm{Cu}$

$\mathrm{Cu}$

$\mathrm{Cu}$

$\mathrm{Cu}$

$\mathrm{Cu}$

$\mathrm{Cu}$

$\mathrm{Cu}$

$\mathrm{Cu}$

$\mathrm{Cu}$

$\mathrm{Cu}$

$\mathrm{Cu}$

$\mathrm{Cu}$

$\mathrm{Cu}$

$\mathrm{Cu}$

$\mathrm{Cu}$

$\mathrm{Cu}$

$\mathrm{Cu}$

$\mathrm{Cu}$

$\mathrm{Cu}$

$\mathrm{Cu}$

$\mathrm{Cu}$

$\mathrm{Cu}$

$\mathrm{Cu}$

$\mathrm{Cu}$

$\mathrm{Cu}$

$\mathrm{Cu}$

O

0

O

O

O

O

0

0
1.28504466

0.00000000

1.30608165

0.00000000

3.85513397

2. 57008931

3. 83259186

2. 33597507

3. 85513397

2. 57008931

3. 87767608

2. 33597507

3. 85513397

2. 57008931

3. 83259186

2. 33597507

3. 85513397

2. 57008931

3. 87767608

2. 33597507

6.42522328

5.14017863

6.40418629

5.37429287

6.42522328

5.14017863

6.42333004

5.37429287

6.42522328

5.14017863

6.40418629

5.37429287

6.42522328

5.14017863

6.42333004

5.37429287

1.09209071

1.09209071

6.61817723

6.61817723

3.60348295

3.60348295

4.10678499

4.10678499
Fri Apr 16 14:51:55 2021

8.99531260

7.71026794

8.99531260

7.71026794

1.28504466

0.00000000

1.28504466

0.00935521

3.85513397

2.57008931

3.85513397

2.56073410

6.42522328

5.14017863

6.42522328

5.14953384

8.99531260

7.71026794

8.99531260

7.70091273

1.28504466

0.00000000

1.28504466

$-0.00935521$

3.85513397

2. 57008931

3.85513397

2.57944453

6.42522328

5.14017863

6.42522328

5.13082341

8.99531260

7.71026794

8.99531260

7.71962315

1.28504466

6.42522328

8.99531260

3.85513397

3.85513397

8.99531260

1.28504466

6.42522328
8.00000000

9.81732758

11.63383379

13.37972717

8.00000000

9.81732758

11.59663528

13.94747306

8.00000000

9.81732758

11.59663528

13.94747306

8.00000000

9.81732758

11.59663528

13.94747306

8.00000000

9.81732758

11.59663528

13.94747306

8.00000000

9.81732758

11.63383379

13.94747306

8.00000000

9.81732758

11.61129154

13.94747306

8.00000000

9.81732758

11.63383379

13.94747306

8.00000000

9.81732758

11.61129154

13.94747306

14.53889131

14.53889131

14.53889131

14.53889131

13.56985709

13.56985709

13.56985709

13.56985709

. /oxygen/Cu/100/iter_9/struct.xyz

57

Lattice $=" 7.710267940093008 \quad 0.0 \quad 0.0 \quad 0.0 \quad 10.280357253457344 \quad 0.0 \quad 0.0 \quad 0.0 \quad 21.451982745204997 " \quad P$ roperties $=$ species: $\mathrm{S}: 1: \mathrm{pos}: \mathrm{R}: 3 \mathrm{pbc}=" \mathrm{~T} \mathrm{~T} \mathrm{~T} "$

$\begin{array}{llrr}\mathrm{Cu} & 1.28504466 & 1.28504466 & 8.00000000 \\ \mathrm{Cu} & 0.00000000 & 0.00000000 & 9.81732758 \\ \mathrm{Cu} & 1.28723392 & 1.28504466 & 11.58975051 \\ \mathrm{Cu} & 0.06699599 & 0.06282033 & 13.39038876 \\ \mathrm{Cu} & 1.28504466 & 3.85513397 & 8.00000000 \\ \mathrm{Cu} & 0.00000000 & 2.57008931 & 9.81732758 \\ \mathrm{Cu} & 1.30140968 & 3.82757950 & 11.63148104 \\ \mathrm{Cu} & 0.06699599 & 2.50726898 & 13.39038876 \\ \mathrm{Cu} & 1.28504466 & 6.42522328 & 8.00000000 \\ \mathrm{Cu} & 0.00000000 & 5.14017863 & 9.81732758 \\ \mathrm{Cu} & 1.29466410 & 6.42522328 & 11.61430963 \\ \mathrm{Cu} & 0.06356575 & 4.94048444 & 13.75140627 \\ \mathrm{Cu} & 1.28504466 & 8.99531260 & 8.00000000 \\ \mathrm{Cu} & 0.00000000 & 7.71026794 & 9.81732758 \\ \mathrm{Cu} & 1.30140968 & 9.02286706 & 11.63148104 \\ \mathrm{Cu} & 0.06356575 & 7.90996213 & 13.75140627 \\ \mathrm{Cu} & 3.85513397 & 1.28504466 & 8.00000000 \\ \mathrm{Cu} & 2.57008931 & 0.00000000 & 9.81732758 \\ \mathrm{Cu} & 3.82931445 & 1.28504466 & 11.54648390 \\ \mathrm{Cu} & 2.30093602 & -0.01844952 & 14.62588067\end{array}$




\section{$\mathrm{Cu}$}

$\mathrm{Cu}$

$\mathrm{Cu}$

$\mathrm{Cu}$

$\mathrm{Cu}$

$\mathrm{Cu}$

$\mathrm{Cu}$

$\mathrm{Cu}$

$\mathrm{Cu}$

$\mathrm{Cu}$

$\mathrm{Cu}$

$\mathrm{Cu}$

$\mathrm{Cu}$

$\mathrm{Cu}$

$\mathrm{Cu}$

$\mathrm{Cu}$

$\mathrm{Cu}$

$\mathrm{Cu}$

$\mathrm{Cu}$

$\mathrm{Cu}$

$\mathrm{Cu}$

$\mathrm{Cu}$

$\mathrm{Cu}$

$\mathrm{Cu}$

$\mathrm{Cu}$

$\mathrm{Cu}$

$\mathrm{Cu}$

$\mathrm{Cu}$

O

O

O

O

O

O

0

0
3. 85513397

2. 57008931

3. 87645589

2. 30093602

3. 85513397

2. 57008931

3. 85023375

2. 51854157

3. 85513397

2. 57008931

3. 87645589

2. 51854157

6. 42522328

5.14017863

6.40668400

5.19019157

6.42522328

5.14017863

6.44133139

5.19019157

6.42522328

5.14017863

6.40500311

5.07871976

6.42522328

5.14017863

6.44133139

5.07871976

0.97108259

1. 26520766

6.50282060

6.50282060

3. 66406105

3. 66406105

3. 81759806

3. 85500134

6.32599575
Fri Apr 16 14:51:55 2021

3.85513397

2. 57008931

3. 86181650

2.58853884

6.42522328

5.14017863

6.42522328

4.85592685

8.99531260

7.71026794

8.98863006

7. 99451972

1. 28504466

0.00000000

1.28504466

0.00815566

3.85513397

2. 57008931

3. 82848332

2. 56193365

6.42522328

5.14017863

6.42522328

5.00746282

8.99531260

7.71026794

9.02196325

7.84298374

1. 28504466

6.42522328

9.15584289

3.69460368

3. 82291249

9.02753408

1. 28504466

6.42522328

6.42522328

. /oxygen/Cu/110/2x1/struct. xyz

12

Lattice $=" 5.140178626728671 \quad 0.0 \quad 0.0 \quad 0.0 \quad 3.63465516347$ ies=species: $\mathrm{S}: 1:$ pos: $\mathrm{R}: 3 \mathrm{pbc}=\mathrm{T} \mathrm{T} \mathrm{T} "$

$\mathrm{Cu} \quad 0.00000000 \quad 0.00000000$

$\mathrm{Cu} \quad 1.28504466 \quad 1.81732758$

$\mathrm{Cu} \quad 0.00000000 \quad 0.00000000$

$\mathrm{Cu} \quad 1.28504466 \quad 1.81732758$

$\mathrm{Cu} \quad-0.04458024 \quad 0.00000000$

$\mathrm{Cu} \quad 2.57008931$

$\mathrm{Cu} \quad 3.85513397$

$\mathrm{Cu} \quad 2.57008931$

$\mathrm{Cu} \quad 3.85513397$

$\mathrm{Cu} \quad 2.61466956$

$\mathrm{Cu} \quad 1.28504466$

O 1.28504466

. /oxygen/Cu/110/c6x2/struct. xyz

0.00000000

1.81732758

0.00000000

1.81732758

0.00000000

1.81732758

0.00000000

90

Lattice $=" 15.420535880186014 \quad 0.0 \quad 0.0 \quad 0.0 \quad 7.26931032694$ rties $=$ species:S:1:pos:R:3 pbc="T T T"

Cu

$\mathrm{Cu}$

$\mathrm{Cu}$

$\mathrm{Cu}$

$\mathrm{Cu}$

$\mathrm{Cu}$

$\mathrm{Cu}$

$\mathrm{Cu}$

$\mathrm{Cu}$

$\mathrm{Cu}$

$\mathrm{Cu}$

0.00000000

1. 28504466

0.00000000

1.28493574

0.01097016

1. 28108668

$-0.26698775$

0.00000000

1. 28504466

0.00000000

1. 28515358

0.00000000

1.81732758

0.00000000

1.81732758

0.00942590

1.81732758

0.04847763

3.63465516

5.45198275

3.63465516

5.45198275

11.66407381

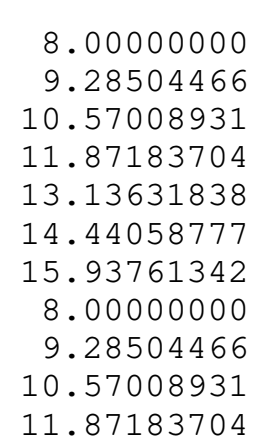

105

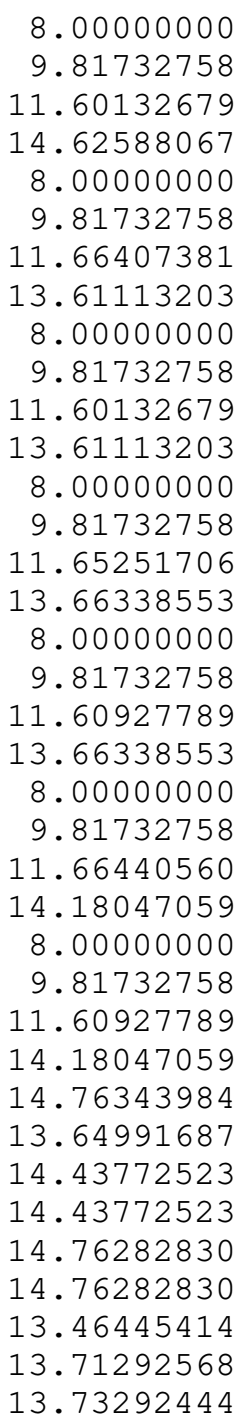

8.00000000

11.6013

14.62588067

8.00000000

13.61113203

8.00000000

11.60132679

13.61113203

8.00000000

9.81732758

11.65251706

11.60927789

13.66338553

8.00000000

14.18047059

8.00000000

9.81732758

14.18047059

14.76343984

13.64991687

14.76282830

14.76282830

13.71292568
13.73292444

$0.00 .00 .021 .14017862672867 "$ Propert

$$
\begin{array}{r}
8.00000000 \\
9.28504466 \\
10.57008931 \\
11.83478357 \\
13.11780131 \\
8.00000000 \\
9.28504466 \\
10.57008931 \\
11.85767306 \\
13.11780131 \\
14.52800391 \\
14.65554247
\end{array}
$$


$\mathrm{Cu}$

$\mathrm{Cu}$

$\mathrm{Cu}$

$\mathrm{Cu}$

$\mathrm{Cu}$

$\mathrm{Cu}$

$\mathrm{Cu}$

$\mathrm{Cu}$

$\mathrm{Cu}$

$\mathrm{Cu}$

$\mathrm{Cu}$

$\mathrm{Cu}$

$\mathrm{Cu}$

$\mathrm{Cu}$

$\mathrm{Cu}$

$\mathrm{Cu}$

$\mathrm{Cu}$

$\mathrm{Cu}$

$\mathrm{Cu}$

$\mathrm{Cu}$

$\mathrm{Cu}$

$\mathrm{Cu}$

$\mathrm{Cu}$

$\mathrm{Cu}$

$\mathrm{Cu}$

$\mathrm{Cu}$

$\mathrm{Cu}$

$\mathrm{Cu}$

$\mathrm{Cu}$

$\mathrm{Cu}$

$\mathrm{Cu}$

$\mathrm{Cu}$

$\mathrm{Cu}$

$\mathrm{Cu}$

$\mathrm{Cu}$

$\mathrm{Cu}$

$\mathrm{Cu}$

$\mathrm{Cu}$

$\mathrm{Cu}$

$\mathrm{Cu}$

$\mathrm{Cu}$

$\mathrm{Cu}$

$\mathrm{Cu}$

$\mathrm{Cu}$

$\mathrm{Cu}$

$\mathrm{Cu}$

$\mathrm{Cu}$

$\mathrm{Cu}$

$\mathrm{Cu}$

$\mathrm{Cu}$

$\mathrm{Cu}$

$\mathrm{Cu}$

$\mathrm{Cu}$

$\mathrm{Cu}$

$\mathrm{Cu}$

$\mathrm{Cu}$

$\mathrm{Cu}$

$\mathrm{Cu}$

$\mathrm{Cu}$

$\mathrm{Cu}$

$\mathrm{Cu}$

$\mathrm{Cu}$

$\mathrm{Cu}$

$\mathrm{Cu}$

$\mathrm{Cu}$

$\mathrm{Cu}$

$\mathrm{Cu}$

$\mathrm{Cu}$
0.01097016

1.28900264

$-0.26698775$

2.57008931

3. 85513397

2. 57008931

3.85300015

2. 55911916

3. 88809230

2.83707706

2. 57008931

3. 85513397

2. 57008931

3.85895980

2. 55911916

3. 85014225

2.83707706

5.14017863

6.42522328

5.14017863

6.42735710

5.14017863

6.39226496

5.14017863

6.42522328

5.14017863

6.42139746

5.14017863

6.43021500

7. 71026794

8.99531260

7.71026794

8.99542152

7. 72123810

8.99927058

7.44328019

7.71026794

8.99531260

7.71026794

8.99520368

7.72123810

8.99135462

7.44328019

10.28035725

11.56540191

10.28035725

11.56922774

10.26938710

11.56041019

10.54734500

10.28035725

11.56540191

10.28035725

11.56326809

10.26938710

11.59836024

10.54734500

12.85044657

14.13549122

12.85044657

14.13166540

12.85044657

14.14048294

12.85044657

14.13549122

12.85044657

14.13762504

12.85044657
Fri Apr 16 14:51:55 2021

106

3.62522927

5.45198275

13.13631838

14.44058777

15.93761342

8.00000000

9.28504466

10.57008931

11.85110072

13.13631838

14.37228009

15.93761342

8.00000000

9.28504466

10.57008931

11.83922299

13.13631838

14.30631676

15.93761342

8.00000000

9.28504466

10.57008931

11.85110072

13.12810740

14.37228009

8.00000000

9.28504466

10.57008931

11.83922299

13.12810740

14.30631676

8.00000000

9.28504466

10.57008931

11.87183704

13.13631838

14.44058777

15.93761342

8.00000000

9.28504466

10.57008931

11.87183704

13.13631838

14.44058777

15.93761342

8.00000000

9.28504466

10.57008931

11.83922299

13.13631838

14.30631676

15.93761342

8.00000000

9.28504466

10.57008931

11.85110072

13.13631838

14.37228009

15.93761342

8.00000000

9.28504466

10.57008931

11.83922299

13.12810740

14.30631676

8.00000000

9.28504466

10.57008931

11.85110072

13.12810740 


\section{$\mathrm{Cu}$}

$\mathrm{Cu}$

$\mathrm{Cu}$

O

O

O

O

O

O

O

0
5.45198275

1.81732758

5.45198275

1.81732758

5.45198275

1.81732758

5.45198275

1.81732758

5.45198275

1.81732758

5.45198275
14.37228009

16.50432980

16.50432980

15.93708810

15.93708810

16.44361178

15.93708810

16.44361178

16.44361178

15.93708810
16.44361178

. /oxygen/Cu/110/iter_1/struct.xyz

46

Lattice $=" 7.710267940093007 \quad 0.0 \quad 0.0 \quad 0.0 \quad 10.90396549041$ ties=species:S:1:pos:R:3 pbc="T T T"

$\mathrm{Cu} \quad 0.00000000 \quad 0.00000000$

$\mathrm{Cu} \quad 1.28504466 \quad 1.81732758$

$\mathrm{Cu} \quad 0.00000000 \quad 0.00000000$

$\mathrm{Cu} \quad 1.28504466 \quad 1.84991409$

$\mathrm{Cu} \quad-0.07475715$

$\mathrm{Cu} \quad 0.00000000$

$-0.04655526$

3.63465516

5.45198275

0.00000000

3.63465516

1.28504466

5.45474705

$-0.02496699$

3.67444781

0.00000000

7.26931033

1.28504466

9.08663791

0.00000000

7.26931033

1.28504466

9.08749496

0.00159849

7.27423169

2.57008931

0.00000000

3. 85513397

1.81732758

2.57008931

0.00000000

3.87214143

1.81759619

2.64484646

$-0.04655526$

2.57008931

3.63465516

3.85513397

5.45198275

2. 57008931

3.63465516

3.85393075

5.45487556

2.59505630

3.67444781

7.26931033

9.08663791

3.85513397

7.26931033

2.57008931

9.08642933

7.27423169

2. 56849082

0.00000000

5.14017863

1.81732758

0.00000000

5.14017863

6.40821582

1.81759619

5.14017863

$-0.01950859$

3.63465516

5.45198275

6.42522328

3.63465516

5.45487556

.14017863

3.63574760

5.14017863

7.26931033

9.08663791

6.42522328

7.26931033

5.14017863

9.08642933

7.26800130

5.14017863

1. 28504466

1.10415594
8.00000000

9.28504466

10.57008931

11.91181256

8.00000000

9.28504466

10.57008931

11.90063499

13.07277550

8.00000000

9.28504466

10.57008931

11.88043718

13.05587113

8.00000000

9.28504466

10.57008931

11.88181829

13.13705077

8.00000000

9.28504466

10.57008931

11.88899346

13.07277550

8.00000000

9.28504466

10.57008931

11.89513747

13.05587113

8.00000000

9.28504466

10.57008931

11.88181829

13.04643162

8.00000000

9.28504466

10.57008931

11.88899346

13.05053174

8.00000000

9.28504466

10.57008931

11.89513747

13.05023965

13.77549727
13.13705077

. /oxygen/Cu/110/iter_10/struct.xyz

55

Lattice $=" 7.710267940093007 \quad 0.0 \quad 0.0 \quad 0.0 \quad 10.90396549041$

ties=species:S:1:pos:R:3 pbc="T T T"

$\begin{array}{lll}\mathrm{Cu} & 0.00000000 & 0.00000000\end{array}$

$\mathrm{Cu} \quad 1.28504466 \quad 1.81732758$

8.00000000

1.81732758
0.00000000

9.28504466

10.57008931

$\mathrm{Cu}$

0.00000000

$0.00 .00 .021 .14017862672867 "$ Proper 
$\mathrm{Cu}$

$\mathrm{Cu}$

$\mathrm{Cu}$

$\mathrm{Cu}$

$\mathrm{Cu}$

$\mathrm{Cu}$

$\mathrm{Cu}$

$\mathrm{Cu}$

$\mathrm{Cu}$

$\mathrm{Cu}$

$\mathrm{Cu}$

$\mathrm{Cu}$

$\mathrm{Cu}$

$\mathrm{Cu}$

$\mathrm{Cu}$

$\mathrm{Cu}$

$\mathrm{Cu}$

$\mathrm{Cu}$

$\mathrm{Cu}$

$\mathrm{Cu}$

$\mathrm{Cu}$

$\mathrm{Cu}$

$\mathrm{Cu}$

$\mathrm{Cu}$

$\mathrm{Cu}$

$\mathrm{Cu}$

$\mathrm{Cu}$

$\mathrm{Cu}$

$\mathrm{Cu}$

$\mathrm{Cu}$

$\mathrm{Cu}$

$\mathrm{Cu}$

$\mathrm{Cu}$

$\mathrm{Cu}$

$\mathrm{Cu}$

$\mathrm{Cu}$

$\mathrm{Cu}$

$\mathrm{Cu}$

$\mathrm{Cu}$

$\mathrm{Cu}$

$\mathrm{Cu}$

$\mathrm{Cu}$

O

O

O

O

O

O

O

O

0

$\mathrm{O}$
1. 26145997

0.10383719

0.00000000

1.28504466

0.00000000

1.29466341

0.06098249

0.00000000

1.28504466

0.00000000

1.32632715

0.02214534

2.57008931

3. 85513397

2. 57008931

3. 79922004

2. 84666528

2.57008931

3. 85513397

2. 57008931

3. 89976874

2. 33238121

2. 57008931

3. 85513397

2. 57008931

3. 84949497

2. 65191257

5. 14017863

6.42522328

5. 14017863

6.32532563

5.03236837

5.14017863

6.42522328

5.14017863

6.45661523

5.07313628

5.14017863

6.42522328

5.14017863

6.45042677

5. 39684871

1. 17474448

6. 30555048

4.03216406

6.35072017

4. 11535006

1. 46534005

3. 36078710

6.72757574

1.15664442

3. 83726651
Fri Apr 16 14:51:55 2021

1.75553473

10.55141239

3.63465516

5.45198275

3.63465516

5.46780706

2.82047443

7.26931033

9.08663791

7. 26931033

9.11911397

7.21211981

0.00000000

1.81732758

0.00000000

1. 81868158

1.30885342

3.63465516

5.45198275

3.63465516

5.48473780

4. 37021725

7.26931033

9.08663791

7.26931033

9.18056093

7.55051142

0.00000000

1. 81732758

0.00000000

1.68591828

10.47919479

3.63465516

5.45198275

3.63465516

5.43283450

3.56247801

7.26931033

9.08663791

7.26931033

9.08999674

7.63750372

1.27108055

0.93159820

0.45405108

9.27590543

6.29053750

6.02573786

2.82395363

4.33009968

8.80754239

9.03038577
11.79938572

13.75215131

8.00000000

9.28504466

10.57008931

11.76323144

13.71704841

8.00000000

9.28504466

10.57008931

11.78566932

13.12434366

8.00000000

9.28504466

10.57008931

11.81495049

14.59884522

8.00000000

9.28504466

10.57008931

11.86143939

13.67890330

8.00000000

9.28504466

10.57008931

11.81850805

13.80402838

8.00000000

9.28504466

10.57008931

11.93686875

14.36621085

8.00000000

9.28504466

10.57008931

11.94132471

13.39157590

8.00000000

9.28504466

10.57008931

11.72793479

13.54093022

13.75054389

13.76806708

15.56988718

13.68227996

13.63028793

13.71867481

13.52103548

13.54242796

13.68857781

13.74815907

. /oxygen/Cu/110/iter_11/struct.xyz

56

Lattice $=" 7.710267940093007 \quad 0.0 \quad 0.0 \quad 0.0 \quad 10.90396549041$

ties=species:S:1:pos:R:3 pbc="T T T"

$\begin{array}{lll}\mathrm{Cu} & 0.00000000 & 0.00000000\end{array}$

$\mathrm{Cu} \quad 1.28504466 \quad 1.81732758$

$\mathrm{Cu} \quad 0.00000000 \quad 0.00000000$

$\begin{array}{lll}\mathrm{Cu} & 1.33365629 & 1.82776356\end{array}$

$\mathrm{Cu} \quad 0.98190214$

$\mathrm{Cu} \quad 0.00000000$

$\mathrm{Cu} \quad 1.28504466$

$\mathrm{Cu} \quad 0.00000000$

$\mathrm{Cu} \quad 1.26416102$

$\mathrm{Cu} \quad 0.13635612$

$\mathrm{Cu} \quad 0.00000000$

$\mathrm{Cu} \quad 1.28504466$

10.51680401

3.63465516

5.45198275

3.63465516

5.45572726

3.08403196

7.26931033

8.00000000

9.28504466

10.57008931

11.85006491

13.75280923

8.00000000

9.28504466

10.57008931

11.78519136

13.77593900

8.00000000

9. 28504466

$0.00 .00 .021 .14017862672867 "$ Proper

9.08663791 
$\mathrm{Cu}$

$\mathrm{Cu}$

$\mathrm{Cu}$

$\mathrm{Cu}$

$\mathrm{Cu}$

$\mathrm{Cu}$

$\mathrm{Cu}$

$\mathrm{Cu}$

$\mathrm{Cu}$

$\mathrm{Cu}$

$\mathrm{Cu}$

$\mathrm{Cu}$

$\mathrm{Cu}$

$\mathrm{Cu}$

$\mathrm{Cu}$

$\mathrm{Cu}$

$\mathrm{Cu}$

$\mathrm{Cu}$

$\mathrm{Cu}$

$\mathrm{Cu}$

$\mathrm{Cu}$

$\mathrm{Cu}$

$\mathrm{Cu}$

$\mathrm{Cu}$

$\mathrm{Cu}$

$\mathrm{Cu}$

$\mathrm{Cu}$

$\mathrm{Cu}$

$\mathrm{Cu}$

$\mathrm{Cu}$

$\mathrm{Cu}$

$\mathrm{Cu}$

$\mathrm{Cu}$

O

O

0

O

o

0

O

O

O

0

0
0.00000000

1.30695832

7. 59109943

2. 57008931

3.85513397

2.57008931

3.85807303

3.10115596

2.57008931

3.85513397

2.57008931

3. 85758203

2.25098603

2. 57008931

3.85513397

2. 57008931

3. 85247460

2. 62206029

5.14017863

6.42522328

5.14017863

6.44190884

5.26272854

5.14017863

6.42522328

5.14017863

6.45742267

5.13696413

5.14017863

6.42522328

5.14017863

6.38305344

4.97535874

1.18622532

6.04881233

2.81013796

7.04779612

4.06377694

1.41160038

3. 47071283

6.77175559

1.12298804

3. 99788771

6.14621654
Fri Apr 16 14:51:55 2021

7.26931033

9.05483510

7.04897091

0.00000000

1.81732758

0.00000000

1.86413714

1.24872364

3.63465516

5.45198275

3.63465516

5.46513757

4.34098102

7.26931033

9.08663791

7.26931033

9.16760672

7.42804105

0.00000000

1.81732758

0.00000000

1.81143288

0.34574810

3.63465516

5.45198275

3.63465516

5.41756318

3.75729542

7.26931033

9.08663791

7.26931033

9.01698474

6.87698017

1.53437973

2.09947860

10.37476381

10.73631931

5.31550583

5.97699916

2.91435673

4.65606380

8.64651589

9.78702969

8.22388767
10.57008931

11.68454293

13.63879439

8.00000000

9.28504466

10.57008931

11.84598226

14.30878270

8.00000000

9.28504466

10.57008931

11.78476116

13.82491653

8.00000000

9.28504466

10.57008931

11.90714581

13.14947686

8.00000000

9.28504466

10.57008931

11.79961864

13.63970332

8.00000000

9.28504466

10.57008931

11.85762278

13.80324055

8.00000000

9.28504466

10.57008931

11.86548514

13.64188953

13.86372119

13.75559845

14.65553481

12.68110544

13.79917450

13.73525917

13.74461067

13.67447120

13.56825492

13.87864615

13.65165241

. /oxygen/Cu/110/iter_12/struct.xyz

57

Lattice $=" 7.710267940093007 \quad 0.0 \quad 0.0 \quad 0.0 \quad 10.90396549041$ ties=species:S:1:pos:R:3 pbc="T T T"

0.00000000

$\mathrm{Cu} \quad 1.28504466 \quad 1.81732758$

$\mathrm{Cu} \quad 0.00000000 \quad 0.00000000$

$\mathrm{Cu} \quad 1.29820458 \quad 1.89267864$

$\mathrm{Cu} \quad 0.06434078$

1.89267864

0.00000000

3.63465516

1.28504466

5.45198275

0.00000000

3.63465516

1. 35884938

0.13619099

5.24363935

3.81860133

0.00000000

7.26931033

9.08663791

7.26931033

1.28504466

9.15703700

1.25114267

$-0.18723761$

7.26735240

0.00000000

2. 57008931

1.81732758

3.85513397

0.00000000

1.83241780

3. 85113908

1.83241780
-0.03144255

\author{
8.00000000 \\ 9.28504466 \\ 10.57008931 \\ 11.83156798 \\ 13.66252334 \\ 8.00000000 \\ 9.28504466 \\ 10.57008931 \\ 11.96251276 \\ 13.94705314 \\ 8.00000000 \\ 9.28504466 \\ 10.57008931 \\ 11.84570864 \\ 14.55123412 \\ 8.00000000 \\ 9.28504466 \\ 10.57008931 \\ 11.79359619 \\ 13.11834268
}




\section{$\mathrm{Cu}$}

$\mathrm{Cu}$

$\mathrm{Cu}$

$\mathrm{Cu}$

$\mathrm{Cu}$

$\mathrm{Cu}$

$\mathrm{Cu}$

$\mathrm{Cu}$

$\mathrm{Cu}$

$\mathrm{Cu}$

$\mathrm{Cu}$

$\mathrm{Cu}$

$\mathrm{Cu}$

$\mathrm{Cu}$

$\mathrm{Cu}$

$\mathrm{Cu}$

$\mathrm{Cu}$

$\mathrm{Cu}$

$\mathrm{Cu}$

$\mathrm{Cu}$

$\mathrm{Cu}$

$\mathrm{Cu}$

$\mathrm{Cu}$

$\mathrm{Cu}$

$\mathrm{Cu}$

O

O

O

O

O

O

O

O

O

O

0

0
2.57008931

3. 85513397

2. 57008931

3. 89847297

2. 94623150

2. 57008931

3. 85513397

2. 57008931

3.79942956

2. 50434700

5.14017863

6.42522328

5. 14017863

6.35461932

4.88428007

5.14017863

6.42522328

5.14017863

6.49141181

5.08635232

5.14017863

6.42522328

5.14017863

6.39852736

5. 17185822

1. 15312098

6.28181823

3. 26690412

6. 32382219

4.06203589

1. 19263639

3. 79231064

6.82318597

1. 23776994

3. 36814940

6.42320622

1.84667404
Fri Apr 16 14:51:55 2021

3.63465516

5.45198275

3.63465516

5. 42095512

3.00555913

7.26931033

9.08663791

7.26931033

9.01983972

6.35989215

0.00000000

1. 81732758

0.00000000

1.94601828

10.39245798

3.63465516

5.45198275

3.63465516

5.45925388

4.20140481

7.26931033

9.08663791

7.26931033

9.08826324

7.24002220

2.19677473

2.81647082

9.58509518

0.05582005

5.75051785

6.88246109

1. 32830895

5.45729427

7.23681653

8.18551668

8.53652061

4.62134072
8.00000000

9.28504466

10.57008931

11.72942681

13.90709625

8.00000000

9.28504466

10.57008931

11.85867049

14.49291182

8.00000000

9.28504466

10.57008931

11.88979643

13.83719989

8.00000000

9.28504466

10.57008931

11.80854558

13.75253787

8.00000000

9.28504466

10.57008931

11.85632228

13.40675172

14.05564354

13.71793262

14.47237416

12.77767249

13.64405857

15.58153483

13.66666312

13.75951362

12.10910422

13.88633330

13.76041128

13.91937125

58

Lattice $=" 7.710267940093007 \quad 0.0 \quad 0.0 \quad 0.0 \quad 10.90396549041$ ties=species:S:1:pos:R:3 pbc="T T T"

$\mathrm{Cu} \quad 0.00000000 \quad 0.00000000$

$\mathrm{Cu} \quad 1.28504466 \quad 1.81732758$

$\mathrm{Cu} \quad 0.00000000 \quad 0.00000000$

$\mathrm{Cu} \quad 1.25361134$

0.06093163

1.80039416

0.00000000

10.69081714

1.28504466

0.00000000

3. 63465516

5.45198275

3.63465516

1. 42581516

5. 31703074

0.04220971

2. 97708804

0.00000000

7.26931033

1. 28504466

0.00000000

1. 24199092

9.08663791

7.26931033

9.18686728

6.08179365

2. 57008931

3. 85513397

6.03436186

0.00000000

1.81732758

0.00000000

1. 84816586

3. 76433421

2. 82151019

0.91034573

3. 63465516

5.45198275

3.85513397

2. 57008931

3.63465516

5. 52440476

4. 30478609

7.26931033

8.00000000

9.28504466

10.57008931

11.80307513

13.61125980

8.00000000

9.28504466

10.57008931

11.79494197

13.76979849

8.00000000

9.28504466

10.57008931

11.78831052

14.60391929

8.00000000

9.28504466

10.57008931

11.84877456

14.16337239

8.00000000

9.28504466

10.57008931

11.92192403

14.13124234

8.00000000

9.28504466 


\section{$\mathrm{Cu}$}

$\mathrm{Cu}$

$\mathrm{Cu}$

$\mathrm{Cu}$

$\mathrm{Cu}$

$\mathrm{Cu}$

$\mathrm{Cu}$

$\mathrm{Cu}$

$\mathrm{Cu}$

$\mathrm{Cu}$

$\mathrm{Cu}$

$\mathrm{Cu}$

$\mathrm{Cu}$

$\mathrm{Cu}$

$\mathrm{Cu}$

$\mathrm{Cu}$

$\mathrm{Cu}$

$\mathrm{Cu}$

O

O

O

O

O

O

O

0

O

O

O

0

$\mathrm{O}$

59

Lattice $=" 7.710267940093007 \quad 0.0 \quad 0.00 .0$ ties $=$ species:S:1:pos:R:3 pbc="T T T"

$\mathrm{Cu}$

$\mathrm{Cu}$

0.00000000

1.28504466

0.00000000

1.32031736

0.01946217

0.00000000

1.28504466

0.00000000

1.22510331

7.06476764

0.00000000

1. 28504466

0.00000000

1.31261220

0.40362932

2.57008931

3.85513397

2.57008931

3.88714128

2. 55509181

2. 57008931

3. 85513397

2. 57008931

3. 77549466

2. 22776980

2. 57008931

3. 85513397

2. 57008931

3. 88380758

2. 99859211

5.14017863

6.42522328

5.14017863
7.26931033

9.09986570

7.14331953

0.00000000

0.00000000

1.81159250

0.16271877

3.63465516

5.45198275

3.63465516

5. 39937558

3.60781838

7.26931033

9.08663791

7.26931033

9.10476027

8.63602375

1. 36484424

1.98608091

10.54386443

8.96439838

5.40318229

6.01784097

2.82438772

4.44654316

6.67822878

8.68642104

8.74757994

5.76398099

7.80550192
1. 81732758
0.00000000

1. 81732758

0.00000000

1.80855322

10.80739863

3. 63465516

5.45198275

3.63465516

5. 40034328

4.35925110

7.26931033

7.26931033

9.11469499

7. 51561308

0.00000000

1.81732758

0.00000000

1.77329830

0.03068412

3. 63465516

5. 45198275

3.63465516

5.46323221

3. 38939078

7.26931033

9.08663791

7.26931033

9.08163025

6.61730212

0.00000000

1. 81732758

0.00000000
9.08663791
10.57008931

11.80563920

13.85154511

8.00000000

9.28504466

10.57008931

11.73176440

13.21972332

8.00000000

9.28504466

10.57008931

11.86674940

13.73275558

8.00000000

9.28504466

10.57008931

11.91901970

14.40123251

13.76318840

13.60943275

14.64394686

13.76911210

13.83484046

15.64266296

13.68312641

13.71160434

12.44437896

13.21027372

15.24301732

15.05405284

15.16124134
$0.0 \quad 0.0 \quad 0.021 .14017862672867 "$ Proper

$$
8.00000000
$$

9.28504466

10.57008931

11.82330304

13.09728362

8.00000000

9.28504466

10.57008931

11.78673471

13.89932286

8.00000000

9.28504466

10.57008931

11.81009733

14.23642328

8.00000000

9.28504466

10.57008931

11.75499077

13.26274082

8.00000000

9.28504466

10.57008931

11.88252052

14.28385562

8.00000000

9.28504466

10.57008931

11.76940413

14.22911821

8.00000000

9.28504466

10.57008931 
$\mathrm{Cu}$

$\mathrm{Cu}$

$\mathrm{Cu}$

$\mathrm{Cu}$

$\mathrm{Cu}$

$\mathrm{Cu}$

$\mathrm{Cu}$

$\mathrm{Cu}$

$\mathrm{Cu}$

$\mathrm{Cu}$

$\mathrm{Cu}$

$\mathrm{Cu}$

O

O

O

O

O

0

O

0

0

O

O

0

O

0
6.47009349

5.19130423

5.14017863

6.42522328

5.14017863

6.34099252

4.94520476

5.14017863

6.42522328

5.14017863

6.42035746

5.33669830

0.84818955

4.54631142

3.81606166

0.31578579

3.50887353

1.42639592

3.15341385

6.71347486

1.34368063

3.71693990

6.74786926

1.00728078

6.41918072

7.07307618
Fri Apr 16 14:51:55 2021

1.81957865

10.82120714

3.63465516

5.45198275

3.63465516

5.45913131

3.63946255

7.26931033

9.08663791

7.26931033

9.10241249

7.39233805

2.07657838

1.80049907

9.80919461

9.58647073

4.77849940

6.04528298

1.71087711

6.15317801

7.26000969

8.31759223

9.48971240

4.68411234

8.12681953

2.48298270
11.90268226

13.18704732

8.00000000

9.28504466

10.57008931

11.78007744

13.06988177

8.00000000

9.28504466

10.57008931

11.79027523

13.59478867

13.75934719

13.76111607

14.26153145

14.56877063

13.76677497

15.24044570

14.36335029

13.57156522

12.47035008

13.63850961

15.39193226

14.96138132

15.29333348

13.84497299
. /oxygen/Cu/110/iter_2/struct.xyz

47

Lattice $=" 7.710267940093007 \quad 0.0 \quad 0.0 \quad 0.0 \quad 10.90396549041$ ties=species:S:1:pos:R:3 pbc="T T T"

$\begin{array}{lll}\mathrm{Cu} & 0.00000000 & 0.00000000\end{array}$

$\mathrm{Cu} \quad 1.28504466 \quad 1.81732758$

$\mathrm{Cu} \quad 0.00000000 \quad 0.00000000$

$\begin{array}{lll}\mathrm{Cu} & 1.28504466 & 1.87029887\end{array}$

$\mathrm{Cu} \quad-0.04967102$

$\mathrm{Cu} \quad 0.00000000$

$-0.13128477$

3.63465516

1.28504466

0.00000000

5.45198275

3. 63465516

1.28504466

0.01464468

5.48768361

3.75875355

7.26931033

0.00000000

1. 28504466

0.00000000

1. 28504466

$-0.00268424$

2.57008931

3. 85513397

2. 57008931

3.81778900

2. 61976033

2. 57008931

3. 85513397

2. 57008931

3. 86667636

2. 55544463

2. 57008931

3. 85513397

2. 57008931

3. 86453077

2. 57277356

5. 14017863

9.08663791

7.26931033

9.06104525

7.27370820

0.00000000

1. 81732758

0.00000000

1.82051476

$-0.13128477$

3. 63465516

5.45198275

3.63465516

5.47385963

3.75875355

7.26931033

9.08663791

7.26931033

9.06475910

7.27370820

0.00000000

6.42522328

5.14017863

6.46256826

5. 14017863

5.14017863

6.42522328

1.81732758

0.00000000

1. 82051476

$-0.09625101$

3. 63465516

5. 45198275

8.00000000

9.28504466

10.57008931

11.93047980

13.12933908

8.00000000

9.28504466

10.57008931

11.86779999

13.07503521

8.00000000

9.28504466

10.57008931

11.85217164

13.06934728

8.00000000

9.28504466

10.57008931

11.89420362

13.12933908

8.00000000

9.28504466

10.57008931

11.87781968

13.07503521

8.00000000

9.28504466

10.57008931

11.88493690

13.06934728

8.00000000

9.28504466

10.57008931

11.89420362

13.10364356

8.00000000

9.28504466

10.57008931

$\mathrm{Cu}$

3.63465516

5.14017863

$0.00 .00 .021 .14017862672867 "$ Proper 


\section{$\mathrm{Cu}$}

$\mathrm{Cu}$

$\mathrm{Cu}$

$\mathrm{Cu}$

$\mathrm{Cu}$

$\mathrm{Cu}$

$\mathrm{Cu}$

O

0
6.41368089

5.14017863

5.14017863

6.42522328

5.14017863

6.41582648

5.14017863

1.28504466

5.14017863
Fri Apr 16 14:51:55 2021

5.47385963

3. 72111325

7.26931033

9.08663791

7. 26931033

9.06475910

7.26739674

1.05872901

1.81465975
11.87781968

13.10675928

8.00000000

9.28504466

10.57008931

11.88493690

13.07600688

13.73699790

13.46416876

48

Lattice $=" 7.710267940093007 \quad 0.0 \quad 0.0 \quad 0.0 \quad 10.90396549041$

ties=species:S:1:pos:R:3 pbc="T T T"

$\mathrm{Cu} \quad 0.00000000 \quad 0.00000000$

$\mathrm{Cu} \quad 1.28504466 \quad 1.81732758$

$\begin{array}{lll}\mathrm{Cu} & 0.00000000 & 0.00000000\end{array}$

$\mathrm{Cu} \quad 1.32310240 \quad 1.81218546$

$\mathrm{Cu} \quad 0.00778648 \quad-0.14323462$

$\mathrm{Cu} \quad 0.00000000 \quad 3.63465516$

$\mathrm{Cu} \quad 1.28504466 \quad 5.45198275$

$\mathrm{Cu} \quad 0.00000000 \quad 3.63465516$

$\mathrm{Cu} \quad 1.25813523 \quad 5.49437519$

$\mathrm{Cu} \quad-0.00480070$

$\mathrm{Cu} \quad 0.00000000$

3.75479989

7.26931033

1.28504466

0.00000000

9.08663791

7.26931033

1.26259687

9.05356415

$-0.07058732$

2. 57008931

7.26955075

0.00000000

3. 85513397

1.81732758

2.57008931

0.00000000

1.81350236

3.81749575

2. 63665356

2. 57008931

$-0.78168280$

3.63465516

5. 45198275

3. 85513397

3.63465516

5. 49708712

3. 84285293

3.76629737

7.26931033

2.52583234

9.08663791

7. 26931033

9.09918281

7. 23417516

0.00000000

1.81732758

0.00000000

1.81977839

$-0.07909532$

3. 63465516

5. 45198275

3. 63465516

5.50556548

3.71889597

7.26931033

9.08663791

7.26931033

9. 07230162

7.34496109

0.74160598

1. 81471408

8.53603134

8.00000000

9.28504466

10.57008931

11.98319266

13.12426516

8.00000000

9.28504466

10.57008931

11.86393831

13.05373851

8.00000000

9.28504466

10.57008931

11.80672740

13.06677546

8.00000000

9.28504466

10.57008931

11.88685228

13.69477496

8.00000000

9.28504466

10.57008931

11.88455359

13.06554660

8.00000000

9.28504466

10.57008931

11.78643766

13.15102067

8.00000000

9.28504466

10.57008931

11.90339214

13.09636111

8.00000000

9.28504466

10.57008931

11.90646065

13.10588921

8.00000000

9.28504466

10.57008931

11.85618218

13.16587246

13.65045978

13.46263942

13.88548506 $0.00 .00 .021 .14017862672867 "$ Proper 1.59007837

5.06980644

3. 65290874

. /oxygen/Cu/110/iter_4/struct.xyz

49

Lattice $=" 7.710267940093007 \quad 0.0 \quad 0.0 \quad 0.0 \quad 10.90396549041$

ties =species: $\mathrm{S}: 1: \operatorname{pos}: \mathrm{R}: 3 \mathrm{pbc}=" \mathrm{~T} \mathrm{~T} \mathrm{~T} "$

$\mathrm{Cu} \quad 0.00000000 \quad 0.00000000$

$\mathrm{Cu} \quad 1.28504466 \quad 1.81732758$

8.00000000

0.00000000

9.28504466

10.57008931

$0.00 .00 .021 .14017862672867 "$ Proper

$\mathrm{Cu}$

0.00000000 


\section{$\mathrm{Cu}$}

$\mathrm{Cu}$

$\mathrm{Cu}$

$\mathrm{Cu}$

$\mathrm{Cu}$

$\mathrm{Cu}$

$\mathrm{Cu}$

$\mathrm{Cu}$

$\mathrm{Cu}$

$\mathrm{Cu}$

$\mathrm{Cu}$

$\mathrm{Cu}$

$\mathrm{Cu}$

$\mathrm{Cu}$

$\mathrm{Cu}$

$\mathrm{Cu}$

$\mathrm{Cu}$

$\mathrm{Cu}$

$\mathrm{Cu}$

$\mathrm{Cu}$

$\mathrm{Cu}$

$\mathrm{Cu}$

$\mathrm{Cu}$

$\mathrm{Cu}$

$\mathrm{Cu}$

$\mathrm{Cu}$

$\mathrm{Cu}$

$\mathrm{Cu}$

$\mathrm{Cu}$

$\mathrm{Cu}$

$\mathrm{Cu}$

$\mathrm{Cu}$

$\mathrm{Cu}$

$\mathrm{Cu}$

$\mathrm{Cu}$

$\mathrm{Cu}$

$\mathrm{Cu}$

$\mathrm{Cu}$

$\mathrm{Cu}$

$\mathrm{Cu}$

$\mathrm{Cu}$

$\mathrm{Cu}$

O

O

O

0

50

Lattice $=" 7.7102679400930070 .00 .00$

0.00000000

$\mathrm{Cu}$

$\mathrm{Cu}$

$\mathrm{Cu}$

$\mathrm{Cu}$

$\mathrm{Cu}$

$\mathrm{Cu}$

$\mathrm{Cu}$

$\mathrm{Cu}$

$\mathrm{Cu}$

$\mathrm{Cu}$

$\mathrm{Cu}$

$\mathrm{Cu}$

$\mathrm{Cu}$

$\mathrm{Cu}$

$\mathrm{Cu}$

$\mathrm{Cu}$

$\mathrm{Cu}$
1.28504466

0.01021701

0.00000000

1.28504466

0.00000000

1.28504466

0.00000000

1.28504466

0.00000000

1.28504466

0.03460682

2. 57008931

3. 85513397

2. 57008931

3. 81842645

2. 55987230

2. 57008931

3. 85513397

2. 57008931

3. 88641700

2.54528902

2. 57008931

3. 85513397

2. 57008931

3. 85588641

2. 53548249

5.14017863

6.42522328

5.14017863

6.46193081

5.14017863

5.14017863

6.42522328

5. 14017863

6.39394026

5.14017863

5.14017863

6.42522328

5.14017863

6.42447084

5.14017863

1. 28504466

5.14017863

3. 72500642

$-1.15491711$
0.02480029
1.75636603

$-0.57686088$

5.45198275

3.63465516

5.56320497

3.73836667

7.26931033

9.08663791

7.26931033

9.08730169

7.37041466

0.00000000

1.81732758

0.00000000

1.75415098

$-0.57686088$

3.63465516

5. 45198275

3.63465516

5.52128051

3.73836667

7.26931033

9.08663791

7.26931033

9.10888567

7.37041466

0.00000000

1. 81732758

0.00000000

1.75415098

$-0.23417866$

3.63465516

5.45198275

3.63465516

5.52128051

3.65563823

7.26931033

9.08663791

7.26931033

9.10888567

7.37548860

0.76597115

1.69901706

8.85069326

8.85069326
3.63465516

Fri Apr 16 14:51:55 2021

114

11.98401235

13.60021590

8.00000000

9.28504466

10.57008931

11.93412746

13.02405787

8.00000000

9.28504466

10.57008931

11.76505110

13.14740305

8.00000000

9.28504466

10.57008931

11.90566980

13.60021590

8.00000000

9.28504466

10.57008931

11.93495470

13.02405787

8.00000000

9.28504466

10.57008931

11.73857519

13.14740305

8.00000000

9.28504466

10.57008931

11.90566980

13.37189176

8.00000000

9.28504466

10.57008931

11.93495470

13.09340256

8.00000000

9.28504466

10.57008931

11.73857519

13.17486815

13.69151164

13.42732876

13.78654504

13.78654504 
$\mathrm{Cu}$

$\mathrm{Cu}$

$\mathrm{Cu}$

$\mathrm{Cu}$

$\mathrm{Cu}$

$\mathrm{Cu}$

$\mathrm{Cu}$

$\mathrm{Cu}$

$\mathrm{Cu}$

$\mathrm{Cu}$

$\mathrm{Cu}$

$\mathrm{Cu}$

$\mathrm{Cu}$

$\mathrm{Cu}$

$\mathrm{Cu}$

$\mathrm{Cu}$

$\mathrm{Cu}$

$\mathrm{Cu}$

$\mathrm{Cu}$

$\mathrm{Cu}$

$\mathrm{Cu}$

$\mathrm{Cu}$

$\mathrm{Cu}$

$\mathrm{Cu}$

$\mathrm{Cu}$

$\mathrm{Cu}$

$\mathrm{Cu}$

O

0

0

0

0
3.82987701

2. 56049085

2. 57008931

3. 85513397

2. 57008931

3.82338156

2. 51242106

2.57008931

3. 85513397

2. 57008931

3. 86530323

2. 51778521

5.14017863

6.42522328

5.14017863

6.45048024

5.14017863

5.14017863

6.42522328

5.14017863

6.45697569

5.14017863

5.14017863

6.42522328

5.14017863

6.41505402

5.14017863

1.28504466

5.14017863

3.73880467

$-1.16871536$

5.14017863
Fri Apr 16 14:51:55 2021

1.75717126

$-0.55191767$

3.63465516

5.45198275

3.63465516

5.51079107

3.66927236

7.26931033

9.08663791

7.26931033

9.10077107

7.41985643

0.00000000

1.81732758

0.00000000

1.75717126

$-0.22498728$

3.63465516

5.45198275

3.63465516

5.51079107

3.58591965

7.26931033

9.08663791

7.26931033

9.10077107

7.39353459

0.78514292

1.71805346

8.89192327

8.89192327

5.44731135
11.89533451

13.59250545

8.00000000

9.28504466

10.57008931

11.92249167

13.01783427

8.00000000

9.28504466

10.57008931

11.74253125

13.14507966

8.00000000

9.28504466

10.57008931

11.89533451

13.34298167

8.00000000

9.28504466

10.57008931

11.92249167

13.36323454

8.00000000

9.28504466

10.57008931

11.74253125

13.37247834

13.71729998

13.44474192

13.78601915

13.78601915

13.47807483

. /oxygen/Cu/110/iter_6/struct.xyz

$$
51
$$

Lattice $=" 7.710267940093007 \quad 0.0 \quad 0.0 \quad 0.0 \quad 10.90396549041$ ties=species: $\mathrm{S}: 1: \mathrm{pos}: \mathrm{R}: 3 \mathrm{pbc}=" \mathrm{~T} \mathrm{~T} \mathrm{~T} "$

$0.00000000 \quad 0.00000000$

$\mathrm{Cu} \quad 1.28504466 \quad 1.81732758$

$\mathrm{Cu} \quad 0.00000000 \quad 0.00000000$

$\mathrm{Cu} \quad 1.26298691 \quad 1.67343097$

$\mathrm{Cu} \quad 0.03320721$

$\mathrm{Cu} \quad 0.00000000$

$\mathrm{Cu} \quad 1.28504466$

$-1.01830488$

3.63465516

5.45198275

0.00000000

3. 63465516

1. 26513331

$-0.05603133$

5.51245793

3.64332532

7.26931033

9.08663791

1.28504466

0.00000000

7.26931033

9.09343530

6.67285162

0.18528866

2.57008931

0.00000000

3.85513397

1.81732758

0.00000000

1.86759010

3.79765154

2. 55061805

2. 57008931

3.85513397

$-0.55446305$

3.63465516

5.45198275

3. 63465516

5.53076402

3.75327885

7.26931033

9.08663791

7.26931033

9.07774419

7.37103732

0.00000000

8.00000000

9.28504466

10.57008931

12.04769249

13.76438776

8.00000000

9.28504466

10.57008931

11.77142469

13.06078838

8.00000000

9.28504466

10.57008931

11.74448690

13.76301893

8.00000000

9.28504466

10.57008931

11.93374813

13.58812880

8.00000000

9.28504466

10.57008931

11.89147468

13.09893559

8.00000000

9.28504466

10.57008931

11.77331498

13.04061980

8.00000000

9.28504466

$0.00 .00 .021 .14017862672867 "$ Proper

1.81732758

5.14017863

6.42522328 


\section{$\mathrm{Cu}$}

$\mathrm{Cu}$

$\mathrm{Cu}$

$\mathrm{Cu}$

$\mathrm{Cu}$

$\mathrm{Cu}$

$\mathrm{Cu}$

$\mathrm{Cu}$

$\mathrm{Cu}$

$\mathrm{Cu}$

$\mathrm{Cu}$

$\mathrm{Cu}$

$\mathrm{Cu}$

$\mathrm{O}$

O

O

O

O

0
5.14017863

6.43609168

5.10167936

5.14017863

6.42522328

5.14017863

6.41538467

5.07027487

5.14017863

6.42522328

5.14017863

6.43972491

4.86445248

1.05674764

5.27378826

4.13556004

$-0.85377342$

5.19235427

1.07594677
Fri Apr 16 14:51:55 2021

0.00000000

1.81723389

0.00185442

3. 63465516

5.45198275

3.63465516

5.49364202

3.74162412

7.26931033

9.08663791

7.26931033

9.02890132

7.52567953

0.52745461

1.88022019

9.33712394

8.23051850

5.62594044

5.00264840

. /oxygen/Cu/110/iter_7/struct.xyz

52

Lattice $=" 7.710267940093007 \quad 0.0 \quad 0.0 \quad 0.0 \quad 10.90396549041$

ties=species:S:1:pos:R:3 pbc="T T T"

0.00000000

$\mathrm{Cu} \quad 1.28504466 \quad 1.81732758$

$\mathrm{Cu} \quad 0.00000000 \quad 0.00000000$

$\mathrm{Cu} \quad 1.26363210$

7.33204820

1.81822264

0.00000000

1.28504466

10.77890427

3.63465516

5.45198275

0.00000000

3.63465516

1. 35088756

5.43103078

7.67607126

3.59972025

0.00000000

1. 28504466

7.26931033

9.08663791

0.00000000

1.27551383

7.26931033

9.09401186

7.34667652

0.00000000

2.57008931

3.85513397

1.81732758

2. 57008931

0.00000000

3.86324634

2.72548716

1.80412239

0.10278006

3.63465516

5.45198275

3. 85513397

2. 57008931

3.91365948

2. 62303280

2. 57008931

3. 85513397

2. 57008931

3. 89587623

2. 62961443

5.14017863

6.42522328

5. 14017863

6.35046782

4. 77222340

5.14017863

6.42522328

5.14017863

6.41358683

5.00321277

5.14017863

6.42522328

5. 14017863

6.40481834

3.63465516

5.43314427

3. 65490980

7.26931033

9.08663791

7.26931033

9.09527625

7.19609520

0.00000000

1.81732758

0.00000000

1.82255033

2.62832200

3.63465516

5.45198275

3.63465516

5.45993050

5.21477007

7.26931033

9.08663791

7.26931033

9.08416724

7.95323503

10.57008931

11.84566290

13.32307279

8.00000000

9.28504466

10.57008931

13.37022655

8.00000000

9.28504466

10.57008931

11.83374690

13.60207986

13.68109774

13.46328539

13.84104676

13.74781627

13.43952556

13.87280663
11.82174725

116

$$
\begin{array}{r}
8.00000000 \\
9.28504466 \\
10.57008931 \\
11.81513109 \\
13.41536028 \\
8.00000000 \\
9.28504466 \\
10.57008931 \\
11.84295287 \\
13.48929969 \\
8.00000000 \\
9.28504466 \\
10.57008931 \\
11.82145759 \\
13.31276377 \\
8.00000000 \\
9.28504466 \\
10.57008931 \\
11.77099935 \\
13.36254161 \\
8.00000000 \\
9.28504466 \\
10.57008931 \\
11.83138604 \\
13.06116329 \\
8.00000000 \\
9.28504466 \\
10.57008931 \\
11.85910003 \\
13.11002944 \\
8.00000000 \\
9.28504466 \\
10.57008931 \\
11.86276705 \\
14.07857486 \\
8.00000000 \\
9.28504466 \\
10.57008931 \\
11.91468256 \\
14.24901446 \\
8.00000000 \\
9.28504466 \\
10.57008931 \\
11.75320926 \\
13.85860773 \\
\end{array}
$$

$0.00 .00 .021 .14017862672867 "$ Proper 


\section{0}

O

0

0

O

0

0
0.00201379

6.06744362

2.50643627

6.82830029

3.99292873

0.20494015

3.13703146
Fri Apr 16 14:51:55 2021

1.68563423

3.76395197

9.19327774

8.99645547

6.72430095

5.48730219

1.86057255
13.32986620

14.60416166

13.35879914

13.74432882

14.35875197

13.41455912

13.75996472

53

Lattice $=" 7.710267940093007 \quad 0.0 \quad 0.0 \quad 0.010 .90396549041$ ties $=$ species: $S: 1:$ pos: $: R: 3$ pbc="T T T"

0.00000000

$\mathrm{Cu} \quad 1.28504466 \quad 1.81732758$

$\mathrm{Cu} \quad 0.00000000 \quad 0.00000000$

$\mathrm{Cu} \quad 1.23311726 \quad 1.75919332$

$\mathrm{Cu} \quad 7.47194429$

$\mathrm{Cu} \quad 0.00000000$

10.33959867

$0.00000000 \quad 3.63465516$

1.28504466

5. 45198275

0.00000000

3.63465516

1. 33054777

5.51535095

0.18038149

2.77873107

0.00000000

7.26931033

1.28504466

9.08663791

0.00000000

7.26931033

1.27631208

9.08567059

0.20312208

7.37909489

2.57008931

0.00000000

3.85513397

1.81732758

2. 57008931

0.00000000

3. 71616368

1.88712347

2. 32184152

2. 57008931

3. 85513397

10.78679263

3.63465516

5.45198275

2. 57008931

3. 92390462

3.63465516

5.48180045

4.47337287

7.26931033

2. 57008931

9.08663791

7.26931033

3.85513397

9.10295630

7.82513373

0.00000000

1.81732758

0.00000000

1.78606444

0.13131547

3.63465516

5.45198275

3.63465516

5.37010314

3.63083855

7.26931033

9.08663791

7.26931033

9.06553110

7.12123895

0.99578458

1.78860125

9.52629484

8.63531224

5.46928739

6.26394936

2.93702557

4.32208565

8.00000000
9.28504466

10.57008931

11.78913907

13.61936138

8.00000000

9.28504466

10.57008931

11.77830367

13.75832908

8.00000000

9.28504466

10.57008931

11.79724181

13.45837543

8.00000000

9.28504466

10.57008931

11.94455374

13.46327279

8.00000000

9.28504466

10.57008931

11.77721744

13.74980139

8.00000000

9.28504466

10.57008931

11.76967322

13.60903502

8.00000000

9.28504466

10.57008931

11.77537572

13.44440669

8.00000000

9.28504466

10.57008931

11.95340579

13.68234104

8.00000000

9.28504466

10.57008931

11.76891709

13.45014209

13.70317369

13.65335257

13.74681855

13.74920857

13.66466089

13.69415412

13.58810339

13.60516280

$0.00 .00 .021 .14017862672867 "$ Proper 117

4.43966669

3.35654089

6.87944665

./oxygen/Cu/110/iter_9/struct.xyz

54

Lattice="7.710267940093007 $0.00 .0 \quad 0.010 .903965490410 .0 \quad 0.00 .021 .14017862672867 "$ Proper ties=species: $\mathrm{S}: 1:$ pos:R:3 pbc="T T T" 


\section{$\mathrm{Cu}$}

$\mathrm{Cu}$

$\mathrm{Cu}$

$\mathrm{Cu}$

$\mathrm{Cu}$

$\mathrm{Cu}$

$\mathrm{Cu}$

$\mathrm{Cu}$

$\mathrm{Cu}$

$\mathrm{Cu}$

$\mathrm{Cu}$

$\mathrm{Cu}$

$\mathrm{Cu}$

$\mathrm{Cu}$

$\mathrm{Cu}$

$\mathrm{Cu}$

$\mathrm{Cu}$

$\mathrm{Cu}$

$\mathrm{Cu}$

$\mathrm{Cu}$

$\mathrm{Cu}$

$\mathrm{Cu}$

$\mathrm{Cu}$

$\mathrm{Cu}$

$\mathrm{Cu}$

$\mathrm{Cu}$

$\mathrm{Cu}$

$\mathrm{Cu}$

$\mathrm{Cu}$

$\mathrm{Cu}$

$\mathrm{Cu}$

$\mathrm{Cu}$

$\mathrm{Cu}$

$\mathrm{Cu}$

$\mathrm{Cu}$

$\mathrm{Cu}$

$\mathrm{Cu}$

$\mathrm{Cu}$

$\mathrm{Cu}$

$\mathrm{Cu}$

$\mathrm{Cu}$

$\mathrm{Cu}$

$\mathrm{Cu}$

$\mathrm{Cu}$

$\mathrm{Cu}$

O

0

O

0

0

o

0

0

0
0.00000000

1.28504466

0.00000000

1.23116853

$-0.23472064$

0.00000000

1.28504466

0.00000000

1.29994273

0.11524598

0.00000000

1.28504466

0.00000000

1.33185894

0.19139738

2.57008931

3.85513397

2.57008931

3. 74068354

2.43086940

2.57008931

3.85513397

2.57008931

3. 90375225

2. 23306011

2.57008931

3.85513397

2.57008931

3.90387838

2.90663833

5.14017863

6.42522328

5.14017863

6.35692301

5.09101078

5.14017863

6.42522328

5.14017863

6.51136279

5.06065069

5.14017863

6.42522328

5.14017863

6.43160572

5.38792798

0.84224164

5.99293511

4.00952875

$-1.08675940$

4.22603903

1.43874008

3.44407330

6.80743188

1.61571549
Fri Apr 16 14:51:55 2021

0.00000000

1.81732758

0.00000000

1.78860018

$-0.50219902$

3.63465516

5.45198275

3.63465516

5.47552096

2.92307279

7.26931033

9.08663791

7.26931033

9.09602899

7.24001511

0.00000000

1.81732758

0.00000000

1.89953525

0.06392256

3.63465516

5.45198275

3.63465516

5.45680670

4.19908603

7.26931033

9.08663791

7.26931033

9.06746563

7.26523376

0.00000000

1.81732758

0.00000000

1.80539036

0.55137307

3.63465516

5.45198275

3. 63465516

5.43816123

3.85896091

7.26931033

9.08663791

7.26931033

9.07483474

7.26138515

1.07970276

2.14210085

10.00774389

8.64972263

5.79692452

5.85142161

2.85822942

4.56116396

8.64714700
8.00000000

9.28504466

10.57008931

11.79474440

13.61760956

8.00000000

9.28504466

10.57008931

11.78190464

13.73560603

8.00000000

9.28504466

10.57008931

11.78712696

13.64913542

8.00000000

9.28504466

10.57008931

11.90966719

13.45966303

8.00000000

9.28504466

10.57008931

11.75671748

13.71265698

8.00000000

9.28504466

10.57008931

11.89216013

13.53125753

8.00000000

9.28504466

10.57008931

11.78036543

13.63708865

8.00000000

9.28504466

10.57008931

11.90103770

13.68908623

8.00000000

9.28504466

10.57008931

11.76082665

13.44578726

13.66057360

13.73550689

13.58577393

13.71721977

13.68442018

13.76149497

13.62973822

13.61494061

13.65224298

. /oxygen/Cu/111/iter_1/struct.xyz

37

Lattice $=" 7.710267940093008 \quad 0.0 \quad 0.0 \quad 3.855133970046504 \quad 6.67728790610526 \quad 0.0 \quad 0.0 \quad 0.0 \quad 22.295407$

411122603" Properties=species:S:1:pos:R:3 pbc="T T T"

$\mathrm{Cu}$

1.28504466

0.00000000

2.56404781

0.74192088

8.00000000

1.48035369

10.09846914

12.20497039

$-0.06797734$

1.28504466

$-0.03924674$

2. 22576264

14.36386096

8.00000000

2.96768351

10.09846914

2.57008931
3.84943861

3. 71289261

1.28504466

2. 30425611

12.17656566

14.36386096

8.00000000

4.45152527

10.09846914

$\mathrm{Cu}$

2.57008931
3.85513397

5.19344615 
$\mathrm{Cu}$

$\mathrm{Cu}$

$\mathrm{Cu}$

$\mathrm{Cu}$

$\mathrm{Cu}$

$\mathrm{Cu}$

$\mathrm{Cu}$

$\mathrm{Cu}$

$\mathrm{Cu}$

$\mathrm{Cu}$

$\mathrm{Cu}$

$\mathrm{Cu}$

$\mathrm{Cu}$

$\mathrm{Cu}$

$\mathrm{Cu}$

$\mathrm{Cu}$

$\mathrm{Cu}$

$\mathrm{Cu}$

$\mathrm{Cu}$

$\mathrm{Cu}$

$\mathrm{Cu}$

$\mathrm{Cu}$

$\mathrm{Cu}$

$\mathrm{Cu}$

$\mathrm{Cu}$

$\mathrm{Cu}$

0

$. / 0 \times 1$

./oxygen/Cu/111/iter_2/struct.xyz
5.14017863

2.56555435

2.57008931

5.14017863

2.63806666

3.85513397

5.14017863

6.43091865

3.88261335

5.14017863

6.42522328

7.68864679

5.14017863

5.14017863

6.42522328

7.71630944

5.14017863

6.42522328

7.71026794

8.99531260

6.39774390

7.71026794

8.99531260

10.30197840

7.71480290

1.28504466
3.85513397
Fri Apr 16 14:51:55 2021

5.94234315

4.45414353

0.00000000

0.74192088

1.47726532

$-0.03924674$

2. 22576264

2. 96768351

3. 71289261

2. 24162786

4.45152527

5.19344615

5.94785000

4.41979482

0.00000000

0.74192088

1.48035369

$-0.00523652$

2. 22576264

2. 96768351

3.68463844

2.24162786

4.45152527

5.19344615

5.94785000

4.45414353

0.74192088
12.20497039

14.23179987

8.00000000

10.09846914

12.17656566

14.36386096

8.00000000

10.09846914

12.17656566

14.23490919

8.00000000

10.09846914

12.19325672

14.23490919

8.00000000

10.09846914

12.20497039

14.23179987

8.00000000

10.09846914

12.19325672

14.23490919

8.00000000

10.09846914

12.19325672

14.23179987

15.44536110

411122603" Properties=species:S: $1:$ pos:R:3 pbc="T T T"

$\mathrm{Cu} \quad 1.28504466 \quad 0.74192088$

$\begin{array}{rr}1.28504466 & 0.74192088 \\ 2.56255305 & 1.47949069\end{array}$

$\mathrm{Cu} \quad 2.56255305$

$\mathrm{Cu} \quad-0.00648526$

$\mathrm{Cu} \quad 1.28504466$

2.57008931

$-0.00374427$

2.22576264

2.96768351

3.84706652

1.25462199

3.72832661

2.28458558

2.57008931

3.85513397

4.45152527

5.19344615

5.14102442

2. 63021513

5.94687332

4.41179733

2. 57008931

0.00000000

3. 85513397

0.74192088

5.15235882

2. 60582015

3. 85513397

5.14017863

6.43543267

3.88175009

5.14017863

6.42522328

7.71083235

5.15784259

5.14017863

6.42522328

7.72065557

1.46749403

$-0.05575828$

2.22576264

2. 96768351

3. 71549878

2.24112946

4.45152527

5.19344615

5.96348231

4.44468881

0.00000000

0.74192088

1.47882109

0.07193445

2.22576264

6.42522328

7.71026794

2. 96768351

3. 69603554

2.24447831

4.45152527

5.19344615

5.94510523

8.00000000

10.09846914

12.17979265

15.31985247

8.00000000

10.09846914

12.17678203

14.29656950

8.00000000

10.09846914

12.19524781

14.29918681

8.00000000

10.09846914

12.17678203

14.29656950

8.00000000

10.09846914

12.17504886

14.18256500

8.00000000

10.09846914

12.20529520

14.22812006

8.00000000

10.09846914

12.19524781

14.29918681

8.00000000

10.09846914

12.20529520

14.22812006

8.00000000

7.71026794

8.99531260

10.29722431

4.44306737

10.09846914

12.18773723

14.20643264

7.69561843
1.56326594

0.90255201

15.55329688

$\begin{array}{llllll}.67728790610526 & 0.0 & 0.0 & 0.0 & 22.295407\end{array}$

$\mathrm{Cu}$ 
o $\quad 9.98615561$

5.76550963

15.57616197

. /oxygen/Cu/111/iter_3/struct.xyz

39

Lattice $=" 7.710267940093008 \quad 0.0 \quad 0.0 \quad 3.855133970046504 \quad 6.67728790610526 \quad 0.0 \quad 0.0 \quad 0.0 \quad 22.295407$

411122603" Properties=species:S:1:pos:R:3 pbc="T T T"

$\begin{array}{lllr}\mathrm{Cu} & 0.00000000 & 0.00000000 & 8.00000000\end{array}$

$\mathrm{Cu} \quad 1.28504466$

0.74192088

2.57984081

1.48947179

10.09846914

$\mathrm{Cu}$

0.00175904

0.00101558

1.28504466

2.22576264

12.19238945

2. 57008931

2.96768351

15.33057341

$\mathrm{Cu}$

3. 85134700

3.72566893

1. 27455079

3.72566893
2.27257923

2. 57008931

4.45152527

3.85513397

5.19344615

5.13286978

5.19344615

2. 58474999

4.42741650

2. 57008931

0.00000000

3. 85513397

0.74192088

5.15219744

1.47252987

8.00000000

10.09846914

12.18784133

14.28248030

8.00000000

10.09846914

12.20959029

14.27612227

8.00000000

2. 60538674

$-0.03249625$

3. 85513397

2. 22576264

2. 96768351

5.14017863

3. 70859117

6.42346833

2. 21716392

3. 84024056

4. 45152527

6.42522328

5.19344615

7. 70067431

5.96465990

5.15873704

4.52584391

0.00000000

5.14017863

0.74192088

6.42522328

1. 47885684

$\begin{array}{ll}5.12663015 & 0.02475091\end{array}$

6.42522328

2. 22576264

7.71026794

2.96768351

9.01588416

3. 68664963

6.49886432

2.20467537

4.45152527

7.71026794

5.19344615

5.94559296

10.29806909

4.45442173

10.09846914

12.18784133

14.28248030

8.00000000

10.09846914

12.17891664

14.24511206

8.00000000

10.09846914

12.19965629

14.28788930

8.00000000

10.09846914

12.20959029

14.27612227

8.00000000

10.09846914

12.19965629

14.28788930

8.00000000

10.09846914

12.18452673

14.17911166

0.90640713

1.56994321

5.77296070

15.55962991

15.57303620

2.97487758

15.39285373

. /oxygen/Cu/111/iter_4/struct . xyz

40

Lattice $=" 7.710267940093008 \quad 0.0 \quad 0.0 \quad 3.855133970046504 \quad 6.67728790610526 \quad 0.0 \quad 0.0 \quad 0.0 \quad 22.295407$

411122603" Properties=species:S:1:pos:R:3 pbc="T T T"

$\mathrm{Cu}$

$\mathrm{Cu}$

0.00000000

0.00000000

1. 28504466

0.74192088

8.00000000

2. 54595664

1. 48865665

10.09846914

$-0.01752849$

1. 28504466

$-0.10670629$

12.17345469

15.42957846

2.22576264

2. 96768351

3.72907764

3. 84972191

2.18394988

4.45152527

2.57008931

3. 85513397

5.19344615

5. 12450598

2. 59881572

2. 57008931

5.94342567

4.41281655

0.00000000

3. 85513397

0.74192088

1.49009708

5.15923148

2. 61547682

3. 85513397

$-0.03494254$

2.22576264

2. 96768351

3. 72113519

6.42030541

3.72113519
2.18823982

8.00000000

10.09846914

12.17222814

14.38786042

8.00000000

10.09846914

12.17242257

14.27991997

8.00000000

10.09846914

12.18477243

14.19020691

8.00000000

10.09846914

12.20582680

14.17796329 


\section{$\mathrm{Cu}$}

$\mathrm{Cu}$

$\mathrm{Cu}$

$\mathrm{Cu}$

$\mathrm{Cu}$

$\mathrm{Cu}$

$\mathrm{Cu}$

$\mathrm{Cu}$

$\mathrm{Cu}$

$\mathrm{Cu}$

$\mathrm{Cu}$

$\mathrm{Cu}$

$\mathrm{Cu}$

$\mathrm{Cu}$

$\mathrm{Cu}$

$\mathrm{Cu}$

O

O

O

0
5.14017863

6.42522328

7.71999381

5.12085763

5.14017863

6.42522328

7.68130924

5.16708220

6.42522328

7.71026794

9.00787374

6.37135076

7.71026794

8.99531260

10.29964610

7.73211689

1.61855341

9.92808757

4.85521989

7.76894530
4.45152527

5.19344615

5.95342044

4.58025014

0.00000000

0.74192088

1.48646519

0.04798705

2.22576264

2.96768351

3.68601115

2.21831649

4. 45152527

5.19344615

5.93633999

4.55058500

0.64811319

5.76393879

3.08453817

3. 12889589
8.00000000

12.17566580

14.25958743

8.00000000

10.09846914

12.26598725

14.22433382

8.00000000

10.09846914

12.21291242

14.47116206

8.00000000

10.09846914

12.18018654

14.23790998

15.59173646

15.56346181

15.39014987

15.41817252
10.09846914

. /oxygen/Cu/111/iter_5/struct.xyz

41

Lattice $=" 7.710267940093008 \quad 0.0 \quad 0.0 \quad 3.855133970046504 \quad 6.67728790610526 \quad 0.0 \quad 0.0 \quad 0.0 \quad 22.295407$

411122603" Properties=species:S:1:pos:R:3 pbc="T T T"

$\mathrm{Cu}$

$\mathrm{Cu}$

$\mathrm{Cu}$

$\mathrm{Cu}$

$\mathrm{Cu}$

$\mathrm{Cu}$

$\mathrm{Cu}$

$\mathrm{Cu}$

$\mathrm{Cu}$

$\mathrm{Cu}$

$\mathrm{Cu}$

$\mathrm{Cu}$

$\mathrm{Cu}$

$\mathrm{Cu}$

$\mathrm{Cu}$

$\mathrm{Cu}$

$\mathrm{Cu}$

$\mathrm{Cu}$

$\mathrm{Cu}$

$\mathrm{Cu}$

$\mathrm{Cu}$

$\mathrm{Cu}$

$\mathrm{Cu}$

$\mathrm{Cu}$

$\mathrm{Cu}$

$\mathrm{Cu}$

$\mathrm{Cu}$

$\mathrm{Cu}$

$\mathrm{Cu}$

$\mathrm{Cu}$

$\mathrm{Cu}$

$\mathrm{Cu}$

$\mathrm{Cu}$

$\mathrm{Cu}$

$\mathrm{Cu}$

$\mathrm{Cu}$

O

0

0

0

0

2.47454963

0.00000000

1. 28504466

2.56648919

$-0.01355409$

1.28504466

2. 57008931

3. 85420403

0.96441423

2. 57008931

3. 85513397

5.12775757

2. 38191394

2. 57008931

3. 85513397

5.11939647

2. 60562131

3. 85513397

5. 14017863

6.43183377

3. 76365276

5.14017863

6.42522328

7. 71101461

5.21077888

5.14017863

6. 42522328

7. 71174265

5.07048014

6.42522328

7. 71026794

9.01884057

6.34095135

7.71026794

8.99531260

10.28114127

7.79976726

1. 62841674

10.14965530

5.01372260

7.58743627

42

42

Lattice $=" 7.710267940093008$

0.00000000

0.74192088

8.00000000

10.09846914

12.18932008

14.22802618

8.00000000

0.18016359

2. 22576264

2. 96768351

3.71835070

1. 62829560

4.45152527

5.19344615

5.91527502

4.53980127

0.00000000

0.74192088

1. 49961401

$-0.07508999$

2. 22576264

2.96768351

3.70091245

2.23574413

4.45152527

5.19344615

5.91003547

4.73899999

0.00000000

0.74192088

1. 46780059

$-0.02212505$

2. 22576264

2.96768351

3.68684580

2.22119139

4. 45152527

5.19344615

5. 91102711

4. 42564392

$-0.13626031$

6.35416587

2. 95803202

3. 05952375

2.73530545

10.09846914

12.18593160

15.72497997

8.00000000

10.09846914

12.16637371

15.44985988

8.00000000

10.09846914

12.20415337

14.16238460

8.00000000

10.09846914

12.19433025

14.26798223

8.00000000

10.09846914

12.16538458

15.58469451

8.00000000

10.09846914

12.25942105

14.20264135

8.00000000

10.09846914

12.17675684

14.31980574

8.00000000

10.09846914

12.17155266

14.22266855

15.80036171

15.59173323

15.48972825

15.50195207

15.54687869 
411122603" Properties=species:S:1:pos:R:3 pbc="T T T"

$\mathrm{Cu} \quad 1.28504466$ 0.74192088 8.00000000 2.57008931 1.47616433 10.09846914

$\begin{array}{lr}\mathrm{Cu} & 2.57008931 \\ \mathrm{Cu} & -0.06479336\end{array}$

$\mathrm{Cu} \quad 1.28504466$ $-0.07510654$ 2.22576264

.57008931

2.96768351

3.93845549

3.68075547

0.71601851

1.23449776

2.57008931

4.45152527

3.85513397

5.19344615

5.18056806

5.93075532

2.57008931

2.92324572

2.57008931

0.00000000

3.85513397

0.74192088

5.15235514

1. 51258304

2.57008931

3. 85513397

$-0.08450092$

5.14017863

2. 22576264

6.42522328

2. 96768351

3. 72809422

4.42416012

1.23449776

4. 45152527

. 14017863

5.19344615

5.93075532

7. 66987850

4.44487327

0.00000000

5.14017863

0.74192088

1.51258304

6.42522328

$-0.07510654$

5.20497199

2. 22576264

6.42522328

2. 96768351

3.68075547

8.91199108

2.15446407

6.42522328

4.45152527

5.19344615

12.21160828

14.08364359

8.00000000

10.09846914

12.20074408

15.93217247

8.00000000

10.09846914

12.17060992

14.41740918

8.00000000

10.09846914

12.21501975

14.31055841

8.00000000

10.09846914

12.34646430

15.93217247

8.00000000

10.09846914

12.17060992

14.62906684

8.00000000

10.09846914

12.21501975

14.08364359

8.00000000

10.09846914

12.20074408

14.42027743

8.00000000

8.99531260

6.00790449

4.44487327

10.28035725

$-0.63122796$

10.09846914

12.05719428

14.62906684

15.65583412

4.48875644

2.89741652

2.89741652

13.43064138

15.67392691

15.67392691

7.07808656
7.77236001

1.54804353

15.63544789

2.57008931

$-0.63122796$

15.65583412

. /oxygen/Cu/111/iter_7/struct.xyz

43

Lattice $=" 7.710267940093008 \quad 0.0 \quad 0.0 \quad 3.855133970046504$

411122603" Properties=species:S:1:pos:R:3 pbc="T T T"

$\mathrm{Cu} \quad 0.00000000$

$\mathrm{Cu} \quad 1.28504466$

0.00000000

0.74192088

2.57969280

1.44208750

8.00000000

3.91887378

6.56798436

1.28504466

2.22576264

2.57008931

3.88220760

2. 96768351

3. 72047824

8.38595827

1.97341770

2.57008931

4. 45152527

3.85513397

5.19344615

5.92860972

5.15549791

4.33055217

0.00000000

0.74192088

1. 50622230

6.65667255

2. 22576264

2.96768351

3. 71275588

2.23982745

4.45152527

10.09846914

12.15941810

14.38928690

8.00000000

10.09846914

12.22187096

15.83870889

8.00000000

10.09846914

12.24510131

14.63992549

8.00000000

10.09846914

12.20399627

14.45440678

8.00000000

10.09846914

12.16806517

14.40908082

8.00000000

$6.67728790610526 \quad 0.0 \quad 0.0 \quad 0.0 \quad 22.295407$

4.03951981 
$\mathrm{Cu}$

$\mathrm{Cu}$

$\mathrm{Cu}$

$\mathrm{Cu}$

$\mathrm{Cu}$

$\mathrm{Cu}$

$\mathrm{Cu}$

$\mathrm{Cu}$

$\mathrm{Cu}$

$\mathrm{Cu}$

$\mathrm{Cu}$

$\mathrm{Cu}$

$\mathrm{Cu}$

$\mathrm{Cu}$

$\mathrm{Cu}$

O

O

0

0

O

0

0
6.42522328

7.70475638

5.22593967

5.14017863

6.42522328

7.71149237

5.20913934

6.42522328

7.71026794

8.95702730

6.52747218

7.71026794

8.99531260

10.30743800

7.71366129

1.31053674

10.23279918

4.72862195

7.30732120

2.15707907

3.96010629

3.54563814
Fri Apr 16 14:51:55 2021

5.19344615

5.96751400

4.40822054

0.00000000

0.74192088

1.44069051

0.02368821

2.22576264

2.96768351

3.74658742

2.24426968

4.45152527

5.19344615

5.92540185

4.55692408

0.30883367

5.92478412

4.12131681

3.39928422

2.75290549

0.69913800

3.37515916
10.09846914

12.18062234

14.29631243

8.00000000

10.09846914

12.17977588

14.25297722

8.00000000

10.09846914

12.14059278

14.23371946

8.00000000

10.09846914

12.22485283

14.19000703

15.81279216

15.51412706

16.17722155

15.60324458

13.61142457

15.47157615

16.05544629

44

Lattice $=" 7.710267940093008 \quad 0.0 \quad 0.0 \quad 3.855133970046504 \quad 6.67728790610526 \quad 0.0 \quad 0.0 \quad 0.0 \quad 22.295407$

411122603" Properties=species:S:1:pos:R:3 pbc="T T T"

$\mathrm{Cu} \quad 0.00000000 \quad 0.00000000 \quad 8.00000000$

Cul 1.28504466

$28504466 \quad 0.74192088$

1.49105743

8.00000000

2.57008931

10.09846914

0.13883719

1.28504466

$-0.07213542$

12.21941943

$\mathrm{Cu}$

2. 22576264

14.20705504

8.00000000

2. 57008931

10.09846914

3.85043524

2.96768351

1.16012596

3. 72561047

2.29746580

2. 57008931

12.16544907

14.20582812

4.45152527

3.85513397

5.19344615

8.00000000

5.17533991

5.95122128

2. 57008931

10.09846914

12.17442540

4.53940559

0.00000000

2.57008931

3. 85513397

0.74192088

1. 47650957

5.14985410

0.09647120

2.22576264

3. 85513397

2.96768351

3. 64420296

6.42522328

3. 98005266

2.29746580

5.14017863

4.45152527

6.42522328

5.19344615

7.67510666

5. 95122128

5.20127304

4.44622866

5.14017863

6.42522328

0.00000000

0.74192088

1.47650957

7.70059246

5.00134143

6.42522328

$-0.07213542$

2.22576264

7.71026794

2.96768351

9.00001132

3.72561047

6.42522328

2.08489614

7.71026794

4.45152527

5.19344615

5.92848617

10.28035725

7.64917353

1.19244051

4.44622866

0.06274311

15.08139192

8.00000000

10.09846914

12.19887869

14.54968503

8.00000000

10.09846914

12.20607721

14.20582812

8.00000000

10.09846914

12.17442540

14.17154321

8.00000000

10.09846914

12.19887869

14.20705504

8.00000000

10.09846914

12.16544907

14.63042785

8.00000000

10.09846914

12.18956426

14.17154321

16.04683801

15.39418562

16.06069419

16.06069419

15.34501241

5.00584944

6.30933117
2.20772413

2.20772413

2.78076168

16.04683801

2.57008931

0.06274311 


$\begin{array}{llll}0 & 4.48325275 & 1.12727701 & 16.84080169 \\ 0 & 0.65692587 & 1.12727701 & 16.84080169\end{array}$

. /oxygen/Cu/111/iter_9/struct.xyz

45

Lattice $=" 7.710267940093008 \quad 0.0 \quad 0.0 \quad 3.855133970046504 \quad 6.67728790610526 \quad 0.0 \quad 0.0 \quad 0.0 \quad 22.295407$

411122603" Properties=species:S:1:pos:R:3 pbc="T T T"

$\mathrm{Cu}$

$\mathrm{Cu}$

$\mathrm{Cu}$

$\mathrm{Cu}$

$\mathrm{Cu}$

$\mathrm{Cu}$

$\mathrm{Cu}$

$\mathrm{Cu}$

$\mathrm{Cu}$

$\mathrm{Cu}$

$\mathrm{Cu}$

$\mathrm{Cu}$

$\mathrm{Cu}$

$\mathrm{Cu}$

$\mathrm{Cu}$

$\mathrm{Cu}$

$\mathrm{Cu}$

$\mathrm{Cu}$

$\mathrm{Cu}$

$\mathrm{Cu}$

$\mathrm{Cu}$

$\mathrm{Cu}$

$\mathrm{Cu}$

$\mathrm{Cu}$

$\mathrm{Cu}$

$\mathrm{Cu}$

$\mathrm{Cu}$

$\mathrm{Cu}$

$\mathrm{Cu}$

$\mathrm{Cu}$

$\mathrm{Cu}$

$\mathrm{Cu}$

$\mathrm{Cu}$

$\mathrm{Cu}$

$\mathrm{Cu}$

$\mathrm{Cu}$

O

0

O

O

O

O

O

O

0

.$/ 0 x$

0.00000000

1. 28504466

2. 57008931

0.03541686

1.28504466

2. 57008931

3. 86460106

1. 15000764

2. 57008931

3. 85513397

5. 14614135

2.57008931

2. 57008931

3. 85513397

5.16161476

2. 57008931

3. 85513397

5. 14017863

6.42522328

3. 99017099

5.14017863

6.42522328

7. 70430521

5.13408246

5.14017863

6.42522328

7. 68883181

5.10476177

6.42522328

7. 71026794

8.98584551

6.42522328

7. 71026794

8.99531260

10.28035725

7.71636411

0.76528876

10.28035725

5.14892150

7.70152507

2. 57008931

4. 37488987

4. 92113728

7. 92930929

6.42522328

84

Lattice $=" 10.280357253457344 \quad 0.0 \quad 0.0 \quad 5.140178626728672 \quad 8.90305054147368 \quad 0.0 \quad 0.0 \quad 0.022 .29540$ 7411122603" Properties=species:S:1:pos:R:3 pbc="T T T"

0

0

0

O

O

0

O

$\mathrm{Cu}$

$\mathrm{Cu}$

$\mathrm{Cu}$

$\mathrm{Cu}$

$\mathrm{Cu}$

3. 85513397

1. 28504466

6.42522328

3. 85513397

8. 99531260

6.42522328

11.56540191

8.99531260

1.28504466

2. 54295766

5. 16731028

3.85513397

5. 11304697

15.70431745

17.91465061

15.70431745

17.91465061

15.70431745

17.91465061

15.70431745

17.91465061

16.79598719

16.79598719

16.79598719

16.79598719

16.79598719 
$\mathrm{Cu}$

$\mathrm{Cu}$

$\mathrm{Cu}$

$\mathrm{Cu}$

$\mathrm{Cu}$

$\mathrm{Cu}$

$\mathrm{Cu}$

$\mathrm{Cu}$

$\mathrm{Cu}$

$\mathrm{Cu}$

$\mathrm{Cu}$

$\mathrm{Cu}$

$\mathrm{Cu}$

$\mathrm{Cu}$

$\mathrm{Cu}$

$\mathrm{Cu}$

$\mathrm{Cu}$

$\mathrm{Cu}$

$\mathrm{Cu}$

$\mathrm{Cu}$

$\mathrm{Cu}$

$\mathrm{Cu}$

$\mathrm{Cu}$

$\mathrm{Cu}$

$\mathrm{Cu}$

$\mathrm{Cu}$

$\mathrm{Cu}$

$\mathrm{Cu}$

$\mathrm{Cu}$

$\mathrm{Cu}$

$\mathrm{Cu}$

$\mathrm{Cu}$

$\mathrm{Cu}$

$\mathrm{Cu}$

$\mathrm{Cu}$

$\mathrm{Cu}$

$\mathrm{Cu}$

$\mathrm{Cu}$

$\mathrm{Cu}$

$\mathrm{Cu}$

$\mathrm{Cu}$

$\mathrm{Cu}$

$\mathrm{Cu}$

$\mathrm{Cu}$

$\mathrm{Cu}$

$\mathrm{Cu}$

$\mathrm{Cu}$

$\mathrm{Cu}$

$\mathrm{Cu}$

$\mathrm{Cu}$

$\mathrm{Cu}$

$\mathrm{Cu}$

$\mathrm{Cu}$

$\mathrm{Cu}$

$\mathrm{Cu}$

$\mathrm{Cu}$

$\mathrm{Cu}$

$\mathrm{Cu}$

$\mathrm{Cu}$

$\mathrm{Cu}$

$\mathrm{Cu}$

$\mathrm{Cu}$

$\mathrm{Cu}$

$\mathrm{Cu}$

$\mathrm{Cu}$

$\mathrm{Cu}$

$\mathrm{Cu}$

$\mathrm{Cu}$
7.73739960

6.42522328

7.68313628

10.30748891

8.99531260

10.25322560

12.87757823

$-0.00175227$

1.28504466

2.55972253

$-0.03309251$

1.28504466

2.56735605

3.85513397

1.28504466

2.56833705

3.85513397

5.12981184

2. 53699680

3.85513397

5.13744536

6.42522328

3. 85513397

2. 57184158

3.85513397

5.15054541

2.60318183

3. 85513397

5.14291189

6.42522328

3. 85513397

5.14193089

6.42522328

7.72063473

5.17327114

6.42522328

7.71300120

8.99531260

6.42522328

5.13842636

6.42522328

7.69990116

5.10708611

6.42522328

7.70753468

8.99531260

6.42522328

7.70851567

8.99531260

10.26999047

7.67717543

8.99531260

10.27762399

11.56540191

8.99531260

7.71202021

8.99531260

10.29072404

7.74336045

8.99531260

10.28309052

11.56540191

8.99531260

10.28210952

11.56540191

12.86081335

10.31344977

11.56540191
Fri Apr 16 14:51:55 2021

125

7.40354431

0.77324982

2. 95201904

2. 95201904

5.22477509

7.40354431

7.40354431

0.00101167

0.74507698

1.48982702

0.01910597

2. 22576264

2.96610546

3.70960439

2.22576264

4.45253694

5.19660225

5.94135229

4.47063124

6.67728791

7.41763073

8.16112966

6.67728791

0.00101167

0.74192088

1.48982702

0.01910597

2.22373929

2.96610546

3. 69763386

2.18755069

4.45253694

5.19344615

5.94135229

4.47063124

6.67526456

7.41763073

8.14915913

6.63907596

0.00101167

0.74507698

1.48982702

0.01910597

2.22576264

2.96610546

3.70960439

2.22576264

4.45253694

5.19660225

5.94135229

4.47063124

6.67728791

7.41763073

8.16112966

6.67728791

0.00101167

0.74192088

1. 48982702

0.01910597

2.22373929

2. 96610546

3. 69763386

2.18755069

4.45253694

5.19344615

5.94135229

4.47063124

6.67526456
16.79598719

16.79598719

16.79598719

16.79598719

16.79598719

16.79598719

16.79598719

7.95705517

10.03486167

12.13028487

14.15909021

7.95184764

10.03486167

12.08476815

14.30502918

7.95705517

10.03486167

12.13028487

14.15909021

7.95184764

10.03486167

12.08476815

14.30502918

7.95705517

10.04232354

12.13028487

14.15909021

7.95705517

10.03486167

12.13028487

14.15909021

7.95705517

10.04232354

12.13028487

14.15909021

7.95705517

10.03486167

12.13028487

14.15909021

7.95705517

10.03486167

12.13028487

14.15909021

7.95184764

10.03486167

12.08476815

14.30502918

7.95705517

10.03486167

12.13028487

14.15909021

7.95184764

10.03486167

12.08476815

14.30502918

7.95705517

10.04232354

12.13028487

14.15909021

7.95705517

10.03486167

12.13028487

14.15909021

7. 95705517

10.04232354

12.13028487

7.95705517
14.15909021 
$\mathrm{Cu} \quad 12.85317983$

$\mathrm{Cu} \quad 14.13549122$

$\mathrm{Cu} \quad 11.56540191$

.$/ 0 x$
Fri Apr 16 14:51:55 2021

126

85

Lattice $=" 10.280357253457344 \quad 0.0 \quad 0.0 \quad 5.140178626728672 \quad 8.90305054147368 \quad 0.0 \quad 0.0 \quad 0.0 \quad 22.29540$

$7411122603 "$ Properties=species:S:1:pos:R:3 pbc="T T T"

$0 \quad 1.2848$

2.22563234

17.98502693

6.42517755

8.18998729

3. 81963062

6.69753997

15.75712971

9. 02028086

3. 69512753

17.92834257

6.42500912

2.18488513

11.56521438

9.03058724

6.42524459

8.16101845

6.69764698

15.75712429

17.92833807

15.66104942

17.92857090

5.19345645

0.78250252

1. 28465039

2.94696883

2.53422058

5.14595706

3.82425556

5.10342790

2.92869474

15.31986775

16.85482010

16.85417670

16.84497681

5.21793680

7. 43288046

16.84497636

7.74668564

7.43317822

6.42493582

0.77520497

7.70373715

2. 92901078

2. 94704441

5. 21798714

9.02562601

10.25117224

12.87912902

7. 40235235

7.40231779

0.00460287

0.74681979

1.28512814

2. 55803197

$-0.01879353$

1.49086498

0.02187295

2. 22580587

2.96530251

3. 70711352

2. 22581358

4.44996152

5.19647078

5.94323899

4.45710778

6.67948051

7.42661574

8.16623410

6.68872388

0.00458868

0.74340508

1.49824498

0.02180530

2. 22285189

2.95741164

3.69395693

2.19851056

4.45006365

5.19348378

5.94321626

4.44149028

6.68034231

7.42657815

8.14999358

6.69746718

0.00107708

0.74794389

1.49824154

0.02745797

2. 22151986

2. 95740787

3. 70710843

16.84481785

16.84528288

16.75684131

16.84481950

16.85480696

16.84527819

16.75680209

16.75683766

7.95506500

10.03958926

12.15008586

14.19196781

7.95218228

10.03958329

12.08453562

14.33873690

7.95506280

10.01908680

12.07823242

14.19191964

7.94645587

10.01910946

12.08457060

14.30570998

7.95509776

10.05691467

12.14840668

14.19208886

7.95506294

10.01908567

12.07823274

14.19192067

7. 94545158

10.00498646

12.07830256

14.08511093

7.94549607

10.01917281

12.14844963

14.08511503

7.97270967

10.05393526

12.14842246

14.17331034

7.94645391

10.01910741

12.08457274

14.30570790 


\section{$\mathrm{Cu}$}

$\mathrm{Cu}$

$\mathrm{Cu}$

$\mathrm{Cu}$

$\mathrm{Cu}$

$\mathrm{Cu}$

$\mathrm{Cu}$

$\mathrm{Cu}$

$\mathrm{Cu}$

$\mathrm{Cu}$

$\mathrm{Cu}$

$\mathrm{Cu}$

$\mathrm{Cu}$

$\mathrm{Cu}$

$\mathrm{Cu}$

$\mathrm{Cu}$

$\mathrm{Cu}$

$\mathrm{Cu}$

$\mathrm{Cu}$

$\mathrm{Cu}$

$\mathrm{Cu}$

$\mathrm{Cu}$

$\mathrm{Cu}$

$\mathrm{Cu}$

.$/ 0 x y$

. / oxygen/Cu/111/p4-OCu3/struct. xyz
Fri Apr 16 14:51:55 2021

127

4.45006143

5.19645578

5.93220877

4.44144411

6.67946340

7. 41626115

8.16118007

6.68866397

0.00107286

0.74341931

1.49088369

0.02746314

2. 22285232

2. 96530102

3. 69564450

2.19854219

4.44996248

5.19059640

5.93220938

4.45699250

6.67526366

7.41625937

8.14999952

6.62250913
7.94549431

10.01917233

12.14845002

14.08511750

7.94647722

10.05394229

12.10702821

14.30569611

7.97273181

10.05692316

12.15008403

14.17345902

7.95506428

10.03958733

12.15008424

14.19196406

10.05691536

12.14840832

14.19208693

7.97271035

10.05393709

12.14842377

14.17331137
7.95509742

7411122603" Properties=species:S:1:pos:R:3 pbc="T T T"

$\begin{array}{lrlr}\mathrm{O} & 3.85513397 & 3.70960439 & 15.81838703 \\ \mathrm{O} & 1.03568575 & 2.08179520 & 17.51019178 \\ \mathrm{O} & 7.05857266 & 7.79546523 & 15.85206104 \\ \mathrm{O} & 3.85513397 & 6.96522277 & 17.51019178 \\ \mathrm{O} & 8.99531260 & 4.44093325 & 15.85206104 \\ \mathrm{O} & 6.67458219 & 2.08179520 & 17.51019178 \\ \mathrm{O} & 10.93205254 & 7.79546523 & 15.85206104 \\ \mathrm{Cu} & 1.43115305 & 0.59075120 & 16.50291324 \\ \mathrm{Cu} & 2.44506694 & 2.89550181 & 16.68571487 \\ \mathrm{Cu} & 5.26520100 & 2.89550181 & 16.68571487 \\ \mathrm{Cu} & 3.85513397 & 5.33780955 & 16.68571487 \\ \mathrm{Cu} & 5.34414961 & 7.36826004 & 16.50291324 \\ \mathrm{Cu} & 6.27911489 & 0.59075120 & 16.50291324 \\ \mathrm{Cu} & 7.76813053 & 3.16980193 & 16.50291324 \\ \mathrm{Cu} & 10.22249467 & 3.16980193 & 16.50291324 \\ \mathrm{Cu} & 12.64647559 & 7.36826004 & 16.50291324 \\ \mathrm{Cu} & -0.00292259 & 0.00168736 & 8.02761708 \\ \mathrm{Cu} & 1.28428973 & 0.74595341 & 10.10192876 \\ \mathrm{Cu} & 2.56232740 & 1.48330579 & 12.19714221 \\ \mathrm{Cu} & -0.03190921 & 0.01842279 & 14.27788821 \\ \mathrm{Cu} & 1.28644911 & 2.22657350 & 8.01934725 \\ \mathrm{Cu} & 2.56711371 & 2.96596555 & 10.10420794 \\ \mathrm{Cu} & 3.85513397 & 3.70960439 & 12.15894489 \\ \mathrm{Cu} & 1.27038264 & 2.21729752 & 14.32519453 \\ \mathrm{Cu} & 2.56913200 & 4.45207798 & 8.02469240 \\ \mathrm{Cu} & 3.85513397 & 5.19688208 & 10.10420794 \\ \mathrm{Cu} & 5.13676183 & 5.94235702 & 12.19714221 \\ \mathrm{Cu} & 2.53043001 & 4.4742258 & 14.21989058 \\ \mathrm{Cu} & 3.85513397 & 6.67566618 & 8.01934725 \\ \mathrm{Cu} & 5.13630889 & 7.41784631 & 10.10192876 \\ \mathrm{Cu} & 6.41838344 & 8.16507865 & 12.15651599 \\ \mathrm{Cu} & 3.85513397 & 6.69421814 & 14.32519453 \\ \mathrm{Cu} & 2.57113109 & 0.00143403 & 8.02133067 \\ \mathrm{Cu} & 3.85513397 & 0.74518099 & 10.09684040 \\ \mathrm{Cu} & 5.14794054 & 1.48330579 & 12.19714221 \\ \mathrm{Cu} & 2.57216138 & 0.01084997 & 14.24084609 \\ \mathrm{Cu} & 3.85513397 & 2.22465723 & 8.02469240 \\ \mathrm{Cu} & 5.14315423 & 2.96596555 & 10.10420794 \\ \mathrm{Cu} & 6.42956840 & 3.70315037 & 12.19714221 \\ \mathrm{Cu} & 3.85513397 & 2.17996802 & 14.21989058\end{array}$




\section{$\mathrm{Cu}$}

$\mathrm{Cu}$

$\mathrm{Cu}$

$\mathrm{Cu}$

$\mathrm{Cu}$

$\mathrm{Cu}$

$\mathrm{Cu}$

$\mathrm{Cu}$

$\mathrm{Cu}$

$\mathrm{Cu}$

$\mathrm{Cu}$

$\mathrm{Cu}$

$\mathrm{Cu}$

$\mathrm{Cu}$

$\mathrm{Cu}$

$\mathrm{Cu}$

$\mathrm{Cu}$

$\mathrm{Cu}$

$\mathrm{Cu}$

$\mathrm{Cu}$

$\mathrm{Cu}$

$\mathrm{Cu}$

$\mathrm{Cu}$

$\mathrm{Cu}$

$\mathrm{Cu}$

$\mathrm{Cu}$

$\mathrm{Cu}$

$\mathrm{Cu}$

$\mathrm{Cu}$

$\mathrm{Cu}$

$\mathrm{Cu}$

$\mathrm{Cu}$

$\mathrm{Cu}$

$\mathrm{Cu}$

$\mathrm{Cu}$

$\mathrm{Cu}$

$\mathrm{Cu}$

$\mathrm{Cu}$

$\mathrm{Cu}$

$\mathrm{Cu}$

. /

33

en/Cu/210/iter_1/struct.xyz
Fri Apr 16 14:51:55 2021

128

4.45207798

8.02469240

5.19181610

5.93282743

4.47442258

6.67566869

7.42002325

8.16620886

6.67006846

0.00143403

0.74595341

1.48639067

0.01084997

2.22657350

2. 96501346

3. 70170642

2.21729752

4.45171046

5.19181723

5.93282743

4.44789475

6.67728791

7.42002325

8.16507865

6.67728791

0.00168736

0.74192088

1. 48639067

0.01842279

2. 22238792

2. 96501346

3.70315037

2.18891706

4.45171046

5.19181610

5.94235702

4.44789475

6.67566869

7.41784631

8.15603184

6.67006846
10.09684040

12.17566362

14.21989058

8.02133067

10.09343286

12.17566362

14.24084609

8.02133067

10.10192876

12.20576929

14.24084609

8.01934725

10.10192876

12.15651599

14.32519453

8.02133067

10.09343286

12.17566362

14.24084609

8.01649048

10.09343286

12.15651599

14.20380202

8.02761708

10.11484310

12.20576929

14.27788821

8.02761708

10.10192876

12.19714221

14.27788821

8.02133067

10.09684040

12.19714221

14.24084609

8.02133067

10.10192876

12.20576929

14.24084609

Lattice $=" 7.269310326940001 \quad 0.0 \quad 0.0 \quad 3.6346551634699993 \quad 8.127336020289532 \quad 0.0 \quad 0.0 \quad 0.021 .6891$ 35214202672" Properties=species:S:1:pos:R:3 pbc="T T T"

$\begin{array}{llll}\mathrm{Cu} & 1.81732758 & 3.25093441 & 8.00000000\end{array}$

$\mathrm{Cu} \quad 3.63465516 \quad 1.62546720$

$1.62546720 \quad 8.81273360$

$1.81732758 \quad 0.00000000$

9.62546720

$1.81732758 \quad 2.43820081$

10.43820081

0.81998578

3.63965906

3.25786876

1.73641496

1.81462670

11.26695014

12.08169102

12.89389234

13.64940009

8.00000000

8.81273360

9.62546720

10.43820081

11.23706343

12.03611905

12.84054513

13.54472907

8.00000000

8.81273360

9.62546720

10.43820081

11.26632540

12.08094964

12.87177418

13.64860042 


\section{$\mathrm{Cu}$}

$\mathrm{Cu}$

$\mathrm{Cu}$

$\mathrm{Cu}$

$\mathrm{Cu}$

$\mathrm{Cu}$

$\mathrm{Cu}$

$\mathrm{Cu}$

$\mathrm{O}$

. /

42 (10/struct.xyz

$\begin{array}{llr}7.26931033 & 7.31460242 & 8.00000000 \\ 9.08663791 & 5.68913521 & 8.81273360 \\ 7.26931033 & 4.06366801 & 9.62546720 \\ 7.26931033 & 6.50186882 & 10.43820081 \\ 9.08408539 & 4.90091716 & 11.22420186 \\ 9.08344444 & 7.31514066 & 12.19422356 \\ 7.25146202 & 5.69272697 & 12.84101975 \\ 5.44802709 & 4.06310768 & 13.55225648 \\ 1.81346974 & 0.38305024 & 14.28055681\end{array}$
35214202672" Properties=species:S:1:pos:R:3 pbc="T T T"

$\begin{array}{llll}\mathrm{Cu} & 1.81732758 & 3.25093441 & 8.00000000\end{array}$

$\begin{array}{llll}\mathrm{Cu} & 3.63465516 & 1.62546720 & 8.81273360\end{array}$

$\begin{array}{llll}\mathrm{Cu} & 1.81732758 & 0.00000000 & 9.62546720\end{array}$

$\begin{array}{llll}\mathrm{Cu} & 1.81732758 & 2.43820081 & 10.43820081\end{array}$

$\begin{array}{llll}\mathrm{Cu} & 3.63896882 & 0.76272384 & 11.18103952\end{array}$

$\begin{array}{llll}\mathrm{Cu} & 3.64342240 & 3.01267128 & 12.15483184\end{array}$

$\begin{array}{llll}\mathrm{Cu} & 1.63337294 & 2.00273238 & 13.97461365\end{array}$

$\mathrm{Cu} \quad 0.02412258 \quad-0.61923725$

$\mathrm{Cu} \quad 3.63465516 \quad 7.31460242$

$5.45198275 \quad 5.68913521$

$3.63465516 \quad 4.06366801$

$3.63465516 \quad 6.50186882$

$5.36180301 \quad 4.86745147$

$\begin{array}{ll}5.51325505 & 7.28322340\end{array}$

$3.82473241 \quad 5.40862922$

$3.61633538 \quad 3.70062446$

$\begin{array}{ll}5.45198275 & 3.25093441\end{array}$

$7.26931033 \quad 1.62546720$

$5.45198275 \quad 0.00000000$

$\begin{array}{ll}5.45198275 & 2.43820081\end{array}$

$7.27837654 \quad 0.89751815$

$\begin{array}{ll}7.19499249 & 3.55287605\end{array}$

$5.71070265 \quad 1.97466650$

$3.63925209 \quad-0.01941499$

$\begin{array}{ll}7.26931033 & 7.31460242\end{array}$

$9.08663791 \quad 5.68913521$

$7.26931033 \quad 4.06366801$

$7.26931033 \quad 6.50186882$

$9.15100636 \quad 4.86351316$

$9.07898119 \quad 7.38388082$

$8.33766426 \quad 5.19555209$

$5.97834867 \quad 5.22782116$

$1.73162058 \quad 0.09294652$

$5.57043464 \quad 0.10804342$

$3.61785238 \quad 1.98890340$

$1.78725199 \quad 3.55470054$

$\begin{array}{ll}5.73075116 & 5.36126701\end{array}$

$9.19745592 \quad 5.55188872$

$7.23918855 \quad 6.57000247$

$5.42915724 \quad 3.47186564$

$7.20911206 \quad 2.04861154$

$4.01782628 \quad 5.70930081$

14.53012334

8.00000000

8.81273360

9.62546720

10.43820081

11.11799533

12.11599911

13.07645051

15.15822949

8.00000000

8.81273360

9. 62546720

10.43820081

11.39626419

12.00393944

14.34989068

14.22568153

8.00000000

8.81273360

9.62546720

10.43820081

11.22643432

12.17019903

14.78705751

15.23495266

13.86874236

14.02487355

13.91184706

15.03041123

12.90075224

13.01733661

15.26964656

15.41358421

13.12502316

15.14407906

. /oxygen/Cu/210/iter_11/struct. xyz

43

Lattice $=" 7.269310326940001 \quad 0.0 \quad 0.0 \quad 3.6346551634699993 \quad 8.127336020289532 \quad 0.0 \quad 0.0 \quad 0.0 \quad 21.6891$ $35214202672 "$ Properties=species:S:1:pos:R:3 pbc="T T T"

$\begin{array}{lllr}\mathrm{Cu} & 1.81732758 & 3.25093441 & 8.00000000 \\ \mathrm{Cu} & 3.63465516 & 1.62546720 & 8.81273360 \\ \mathrm{Cu} & 1.81732758 & 0.00000000 & 9.62546720 \\ \mathrm{Cu} & 1.81732758 & 2.43820081 & 10.43820081 \\ \mathrm{Cu} & 3.63465516 & 0.84249190 & 11.15060087 \\ \mathrm{Cu} & 3.63465516 & 3.18422140 & 11.98133900 \\ \mathrm{Cu} & 1.84798648 & 2.72078734 & 14.24031422 \\ \mathrm{Cu} & 7.26931033 & 0.42063457 & 14.41440874 \\ \mathrm{Cu} & 3.63465516 & 7.31460242 & 8.00000000\end{array}$




\section{$\mathrm{Cu}$}

$\mathrm{Cu}$

$\mathrm{Cu}$

$\mathrm{Cu}$

$\mathrm{Cu}$

$\mathrm{Cu}$

$\mathrm{Cu}$

$\mathrm{Cu}$

$\mathrm{Cu}$

$\mathrm{Cu}$

$\mathrm{Cu}$

$\mathrm{Cu}$

$\mathrm{Cu}$

$\mathrm{Cu}$

$\mathrm{Cu}$

$\mathrm{Cu}$

$\mathrm{Cu}$

$\mathrm{Cu}$

$\mathrm{Cu}$

$\mathrm{Cu}$

$\mathrm{Cu}$

$\mathrm{Cu}$

$\mathrm{Cu}$

O

O

O

O

O

O

O

O

0

0

0

44

gen/Cu/210/iter_12/struct.xyz
Fri Apr 16 14:51:55 2021

130

5.68913521

4.06366801

6.50186882

4.80177447

7.73455674

5.19909442

6.01360123

3.25093441

1.62546720

0.00000000

2.43820081

0.85901317

3.12833653

2.72078734

0.48801947

7.31460242

5.68913521

4.06366801

6.50186882

4.80177447

7.73455674

5.68277001

6.01360123

0.65802734

0.65802734

2.34636848

5.07770452

5.88939456

5.88939456

7.25463807

4.39138413

2.39917229

6.86086561

4.39138413
8.81273360

9.62546720

10.43820081

11.21398801

12.21263492

13.84736026

15.34995788

8.00000000

8.81273360

9.62546720

10.43820081

11.12671739

12.13324740

14.24031422

14.39736507

8.00000000

8.81273360

9.62546720

10.43820081

11.21398801

12.21263492

12.87471429

15.34995788

13.98726232

13.98726232

13.71189078

14.74241534

12.75391209

12.75391209

15.67426274

14.99700935

13.98517038

15.23588746

14.99700935

attice $=" 7.269310326940001 \quad 0.0 \quad 0.0 \quad 3.6346551634699993 \quad 8.127336020289532 \quad 0.0 \quad 0.0 \quad 0.021 .6891$ 35214202672" Properties=species:S:1:pos:R:3 pbc="T T T"

$\begin{array}{llll}\mathrm{Cu} & 1.81732758 & 3.25093441 & 8.00000000\end{array}$

$\mathrm{Cu} \quad 3.63465516$

1.62546720

8.00000000

0.00000000

8.81273360

1.81732758

2.43820081

9.62546720

1.81732758

0.80339951

10.43820081

3.63465516

3. 31911161

2.08798437

0.00000000

$-0.10412680$

7. 31460242

5.68913521

4.06366801

6.50186882

4.88990882

7.43260995

5.37217971

4.61720502

3.25093441

1. 62546720

0.00000000

2.43820081

0.76466372

3.18557798

2.08798437

$-0.53874803$

7.31460242

5.68913521

4.06366801

6.50186882

11.25851240

11.95598555

14.07245555

14.47964606

8.00000000

8.81273360

9.62546720

10.43820081

11.22261903

12.24963585

13.34779727

15.39985015

8.00000000

8.81273360

9.62546720

10.43820081

11.18397084

12.07981042

14.07245555

14.80732830

8.00000000

8.81273360

9.62546720

10.43820081

11.22261903

4.88990882

12.24963585 


\section{$\mathrm{Cu}$}

$\mathrm{Cu}$

O

O

0

O

O

O

O

O

O

O

O

0
7.26931033

5.77812010

1. 91248992

5. 35682041

3.63465516

7.26931033

5. 42865022

9.10997043

7.26931033

4. 71530825

7.26931033

3.63465516

2. 55400208

3.63465516
Fri Apr 16 14:51:55 2021

5.46479799

4. 61720502

0.08714419

0.08714419

1.75289875

3.43619321

5.59497575

5.59497575

5.79510389

3.16001837

1.30358547

6.88532825

3.16001837

5.54237476
13.20140272

15.39985015

14.25529524

14.25529524

12.88706641

14.28723172

13.06775747

13.06775747

15.57554397

15.17606850

13.05190125

15.97082941

15.17606850

15.55746040

. /oxygen/Cu/210/iter_2/struct.xyz 34

Lattice $=" 7.269310326940001 \quad 0.0 \quad 0.0 \quad 3.6346551634699993 \quad 8.127336020289532 \quad 0.0 \quad 0.0 \quad 0.0 \quad 21.6891$ 35214202672" Properties=species:S:1:pos:R:3 pbc="T T T"

$\begin{array}{lrrr}\mathrm{Cu} & 1.81732758 & 3.25093441 & 8.00000000 \\ \mathrm{Cu} & 3.63465516 & 1.62546720 & 8.81273360 \\ \mathrm{Cu} & 1.81732758 & 0.00000000 & 9.62546720 \\ \mathrm{Cu} & 1.81732758 & 2.43820081 & 10.43820081 \\ \mathrm{Cu} & 3.63465516 & 0.81510413 & 11.27532686 \\ \mathrm{Cu} & 3.63465516 & 3.25979608 & 12.07370841 \\ \mathrm{Cu} & 1.81732758 & 1.70310982 & 12.87978920 \\ \mathrm{Cu} & -0.00000000 & -0.08086452 & 13.83985351 \\ \mathrm{Cu} & 3.63465516 & 7.31460242 & 8.00000000 \\ \mathrm{Cu} & 5.45198275 & 5.68913521 & 8.81273360 \\ \mathrm{Cu} & 3.63465516 & 4.06366801 & 9.62546720 \\ \mathrm{Cu} & 3.63465516 & 6.50186882 & 10.43820081 \\ \mathrm{Cu} & 5.45198275 & 4.88730044 & 11.21700294 \\ \mathrm{Cu} & 5.45198275 & 7.29763557 & 12.10042474 \\ \mathrm{Cu} & 3.63465516 & 5.67257805 & 12.81375149 \\ \mathrm{Cu} & 1.81732758 & 4.13163731 & 13.54139380 \\ \mathrm{Cu} & 5.45198275 & 3.25093441 & 8.00000000 \\ \mathrm{Cu} & 7.26931033 & 1.62546720 & 8.81273360 \\ \mathrm{Cu} & 5.45198275 & 0.00000000 & 9.62546720 \\ \mathrm{Cu} & 5.45198275 & 2.43820081 & 10.43820081 \\ \mathrm{Cu} & 7.26931033 & 0.81510413 & 11.27532686 \\ \mathrm{Cu} & 7.26931033 & 3.25979608 & 12.07370841 \\ \mathrm{Cu} & 5.45198275 & 1.70310982 & 12.87978920 \\ \mathrm{Cu} & 3.63465516 & -0.08086452 & 13.83985351 \\ \mathrm{Cu} & 7.26931033 & 7.31460242 & 8.00000000 \\ \mathrm{Cu} & 9.08663791 & 5.68913521 & 8.81273360 \\ \mathrm{Cu} & 7.26931033 & 4.06366801 & 9.62546720 \\ \mathrm{Cu} & 7.26931033 & 6.50186882 & 10.43820081 \\ \mathrm{Cu} & 9.08663791 & 4.88730044 & 11.21700294 \\ \mathrm{Cu} & 9.08663791 & 7.29763557 & 12.10042474 \\ \mathrm{Cu} & 7.26931033 & 5.67257805 & 12.81375149 \\ \mathrm{Cu} & 5.45198275 & 4.13163731 & 13.54139380 \\ \mathrm{O} & 1.81732758 & 0.17762007 & 14.12407168 \\ \mathrm{O} & 5.45198275 & 0.17762007 & 14.12407168\end{array}$

. /oxygen/Cu/210/iter_3/struct.xyz

35

Lattice $=" 7.269310326940001 \quad 0.0 \quad 0.0 \quad 3.6346551634699993 \quad 8.127336020289532 \quad 0.0 \quad 0.0 \quad 0.021 .6891$ 35214202672 " Properties=species:S:1:pos:R:3 pbc="T T T"

$\begin{array}{lll}\mathrm{Cu} & 1.81732758 & 3.25093441\end{array}$

$\mathrm{Cu} \quad 3.63465516 \quad 1.62546720$

$\mathrm{Cu} \quad 1.81732758 \quad 0.00000000$

$\mathrm{Cu} \quad 1.81732758 \quad 2.43820081$

$\mathrm{Cu} \quad 3.63465516 \quad 0.81450131$

$\begin{array}{lll}\mathrm{Cu} & 3.63465516 & 3.23444440\end{array}$

$\mathrm{Cu} \quad 1.78327011 \quad 1.67982970$

$\mathrm{Cu} \quad-0.00000000 \quad-0.04401541$

$\begin{array}{lll}\mathrm{Cu} & 3.63465516 & 7.31460242\end{array}$

$\mathrm{Cu} \quad 5.45198275 \quad 5.68913521$

$\mathrm{Cu} \quad 3.63465516 \quad 4.06366801$

$\mathrm{Cu} \quad 3.63465516$

4. 06366801

8.00000000

8.81273360

9.62546720

10.43820081

11.25891356

12.06476251

12.85046442

13.82090943

8.00000000

8.81273360

9.62546720

10.43820081 


\section{$\mathrm{Cu}$}

$\mathrm{Cu}$

$\mathrm{Cu}$

$\mathrm{Cu}$

$\mathrm{Cu}$

$\mathrm{Cu}$

$\mathrm{Cu}$

$\mathrm{Cu}$

$\mathrm{Cu}$

$\mathrm{Cu}$

$\mathrm{Cu}$

$\mathrm{Cu}$

$\mathrm{Cu}$

$\mathrm{Cu}$

$\mathrm{Cu}$

$\mathrm{Cu}$

$\mathrm{Cu}$

$\mathrm{Cu}$

$\mathrm{Cu}$

$\mathrm{Cu}$

O

0

0
5.45176481

5.46015845

3. 63465516

1. 77857895

5.45198275

7.26931033

5.45198275

5.45198275

7.26931033

7.26931033

5.48604022

3. 63465516

7.26931033

9.08663791

7.26931033

7.26931033

9.08685584

9.07846221

7.26931033

5.49073137

1. 82215841

5.44715192

3.63465516
4.87979577

7.30325214

5.73064411

4.09628709

3. 25093441

1. 62546720

0.00000000

2. 43820081

0.81620037

3.27273511

1.67982970

$-0.07710261$

7.31460242

5.68913521

4.06366801

6.50186882

4.87979577

7.30325214

5.65420900

4.09628709

0.15479013

0.15479013

4.25752657
11.21753843

12.07661307

12.81058452

13.61309101

8.00000000

8.81273360

9.62546720

10.43820081

11.24826155

12.08757751

12.85046442

13.81393962

8.00000000

8.81273360

9. 62546720

10.43820081

11.21753843

12.07661307

12.80288305

13.61309101

14.09166706

14.09166706

14.09859838

. /oxygen/Cu/210/iter_4/struct.xyz

36

Lattice $=" 7.269310326940001 \quad 0.0 \quad 0.0 \quad 3.6346551634699993 \quad 8.127336020289532 \quad 0.0 \quad 0.0 \quad 0.021 .6891$ 35214202672" Properties=species:S:1:pos:R:3 pbc="T T T"

$\begin{array}{lrrr}\mathrm{Cu} & 1.81732758 & 3.25093441 & 8.00000000 \\ \mathrm{Cu} & 3.63465516 & 1.62546720 & 8.81273360 \\ \mathrm{Cu} & 1.81732758 & 0.00000000 & 9.62546720 \\ \mathrm{Cu} & 1.81732758 & 2.43820081 & 10.43820081 \\ \mathrm{Cu} & 3.63465516 & 0.81361882 & 11.23908503 \\ \mathrm{Cu} & 3.63465516 & 3.25465634 & 12.06431869 \\ \mathrm{Cu} & 1.81475060 & 1.68254998 & 12.81213408 \\ \mathrm{Cu} & -0.00000000 & -0.00909323 & 13.81060735 \\ \mathrm{Cu} & 3.63465516 & 7.31460242 & 8.00000000 \\ \mathrm{Cu} & 5.45198275 & 5.68913521 & 8.81273360 \\ \mathrm{Cu} & 3.63465516 & 4.06366801 & 9.62546720 \\ \mathrm{Cu} & 3.63465516 & 6.50186882 & 10.43820081 \\ \mathrm{Cu} & 5.45302613 & 4.87586218 & 11.23984982 \\ \mathrm{Cu} & 5.45042255 & 7.31991433 & 12.06267461 \\ \mathrm{Cu} & 3.63465516 & 5.74622795 & 12.81225622 \\ \mathrm{Cu} & 1.81647582 & 4.05800475 & 13.80849325 \\ \mathrm{Cu} & 5.45198275 & 3.25093441 & 8.00000000 \\ \mathrm{Cu} & 7.26931033 & 1.62546720 & 8.81273360 \\ \mathrm{Cu} & 5.45198275 & 0.00000000 & 9.62546720 \\ \mathrm{Cu} & 5.45198275 & 2.43820081 & 10.43820081 \\ \mathrm{Cu} & 7.26931033 & 0.81215552 & 11.23962072 \\ \mathrm{Cu} & 7.26931033 & 3.25699784 & 12.06281024 \\ \mathrm{Cu} & 5.45455972 & 1.68254998 & 12.81213408 \\ \mathrm{Cu} & 3.63465516 & -0.00678040 & 13.80882149 \\ \mathrm{Cu} & 7.26931033 & 7.31460242 & 8.00000000 \\ \mathrm{Cu} & 9.08663791 & 5.68913521 & 8.81273360 \\ \mathrm{Cu} & 7.26931033 & 4.06366801 & 9.62546720 \\ \mathrm{Cu} & 7.26931033 & 6.50186882 & 10.43820081 \\ \mathrm{Cu} & 9.08559452 & 4.87586218 & 11.23984982 \\ \mathrm{Cu} & 9.08819811 & 7.31991433 & 12.06267461 \\ \mathrm{Cu} & 7.26931033 & 5.74810713 & 12.81028956 \\ \mathrm{Cu} & 5.45283451 & 4.05800475 & 13.80849325 \\ \mathrm{O} & 1.81736454 & 0.19920197 & 14.09405206 \\ \mathrm{O} & 5.45194579 & 0.19920197 & 14.09405206 \\ \mathrm{O} & 3.63465516 & 4.26298363 & 14.09214343 \\ \mathrm{O} & 7.26931033 & 4.27254899 & 14.09765533\end{array}$

. /oxygen/Cu/210/iter_5/struct.xyz 37

Lattice $=" 7.269310326940001 \quad 0.0 \quad 0.0 \quad 3.6346551634699993 \quad 8.127336020289532 \quad 0.0 \quad 0.0 \quad 0.021 .6891$ 35214202672" Properties=species:S:1:pos:R:3 pbc="T T T"

$\mathrm{Cu}$

1.81732758

3.25093441

8.00000000 


\section{$\mathrm{Cu}$}

$\mathrm{Cu}$

$\mathrm{Cu}$

$\mathrm{Cu}$

$\mathrm{Cu}$

$\mathrm{Cu}$

$\mathrm{Cu}$

$\mathrm{Cu}$

$\mathrm{Cu}$

$\mathrm{Cu}$

$\mathrm{Cu}$

$\mathrm{Cu}$

$\mathrm{Cu}$

$\mathrm{Cu}$

$\mathrm{Cu}$

$\mathrm{Cu}$

$\mathrm{Cu}$

$\mathrm{Cu}$

$\mathrm{Cu}$

$\mathrm{Cu}$

$\mathrm{Cu}$

$\mathrm{Cu}$

$\mathrm{Cu}$

$\mathrm{Cu}$

$\mathrm{Cu}$

$\mathrm{Cu}$

$\mathrm{Cu}$

$\mathrm{Cu}$

$\mathrm{Cu}$

$\mathrm{Cu}$

$\mathrm{Cu}$

0

0

0

0

0

.$/ 0 x$

38

Lattice $=" 7.269310326940001 \quad 0.0 \quad 0.0 \quad 3.6346551634699993 \quad 8.1273360202895320 .0 \quad 0.0 \quad 0.021 .6891$

35214202672" Properties=species:S:1:pos:R:3 pbc="T T T"

$\mathrm{Cu}$

$\mathrm{Cu}$

$\mathrm{Cu}$

$\mathrm{Cu}$

$\mathrm{Cu}$

$\mathrm{Cu}$

$\mathrm{Cu}$

$\mathrm{Cu}$

$\mathrm{Cu}$

$\mathrm{Cu}$

$\mathrm{Cu}$

$\mathrm{Cu}$

$\mathrm{Cu}$

$\mathrm{Cu}$

$\mathrm{Cu}$

$\mathrm{Cu}$

$\mathrm{Cu}$

$\mathrm{Cu}$

$\mathrm{Cu}$

$\mathrm{Cu}$

$\mathrm{Cu}$

$\mathrm{Cu}$

$\mathrm{Cu}$

$\mathrm{Cu}$

$\mathrm{Cu}$

$\mathrm{Cu}$

$\mathrm{Cu}$

$\mathrm{Cu}$
3.63465516

1.81732758

3.63550835

3.65961536

1.81802912

0.08741684

3.63465516

5.45198275

3.63465516

3.63465516

5.45225009

3.64056562

1.81734336

5.45198275

5.45198275

5.45198275

7.27030668

7.24653734

5.45601455

3.56358255

7.26931033

9.08663791

7.26931033

9.08543680

7.25819134

5.45260804

1.82645544

5.45998872

3.61661986

7.28757638

5.44720368
1.81732758

5.45350741

7.26931033

7.26931033

9.08631029
Fri Apr 16 14:51:55 2021

1.62546720

0.00000000

2.43820081

0.82251046

3.28086512

1.72277777

0.26190608

7.31460242

5.68913521

4.06366801

6.50186882

4.87719380

7.37043264

5.77751035

4.05010684

3.25093441

1.62546720

0.00000000

2.43820081

0.82236231

3.28121400

1.66719856

0.26951037

7.31460242

5.68913521

4.06366801

6.50186882

4.91125537

7.33249086

5.78171206

3. 98481067

0.82291014

0.02660642

4.05928962

4.07205784

6.20795887
8.81273360

9.62546720

10.43820081

11.25490205

12.06754939

12.76760245

13.98940223

8.00000000

8.81273360

9.62546720

10.43820081

11.20286096

12.01498168

12.87608025

13.79246085

8.00000000

8.81273360

9.62546720

10.43820081

11.25527249

12.06906189

12.83884158

13.98927896

8.00000000

8.81273360

10.43820081

11.29027764

12.87746705

13.85278522

14.42308746

13.96961048

14.03475713

14.03793721

13.58374027
9.62546720

12.10774633
8.00000000

8.81273360

9.62546720

10.43820081

11.22933420

12.13169163

12.76350846

14.00026874

8.00000000

8.81273360

9.62546720

10.43820081

11.22563108

12.06200641

14.24069331

8.00000000

8.81273360

9. 62546720

10.43820081

11.22818177

12.12317141

12.76350846

13.99817489

8.00000000

8.81273360

9.62546720

10.43820081
13.10101259 


\section{$\mathrm{Cu}$}

$\mathrm{Cu}$

$\mathrm{Cu}$

$\mathrm{Cu}$

0

O

0

O

O

0
9.08632229

9.08745955

9.08656701

7.26931033

5.45159984

1.81737170

5.45193863

3.63465516

7.26931033

5.45229836
Fri Apr 16 14:51:55 2021

4.85874682

7.38609563

5.79946806

3.56887198

0.43329689

0.43329689

3.79341325

3.78106255

5.67997555

5.67997555
11.22563108

12.06200641

13.09973286

14.24069331

14.29146161

14.29146161

14.08921594

14.07874705

13.11051931

13.11051931

39

Lattice $=" 7.269310326940001 \quad 0.0 \quad 0.0 \quad 3.6346551634699993 \quad 8.127336020289532 \quad 0.0 \quad 0.0 \quad 0.0 \quad 21.6891$ 35214202672" Properties=species:S:1:pos:R:3 pbc="T T T"

$\begin{array}{lrlr}\mathrm{Cu} & 1.81732758 & 3.25093441 & 8.00000000 \\ \mathrm{Cu} & 3.63465516 & 1.62546720 & 8.81273360 \\ \mathrm{Cu} & 1.81732758 & 0.0000000 & 9.62546720 \\ \mathrm{Cu} & 1.81732758 & 2.43820081 & 10.43820081 \\ \mathrm{Cu} & 3.63465516 & 0.82090980 & 11.22062179 \\ \mathrm{Cu} & 3.63465516 & 3.24623265 & 12.08417773 \\ \mathrm{Cu} & 1.75454410 & 1.53088316 & 12.72894522 \\ \mathrm{Cu} & 7.26931033 & 1.43240434 & 14.66353348 \\ \mathrm{Cu} & 3.63465516 & 7.31460242 & 8.00000000 \\ \mathrm{Cu} & 5.45198275 & 5.68913521 & 8.81273360 \\ \mathrm{Cu} & 3.63465516 & 4.06366801 & 9.62546720 \\ \mathrm{Cu} & 3.63465516 & 6.50186882 & 10.43820081 \\ \mathrm{Cu} & 5.43563642 & 4.88314393 & 11.27680900 \\ \mathrm{Cu} & 5.52841064 & 7.31777131 & 11.99801621 \\ \mathrm{Cu} & 3.63465516 & 6.11957296 & 13.21455618 \\ \mathrm{Cu} & 1.81033713 & 3.86078476 & 14.09496420 \\ \mathrm{Cu} & 5.45198275 & 3.25093441 & 8.00000000 \\ \mathrm{Cu} & 7.26931033 & 1.62546720 & 8.81273360 \\ \mathrm{Cu} & 5.45198275 & 0.0000000 & 9.62546720 \\ \mathrm{Cu} & 5.45198275 & 2.43820081 & 10.43820081 \\ \mathrm{Cu} & 7.26931033 & 0.78731994 & 11.20699481 \\ \mathrm{Cu} & 7.26931033 & 3.30584184 & 12.08451711 \\ \mathrm{Cu} & 5.51476623 & 1.53088316 & 12.72894522 \\ \mathrm{Cu} & 3.63465516 & 1.48269398 & 14.63547452 \\ \mathrm{Cu} & 7.26931033 & 7.31460242 & 8.00000000 \\ \mathrm{Cu} & 9.08663791 & 5.68913521 & 8.81273360 \\ \mathrm{Cu} & 7.26931033 & 4.06366801 & 9.62546720 \\ \mathrm{Cu} & 7.26931033 & 6.50186882 & 10.43820081 \\ \mathrm{Cu} & 9.10298423 & 4.88314393 & 11.27680900 \\ \mathrm{Cu} & 9.01021002 & 7.31777131 & 11.99801621 \\ \mathrm{Cu} & 7.26931033 & 6.24640804 & 13.97767506 \\ \mathrm{Cu} & 5.45897320 & 3.86078476 & 14.09496420 \\ \mathrm{O} & 1.75938086 & 1.88487499 & 14.66190111 \\ \mathrm{O} & 5.50992946 & 1.88487499 & 14.66190111 \\ \mathrm{O} & 3.63465516 & 3.43973484 & 14.13216235 \\ \mathrm{O} & 7.26931033 & 4.24333229 & 13.91985104 \\ \mathrm{O} & 5.42444836 & 5.80556338 & 13.32843579 \\ \mathrm{O} & 9.11417230 & 5.80556338 & 13.32843579 \\ \mathrm{O} & 7.26931033 & 7.80985669 & 14.88137053 \\ \mathrm{O} & \mathrm{Cu} & & \end{array}$

. /oxygen/Cu/210/iter_8/struct.xyz

40

Lattice $=" 7.269310326940001 \quad 0.0 \quad 0.0 \quad 3.6346551634699993 \quad 8.1273360202895320 .0 \quad 0.0 \quad 0.021 .6891$ $35214202672 "$ Properties=species:S:1:pos:R:3 pbc="T T T"

$\begin{array}{lllr}\mathrm{Cu} & 1.81732758 & 3.25093441 & 8.00000000 \\ \mathrm{Cu} & 3.63465516 & 1.62546720 & 8.81273360 \\ \mathrm{Cu} & 1.81732758 & 0.00000000 & 9.62546720 \\ \mathrm{Cu} & 1.81732758 & 2.43820081 & 10.43820081 \\ \mathrm{Cu} & 3.64619118 & 0.82278740 & 11.20147449 \\ \mathrm{Cu} & 3.58725586 & 3.21792876 & 12.04623067 \\ \mathrm{Cu} & 1.74912213 & 1.65775147 & 12.74980887 \\ \mathrm{Cu} & 7.09815658 & 0.20131226 & 14.22672981 \\ \mathrm{Cu} & 3.63465516 & 7.31460242 & 8.00000000 \\ \mathrm{Cu} & 5.45198275 & 5.68913521 & 8.81273360 \\ \mathrm{Cu} & 3.63465516 & 4.06366801 & 9.62546720\end{array}$


$\mathrm{Cu}$

$\mathrm{Cu}$

$\mathrm{Cu}$

$\mathrm{Cu}$

$\mathrm{Cu}$

$\mathrm{Cu}$

$\mathrm{Cu}$

$\mathrm{Cu}$

$\mathrm{Cu}$

$\mathrm{Cu}$

$\mathrm{Cu}$

$\mathrm{Cu}$

$\mathrm{Cu}$

$\mathrm{Cu}$

$\mathrm{Cu}$

$\mathrm{Cu}$

$\mathrm{Cu}$

$\mathrm{Cu}$

$\mathrm{Cu}$

$\mathrm{Cu}$

$\mathrm{Cu}$

O

O

0

O

O

0

O

0
3.63465516

5.45978268

5.52820001

3.82864591

2.48843106

5.45198275

7.26931033

5.45198275

5.45198275

7.24098201

7.29177741

5.45089823

3. 39816532

7.26931033

9.08663791

7.26931033

7.26931033

9.06937248

9.04621562

8.89958302

6.88147626

1.61446079

5.31543109

3.63086837

8.56717926

5.66869600

9.28779634

7.04936136

6.87802465
Fri Apr 16 14:51:55 2021

135

6.50186882

4.85722403

7.29175316

5.48916976

3.33532050

3.25093441

1.62546720

0.00000000

2.43820081

0.80772134

3.26790723

1.89020395

0.14323281

7.31460242

5.68913521

4.06366801

6.50186882

4.86512025

7.48801901

6.16978317

4.77539552

0.12890476

$-0.13032471$

2.03318560

4.42506657

5.44978666

5.69898464

6.50678448

3.00515923
10.43820081

11.23213592

12.16370936

13.15466879

14.60728068

8.00000000

8.81273360

9.62546720

10.43820081

11.25423017

11.97660185

13.04639581

14.56223613

8.00000000

8.81273360

9.62546720

10.43820081

11.21484600

12.13234578

14.90283982

14.46835490

13.96229729

14.33508769

13.95869389

15.43395527

13.12083706

12.94817051

15.31515067

13.80499808

. / oxygen/Cu/210/iter_9/struct.xyz

41

Lattice $=" 7.269310326940001 \quad 0.0 \quad 0.0 \quad 3.6346551634699993 \quad 8.1273360202895320 .0 \quad 0.0 \quad 0.021 .6891$ $35214202672 "$ Properties=species:S:1:pos:R:3 pbc="T T T"

$\begin{array}{llll}\mathrm{Cu} & 1.81732758 & 3.25093441 & 8.00000000\end{array}$

$\mathrm{Cu}$

1.81732758

25093441

.

.63465516

1.62546720

8.81273360

1.81732758

0.00000000

2. 43820081

1.81732758
3.66974285

0.82414307

9.62546720

$\mathrm{Cu}$

3. 64261632

3. 24410589

1. 56317237

2.00472485

10.58779670

7.97175903

3.63465516

5. 45198275

7.31460242

5.68913521

3.63465516

4.06366801

3.63465516

6.50186882

4.84875698

10.43820081

11.29560080

12.04724470

13.08540927

14.23752010

8.00000000

8.81273360

9.62546720

10.43820081

5.42805838

7.42243662

11.13167709

5.42152719

5.48834201

3.73230141

2.74310058

3.25093441

1.62546720

0.00000000

2.43820081

0.83089273

3.94746069

2.55194800

8.03657023

7. 31460242

5.68913521

4.06366801

6.50186882

4.80910738

7.31558580

12.06245551

13.21458174

14.92237853

8.00000000

8.81273360

9.62546720

10.43820081

11.25830091

12.78000363

13.94139732

14.37951166

8.00000000

8.81273360

9.62546720

10.43820081

11.23384453

12.07391867

14.46346762

15.34331417

13.92799562

14.16885109

0.13116921

7.66488397

13.21926848

8.77553096

1.57883789 


\section{0}

O

0

○

O

0
14.61977628

12.81072777

12.96311569

15.48908414

15.27938788

12.82729966

oxygen/Cu/211/iter_1/struct.xyz

33

Lattice $=" 5.1401786267286730 .0 \quad 0.0 \quad 0.0 \quad 12.5908148222452070 .0 \quad 0.0 \quad 0.021 .19344614919298 "$ Pr operties=species:S:1:pos:R:3 pbc="T T T"

$\mathrm{Cu} \quad 0.00000000 \quad 0.00000000$

$\mathrm{Cu} \quad 1.28504466 \quad 2.09846914$

$\mathrm{Cu} \quad 0.00000000 \quad 4.19693827$

$\mathrm{Cu} \quad 1.28504466 \quad 0.05609426$

$\mathrm{Cu} \quad 0.00357906 \quad 2.14335588$

$\mathrm{Cu} \quad 1.28504466 \quad 4.17304263$

$\mathrm{Cu} \quad-0.00064601 \quad 0.00015774$

$\mathrm{Cu} \quad 1.28504466$

10.49234569

0.00000000

6.29540741

$\begin{array}{ll}\mathrm{Cu} & 1.28504466 \\ \mathrm{Cu} & 0.00000000\end{array}$

8.39387655

10.49234569

1. 28504466

6.35472366

$-0.00567634$

8.45129967

1. 28504466

10.53479457

$-0.06356588$

6.35278306

3. 85513397

4.19693827

2. 57008931

0.00000000

3. 85513397

2.09846914

2. 57008931

4.19693827

3. 85513397

0.05846157

2. 56651026

2.14335588

3. 85513397

4.21638175

2. 57073533

0.00015774

3. 85513397

10.49234569

2. 57008931

6.29540741

3. 85513397

8.39387655

2. 57008931

10.49234569

3. 85513397

6.37519819

8.45129967

2.57576565

10.54179953

6.35278306

8.00000000

8.74192088

9.48384176

10.22576264

10.93460226

11.75051876

12.52874447

13.06420059

8.00000000

8.74192088

9.48384176

10.22576264

10.94654370

11.77637394

12.42088151

13.13937129

8.00000000

8.74192088

9.48384176

10.22576264

10.93828032

11.75051876

12.35347254

13.06420059

8.00000000

8.74192088

9.48384176

10.22576264

10.97273086

11.77637394

12.42694871

2. 63365519

5.40617744

13.13937129

14.03249911

. /oxygen/Cu/211/iter_10/struct.xyz

42

Lattice $=" 5.140178626728673 \quad 0.0 \quad 0.0 \quad 0.0 \quad 12.5908148222452070 .0 \quad 0.0 \quad 0.021 .19344614919298 "$ Pr operties=species:S:1:pos:R:3 pbc="T T T"

$\begin{array}{lrrr}\mathrm{Cu} & 1.28504466 & 4.19693827 & 8.00000000 \\ \mathrm{Cu} & 0.00000000 & 0.00000000 & 8.74192088 \\ \mathrm{Cu} & 1.28504466 & 2.09846914 & 9.48384176 \\ \mathrm{Cu} & 0.00000000 & 4.19693827 & 10.22576264 \\ \mathrm{Cu} & 1.30449920 & 0.02914313 & 11.05148651 \\ \mathrm{Cu} & 0.14776063 & 2.26210323 & 11.77280345 \\ \mathrm{Cu} & 1.41125557 & 4.45186773 & 12.71895011 \\ \mathrm{Cu} & -0.14886649 & 12.42842441 & 14.07365964 \\ \mathrm{Cu} & 1.28504466 & 10.49234569 & 8.00000000 \\ \mathrm{Cu} & 0.00000000 & 6.29540741 & 8.74192088 \\ \mathrm{Cu} & 1.28504466 & 8.39387655 & 9.48384176 \\ \mathrm{Cu} & 0.00000000 & 10.49234569 & 10.22576264 \\ \mathrm{Cu} & 1.33113680 & 6.36020689 & 10.91223389 \\ \mathrm{Cu} & -0.02631976 & 8.39510923 & 11.77580263 \\ \mathrm{Cu} & 1.20250901 & 10.55298138 & 12.43534461 \\ \mathrm{Cu} & -0.06161409 & 9.47801646 & 14.79270274 \\ \mathrm{Cu} & 3.85513397 & 4.19693827 & 8.00000000 \\ \mathrm{Cu} & 2.57008931 & 0.00000000 & 8.74192088 \\ \mathrm{Cu} & 3.85513397 & 2.09846914 & 9.48384176 \\ \mathrm{Cu} & 2.57008931 & 4.19693827 & 10.22576264 \\ \mathrm{Cu} & 3.89551666 & 0.09890982 & 10.83878202\end{array}$


$\mathrm{Cu}$

$\mathrm{Cu}$

$\mathrm{Cu}$

$\mathrm{Cu}$

$\mathrm{Cu}$

$\mathrm{Cu}$

$\mathrm{Cu}$

$\mathrm{Cu}$

$\mathrm{Cu}$

$\mathrm{Cu}$

$\mathrm{Cu}$

O

0

O

0

O

O

0

0

0

0

.$/ 10 x$

43

oxy/Cu/211/iter_11/struct.xyz
2.72549428

4.00300498

2.14206983

3.85513397

2.57008931

3.85513397

2.57008931

3.83707606

2.55384179

3.74184073

2.71042370

4.28934762

0.97600829

3.87635122

1.74718778

3.56167306

2.70641336

2.81706485

1.27915784

0.83934467

0.10581308
Fri Apr 16 14:51:55 2021

137

2.15048476

4.42651860

$-0.17545455$

10.49234569

6.29540741

8.39387655

10.49234569

6.34415994

8.43438954

10.78501352

8.64224335

11.31828494

0.87800978

9.08543913

8.25235911

0.16777995

3.30109228

11.24530807

8.78347755

9.31394588

5.77712658
11.77540170

12.72661480

14.05234761

8.00000000

8.74192088

9.48384176

10.22576264

10.94618098

11.72662036

12.42436605

14.73836196

15.40575729

12.97097156

13.33026316

16.23541888

12.75033809

13.33233937

15.31389581

13.35212885

16.40103426

12.39676103

Lattice $=" 5.140178626728673 \quad 0.0 \quad 0.0 \quad 0.0 \quad 12.5908148222452070 .0 \quad 0.0 \quad 0.021 .19344614919298 "$ Pr operties $=$ species:S:1:pos:R:3 pbc="T T T"

Cu $\quad 1.28504466$

$\mathrm{Cu} \quad 0.00000000$

$\mathrm{Cu} \quad 1.28504466$

$\mathrm{Cu} \quad 0.00000000$

$\mathrm{Cu} \quad 1.32368146$

$\mathrm{Cu} \quad 0.09863343$

$\mathrm{Cu} \quad 2.67353812$

$\mathrm{Cu} \quad 0.12272123$

$\mathrm{Cu} \quad 1.28504466$

$\mathrm{Cu} \quad 0.00000000$

$\mathrm{Cu} \quad 1.28504466$

$\mathrm{Cu} \quad 0.00000000$

$\mathrm{Cu} \quad 1.29599734$

$\mathrm{Cu} \quad 0.03301221$

$\mathrm{Cu} \quad 1.34580198$

$\mathrm{Cu} \quad 4.83992797$

$\mathrm{Cu} \quad 3.85513397$

$\mathrm{Cu} \quad 2.57008931$

$\mathrm{Cu} \quad 3.85513397$

$\mathrm{Cu} \quad 2.57008931$

$\mathrm{Cu} \quad 3.91697871$

$\mathrm{Cu} \quad 2.64303557$

$\mathrm{Cu} \quad 0.06169366$

$\mathrm{Cu} \quad 2.51657994$

$\mathrm{Cu} \quad 3.85513397$

$\mathrm{Cu} \quad 2.57008931$

$\mathrm{Cu} \quad 3.85513397$

$\mathrm{Cu} \quad 2.57008931$

$\mathrm{Cu} \quad 3.83224133$

$\mathrm{Cu} \quad 2.52130201$

$\mathrm{Cu} \quad 3.86038032$

$\mathrm{Cu} \quad 2.46739041$

O $\quad 4.56961458$

O 1.32309706

o $\quad 3.73029345$

O $\quad 1.37015771$

O $\quad 3.91296252$

O 4.41156990

O $\quad 3.16639785$

O $\quad 1.16699591$

o $\quad 0.75760012$

O $\quad 1.26430179$

O $\quad 3.92994646$
4.19693827

0.00000000

2.09846914

4.19693827

12.58365447

2.64794460

4.55879813

12.40258016

10.49234569

6.29540741

8.39387655

10.49234569

6.26677444

8.34754335

10.47450498

8.25019831

4.19693827

0.00000000

2.09846914

4.19693827

0.06452915

2.24041042

5.36795875

12.53823696

10.49234569

6.29540741

8.39387655

10.49234569

6.35587872

8.38056226

10.55101002

7.45236558

10.98328705

1.10012674

8.66013116

6.46556434

1. 33077890

4.96178573

11.32288275

8.72344118

7.49579016

3.98271906

6.52707288
8.00000000

8.74192088

9.48384176

10.22576264

10.99259225

13.12571180

12.72797855

13.49357628

8.00000000

8.74192088

9.48384176

10.22576264

10.96184867

11.70523679

12.48756127

14.66723339

8.00000000

8.74192088

9.48384176

10.22576264

11.04909429

11.61984925

13.99214607

13.64678871

8.00000000

8.74192088

9.48384176

10.22576264

10.90797453

11.56769726

12.44896183

14.01958822

14.48691013

12.71070809

13.11955764

15.27412454

12.82440286

12.12127587

14.85549532

13.44295774

16.01154124

13.72219038

14.64356819 
. /oxygen/Cu/211/iter_2/struct.xyz

34

Lattice $=" 5.140178626728673 \quad 0.0 \quad 0.0 \quad 0.0 \quad 12.5908148222452070 .0 \quad 0.0 \quad 0.021 .19344614919298 "$ Pr operties=species:S:1:pos:R:3 pbc="T T T"

$\mathrm{Cu} \quad 0.00000000 \quad 0.00000000$

$\begin{array}{lll}\mathrm{Cu} & 1.28504466 & 2.09846914\end{array}$

$\mathrm{Cu} \quad 0.00000000 \quad 4.19693827$

$\mathrm{Cu} \quad 1.34701984 \quad 0.02643958$

$\mathrm{Cu} \quad-0.11348523 \quad 2.17535940$

$\mathrm{Cu} \quad 1.25833664 \quad 4.26079867$

$\mathrm{Cu} \quad 0.67286072 \quad 0.56096887$

$\mathrm{Cu} \quad 1.28504466$

$\mathrm{Cu} \quad 0.00000000$

$\mathrm{Cu} \quad 1.28504466$

10.49234569

8.39387655

0.00000000

10.49234569

1. 26844828

6.31803596

0.02074034

8.43994205

1. 27952947

$-0.03433695$

10.67935967

3. 85513397

6.34015702

4.19693827

2. 57008931

0.00000000

3. 85513397

2.09846914

2. 57008931

4.19693827

3. 89804180

0.04285822

2. 58495775

2.08010736

3. 81817020

4.32743143

3. 15238378

$-0.03353513$

3. 85513397

10.49234569

2. 57008931

6.29540741

3. 85513397

8.39387655

2. 57008931

10.49234569

3. 83230342

6.38953408

8.48498006

2.52983269

10.40403753

2. 53811490

6.35391694

$-0.27027355$

1.17606497

11.55976698

2.37771232

8.00000000

8.74192088

9.48384176

10.22576264

10.97128432

11.68396354

12.54984132

13.42243767

8.00000000

8.74192088

9.48384176

10.22576264

10.96139567

11.71921140

12.53604157

13.07636261

8.00000000

8.74192088

9.48384176

10.22576264

10.88839785

11.83302823

12.38638727

13.18302523

8.00000000

8.74192088

9.48384176

10.22576264

10.94018495

11.74668408

12.40356998

13.07577363

13.68659059

13.21610215

. /oxygen/Cu/211/iter_3/struct.xyz

35

Lattice $=" 5.140178626728673 \quad 0.0 \quad 0.0 \quad 0.0 \quad 12.590814822245207 \quad 0.0 \quad 0.0 \quad 0.021 .19344614919298 "$ Pr operties=species:S:1:pos:R:3 pbc="T T T"

$\begin{array}{lrrr}\mathrm{Cu} & 1.28504466 & 4.19693827 & 8.00000000 \\ \mathrm{Cu} & 0.00000000 & 0.00000000 & 8.74192088 \\ \mathrm{Cu} & 1.28504466 & 2.09846914 & 9.48384176 \\ \mathrm{Cu} & 0.00000000 & 4.19693827 & 10.22576264 \\ \mathrm{Cu} & 1.35947855 & 0.06399461 & 10.98318110 \\ \mathrm{Cu} & -0.10720186 & 2.20663546 & 11.68457592 \\ \mathrm{Cu} & 1.26360865 & 4.29899053 & 12.64766488 \\ \mathrm{Cu} & 0.62081694 & 0.59842625 & 13.44298639 \\ \mathrm{Cu} & 1.28504466 & 10.49234569 & 8.00000000 \\ \mathrm{Cu} & 0.00000000 & 6.29540741 & 8.74192088 \\ \mathrm{Cu} & 1.28504466 & 8.39387655 & 9.48384176 \\ \mathrm{Cu} & 0.00000000 & 10.49234569 & 10.22576264 \\ \mathrm{Cu} & 1.28502002 & 6.32441596 & 10.97957762 \\ \mathrm{Cu} & 0.02226833 & 8.49600843 & 11.75043844 \\ \mathrm{Cu} & 1.25227413 & 10.75238153 & 12.55456456 \\ \mathrm{Cu} & 0.02023932 & 6.35896503 & 13.12980241 \\ \mathrm{Cu} & 3.85513397 & 4.19693827 & 8.00000000 \\ \mathrm{Cu} & 2.57008931 & 0.00000000 & 8.74192088 \\ \mathrm{Cu} & 3.85513397 & 2.09846914 & 9.48384176 \\ \mathrm{Cu} & 2.57008931 & 4.19693827 & 10.22576264 \\ \mathrm{Cu} & 3.90575817 & 0.06260626 & 10.89933668 \\ \mathrm{Cu} & 2.57862291 & 2.13862355 & 11.82945821 \\ \mathrm{Cu} & 3.83041763 & 4.34148952 & 12.38695123 \\ \mathrm{Cu} & 3.11801890 & 0.03859491 & 13.19289289 \\ \mathrm{Cu} & 3.85513397 & 10.49234569 & 8.00000000 \\ \mathrm{Cu} & 2.57008931 & 6.29540741 & 8.74192088\end{array}$




\section{$\mathrm{Cu}$}

$\mathrm{Cu}$

$\mathrm{Cu}$

$\mathrm{Cu}$

$\mathrm{Cu}$

$\mathrm{Cu}$

O

O

O
3.85513397

2. 57008931

3. 85450151

2.52680640

3. 83209269

2.53125286

$-0.28216162$

1.17104890

3. 84497621
Fri Apr 16 14:51:55 2021

139
8.39387655

10.49234569

6.33834664

8.52746988

10.51258429

6.35897665

11.57962563

2.39628298

7.79813792
9.48384176

10.22576264

10.88574769

11.77741492

12.37934137

13.12494935

13.70549782

13.22968137

13.21578863

36

Lattice $=" 5.140178626728673 \quad 0.0 \quad 0.0 \quad 0.0 \quad 12.590814822245207 \quad 0.0 \quad 0.0 \quad 0.021 .19344614919298 "$ Pr operties=species:S:1:pos:R:3 pbc="T T T"

$\mathrm{C}$

$\mathrm{Cu}$

1.28504466

4.19693827

0.00000000

0.00000000

1. 28504466

2.09846914

0.00000000

4.19693827

1. 31477864

0.01407518

$-0.04443471$

2.17650451

1. 18335868

4. 34026405

0.15829435

0.13040974

1. 28504466

0.00000000

10.49234569

1. 28504466

0.00000000

6.29540741

8.39387655

1. 33038237

10.49234569

6.35954684

8.51391146

$-0.00813190$

10.58436224

0.06598470

6.46646174

4.19693827

3. 85513397

0.00000000

57008931

2.09846914

4.19693827

2. 57008931

0.06413943

3. 90047169

2.21850404

4.28895483

3. 86219002

2. 63607401

3. 85513397

0.17105433

10.49234569

6.29540741

8.39387655

3. 85513397

10.49234569

2. 57008931

3. 88486796

6.30948259

2.52565460

8.47191192

3. 75344799

10.63567146

6.42581715

2. 72838366

$-0.06095335$

1. 26877643

3. 83886574

10.93224947

1. 66064186

7.95604928

2. 50913596

4.63684206

8.00000000

8.74192088

9.48384176

10.22576264

10.91008248

11.76402550

12.48135122

13.39007246

8.00000000

8.74192088

9.48384176

10.22576264

10.96852172

11.69641507

12.47273183

13.07666860

8.00000000

8.74192088

9.48384176

10.22576264

10.96852172

11.69641507

12.47273183

13.07666860

8.00000000

8.74192088

9.48384176

10.22576264

10.91008248

11.76402550

12.48135122

13.39007246

13.79898111

13.20218011

13.20218011

13.79898111

. /oxygen/Cu/211/iter_5/struct.xyz

37

Lattice $=" 5.1401786267286730 .0 \quad 0.0 \quad 0.0 \quad 12.5908148222452070 .00 .0 \quad 0.021 .19344614919298 "$ Pr operties=species:S:1:pos:R:3 pbc="T T T"

$\begin{array}{lrrr}\mathrm{Cu} & 1.28504466 & 4.19693827 & 8.00000000 \\ \mathrm{Cu} & 0.00000000 & 0.00000000 & 8.74192088 \\ \mathrm{Cu} & 1.28504466 & 2.09846914 & 9.48384176 \\ \mathrm{Cu} & 0.00000000 & 4.19693827 & 10.22576264 \\ \mathrm{Cu} & 1.24819868 & 0.03315768 & 10.90670983 \\ \mathrm{Cu} & -0.17403481 & 2.27543650 & 11.79986726 \\ \mathrm{Cu} & 1.15266510 & 4.37494336 & 12.45427727 \\ \mathrm{Cu} & -0.30353214 & 0.11765879 & 13.68779234 \\ \mathrm{Cu} & 1.28504466 & 10.49234569 & 8.00000000 \\ \mathrm{Cu} & 0.00000000 & 6.29540741 & 8.74192088 \\ \mathrm{Cu} & 1.28504466 & 8.39387655 & 9.48384176 \\ \mathrm{Cu} & 0.00000000 & 10.49234569 & 10.22576264 \\ \mathrm{Cu} & 1.33732177 & 6.37404737 & 10.96232294 \\ \mathrm{Cu} & 0.06198620 & 8.48901471 & 11.68264406 \\ \mathrm{Cu} & 1.41427561 & 10.53847207 & 12.44093754\end{array}$




\section{$\mathrm{Cu}$}

$\mathrm{Cu}$

$\mathrm{Cu}$

$\mathrm{Cu}$

$\mathrm{Cu}$

$\mathrm{Cu}$

$\mathrm{Cu}$

$\mathrm{Cu}$

$\mathrm{Cu}$

$\mathrm{Cu}$

$\mathrm{Cu}$

$\mathrm{Cu}$

$\mathrm{Cu}$

$\mathrm{Cu}$

$\mathrm{Cu}$

$\mathrm{Cu}$

$\mathrm{Cu}$

O

O

O

O

0
0.06991998

3.85513397

2. 57008931

3. 85513397

2. 57008931

3. 82106855

2. 51022398

3. 80597557

2. 61078405

3. 85513397

2. 57008931

3.85513397

2. 57008931

3.89535385

2. 59789870

3. 91394391

2. 73331393

0.19577944

1. 15253149

3. 88302610

2.39302003

3. 77792506
Fri Apr 16 14:51:55 2021

13.09662857

6.47977972

4.19693827

0.00000000

2.09846914

4.19693827

$-0.00144229$

2. 26797242

4.33476685

$-0.00962310$

10.49234569

6.29540741

8.39387655

10.49234569

6.29602435

8.46207833

10.54220386

6.51086451

10.92536535

1.07786944

8.01224682

4.75255344

1.65450573
8.00000000

8.74192088

9.48384176

10.22576264

10.93983381

11.75732025

12.55102527

13.20224405

8.00000000

8.74192088

9.48384176

10.22576264

10.92117660

11.72751480

12.54062137

13.40474011

13.80768136

12.63515242

13.21826826

13.82285010

13.38435637

38

Lattice $=" 5.1401786267286730 .0 \quad 0.0 \quad 0.0 \quad 12.590814822245207 \quad 0.0 \quad 0.0 \quad 0.021 .19344614919298 "$ Pr operties=species:S:1:pos:R:3 pbc="T T T"

$\begin{array}{llll}\mathrm{Cu} & 1.28504466 & 4.19693827 & 8.00000000 \\ \mathrm{Cu} & 0.00000000 & 0.00000000 & 8.74192088\end{array}$

$\mathrm{Cu} \quad 0.00000000 \quad 0.00000000$

$\mathrm{Cu} \quad 1.28504466 \quad 2.09846914$

$\mathrm{Cu} \quad 0.00000000 \quad 4.19693827$

$\mathrm{Cu} \quad 1.32600054 \quad 0.04143346$

$\mathrm{Cu} \quad 2.75615818 \quad 1.84976428$

$\mathrm{Cu} \quad-1.14191398 \quad 4.41929930$

$\mathrm{Cu} \quad 4.32322017$

12.10479961

1.28504466

10.49234569

0.00000000

6.29540741

8.39387655

1.28504466

10.49234569

1.30777026

6.32457017

8.37918242

0.02839226

10.46046272

1. 25118548

6.86638761

4.19693827

3. 85513397

0.00000000

2.09846914

2.57008931

4.19693827

2. 57008931

0.01815914

3. 78196748

2. 26377224

4.44119791

1.32019863

11.89465006

1.77614094

3. 85513397

2. 57008931

10.49234569

6.29540741

8.39387655

3. 85513397

10.49234569

.74192088

9.48384176

10.22576264

11.01373944

13.22396827

12.74291999

14.15037148

8.00000000

8.74192088

9.48384176

10.22576264

10.93482470

11.67561560

12.35782905

13.54835188

8.00000000

8.74192088

9.48384176

10.22576264

10.96514503

11.72872970

12.77285025

14.38480722

8.00000000

8.74192088

9.48384176

10.22576264

10.93675650

11.70556159

13.76964406

13.48392871

14.12648628

12.34694813

13.80221277

13.79023548

14.60610346

12.08578565

. /oxygen/Cu/211/iter_7/struct.xyz

5.49412958

0.66960639

3.30233053

39

Lattice $=" 5.140178626728673 \quad 0.0 \quad 0.0 \quad 0.0 \quad 12.590814822245207 \quad 0.0 \quad 0.0 \quad 0.021 .19344614919298 "$ Pr operties=species:S:1:pos:R:3 pbc="T T T" 
$\mathrm{Cu}$

$\mathrm{Cu}$

$\mathrm{Cu}$

$\mathrm{Cu}$

$\mathrm{Cu}$

$\mathrm{Cu}$

$\mathrm{Cu}$

$\mathrm{Cu}$

$\mathrm{Cu}$

$\mathrm{Cu}$

$\mathrm{Cu}$

$\mathrm{Cu}$

$\mathrm{Cu}$

$\mathrm{Cu}$

$\mathrm{Cu}$

$\mathrm{Cu}$

$\mathrm{Cu}$

$\mathrm{Cu}$

$\mathrm{Cu}$

$\mathrm{Cu}$

$\mathrm{Cu}$

$\mathrm{Cu}$

$\mathrm{Cu}$

$\mathrm{Cu}$

$\mathrm{Cu}$

$\mathrm{Cu}$

$\mathrm{Cu}$

$\mathrm{Cu}$

$\mathrm{Cu}$

$\mathrm{Cu}$

$\mathrm{Cu}$

$\mathrm{Cu}$

O

O

O

0

O

0

0

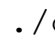

40

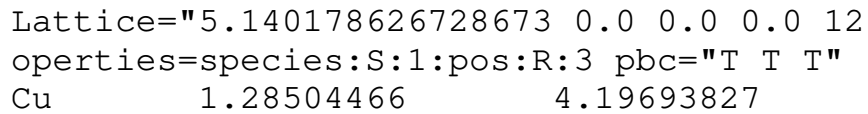

$\mathrm{Cu} \quad 0.00000000 \quad 0.00000000$

$\begin{array}{lll}\mathrm{Cu} & 1.28504466 & 2.09846914\end{array}$

$\mathrm{Cu} \quad 0.00000000$

$\mathrm{Cu} \quad 1.28811154$

$\mathrm{Cu} \quad 0.05491769$

$\mathrm{Cu} \quad 0.70431294$

$\mathrm{Cu} \quad 5.13365947$

$\mathrm{Cu} \quad 1.28504466$

$\mathrm{Cu} \quad 0.00000000$

$\mathrm{Cu} \quad 1.28504466$

$\mathrm{Cu} \quad 0.00000000$

$\mathrm{Cu} \quad 1.36173832$

$\mathrm{Cu} \quad 0.03422754$

$\mathrm{Cu} \quad 1.21843613$

$\mathrm{Cu} \quad 4.03652173$

$\mathrm{Cu} \quad 3.85513397$

$\mathrm{Cu} \quad 2.57008931$

$\mathrm{Cu} \quad 3.85513397$

$\mathrm{Cu} \quad 2.57008931$

$\mathrm{Cu} \quad 3.88477493$

$\mathrm{Cu} \quad 2.82491476$

$\mathrm{Cu} \quad 3.56609679$

$\mathrm{Cu} \quad 2.28644264$

$\mathrm{Cu} \quad 3.85513397$
Fri Apr 16 14:51:55 2021

141

4.19693827

8.00000000

8.74192088

9.48384176

10.22576264

10.95795565

11.77868612

12.63432342

13.65633042

8.00000000

8.74192088

9.48384176

10.22576264

11.02189804

11.65441619

12.43496415

13.51558425

8.00000000

8.74192088

9.48384176

10.22576264

10.97365146

11.77991527

12.45430839

13.67798318

8.00000000

8.74192088

9.48384176

10.22576264

10.90470735

11.61093255

12.45599219

13.56007564

14.18799992

12.81240346

13.20963693

14.14662792

12.81631263

13.33742760

14.21165392

$4.19693827 \quad 10.22576264$

$-0.00431846 \quad 11.01926405$

$2.31405264 \quad 11.79415618$

$5.00564538 \quad 12.86096611$

$-0.20391980 \quad 14.03395982$

$10.49234569 \quad 8.00000000$

$6.29540741 \quad 8.74192088$

$8.39387655 \quad 9.48384176$

$10.49234569 \quad 10.22576264$

$6.38699776 \quad 10.92122505$

$8.37650198 \quad 11.70288360$

$10.47149092 \quad 12.40308799$

$6.58385189 \quad 13.41209636$

$4.19693827 \quad 8.00000000$

$0.00000000 \quad 8.74192088$

$2.09846914 \quad 9.48384176$

$4.19693827 \quad 10.22576264$

$0.01880707 \quad 10.90487132$

$1.93642784 \quad 12.36484862$

$4.38638748 \quad 12.51324593$

$-0.38791090 \quad 13.96554476$

$10.49234569 \quad 8.00000000$ 


\section{$\mathrm{Cu}$}

$\mathrm{Cu}$

$\mathrm{Cu}$

$\mathrm{Cu}$

$\mathrm{Cu}$

$\mathrm{Cu}$

$\mathrm{Cu}$

O

O

O

O

O

O

O

0

.$/$

41

gen/Cu/211/iter_9/struct.xyz
2.57008931

2. 57008931

3. 94345861

2. 67996877

4.05684501

1. 33479725

4.48894485

1.16056860

3.10863638

2. 28257306

3. 73513426

1. 81731725

2. 95478117

0.48620045
3.85513397
6.29540741

8.39387655

10.49234569

6.31043390

8.46850771

9.70461614

7.33820849

11.30804288

0.78506720

8.30229013

5.66642467

0.36294265

3.47890030

11.06484534

9.07503419
8.74192088

9.48384176

10.22576264

10.93156795

11.69812030

14.39658176

13.71850251

15.40092142

12.92830016

13.63540768

13.80853708

12.82101790

12.00037291

15.27593969

13.57732709

Lattice $=" 5.140178626728673 \quad 0.0 \quad 0.0 \quad 0.0 \quad 12.5908148222452070 .0 \quad 0.0 \quad 0.021 .19344614919298 "$ Pr operties=species:S:1:pos:R:3 pbc="T T T"

$\mathrm{Cu} \quad 0.00000000$

1.28504466

0.00000000

1. 25434695

0.06615086

1. 28558705

0.23896869

1. 28504466

0.00000000

1.28504466

0.00000000

1. 25439099

5. 10257186

1. 28814157

5.00757190

3. 85513397

2. 57008931

3. 85513397

2. 57008931

3. 79056241

2. 39263055

3. 99866026

2.75240259

3. 85513397

2. 57008931

3. 85513397

2. 57008931

3. 82910134

2. 58137011

3. 81434281

2. 30945835

0.17272025

1. 77430397

3.80257106

1. 84466012

3. 87654458

2. 67554449

3. 98721475

1. 38497615

0.78046222
0.00000000

2.09846914

4.19693827

12.57664487

2.16533576

4.26017813

0.54678429

10.49234569

6.29540741

8.39387655

10.49234569

6.28368149

8.35957892

10.53480539

6.02175257

4.19693827

0.00000000

2.09846914

4.19693827

0.07301905

2.02822163

4.27399469

11.64439987

10.49234569

6.29540741

8.39387655

10.49234569

6.30438741

8.43228191

10.52270768

7.22713201

11.10726764

0.55268145

6.96872299

6.30647113

1.12640559

3.82493465

10.28167168

8.56040885

5.41321712
8.00000000

8.74192088

9.48384176

10.22576264

11.02860206

11.68041837

12.41249627

13.66581609

8.00000000

8.74192088

9.48384176

10.22576264

10.93903092

11.75769964

12.57311106

13.92664082

8.00000000

8.74192088

9.48384176

10.22576264

11.01355412

13.80434911

12.37055075

14.44933446

8.00000000

8.74192088

9.48384176

10.22576264

10.95458857

11.59047620

12.33380411

14.14914781

14.14187128

14.69883743

12.89258952

15.66786742

12.68255575

13.57421812

14.27868510

13.09905595

15.39376557

. /oxygen/Cu/221/iter_1/struct . xyz

41

Lattice $=" 5.140178626728671 \quad 0.0 \quad 0.0 \quad 0.0$ rties $=$ species:S:1:pos:R:3 pbc="T T T"

$\begin{array}{lll}\mathrm{Cu} & 1.28504466 & 3.85513397\end{array}$

$\mathrm{Cu} \quad 0.00000000 \quad 5.99687506$

$\mathrm{Cu} \quad 1.28504466 \quad 0.42834822$

$\mathrm{Cu} \quad 0.00000000$

2.57008931

\author{
8.00000000 \\ 8.60577586 \\ 9.21155172 \\ 9. 81732758
}


$\mathrm{Cu}$

$\mathrm{Cu}$

$\mathrm{Cu}$

$\mathrm{Cu}$

$\mathrm{Cu}$

$\mathrm{Cu}$

$\mathrm{Cu}$

$\mathrm{Cu}$

$\mathrm{Cu}$

$\mathrm{Cu}$

$\mathrm{Cu}$

$\mathrm{Cu}$

$\mathrm{Cu}$

$\mathrm{Cu}$

$\mathrm{Cu}$

$\mathrm{Cu}$

$\mathrm{Cu}$

$\mathrm{Cu}$

$\mathrm{Cu}$

$\mathrm{Cu}$

$\mathrm{Cu}$

$\mathrm{Cu}$

$\mathrm{Cu}$

$\mathrm{Cu}$

$\mathrm{Cu}$

$\mathrm{Cu}$

$\mathrm{Cu}$

$\mathrm{Cu}$

$\mathrm{Cu}$

$\mathrm{Cu}$

$\mathrm{Cu}$

$\mathrm{Cu}$

$\mathrm{Cu}$

$\mathrm{Cu}$

$\mathrm{Cu}$

$\mathrm{Cu}$

$.10 x$

50

Lattice $=" 5.1401786267286710 .0 \quad 0.0 \quad 0.0$ rties $=$ species: $\mathrm{S}: 1:$ pos: $\mathrm{R}: 3$ pbc $=$ "T T T"

3.85513397

$\mathrm{Cu} \quad 0.00000000$

$\mathrm{Cu} \quad 1.28504466$

$\mathrm{Cu} \quad 0.00000000$

$\mathrm{Cu} \quad 1.28504466$

$\mathrm{Cu} \quad 5.09299391$

$\mathrm{Cu} \quad 1.28504466$

$\mathrm{Cu} \quad 5.09573729$

$\mathrm{Cu} \quad 1.28504466$

$\mathrm{Cu} \quad 0.00930557$

$\mathrm{Cu} \quad 1.28504466$

$\mathrm{Cu} \quad 0.00000000$

$\mathrm{Cu} \quad 1.28504466$

$\mathrm{Cu} \quad 0.00000000$

$\mathrm{Cu} \quad 1.28504466$

$\mathrm{Cu} \quad 5.12723412$

$\mathrm{Cu} \quad 1.28504466$

$\mathrm{Cu} \quad 0.01522819$

$\mathrm{Cu} \quad 1.28504466$

$\mathrm{Cu} \quad 5.13051796$

$\mathrm{Cu} \quad 3.85513397$

$\mathrm{Cu} \quad 2.57008931$

$\mathrm{Cu} \quad 3.85513397$

$\mathrm{Cu} \quad 2.57008931$

$\mathrm{Cu} \quad 3.85513397$

$\mathrm{Cu} \quad 2.61727403$

$\mathrm{Cu}$
Fri Apr 16 14:51:55 2021

4.71183041

6.88414396

1.35746058

3.45215440

5.50695698

0.00119193

11.56540191

13.70714300

8.13861616

10.28035725

12.42209835

14.59037231

9.09641870

11.18989247

13.31159084

7.77434377

3.85513397

5.99687506

0.42834822

2.57008931

4.71183041

6.88428907

1.35984595

3. 45245080

5.57162445

$-0.00033153$

11.56540191

13.70714300

8.13861616

10.28035725

12.42209835

14.58965695

9.07343461

11.19062466

13.31324215

7.77197548

6.88239183
10.42310344

11.01444529

11.67466574

12.26087453

12.93379603

13.32159055

8.00000000

8.60577586

9.21155172

9.81732758

10.42310344

10.97583008

11.64866545

12.27130700

12.80133407

13.37380955

8.00000000

8.60577586

9. 21155172

9.81732758

10.42310344

11.01107820

11.66730406

12.25992447

12.74774246

13.32141016

8.00000000

8.60577586

9.21155172

9.81732758

10.42310344

10.97603240

11.67305572

12.27037919

12.79428045

13.37133601

14.30131309
143 


$\begin{array}{ll}\mathrm{Cu} & 2.61453065 \\ \mathrm{Cu} & 3.85513397 \\ \mathrm{Cu} & 2.56078374 \\ \mathrm{Cu} & 3.85513397 \\ \mathrm{Cu} & 2.57008931 \\ \mathrm{Cu} & 3.85513397 \\ \mathrm{Cu} & 2.57008931 \\ \mathrm{Cu} & 3.85513397 \\ \mathrm{Cu} & 2.58303382 \\ \mathrm{Cu} & 3.85513397 \\ \mathrm{Cu} & 2.55486112 \\ \mathrm{Cu} & 3.85513397 \\ \mathrm{Cu} & 2.57974998 \\ \mathrm{O} & 1.28504466 \\ \mathrm{O} & 3.85513397 \\ \mathrm{O} & 1.28504466 \\ \mathrm{O} & 3.85513397 \\ \mathrm{O} & 1.28504466 \\ \mathrm{O} & 3.85513397 \\ \mathrm{O} & 3.85513397 \\ \mathrm{O} & 1.28504466 \\ \mathrm{O} & 1.28504466 \\ \mathrm{O} & 3.85513397\end{array}$

Fri Apr 16 14:51:55 2021

144
3.49264182

6.01617996

1.26117268

11.56540191

13.70714300

8.13861616

10.28035725

12.42209835

14.64666240

9.08539185

11.36718027

14.28260513

8.73905020

7.63010849

0.15045730

2.56199709

9.90421722

12.75598260

4.35978557

12.68196054

9.94186443

5.82103602

7.56313139
12.27525189

14.16284138

14.00563135

8.00000000

8.60577586

9.21155172

9.81732758

10.96774428

11.63347986

12.95008569

13.37479965

14.44160951

15.20232077

14.70540122

13.59490518

13.48394892

12.47136429

13.48073419

12.40292514

13.55497352

12.02913537

15.12027626
10.42310344

. /oxygen/Cu/221/iter_11/struct.xyz 51

Lattice $=" 5.140178626728671 \quad 0.0 \quad 0.0 \quad 0.0 \quad 15.420535880186020 .0 \quad 0.0 \quad 0.021 .451982745205 "$ Prope rties $=$ species:S:1:pos:R:3 pbc="T T T"

$\mathrm{Cu} \quad 1.28504466$

$\mathrm{Cu} \quad 0.00000000$

$\mathrm{Cu} \quad 1.28504466$

0.00000000

1.28504466

0.05256290

1.28504466

$-0.01489033$

1. 28504466

$-0.01887445$

1. 28504466

0.00000000

1.28504466

0.00000000

1.28504466

0.04292232

1.28504466

$-0.06537240$

1.28504466

$-0.02184330$

3.85513397

2.57008931

3.85513397

3.85513397

5.99687506

0.42834822

2.57008931

4.71183041

7.02609937

1. 32375770

3. 33105737

6.04089538

$-0.04088082$

11.56540191

13.70714300

8.13861616

10.28035725

12.42209835

14.69097581

9.14057392

11.30746684

14.25202214

8.59646598

3.85513397

5.99687506

0.42834822

2. 57008931

4.71183041

7.02609937

1.35681425

3. 33105737

6.39062439

$-0.04088082$

11.56540191

13.70714300

8.13861616

10.28035725

12.42209835

14.69097581

9.24569675

11.30746684

13.50044370

8.59646598

7.33201396
8.00000000
8.60577586
9.21155172
9.81732758
10.42310344
11.03345648
11.80642989
12.83209003
13.62112410
14.87933711
8.00000000
8.60577586
9.21155172
9.81732758
10.42310344
10.94837471
11.65400642
13.08116818
13.09946861
14.45933719
8.00000000
8.60577586
9.21155172
9.81732758
10.42310344
11.03345648
11.57835430
12.83209003
13.73185993
14.87933711
8.00000000
8.60577586
9.21155172
9.81732758
10.42310344
10.94837471
11.66805064
13.08116818
13.85126366
14.45933719
14.90615425 


\section{0}

O

0

O

O

0

0

0

0

0
3.85513397

3.85513397

1.28504466

3. 85513397

1.28504466

3.85513397

3.85513397

1.28504466

1.28504466

3.85513397
Fri Apr 16 14:51:55 2021

14.26408585

0.68054905

9.91784188

12.46367639

5.93104117

13.65782142

9.65151375

4.59739128

7.46814173

2.10798194
15.61750102

13.76093033

13.66834387

12.46045311

11.93573619

11.94689215

13.55483524

15.24594807

13.34477907
12.44803692

52

Lattice $=" 5.1401786267286710 .00 .00 .015 .420535880186020 .00 .00 .021 .451982745205 "$ Prope rties=species:S:1:pos:R:3 pbc="T T T"

$\mathrm{Cu} \quad 1.28504466$

$\mathrm{Cu} \quad 0.00000000$

3.85513397

1.28504466

5.99687506

0.00000000

0.42834822

Cu

2.57008931

1.28504466

4.71183041

0.00000000

6.92837231

1.28504466

1. 32479704

0.00000000

3.51428323

1.28504466

6.31789631

0.00000000

0.91437153

1.28504466

0.00000000

1.28504466

0.00000000

1.28504466

0.00000000

1.28504466

0.00000000

1.28504466

0.00000000

3.85513397

2. 57008931

3.85513397

11.56540191

13.70714300

8.13861616

10.28035725

12.42209835

14.63864025

9.03506498

11.22455117

14.02816425

8.62463947

3.85513397

5.99687506

0.42834822

2.57008931

4.71183041

3. 85513397

2. 57008931

3.85513397

6.92837231

1. 32479704

3. 51428323

6.31789631

0.91437153

3. 85513397

2. 57008931

3. 85513397

2. 57008931

3. 85513397

2. 57008931

3.85513397

2. 57008931

3. 85513397

2. 57008931

3.85513397

2. 57008931

1.28504466

3.85513397

1.28504466

3.85513397

1.28504466

3. 85513397

3. 85513397

1.28504466

1.28504466

3. 85513397

3. 85513397

o $\quad 1.28504466 \quad 15.1262975$

11.56540191

13.70714300

8.13861616

10.28035725

12.42209835

14.63864025

9.03506498

11.22455117

14.02816425

8.62463947

7.41602958

15.12629752

2.12638769

9.83665563

12.55731992

4.84705198

12.55731992

9.83665563

4.84705198

7.41602958

2.12638769

o $\quad 1.28504466 \quad 15.12629752$
8.00000000

8.60577586

9.21155172

9.81732758

10.42310344

11.07029408

11.63799748

12.93425899

13.50118610

14.39979508

8.00000000

8.60577586

9.21155172

9.81732758

11.07029408

11.63799748

12.93425899

13.50118610

14.39979508

8.00000000

8.60577586

9.21155172

9.81732758

10.42310344

11.07029408

11.63799748

12.93425899

13.50118610

14.39979508

8.00000000

8.60577586

9.21155172

9.81732758

10.42310344

11.07029408

11.63799748

12.93425899

13.50118610

14.39979508

14.97487785

14.97487785

13.55719913

13.55719913

12.38308266

12.38308266

12.38308266

13.55719913

12.38308266

14.97487785

13.55719913

14.97487785
10.42310344

145

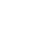

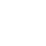

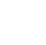

. / oxygen/Cu/221/iter_13/struct.xyz

53 
Lattice $=" 5.140178626728671 \quad 0.0 \quad 0.0 \quad 0.0 \quad 15.420535880186020 .00 .0 \quad 0.021 .451982745205 "$ Prope rties=species:S:1:pos:R:3 pbc="T T T"

$\mathrm{Cu} \quad 1.28504466 \quad 3.85513397$

$\mathrm{Cu} \quad 0.00000000 \quad 5.99687506$

$\mathrm{Cu} \quad 1.28504466 \quad 0.42834822$

$\begin{array}{lll}\mathrm{Cu} & 0.00000000 & 2.57008931\end{array}$

$\begin{array}{lll}\mathrm{Cu} & 1.28504466 & 4.71183041\end{array}$

$\mathrm{Cu} \quad 0.00000000 \quad 6.84257499$

$\mathrm{Cu} \quad 1.28428477 \quad 1.32825356$

$\mathrm{Cu} \quad 0.00000000 \quad 3.63105611$

$\mathrm{Cu} \quad 1.27880564 \quad 6.07600228$

$\mathrm{Cu} \quad 0.00000000$

$\mathrm{Cu} \quad 1.28504466$

$\mathrm{Cu} \quad 0.00000000$

$\mathrm{Cu} \quad 1.28504466$

$\mathrm{Cu} \quad 0.00000000$

$\mathrm{Cu} \quad 1.28504466$

$\mathrm{Cu} \quad 0.00000000$

$\mathrm{Cu} \quad 1.31202632$

$\mathrm{Cu} \quad 0.00000000$

$\mathrm{Cu} \quad 1.28274332$

$\mathrm{Cu} \quad 0.00000000$

$\mathrm{Cu} \quad 3.85513397$

$\mathrm{Cu} \quad 2.57008931$

3.85513397

6.07600228
0.91117062

11.56540191

13.70714300

8.13861616

10.28035725

12.42209835

14.62166665

8.99337261

10.96251233

13.87742510

8.03758147

3.85513397

5.99687506

0.42834822

2.57008931

4.71183041

3.85513397

6.83780268

1. 32825356

3.85589386

3.59650428

6.07600228

0.91824877

3.86137299

11.56540191

2.57008931

13.70714300

2. 57008931

3. 85513397

8.13861616

2. 57008931

3. 85513397

10.28035725

12.42209835

2. 57008931

14.61878212

8.99337261

3.82815230

2. 57008931

10.95281871

3. 85743531

13.87742510

8.39541089

7.01429509

1.41339947

15.09558581

3.85327437

1. 28289768

2.18892710

9.57297654

3. 82770224

12.25591019

5.06545842

3. 85294817

3. 86295086

12.25591019

9.57297654

5.06545842

1.28723046

3.72677915

7.01429509

2.18892710

3. 85728095

1. 28690425

2. 57008931

15.09558581

6.36248934

. / oxygen/Cu/221/iter_14/struct. xyz

54

Lattice $=" 5.140178626728671 \quad 0.0 \quad 0.0 \quad 0.0 \quad 15.420535880186020 .0 \quad 0.0 \quad 0.021 .451982745205 "$ Prope rties $=$ species: $\mathrm{S}: 1:$ pos:R: 3 pbc="T T T"

$\begin{array}{lllr}\mathrm{Cu} & 1.28504466 & 3.85513397 & 8.00000000 \\ \mathrm{Cu} & 0.00000000 & 5.99687506 & 8.60577586 \\ \mathrm{Cu} & 1.28504466 & 0.42834822 & 9.21155172 \\ \mathrm{Cu} & 0.00000000 & 2.57008931 & 9.81732758 \\ \mathrm{Cu} & 1.28504466 & 4.71183041 & 10.42310344 \\ \mathrm{Cu} & 0.00000000 & 6.81154640 & 10.99871903 \\ \mathrm{Cu} & 1.28247038 & 1.28201463 & 11.56583129 \\ \mathrm{Cu} & 0.00000000 & 3.60114746 & 12.29897184 \\ \mathrm{Cu} & 1.30745617 & 5.73629360 & 12.82140909\end{array}$
8.00000000
8.60577586
9.21155172
9.81732758
10.42310344
11.14195252
11.59355452
12.84939052
13.98017594
14.18577493
8.00000000
8.60577586
9.21155172
9.81732758
10.42310344
11.05074364
11.53872492
13.07712415
13.31593737
13.41854119
8.00000000
8.60577586
9.21155172
9.81732758
10.42310344
11.08406592
11.59355452
12.74170240
13.98017594
14.18952191
8.00000000
8.60577586
9.21155172
9.81732758
10.42310344
11.05192647
11.53872492
12.96202801
13.31593737
14.56929911
15.62221151
14.69138334
13.44675052
13.55954927
12.41546797
12.41176591
12.41546797
13.55954927
12.41176591
15.62221151
13.44675052
14.69138334
16.30595070 
$\mathrm{Cu}$

$\mathrm{Cu}$

$\mathrm{Cu}$

$\mathrm{Cu}$

$\mathrm{Cu}$

$\mathrm{Cu}$

$\mathrm{Cu}$

$\mathrm{Cu}$

$\mathrm{Cu}$

$\mathrm{Cu}$

$\mathrm{Cu}$

$\mathrm{Cu}$

$\mathrm{Cu}$

$\mathrm{Cu}$

$\mathrm{Cu}$

$\mathrm{Cu}$

$\mathrm{Cu}$

$\mathrm{Cu}$

$\mathrm{Cu}$

$\mathrm{Cu}$

$\mathrm{Cu}$

$\mathrm{Cu}$

$\mathrm{Cu}$

$\mathrm{Cu}$

$\mathrm{Cu}$

$\mathrm{Cu}$

$\mathrm{Cu}$

$\mathrm{Cu}$

$\mathrm{Cu}$

$\mathrm{Cu}$

$\mathrm{Cu}$

0

0

o

0

0

O

o

0

0

O

O

0

O

0
0.00000000

1.28504466

0.00000000

1.28504466

1.28504466

0.00000000

1.26539752

0.00000000

1.28334253

0.00000000

3.85513397

2. 57008931

2. 57008931

3. 85513397

2. 57008931

3. 85770824

2. 57008931

3. 83272246

2. 57008931

3.85513397

2. 57008931

3. 85513397

2. 57008931

3. 85513397

2. 57008931

3. 87478111

2. 57008931

3. 85683610

2.57008931

1.36834966

3.85936326

1. 28153299

1.30889190

3.76955125

3. 83128673

1.27518989

1.37062738

3.77182897

3.85864564

1.28081537

2.57008931

2.57008931
0.00000000

3.85513397

3.86498874

Fri Apr 16 14:51:55 2021

147

1.06416128

11.56540191

13.70714300

8.13861616

10.28035725

12.42209835

14.60538025

8.94211708

10.92045123

13.95123668

9.02744609

3.85513397

5.99687506

0.42834822

2.57008931

4.71183041

6.86514477

1.28201463

3. 54620994

5.73629360

1.06419079

11.56540191

13.70714300

8.13861616

10.28035725

12.42209835

14.60601160

8. 94211708

10.99613064

13.95123668

7.85072440

8.60134616

$-0.17701207$

2.26897985

9.54081376

12.30318624

5.27268490

12.30318624

9.54081376

5.27268490

8.60134616

2.26897985

$-0.17701207$

7.99062262

5.12642525

struct. xyz

55

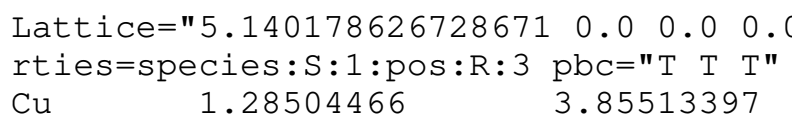

$\mathrm{Cu} \quad 0.00000000 \quad 5.99687506$

$\mathrm{Cu} \quad 1.28504466$

$\mathrm{Cu} \quad 0.00000000$

$\mathrm{Cu} \quad 1.28504466$

$\mathrm{Cu} \quad 0.00000000$

$\mathrm{Cu} \quad 1.24569675$

$\mathrm{Cu} \quad 0.00000000$

$\mathrm{Cu} \quad 1.21821956$

$\mathrm{Cu} \quad 0.00000000$

$\mathrm{Cu} \quad 1.28504466$

$\mathrm{Cu} \quad 0.00000000$

$\mathrm{Cu} \quad 1.28504466$

$\mathrm{Cu} \quad 0.00000000$

$\mathrm{Cu} \quad 1.28504466$

$\mathrm{Cu} \quad 0.00000000$

$\mathrm{Cu} \quad 1.25368049$

$\mathrm{Cu} \quad 0.00000000$

$\mathrm{Cu} \quad 1.30086274$
14.09867914

8.00000000

8.60577586

9.21155172

9.81732758

10.42310344

11.02933165

11.55264547

12.71075164

13.30056109

8.00000000

8.60577586

9.21155172

9.81732758

10.42310344

11.00517034

11.56583129

12.25555145

12.82140909

14.10640735

8.60577586

9.21155172

9.81732758

10.42310344

11.03007202

11.55264547

13.13791225

13.30056109

13.42716301

16.13453074

14.61295472

13.33480483

13.48311038

12.43547097

14.66201835

12.43547097

13.48311038

14.66201835

16.13453074

13.33480483

14.61295472

15.61861800

15.39890922
14.85228027

8.00000000

0.42834822

2.57008931

4.71183041

6.83563519

1.27255654

3.57375908

5.68559731

1.20447665

11.56540191

13.70714300

8.13861616

10.28035725

12.42209835

14.54189372

8.99850081

11.29757454

14.08197395
8.00000000
8.60577586
9.21155172
9.81732758
10.42310344
10.98568209
11.51958761
12.30717259
12.87891021
14.30633820
8.00000000
8.60577586
9.21155172
9.81732758
10.42310344
11.09550913
11.59660588
12.95746988
13.62117217 


\section{$\mathrm{Cu}$}

$\mathrm{Cu}$

$\mathrm{Cu}$

$\mathrm{Cu}$

$\mathrm{Cu}$

$\mathrm{Cu}$

$\mathrm{Cu}$

$\mathrm{Cu}$

$\mathrm{Cu}$

$\mathrm{Cu}$

$\mathrm{Cu}$

$\mathrm{Cu}$

$\mathrm{Cu}$

$\mathrm{Cu}$

$\mathrm{Cu}$

$\mathrm{Cu}$

$\mathrm{Cu}$

$\mathrm{Cu}$

$\mathrm{Cu}$

$\mathrm{Cu}$

$\mathrm{Cu}$

o

O

0

O

O

O

0

O

O

O

O

O

O

O

O
0.00000000

3.85513397

2. 57008931

3. 85513397

2. 57008931

3. 85513397

2. 57008931

3. 89448187

2. 57008931

3. 92195907

2. 57008931

3. 85513397

2. 57008931

3. 85513397

2. 57008931

3. 85513397

2. 57008931

3. 88649814

2. 57008931

3. 83931588

2. 57008931

0.72949822

3.98498166

1. 26285883

3. 86548452

1. 26694280

3. 75194732

3. 87323583

1.27469411

1. 38823131

4.41068040

3. 87731980

1.15519697

2.57008931

2. 57008931

0.00000000
Fri Apr 16 14:51:55 2021

148

9.11735765

3. 85513397

5.99687506

0.42834822

2.57008931

4.71183041

6.87939659

1.27255654

3.52726043

5.68559731

0.75236640

11.56540191

13.70714300

8.13861616

10.28035725

12.42209835

14.60420138

8.99850081

11.08578586

14.08197395

8.47248517

8.15163454

15.17251850

2.25597981

9.83879645

12.64515800

5.01847351

12.64515800

9.83879645

5.01847351

8.15163454

2.25597981

15.17251850

7.11501276

5.45324760

14.51397002
14.62704899

8.00000000

8.60577586

9.21155172

9.81732758

10.42310344

10.96396725

11.51958761

12.29452312

12.87891021

13.54166676

8.00000000

8.60577586

9.21155172

9.81732758

10.42310344

11.14889773

11.59660588

12.29551811

13.62117217

14.37272994

16.04508469

15.20408255

13.27348871

13.42774663

12.43549962

14.69218487

12.43549962

13.42774663

14.69218487

16.04508469

13.27348871

15.20408255

12.94459581

15.33786626

15.91431491

56

Lattice="5.140178626728671 0.00 .00 .0
rties=species:S:1:pos:R:3 pbc="T T T"

$\mathrm{Cu} \quad 1.28504466 \quad 3.85513397$

$\mathrm{Cu} \quad 0.00000000 \quad 5.99687506$

0.42834822

1.28504466

2.57008931

0.00000000

4.71183041

1.28504466

0.00000000

6.84411421

1. 30290222

0.00000000

1.27092539

0.00000000

1. 28504466

0.00000000

1. 28504466

0.00000000

1. 28504466

0.00000000

1. 26718709

0.00000000

1. 29916393

0.00000000

3. 85513397

1. 30946892

2. 92284024

5.65874530

15.39496765

11.56540191

13.70714300

8.13861616

10.28035725

12.42209835

14.59749128

9.01973686

11.11438861

13.36901324

8.73360951

3.85513397

5.99687506

0.42834822

2.57008931

4.71183041

6.88722334

1. 30946892

3.40412067
8.00000000
8.60577586
9.21155172
9.81732758
10.42310344
11.00475930
11.59509806
14.08801941
12.75041256
13.35094357
8.00000000
8.60577586
9.21155172
9.81732758
10.42310344
11.00376280
11.59509806
12.24951074
12.75041256
14.79855813
8.00000000
8.60577586
9.21155172
9.81732758
10.42310344
11.00376280
11.59509806
12.24951074 
$\mathrm{Cu}$

$\mathrm{Cu}$

$\mathrm{Cu}$

$\mathrm{Cu}$

$\mathrm{Cu}$

$\mathrm{Cu}$

$\mathrm{Cu}$

$\mathrm{Cu}$

$\mathrm{Cu}$

$\mathrm{Cu}$

$\mathrm{Cu}$

$\mathrm{Cu}$

O

0

0

O

O

0

O

O

0

O

O

0

0

0

0

0
3.86925324

2. 57008931

3. 85513397

2. 57008931

3. 85513397

2. 57008931

3. 85513397

2. 57008931

3. 87299154

2. 57008931

3. 84101470

2. 57008931

1. 36032596

3. 93041527

1.28901037

3.85909968

1.19865674

3.76874605

3. 94152189

1.28107895

1.37143257

3. 77985267

3. 85116826

1.20976335

2. 57008931

2.57008931

0.00000000

0.00000000
Fri Apr 16 14:51:55 2021

5.65874530

1.02334157

11.56540191

13.70714300

8.13861616

10.28035725

12.42209835

14.55438215

9.01973686

10.63310818

13.36901324

7.68469971

8.15830892

0.44804098

1.61496376

9.32523170

12.03658165

4.32631371

12.03658165

9.32523170

4.32631371

8.15830892

1.61496376

0.44804098

7.56744733

4.25560441

15.27771527

11.96587235
12.75041256

14.79855813

8.00000000

8.60577586

9.21155172

9.81732758

10.42310344

11.00475930

11.59509806

14.08801941

12.75041256

13.35094357

16.04231548

16.04231548

13.53678236

13.53678236

14.16272526

14.16272526

14.16272526

13.53678236

14.16272526

16.04231548

13.53678236

16.04231548

15.48806199

15.05629321

15.48806199

15.05629321

42

Lattice $=" 5.1401786267286710 .0 \quad 0.0 \quad 0.015 .420535880186020 .0 \quad 0.00 .021 .451982745205 "$ Prope rties=species:S: $1:$ pos:R: 3 pbc="T T T"

3.85513397

$\mathrm{Cu} \quad 0.00000000 \quad 5.99687506$

$\mathrm{Cu} \quad 1.28504466 \quad 0.42834822$

$\mathrm{Cu} \quad 0.00000000 \quad 2.57008931$

$\mathrm{Cu} \quad 1.28504466$

0.00314865

1.28504466

0.00308303

1.28504466

0.06780845

1. 28504466

0.00000000

1.28504466

0.00000000

1.28504466

$-0.00314865$

1. 28504466

$-0.00308303$

1.28504466

$-0.06780845$

3.85513397

2. 57008931

3.85513397

2. 57008931

3. 85513397

2. 56694067

3.85513397

2. 56700629

3. 85513397

2. 50228087

3.85513397

2. 57008931

3. 85513397

2. 57008931

3.85513397

4.71183041

6.87522949

1.34578369

3. 45014541

5.49894620

0.06996433

11.56540191

13.70714300

8.13861616

10.28035725

12.42209835

14.58549743

9.07634186

11.16041335

13.27568258

7.78023227

3.85513397

5.99687506

0.42834822

2.57008931

4.71183041

6.87522949

1. 36607392

3.45014541

5.56541464

0.06996433

11.56540191

13.70714300

8.13861616

10.28035725

12.42209835

14.58549743

8.00000000

8.60577586

9. 21155172

9.81732758

10.42310344

11.01269007

11.65606419

12.25704283

12.93993837

13.36784068

8.00000000

8.60577586

9.21155172

9.81732758

10.42310344

11.01269007

11.63145439

12.25704283

12.75761488

13.36784068

8.00000000

8.60577586

9.21155172

9.81732758

10.42310344

11.01269007

11.63145439

12.25704283

12.75761488

13.36784068

8.00000000

8.60577586

9.21155172

9.81732758

10.42310344

11.01269007 


\section{$\mathrm{Cu}$}

$\mathrm{Cu}$

$\mathrm{Cu}$

$\mathrm{Cu}$

O

0

. $/$

43

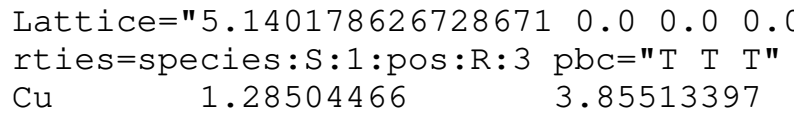

$\mathrm{Cu}$

$\mathrm{Cu}$

$\mathrm{Cu}$

$\mathrm{Cu}$

$\mathrm{Cu}$

$\mathrm{Cu}$

$\mathrm{Cu}$

$\mathrm{Cu}$

$\mathrm{Cu}$

$\mathrm{Cu}$

$\mathrm{Cu}$

$\mathrm{Cu}$

$\mathrm{Cu}$

$\mathrm{Cu}$

$\mathrm{Cu}$

$\mathrm{Cu}$

$\mathrm{Cu}$

$\mathrm{Cu}$

$\mathrm{Cu}$

$\mathrm{Cu}$

$\mathrm{Cu}$

$\mathrm{Cu}$

$\mathrm{Cu}$

$\mathrm{Cu}$

$\mathrm{Cu}$

$\mathrm{Cu}$

$\mathrm{Cu}$

$\mathrm{Cu}$

$\mathrm{Cu}$

$\mathrm{Cu}$

$\mathrm{Cu}$

$\mathrm{Cu}$

$\mathrm{Cu}$

$\mathrm{Cu}$

$\mathrm{Cu}$

$\mathrm{Cu}$

$\mathrm{Cu}$

$\mathrm{Cu}$

$\mathrm{Cu}$

O

0

0
3. 85513397

2. 57317234

3. 85513397

2. 63789776

1. 28504466

3. 85513397
9.05605163

11.16041335

13.20921414

6.88305943

14.59332737
7.78023227

oxygen/Cu/221/iter_3/struct.xyz

$\begin{array}{rr}1.28504466 & 3.85513397 \\ 0.00000000 & 5.99687506 \\ 1.28504466 & 0.42834822 \\ 0.00000000 & 2.57008931 \\ 1.28504466 & 4.71183041 \\ 0.00049160 & 6.87766369 \\ 1.28504466 & 1.32266356 \\ -0.01323958 & 3.46745679 \\ 1.28504466 & 5.51790155\end{array}$

$-0.00417865$

1.28504466

0.00000000

1. 28504466

0.00000000

1.28504466

1.28504466

0.00777767

$-0.07041035$

3.85513397

3.85513397

2.57008931

2. 56959771

3.85513397

2.58332890

3.85513397

2.57426797

3. 85513397

2. 57008931

3.85513397

2.57008931

3. 85513397

2.57703852

3. 85513397

2. 56231164

3.85513397

2. 64049967

1. 28504466

3. 85513397

0.12402955

11.56540191

13.70714300

8.13861616

10.28035725

12.42209835

14.58380259

9.06727446

11.15229283

13.30444005

7.78356962

5.99687506

0.42834822

2.57008931

4.71183041

6.87766369

1. 31564860

3.46745679

5.57767966

0.12402955

11.56540191

13.70714300

8.13861616

10.28035725

12.42209835

14.58380259

9.05378266

11.15229283

13.20176346

7.78356962

6.87795713

14.37458630

1.44315814
Fri Apr 16 14:51:55 2021

150
11.65606419

12.25704283

12.93993837

13.36784068

14.29283503
14.29283503

$-0.00694921$

1.28504466

2.57008931

3.85513397

3.85513397

$15.420535880186020 .0 \quad 0.0 \quad 0.021 .451982745205 "$ Prope

8.00000000

8.60577586

9.21155172

9.81732758

10.42310344

11.00486341

11.60791795

12.24229749

12.90851089

13.71820114

8.00000000

8.60577586

9. 21155172

9.81732758

10.42310344

11.03050128

11.63261506

12.23315099

12.76525853

13.36993458

8.00000000

8.60577586

9. 21155172

9.81732758

10.42310344

11.00486341

11.64801805

12.24229749

12.75306498

13.71820114

8.00000000

8.60577586

9. 21155172

9. 81732758

10.42310344

11.03050128

11.65678109

12.23315099

12.83548499

13.36993458

14.28341372

14.32917521

13.56240295

. /oxygen/Cu/221/iter_4/struct.xyz

44

Lattice $=" 5.140178626728671 \quad 0.0 \quad 0.0 \quad 0.0 \quad 15.42053588018602 \quad 0.0 \quad 0.0 \quad 0.021 .451982745205 "$ Prope rties=species:S:1:pos:R:3 pbc="T T T"

$\begin{array}{lrr}\mathrm{Cu} & 1.28504466 & 3.85513397 \\ \mathrm{Cu} & 0.00000000 & 5.99687506 \\ \mathrm{Cu} & 1.28504466 & 0.42834822 \\ \mathrm{Cu} & 0.00000000 & 2.57008931 \\ \mathrm{Cu} & 1.28504466 & 4.71183041 \\ \mathrm{Cu} & 0.00309801 & 6.87581865 \\ \mathrm{Cu} & 1.28504466 & 1.31946052 \\ \mathrm{Cu} & -0.02242800 & 3.45373952 \\ \mathrm{Cu} & 1.28504466 & 5.50690336 \\ \mathrm{Cu} & -0.00268399 & 0.12477004 \\ \mathrm{Cu} & 1.28504466 & 11.56540191\end{array}$


$\mathrm{Cu}$

$\mathrm{Cu}$

$\mathrm{Cu}$

$\mathrm{Cu}$

$\mathrm{Cu}$

$\mathrm{Cu}$

$\mathrm{Cu}$

$\mathrm{Cu}$

$\mathrm{Cu}$

$\mathrm{Cu}$

$\mathrm{Cu}$

$\mathrm{Cu}$

$\mathrm{Cu}$

$\mathrm{Cu}$

$\mathrm{Cu}$

$\mathrm{Cu}$

$\mathrm{Cu}$

$\mathrm{Cu}$

$\mathrm{Cu}$

$\mathrm{Cu}$

$\mathrm{Cu}$

$\mathrm{Cu}$

$\mathrm{Cu}$

$\mathrm{Cu}$

$\mathrm{Cu}$

$\mathrm{Cu}$

$\mathrm{Cu}$

$\mathrm{Cu}$

$\mathrm{Cu}$

O

O

O

0

$.10 x y+2$

45

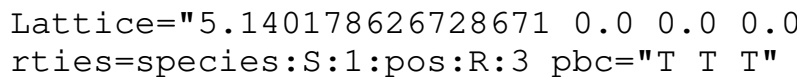

$\mathrm{Cu} \quad 1.28504466 \quad 3.85513397$

$\begin{array}{lll}\mathrm{Cu} & 0.00000000 & 5.99687506\end{array}$

$\mathrm{Cu} \quad 1.28504466$

$\mathrm{Cu} \quad 0.00000000$

$\mathrm{Cu} \quad 1.28504466$

$\mathrm{Cu} \quad-0.00170256$

$\mathrm{Cu} \quad 1.28504466$

$\mathrm{Cu} \quad-0.01357827$

$\mathrm{Cu} \quad 1.28504466$

$\mathrm{Cu} \quad 0.00409664$

$\mathrm{Cu} \quad 1.28504466$

$\mathrm{Cu} \quad 0.00000000$

$\mathrm{Cu} \quad 1.28504466$

$\mathrm{Cu} \quad 0.00000000$

$\mathrm{Cu} \quad 1.28504466$

$\mathrm{Cu} \quad-0.00332355$

$\mathrm{Cu} \quad 1.28504466$

$\mathrm{Cu} \quad-0.03039938$

$\mathrm{Cu} \quad 1.28504466$

$\mathrm{Cu} \quad 0.00148713$

$\mathrm{Cu} \quad 3.85513397$

$\mathrm{Cu} \quad 2.57008931$

$\begin{array}{ll}\mathrm{Cu} & 3.85513397\end{array}$

$\mathrm{Cu} \quad 2.57008931$

$\mathrm{Cu} \quad 3.85513397$

$\mathrm{Cu} \quad 2.57179188$

$\mathrm{Cu} \quad 3.85513397$

$\mathrm{Cu} \quad 2.58366758$

$\mathrm{Cu} \quad 3.85513397$

$\mathrm{Cu} \quad 2.56599267$

$\mathrm{Cu}$
0.42834822

2.57008931

4.71183041

6.89253272

1.23410051

3.38713568

5.48503992

0.55832056

11.56540191

13.70714300

8.13861616

10.28035725

12.42209835

14.57036149

9.02072014

11.22809779

13.58051276

7.73471881

3.85513397

0.42834822

2.57008931

4. 71183041

6.89253272

1.29858554

5.54956858

0.55832056

11.56540191
5.99687506

3. 38713568
Fri Apr 16 14:51:55 2021

151

13.70714300

8.13861616

8. 60577586

9.21155172

9.81732758

10.42310344

11.02432372

11.64430176

12.21981600

12.76109289

13.71795280

8.00000000

8.60577586

9.21155172

9.81732758

10.42310344

11.02432372

11.64430176

12.21981600

12.76109289

13.71795280

8.00000000

8.60577586

9.21155172

9.81732758

10.42310344

11.02432372

11.60948939

12.21981600

12.82193045

13.71795280

14.32518604

14.32518604

13.56905302

13.56905302
8.00000000
8.60577586
9.21155172
9.81732758
10.42310344
11.03337859
11.53283220
12.26670214
12.78297101
13.71254992
8.00000000
8.60577586
9.21155172
9.81732758
10.42310344
10.98950034
11.64235079
12.29033831
12.85953430
13.72240761
8.00000000
8.60577586
9.21155172
9.81732758
10.42310344
11.03337859
11.59522701
12.26670214
12.74698541
13.71254992
8.00000000 


$\begin{array}{ll}\mathrm{Cu} & 2.57008931 \\ \mathrm{Cu} & 3.85513397 \\ \mathrm{Cu} & 2.57008931 \\ \mathrm{Cu} & 3.85513397 \\ \mathrm{Cu} & 2.57341287 \\ \mathrm{Cu} & 3.85513397 \\ \mathrm{Cu} & 2.60048870 \\ \mathrm{Cu} & 3.85513397 \\ \mathrm{Cu} & 2.56860218 \\ \mathrm{O} & 1.28504466 \\ \mathrm{O} & 3.85513397 \\ \mathrm{O} & 1.28504466 \\ \mathrm{O} & 3.85513397 \\ \mathrm{O} & 1.28504466\end{array}$

Fri Apr 16 14:51:55 2021

13.70714300

10.28035725

12.42209835

14.57036149

11.22809779

13.42542296

14.76722375

11.79786512

/ oxygen/Cu/221/iter_6/struct.xyz

46

Lattice $=" 5.140178626728671 \quad 0.0 \quad 0.0 \quad 0.0 \quad 15.42053588018602$ rties=species:S:1:pos:R:3 pbc="T T T"

$\mathrm{Cu} \quad 1.28504466$

$\mathrm{Cu} \quad 0.00000000$

$\mathrm{Cu} \quad 1.28504466$

0.00000000

1.28504466

$-0.00903751$

1.28504466

0.03144473

1.28504466

$-0.00157118$

1.28504466

0.00000000

1.28504466

0.00000000

1.28504466

0.00903751

1.28504466

$-0.03144473$

1. 28504466

0.00157118

3.85513397

2.57008931

3. 85513397

2. 57008931

3. 85513397

2.57912683

3. 85513397

2.53864458

3.85513397

2. 57166049

3.85513397

2.57008931

3. 85513397

2.57008931

3.85513397

2. 56105180

3.85513397

2.60153404

3. 85513397

2.56851814

1.28504466

3. 85513397

1.28504466

3.85513397

1.28504466

3. 85513397

10.42310344
8.13861616

9.09009236

7.73471881

6.56786222

1.93285626

9.04268562
8. 60577586

9.21155172

9.81732758

10.98950034

11.62349077

12.29033831

12.86273389

13.72240761

14.33658556

14.22429098

13.46524772

13.56740084

13.54584488

\section{2}

5.99687506

8.00000000

8.60577586

9.21155172

9.81732758

10.42310344

11.01977781

11.57800677

12.27748352

12.81093597

13.71550663

8.00000000

8.60577586

9.21155172

9.81732758

10.42310344

11.01977781

11.62509655

12.27748352

12.83965072

13.71550663

8.00000000

8.60577586

9. 21155172

9.81732758

10.42310344

11.01977781

11.62509655

12.27748352

12.83965072

13.71550663

8.00000000

8.60577586

9. 21155172

9.81732758

10.42310344

11.01977781

11.57800677

12.27748352

12.81093597

13.71550663

14.29943335

14.29943335

13.53423315

13.53423315

13.54624211

13.54624211

9.23834937

3. 94250654
1.52808143

11.65277448

. /oxygen/Cu/221/iter_7/struct. xyz $0.0 \quad 0.00 .021 .451982745205 "$ Prope

47

Lattice $=" 5.140178626728671 \quad 0.0 \quad 0.0 \quad 0.0 \quad 15.420535880186020 .0 \quad 0.0 \quad 0.021 .451982745205 "$ Prope rties=species:S:1:pos:R:3 pbc="T T T" 
$\mathrm{Cu}$

$\mathrm{Cu}$

$\mathrm{Cu}$

$\mathrm{Cu}$

$\mathrm{Cu}$

$\mathrm{Cu}$

$\mathrm{Cu}$

$\mathrm{Cu}$

$\mathrm{Cu}$

$\mathrm{Cu}$

$\mathrm{Cu}$

$\mathrm{Cu}$

$\mathrm{Cu}$

$\mathrm{Cu}$

$\mathrm{Cu}$

$\mathrm{Cu}$

$\mathrm{Cu}$

$\mathrm{Cu}$

$\mathrm{Cu}$

$\mathrm{Cu}$

$\mathrm{Cu}$

$\mathrm{Cu}$

$\mathrm{Cu}$

$\mathrm{Cu}$

$\mathrm{Cu}$

$\mathrm{Cu}$

$\mathrm{Cu}$

$\mathrm{Cu}$

$\mathrm{Cu}$

$\mathrm{Cu}$

$\mathrm{Cu}$

$\mathrm{Cu}$

$\mathrm{Cu}$

$\mathrm{Cu}$

$\mathrm{Cu}$

$\mathrm{Cu}$

$\mathrm{Cu}$

$\mathrm{Cu}$

$\mathrm{Cu}$

$\mathrm{Cu}$

$\mathrm{O}$

0

0

0

0

0

O
1.28504466

0.00000000

1. 28504466

0.00000000

1.28504466

$-0.00687813$

0.00505424

1. 28504466

0.00727193

1. 28504466

0.00000000

1. 28504466

0.00000000

1. 28504466

0.00568949

0.10240949

1. 28504466

$-0.00373003$

3. 85513397

2. 57008931

3. 85513397

2. 57008931

3. 85513397

2.57696744

3. 85513397

2. 56503508

3. 85513397

2. 56281738

3. 85513397

2. 57008931

3. 85513397

2. 57008931

2. 56439982

3. 85513397

2. 46767982

3. 85513397

2. 57381934

1. 28504466

3. 85513397

1. 28504466

3. 85513397

1. 28504466

3. 85513397

3. 85513397
1. 28504466

1. 28504466

3. 85513397

Fri Apr 16 14:51:55 2021

3.85513397

5.99687506

0.42834822

2.57008931

4.71183041

6.88316048

1.24932503

3.47120359

5.69619079

0.90966033

11.56540191

13.70714300

8.13861616

10.28035725

12.42209835

14.59216065

8.99371095

11.06073240

14.03977249

8.08979590

3.85513397

5.99687506

0.42834822

2.57008931

4.71183041

6.88316048

1.28554570

3.47120359

5.85780520

0.90966033

11.56540191

13.70714300

8.13861616

10.28035725

12.42209835

14.59216065

9.03376366

11.06073240

13.95703084

8.08979590

6.90300327

15.26140751

2.18472658

9.43734942

12.18987245

4.11452515

12.44622243

. /oxygen/Cu/221/iter_8/struct.xyz

48

Lattice $=" 5.140178626728671 \quad 0.0 \quad 0.0 \quad 0.0 \quad 15.42053588018602$ rties $=$ species:S:1:pos:R:3 pbc="T T T"

$\mathrm{Cu}$

$\mathrm{Cu}$

$\mathrm{Cu}$

$\mathrm{Cu}$

$\mathrm{Cu}$

$\mathrm{Cu}$

$\mathrm{Cu}$

$\mathrm{Cu}$

$\mathrm{Cu}$

$\mathrm{Cu}$

$\mathrm{Cu}$

$\mathrm{Cu}$

$\mathrm{Cu}$

$\mathrm{Cu}$

$\mathrm{Cu}$

$\mathrm{Cu}$

$\mathrm{Cu}$
3. 85513397

5.99687506

0.42834822

2.57008931

4.71183041

6.88182900

1.37690347

3. 47532190

5.63018197

1. 34490335

11.56540191

13.70714300

8.13861616

10.28035725

12.42209835

14.67033274

8.98190293
153
8.00000000

8.60577586

9. 21155172

9.81732758

10.42310344

10.98876618

11.61679631

12.27948869

12.80338949

13.94532332

8.00000000

8.60577586

9.21155172

9.81732758

10.42310344

11.01798729

11.61235006

12.38639539

13.09837689

13.72767911

8.00000000

8.60577586

9.21155172

9.81732758

10.42310344

10.98876618

11.63143641

12.27948869

12.86057217

13.94532332

8.00000000

8.60577586

9.21155172

9.81732758

10.42310344

11.01798729

11.58310632

12.38639539

13.39171894

13.72767911

14.27695991

14.68668069

13.48723416

13.54387128

13.43849394

13.56429799

12.34299842
8.00000000
8.60577586
9.21155172
9.81732758
10.42310344
11.00779777
11.61649328
12.26531953
12.77857807
14.13704198
8.00000000
8.60577586
9.21155172
9. 81732758
10.42310344
10.99614831
11.60008434 
$\mathrm{Cu}$

$\mathrm{Cu}$

$\mathrm{Cu}$

$\mathrm{Cu}$

$\mathrm{Cu}$

$\mathrm{Cu}$

$\mathrm{Cu}$

$\mathrm{Cu}$

$\mathrm{Cu}$

$\mathrm{Cu}$

$\mathrm{Cu}$

$\mathrm{Cu}$

$\mathrm{Cu}$

$\mathrm{Cu}$

$\mathrm{Cu}$

$\mathrm{Cu}$

$\mathrm{Cu}$

$\mathrm{Cu}$

$\mathrm{Cu}$

$\mathrm{Cu}$

$\mathrm{Cu}$

$\mathrm{Cu}$

$\mathrm{Cu}$

O

0

O

0

0

O

0

0

1.28504466

49

Lattice $=" 5.140178626728671 \quad 0.0 \quad 0.0 \quad 0.0$ rties=species: $\mathrm{S}: 1:$ pos $: \mathrm{R}: 3$ pbc $=" \mathrm{~T} T \mathrm{~T}$ T"

$1.28504466 \quad 3.85513397$

$\mathrm{Cu} \quad 0.00000000$

$\mathrm{Cu} \quad 1.28504466$

$\mathrm{Cu} \quad 0.00000000$

$\mathrm{Cu} \quad 1.28504466$

$\mathrm{Cu} \quad 5.07976056$

$\mathrm{Cu} \quad 1.28504466$

$\mathrm{Cu} \quad 0.03520518$

$\mathrm{Cu} \quad 1.28504466$

$\mathrm{Cu} \quad 0.01369297$

$\mathrm{Cu} \quad 1.28504466$

$\mathrm{Cu} \quad 0.00000000$

$\mathrm{Cu} \quad 1.28504466$

$\mathrm{Cu} \quad 0.00000000$

$\mathrm{Cu} \quad 1.28504466$

$\mathrm{Cu} \quad 5.09149491$

$\mathrm{Cu} \quad 1.28504466$

$\mathrm{Cu} \quad 0.02563553$

$\mathrm{Cu} \quad 1.28504466$

$\mathrm{Cu} \quad 5.09917345$

$\mathrm{Cu} \quad 3.85513397$

$\mathrm{Cu} \quad 2.57008931$

$\mathrm{Cu} \quad 3.85513397$

$\mathrm{Cu} \quad 2.57008931$

$\mathrm{Cu} \quad 3.85513397$

$\mathrm{Cu} \quad 2.63050738$

$\mathrm{Cu} \quad 3.85513397$

$\mathrm{Cu} \quad 2.53488414$

$\mathrm{Cu} \quad 3.85513397$

$\mathrm{Cu} \quad 2.55639634$

$\mathrm{Cu} \quad 3.85513397$

Cu 2.57008931

$\mathrm{Cu}$

3.85513397
Fri Apr 16 14:51:55 2021

154

11.27618603

14.90983986

7.75037752

3.85513397

5.99687506

0.42834822

2.57008931

4.71183041

6.88182900

1. 32545059

3.47532190

5.77214738

1.34490335

11.56540191

13.70714300

8.13861616

10.28035725

12.42209835

14.67033274

9.05755324

11.27618603

14.19983099

7.75037752

6.57388489

0.14184510

2.58763029

9.02036466

13.55933221

4.04368834

12.58641229

10.05750686

5.99687506

0.42834822

2.57008931

4.71183041

7.00140957

1.39385679

3.43553167

6.52774574

1.53851157

11.56540191

13.70714300

8.13861616

10.28035725

12.42209835

14.67888663

9.09725823

11.37272301

15.03333871

8.53009182

5.99687506

0.42834822

2.57008931

4.71183041

7.00140957

1. 34173458

3.43553167

6.59889153

1.53851157

11.56540191

13.70714300

8.13861616
12.71364005

13.29090267

13.86874372

8.00000000

8.60577586

9.21155172

9.81732758

10.42310344

11.00779777

11.68890042

12.26531953

12.86480252

14.13704198

8.00000000

8.60577586

9.21155172

9.81732758

10.42310344

10.99614831

11.62333352

12.71364005

13.45023686

13.86874372

14.42326118

14.71287137

13.74245569

13.56416359

11.99338615

13.60001007

12.46891778

13.19760844

$$
8.00000000
$$

8.60577586

9.21155172

9.81732758

10.42310344

11.00938575

11.58544708

12.30744307

13.66690617

14.16437023

8.00000000

8.60577586

9.21155172

9.81732758

10.42310344

10.98531095

11.56960878

12.71629265

13.28435597

14.49486637

8.00000000

8.60577586

9.21155172

9. 81732758

10.42310344

11.00938575

11.71503444

12.30744307

13.31133425

14.16437023

8.00000000

8.60577586

9.21155172
$15.420535880186020 .0 \quad 0.0 \quad 0.021 .451982745205 "$ Prope 


\section{$\mathrm{Cu}$}

$\mathrm{Cu}$

$\mathrm{Cu}$

$\mathrm{Cu}$

$\mathrm{Cu}$

$\mathrm{Cu}$

$\mathrm{Cu}$

O

O

O

O

0

O

O

0

0

1. 28504466

37

$8827071757 "$ Properties=spe

Cu

$\mathrm{Cu}$

$\mathrm{Cu}$

$\mathrm{Cu}$

$\mathrm{Cu}$

$\mathrm{Cu}$

$\mathrm{Cu}$

$\mathrm{Cu}$

$\mathrm{Cu}$

$\mathrm{Cu}$

$\mathrm{Cu}$

$\mathrm{Cu}$

$\mathrm{Cu}$

$\mathrm{Cu}$

$\mathrm{Cu}$

$\mathrm{Cu}$

$\mathrm{Cu}$

$\mathrm{Cu}$

$\mathrm{Cu}$

$\mathrm{Cu}$

$\mathrm{Cu}$

$\mathrm{Cu}$

$\mathrm{Cu}$

$\mathrm{Cu}$

$\mathrm{Cu}$

$\mathrm{Cu}$

$\mathrm{Cu}$

$\mathrm{Cu}$

$\mathrm{Cu}$

$\mathrm{Cu}$

$\mathrm{Cu}$

$\mathrm{Cu}$

$\mathrm{Cu}$

$\mathrm{Cu}$

$\mathrm{Cu}$

$\mathrm{Cu}$

O

3. 85147131
Fri Apr 16 14:51:55 2021

155

12.42209835

14.67888663

9.07758168

11.37272301

14.33616587

8.53009182

7.46961306

0.31484710

2.88034740

9.66827785

13. 61717472

4.82880403

12.68909020

10.10165988

5.91654111
uct. xyz

ruct. xyz
9.81732758

10.42310344

10.98531095

11.79416206

12.71629265

13.41317699

14.49486637

15.25614665

14.64192117

13.94843172

13.73288707

12.02050769

12.57698722

12.47874448

13.21160799

11.93430719
10.28035725

$\begin{array}{lllllllllllll}0.0 & 0.0 & 2.5700893133643348 & 8.524021930131648 & 0.0 & 0.0 & 0.0 & 21.47944\end{array}$ : $: 1: \mathrm{pos}: \mathrm{R}: 3 \mathrm{pbc}=" \mathrm{~T} \mathrm{~T} \mathrm{~T} "$

$\begin{array}{lll}2.57008931 & 0.77491108 & 9.09588977\end{array}$

$\begin{array}{lll}1.28504466 & 2.71218880 & 10.19177953\end{array}$

$\begin{array}{lll}1.29009873 & 0.38577134 & 11.22743844\end{array}$

$2.58808082 \quad 2.35003165$

$-0.05029168 \quad 0.01266589$

$3.85513397 \quad 7.36165530$

$3.85513397 \quad 5.03692205$

$2.57008931 \quad 6.97419976$

$2.57330057 \quad 4.66015977$

6.59857211

4.35468649

3.09964434

0.77491108

2.71218880

0.41273198

2. 34419111

$-0.01780908$

7.36165530

5.03692205

6.97419976

4.67539295

6.60256569

4.29817797

3.09964434

0.77491108

2.71218880

0.41841821

2. 37451539

$-0.02640689$

7. 36165530

5.03692205

6.97419976

4.68119342

6.61581471

4.30631047

1. 37815313

12.44690233

13.41408892

8.00000000

9.09588977

10.19177953

11.29360463

12.40674691

13.39506037

8.00000000

9.09588977

10.19177953

11.28416514

12.41746495

13.41985581

8.00000000

9.09588977

10.19177953

11.28247872

12.42381256

13.37973334

8.00000000

9.09588977

10.19177953

11.28304168

12.43878579

13.35358615

8.00000000

9.09588977

10.19177953

11.29572684

12.41125786

13.38411867

13.76630464

46

Lattice $=" 7.71026794009301 \quad 0.0 \quad 0.0 \quad 2.5700893133643348 \quad 8.524021930131648 \quad 0.0 \quad 0.0 \quad 0.0 \quad 21.47944$ $8827071757 "$ Properties=species:S:1:pos:R:3 pbc="T T T"

$\mathrm{Cu}$

..57008931

3.09964434

0.77491108

2.71218880

1. 28504466

0.32954772

2. 22322905

0.73184300

9.09588977

10.19177953

1. 24269413

11.28593816

$\mathrm{Cu} \quad 2.56794302$

0.68145095

7.36165530

12.34532621

14.75402646

8.00000000

$\mathrm{Cu}$

3.85513397 


\section{$\mathrm{Cu}$}

$\mathrm{Cu}$

$\mathrm{Cu}$

$\mathrm{Cu}$

$\mathrm{Cu}$

$\mathrm{Cu}$

$\mathrm{Cu}$

$\mathrm{Cu}$

$\mathrm{Cu}$

$\mathrm{Cu}$

$\mathrm{Cu}$

$\mathrm{Cu}$

$\mathrm{Cu}$

$\mathrm{Cu}$

$\mathrm{Cu}$

$\mathrm{Cu}$

$\mathrm{Cu}$

$\mathrm{Cu}$

$\mathrm{Cu}$

$\mathrm{Cu}$

$\mathrm{Cu}$

$\mathrm{Cu}$

$\mathrm{Cu}$

$\mathrm{Cu}$

$\mathrm{Cu}$

$\mathrm{Cu}$

$\mathrm{Cu}$

$\mathrm{Cu}$

$\mathrm{Cu}$

O

O

0

0

O

0

0

0

O

0

$. / 0 \times 1$

47
Fri Apr 16 14:51:55 2021

156

5.03692205

6.97419976

4.64677973

7.14223726

4.02036027

3.09964434

0.77491108

2.71218880

0.36303847

2.28445137

1.37096448

7.36165530

5.03692205

6.97419976

4.72016776

6.89659150

4.16964967

3.09964434

0.77491108

2.71218880

0.44865833

2.28667894

8.37888517

7.36165530

5.03692205

6.97419976

4.70758425

6.88184962

3.90962116

0.47018523

8.23596177

2.66139926

5.37711527

2.54707233

0.10619846

3.07006732

7.72059755

5.17767212

5.31766369
9.09588977

10.19177953

11.39862383

12.98930119

14.06840274

8.00000000

9.09588977

10.19177953

11.25141738

12.30448546

14.88637708

8.00000000

9.09588977

10.19177953

11.27295776

13.98469524

14.00795426

8.00000000

9.09588977

10.19177953

11.22529384

12.27139564

15.69112436

8.00000000

9.09588977

10.19177953

11.33088947

12.61397758

13.89114247

13.23661018

15.17490301

14.20809566

13.46034776

14.20671527

16.20057303

14.90861591

12.14974339

13.31880481

13.15946309

Lattice $=" 7.71026794009301 \quad 0.0 \quad 0.0 \quad 2.5700893133643348 \quad 8.524021930131648 \quad 0.0 \quad 0.0 \quad 0.021 .47944$ 8827071757" Properties=species:S:1:pos:R:3 pbc="T T T"

$\begin{array}{llll}\mathrm{Cu} & 2.57008931 & 3.09964434 & 8.00000000\end{array}$

$\mathrm{Cu} \quad 2.57008931 \quad 0.77491108$

$1.28504466 \quad 2.71218880$

9.09588977

$2.71218880 \quad 10.19177953$

$1.31114183 \quad 0.46560605$

$2.63194115 \quad 2.41726909$

11.27947271

$\mathrm{Cu} \quad 2.63194115$

9.64289511

7.36165530

12.51408487

14.73458230

8.00000000

3. 85513397

5.03692205

2.57008931

6.97419976

4.64273399

2.56759865

6.78007329

3. 81688510

4.68142121

9.10680979
5.14017863

3.09964434

5.14017863

0.77491108

2.71218880

3.85513397

0.36685069

2.29494694

5.17186657

0.14454404

2.03746080

7. 36165530

5.03692205

6.97419976

4.69308182

7.36026740

9.09588977

10.19177953

11.27468303

12.42821528

14.43598417

8.00000000

9.09588977

10.19177953

11.28113801

12.36329025

14.21116605

8.00000000

9.09588977

10.19177953

11.32390736

14.23166233

14.36971606

3. 94757316

4.68514401

8.00000000

$\mathrm{Cu}$

3.09964434 


\section{$\mathrm{Cu}$}

$\mathrm{Cu}$

$\mathrm{Cu}$

$\mathrm{Cu}$

$\mathrm{Cu}$

$\mathrm{Cu}$

$\mathrm{Cu}$

$\mathrm{Cu}$

$\mathrm{Cu}$

$\mathrm{Cu}$

$\mathrm{Cu}$

O

O

O

0

0

O

0

O

O

O

0
7.71026794

6.42522328

6.47762177

7.81838559

4.53224904

8.99531260

8.99531260

7.71026794

7.70809777

8.99567166

6.51690239

0.51948647

6.06980205

5.12564600

2. 62265236

7. 95277052

6.11432594

2. 68881746

5.11186327

7.79871510

5.12950774

2.77376502
Fri Apr 16 14:51:55 2021

0.77491108

2.71218880

0.40189032

2. 32783977

1. 49930305

7.36165530

5.03692205

6.97419976

4.63087442

6.56852601

4.33556336

0.10170368

8.45831581

3.12668384

6.16803276

3.22835803

0.70965461

3. 61348503

0.18692714

5.78097928

5.54986463

2.24850873
9.09588977

10.19177953

11.25169919

12.43394517

15.03206210

8.00000000

9.09588977

10.19177953

11.23952897

12.25864537

13.66940077

13.15759560

15.29088363

14.17024094

14.47612785

14.09764839

15.52848017

15.31499140

12.64143526

13.58565837

13.16060547

14.62490656

48

Lattice $=" 7.71026794009301 \quad 0.0 \quad 0.0 \quad 2.5700893133643348 \quad 8.524021930131648 \quad 0.0 \quad 0.0 \quad 0.0 \quad 21.47944$ 8827071757" Properties=species:S:1:pos:R:3 pbc="T T T"

$\mathrm{Cu} \quad 2.57008931 \quad 3.09964434 \quad 8.00000000$

$\mathrm{Cu} \quad 2.57008931$

0.77491108

1.28504466

2.71218880

9.09588977

1. 30982417

0.42873307

2.63947314

2.35711227

9.37073231

8.29102810

3. 85513397

7. 36165530

3.85513397

5.03692205

2. 57008931

6.97419976

2. 58627633

4.66952350

3.88697825

6.77372331

1. 35027960

4.23925262

5.14017863

3.09964434

0.77491108

5.14017863

3. 85513397

2.71218880

10.19177953

11.28401155

12.26328194

14.29230519

8.00000000

9.09588977

10.19177953

11.30220474

12.36138295

13.66177938

8.00000000

9.09588977

0.37509837

2.42040604

5.22984408

1. 62693843

0.48818502

7.36165530

5.03692205

6.42522328

6.97419976

4.64207930

5.13637366

6.40186186

6.57016791

3. 90772332

7. 71026794

7.71026794

4.41952247

3.09964434

0.77491108

6.42522328

6.43145220

7.77458212

2.71218880

0.40492756

2. 33201490

4. 18575395

0.32886338

8.99531260

8.99531260

7. 36165530

5.03692205

7.71026794

7.70054492

6.97419976

4.59265660

6.58342615

8.90969613

6.58342615
4.59153244

10.19177953

11.33000727

12.43579166

14.84749010

8.00000000

9.09588977

10.19177953

11.26838072

12.32532570

14.10831609

8.00000000

9.09588977

10.19177953

11.28319211

12.43945632

14.69895189

8.00000000

9.09588977

10.19177953

11.29436189

12.28107570

14.20113862

13.21369154

16.02003538

0.09882083

3.21642759

5.58061073

3.14721947

14.20125091

13.34512074

14.21375280

15.12294731

7. 34190968 


\section{0}

O

0

0

O

0

\begin{abstract}
2.74104628
5.33369204

7.73117971

5.16762727

2.88508219

2.97365077
\end{abstract}

14.90414056 13.10980753 14.08228205 13.67713837 14.15113697 15.47845371

38

Lattice $=" 7.71026794009301 \quad 0.0 \quad 0.0 \quad 2.5700893133643348 \quad 8.524021930131648 \quad 0.0 \quad 0.0 \quad 0.0 \quad 21.47944$ $8827071757 "$ Properties=species:S:1:pos:R:3 pbc="T T T"

$\mathrm{Cu} \quad 2.57008931 \quad 3.09964434 \quad 8.00000000$

$\begin{array}{llll}\mathrm{Cu} & 2.57008931 & 0.77491108 & 9.09588977\end{array}$

$\begin{array}{llll}\mathrm{Cu} & 1.28504466 & 2.71218880 & 10.19177953\end{array}$

$\begin{array}{llll}\mathrm{Cu} & 1.30772821 & 0.38396565 & 11.24376214\end{array}$

$\begin{array}{llll}\mathrm{Cu} & 2.58757257 & 2.34528394 & 12.42270897\end{array}$

$\begin{array}{llll}\mathrm{Cu} & -0.01372525 & 0.05039160 & 13.39997165\end{array}$

$\mathrm{Cu} \quad 3.85513397$

$\mathrm{Cu} \quad 3.85513397$

7.36165530

5.03692205

$\begin{array}{ll}2.57008931 & 6.97419976\end{array}$

$2.57305039 \quad 4.66587461$

8.00000000

9.09588977

$\mathrm{Cu} \quad 2.57305039$

3.83506721

6.62727023

1.28786387

4.35291141

5.14017863

3.09964434

5.14017863

0.77491108

3.85513397

2.71218880

3.84867870

0.40515228

5.14719503

2.34254696

2.56210471

0.09239919

6.42522328

7.36165530

6.42522328

5.03692205

5.14017863

6.97419976

5.14798194

4.67912817

6.39501561

6.56078418

3.87023451

4.30636618

7.71026794

3. 09964434

7.71026794

0.77491108

6.42522328

2.71218880

6.42893608

0.42053589

7.68639497

2.38117203

5.23009711

0.00567738

8.99531260

7.36165530

8.99531260

5.03692205

7.71026794

6.97419976

7.70815946

4.68470161

9.04676458

6.62212921

10.19177953

11.29324190

12.40138322

13.39445007

8.00000000

9.09588977

10.19177953

11.30856310

12.40014719

13.76556600

8.00000000

9.09588977

10.19177953

11.28092069

12.45549823

13.36789882

8.00000000

9.09588977

10.19177953

11.29791442

12.41168174

13.43213219

8.00000000

9.09588977

10.19177953

11.29718109

12.39690417

13.40510666

13.77412608

4.25150946
1.47780601

14.19845820

. /oxygen/Cu/311/iter_3/struct.xyz

1.30219688

7.43442050

39

Lattice $=" 7.71026794009301 \quad 0.0 \quad 0.0 \quad 2.5700893133643348 \quad 8.524021930131648 \quad 0.0 \quad 0.0 \quad 0.021 .47944$ 8827071757 " Properties=species:S:1:pos:R:3 pbc="T T T"

$\mathrm{Cu}$

2.57008931

0.77491108

1.28504466

2.71218880

1.30137189

0.38459128

2. 56260515

2. 35389803

$-0.04549620$

0.07120359

3.85513397

7.36165530

3. 85513397

5.03692205

2. 57008931

6.97419976

2.58088415

4.67458732

3. 83563948

6.63823938

1. 27748451

4. 37269800

5.14017863

3.09964434

5.14017863

0.77491108

3.85513397

2.71218880

3. 84778684

0.40448484

9.09588977

10.19177953

11.23844659

12.38946227

13.40409921

8.00000000

9.09588977

10.19177953

11.28768052

12.38860948

13.37775803

8.00000000

9.09588977

10.19177953

11.31708922 


\section{$\mathrm{Cu}$}

$\mathrm{Cu}$

$\mathrm{Cu}$

$\mathrm{Cu}$

$\mathrm{Cu}$

$\mathrm{Cu}$

$\mathrm{Cu}$

$\mathrm{Cu}$

$\mathrm{Cu}$

$\mathrm{Cu}$

$\mathrm{Cu}$

$\mathrm{Cu}$

$\mathrm{Cu}$

$\mathrm{Cu}$

$\mathrm{Cu}$

$\mathrm{Cu}$

$\mathrm{Cu}$

$\mathrm{Cu}$

$\mathrm{Cu}$

$\mathrm{Cu}$

O

o

0
5.14577482

2.53843903

6.42522328

6.42522328

5.14017863

5.14195242

6.39530043

3. 76361057

7.71026794

7.71026794

6.42522328

6.41004356

7.71266099

5.22899409

8.99531260

8.99531260

7.71026794

7.70110607

9.06353091

6.50668108

1.29183422

6.39853717

5.08584567
2.25096625

0.08292670

7.36165530

5.03692205

6.97419976

4.66325029

6.53727202

4.36431342

3.09964434

0.77491108

2.71218880

0.41272726

2.38647986

$-0.10733608$

7.36165530

5.03692205

6.97419976

4.69345537

6.60586282

4.26201243

1.47585463

7. 38912486

3.29442029
12.52834484

13.74942449

8.00000000

9.09588977

10.19177953

11.29102057

12.43798501

13.44542223

8.00000000

9.09588977

10.19177953

11.30309280

12.35620736

13.46504453

8.00000000

9.09588977

10.19177953

11.29044711

12.38877206

13.47082112

13.73921166

14.19179096

14.20210210

. /oxygen/Cu/311/iter_4/struct.xyz

40

Lattice $=" 7.71026794009301 \quad 0.0 \quad 0.0 \quad 2.5700893133643348 \quad 8.524021930131648 \quad 0.0 \quad 0.0 \quad 0.021 .47944$ 8827071757 " Properties=species:S:1:pos:R:3 pbc="T T T"

$\mathrm{Cu}$

2.57008931

3.09964434

2.57008931

0.77491108

8.00000000

1.28504466

2.71218880

1. 29845961

0.38335829

2. 57575587

2.39739288

$-0.07865697$

3.85513397

0.17748492

7.36165530

3. 85513397

5.03692205

2. 57008931

6.97419976

2. 58350426

4.64536926

3.86080053

6.65940385

1.20638769

4.43949589

5. 14017863

3.09964434

5.14017863

0.77491108

3. 85513397

2.71218880

9.09588977

10.19177953

11.25452770

12.42482137

13.41334055

8.00000000

9.09588977

10.19177953

11.25452770

12.42482137

13.41334055

8.00000000

9.09588977

3. 83402195

0.40695914

5.13886069

2.26913069

2. 54512879

0.11418950

6.42522328

7.36165530

6.42522328

5.03692205

5.14017863

6.97419976

5.11906660

4.66897010

6.42390535

6.53114165

3. 83017345

4.37620047

7. 71026794

3.09964434

7.71026794

0.77491108

6.42522328

2.71218880

6.41289056

0.44144972

7.75064160

2.43372973

5.19370241

$-0.04272841$

8.99531260

7.36165530

8.99531260

5.03692205

7.71026794

6.97419976

7.69793522

4.70346069

9.03568626

6.69574069

6.47874706

4.21928255

1. 31052094

1.49932966

6.39461118

7. 40599308

5.10956652

3.14398211

2. 59556559

5.76134062

. / oxygen/Cu/311/iter_5/struct.xyz

10.19177953

11.31069046

12.45171325

13.76810566

8.00000000

9.09588977

10.19177953

11.31069046

12.45171325

13.76810566

8.00000000

9.09588977

10.19177953

11.28845068

12.35650306

13.45833366

8.00000000

9.09588977

10.19177953

11.28845068

12.35650306

13.45833366

13.76860279

14.18904618

14.18904618

13.76860279 
41

Lattice $=" 7.71026794009301 \quad 0.0 \quad 0.0 \quad 2.5700893133643348 \quad 8.524021930131648 \quad 0.0 \quad 0.0 \quad 0.021 .47944$ 8827071757" Properties=species:S:1:pos:R:3 pbc="T T T"

2.57008931

3.09964434

1.28504466

0.77491108

2.71218880

1.28707243

0.44220598

2.60974554

0.01344142

3. 85513397

2.41868769

$-0.25818320$

3. 85513397

7.36165530

5.03692205

2. 57008931

6.97419976

2. 57433165

4.65510121

3. 90760587

6.59781985

0.29680480

5.59310451

5.14017863

3.09964434

5.14017863

0.77491108

3. 85513397

2.71218880

0.38187236

2.21562260

5.10872386

2. 57128408

6.42522328

$-0.03173700$

7.36165530

6.42522328

5.03692205

5.14017863

6.97419976

4.65298241

6.39678508

6.57648591

2. 78106856

5.18336919

3.09964434

0.77491108

7.71026794

2.71218880

6.42522328

0.40494239

6.46500363

2.30614689

0.04086199

5. 19324257

7. 36165530

8.99531260

5.03692205

6.97419976

7.71026794

4.62732158

7.70623364

6.62552403

4.08728093

5. 32740282

1.23577920

7.34724936

6.51848579

3.54478542

3. 57821389

1. 82991509

6.70138811

8.00000000
9.09588977

10.19177953

11.31797341

12.52664813

13.84559818

8.00000000

9.09588977

10.19177953

11.29312701

12.30772024

14.20401423

8.00000000

9.09588977

10.19177953

11.28709542

12.23029126

13.73374369

8.00000000

9.09588977

10.19177953

11.23621938

12.35691332

14.04492828

8.00000000

9.09588977

10.19177953

11.29098138

12.53162605

13.37522436

8.00000000

9.09588977

10.19177953

11.41203315

12.26724451

13.68638589

13.65639822

14.19837335

13.90173955

14.58332355

4.08918020

13.49789020

. /oxygen/Cu/311/iter_6/struct.xyz

42

Lattice $=" 7.71026794009301 \quad 0.0 \quad 0.0 \quad 2.5700893133643348 \quad 8.524021930131648 \quad 0.0 \quad 0.0 \quad 0.021 .47944$ 8827071757 " Properties=species:S:1:pos:R:3 pbc="T T T"

$\mathrm{Cu}$

$\mathrm{Cu}$

2.57008931

3.09964434

0.77491108

.57008931

2.71218880

0.35631444

1. 27609653

2. 27519210

2. 56127450

0.12904221

7.36165530

3. 85513397

3. 85513397

5.03692205

2. 57008931

6.97419976

2. 56114119

4.61832540

3. 84631915

6.53720307

4.39105317

3.09964434

5.14017863

0.77491108

2.71218880

0.42551504

2.45130896

3.86964026

$-0.24612567$

2. 47175518

6.42522328

7. 36165530

6.42522328

5.03692205

8.00000000

9.09588977

10.19177953

11.30581503

12.39030733

13.68792575

8.00000000

9.09588977

10.19177953

11.30581503

12.39030733

13.68792575

8.00000000

9.09588977

10.19177953

11.28245138

12.28516392

13.72730292

8.00000000

9.09588977 
$\mathrm{Cu}$

$\mathrm{Cu}$

$\mathrm{Cu}$

$\mathrm{Cu}$

$\mathrm{Cu}$

$\mathrm{Cu}$

$\mathrm{Cu}$

$\mathrm{Cu}$

$\mathrm{Cu}$

$\mathrm{Cu}$

$\mathrm{Cu}$

$\mathrm{Cu}$

$\mathrm{Cu}$

$\mathrm{Cu}$

$\mathrm{Cu}$

$\mathrm{Cu}$

O

0

O

0

0

0
5.14017863

5.15468492

6.50468045

3.75679983

7.71026794

7.71026794

6.42522328

6.42423209

7.69400637

4.98749804

8.99531260

8.99531260

7.71026794

7.70927674

8.97905102

6.27254270

1.37576939

5.41832470

4.13328005

2.66081405

7.36220589

8.64725054
Fri Apr 16 14:51:55 2021

6.97419976

4.68752600

6.71331992

4.01588530

3.09964434

0.77491108

2.71218880

0.40975439

2.36752858

0.57807741

7.36165530

5.03692205

6.97419976

4.67176536

6.62953955

4.84008838

1.23928374

6.47318523

2. 21117426

5.50129471

3.39897320

7.66098416
10.19177953

11.28245138

12.28516392

13.72730292

8.00000000

9.09588977

10.19177953

11.30951929

12.39082907

13.85630806

8.00000000

9.09588977

10.19177953

11.30951929

12.39082907

13.85630806

13.66627607

13.90718321

13.90718321

13.66627607

14.03205601

14.03205601

43

Lattice $=" 7.71026794009301 \quad 0.0 \quad 0.0 \quad 2.5700893133643348 \quad 8.524021930131648 \quad 0.0 \quad 0.0 \quad 0.0 \quad 21.47944$ $8827071757 "$ Properties=species:S:1:pos:R:3 pbc="T T T"

$\mathrm{Cu} \quad 2.57008931 \quad 3.09964434 \quad 8.00000000$

$\mathrm{Cu} \quad 2.57008931$

0.77491108

$1.28504466 \quad 2.71218880$

9.09588977

1.28698532

0.43043323

2. 80790988

0.36827320

1.74194986

3.85513397

0.14390984

7.36165530

3.85513397

5.03692205

2. 57008931

6.97419976

2. 57403355

4.63571467

3. 86327641

6.62525224

2. 45461093

4.65607775

5.14017863

5.14017863

3.09964434

0.77491108

3.85513397

2.71218880

3. 81434588

5. 11452259

0.43127478

2.39496127

5.44492352

7.46974018

6.42522328

6.42522328

7.36165530

5.03692205

5.14017863

5.14290527

6.44577356

6.97419976

4.69000047

6.59966668

5.14236138

4.50844205

7.71026794

7.71026794

3.09964434

0.77491108

6.42522328

6.43292762

7.80226061

2.71218880

0.42998698

2.38114864

4.68472710

0.28515838

8.99531260

8.99531260

7.36165530

5.03692205

7.71026794

6.97419976

4.65562559

6.44574949

8.99928199

7.79708357

1.83003943

6.45142825

4.64381369

0.39907342

10.19177953

11.31350068

14.17089891

13.88692332

8.00000000

9.09588977

10.19177953

11.22290362

12.34169506

13.78563279

8.00000000

9.09588977

10.19177953

11.28153101

12.31624833

14.56680187

8.00000000

9.09588977

10.19177953

11.21777126

12.33636446

14.03111537

8.00000000

9.09588977

10.19177953

11.38306892

12.42070764

13.62878875

8.00000000

9.09588977

10.19177953

11.23429847

12.37288310

13.94480754

14.95109995

5.91735435

14.26572959

13.67691142

14.15368727

5.88050845

13.80752589

3.79270518

1.28626135

3.21427416

13.30217068 

o $\quad 3.84064258$
3.16185978
13.61034573
. /oxygen/Cu/311/iter_8/struct.xyz

44

Lattice $=" 7.71026794009301 \quad 0.0 \quad 0.0 \quad 2.5700893133643348 \quad 8.524021930131648 \quad 0.0 \quad 0.0 \quad 0.0 \quad 21.47944$ 8827071757 " Properties=species:S:1:pos:R:3 pbc="T T T"

$\begin{array}{llll}\mathrm{Cu} & 2.57008931 & 3.09964434 & 8.00000000 \\ \mathrm{Cu} & 2.57008931 & 0.77491108 & 9.09588977\end{array}$

$\mathrm{Cu} \quad 2.57008931$

77491108

1.28504466

2.71218880

1.34176860

0.37422007

2. 51177677

2.46178555

$-0.07823139$

3.85513397

$-0.15093308$

3. 85513397

7.36165530

2. 57008931

5.03692205

2. 55818861

6.97419976

4.65094078

10.19177953

11.36072962

12.42255933

13.63142011

8.00000000

9.09588977

10.19177953

11.24893307

3. 85790215

6.47814083

1. 92072872

5. 25304435

5.14017863

3.09964434

5.14017863

0.77491108

3. 85513397

2.71218880

3. 86917002

0.43816086

2.58007361

5.27442749

$-0.18786366$

2. 32727887

7.36165530

6.42522328

5.03692205

6.42522328

6.97419976

5.12619134

4.68478011

12.45470550

14.38499762

8.00000000

9.09588977

10.19177953

11.39929399

12.34807253

14.09751683

8.00000000

9.09588977

10.19177953

11.21575892

12.26133973

65228086

4.01896408

3. 34805963

7.71026794

3. 09964434

14.35000392

8.00000000

9.09588977

7.71026794

0.77491108

10.19177953

6.52484784

2. 71218880

11.23650095

14.09051937

2.90705670

7. 95279664

$-0.57489164$

8.73931636

7.36165530

14.07485432

8.00000000

9.09588977

5.03692205

10.19177953

11.30415930

12.27296980

14.44795987

13.41722027

14.99545290

14.09133847

14.13308828

14.48142263

14.21541979

14.24970666

12.78457063

. /oxygen/Cu/311/iter_9/struct. xyz

45

Lattice $=" 7.71026794009301 \quad 0.0 \quad 0.0 \quad 2.5700893133643348 \quad 8.524021930131648 \quad 0.0 \quad 0.0 \quad 0.021 .47944$ 8827071757 " Properties=species:S:1:pos:R:3 pbc="T T T"

$\begin{array}{lrrr}\mathrm{Cu} & 2.57008931 & 3.09964434 & 8.00000000 \\ \mathrm{Cu} & 2.57008931 & 0.77491108 & 9.09588977 \\ \mathrm{Cu} & 1.28504466 & 2.71218880 & 10.19177953 \\ \mathrm{Cu} & 1.31396031 & 0.42836173 & 11.27714273 \\ \mathrm{Cu} & 2.62411340 & 2.37214550 & 12.38650033 \\ \mathrm{Cu} & -0.36371595 & 1.45992720 & 14.86526700 \\ \mathrm{Cu} & 3.85513397 & 7.36165530 & 8.00000000 \\ \mathrm{Cu} & 3.85513397 & 5.03692205 & 9.09588977 \\ \mathrm{Cu} & 2.57008931 & 6.97419976 & 10.19177953 \\ \mathrm{Cu} & 2.54238373 & 4.62852736 & 11.23996226 \\ \mathrm{Cu} & 3.82585048 & 6.55047657 & 12.27603562 \\ \mathrm{Cu} & 1.50486702 & 4.50944850 & 14.10452512 \\ \mathrm{Cu} & 5.14017863 & 3.09964434 & 8.00000000 \\ \mathrm{Cu} & 5.14017863 & 0.77491108 & 9.09588977 \\ \mathrm{Cu} & 3.85513397 & 2.71218880 & 10.19177953\end{array}$




\section{$\mathrm{Cu}$}

$\mathrm{Cu}$

$\mathrm{Cu}$

$\mathrm{Cu}$

$\mathrm{Cu}$

$\mathrm{Cu}$

$\mathrm{Cu}$

$\mathrm{Cu}$

$\mathrm{Cu}$

$\mathrm{Cu}$

$\mathrm{Cu}$

$\mathrm{Cu}$

$\mathrm{Cu}$

$\mathrm{Cu}$

$\mathrm{Cu}$

$\mathrm{Cu}$

$\mathrm{Cu}$

$\mathrm{Cu}$

$\mathrm{Cu}$

$\mathrm{Cu}$

$\mathrm{Cu}$

O

O

0

O

O

0

O

O

0

$. / 10 \times$ 18

$\begin{array}{lllll}\text { Lattice }=" 4.974854628764761 & 0.0 & 0.0 & 0.0 \\ \text { perties }=\text { species:S:1:pos:R:3 } & \text { pbc }=" \text { T } & \text { T } & \text { T" }\end{array}$

$\begin{array}{lll}\mathrm{Ni} & 1.24371366 & 1.24371366 \\ \mathrm{Ni} & 0.00000000 & 0.00000000 \\ \mathrm{Ni} & 1.24371366 & 1.24371366 \\ \mathrm{Ni} & 0.00000000 & 0.00000000 \\ \mathrm{Ni} & 1.24371366 & 3.73114097 \\ \mathrm{Ni} & 0.0000000 & 2.48742731 \\ \mathrm{Ni} & 1.24371366 & 3.73114097 \\ \mathrm{Ni} & 0.0000000 & 2.48742731 \\ \mathrm{Ni} & 3.73114097 & 1.24371366 \\ \mathrm{Ni} & 2.48742731 & 0.00000000 \\ \mathrm{Ni} & 3.73114097 & 1.24371366 \\ \mathrm{Ni} & 2.48742731 & 0.00000000 \\ \mathrm{Ni} & 3.73114097 & 3.73114097 \\ \mathrm{Ni} & 2.48742731 & 2.48742731 \\ \mathrm{Ni} & 3.73114097 & 3.73114097 \\ \mathrm{Ni} & 2.48742731 & 2.48742731 \\ \mathrm{O} & 1.24371366 & 1.24371366 \\ \mathrm{O} & 3.73114097 & 3.73114097\end{array}$

Fri Apr 16 14:51:55 2021

163

0.39539586

11.28880498

12.38586399

13.70882533

8.00000000

9.09588977

10.19177953

11.33180923

12.51206037

13.88112883

8.00000000

9.09588977

10.19177953

11.34304593

14.63284846

8.00000000

9.09588977

10.19177953

11.25578099

12.37414618

13.92084203

13.12598725

14.26714790

14.12421871

13.80665729

14.34831087

15.61169070

14.12635220

12.76134317

13.86990876
12.34954261

. /oxygen/Ni/100/iter_1/struct. xyz

49

Lattice $=" 7.462281943147142 \quad 0.0 \quad 0.0 \quad 0.0 \quad 9.9497092575295230 .0 \quad 0.0 \quad 0.021 .27663016512527 "$ Pro perties=species:S:1:pos:R:3 pbc="T T T"

$\begin{array}{lllr}\mathrm{Ni} & 1.24371366 & 1.24371366 & 8.00000000 \\ \mathrm{Ni} & 0.00000000 & 0.00000000 & 9.75887672 \\ \mathrm{Ni} & 1.24371366 & 1.24371366 & 11.46215162 \\ \mathrm{Ni} & 0.00547197 & 0.00400241 & 13.30318048 \\ \mathrm{Ni} & 1.24371366 & 3.73114097 & 8.00000000 \\ \mathrm{Ni} & 0.00000000 & 2.48742731 & 9.75887672 \\ \mathrm{Ni} & 1.24371366 & 3.73049616 & 11.53131743 \\ \mathrm{Ni} & 0.00547197 & 2.48342491 & 13.30318048 \\ \mathrm{Ni} & 1.24371366 & 6.21856829 & 8.00000000 \\ \mathrm{Ni} & 0.00000000 & 4.97485463 & 9.75887672 \\ \mathrm{Ni} & 1.24371366 & 6.21856829 & 11.53121520 \\ \mathrm{Ni} & 0.00071403 & 4.97715603 & 13.22511088\end{array}$

8.00000000

9.75887672

11.48610895

13.37065753

8.00000000

9.75887672

11.52387830

13.37065753

8.00000000

9.75887672

11.52387830

13.37065753

8.00000000

9.75887672

11.48610895

13.37065753

14.15756711

14.15756711
$620.0 \quad 0.0 \quad 0.021 .27663016512527 "$ Pro 
$\mathrm{Ni}$

$\mathrm{Ni} \quad 0.00000000$

$\mathrm{Ni} \quad 1.24371366$

$\mathrm{Ni} \quad 0.00071403$

$\mathrm{Ni} \quad 3.73114097$

$\mathrm{Ni} \quad 2.48742731$

$\mathrm{Ni} \quad 3.73052211$

$\mathrm{Ni} \quad 2.48195534$

$\mathrm{Ni} \quad 3.73114097$

$\mathrm{Ni} \quad 2.48742731$

$\mathrm{Ni} \quad 3.72432346$

$\mathrm{Ni} \quad 2.48195534$

$\mathrm{Ni} \quad 3.73114097$

$\mathrm{Ni} \quad 2.48742731$

$\mathrm{Ni} \quad 3.73239957$

$\mathrm{Ni} \quad 2.48671329$

$\mathrm{Ni} \quad 3.73114097$

$\mathrm{Ni} \quad 2.48742731$

$\mathrm{Ni} \quad 3.72432346$

$\mathrm{Ni} \quad 2.48671329$

$\mathrm{Ni} \quad 6.21856829$

$\mathrm{Ni} \quad 4.97485463$

$\mathrm{Ni}$

$\mathrm{Ni}$

$\mathrm{Ni}$

$\mathrm{Ni}$

$\mathrm{Ni}$

$\mathrm{Ni}$

$\mathrm{Ni}$

$\mathrm{Ni}$

$\mathrm{Ni}$

$\mathrm{Ni}$

$\mathrm{Ni}$

$\mathrm{Ni}$

$\mathrm{Ni}$

$\mathrm{Ni}$

58

ygen/Ni/100/iter_10/struct.xyz
perties=species:S:1:pos:R:3 pbc="T T T"

$\mathrm{Ni}$

$\mathrm{Ni}$

$\mathrm{Ni}$

$\mathrm{Ni}$

$\mathrm{Ni}$

$\mathrm{Ni}$

$\mathrm{Ni}$

$\mathrm{Ni}$

$\mathrm{Ni}$

$\mathrm{Ni}$

$\mathrm{Ni}$

$\mathrm{Ni}$

$\mathrm{Ni}$

$\mathrm{Ni}$

$\mathrm{Ni}$

$\mathrm{Ni}$

$\mathrm{Ni}$

$\mathrm{Ni}$

$\mathrm{Ni}$

$\mathrm{Ni}$

$\mathrm{Ni}$

$\mathrm{Ni}$

$\mathrm{Ni}$

$\mathrm{Ni}$

$\mathrm{Ni}$

$\mathrm{Ni}$

\section{Fri Apr 16 14:51:55 2021}

8.70599560

7.46228194

8.70664041

7.45998054

1.24371366

0.00000000

1.24371366

0.00400241

3.73114097

2.48742731

3.72072085

2. 48342491

6.21856829

4.97485463

6.21856829

4. 97715603

8.70599560

7.46228194

8.71641572

7.45998054

1.24371366

0.00000000

1.24371366

0.00133619

3.73114097

2.48742731

3.72072085

2. 48609112

6.21856829

4.97485463

6.21856829

4.96943632

8.70599560

7.46228194

8.71641572

7.46770026

1.24371366
8.00000000

9.75887672

11.53131743

13.22511088

8.00000000

9.75887672

11.53050786

13.30318048

8.00000000

9.75887672

11.54657994

13.30318048

8.00000000

9.75887672

11.53433958

13.22511088

8.00000000

9.75887672

11.54657994

13.22511088

8.00000000

9.75887672

11.53050786

13.22312158

8.00000000

9.75887672

11.54657994

13.22312158

8.00000000

9.75887672

11.53433958

13.22616131

8.00000000

9.75887672

13.22616131

14.14824448
11.54657994
164 
$\mathrm{Ni}$

$\mathrm{Ni}$

2.02350057

3.73114097

2.48742731

3.77224016

2.54151118

6.21856829

4.97485463

6.25776807

5.64920324

6.21856829

4.97485463

6.15469946

4.97485463

6.21856829

4.97485463

6.17746910

4.30050601

6.21856829

4.97485463

6.23904919

4.97485463

2.02903192

1.17875137

3.59732531

3.83930172

6.11040754

6.35238394

6.63480849

1.30867595

3.31490077

0.45839539
Fri Apr 16 14:51:55 2021

4.66311789

8.70599560

7.46228194

8.73386786

7.39516084

1.24371366

0.00000000

1.26340995

0.02511254

3.73114097

2.48742731

3.75783207

2.48742731

6.21856829

4.97485463

6.19069603

4.94974209

8.70599560

7.46228194

8.66970649

7.46228194

1.15597700

6.18879271

3.44358487

8.54524214

6.37932175

1.53126976

8.99942955

8.73577118

5.92513434

3.81887763
14.32239881

8.00000000

9.75887672

11.55017273

13.90751483

8.00000000

9.75887672

11.46664809

13.42472536

8.00000000

9.75887672

11.51227600

13.41172012

8.00000000

9.75887672

11.55017273

13.42472536

8.00000000

9.75887672

11.47529713

13.30842807

14.53490705

13.40872061

14.37586337

14.41114939

14.41114939

14.37586337

14.67268976

13.40872061

14.67268976

14.53490705

. /oxygen/Ni/100/iter_11/struct.xyz

59

Lattice $=" 7.462281943147142 \quad 0.0 \quad 0.0 \quad 0.0 \quad 9.949709257529523 \quad 0.0 \quad 0.0 \quad 0.021 .27663016512527 " \quad$ Pro perties=species:S:1:pos:R:3 pbc="T T T"

$\mathrm{Ni} \quad 1.24371366 \quad 1.24371366$

$\mathrm{Ni} \quad 0.00000000 \quad 0.00000000$

$\mathrm{Ni} \quad 1.25977828 \quad 1.25093537$

$\mathrm{Ni} \quad 0.00381964 \quad 0.21899313$

$\begin{array}{lll}\mathrm{Ni} & 1.24371366 & 3.73114097\end{array}$

$\mathrm{Ni} \quad 0.00000000 \quad 2.48742731$

$\mathrm{Ni} \quad 1.27348226 \quad 3.73114097$

$\begin{array}{lll}\mathrm{Ni} & 0.01949007 & 2.54976697\end{array}$

$\mathrm{Ni} \quad 1.24371366 \quad 6.21856829$

$\mathrm{Ni} \quad 0.00000000 \quad 4.97485463$

$\begin{array}{lll}\mathrm{Ni} & 1.25977828 & 6.21134657\end{array}$

$\mathrm{Ni} \quad 0.01949007 \quad 4.91251497$

$\mathrm{Ni} \quad 1.24371366 \quad 8.70599560$

$\begin{array}{lll}\mathrm{Ni} & 0.00000000 & 7.46228194\end{array}$

$\mathrm{Ni} \quad 1.25539558$

$\mathrm{Ni} \quad 0.00381964$

$\mathrm{Ni} \quad 3.73114097$

8.70599560

7.24328882

1.24371366

2.48742731

0.00000000

3.71507634

2.48742731

1.25093537

$-0.04048659$

3. 73114097

3.73114097

2. 48742731

3.70137237

2. 48742731

3.73114097

2. 48742731

3. 73114097

2.42098258

6.21856829

4.97485463

8.00000000

9.75887672

11.51442001

13.96361339

8.00000000

9.75887672

11.46800937

13.20508973

8.00000000

9.75887672

11.51442001

13.20508973

8.00000000

9.75887672

11.53122389

13.96361339

8.00000000

9.75887672

11.51442001

14.29400322

8.00000000

9.75887672

11.46800937

14.50650254

8.00000000

9.75887672

2.48742731
3.71507634

6.21134657

5.04129936

8.70599560

7.46228194

3. 73114097

11.51442001

14.50650254

8.00000000

9.75887672

8.70599560

7.50276854

11.53122389

14.29400322

8.00000000 
$\mathrm{Ni}$

$\mathrm{Ni}$

$\mathrm{Ni}$

$\mathrm{Ni}$

$\mathrm{Ni}$

$\mathrm{Ni}$

$\mathrm{Ni}$

$\mathrm{Ni}$

$\mathrm{Ni}$

$\mathrm{Ni}$

$\mathrm{Ni}$

$\mathrm{Ni}$

$\mathrm{Ni}$

$\mathrm{Ni}$

$\mathrm{Ni}$

0

O

0

O

0

0

O

O

0

O

0
4.97485463

6.21856829

4.97103499

6.21856829

4.97485463

6.21856829

4.95536456

6.21856829

4.97485463

6.21856829

4.95536456

6.21856829

4.97485463

6.21856829

4.97103499

1.26536354

1.26536354

3.82789606

3.75190113

6.21856829

6.21856829

6.21856829

1.22295350

3.70949108

1.14695857

3.70949108
Fri Apr 16 14:51:55 2021

0.00000000

1.24096709

0.21899313

3.73114097

2.48742731

3.73114097

2.54976697

6.21856829

4.97485463

6.22131485

4.91251497

8.70599560

7.46228194

8.70599560

7.24328882

1.06482980

6.39745215

3.73114097

8.70599560

5.93106261

1.53121933

8.70599560

8.70599560

6.39745215

3.73114097

1.06482980
9.75887672

11.49004230

13.96361339

8.00000000

9.75887672

11.46367990

13.20508973

8.00000000

9.75887672

11.49004230

13.20508973

8.00000000

9.75887672

11.48902513

13.96361339

14.99611635

14.99611635

14.30723504

13.47511361

14.32963125

14.32963125

13.37303001

13.47511361

14.99611635

14.30723504

14.99611635

. /oxygen/Ni/100/iter_12/struct.xyz

60

Lattice $=" 7.462281943147142 \quad 0.0 \quad 0.0 \quad 0.0 \quad 9.949709257529523 \quad 0.0 \quad 0.0 \quad 0.021 .27663016512527 "$ Pro perties=species:S:1:pos:R:3 pbc="T T T"

$\mathrm{Ni}$

1.24371366

1.24371366

$0.00000000 \quad 0.00000000$

$1.24371366 \quad 1.24371366$

0.00000000

1.24371366

3.73114097

0.00000000

2.48742731

1.24371366

3.73114097

0.00000000

2.48742731

1. 24371366

6.21856829

0.00000000

4.97485463

1. 24371366

6.21856829

0.00000000

4.97485463

1. 24371366

0.00000000

8.70599560

7.46228194

1. 24371366

0.00000000

8.70599560

7.46228194

3. 73114097

1. 24371366

2. 48742731

3.73114097

0.00000000

1.24371366

2. 48742731

0.00000000

3. 73114097

3.73114097

2.48742731

2.48742731

3. 73114097

3.73114097

2.48742731

3. 73114097

2. 48742731

6.21856829

2. 48742731

4. 97485463

3. 73114097

6. 21856829

2. 48742731

4.97485463

3. 73114097

8.70599560

7.46228194

8.70599560

3. 73114097

8.70599560
7.46228194

2. 48742731

6.21856829

4. 97485463

1. 24371366

0.00000000

1. 24371366

6.21856829

0.00000000

3.73114097

6.21856829

2. 48742731

8.00000000
9.75887672
11.49347185
13.54038857
8.00000000
9.75887672
11.49347185
13.54038857
8.00000000
9.75887672
11.49347185
13.54038857
8.00000000
9.75887672
11.49347185
13.54038857
8.00000000
9.75887672
11.49347185
13.54038857
8.00000000
9.75887672
11.49347185
13.54038857
8.00000000
9.75887672
11.49347185
13.54038857
8.00000000
9.75887672
11.49347185
13.54038857
8.00000000
9.75887672
11.49347185
13.54038857
8.00000000
9.75887672 
$\mathrm{Ni}$

$\mathrm{Ni}$

6.21856829

4.97485463

6.21856829

4.97485463

6.21856829

4.97485463

6.21856829

4.97485463

6.21856829

4.97485463

1.24371366

1.24371366

3.73114097

3.73114097

6.21856829

6.21856829

6.21856829

1.24371366

3.73114097

1.24371366

3.73114097

6.21856829
Fri Apr 16 14:51:55 2021

3.73114097

2.48742731

6.21856829

4.97485463

6.21856829

4.97485463

8.70599560

7.46228194

8.70599560

7.46228194

1.24371366

6.21856829

3.73114097

8.70599560

6.21856829

1.24371366

8.70599560

8.70599560

6.21856829

3.73114097

1.24371366

3.73114097
11.49347185

13.54038857

8.00000000

9.75887672

11.49347185

13.54038857

8.00000000

9.75887672

11.49347185

13.54038857

14.20841912

14.20841912

14.20841912

14.20841912

14.20841912

14.20841912

14.20841912

14.20841912

14.20841912

14.20841912

14.20841912

14.20841912

. /oxygen/Ni/100/iter_2/struct.xyz

50

Lattice $=" 7.462281943147142 \quad 0.0 \quad 0.0 \quad 0.0 \quad 9.9497092575295230 .0 \quad 0.0 \quad 0.021 .27663016512527 "$ Pro perties=species:S:1:pos:R:3 pbc="T T T"

$\mathrm{N} i$

$\mathrm{Ni}$

$\mathrm{Ni}$

$\mathrm{Ni}$

$\mathrm{Ni}$

$\mathrm{Ni}$

$\mathrm{Ni}$

$\mathrm{Ni}$

$\mathrm{Ni}$

$\mathrm{Ni}$

$\mathrm{Ni}$

$\mathrm{Ni}$

$\mathrm{Ni}$

$\mathrm{Ni}$

$\mathrm{Ni}$

$\mathrm{Ni}$

$\mathrm{Ni}$

$\mathrm{Ni}$

$\mathrm{Ni}$

$\mathrm{Ni}$

$\mathrm{Ni}$

$\mathrm{Ni}$

$\mathrm{Ni}$

$\mathrm{Ni}$

$\mathrm{Ni}$

$\mathrm{Ni}$

$\mathrm{Ni}$

$\mathrm{Ni}$

$\mathrm{Ni}$

$\mathrm{Ni}$

$\mathrm{Ni}$

$\mathrm{Ni}$

$\mathrm{Ni}$

$\mathrm{Ni}$

$\mathrm{Ni}$

$\mathrm{Ni}$

$\mathrm{Ni}$

$\mathrm{Ni}$

$\mathrm{Ni}$

$\mathrm{Ni}$

$\mathrm{Ni}$

$\mathrm{Ni}$
1.24371366

0.00000000

1.24371366

0.00301801

1.24371366

0.00000000

1.24371366

0.00301801

1.24371366

0.00000000

1.24371366

0.00301801

1.24371366

0.00000000

1.24371366

0.00301801

3.73114097

2. 48742731

3.73206990

2.48440931

3. 73114097

2. 48742731

3.71604739

2. 48440931

3.73114097

2. 48742731

3.73206990

2. 48440931

3.73114097

2. 48742731

3.71604739

2. 48440931

6.21856829

4.97485463

6.21763936

4.97485463

6.21856829

4.97485463

6.23366187

4.97485463

6.21856829

4.97485463
1.24371366

0.00000000

1. 24371366

0.00178995

3.73114097

2. 48742731

3.73114097

2. 48563736

6.21856829

4.97485463

6.21856829

4.97664458

8.70599560

7.46228194

8.70599560

7.46049199

1.24371366

0.00000000

1.24371366

0.00178995

3.73114097

2. 48742731

3. 73114097

2.48563736

6.21856829

4.97485463

6.21856829

4.97664458

8.70599560

7.46228194

8.70599560

7.46049199

1.24371366

0.00000000

1.24371366

$-0.00260888$

3.73114097

2. 48742731

3.73114097

2.49003620

6.21856829

4.97485463
8.00000000

9.75887672

11.45132390

13.28504358

8.00000000

9.75887672

11.52305352

13.28504358

8.00000000

9.75887672

11.45132390

13.28504358

8.00000000

9.75887672

11.52305352

13.28504358

8.00000000

9.75887672

11.52809671

13.28504358

8.00000000

9.75887672

11.55488236

13.28504358

8.00000000

9.75887672

11.52809671

13.28504358

8.00000000

9.75887672

11.55488236

13.28504358

8.00000000

9.75887672

11.52809671

13.21843361

8.00000000

9.75887672

11.55488236

13.21843361

8.00000000

9.75887672 
$\mathrm{Ni}$

$\mathrm{Ni}$

$\mathrm{Ni}$

$\mathrm{Ni}$

$\mathrm{Ni}$

$\mathrm{Ni}$

0

0
6.21763936

4. 97485463

6.21856829

4. 97485463

6.23366187

4.97485463

1. 24371366

1.24371366

Fri Apr 16 14:51:55 2021

168

6.21856829

4. 97224575

8.70599560

7.46228194

8.70599560

7.46489083

1.24371366

6.21856829
11.52809671

13.21843361

8.00000000

9.75887672

11.55488236

13.21843361

14.13191914

14.13191914

51

Lattice $=" 7.462281943147142 \quad 0.0 \quad 0.0 \quad 0.0 \quad 9.9497092575295230 .0 \quad 0.0 \quad 0.021 .27663016512527 "$ Pro perties=species:S:1:pos:R:3 pbc="T T T"

\begin{tabular}{|c|c|c|c|}
\hline $\mathrm{Ni}$ & 1.24371366 & 1.24371366 & 8.00000000 \\
\hline $\mathrm{Ni}$ & 0.00000000 & 0.00000000 & 9.75887672 \\
\hline $\mathrm{Ni}$ & 1.25181970 & 1.25342789 & 11.45922921 \\
\hline $\mathrm{Ni}$ & -0.02518822 & -0.00493692 & 13.27652126 \\
\hline $\mathrm{Ni}$ & 1.24371366 & 3.73114097 & 8.00000000 \\
\hline $\mathrm{Ni}$ & 0.00000000 & 2.48742731 & 9.75887672 \\
\hline $\mathrm{Ni}$ & 1.26430271 & 3.73114097 & 11.53491469 \\
\hline $\mathrm{Ni}$ & 0.01932638 & 2.47837978 & 13.26598643 \\
\hline $\mathrm{Ni}$ & 1.24371366 & 6.21856829 & 8.00000000 \\
\hline $\mathrm{Ni}$ & 0.00000000 & 4.97485463 & 9.75887672 \\
\hline $\mathrm{Ni}$ & 1.25181970 & 6.20885405 & 11.45922921 \\
\hline $\mathrm{Ni}$ & 0.01932638 & 4.98390216 & 13.26598643 \\
\hline $\mathrm{Ni}$ & 1.24371366 & 8.70599560 & 8.00000000 \\
\hline $\mathrm{Ni}$ & 0.00000000 & 7.46228194 & 9.75887672 \\
\hline $\mathrm{Ni}$ & 1.23845547 & 8.70599560 & 11.51561815 \\
\hline $\mathrm{Ni}$ & -0.02518822 & 7.46721887 & 13.27652126 \\
\hline $\mathrm{Ni}$ & 3.73114097 & 1.24371366 & 8.00000000 \\
\hline $\mathrm{Ni}$ & 2.48742731 & 0.00000000 & 9.75887672 \\
\hline $\mathrm{Ni}$ & 3.72433437 & 1.24657453 & 11.52728773 \\
\hline $\mathrm{Ni}$ & 2.46816821 & 0.00466792 & 13.27305959 \\
\hline $\mathrm{Ni}$ & 3.73114097 & 3.73114097 & 8.00000000 \\
\hline $\mathrm{Ni}$ & 2.48742731 & 2.48742731 & 9.75887672 \\
\hline $\mathrm{Ni}$ & 3.71989544 & 3.73114097 & 11.47595888 \\
\hline $\mathrm{Ni}$ & 2.50445266 & 2.48371788 & 13.44856217 \\
\hline $\mathrm{Ni}$ & 3.73114097 & 6.21856829 & 8.00000000 \\
\hline $\mathrm{Ni}$ & 2.48742731 & 4.97485463 & 9.75887672 \\
\hline $\mathrm{Ni}$ & 3.72433437 & 6.21570741 & 11.52728773 \\
\hline $\mathrm{Ni}$ & 2.50445266 & 4.97856406 & 13.44856217 \\
\hline $\mathrm{Ni}$ & 3.73114097 & 8.70599560 & 8.00000000 \\
\hline $\mathrm{Ni}$ & 2.48742731 & 7.46228194 & 9.75887672 \\
\hline $\mathrm{Ni}$ & 3.71060436 & 8.70599560 & 11.54247423 \\
\hline $\mathrm{Ni}$ & 2.46816821 & 7.45761403 & 13.27305959 \\
\hline $\mathrm{Ni}$ & 6.21856829 & 1.24371366 & 8.00000000 \\
\hline $\mathrm{Ni}$ & 4.97485463 & 0.00000000 & 9.75887672 \\
\hline $\mathrm{Ni}$ & 6.20794863 & 1.25181286 & 11.52549986 \\
\hline $\mathrm{Ni}$ & 4.96530644 & -0.00800218 & 13.20012612 \\
\hline $\mathrm{Ni}$ & 6.21856829 & 3.73114097 & 8.00000000 \\
\hline $\mathrm{Ni}$ & 4.97485463 & 2.48742731 & 9.75887672 \\
\hline $\mathrm{Ni}$ & 6.24967234 & 3.73114097 & 11.53871074 \\
\hline $\mathrm{Ni}$ & 4.99157662 & 2.48468525 & 13.26533155 \\
\hline $\mathrm{Ni}$ & 6.21856829 & 6.21856829 & 8.00000000 \\
\hline $\mathrm{Ni}$ & 4.97485463 & 4.97485463 & 9.75887672 \\
\hline $\mathrm{Ni}$ & 6.20794863 & 6.21046909 & 11.52549986 \\
\hline $\mathrm{Ni}$ & 4.99157662 & 4.97759669 & 13.26533155 \\
\hline $\mathrm{Ni}$ & 6.21856829 & 8.70599560 & 8.00000000 \\
\hline $\mathrm{Ni}$ & 4.97485463 & 7.46228194 & 9.75887672 \\
\hline $\mathrm{Ni}$ & 6.22414675 & 8.70599560 & 11.54081098 \\
\hline $\mathrm{Ni}$ & 4.96530644 & 7.47028412 & 13.20012612 \\
\hline 0 & 1.20113922 & 1.20511532 & 14.13854970 \\
\hline O & 1.20113922 & 6.25716662 & 14.13854970 \\
\hline O & 3.81598558 & 3.73114097 & 14.14927743 \\
\hline
\end{tabular}

. /oxygen/Ni/100/iter_4/struct.xyz 52

Lattice $=" 7.462281943147142 \quad 0.0 \quad 0.0 \quad 0.0 \quad 9.9497092575295230 .0 \quad 0.0 \quad 0.021 .27663016512527 "$ Pro perties =species:S:1:pos:R:3 pbc="T T T"

$\mathrm{Ni}$

1.24371366

1. 24371366

8.00000000 
$\mathrm{Ni}$

$\mathrm{Ni}$

$\mathrm{Ni}$

$\mathrm{Ni}$

$\mathrm{Ni}$

$\mathrm{Ni}$

$\mathrm{Ni}$

$\mathrm{Ni}$

$\mathrm{Ni}$

$\mathrm{Ni}$

$\mathrm{Ni}$

$\mathrm{Ni}$

$\mathrm{Ni}$

$\mathrm{Ni}$

$\mathrm{Ni}$

$\mathrm{Ni}$

$\mathrm{Ni}$

$\mathrm{Ni}$

$\mathrm{Ni}$

$\mathrm{Ni}$

$\mathrm{Ni}$

$\mathrm{Ni}$

$\mathrm{Ni}$

$\mathrm{Ni}$

$\mathrm{Ni}$

$\mathrm{Ni}$

$\mathrm{Ni}$

$\mathrm{Ni}$

$\mathrm{Ni}$

$\mathrm{Ni}$

$\mathrm{Ni}$

$\mathrm{Ni}$

$\mathrm{Ni}$

$\mathrm{Ni}$

$\mathrm{Ni}$

$\mathrm{Ni}$

$\mathrm{Ni}$

$\mathrm{Ni}$

$\mathrm{Ni}$

$\mathrm{Ni}$

$\mathrm{Ni}$

$\mathrm{Ni}$

$\mathrm{Ni}$

$\mathrm{Ni}$

$\mathrm{Ni}$

$\mathrm{Ni}$

$\mathrm{Ni}$

0

0

0

0
0.00000000

1.26306558

$-0.00586640$

1.24371366

0.00000000

1.25373590

$-0.00586640$

1.24371366

0.00000000

1.26306558

$-0.00586640$

1.24371366

0.00000000

1.25373590

$-0.00586640$

3. 73114097

2. 48742731

3. 72111873

2.48742731

3. 73114097

2.48742731

3.71178905

2.48742731

3.73114097

2. 48742731

3.72111873

2.48742731

3.73114097

2.48742731

3.71178905

2.48742731

6.21856829

4.97485463

6.19936890

4.98072103

6.21856829

4.97485463

6.23776767

4.98072103

6.21856829

4.97485463

6.19936890

4.98072103

6.21856829

4.97485463

6.23776767

4.98072103

1.16588342

1.16588342

3.80897121

3.80897121
Fri Apr 16 14:51:55 2021

0.00000000

1.24371366

$-0.00034827$

3.73114097

2.48742731

3.73114097

2.48777558

6.21856829

4.97485463

6.21856829

4.97450636

8.70599560

7.46228194

8.70599560

7.46263021

1.24371366

0.00000000

1.24371366

0.00000000

3.73114097

2.48742731

3.73114097

2.48742731

6.21856829

4.97485463

6.21856829

4.97485463

8.70599560

7.46228194

8.70599560

7.46228194

1.24371366

0.00000000

1. 24371366

0.00034827

3.73114097

2. 48742731

3.73114097

2.48707905

6.21856829

4.97485463

6.21856829

4.97520290

8.70599560

7.46228194

8.70599560

7.46193368

1.24371366

6.21856829

3.73114097

8.70599560
9.75887672

11.46869053

13.25446898

8.00000000

9.75887672

11.52360975

13.25446898

8.00000000

9.75887672

11.46869053

13.25446898

8.00000000

9.75887672

11.52360975

13.25446898

8.00000000

9.75887672

11.52360975

13.41948541

8.00000000

9.75887672

11.46869053

13.41948541

8.00000000

9.75887672

11.52360975

13.41948541

8.00000000

9.75887672

11.46869053

13.41948541

8.00000000

9.75887672

11.52668865

13.25446898

8.00000000

9.75887672

11.52668865

13.25446898

8.00000000

9.75887672

11.52668865

13.25446898

8.00000000

9.75887672

11.52668865

13.25446898

14.13704394

14.13704394

14.13704394

14.13704394

53

Lattice $=" 7.462281943147142 \quad 0.0 \quad 0.0 \quad 0.0 \quad 9.949709257529523 \quad 0.0 \quad 0.0 \quad 0.0 \quad 21.27663016512527 "$ Pro perties=species:S:1:pos:R:3 pbc="T T T"

$\mathrm{Ni} \quad 1.24371366 \quad 1.24371366$

$\mathrm{Ni} \quad 0.00000000 \quad 0.00000000$

$\mathrm{Ni} \quad 1.25663330 \quad 1.24371366$

$\mathrm{Ni} \quad-0.02782673 \quad 0.00621388$

$\mathrm{Ni} \quad 1.24371366 \quad 3.73114097$

$\begin{array}{lll}\mathrm{Ni} & 0.00000000 & 2.48742731\end{array}$

$\mathrm{Ni} \quad 1.24536204 \quad 3.74193572$

$\mathrm{Ni} \quad-0.02782673 \quad 2.48121343$

$\begin{array}{lll}\mathrm{Ni} & 1.24371366 & 6.21856829\end{array}$

$\mathrm{Ni} \quad 0.00000000 \quad 4.97485463$

$\mathrm{Ni} \quad 1.26783215 \quad 6.21856829$

$\mathrm{Ni} \quad 0.02037443 \quad 4.98455517$

$\mathrm{Ni}$

1. 24371366

8.70599560

8.00000000

9.75887672

11.46536764

13.25059614

8.00000000

9.75887672

11.53838390

13.25059614

8.00000000

9.75887672

11.47866256

13.33886348

8.00000000 
$\mathrm{Ni}$

$\mathrm{Ni}$

$\mathrm{Ni}$

$\mathrm{Ni}$

$\mathrm{Ni}$

$\mathrm{Ni}$

$\mathrm{Ni}$

$\mathrm{Ni}$

$\mathrm{Ni}$

$\mathrm{Ni}$

$\mathrm{Ni}$

$\mathrm{Ni}$

$\mathrm{Ni}$

$\mathrm{Ni}$

$\mathrm{Ni}$

$\mathrm{Ni}$

$\mathrm{Ni}$

$\mathrm{Ni}$

$\mathrm{Ni}$

$\mathrm{Ni}$

$\mathrm{Ni}$

$\mathrm{Ni}$

$\mathrm{Ni}$

$\mathrm{Ni}$

$\mathrm{Ni}$

$\mathrm{Ni}$

$\mathrm{Ni}$

$\mathrm{Ni}$

$\mathrm{Ni}$

$\mathrm{Ni}$

$\mathrm{Ni}$

$\mathrm{Ni}$

$\mathrm{Ni}$

$\mathrm{Ni}$

$\mathrm{Ni}$

0

0

o

0

0

6.14146287

0.00000000

1.24536204

0.02037443

3. 73114097

2. 48742731

3.71215715

2. 44549663

3. 73114097

2.48742731

3.72128359

2.44549663

3. 73114097

2. 48742731

3.74129550

2. 51993811

3. 73114097

2. 48742731

3. 72128359

2. 51993811

6.21856829

4.97485463

6.19142392

4.96341887

6.21856829

4.97485463

6.23278320

4.96341887

6.21856829

4.97485463

6.20785426

5.00098510

6.21856829

4.97485463

6.23278320

5.00098510

1.12456953

1.35971157

3.77007554

3.77007554

/Ni/100/iter

54

gen/Ni/100/iter_6/struct.xyz

Lattice $=" 7.462281943147142$

perties=species:S:1:pos:R:3 pbc="T T T"

$\mathrm{Ni}$

$\mathrm{Ni}$

1.24371366

0.00000000

1.26037396

0.00000000

1.24371366

0.00000000

1.22994895

0.00000000

1.24371366

0.00000000

1.26037396

0.00000000

1.24371366

0.00000000

1.22994895

0.00000000

3.73114097

2. 48742731

3.73114097

2.48262363

3.73114097

2. 48742731

3.73114097

2.48262363

0.01484738

7.46228194
Fri Apr 16 14:51:55 2021

170

7.46228194

8.69520086

7.45258140

1.24371366

0.00000000

1.24371366

3.73114097

2.48742731

3. 74659124

2.47257993

6.21856829

4.97485463

6.21856829

4.95023728

8.70599560

8.69054533

7.48689929

1.24371366

0.00000000

1.24371366

0.01090064

3.73114097

2. 48742731

3.73840596

2.47652667

6.21856829

4.97485463

6.21856829

4.95143848

8.70599560

7.46228194

8.69873061

7.48569809

1.24371366

6.21856829

3. 65087694

8.78625964

8.78625964
6.21856829

9.75887672

11.53838390

13.33886348

8.00000000

9.75887672

11.51550522

13.41205696

8.00000000

9.75887672

11.47607559

13.41205696

8.00000000

9.75887672

11.52940205

13.38982036

8.00000000

9.75887672

11.47607559

13.38982036

8.00000000

9.75887672

11.51623125

13.24062901

8.00000000

9.75887672

11.53854533

13.24062901

8.00000000

9.75887672

11.47851108

13.39535863

8.00000000

9.75887672

11.53854533

13.39535863

14.15522948

14.16027413

14.13995468

14.13995468

14.18621133 
$\mathrm{Ni}$

$\mathrm{Ni}$

$\mathrm{Ni}$

$\mathrm{Ni}$

$\mathrm{Ni}$

$\mathrm{Ni}$

$\mathrm{Ni}$

$\mathrm{Ni}$

$\mathrm{Ni}$

$\mathrm{Ni}$

$\mathrm{Ni}$

$\mathrm{Ni}$

$\mathrm{Ni}$

$\mathrm{Ni}$

$\mathrm{Ni}$

$\mathrm{Ni}$

$\mathrm{Ni}$

$\mathrm{Ni}$

$\mathrm{Ni}$

$\mathrm{Ni}$

$\mathrm{Ni}$

$\mathrm{Ni}$

$\mathrm{Ni}$

$\mathrm{Ni}$

O

O

0

0

O

0

$.10 x y+2$

55

$\begin{array}{llllll}\text { Lattice }=" 7.462281943147142 & 0.0 & 0.0 & 0.0 & 9.9 \\ \text { perties }=\text { species:S: }: 1: \text { pos }: R: 3 & \text { pbc="T } & \text { T } & \text { T" }\end{array}$

$\mathrm{Ni} \quad 1.24371366 \quad 1.24371366$

$\mathrm{Ni} \quad 0.00000000 \quad 0.00000000$

$\mathrm{Ni} \quad 1.25388120$

$\mathrm{Ni} \quad 0.14121241$

$\mathrm{Ni} \quad 1.24371366$

$\mathrm{Ni} \quad 0.00000000$

$\mathrm{Ni} \quad 1.17019352$

$\mathrm{Ni} \quad-0.10083123$

$\mathrm{Ni} \quad 1.24371366$

$\mathrm{Ni} \quad 0.00000000$

$\mathrm{Ni} \quad 1.25388120$

$\mathrm{Ni} \quad-0.10083123$

$\mathrm{Ni} \quad 1.24371366$

$\mathrm{Ni} \quad 0.00000000$

$\mathrm{Ni} \quad 1.29544419$

$\mathrm{Ni} \quad 0.14121241$

$\mathrm{Ni} \quad 3.73114097$

$\mathrm{Ni} \quad 2.48742731$

$\mathrm{Ni} \quad 3.73677938$

$\mathrm{Ni} \quad 2.57837679$

$\mathrm{Ni} \quad 3.73114097$

$\mathrm{Ni} \quad 2.48742731$

$\mathrm{Ni} \quad 3.68339451$

$\mathrm{Ni} \quad 2.41777347$

$\mathrm{Ni} \quad 3.73114097$

$\mathrm{Ni} \quad 2.48742731$

$\mathrm{Ni} \quad 3.73677938$

$\mathrm{Ni} \quad 2.41777347$

$\mathrm{Ni} \quad 3.73114097$

$\mathrm{Ni} \quad 2.48742731$

$\mathrm{Ni} \quad 3.78949772$

$\mathrm{Ni} \quad 2.57837679$

$\mathrm{Ni} \quad 6.21856829$

$\mathrm{Ni}$

4.96664389

8.70599560

2.49563805
1. 24788653

$-0.02939753$

3.73114097

2.48742731

3. 73114097

2. 41729507

6.21856829

4.97485463

6.21439541

5.04498688

8.70599560

7.46228194

8.70599560

7.49167948

1. 24371366

0.00000000

1.22494164

0.02707943

3.73114097

2.48742731

3. 73114097

2.51514265

6.21856829

4. 97485463

6.23734030

4.94713929

8.70599560

7.46228194

8.70599560

7.43520251

1.24371366

0.00000000
4.97485463
Fri Apr 16 14:51:55 2021

6.21856829

4.97485463

6.21856829

8.70599560

7.46228194

7.47049268

1.24371366

0.00000000

1.24371366

$-0.00821074$

3.73114097

2.48742731

3.73114097

6.21856829

4.97485463

6.21856829

4.96664389

8.70599560

7.46228194

8.70599560

7.47049268

1.24371366

6.21856829

3.73114097

8.70599560

6.21856829

1.24371366
8.00000000

9.75887672

11.50944606

13.36418807

8.00000000

9.75887672

11.48763813

13.36418807

8.00000000

9.75887672

11.46868610

13.36418807

8.00000000

9.75887672

11.54514522

13.36418807

8.00000000

9.75887672

11.46868610

13.36418807

8.00000000

9.75887672

11.54514522

13.36418807

14.17551978

14.17551978

14.13904242

14.13904242

14.17551978

14.17551978
8.00000000

9.75887672

11.47837140

13.35189707

8.00000000

9.75887672

11.54710140

13.40110336

8.00000000

9.75887672

11.47837140

13.40110336

8.00000000

9.75887672

11.50794195

13.35189707

8.00000000

9.75887672

11.50793427

13.38270819

8.00000000

9.75887672

11.46520561

13.32429936

8.00000000

9.75887672

11.50793427

13.32429936

8.00000000

9.75887672

11.52417538

13.38270819

8.00000000

9.75887672

$30.00 .00 .021 .27663016512527 "$ Pro 
$\mathrm{Ni}$

$\mathrm{Ni}$

$\mathrm{Ni}$

$\mathrm{Ni}$

$\mathrm{Ni}$

$\mathrm{Ni}$

$\mathrm{Ni}$

$\mathrm{Ni}$

$\mathrm{Ni}$

$\mathrm{Ni}$

$\mathrm{Ni}$

$\mathrm{Ni}$

$\mathrm{Ni}$

$\mathrm{Ni}$

0

0

O

0

0

0

0

$.10 \times$

6.20575248

5.12547791

6.21856829

4.97485463

6.20647850

4.85780727

6.21856829

4.97485463

6.20575248

4.85780727

6.21856829

4.97485463

6.26370267

5.12547791

1.32254357

1.32254357

3.60393270

3.72570186

5.96968999

5.96968999

6.50234436
Fri Apr 16 14:51:55 2021

1.23419155

0.05484737

3.73114097

2.48742731

3.73114097

2.53194045

6.21856829

4.97485463

6.22809039

4.93034149

8.70599560

7.46228194

8.70599560

7.40743457

1.26403072

6.19825122

3.73114097

8.70599560

5.87925859

1.58302335

8.70599560
11.49658787

13.53006378

8.00000000

9.75887672

11.53479888

13.28089286

8.00000000

9.75887672

11.49658787

13.28089286

8.00000000

9.75887672

11.50480093

13.53006378

14.16659009

14.16659009

14.18173671

14.14566612

14.34451441

14.34451441

14.27211013

56

$\begin{array}{lllll}\text { Lattice }=" 7.462281943147142 & 0.0 & 0.0 & 0.0 \\ \text { perties }=\text { species:S:1:pos:R:3 pbc="T } & \text { T T" }\end{array}$

$\begin{array}{lll}\mathrm{Ni} & 1.24371366 & 1.24371366\end{array}$

Ni $\quad 0.00000000 \quad 0.00000000$

1.22723170

1.24893623

$\begin{array}{ll}0.00000000 & 0.08811571 \\ 1.24371366 & 3.73114097\end{array}$

$0.00000000 \quad 2.48742731$

$\begin{array}{ll}1.22833276 & 3.73114097\end{array}$

$0.00000000 \quad 2.42777305$

$1.24371366 \quad 6.21856829$

$0.00000000 \quad 4.97485463$

$1.24893623 \quad 6.23505024$

$0.00000000 \quad 5.03450889$

$1.24371366 \quad 8.70599560$

$0.00000000 \quad 7.46228194$

$\begin{array}{ll}1.21037529 & 8.70599560\end{array}$

$0.00000000 \quad 7.37416623$

$3.73114097 \quad 1.24371366$

$\begin{array}{ll}2.48742731 & 0.00000000\end{array}$

$3.73114097 \quad 1.23521986$

$\begin{array}{ll}2.32812096 & 0.07452412\end{array}$

$\begin{array}{ll}3.73114097 & 3.73114097\end{array}$

$\begin{array}{ll}2.48742731 & 2.48742731\end{array}$

$\begin{array}{ll}3.73114097 & 3.73114097\end{array}$

$\begin{array}{ll}2.50421312 & 2.52801771\end{array}$

$\begin{array}{ll}3.73114097 & 6.21856829\end{array}$

$\begin{array}{ll}2.48742731 & 4.97485463\end{array}$

$\begin{array}{ll}3.73114097 & 6.22706209\end{array}$

$2.50421312 \quad 4.93426423$

$\begin{array}{ll}3.73114097 & 8.70599560\end{array}$

$\begin{array}{ll}2.48742731 & 7.46228194\end{array}$

$\begin{array}{ll}3.73114097 & 8.70599560\end{array}$

$\begin{array}{ll}2.32812096 & 7.38775782\end{array}$

$6.21856829 \quad 1.24371366$

$\begin{array}{ll}4.97485463 & 0.00000000\end{array}$

$6.21334572 \quad 1.22723170$

$\begin{array}{ll}5.13416098 & 0.07452412\end{array}$

$\begin{array}{ll}6.21856829 & 3.73114097\end{array}$

$\begin{array}{ll}4.97485463 & 2.48742731\end{array}$

$\begin{array}{ll}6.23394918 & 3.73114097\end{array}$

$\begin{array}{ll}4.95806882 & 2.52801771\end{array}$

$6.21856829 \quad 6.21856829$

$4.97485463 \quad 4.97485463$

$6.21334572 \quad 6.23505024$

8.00000000

9.75887672

11.48100513

14.45702354

8.00000000

9.75887672

11.52002006

13.25294240

8.00000000

9.75887672

11.48100513

13.25294240

8.00000000

9.75887672

11.49424074

14.45702354

8.00000000

9.75887672

11.54422044

13.43758209

8.00000000

9.75887672

11.48318314

13.31085062

8.00000000

9.75887672

11.54422044

13.31085062

8.00000000

9.75887672

11.56163444

13.43758209

8.00000000

9.75887672

11.48100513

13.43758209

8.00000000

9.75887672

11.52002006

13.31085062

8.00000000

9.75887672

11.48100513 
$\mathrm{Ni}$

$\mathrm{Ni}$

$\mathrm{Ni}$

$\mathrm{Ni}$

$\mathrm{Ni}$

O

0

0

O

0

O

O

0

. / 57

perties=species:S:1:pos:R:3 pbc="T T T"

$\mathrm{Ni}$

$\mathrm{Ni}$

$\mathrm{Ni}$

$\mathrm{Ni}$

$\mathrm{Ni}$

$\mathrm{Ni}$

$\mathrm{Ni}$

$\mathrm{Ni}$

$\mathrm{Ni}$

$\mathrm{Ni}$

$\mathrm{Ni}$

$\mathrm{Ni}$

$\mathrm{Ni}$

$\mathrm{Ni}$

$\mathrm{Ni}$

$\mathrm{Ni}$

$\mathrm{Ni}$

$\mathrm{Ni}$

$\mathrm{Ni}$

$\mathrm{Ni}$

$\mathrm{Ni}$

$\mathrm{Ni}$

$\mathrm{Ni}$

$\mathrm{Ni}$

$\mathrm{Ni}$

$\mathrm{Ni}$

$\mathrm{Ni}$

$\mathrm{Ni}$

$\mathrm{Ni}$

$\mathrm{Ni}$

$\mathrm{Ni}$

$\mathrm{Ni}$

$\mathrm{Ni}$

$\mathrm{Ni}$

$\mathrm{Ni}$

$\mathrm{Ni}$

$\mathrm{Ni}$

$\mathrm{Ni}$

$\mathrm{Ni}$

$\mathrm{Ni}$

$\mathrm{Ni}$

$\mathrm{Ni}$

$\mathrm{Ni}$

$\mathrm{Ni}$

$\mathrm{Ni}$

$\mathrm{Ni}$

$\mathrm{Ni}$

0

0

o
1.30746952

4.95806882

6.21856829

4.97485463

6.25190665

5.13416098

1.33059971

1.33059971

3.73114097

3.73114097

6.13168223

6.13168223

6.15481242
Fri Apr 16 14:51:55 2021

4.93426423

8.70599560

7.46228194

8.70599560

7.38775782

1.42490129

6.03738066

3.73114097

8.70599560

6.03738066

1.42490129

8.70599560

8.70599560
13.31085062

8.00000000

9.75887672

11.49424074

13.43758209

14.33092426

14.33092426

14.21373209

13.47754627

14.33092426

14.33092426

14.65796677

14.65796677

$949709257529523 \quad 0.0 \quad 0.0 \quad 0.021 .27663016512527 "$ Pro

8.00000000

9.75887672

11.44745527

14.59340324

8.00000000

9.75887672

11.54563075

13.26621076

8.00000000

9.75887672

11.49722829

13.31927137

8.00000000

9.75887672

11.51147568

14.93744834

8.00000000

9.75887672

11.49729937

13.25078874

8.00000000

9.75887672

11.49808866

13.33391001

8.00000000

9.75887672

11.48917443

13.35813791

8.00000000

9.75887672

11.59646607

13.97419741

8.00000000

9.75887672

11.44745527

13.25078874

8.00000000

9.75887672

11.54563075

13.33391001

8.00000000

9.75887672

11.49722829

13.35813791

8.00000000

9.75887672

11.51147568

13.97419741

14.29019672

14.52522199

14.25071300 


\section{0}

O

0

0

O

0

\subsection{7 \\ 6.12409072 \\ 6.16077776 \\ 6.22689637 \\ 1.23538557 \\ 3.73114097}

Fri Apr 16 14:51:55 2021

$\begin{array}{ll}8.83301038 & 13.63367259 \\ 5.95010415 & 14.52522199 \\ 1.25857708 & 14.29019672 \\ 8.51033343 & 15.09120723 \\ 8.51033343 & 15.09120723 \\ 6.38696321 & 13.36740383\end{array}$

174

17

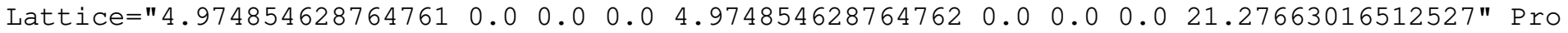
perties=species:S:1:pos:R:3 pbc="T T T"

$\mathrm{Ni} \quad 0.00000000 \quad 0.00000000$

$\mathrm{Ni} \quad 1.24371366 \quad 1.24371366$

$\mathrm{Ni} \quad 0.00154826 \quad 0.00154826$

$\mathrm{Ni} \quad 1.24371366 \quad 3.73114097$

$\mathrm{Ni} \quad 0.00000000 \quad 2.48742731$

$\mathrm{Ni} \quad 1.24371366 \quad 3.73114097$

$\mathrm{Ni} \quad 0.00154826 \quad 2.48587905$

$\mathrm{Ni} \quad 3.73114097 \quad 1.24371366$

$\mathrm{Ni} \quad 2.48742731 \quad 0.00000000$

$\mathrm{Ni} \quad 3.73114097 \quad 1.24371366$

$\begin{array}{lll}\mathrm{Ni} & 2.48587905 & 0.00154826\end{array}$

$\begin{array}{lll}\mathrm{Ni} & 3.73114097 & 3.73114097\end{array}$

$\mathrm{Ni} \quad 2.48742731 \quad 2.48742731$

$\begin{array}{lll}\mathrm{Ni} & 3.73114097 & 3.73114097\end{array}$

$\begin{array}{lll}\mathrm{Ni} & 2.48587905 & 2.48587905\end{array}$

o $1.24371366 \quad 1.24371366$

./oxygen/Ni/110/iter_1/struct.xyz

46

Lattice $=" 7.462281943147140 .0 \quad 0.0 \quad 0.0 \quad 10.5532603302505410 .00 .0 \quad 0.020 .97485462876476 "$ Pro perties=species:S:1:pos:R:3 pbc="T T T"

$\mathrm{N}$

$\mathrm{Ni}$

0.00000000

1.24371366

0.00000000

1.24371366

$-0.05812093$

0.00000000

1.24371366

0.00000000

1.24371366

$-0.02924357$

0.00000000

1. 24371366

0.00000000

1.24371366

0.00100077

2.48742731

3.73114097

2. 48742731

3.75200202

2.54554825

2.48742731

3.73114097

2.48742731

3.73526760

2. 51667088

2.48742731

3. 73114097

2. 48742731

3. 73642077

2.48642654

4.97485463

6.21856829

4.97485463

6.19770724

4.97485463

4.97485463

6.21856829
0.00000000

1.75887672

0.00000000

1.78036778

$-0.04415444$

3.51775344

5.27663017

3.51775344

5.28976375

3.58285141

7.03550689

8.79438361

7.03550689

8.77993847

7.04325315

0.00000000

1.75887672

0.00000000

1.77301498

$-0.04415444$

3.51775344

5.27663017

3.51775344

5.29684851

3.58285141

7.03550689

8.79438361

7.03550689

8.78746899

7.04325315

0.00000000

1.75887672

0.00000000

$-0.02867611$

3.51775344

5.27663017
1.77301498
8.00000000

9.75887672

11.44929409

13.28047295

8.00000000

9.75887672

11.52105518

13.28047295

8.00000000

9.75887672

11.52105518

13.28047295

8.00000000

9.75887672

11.58008361

13.28047295

14.12619531
$\mathrm{Ni}$

$\mathrm{Ni}$

$$
\begin{array}{r}
8.00000000 \\
9.24371366 \\
10.48742731 \\
11.88500994 \\
12.94157073 \\
8.00000000 \\
9.24371366 \\
10.48742731 \\
11.77209356 \\
12.88144430 \\
8.0000000 \\
9.24371366 \\
10.48742731 \\
11.75034573 \\
12.88162019 \\
8.00000000 \\
9.24371366 \\
10.48742731 \\
11.76996559 \\
12.94157073 \\
8.00000000 \\
9.24371366 \\
10.48742731 \\
11.77158132 \\
12.88144430 \\
8.00000000 \\
9.24371366 \\
10.48742731 \\
11.77103256 \\
12.88162019 \\
8.00000000 \\
9.24371366 \\
10.48742731 \\
11.76996559 \\
12.86651525 \\
8.00000000 \\
9.24371366
\end{array}
$$


$\mathrm{Ni}$

$\mathrm{Ni}$

$\mathrm{Ni}$

$\mathrm{Ni}$

$\mathrm{Ni}$

$\mathrm{Ni}$

$\mathrm{Ni}$

$\mathrm{Ni}$

0

\subsection{3}

6.21444166

4.97485463

4.97485463

6.21856829

4.97485463

6.21328849

4.97485463

1.24371366
Fri Apr 16 14:51:55 2021

3.51775344

5.29684851

3.54998015

7.03550689

8.79438361

7.03550689

8.78746899

7.04537495

1.01644149
10.48742731

11.77158132

12.87840328

8.00000000

9.24371366

10.48742731

11.77103256

12.88491889

13.66311608

55

Lattice $=" 7.46228194314714 \quad 0.0 \quad 0.0 \quad 0.0 \quad 10.553260330250541 \quad 0.0 \quad 0.0 \quad 0.020 .97485462876476 "$ Pro perties=species:S:1:pos:R:3 pbc="T T T"

$\mathrm{N}$

$$
0.00000000 \quad 0.00000000
$$

$\mathrm{Ni}$

1.24371366

1.75887672

0.00000000

0.00000000

1.13015128

1.72187048

7.37518095

9.81196045

0.00000000

1.24371366

0.00000000

3.51775344

5.27663017

3.51775344

1.24531561

$-0.00080930$

0.00000000

5.29972418

3.57923725

7.03550689

1. 24371366

0.00000000

8.79438361

7.03550689

1.27142521

8.76347643

0.07956251

2. 48742731

3.73114097

6.66869910

0.00000000

1.75887672

2.48742731

3. 69442243

0.00000000

1.77711970

2. 45260464

2. 48742731

3. 73114097

2. 48742731

3.75101927

2. 38494815

2. 48742731

3. 73114097

2. 48742731

3. 81588071

2. 61071120

4.97485463

6.21856829

4. 97485463

6.16220857

5.01296610

4.97485463

6.21856829

4.97485463

6.22730629

4. 81715321

4. 97485463

6.21856829

4. 97485463

6.26647429

4. 99322119

0.91731454

3. 83515973

6.29408327

6.35706001

1. 23966646

3. 99465062

1. 29998784

3. 63455180

0.00682449

3.51775344

5.27663017

3. 51775344

5.26123952

3.54606029

7.03550689

8.79438361

7.03550689

8.86205744

6.97521101

0.00000000

1.75887672

0.00000000

1.78623361

0.77087206

3.51775344

5.27663017

3. 51775344

5.27274493

3.86994867

7.03550689

8.79438361

7.03550689

8.77982471

7.02163888

0.79404794

5.65480303

5.08193445

8.11789138

4.89703697

8.00000000

9.24371366

10.48742731

11.82002319

13.72072973

8.00000000

9.24371366

10.48742731

11.67146254

13.29064513

8.00000000

9.24371366

10.48742731

11.76380525

13.51583884

8.00000000

9.24371366

10.48742731

11.75420018

13.04469627

8.00000000

9.24371366

10.48742731

11.68278863

12.97939049

8.00000000

9.24371366

10.48742731

11.82292023

13.03589290

8.00000000

9.24371366

10.48742731

11.74933975

13.74911639

8.00000000

9.24371366

10.48742731

11.70302963

13.55975503

8.00000000

9.24371366

10.48742731

11.73977061

13.06249704

13.45012673

13.56156010

13.64036357

13.55343392

13.53119823

13.46174689

13.56797671

13.58912182

13.54512933

14.76950481

8.09175908

2.40078421

2. 44229148

10.55049294

6.19453472 
. /oxygen/Ni/110/iter_11/struct.xyz

56

Lattice $=" 7.46228194314714 \quad 0.0 \quad 0.0 \quad 0.0 \quad 10.5532603302505410 .0 \quad 0.0 \quad 0.020 .97485462876476 "$ Pro perties=species:S:1:pos:R: 3 pbc="T T T"

$\mathrm{Ni}$ $0.00000000 \quad 0.00000000$

$\begin{array}{lll}\mathrm{Ni} & 1.24371366 & 1.75887672 \\ \mathrm{Ni} & 0.00000000 & 0.00000000\end{array}$

$\mathrm{Ni} \quad 1.24524597$

1.73112449

7.31854288

$\mathrm{Ni} \quad 0.00000000$

10.41628936

1. 24371366

0.00000000

3.51775344

5.27663017

3.51775344

1. 24557749

0.09051658

0.00000000

5.30312645

3. 60813272

7.03550689

1. 24371366

0.00000000

1. 24414108

8.79438361

7.03550689

8.80894468

0.03676693

6.79050821

2. 48742731

0.00000000

3. 73114097

1. 75887672

2. 48742731

3. 74633680

2. 64328602

2. 48742731

3. 73114097

2. 48742731

3. 74136682

2. 40786212

2. 48742731

3. 73114097

2. 48742731

0.00000000

1.75046798

$-0.13148757$

3. 51775344

5.27663017

3.51775344

5.28714375

3. 61094280

7. 03550689

8.79438361

7.03550689

8.78797274

3. 74107483

2. 45122423

6.79295562

0.00000000

4.97485463

1.75887672

0.00000000

4. 97485463

1. 74874661

1.12334438

4. 98089378

3.51775344

5.27663017

3. 51775344

5.28505577

3. 93939069

7.03550689

8.79438361

7.03550689

8.78994063

6.87818728

0.99094851

5.44836486

5.44721843

8.13239453

5.13104503

0.04083517

8.10465195

2. 55012616

2. 55099961

8.00000000

9.24371366

10.48742731

11.77482058

13.18879061

8.00000000

9.24371366

10.48742731

11.64174688

12.98270139

8.00000000

9.24371366

10.48742731

11.75631951

13.45518862

8.00000000

9.24371366

10.48742731

11.79499272

13.18186867

8.00000000

9.24371366

10.48742731

11.68469282

12.98404749

8.00000000

9.24371366

10.48742731

11.73339437

13.45860490

8.00000000

9.24371366

10.48742731

11.79603394

14.12442922

8.00000000

9.24371366

10.48742731

11.68220018

13.58244576

8.00000000

9. 24371366

10.48742731

11.73393289

13.46576797

13.47414534

13.64182414

13.63831952

13.45375241

13.53738189

14.57250115

13.47807136

13.59527948

13.59408182

14.57841403

0.04298424

8.13235515

13.45407738

. /oxygen/Ni/110/iter_12/struct.xyz 57

Lattice $=" 7.46228194314714 \quad 0.0 \quad 0.0 \quad 0.0 \quad 10.5532603302505410 .0 \quad 0.0 \quad 0.020 .97485462876476 "$ Pro perties=species:S:1:pos:R:3 pbc="T T T"
$\mathrm{Ni}$
$\mathrm{Ni}$
0.00000000
0.00000000
1. 75887672
8.00000000
0.00000000
9.24371366
0.00000000
1.91441871
10.48742731
$\mathrm{Ni}$
1.25024327
11.93598963 
$\mathrm{Ni}$

$\mathrm{Ni}$

$\mathrm{Ni}$

$\mathrm{Ni}$

$\mathrm{Ni}$

$\mathrm{Ni}$

$\mathrm{Ni}$

$\mathrm{Ni}$

$\mathrm{Ni}$

$\mathrm{Ni}$

$\mathrm{Ni}$

$\mathrm{Ni}$

$\mathrm{Ni}$

$\mathrm{Ni}$

$\mathrm{Ni}$

$\mathrm{Ni}$

$\mathrm{Ni}$

$\mathrm{Ni}$

$\mathrm{Ni}$

$\mathrm{Ni}$

$\mathrm{Ni}$

$\mathrm{Ni}$

$\mathrm{Ni}$

$\mathrm{Ni}$

$\mathrm{Ni}$

$\mathrm{Ni}$

$\mathrm{Ni}$

$\mathrm{Ni}$

$\mathrm{Ni}$

$\mathrm{Ni}$

$\mathrm{Ni}$

$\mathrm{Ni}$

$\mathrm{Ni}$

$\mathrm{Ni}$

$\mathrm{Ni}$

$\mathrm{Ni}$

$\mathrm{Ni}$

$\mathrm{Ni}$

$\mathrm{Ni}$

$\mathrm{Ni}$

$\mathrm{Ni}$

0

0

0

0

0

o

0

0

o

0

0

0
6.26222232

7.32759611

0.00000000

1.24371366

0.00000000

1.24428396

7.14625818

0.00000000

1.24371366

0.00000000

1.24527521

0.00579421

2. 48742731

3.73114097

2. 48742731

3. 67636329

2. 64365930

2. 48742731

3.73114097

2. 48742731

3. 72581020

2. 82996820

2. 48742731

3. 73114097

2.48742731

3.70695252

2.46897799

4.97485463

6.21856829

4.97485463

6.28398671

4.97673859

4.97485463

6.21856829

4.97485463

6.21825521

4. 98553488

4.97485463

6.21856829

4. 97485463

6.24327431

4. 96795781

1.25279547

3. 67530525

6.33443693

6.17848221

1. 23541816

3. 75063839

1.23789193

3. 64125408

4. 97840955

6.21311452

3. 75926254
Fri Apr 16 14:51:55 2021

0.02211242

3.51775344

5.27663017

3.51775344

5.16976928

3.59934413

7.03550689

8.79438361

7.03550689

8.82352707

6.85863603

0.00000000

1.75887672

0.00000000

1.83448648

0.02764222

3.51775344

5.27663017

3.51775344

5.25734559

3.58841636

7.03550689

8.79438361

7.03550689

8.75626931

6.85593838

0.00000000

1.75887672

0.00000000

1.83611547

9.46713703

3. 51775344

5.27663017

3.51775344

5.25707350

1.58939045

7.03550689

8.79438361

7.03550689

8.75765143

6.75377178

1.09727851

5.46810552

2.73229060

8.18147312

5.50784989

0.15304242

8.20489129

2.74101222

0.65094028

0.14392129

8.18453309

5.47067487
13.18327257

8.00000000

9.24371366

10.48742731

11.75179205

13.16364246

8.00000000

9.24371366

10.48742731

11.79466513

13.54223366

8.00000000

9.24371366

10.48742731

11.71824733

13.18129506

8.00000000

9.24371366

10.48742731

11.65135869

13.16832282

8.00000000

9.24371366

10.48742731

11.71575810

13.54051659

8.00000000

9.24371366

10.48742731

11.72516518

14.21229931

8.00000000

9.24371366

10.48742731

11.65309461

14.58295544

8.00000000

9.24371366

10.48742731

11.71425846

13.39915887

13.58623593

13.50840735

14.43017910

13.52361051

13.59640825

14.67735784

13.55554622

14.44953866

12.23857251

14.67297785

13.52831685

13.50988252

58

Lattice $=" 7.46228194314714 \quad 0.0 \quad 0.0 \quad 0.0 \quad 10.5532603302505410 .0 \quad 0.0 \quad 0.020 .97485462876476 "$ Pro perties=species:S:1:pos:R:3 pbc="T T T"

$\begin{array}{lllr}\mathrm{Ni} & 0.00000000 & 0.00000000 & 8.00000000 \\ \mathrm{Ni} & 1.24371366 & 1.75887672 & 9.24371366 \\ \mathrm{Ni} & 0.00000000 & 0.00000000 & 10.48742731 \\ \mathrm{Ni} & 1.24996191 & 1.97665040 & 11.93024848 \\ \mathrm{Ni} & 0.00865219 & 0.10863213 & 13.10494122 \\ \mathrm{Ni} & 0.00000000 & 3.51775344 & 8.00000000 \\ \mathrm{Ni} & 1.24371366 & 5.27663017 & 9.24371366 \\ \mathrm{Ni} & 0.00000000 & 3.51775344 & 10.48742731 \\ \mathrm{Ni} & 1.24466205 & 5.24678490 & 11.63339170 \\ \mathrm{Ni} & 7.32791779 & 3.92700567 & 14.05762784 \\ \mathrm{Ni} & 0.00000000 & 7.03550689 & 8.00000000\end{array}$


$\mathrm{Ni}$

$\mathrm{Ni} \quad 0.00000000$

$\mathrm{Ni} \quad 1.24480271$

$\mathrm{Ni} \quad 0.02150763$

$\mathrm{Ni} \quad 2.48742731$

$\mathrm{Ni} \quad 3.73114097$

$\mathrm{Ni} \quad 2.48742731$

$\mathrm{Ni} \quad 3.69552109$

$\mathrm{Ni} \quad 2.48453289$

$\mathrm{Ni} \quad 2.48742731$

$\mathrm{Ni} \quad 3.73114097$

$\mathrm{Ni} \quad 2.48742731$

$\mathrm{Ni} \quad 3.72153405$

$\mathrm{Ni} \quad 2.61513447$

$\mathrm{Ni} \quad 2.48742731$

$\begin{array}{ll}\mathrm{Ni} & 3.73114097\end{array}$

$\mathrm{Ni} \quad 2.48742731$

$\mathrm{Ni} \quad 3.72464369$

$\mathrm{Ni} \quad 2.45507527$

$\mathrm{Ni} \quad 4.97485463$

$\mathrm{Ni} \quad 6.21856829$

$\mathrm{Ni} \quad 4.97485463$

$\mathrm{Ni}$

$\mathrm{Ni}$

$\mathrm{Ni}$

$\mathrm{Ni}$

$\mathrm{Ni}$

$\mathrm{Ni}$

$\mathrm{Ni}$

$\mathrm{Ni}$

$\mathrm{Ni}$

$\mathrm{Ni}$

$\mathrm{Ni}$

$\mathrm{Ni}$

0

0

0

O

O

O

0

0

O

O

O

0

0

4. 98025070

4.97485463

6.21856829

4.97485463

6.22214686

4.96714702

4.97485463

6.21856829

6.22365144

4.96956041

1. 23668375

3. 68010648

6.31331545

6.20525266

1.23752301

3.81697265

1. 23920826

3.61817932

4. 97447587

6.13986166

6.25930612

1. 24796949
Fri Apr 16 14:51:55 2021

178

8.79438361

7.03550689

8.83054246

6.83563283

0.00000000

1.75887672

0.00000000

1.74690519

0.10868313

3.51775344

5.27663017

3.51775344

5.25833883

3.91087952

7.03550689

8.79438361

7.03550689

8.77834304

6.83380415

0.00000000

1.75887672

0.00000000

1.75146117

4.97485463

3.73284945
$-0.06467671$

3.51775344

5.27663017

3.51775344

5.25736317

1.98882074

7.03550689

8.79438361

7.03550689

8.77600149

6.77830322

2.77082320

5.43419084

3.02329010

8.09632684

5.28012074

0.51672927

3.01637619

2.97109881

0.51705409

8.09613761

5.43379525

0.17259444
8.12925903
9.24371366

10.48742731

11.76505768

13.45689291

8.00000000

9.24371366

10.48742731

11.70221732

13.10240519

8.00000000

9.24371366

10.48742731

11.70552453

14.04103530

8.00000000

9.24371366

10.48742731

11.76564541

13.46976544

8.00000000

9.24371366

10.48742731

11.70835517

12.99342057

8.00000000

9.24371366

10.48742731

11.70296020

14.68884651

8.00000000

9.24371366

10.48742731

11.76477340

13.58611685

13.62481838

13.59586001

15.11287447

13.49544274

13.52284709

14.25092318

13.47429682

15.11964141

12.27916085

14.25229037

13.49444603

13.59010860

14.31838874

59

Lattice $=" 7.46228194314714 \quad 0.0 \quad 0.0 \quad 0.0 \quad 10.5532603302505410 .0 \quad 0.0 \quad 0.020 .97485462876476 " \quad$ Pro perties=species:S:1:pos:R:3 pbc="T T T"

$\begin{array}{lrrr}\mathrm{Ni} & 0.00000000 & 0.00000000 & 8.00000000 \\ \mathrm{Ni} & 1.24371366 & 1.75887672 & 9.24371366 \\ \mathrm{Ni} & 0.00000000 & 0.00000000 & 10.48742731 \\ \mathrm{Ni} & 1.24371366 & 1.83487527 & 11.82358760 \\ \mathrm{Ni} & -0.01935469 & 10.47729865 & 13.08181579 \\ \mathrm{Ni} & 0.00000000 & 3.51775344 & 8.00000000 \\ \mathrm{Ni} & 1.24371366 & 5.27663017 & 9.24371366 \\ \mathrm{Ni} & 0.00000000 & 3.51775344 & 10.48742731 \\ \mathrm{Ni} & 1.24371366 & 5.31267157 & 11.78258304 \\ \mathrm{Ni} & 7.29760910 & 2.09094121 & 14.25108853 \\ \mathrm{Ni} & 0.00000000 & 7.03550689 & 8.00000000 \\ \mathrm{Ni} & 1.24371366 & 8.79438361 & 9.24371366 \\ \mathrm{Ni} & 0.00000000 & 7.03550689 & 10.48742731 \\ \mathrm{Ni} & 1.24371366 & 8.80751389 & 11.72307128 \\ \mathrm{Ni} & 7.44725022 & 7.08681435 & 13.64602392 \\ \mathrm{Ni} & 2.48742731 & 0.00000000 & 8.00000000 \\ \mathrm{Ni} & 3.73114097 & 1.75887672 & 9.24371366\end{array}$


$\mathrm{Ni}$

$\mathrm{Ni}$

2.48742731

3.72426363

2.50678200

2.48742731

3.73114097

2.48742731

3.70574459

2.65210016

2.48742731

3.73114097

2.48742731

3.73245407

2.50245903

4.97485463

6.21856829

4.97485463

6.22544563

4.97485463

4.97485463

6.21856829

4.97485463

6.24396467

4.97485463

4.97485463

6.21856829

4.97485463

6.21725519

4.97485463

1.24371366

3.78497945

6.35605513

6.19001794

1.24371366

3.61820145

1.24371366

3.59365413

6.29738117

6.33150781

3.75969131

6.16472981

1.24371366

3.65232809
Fri Apr 16 14:51:55 2021

179

0.00000000

1.62258284

10.47729865

3.51775344

5.27663017

3.51775344

5.36466870

2.09094121

7.03550689

8.79438361

7.03550689

8.82601124

7.08681435

0.00000000

1. 75887672

0.00000000

1.62258284

10.00203070

3. 51775344

5.27663017

3.51775344

5.36466870

4.29102878

7.03550689

8.79438361

7.03550689

8.82601124

7.10752897

2.96737673

5.74233446

3. 38108267

8.51443124

5.80866505

0.50481873

8.40721048

3. 38108267

3. 47609200

0.50481873

8.51443124

5.74233446

0.70066713

3.47609200
10.48742731

11.78825525

13.08181579

8.00000000

9.24371366

10.48742731

11.80105343

14.25108853

8.00000000

9.24371366

10.48742731

11.71793127

13.64602392

8.00000000

9.24371366

10.48742731

11.78825525

14.07194661

8.00000000

9.24371366

10.48742731

11.80105343

14.47030197

8.00000000

9.24371366

10.48742731

11.71793127

13.47626643

13.38512361

13.66271556

14.94978496

13.61132801

13.54359775

14.48714953

13.54784175

14.94978496

11.93145309

14.48714953

13.61132801

13.66271556

14.29684604

11.93145309

. /oxygen/Ni/110/iter_2/struct.xyz

47

Lattice $=" 7.46228194314714 \quad 0.0 \quad 0.0 \quad 0.0 \quad 10.5532603302505410 .0 \quad 0.0 \quad 0.020 .97485462876476 "$ Pro perties=species: $\mathrm{S}: 1:$ pos:R:3 pbc="T T T"

$\mathrm{Ni}$

$\mathrm{Ni}$

$\mathrm{Ni}$

$\mathrm{Ni}$

$\mathrm{Ni}$

$\mathrm{Ni}$

$\mathrm{Ni}$

$\mathrm{Ni}$

$\mathrm{Ni}$

$\mathrm{Ni}$

$\mathrm{Ni}$

$\mathrm{Ni}$

$\mathrm{Ni}$

$\mathrm{Ni}$

$\mathrm{Ni}$

$\mathrm{Ni}$

$\mathrm{Ni}$

$\mathrm{Ni}$

$\mathrm{Ni}$

$\mathrm{Ni}$

$\mathrm{Ni}$

$\mathrm{Ni}$
$0.00000000 \quad 0.00000000$

$1.24371366 \quad 1.75887672$

$0.00000000 \quad 0.00000000$

$1.24203409 \quad 1.77452355$

$-0.05227503 \quad-0.04860221$

$0.00000000 \quad 3.51775344$

$\begin{array}{ll}1.24371366 & 5.27663017\end{array}$

$0.00000000 \quad 3.51775344$

$1.22151332 \quad 5.30795203$

$\begin{array}{ll}-0.02952867 & 3.56676638\end{array}$

$0.00000000 \quad 7.03550689$

$1.24371366 \quad 8.79438361$

$0.00000000 \quad 7.03550689$

$\begin{array}{ll}1.24371165 & 8.79476444\end{array}$

$0.00032954 \quad 7.07678236$

$2.48742731 \quad 0.00000000$

$3.73114097 \quad 1.75887672$

$\begin{array}{ll}2.48742731 & 0.00000000\end{array}$

$\begin{array}{lr}3.74992312 & 1.75445037\end{array}$

$2.55017708 \quad-0.05246984$

$\begin{array}{ll}2.48742731 & 3.51775344\end{array}$

$\begin{array}{ll}3.73114097 & 5.27663017\end{array}$
8.00000000

9.24371366

10.48742731

11.86594443

12.94622656

8.00000000

9.24371366

10.48742731

11.76021814

12.86888676

8.00000000

9.24371366

10.48742731

11.73470543

12.88323483

8.00000000

9.24371366

10.48742731

11.74532859

12.94384429

8.00000000

9.24371366 
$\mathrm{Ni}$

$\mathrm{Ni}$

$\mathrm{Ni}$

$\mathrm{Ni}$

$\mathrm{Ni}$

$\mathrm{Ni}$

$\mathrm{Ni}$

$\mathrm{Ni}$

$\mathrm{Ni}$

$\mathrm{Ni}$

$\mathrm{Ni}$

$\mathrm{Ni}$

$\mathrm{Ni}$

$\mathrm{Ni}$

$\mathrm{Ni}$

$\mathrm{Ni}$

$\mathrm{Ni}$

$\mathrm{Ni}$

$\mathrm{Ni}$

$\mathrm{Ni}$

$\mathrm{Ni}$

$\mathrm{Ni}$

$\mathrm{Ni}$

O

0
Fri Apr 16 14:51:55 2021

2. 48742731

3. 73442974

2. 44026482

2. 48742731

3. 73114097

2. 48742731

3. 74040858

2. 45711227

4.97485463

6.21856829

4.97485463

6.20250016

4.98100140

4.97485463

6.21856829

4.97485463

6.23369045

5.01465658

4.97485463

6.21856829

4.97485463

6.21494342

5.00527642

1.25215796

3. 74455487
3.51775344

5.32569993

3.55132440

7.03550689

8.79438361

7.03550689

8.79872845

7.11200529

0.00000000

1.75887672

0.00000000

1.76748169

$-0.01973603$

3.51775344

5.27663017

3. 51775344

5. 30978214

3. 50250763

7.03550689

8.79438361

7.03550689

8.80444697

7.11068896

1.03253536

4.60902349
10.48742731

11.84645781

12.94258912

8.00000000

9.24371366

10.48742731

11.75851176

12.88920840

8.00000000

9.24371366

10.48742731

11.76530417

12.87116244

8.00000000

9.24371366

10.48742731

11.76043727

12.93485904

8.00000000

9.24371366

10.48742731

11.75744421

12.89098825

13.64635892

13.66311926

48

Lattice $=" 7.46228194314714 \quad 0.0 \quad 0.0 \quad 0.0 \quad 10.553260330250541 \quad 0.0 \quad 0.0 \quad 0.020 .97485462876476 "$ Pro perties=species:S:1:pos:R:3 pbc="T T T"

$\mathrm{Ni}$

$\mathrm{Ni}$

$\mathrm{Ni}$

$\mathrm{Ni}$

$\mathrm{Ni}$

$\mathrm{Ni}$

$\mathrm{Ni}$

$\mathrm{Ni}$

$\mathrm{Ni}$

$\mathrm{Ni}$

$\mathrm{Ni}$

$\mathrm{Ni}$

$\mathrm{Ni}$

$\mathrm{Ni}$

$\mathrm{Ni}$

$\mathrm{Ni}$

$\mathrm{Ni}$

$\mathrm{Ni}$

$\mathrm{Ni}$

$\mathrm{Ni}$

$\mathrm{Ni}$

$\mathrm{Ni}$

$\mathrm{Ni}$

$\mathrm{Ni}$

$\mathrm{Ni}$

$\mathrm{Ni}$

$\mathrm{Ni}$

$\mathrm{Ni}$

$\mathrm{Ni}$

$\mathrm{Ni}$

$\mathrm{Ni}$

$\mathrm{Ni}$

$\mathrm{Ni}$

$\mathrm{Ni}$

$\mathrm{Ni}$

$\mathrm{Ni}$

$\mathrm{Ni}$

$\mathrm{Ni}$

$\mathrm{Ni}$
0.00000000

1. 24371366

0.00000000

1.26818857

$-0.00721410$

0.00000000

1.24371366

0.00000000

1. 21047488

0.00459500

0.00000000

1. 24371366

0.00000000

1. 25584190

0.00088530

2. 48742731

3. 73114097

2. 48742731

3. 73368411

2. 57512531

2. 48742731

3. 73114097

2. 48742731

3. 73911758

2. 41514743

2. 48742731

3. 73114097

2. 48742731

3. 74571950

2. 46973239

4. 97485463

6.21856829

4. 97485463

6.18611367

4.97933670

4.97485463

6.21856829

4.97485463

6. 22886504
0.00000000

1.75887672

0.00000000

1.76738879

$-0.10489053$

3.51775344

5.27663017

3.51775344

5. 30948585

3. 60445146

7.03550689

8.79438361

7.03550689

8.76622487

7.05743660

0.00000000

1.75887672

0.00000000

1. 76805837

$-0.08803038$

3.51775344

5.27663017

3.51775344

5. 31114376

3.59670630

7.03550689

8.79438361

7.03550689

8.77395626

7.06669392

0.00000000

1.75887672

0.00000000

1.76116736

$-0.07378105$

3.51775344

5. 27663017

3.51775344

5.30568607
8.00000000

9.24371366

10.48742731

11.86602918

12.94644766

8.00000000

9.24371366

10.48742731

11.73735103

12.93660380

8.00000000

9.24371366

10.48742731

11.73726463

12.88651156

8.00000000

9.24371366

10.48742731

11.74758816

12.93464025

8.00000000

9.24371366

10.48742731

11.76670644

12.93199312

8.00000000

9.24371366

10.48742731

11.75070227

12.89385146

8.00000000

9.24371366

10.48742731

11.83686112

12.87380238

8.00000000

9.24371366

10.48742731

11.74031936 
$\mathrm{Ni}$

$\mathrm{Ni}$

$\mathrm{Ni}$

$\mathrm{Ni}$

$\mathrm{Ni}$

$\mathrm{Ni}$

o

0

0
3.59475771

7.03550689

8.79438361

7.03550689

8.78406287

7.08224133

0.99191438

4.79750783

2.44266304
13.11834032

8.00000000

9.24371366

10.48742731

11.75212448

12.88992968

13.62881420

13.62632777

13.62475848

49

Lattice $=" 7.46228194314714 \quad 0.0 \quad 0.0 \quad 0.0 \quad 10.5532603302505410 .00 .0 \quad 0.020 .97485462876476 "$ Pro perties=species:S:1:pos:R:3 pbc="T T T"

$\mathrm{Ni}$

$0.00000000 \quad 0.00000000$

1.75887672

1.24371366

0.00000000

0.00000000

1.83615686

1.28364746

10.50689382

$-0.02414471$

3.51775344

1.24371366

5.27663017

0.00000000

3.51775344

1.21382141

5.29570244

0.03114094

3.67942034

0.00000000

7.03550689

1.24371366

8.79438361

7.03550689

0.00000000

8.74387776

1.26781443

6.94507540

05477110

0.00000000

3.73114097

1.75887672

0.00000000

1.81700716

.48742731

3.73485066

10.49097737

2.54943881

2.48742731

3. 51775344

3.73114097

5.27663017

3. 51775344

5.29246571

3. 73818711

3.71652032

7.03550689

2.48742731

3. 73114097

8.79438361

7.03550689

8.73802466

3.71997369

2.46408432

6.95274952

0.00000000

1.75887672

6.21856829

0.00000000

1.81873568

4.97485463
6.17659798

$-0.01260590$

4.93535981

4.97485463

3. 51775344

6.21856829

5.27663017

3.51775344

5.29132857

6.24356343

3.76621710

7.03550689

8.79438361

7.03550689

8.72911607

6.78472198

8.00000000

9.24371366

10.48742731

11.90859167

12.91040261

8.00000000

9.24371366

10.48742731

11.68439841

12.96961835

8.00000000

9.24371366

10.48742731

11.75201949

12.95913481

8.00000000

9.24371366

10.48742731

11.77043433

12.91722531

8.00000000

9.24371366

10.48742731

11.62116314

12.93624214

8.00000000

9.24371366

10.48742731

11.77693604

12.90700627

8.00000000

9.24371366

10.48742731

11.87450950

12.85278103

8.00000000

9.24371366

10.48742731

11.67256708

13.14654114

8.00000000

9.24371366

6.21856829

10.48742731

11.88980897

13.16574422

1.00207573

5.24198422

13.64375775

13.57745674

13.63497735

3. 76156535

2.57034819

13.65445584

. /oxygen/Ni/110/iter_5/struct.xyz

50

Lattice $=" 7.46228194314714 \quad 0.0 \quad 0.0 \quad 0.0 \quad 10.5532603302505410 .0 \quad 0.0 \quad 0.020 .97485462876476 "$ Pro perties $=$ species:S:1:pos:R:3 pbc="T T T"
$\mathrm{Ni}$
0.00000000
0.00000000
8.00000000
$\mathrm{Ni}$
1. 24371366
1. 75887672
9.24371366 
$\mathrm{Ni}$

$\mathrm{Ni}$

$\mathrm{Ni}$

$\mathrm{Ni}$

$\mathrm{Ni}$

$\mathrm{Ni}$

$\mathrm{Ni}$

$\mathrm{Ni}$

$\mathrm{Ni}$

$\mathrm{Ni}$

$\mathrm{Ni}$

$\mathrm{Ni}$

$\mathrm{Ni}$

$\mathrm{Ni}$

$\mathrm{Ni}$

$\mathrm{Ni}$

$\mathrm{Ni}$

$\mathrm{Ni}$

$\mathrm{Ni}$

$\mathrm{Ni}$

$\mathrm{Ni}$

$\mathrm{Ni}$

$\mathrm{Ni}$

$\mathrm{Ni}$

$\mathrm{Ni}$

$\mathrm{Ni}$

$\mathrm{Ni}$

$\mathrm{Ni}$

$\mathrm{Ni}$

$\mathrm{Ni}$

$\mathrm{Ni}$

$\mathrm{Ni}$

$\mathrm{Ni}$

$\mathrm{Ni}$

$\mathrm{Ni}$

$\mathrm{Ni}$

$\mathrm{Ni}$

$\mathrm{Ni}$

$\mathrm{Ni}$

$\mathrm{Ni}$

$\mathrm{Ni}$

$\mathrm{Ni}$

$\mathrm{Ni}$

0

0

O

0

0
0.00000000

1.27792375

7. 44641179

0.00000000

1.24371366

0.00000000

1.21400105

0.00000000

1.24371366

0.00000000

0.02498523

2. 48742731

3. 73114097

2. 48742731

2. 55290708

2. 48742731

3. 73114097

3.75337396

2. 50634316

2.48742731

3.73114097

2. 48742731

3. 72212854

2. 46945982

4.97485463

6.21856829

4.97485463

6.19952487

4.95204624

4.97485463

6.21856829

4.97485463

6.21223958

4.93960537

4.97485463

6.21856829

4.97485463

6.20843593

4.91280454

3. 92061610

6.20320553

6.19339217

1.02135077
7.46173316

1.25594084

3.72909801

2.48742731

1. 29250909

Fri Apr 16 14:51:55 2021

182

0.00000000

1.85649228

10.50789315

3.51775344

5.27663017

3.51775344

5.28845233

3.73481006

7.03550689

8.79438361

7.03550689

8.73669806

6.83650727

0.00000000

1. 75887672

0.00000000

1.81557852

10.49257880

3.51775344

5.27663017

3.51775344

5.29602376

3.70086283

7.03550689

8.79438361

7.03550689

8.74099274

7.01153034

0.00000000

1.75887672

0.00000000

1.82277240

10.53580925

3.51775344

5.27663017

3.51775344

5. 28284308

3.68501286

7.03550689

8.79438361

7.03550689

8.70103158

6.81479224

1.00671198

5.20572581

2. 49634361

8.01982054

5.26870190

5.26870190
ruct. xyz

51

Lattice $=" 7.46228194314714 \quad 0.0 \quad 0.0 \quad 0.0 \quad 10.553260330250541$

perties =species: $:: 1:$ pos $: R: 3$ pbc $=" T$ T T"

$\mathrm{Ni} \quad 0.00000000 \quad 0.00000000$

$\mathrm{Ni} \quad 1.24371366 \quad 1.75887672$

$\mathrm{Ni} \quad 0.00000000$

1.81175497

1.24958931

7.42676527

0.00000000

10.52934981

3.51775344

5.27663017

3.51775344

5.27672532

3.67539893

7.03550689

8.79438361

7.03550689

8.72363276

6.75242813

0.00000000
10.48742731

11.91215850

12.91021984

8.00000000

10.48742731

11.65173276

13.14996962

8.00000000

9.24371366

11.75052794

13.14801736

9.24371366

10.48742731

11.77002443

12.91108205

8.00000000

9.24371366

10.48742731

11.64343172

12.97148275

8.00000000

9.24371366

10.48742731

11.77358444

12.90554182

9.24371366

10.48742731

11.85195594

12.84179259

8.00000000

9.24371366

10.48742731

11.65568818

13.10591780

8.00000000

9.24371366

10.48742731

11.89621442

13.15866335

13.64184836

13.57028237

13.62253408

13.65531081

13.57619110
9.24371366

10.48742731

8.00000000

8.00000000
0.00000000

1.21826326
7.36225344

0.00000000

1.24371366

1.26652644

0.13733871

2. 48742731
8.00000000
9.24371366
10.48742731
11.82266815
12.90959361
8.00000000
9.24371366
10.48742731
11.64054749
13.11968718
8.00000000
9.24371366
10.48742731
11.76771780
13.18808463
8.00000000
$\mathrm{Ni}$

$\mathrm{Ni}$

$10.00 .00 .020 .97485462876476 "$ Pro 
$\mathrm{Ni}$

$\mathrm{Ni}$

$\mathrm{Ni}$

$\mathrm{Ni}$

$\mathrm{Ni}$

$\mathrm{Ni}$

$\mathrm{Ni}$

$\mathrm{Ni}$

$\mathrm{Ni}$

$\mathrm{Ni}$

$\mathrm{Ni}$

$\mathrm{Ni}$

$\mathrm{Ni}$

$\mathrm{Ni}$

$\mathrm{Ni}$

$\mathrm{Ni}$

$\mathrm{Ni}$

$\mathrm{Ni}$

$\mathrm{Ni}$

$\mathrm{Ni}$

$\mathrm{Ni}$

$\mathrm{Ni}$

$\mathrm{Ni}$

$\mathrm{Ni}$

$\mathrm{Ni}$

$\mathrm{Ni}$

$\mathrm{Ni}$

$\mathrm{Ni}$

$\mathrm{Ni}$

0

O

o

0

0

0
3.73114097

2. 48742731

3.70983399

2. 48597796

2. 48742731

3.73114097

2. 48742731

3. 75265858

2.40521251

2. 48742731

3. 73114097

2. 48742731

3. 72863681

2. 56448799

4.97485463

6.21856829

4.97485463

6.17225690

5.01526798

4.97485463

6.21856829

4.97485463

6.21406188

4.82724422

4.97485463

6.21856829

4.97485463

6.26965199

5.06543973

1.19133962

3.92190891

6.02680989

6.40012891

1. 04183509

3.78380870
Fri Apr 16 14:51:55 2021

1.75887672

0.00000000

1.83093965

0.00331983

3.51775344

5.27663017

3.51775344

5.27717453

3.62941307

7.03550689

8.79438361

7.03550689

8.74193363

6.92299743

0.00000000

1.75887672

0.00000000

1.82984181

0.02706758

3.51775344

5.27663017

3.51775344

5.27769413

3.79984346

7.03550689

8.79438361

7.03550689

8.72061978

6.87514904

1.14699104

5.46926221

2.50961490

8.03921126

5.07999906

9.41664548
9.24371366

10.48742731

11.76556672

13.09065782

8.00000000

9.24371366

10.48742731

11.63880542

12.92940665

8.00000000

9.24371366

10.48742731

11.82998199

12.92980651

8.00000000

9.24371366

10.48742731

11.82775692

12.91057595

8.00000000

9.24371366

10.48742731

11.65521511

13.19091654

8.00000000

9.24371366

10.48742731

11.83794642

13.12570523

13.61135381

13.57466362

13.58376289

13.59372673

13.57300473

13.61289330

52

Lattice $=" 7.46228194314714 \quad 0.0 \quad 0.0 \quad 0.0 \quad 10.5532603302505410 .0 \quad 0.0 \quad 0.020 .97485462876476 "$ Pro perties=species:S:1:pos:R:3 pbc="T T T"

$\begin{array}{lllr}\mathrm{Ni} & 0.00000000 & 0.00000000 & 8.00000000 \\ \mathrm{Ni} & 1.24371366 & 1.75887672 & 9.24371366 \\ \mathrm{Ni} & 0.00000000 & 0.00000000 & 10.48742731 \\ \mathrm{Ni} & 1.24666074 & 1.80361676 & 11.78058930 \\ \mathrm{Ni} & 7.44549061 & 0.00591091 & 12.90710420 \\ \mathrm{Ni} & 0.00000000 & 3.51775344 & 8.00000000 \\ \mathrm{Ni} & 1.24371366 & 5.27663017 & 9.24371366 \\ \mathrm{Ni} & 0.00000000 & 3.51775344 & 10.48742731 \\ \mathrm{Ni} & 1.20132849 & 5.29239373 & 11.68156557 \\ \mathrm{Ni} & 7.29313642 & 3.59955633 & 13.13126795 \\ \mathrm{Ni} & 0.00000000 & 7.03550689 & 8.00000000 \\ \mathrm{Ni} & 1.24371366 & 8.79438361 & 9.24371366 \\ \mathrm{Ni} & 0.00000000 & 7.03550689 & 10.48742731 \\ \mathrm{Ni} & 1.26767763 & 8.74112438 & 11.83469237 \\ \mathrm{Ni} & 0.08645907 & 6.66000232 & 13.45106606 \\ \mathrm{Ni} & 2.48742731 & 0.00000000 & 8.00000000 \\ \mathrm{Ni} & 3.73114097 & 1.75887672 & 9.24371366 \\ \mathrm{Ni} & 2.48742731 & 0.00000000 & 10.48742731 \\ \mathrm{Ni} & 3.70900251 & 1.84242630 & 11.75117189 \\ \mathrm{Ni} & 2.53779069 & 0.08313700 & 13.10093532 \\ \mathrm{Ni} & 2.48742731 & 3.51775344 & 8.00000000 \\ \mathrm{Ni} & 3.73114097 & 5.27663017 & 9.24371366 \\ \mathrm{Ni} & 2.48742731 & 3.51775344 & 10.48742731 \\ \mathrm{Ni} & 3.73672392 & 5.26095325 & 11.62117911 \\ \mathrm{Ni} & 2.36021018 & 3.59892943 & 12.94298760 \\ \mathrm{Ni} & 2.48742731 & 7.03550689 & 8.00000000 \\ \mathrm{Ni} & 3.73114097 & 8.79438361 & 9.24371366 \\ \mathrm{Ni} & 2.48742731 & 7.03550689 & 10.48742731 \\ \mathrm{Ni} & 3.76409525 & 8.74250803 & 11.84229249\end{array}$


$\mathrm{Ni}$

$\mathrm{Ni}$

$\mathrm{Ni}$

$\mathrm{Ni}$

$\mathrm{Ni}$

$\mathrm{Ni}$

$\mathrm{Ni}$

$\mathrm{Ni}$

$\mathrm{Ni}$

$\mathrm{Ni}$

$\mathrm{Ni}$

$\mathrm{Ni}$

$\mathrm{Ni}$

$\mathrm{Ni}$

$\mathrm{Ni}$

$\mathrm{Ni}$

0

0

O

0

0

0

0
2.67608963

4.97485463

6.21856829

4.97485463

6.18601779

5.05002600

4.97485463

6.21856829

4.97485463

6.22707471

4.78967414

4.97485463

6.21856829

4.97485463

6.26289409

5.07351970

1.20666731

3. 90496104

5.95081750

6.31534359

1.01025595

3.83629048

1.39396577
Fri Apr 16 14:51:55 2021

6.84209470

0.00000000

1.75887672

0.00000000

1.82143735

0.10354717

3.51775344

5.27663017

3.51775344

5.26782329

3.72967201

7.03550689

8.79438361

7.03550689

8.74014754

6.78517963

1.20665744

5.40375884

2.39247724

7.98554081

4.91728399

9.49008890

7.97847752
13.09693589

8.00000000

9.24371366

10.48742731

11.76029397

12.93515662

8.00000000

9.24371366

10.48742731

11.66347315

13.15454018

8.00000000

9.24371366

10.48742731

11.82303787

13.13166742

13.59370322

13.56019681

13.54848930

13.53658264

13.62287394

13.58910897

13.55931595

. /oxygen/Ni/110/iter_8/struct.xyz 53

Lattice $=" 7.46228194314714 \quad 0.0 \quad 0.0 \quad 0.0 \quad 10.5532603302505410 .0 \quad 0.0 \quad 0.020 .97485462876476 "$ Pro perties=species:S:1:pos:R:3 pbc="T T T"

$\begin{array}{lllr}\mathrm{Ni} & 0.00000000 & 0.00000000 & 8.00000000 \\ \mathrm{Ni} & 1.24371366 & 1.75887672 & 9.24371366 \\ \mathrm{Ni} & 0.00000000 & 0.00000000 & 10.48742731 \\ \mathrm{Ni} & 1.20397795 & 1.87415307 & 11.82381753 \\ \mathrm{Ni} & 7.34278353 & 0.01134199 & 12.90123440 \\ \mathrm{Ni} & 0.00000000 & 3.51775344 & 8.00000000 \\ \mathrm{Ni} & 1.24371366 & 5.27663017 & 9.24371366 \\ \mathrm{Ni} & 0.00000000 & 3.51775344 & 10.48742731 \\ \mathrm{Ni} & 1.21353512 & 5.26892961 & 11.67352835 \\ \mathrm{Ni} & 7.26182800 & 3.68121656 & 13.15685344 \\ \mathrm{Ni} & 0.00000000 & 7.03550689 & 8.00000000 \\ \mathrm{Ni} & 1.24371366 & 8.79438361 & 9.24371366 \\ \mathrm{Ni} & 0.00000000 & 7.03550689 & 10.48742731 \\ \mathrm{Ni} & 1.24559483 & 8.76766460 & 11.80934595 \\ \mathrm{Ni} & 0.04854007 & 6.73374495 & 13.38134928 \\ \mathrm{Ni} & 2.48742731 & 0.00000000 & 8.00000000 \\ \mathrm{Ni} & 3.73114097 & 1.75887672 & 9.24371366 \\ \mathrm{Ni} & 2.48742731 & 0.00000000 & 10.48742731 \\ \mathrm{Ni} & 3.72251393 & 1.84355366 & 11.79952088 \\ \mathrm{Ni} & 2.45245090 & 0.04896247 & 13.13989215 \\ \mathrm{Ni} & 2.48742731 & 3.51775344 & 8.00000000 \\ \mathrm{Ni} & 3.73114097 & 5.27663017 & 9.24371366 \\ \mathrm{Ni} & 2.48742731 & 3.51775344 & 10.48742731 \\ \mathrm{Ni} & 3.76518938 & 5.21389340 & 11.68016179 \\ \mathrm{Ni} & 2.44152786 & 3.74370766 & 13.18914084 \\ \mathrm{Ni} & 2.48742731 & 7.03550689 & 8.00000000 \\ \mathrm{Ni} & 3.73114097 & 8.79438361 & 9.24371366 \\ \mathrm{Ni} & 2.48742731 & 7.03550689 & 10.48742731 \\ \mathrm{Ni} & 3.75293224 & 8.76210358 & 11.81279852 \\ \mathrm{Ni} & 2.61528625 & 6.93458401 & 13.05342503 \\ \mathrm{Ni} & 4.97485463 & 0.00000000 & 8.00000000 \\ \mathrm{Ni} & 6.21856829 & 1.75887672 & 9.24371366 \\ \mathrm{Ni} & 4.97485463 & 0.00000000 & 10.48742731 \\ \mathrm{Ni} & 6.19881134 & 1.82033115 & 11.68231022 \\ \mathrm{Ni} & 5.03228637 & 0.08906866 & 12.92090720 \\ \mathrm{Ni} & 4.97485463 & 3.51775344 & 8.00000000 \\ \mathrm{Ni} & 6.21856829 & 5.27663017 & 9.24371366 \\ \mathrm{Ni} & 4.97485463 & 3.51775344 & 10.48742731 \\ \mathrm{Ni} & 6.21415087 & 5.25211773 & 11.66038381 \\ \mathrm{Ni} & 5.23633691 & 2.43017098 & 13.81228261 \\ \mathrm{Ni} & 4.97485463 & 7.03550689 & 8.00000000\end{array}$


$\mathrm{Ni}$

$\mathrm{Ni}$

$\mathrm{Ni}$

$\mathrm{Ni}$

0

O

0

O

0

0

0

0

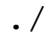

54

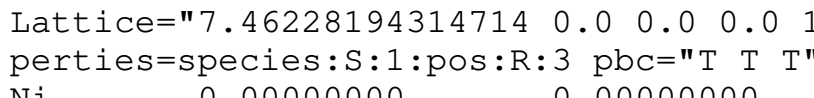

$\begin{array}{lll}\mathrm{Ni} & 0.00000000 & 0.00000000\end{array}$

$\mathrm{Ni} \quad 1.24371366 \quad 1.75887672$

$\begin{array}{lll}\mathrm{Ni} & 0.00000000 & 0.00000000\end{array}$

$\mathrm{Ni} \quad 1.19286394$

$\mathrm{Ni} \quad 0.00611087$

$\mathrm{Ni} \quad 0.00000000$

$\mathrm{Ni} \quad 1.24371366$

$\mathrm{Ni} \quad 0.00000000$

$\mathrm{Ni} \quad 1.23641291$

$\mathrm{Ni} \quad 7.35992965$

$\mathrm{Ni} \quad 0.00000000$

$\mathrm{Ni} \quad 1.24371366$

$\mathrm{Ni} \quad 0.00000000$

$\mathrm{Ni} \quad 1.25803775$

$\mathrm{Ni} \quad 0.07041546$

$\mathrm{Ni} \quad 2.48742731$

$\mathrm{Ni} \quad 3.73114097$

$\mathrm{Ni} \quad 2.48742731$

$\mathrm{Ni} \quad 3.69439397$

$\mathrm{Ni} \quad 2.53325454$

$\mathrm{Ni} \quad 2.48742731$

$\mathrm{Ni} \quad 3.73114097$

$\mathrm{Ni} \quad 2.48742731$

$\mathrm{Ni} \quad 3.75999058$

$\mathrm{Ni} \quad 2.33286371$

$\mathrm{Ni} \quad 2.48742731$

$\mathrm{Ni} \quad 3.73114097$

$\mathrm{Ni} \quad 2.48742731$

$\mathrm{Ni} \quad 3.75094118$

$\mathrm{Ni} \quad 2.58636126$

$\mathrm{Ni} \quad 4.97485463$

$\mathrm{Ni} \quad 6.21856829$

$\mathrm{Ni} \quad 4.97485463$

$\mathrm{Ni} \quad 6.24388988$

$\mathrm{Ni} \quad 4.94672368$

$\mathrm{Ni} \quad 4.97485463$

$\mathrm{Ni} \quad 6.21856829$

$\mathrm{Ni} \quad 4.97485463$

$\mathrm{Ni} \quad 6.20733633$

$\mathrm{Ni} \quad 4.98153450$

$\mathrm{Ni} \quad 4.97485463$

$\mathrm{Ni} \quad 6.21856829$

$\mathrm{Ni} \quad 4.97485463$

$\mathrm{Ni} \quad 6.25204380$

$\mathrm{Ni} \quad 5.09155857$

O $\quad 1.19847714$

o $\quad 3.83060544$

$0 \quad 6.16800417$

o $\quad 6.35567846$

$0 \quad 1.10047772$

$0 \quad 3.76931446$

o $\quad 1.29077381$

3. 74449798
Fri Apr 16 14:51:55 2021

185

8.79438361

7.03550689

8.73617036

6.78393521

1.14408158

5.55633151

3.10933106

8.01746011

5.00744855

9.48297064

8.06785806

2.32978709

1.79670057

10.11715803

3.51775344

5.27663017

3.51775344

5.31611893

7.03550689

8.79438361

7.03550689

8.76383823

6.84029526

0.00000000

1.75887672

0.00000000

1.78928378

10.47510768

3.51775344

5.27663017

3.51775344

5.26166711

3. 71038898

7.03550689

8.79438361

7.03550689

8.74700438

7.06320193

0.00000000

1. 75887672

0.00000000

1. 76902025

10.48128170

3.51775344

5. 27663017

3. 51775344

5.29983532

3. 47371994

7.03550689

8.79438361

7.03550689

8.76884330

6.99980409

0.95369479

5.81057293

3.79365862

8.23868428

5.13294149

9.24085673

8.27422315
9.24371366

10.48742731

11.79916927

13.12520377

13.55902291

13.51766695

14.65487646

13.55659812

13.55451549

13.54927153

13.63213472
13.52416737 8.00000000

9.24371366

10.48742731

11.85706827

13.49851561

8.00000000

9.24371366

10.48742731

11.66374818

13.10120643

8.00000000

9.24371366

10.48742731

11.72339108

13.34147343

9.24371366

10.48742731

11.79144076

13.04736998

8.00000000

9.24371366

10.48742731

11.75596374

13.13869933

8.00000000

9.24371366

10.48742731

11.70530707

13.12329268

8.00000000

9.24371366

10.48742731

11.83643805

12.97223423

8.00000000

9.24371366

10.48742731

11.66966978

13.15224628

8.00000000

9.24371366

10.48742731

11.70297528

13.09831802

13.53376311

13.56724145

14.38265648

13.53850022

13.57078927

13.53095755

13.57715759
$10.5532603302505410 .00 .00 .020 .97485462876476 "$ Pro

8.00000000 
O $\quad 3.53780935$ O $\quad 6.25390254$
Fri Apr $16 \quad 14: 51: 552021$
2.45209769
13.55264467
0.94096902
13.53416937

. /oxygen/Ni/110/MR-p2x1/struct.xyz 12

Lattice $=" 4.97485462876476 \quad 0.0 \quad 0.0 \quad 0.0 \quad 3.517753443416847 \quad 0.0 \quad 0.0 \quad 0.0 \quad 20.97485462876476 "$ Prop erties=species:S:1:pos:R:3 pbc="T T T"

$\begin{array}{lllr}\mathrm{Ni} & 0.00000000 & 0.00000000 & 8.00000000 \\ \mathrm{Ni} & 1.24371366 & 1.75887672 & 9.24371366 \\ \mathrm{Ni} & 0.00000000 & 0.00000000 & 10.48742731 \\ \mathrm{Ni} & 1.24371366 & 1.75887672 & 11.69326565 \\ \mathrm{Ni} & -0.02442440 & 0.00000000 & 12.95039323 \\ \mathrm{Ni} & 2.48742731 & 0.00000000 & 8.00000000 \\ \mathrm{Ni} & 3.73114097 & 1.75887672 & 9.24371366 \\ \mathrm{Ni} & 2.48742731 & 0.00000000 & 10.48742731 \\ \mathrm{Ni} & 3.73114097 & 1.75887672 & 11.78249372 \\ \mathrm{Ni} & 2.51185172 & 0.00000000 & 12.95039323 \\ \mathrm{Ni} & 1.24371366 & 1.75887672 & 14.21630105 \\ \mathrm{O} & 1.24371366 & 0.00000000 & 14.51439016\end{array}$

. /oxygen/Ni/110/MR-p3x1/struct. xyz

19

Lattice $=" 7.46228194314714 \quad 0.0 \quad 0.0 \quad 0.0 \quad 3.517753443416847 \quad 0.0 \quad 0.0 \quad 0.0 \quad 20.97485462876476 "$ Prop erties $=$ species: $\mathrm{S}: 1:$ pos: $\mathrm{R}: 3 \mathrm{pbc}=" \mathrm{~T} \mathrm{~T} \mathrm{~T} "$

$0.00000000 \quad 0.00000000$

$\mathrm{Ni} \quad 1.24371366 \quad 1.75887672$

$\begin{array}{lll}\mathrm{Ni} & 0.00000000 & 0.00000000\end{array}$

$\begin{array}{lll}\mathrm{Ni} & 1.24749326 & 1.75887672\end{array}$

$\mathrm{Ni} \quad-0.03333877 \quad 0.00000000$

$\begin{array}{lll}\mathrm{Ni} & 2.48742731 & 0.00000000\end{array}$

$\mathrm{Ni} \quad 3.73114097 \quad 1.75887672$

$\mathrm{Ni} \quad 2.48742731 \quad 0.00000000$

$\begin{array}{lll}\mathrm{Ni} & 3.72736137 & 1.75887672\end{array}$

$\mathrm{Ni} \quad 2.48742731 \quad 0.00000000$

$\mathrm{Ni} \quad 4.97485463 \quad 0.00000000$

$\begin{array}{lll}\mathrm{Ni} & 6.21856829 & 1.75887672\end{array}$

$\mathrm{Ni} \quad 4.97485463 \quad 0.00000000$

$\mathrm{Ni} \quad 6.21856829 \quad 1.75887672$

$\mathrm{Ni} \quad 5.00819340 \quad 0.00000000$

$\mathrm{Ni} \quad 1.20701740 \quad 1.75887672$

$\mathrm{Ni} \quad 3.76783723 \quad 1.75887672$

$\begin{array}{lll}0 & 0.87162677 & 0.00000000\end{array}$

o 4.10322785

0.00000000

8.00000000

9.24371366

10.48742731

11.72504143

12.94987380

8.00000000

9.24371366

10.48742731

11.72504143

12.98453093

8.00000000

9.24371366

10.48742731

11.76289465

12.94987380

14.32511375

14.32511375

14.60991171

14.60991171

. /oxygen/Ni/110/p2x1/struct. xyz

11

Lattice $=" 4.97485462876476 \quad 0.0 \quad 0.0 \quad 0.0 \quad 3.5177534434168470 .0 \quad 0.0 \quad 0.020 .97485462876476 "$ Prop erties=species:S:1:pos:R:3 pbc="T T T"

$\mathrm{N}$

$\mathrm{Ni}$

0.00000000

0.00000000

1.24371366

1.75887672

0.00000000

0.00000000

1.24512155

1.75887672

0.00000000

0.00000000

2. 48742731

0.00000000

3. 73114097

1. 75887672

2.48742731

0.00000000

3.72973308

1. 75887672

2. 48742731

0.00000000

0.00000000

1.75887672

8.00000000
9.24371366
10.48742731
11.76889526
13.19135657
8.00000000
9.24371366
10.48742731
11.76889526
12.86402041
13.34213596

. /oxygen/Ni/110/p3x1/struct. xyz

17

Lattice $=" 7.46228194314714 \quad 0.0 \quad 0.0 \quad 0.0 \quad 3.517753443416847 \quad 0.0 \quad 0.0 \quad 0.0 \quad 20.97485462876476 "$ Prop erties=species:S:1:pos:R:3 pbc="T T T"

$\mathrm{Ni}$

$\mathrm{Ni}$

$\mathrm{Ni}$

$\mathrm{Ni}$

$\mathrm{Ni}$

$\mathrm{Ni}$

$\mathrm{Ni}$

$\mathrm{Ni}$
0.00000000

1. 24371366

0.00000000

1. 24371366

$-0.10696038$

2.48742731

3. 73114097

2. 48742731
0.00000000

1. 75887672

0.00000000

1. 75887672

0.00000000

0.00000000

1. 75887672

0.00000000
8.00000000

9. 24371366

10.48742731

11.84074637

13.20879359

8.00000000

9.24371366

10.48742731 
$\mathrm{Ni}$

$\mathrm{Ni}$

$\mathrm{Ni}$

$\mathrm{Ni}$

$\mathrm{Ni}$

$\mathrm{Ni}$

$\mathrm{Ni}$

0

0
1.75887672

0.00000000

0.00000000

1.75887672

0.00000000

1.75887672

0.00000000

1.75887672

1.75887672
11.74164466

13.20879359

8.00000000

9.24371366

10.48742731

11.74164466

12.84689939

13.41177254

13.41177254

37

Lattice $=" 7.4622819431471425 \quad 0.0 \quad 0.0 \quad 3.731140971573571 \quad 6.462525732967329 \quad 0.0 \quad 0.0 \quad 0.0 \quad 22.0929$

2769249835 " Properties=species:S:1:pos:R:3 pbc="T T T"

$\mathrm{Ni}$

0.00000000

0.00000000

1. 24371366

0.71805841

2. 48246571

$\mathrm{Ni}$

$-0.03301252$

1.24371366

1. 43325225

Ni

$-0.01905979$

2. 48742731

2.15417524

3. 72676092

2.87223366

1.24371366

3. 59282090

2. 48742731

2.19229482

3.73114097

4.30835049

5.02640890

4.97485463

5.75019649

2. 48487974

4. 30982133

2. 48742731

0.00000000

3.73114097

0.71805841

4.97485463

2. 52043984

3. 73114097

1.43105918

$-0.01905979$

2.15417524

4.97485463

2.87223366

6.22294834

3. 59282090

3. 76285399

2.17248477

4.30835049

5.02640890

8.00000000

10.03097590

12.06453057

14.15792754

8.00000000

10.03097590

12.04622404

14.15792754

8.00000000

10.03097590

12.06453057

14.05250741

8.00000000

10.03097590

12.04622404

14.15792754

8.00000000

10.03097590

12.04622404

14.04258458

8.00000000

6.21856829

5.74856459

7. 45518526

4.97485463

4.27173144

4.97485463

0.00000000

6.21856829

0.71805841

7. 46724355

1. 43325225

4.97485463

$-0.00294168$

6.21856829

2.15417524

2. 87223366

7. 46228194

3. 58209754

2. 17248477

4.30835049

5.02640890

7. 46228194

5.74856459

4.30982133

10.03097590

12.06956375

14.04258458

8.00000000

10.03097590

12.06453057

14.05250741

8.00000000

10.03097590

12.06956375

14.04258458

8.00000000

10.03097590

12.06956375

14.05250741

7.46482952

0.71805841

15.25750793

/ oxygen/Ni/111/iter_2/struct.xyz

38

Lattice $=" 7.4622819431471425 \quad 0.0 \quad 0.0 \quad 3.731140971573571 \quad 6.462525732967329 \quad 0.0 \quad 0.0 \quad 0.0 \quad 22.0929$ $2769249835 "$ Properties=species:S:1:pos:R:3 pbc="T T T"

$\mathrm{Ni}$

$\mathrm{Ni}$

$\mathrm{Ni}$

0.00000000

0.00000000

8.00000000

1. 24371366

0.71805841

2. 49203979

1.43877984

$-0.02951836$

1. 24371366

$-0.01704243$

2. 48742731

2.15417524

2. 87223366

3. 73337303

3.58900339

1.24371366

2.18826011

2. 48742731

3. 73114097

4.30835049

5.02640890

4.97485463

5.02640890

4. 32636144

0.00000000

10.03097590

12.06759450

14.12589291

8.00000000

10.03097590

12.04854549

14.12589291

8.00000000

10.03097590

12.06759450

14.02208006

8.00000000

2. 48742731

0.71805841

10.03097590 
$\mathrm{Ni}$

$\mathrm{Ni}$

$\mathrm{Ni}$

$\mathrm{Ni}$

$\mathrm{Ni}$

$\mathrm{Ni}$

$\mathrm{Ni}$

$\mathrm{Ni}$

$\mathrm{Ni}$

$\mathrm{Ni}$

$\mathrm{Ni}$

$\mathrm{Ni}$

$\mathrm{Ni}$

$\mathrm{Ni}$

$\mathrm{Ni}$

$\mathrm{Ni}$

$\mathrm{Ni}$

$\mathrm{Ni}$

$\mathrm{Ni}$

$\mathrm{Ni}$

$\mathrm{Ni}$

$\mathrm{Ni}$

o

0

.1

4.97485463

2.51694568

3.73114097

4.97485463

6.21633623

3.72812385

4.97485463

6.21856829

7.45052485

4.97485463

4.97485463

6.21856829

7.45766947

4.97485463

6.21856829

7.46228194

8.70599560

6.22158540

7.46228194

8.70599560

9.96146635

7.49347783

1.24371366

4.97485463
Fri Apr 16 14:51:55 2021

188

1.43869419

$-0.01704243$

2. 15417524

2.87223366

3.58900339

2.15243331

4. 30835049

5.02640890

5.75125528

4.31183436

0.00000000

0.71805841

1.43877984

$-0.03602191$

2.15417524

2.87223366

3. 57671616

2.15243331

4.30835049

5.02640890

5.75125528

4.32636144

0.71805841

2.87223366
12.04854549

14.12589291

8.00000000

10.03097590

12.04854549

14.12890993

8.00000000

10.03097590

12.04967205

14.12890993

8.00000000

10.03097590

12.06759450

14.02208006

8.00000000

10.03097590

12.04967205

14.12890993

8.00000000

10.03097590

12.04967205

14.02208006

15.23128201

15.26004322

39

Lattice="7.4622819431471425 0.0 0.0 3.731140971573571
$2769249835 "$ Properties=species:S:1:pos:R:3 pbc="T T T"

$\begin{array}{llll}\mathrm{Ni} & 0.00000000 & 0.00000000 & 8.00000000\end{array}$

$\mathrm{Ni} \quad 1.24371366 \quad 0.71805841$

$\mathrm{Ni} \quad 2.49327621 \quad 1.43949369$

$\mathrm{Ni} \quad-0.00356686$

$\mathrm{Ni} \quad 1.24371366$

$-0.00205933$

$1.24371366 \quad 2.15417524$

$2.48742731 \quad 2.87223366$

$3.72529208 \quad 3.59366893$

$1.24371366 \quad 2.15829390$

$\begin{array}{ll}2.48742731 & 4.30835049\end{array}$

$3.73114097 \quad 5.02640890$

$4.97485463 \quad 5.73771360$

$2.49099418 \quad 4.30629116$

$\begin{array}{ll}2.48742731 & 0.00000000\end{array}$

$\begin{array}{ll}3.73114097 & 0.71805841\end{array}$

$\begin{array}{ll}4.97485463 & 1.42936311\end{array}$

$2.49099418 \quad-0.00205933$

$\begin{array}{ll}3.73114097 & 2.15417524\end{array}$

$\begin{array}{ll}4.97485463 & 2.87223366\end{array}$

$\begin{array}{ll}6.22441718 & 3.59366893\end{array}$

$3.72757411 \quad 2.15211591$

$\begin{array}{ll}4.97485463 & 4.30835049\end{array}$

$\begin{array}{ll}6.21856829 & 5.02640890\end{array}$

$7.45643305 \quad 5.74784418$

$4.97485463 \quad 4.31246915$

$\begin{array}{ll}4.97485463 & 0.00000000\end{array}$

$\begin{array}{ll}6.21856829 & 0.71805841\end{array}$

$7.45643305 \quad 1.43949369$

$\begin{array}{ll}4.97485463 & 0.00411866\end{array}$

$6.21856829 \quad 2.15417524$

$\begin{array}{ll}7.46228194 & 2.87223366\end{array}$

$\begin{array}{ll}8.70599560 & 3.58353835\end{array}$

$\begin{array}{ll}6.22213515 & 2.15211591\end{array}$

$\begin{array}{ll}7.46228194 & 4.30835049\end{array}$

$8.70599560 \quad 5.02640890$

$9.95555815 \quad 5.74784418$

$\begin{array}{ll}7.45871508 & 4.30629116\end{array}$

10.03097590

12.05761836

14.10475564

8.00000000

10.03097590

12.05761836

14.10475564

8.00000000

10.03097590

12.05761836

14.10475564

8.00000000

10.03097590

12.05761836

14.10475564

8.00000000

10.03097590

12.05761836

14.10475564

8.00000000

10.03097590

12.05761836

14.10475564

8.00000000

10.03097590

12.05761836

14.10475564

8.00000000

10.03097590

12.05761836

14.10475564

8.00000000

10.03097590

12.05761836

14.10475564

15.23559972

15.23559972

$4.97485463 \quad 2.87223366$

$8.70599560 \quad 5.02640890$

15.23559972

. /oxygen/Ni/111/iter_4/struct.xyz 
40

Lattice $=" 7.4622819431471425 \quad 0.0 \quad 0.0 \quad 3.731140971573571 \quad 6.462525732967329 \quad 0.0 \quad 0.0 \quad 0.022 .0929$ 2769249835 " Properties=species:S:1:pos:R:3 pbc="T T T"

$\mathrm{Ni}$

$\mathrm{Ni}$

$\mathrm{Ni}$

$\mathrm{Ni}$

$\mathrm{Ni}$

$\mathrm{Ni}$

$\mathrm{Ni}$

$\mathrm{Ni}$

$\mathrm{Ni}$

$\mathrm{Ni}$

$\mathrm{Ni}$

$\mathrm{Ni}$

$\mathrm{Ni}$

$\mathrm{Ni}$

$\mathrm{Ni}$

$\mathrm{Ni}$

$\mathrm{Ni}$

$\mathrm{Ni}$

$\mathrm{Ni}$

$\mathrm{Ni}$

$\mathrm{Ni}$

$\mathrm{Ni}$

$\mathrm{Ni}$

$\mathrm{Ni}$

$\mathrm{Ni}$

$\mathrm{Ni}$

$\mathrm{Ni}$

$\mathrm{Ni}$

$\mathrm{Ni}$

$\mathrm{Ni}$

$\mathrm{Ni}$

$\mathrm{Ni}$

$\mathrm{Ni}$

$\mathrm{Ni}$

$\mathrm{Ni}$

$\mathrm{Ni}$

0

O

O

/ $/ 1$

0.00000000

1. 24371366

2.47830065

$-0.01607525$

1. 24371366

2. 48742731

3. 72157173

1.29077320

2. 48742731

3.73114097

4.97389131

2.52084729

2. 48742731

3. 73114097

4.98365724

2. 52173200

3. 73114097

4.97485463

6.23049556

3. 68636379

4.97485463

6.21856829

7. 46260600

4.95750991

4.97485463

6.21856829

7. 45232719

4.98532713

6.21856829

7. 46228194

8.70402308

6.17596117

7. 46228194

8.70599560

9.96024182

7. 45782952

1.29637584

4.78669451

8.84149354

7.46228194

41

Lattice $=" 7.4622819431471425 \quad 0.0 \quad 0.0 \quad 3.731140971573571 \quad 6.462525732967329 \quad 0.0 \quad 0.0 \quad 0.0 \quad 22.0929$ 2769249835 " Properties=species:S:1:pos:R:3 pbc="T T T"

$\mathrm{Ni} \quad 0.00000000$

$\mathrm{Ni} \quad 1.24371366$

$\mathrm{Ni} \quad 2.49564581$

$\mathrm{Ni} \quad 0.00727212$

$\mathrm{Ni} \quad 1.24371366$

$\mathrm{Ni} \quad 2.48742731$

$\mathrm{Ni} \quad 3.71960687$

$\mathrm{Ni} \quad 1.25818717$

$\mathrm{Ni} \quad 2.48742731$

$\mathrm{Ni} \quad 3.73114097$

$\mathrm{Ni} \quad 4.97809973$

$\mathrm{Ni} \quad 2.49785555$

$\mathrm{Ni} \quad 2.48742731$

$\mathrm{Ni} \quad 3.73114097$

$\mathrm{Ni} \quad 4.96924906$

$\mathrm{Ni} \quad 2.49030741$

$\mathrm{Ni} \quad 3.73114097$

$\mathrm{Ni} \quad 4.97485463$

$\mathrm{Ni} \quad 6.23560795$

$\mathrm{Ni}$

$\mathrm{Ni}$
0.0000000

0.71805841

1. 44101192

$-0.02930901$

2.15417524

2.87223366

3.59692922

2.13214662

4.30835049

5.02640890

5.73286153

4.30908342

0.00000000

0.71805841

1. 44157321

$-0.03189842$

2.15417524

2.87223366

3.59490062

2.14041573

4.30835049

5.02640890

5.73411584

4.33692657

0.00000000

0.71805841

1. 44414188

0.04565794

2.15417524

2. 87223366

3.57765848

2.12443479

4.30835049

5.02640890

5.74943595

4.36011956

0.53119435

2.92005890

5.16544773

2.87223366
8.00000000

10.03097590

12.05435160

14.09090533

8.00000000

10.03097590

14.26433792

8.00000000

10.03097590

12.03731569

14.09090533

8.00000000

10.03097590

12.05435160

14.03551738

8.00000000

10.03097590

12.08103449

14.03551738

8.00000000

10.03097590

12.05435160

14.09090533

8.00000000

12.08103449

14.03551738

8.00000000

10.03097590

12.08103449

14.26433792

8.00000000

10.03097590

12.03731569

14.26433792

15.20929315

15.20929315

15.20929315

15.29956456
12.03731569

10.03097590
3. 71564217

4.97485463
0.00000000

0.71805841

1.45945339

$-0.02555700$

2. 15417524

2.87223366

3. 59152296

2.11126125

4.30835049

5.02640890

5.74365558

4. 32097844

0.00000000

0.71805841

$-0.03132481$

2. 15417524

2.87223366

3.58943453

2.18401888

4.30835049
1. 43963128
8.00000000

10.03097590

12.08782204

14.02820581

8.00000000

10.03097590

12.05038473

14.26774464

8.00000000

10.03097590

12.00771408

14.18216149

8.00000000

10.03097590

14.02134445

8.00000000

10.03097590

12.06334684

14.26628865
12.05982648

8.00000000 
$\mathrm{Ni}$

$\mathrm{Ni}$

$\mathrm{Ni}$

$\mathrm{Ni}$

$\mathrm{Ni}$

$\mathrm{Ni}$

$\mathrm{Ni}$

$\mathrm{Ni}$

$\mathrm{Ni}$

$\mathrm{Ni}$

$\mathrm{Ni}$

$\mathrm{Ni}$

$\mathrm{Ni}$

$\mathrm{Ni}$

$\mathrm{Ni}$

0

O

0

0

0
6.21856829

7.46475723

4.99184508

4.97485463

6.21856829

7.46327740

4.95102526

6.21856829

7.46228194

8.71318542

6.19260458

7.46228194

8.70599560

9.94021877

7.44508253

1.28764785

3.80907236

8.70828870

7.39098717

4.98724437
Fri Apr 16 14:51:55 2021

190

5.02640890

5.73591531

4.32620165

0.00000000

0.71805841

1.44476990

0.02053365

2.15417524

2.87223366

3.57840068

2.14154090

4.30835049

5.02640890

5.74051099

4.32973657

0.52858640

3.72847875

5.09746922

2.89030228

1.36235027
10.03097590

12.05116224

14.02847299

8.00000000

10.03097590

12.05150382

14.09953765

8.00000000

10.03097590

12.07916923

14.15839658

8.00000000

10.03097590

12.04865877

14.17547295

15.22932611

15.27713522

15.25597343

15.26802453

15.29216684

42

Lattice $=" 7.4622819431471425 \quad 0.0 \quad 0.0 \quad 3.731140971573571 \quad 6.462525732967329 \quad 0.0 \quad 0.0 \quad 0.0 \quad 22.0929$

$2769249835 "$ Properties=species:S:1:pos:R:3 pbc="T T T"

$$
2769
$$

$\mathrm{N}$

0.00000000

0.00000000

1.24371366

0.71805841

8.00000000

2. 49552589

1. 43736384

$-0.02176518$

1. 24371366

$-0.01776895$

2.15417524

2. 48742731

2. 87223366

3.73363220

3. 58467241

1. 25260554

2.15250542

2. 48742731

4.30835049

5.02640890

.73114097

5.73606298

4.29791049

2. 51712737

0.00000000

.48742731

0.71805841

4.97230516

1. 43517469

0.00052017

2.49412844

2.15417524

3. 73114097

2. 87223366

3.59170277

6.22724178

2.14875729

3. 72953194

4.30835049

5.02640890

6.21856829

5.73734288

7. 45706620

4.30378009

0.00000000

4.97485463

0.71805841

6.21856829

1. 44098585

0.02027855

4.98440334

2.15417524

2.87223366

7. 46228194

3.58515345

8.70125760

2.12927397

4.30835049

7.46228194

5.02640890

8.70599560

5.73464071

4.32212125

0.67395341

5.04235450

5.11771529

2.80459649

1. 36355401

3. 62156082

10.03097590

12.09649157

13.99612695

8.00000000

10.03097590

12.05525369

14.15238918

8.00000000

10.03097590

12.02880833

14.09011399

8.00000000

10.03097590

12.05713512

14.14959788

8.00000000

10.03097590

12.08111617

14.21157187

8.00000000

10.03097590

12.06465469

14.16532346

8.00000000

10.03097590

12.03031338

14.09054646

8.00000000

10.03097590

12.06582135

14.16844738

8.00000000

10.03097590

12.03024902

14.28671546

15.18777336

15.27776293

15.25498426

15.27873091

15.28716089

15.28654838

/oxygen/Ni/111/iter_7/struct. xyz

43 
Lattice $=" 7.4622819431471425 \quad 0.0 \quad 0.0 \quad 3.7311409715735716 .4625257329673290 .0 \quad 0.0 \quad 0.022 .0929$ $2769249835 "$ Properties=species:S:1:pos:R:3 pbc="T T T"

$\begin{array}{lrrr}\mathrm{Ni} & 0.00000000 & 0.00000000 & 8.00000000 \\ \mathrm{Ni} & 1.24371366 & 0.71805841 & 10.03097590 \\ \mathrm{Ni} & 2.48277432 & 1.44158972 & 12.04360292 \\ \mathrm{Ni} & -0.07712449 & 0.04903954 & 14.11046872 \\ \mathrm{Ni} & 1.24371366 & 2.15417524 & 8.00000000 \\ \mathrm{Ni} & 2.48742731 & 2.87223366 & 10.03097590 \\ \mathrm{Ni} & 3.73388802 & 3.59418083 & 12.02964641 \\ \mathrm{Ni} & 1.26122400 & 2.11633590 & 14.10707578 \\ \mathrm{Ni} & 2.48742731 & 4.30835049 & 8.00000000 \\ \mathrm{Ni} & 3.73114097 & 5.02640890 & 10.03097590 \\ \mathrm{Ni} & 4.96171820 & 5.75273200 & 12.05893251 \\ \mathrm{Ni} & 2.49561283 & 4.29961323 & 14.06008512 \\ \mathrm{Ni} & 2.48742731 & 0.00000000 & 8.00000000 \\ \mathrm{Ni} & 3.73114097 & 0.71805841 & 10.03097590 \\ \mathrm{Ni} & 4.97951802 & 1.43372407 & 12.04838943 \\ \mathrm{Ni} & 2.51531161 & -0.06121604 & 14.09478670 \\ \mathrm{Ni} & 3.73114097 & 2.15417524 & 8.00000000 \\ \mathrm{Ni} & 4.97485463 & 2.87223366 & 10.03097590 \\ \mathrm{Ni} & 6.21866007 & 3.59152876 & 12.09957758 \\ \mathrm{Ni} & 3.78616378 & 2.17467531 & 14.06342808 \\ \mathrm{Ni} & 4.97485463 & 4.30835049 & 8.00000000 \\ \mathrm{Ni} & 6.21856829 & 5.02640890 & 10.03097590 \\ \mathrm{Ni} & 7.46692728 & 5.73074456 & 12.08223301 \\ \mathrm{Ni} & 4.92769861 & 4.33343450 & 14.09363803 \\ \mathrm{Ni} & 4.97485463 & 0.00000000 & 8.00000000 \\ \mathrm{Ni} & 6.21856829 & 0.71805841 & 10.03097590 \\ \mathrm{Ni} & 7.44934985 & 1.45000552 & 12.04195069 \\ \mathrm{Ni} & 4.98668982 & 0.06088116 & 14.19535239 \\ \mathrm{Ni} & 6.21856829 & 2.15417524 & 8.00000000 \\ \mathrm{Ni} & 7.46228194 & 2.87223366 & 10.03097590 \\ \mathrm{Ni} & 8.68170389 & 3.58819636 & 12.06733193 \\ \mathrm{Ni} & 6.03841813 & 2.54527669 & 15.45407081 \\ \mathrm{Ni} & 7.46228194 & 4.30835049 & 8.00000000 \\ \mathrm{Ni} & 8.70599560 & 5.02640890 & 10.03097590 \\ \mathrm{Ni} & 9.93700912 & 5.75930315 & 12.04660462 \\ \mathrm{Ni} & 7.44610722 & 4.28817064 & 14.32688395 \\ \mathrm{O} & 1.24856259 & 0.60427953 & 15.15269869 \\ \mathrm{O} & 6.00747090 & 4.41285191 & 15.55100677 \\ \mathrm{O} & 8.84444794 & 5.01791944 & 15.24212482 \\ \mathrm{O} & 7.81209243 & 2.84949041 & 15.45482338 \\ \mathrm{O} & 6.22562703 & 0.78813853 & 15.33662907 \\ \mathrm{O} & 3.69203624 & 3.56173509 & 15.23249188 \\ \mathrm{O} & 3.67923740 & 0.79653536 & 15.21871106 \\ \mathrm{~N} & 1.61 .111169 & & \end{array}$

. /oxygen/Ni/111/iter_8/struct.xyz 44

Lattice $=" 7.4622819431471425 \quad 0.0 \quad 0.0 \quad 3.731140971573571 \quad 6.462525732967329 \quad 0.0 \quad 0.0 \quad 0.022 .0929$ 2769249835 " Properties=species:S:1:pos:R:3 pbc="T T T"

$\mathrm{Ni} \quad 1.24371366 \quad 0.71805841 \quad 10.03097590$

$\mathrm{Ni} \quad 2.48466805 \quad 1.44541997$

$\mathrm{Ni} \quad 0.00297911 \quad-0.04765014$

$\mathrm{Ni} \quad 1.24371366 \quad 2.15417524$

$\mathrm{Ni} \quad 2.48742731 \quad 2.87223366$

$\mathrm{Ni} \quad 3.73390584 \quad 3.59333657$

$\mathrm{Ni} \quad 1.24311931 \quad 2.16516129$

$\mathrm{Ni} \quad 2.48742731 \quad 4.30835049$

$\mathrm{Ni} \quad 3.73114097 \quad 5.02640890$

$\mathrm{Ni} \quad 4.97813805 \quad 5.74909924$

$\mathrm{Ni} \quad 2.53810296 \quad 4.34057010$

$\mathrm{Ni} \quad 2.48742731 \quad 0.00000000$

$\mathrm{Ni} \quad 3.73114097 \quad 0.71805841$

$\mathrm{Ni} \quad 4.98065581 \quad 1.44403772$

$\mathrm{Ni} \quad 2.47839197 \quad-0.00064033$

$\mathrm{Ni} \quad 3.73114097 \quad 2.15417524$

$\mathrm{Ni} \quad 4.97485463 \quad 2.87223366$

Ni $\quad 6.22804273 \quad 3.59087345$

12.07176640

14.10242206

8.00000000

10.03097590

12.05448850

14.18110718

8.00000000

10.03097590

12.05444419

14.10671768

8.00000000

10.03097590

12.07366865

14.14374973

8.00000000

10.03097590

12.05730773 
$\mathrm{Ni}$

$\mathrm{Ni}$

$\mathrm{Ni}$

$\mathrm{Ni}$

$\mathrm{Ni}$

$\mathrm{Ni}$

$\mathrm{Ni}$

$\mathrm{Ni}$

$\mathrm{Ni}$

$\mathrm{Ni}$

$\mathrm{Ni}$

$\mathrm{Ni}$

$\mathrm{Ni}$

$\mathrm{Ni}$

$\mathrm{Ni}$

$\mathrm{Ni}$

$\mathrm{Ni}$

O

O

O

O

O

O

0

0

45

Lattice="7.4622819431471425 $0.0 \quad 0.0 \quad 3.731140971573571$

2769249835" Properties=species:S:1:pos:R: 3 pbc="T T T"

$\mathrm{Ni}$

$\mathrm{Ni}$

$\mathrm{Ni}$

$\mathrm{Ni}$

$\mathrm{Ni}$

$\mathrm{Ni}$

$\mathrm{Ni}$

$\mathrm{Ni}$

$\mathrm{Ni}$

$\mathrm{Ni}$

$\mathrm{Ni}$

$\mathrm{Ni}$

$\mathrm{Ni}$

$\mathrm{Ni}$

$\mathrm{Ni}$

$\mathrm{Ni}$

$\mathrm{Ni}$

$\mathrm{Ni}$

$\mathrm{Ni}$

$\mathrm{Ni}$

$\mathrm{Ni}$

$\mathrm{Ni}$

$\mathrm{Ni}$

$\mathrm{Ni}$

$\mathrm{Ni}$

$\mathrm{Ni}$

$\mathrm{Ni}$

$\mathrm{Ni}$

$\mathrm{Ni}$

$\mathrm{Ni}$

$\mathrm{Ni}$

$\mathrm{Ni}$

$\mathrm{Ni}$

$\mathrm{Ni}$

$\mathrm{Ni}$

$\mathrm{Ni}$

0

0

O
3.73370250

4.97485463

6.21856829

7. 46461124

4.92914522

4.97485463

6.21856829

7.46290604

4.99241969

6.21856829

7.46228194

8.69888637

6.22663094

7.46228194

8.70599560

9.95106565

7.46620790

1.19619899

4.93703702

8.80254405

7.46595943

6.27401250

2.53310410

3.73539297

6.13146668
Fri Apr 16 14:51:55 2021

2.17570080

4.30835049

5.02640890

5.74605064

4.34229309

0.00000000

0.71805841

1.45271948

$-0.00152977$

2.15417524

2.87223366

3.59037015

2.16295000

4.30835049

5.02640890

5.74761524

4.31035490

0.64750681

2.96009419

5.02779822

2.88009981

0.64539768

2.96290750

0.72363295

5.02942021
14.14819073

8.00000000

10.03097590

12.07181476

14.11085485

8.00000000

10.03097590

12.05324127

14.14562649

8.00000000

12.05322164

14.18272375

10.03097590

12.05193091

15.23784616

15.24356969

15.24201410

15.27044339

15.24233183

15.24515722

15.23205357

15.24108241
10.03097590

8.00000000

14.17953898

$\begin{array}{rrr}0.00000000 & 0.00000000 & 8.00000000 \\ 1.24371366 & 0.71805841 & 10.03097590 \\ 2.47563791 & 1.42931021 & 12.06297087 \\ -0.02183739 & -0.01260782 & 14.15099914 \\ 1.24371366 & 2.15417524 & 8.00000000 \\ 2.48742731 & 2.87223366 & 10.03097590 \\ 3.71723762 & 3.58229704 & 12.06341507 \\ 1.22260324 & 2.14224846 & 14.14996354 \\ 2.48742731 & 4.30835049 & 8.00000000 \\ 3.73114097 & 5.02640890 & 10.03097590 \\ 4.96190432 & 5.73600733 & 12.06455379 \\ 2.46642647 & 4.29672716 & 14.15198205 \\ 2.48742731 & 0.00000000 & 8.00000000 \\ 3.73114097 & 0.71805841 & 10.03097590 \\ 4.96097905 & 1.42807369 & 12.06341507 \\ 2.46654320 & -0.01231876 & 14.14996354 \\ 3.73114097 & 2.15417524 & 8.00000000 \\ 4.97485463 & 2.87223366 & 10.03097590 \\ 6.20455898 & 3.58220379 & 12.06560690 \\ 3.71152032 & 2.14284725 & 14.15148349 \\ 4.97485463 & 4.30835049 & 8.00000000 \\ 6.21856829 & 5.02640890 & 10.03097590 \\ 7.44934083 & 5.73689265 & 12.06432427 \\ 4.95308108 & 4.29740561 & 14.15008396 \\ 4.97485463 & 0.00000000 & 8.00000000 \\ 6.21856829 & 0.71805841 & 10.03097590 \\ 7.44848022 & 1.42913152 & 12.06455379 \\ 4.95428811 & -0.01237560 & 14.15198205 \\ 6.21856829 & 2.15417524 & 8.00000000 \\ 7.46228194 & 2.87223366 & 10.03097590 \\ 8.69296519 & 3.58287208 & 12.06432427 \\ 6.19820297 & 2.14079123 & 14.15008396 \\ 7.46228194 & 4.30835049 & 8.00000000 \\ 8.70599560 & 5.02640890 & 10.03097590 \\ 9.93586848 & 5.73647634 & 12.06350653 \\ 7.44283096 & 4.29712046 & 14.15007983 \\ 1.22244022 & 0.70577619 & 15.26581636 \\ 4.95604793 & 2.86137560 & 15.26562023 \\ 8.68377841 & 5.01358180 & 15.26796594\end{array}$

.00000000

12.06297087

4.15099914

2.06341507

14.14996354

12.06455379

14.15198205

12.06341507

4.14996354

10.03097590

12.06560690

.

000000

12.06432427

14.15008396

12.06455379

14.15198205

12.06432427

14.15008396

8.00000000

14.15007983

15.26562

15.26796594

$6.462525732967329 \quad 0.0 \quad 0.0 \quad 0.0 \quad 22.0929$ 


\section{0}

O

0

0

O

0

$$
\begin{aligned}
& 7.44102136 \\
& 6.19769044 \\
& 3.70997300 \\
& 3.70901156 \\
& 6.19771304 \\
& 2.46626275
\end{aligned}
$$

$\begin{array}{ll}2.86042690 & 15.26635020 \\ 0.70566957 & 15.26780352 \\ 5.01452258 & 15.26780352 \\ 0.70639611 & 15.26667995 \\ 5.01390007 & 15.26635020 \\ 2.85890018 & 15.26667995\end{array}$

17

Lattice $=" 4.974854628764762 \quad 0.0 \quad 0.0 \quad 2.4874273143823804 \quad 4.3083504886448860 .0 \quad 0.0 \quad 0.022 .0929$

$2769249835 "$ Properties=species:S:1:pos:R:3 pbc="T T T"

Ni

0.00000000

0.00000000

1.24371366

0.71805841

8.00000000

$\mathrm{Ni} \quad 2.48503504$

1.43473565

$-0.02955781$

1.24371366

$-0.01706521$

$\mathrm{Ni}$

2.15417524

2. 48742731

2.87223366

3.73114097

3.59305444

1.24371366

2.18830567

2. 48742731

0.00000000

3.73114097

0.71805841

4.97724690

1.43473565

2. 51698513

$-0.01706521$

3. 73114097

2.15417524

4.97485463

2.87223366

6.21856829

3.59029207

10.03097590

12.04006719

14.13247842

8.00000000

10.03097590

12.04006719

14.13247842

8.00000000

10.03097590

12.04006719

14.13247842

8.00000000

10.03097590

3.73114097

12.08350714

2.15417524

14.00889073

1.24371366

0.71805841

. / oxygen/Ni/111/r3xr3R30/graph_code/1/struct.xyz

62

Lattice $=" 9.95116096182474 \quad 0.0 \quad 0.0 \quad-4.97558048091237 \quad 8.617958190088215 \quad 0.0 \quad 0.0 \quad 0.022 .382044$

$53 "$ Properties=species:S:1:pos:R:3 pbc="T T T"

$\mathrm{Sn}$ 3.73168536

0.71816318

6.50000000

$-1.24389512$

3.59081591

6.50000000

3.73168536

6.46346864

6.50000000

0.41463171

0.71816318

8.84551113

5.39021219

3.59081591

8.84551113

0.41463171

6.46346864

7.04873901

8.84551113

2.07315853

0.71816318

3.59081591

11.19102226

$-2.90242195$

6.46346864

11.19102226

3.73168536

$-1.24389512$

0.71816318

11.19102226

3.59081591

13.53653340

13.53653340

13.53653340

3.
0.41463171

6.46346864

0.71816318

5. 39021219

3.59081591

15.88204453

15.88204453

0.41463171

6.46346864

8.70726584

0.71816318

1. 24389512

6.21947560

2.15448955

2.15448955

3. 73168536

1. 24389512

6.21947560

3.59081591

5.02714228

5.02714228

$-1.24389512$

$-3.73168536$

1.24389512

5. 39021219

2. 90242195

7.87800243

0.41463171

$-2.07315853$

2. 90242195

5.39021219

$-2.07315853$

2. 90242195

2. 07315853

6.46346864

7.89979501

7.89979501

0.71816318

2.15448955

2.15448955

3. 59081591

5. 02714228

5.02714228

6.46346864

7.89979501

15.88204453

6.50000000

6.50000000

6.50000000

6.50000000

6.50000000

6.50000000

6.50000000

6.50000000

6.50000000

8.84551113

8.84551113

8.84551113

8.84551113

8.84551113

8.84551113

8.84551113

8.84551113

8.84551113

7.89979501

0.71816318

2.15448955

11.19102226

11.19102226

2.15448955

11.19102226

4. 56094877

3. 59081591

11.19102226 
Pt

Pt

Pt

Pt

Pt

Pt

Pt

Pt

Pt

Pt

Pt

Pt

Pt

Pt

Pt

Pt

Pt

Pt

Pt

Pt

Pt

Pt

Pt

$\mathrm{N}$

0
$-0.41463171$

4. 56094877

2.07315853

$-0.41463171$

4.56094877

8.70726584

1. 24389512

6.21947560

3. 73168536

1. 24389512

6.21947560

$-1.24389512$

$-3.73168536$

1. 24389512

5.39021219

2.90242195

7.87800243

0.41463171

$-2.07315853$

2. 90242195

5. 39021219

$-2.07315853$

2. 90242195

1.65852683

1. 65852683
Fri Apr 16 14:51:55 2021

5.02714228

5.02714228

6.46346864

7.89979501

7.89979501

0.71816318

2.15448955

2.15448955

3. 59081591

5. 02714228

5.02714228

6.46346864

7.89979501

7.89979501

0.71816318

2.15448955

2.15448955

3. 59081591

5. 02714228

5.02714228

6.46346864

7.89979501

7.89979501

4.30897909

4.30897909
194

11.19102226

11.19102226

11.19102226

11.19102226

11.19102226

13.53653340

13.53653340

13.53653340

13.53653340

13.53653340

13.53653340

13.53653340

13.53653340

13.53653340

15.88204453

15.88204453

15.88204453

15.88204453

15.88204453

15.88204453

15.88204453

15.88204453

15.88204453

17.08204453

18.48204453

. /oxygen/Ni/111/r3xr3R30/graph_code/2/struct.xyz

62

Lattice $=" 9.95116096182474 \quad 0.0 \quad 0.0 \quad-4.97558048091237 \quad 8.6179581900882150 .0 \quad 0.0 \quad 0.022 .382044$ $53 "$ Properties=species:S:1:pos:R:3 pbc="T T T"

$\mathrm{Sn} \quad 3.73168536 \quad 0.71816318$

Sn $\quad-1.24389512 \quad 3.59081591$

6.50000000

6.46346864

6.50000000

3168536

0.71816318

6.50000000

0.41463171

3.59081591

8.84551113

5. 39021219

6.46346864

8.84551113

0.41463171

8.84551113

7.04873901

0.71816318

2.07315853

3.59081591

11.19102226

11.19102226

$-2.90242195$

6.46346864

11.19102226

3.73168536

0.71816318

13.53653340

13.53653340

3.73168536

3.59081591

13.53653340

0.41463171

5.39021219

.46346864

15.88204453

15.88204453

15.88204453

6.50000000

6.50000000

6.50000000

6.50000000

6.50000000

6.50000000

6.50000000

6.50000000

6.50000000

8.84551113

8.84551113

8.84551113

8.84551113

8.84551113

8.84551113

8.84551113

8.84551113

8.84551113

11.19102226

11.19102226

11.19102226

11.19102226

11.19102226

11.19102226 
all_structures.xyz

$\begin{array}{lr}\text { Pt } & 2.07315853 \\ \text { Pt } & -0.41463171 \\ \text { Pt } & 4.56094877 \\ \text { Pt } & 8.70726584 \\ \text { Pt } & 1.24389512 \\ \text { Pt } & 6.21947560 \\ \text { Pt } & 3.73168536 \\ \text { Pt } & 1.24389512 \\ \text { Pt } & 6.21947560 \\ \text { Pt } & -1.24389512 \\ \text { Pt } & -3.73168536 \\ \text { Pt } & 1.24389512 \\ \text { Pt } & 5.39021219 \\ \text { Pt } & 2.90242195 \\ \text { Pt } & 7.87800243 \\ \text { Pt } & 0.41463171 \\ \text { Pt } & -2.07315853 \\ \text { Pt } & 2.90242195 \\ \text { Pt } & 5.39021219 \\ \text { Pt } & -2.07315853 \\ \text { Pt } & 2.90242195 \\ \text { N } & 7.04873902 \\ \text { O } & 7.04873902\end{array}$

Fri Apr 16 14:51:55 2021

6.46346864

7.89979501

7.89979501

0.71816318

2.15448955

2.15448955

3.59081591

5.02714228

5.02714228

6.46346864

7.89979501

7.89979501

0.71816318

2.15448955

2.15448955

3.59081591

5.02714228

5.02714228

6.46346864

7.89979501

7.89979501

0.71816318

0.71816318
11.19102226

11.19102226

11.19102226

13.53653340

13.53653340

13.53653340

13.53653340

13.53653340

13.53653340

13.53653340

13.53653340

15.88204453

15.88204453

15.88204453

15.88204453

15.88204453

15.88204453

15.88204453

15.88204453

15.88204453

17.08204453

18.48204453
13.53653340

195

. /oxygen/Ni/111/r3xr3R30/struct.xyz 13

Lattice $=" 4.308350488644886 \quad 0.0 \quad 0.0 \quad 2.1541752443224427 \quad 3.7311409715735704 \quad 0.0 \quad 0.0 \quad 0.0 \quad 22.092$ 92769249835" Properties=species:S:1:pos:R:3 pbc="T T T"

$\begin{array}{llll}\mathrm{Ni} & 2.51320445 & 0.62185683 & 8.00000000 \\ \mathrm{Ni} & 4.66737970 & 1.86557049 & 8.00000000 \\ \mathrm{Ni} & 2.51320445 & 3.10928414 & 8.00000000 \\ \mathrm{Ni} & 3.94932128 & 0.62185683 & 10.03097590 \\ \mathrm{Ni} & 1.79514604 & 1.86557049 & 10.03097590 \\ \mathrm{Ni} & 3.94932128 & 3.10928414 & 10.03097590 \\ \mathrm{Ni} & 1.08905352 & 0.62870902 & 12.01182439 \\ \mathrm{Ni} & 3.24380489 & 1.87402925 & 12.07197529 \\ \mathrm{Ni} & 5.39930885 & 3.11560928 & 12.07146432 \\ \mathrm{Ni} & 2.53722396 & 0.63215405 & 14.10570814 \\ \mathrm{Ni} & 4.68376069 & 1.88149603 & 14.10650375 \\ \mathrm{Ni} & 2.52951807 & 3.11322164 & 14.10737669 \\ \mathrm{O} & 1.09402933 & 0.63178562 & 15.23938240\end{array}$

. /oxygen/Ni/210/iter_1/struct.xyz

33

Lattice $=" 7.035506886833694 \quad 0.0 \quad 0.0 \quad 3.5177534434168454$

507929482" Properties=species:S:1:pos:R:3 pbc="T T T"

$3.51775344 \quad 1.57318717$

$1.75887672 \quad 0.00000000$

$\begin{array}{ll}1.75887672 & 2.35978075\end{array}$

$3.51775344 \quad 0.76567740$

$3.51775344 \quad 3.09167209$

$1.77082393 \quad 1.55624483$

$-0.00000000 \quad 0.08932155$

$3.51775344 \quad 7.07934224$

$\begin{array}{ll}5.27663017 & 5.50615508\end{array}$

$3.51775344 \quad 3.93296791$

$3.51775344 \quad 6.29274866$

$5.29212536 \quad 4.70926296$

$5.28192599 \quad 7.05687504$

$3.51775344 \quad 5.59322656$

$\begin{array}{ll}1.87611873 & 3.91294365\end{array}$

$\begin{array}{ll}5.27663017 & 3.14637433\end{array}$

$\begin{array}{ll}7.03550689 & 1.57318717\end{array}$

$5.27663017 \quad 0.00000000$

$\begin{array}{ll}5.27663017 & 2.35978075\end{array}$

$\begin{array}{ll}7.03550689 & 0.79171866\end{array}$

$\begin{array}{ll}7.03550689 & 3.11704311\end{array}$

$5.26468296 \quad 1.55624483$

8.00000000

8.78659358

9.57318717

10.35978075

11.13342430

11.87487390

12.67726695

13.38234894

8.00000000

8.78659358

9.57318717

10.35978075

11.14235452

11.96131261

12.74477537

13.44389600

8.00000000

8.78659358

9.57318717

10.35978075

11.13339279

12.06521201

12.67726695

13.38455079 
$\mathrm{Ni}$

$\mathrm{Ni}$

$\mathrm{Ni}$

$\mathrm{Ni}$

$\mathrm{Ni}$

$\mathrm{Ni}$

$\mathrm{Ni}$

$\mathrm{Ni}$

0
Fri Apr 16 14:51:55 2021

7.03550689

8.79438361

7.03550689

7.03550689

8.77888841

8.78908778

7.03550689

5.15938816

3.51775344
7.07934224

5.50615508

3.93296791

6.29274866

4.70926296

7.05687504

5.46498192

3.91294365

4.39752826
8.00000000
8.78659358
9.57318717
10.35978075
11.14235452
11.96131261
12.69785877
13.44389600
14.17754237

42

Lattice $=" 7.035506886833694 \quad 0.0 \quad 0.0 \quad 3.5177534434168454$

$\mathrm{Ni} \quad 1.75887672 \quad 3.14637433 \quad 8.00000000$

$\mathrm{Ni} \quad 3.51775344 \quad 1.57318717$

$\mathrm{Ni} \quad 1.75887672 \quad 0.00000000$

$\mathrm{Ni} \quad 1.75887672 \quad 2.35978075$

$\mathrm{Ni} \quad 3.47179244 \quad 0.76245218$

$\mathrm{Ni} \quad 3.46424772 \quad 3.11315717$

$\mathrm{Ni} \quad 1.65744122$

$\mathrm{Ni} \quad 6.80033782$

$\mathrm{Ni} \quad 3.51775344$

$\mathrm{Ni} \quad 5.27663017$

$\mathrm{Ni} \quad 3.51775344$

$\mathrm{Ni} \quad 3.51775344$

$\mathrm{Ni} \quad 5.31502194$

$\mathrm{Ni} \quad 5.27684492$

1.53056827

$-0.05476639$

7.07934224

5.50615508

3.93296791

6.29274866

4.65916714

7.13643830

3. 58611312

5.47684283

3.87281424

3.49355118

3.14637433

5.27663017

1.57318717

7.03550689

0.00000000

5.27663017

2.35978075

7.07247111

0.81896549

3.22222462

7.14140031

1.55916650

0.74259214

3.60931668

7.07934224

7.03550689

5.50615508

3.93296791

7.03550689

6.29274866

7.03550689

4.69753229

7.05800260

8.72670704

5.47208693

2.75637224

7.63761904

3. 62237929

2. 53849269

6.27051341

1.10371804

6.96060172

4.60985368

4.02373334

5.51528498

0.90640818

8.78659358

9.57318717

10.35978075

11.14434893

11.98939917

12.98401519

13.63006028

8.00000000

8.78659358

9.57318717

10.35978075

11.16740156

11.93789797

12.83640816

15.10190416

8.00000000

8. 78659358

9.57318717

10.35978075

11.15626403

12.03422944

12.54867696

14.52338895

8.00000000

8.78659358

9.57318717

10.35978075

11.08657620

11.83023117

14.71588747

14.39961370

15.06337302

15.42129878

13.78953594

13.44833747

14.37827766

15.14923625

15.67622927

13.69240039

12.76850977

14.83214876

$\begin{array}{llllll}7.86593582756403 & 0.0 & 0.0 & 0.0 & 21.50615\end{array}$ (1)

. /oxygen/Ni/210/iter_11/struct.xyz

43

Lattice $=" 7.035506886833694 \quad 0.0 \quad 0.0 \quad 3.5177534434168454$

$507929482 "$ Properties=species:S:1:pos:R:3 pbc="T T T"

$\begin{array}{llll}\mathrm{Ni} & 1.75887672 & 3.14637433 & 8.00000000\end{array}$

$\mathrm{Ni} \quad 3.51775344 \quad 1.57318717$

$\mathrm{Ni} \quad 1.75887672 \quad 0.00000000$

$\begin{array}{lll}\mathrm{Ni} & 1.75887672 & 2.35978075\end{array}$

$\mathrm{Ni} \quad 3.55121525 \quad 0.79249150$

$\begin{array}{lll}\mathrm{Ni} & 3.60009680 & 3.20418627\end{array}$

$\mathrm{Ni} \quad 1.87714412 \quad 1.68690057$

$\mathrm{Ni} \quad 7.30929508 \quad 1.02275287$

$\mathrm{Ni}$

1.02275287
7.07934224

8.78659358

9.57318717

10.35978075

11.11043783

11.84629290

12.66228530

14.37890753

8.00000000

$\begin{array}{llllll}7.86593582756403 & 0.0 & 0.0 & 0.0 & 21.50615\end{array}$ 
$\mathrm{Ni}$

$\mathrm{Ni}$

$\mathrm{Ni}$

$\mathrm{Ni}$

$\mathrm{Ni}$

$\mathrm{Ni}$

$\mathrm{Ni}$

$\mathrm{Ni}$

$\mathrm{Ni}$

$\mathrm{Ni}$

$\mathrm{Ni}$

$\mathrm{Ni}$

$\mathrm{Ni}$

$\mathrm{Ni}$

$\mathrm{Ni}$

$\mathrm{Ni}$

$\mathrm{Ni}$

$\mathrm{Ni}$

$\mathrm{Ni}$

$\mathrm{Ni}$

$\mathrm{Ni}$

$\mathrm{Ni}$

$\mathrm{Ni}$

0

0

0

0

O

0

O

O

O

0

0

34

Lattice $=" 7.035506886833694 \quad 0.0 \quad 0.0 \quad 3.5177534434168454$ 507929482 " Properties=species:S:1:pos:R: 3 pbc="T T T" $\mathrm{Ni}$

$\mathrm{Ni}$

$\mathrm{Ni}$

$\mathrm{Ni}$

$\mathrm{Ni}$

$\mathrm{Ni}$

$\mathrm{Ni}$

$\mathrm{Ni}$

$\mathrm{Ni}$

$\mathrm{Ni}$

$\mathrm{Ni}$

$\mathrm{Ni}$

$\mathrm{Ni}$

$\mathrm{Ni}$

$\mathrm{Ni}$

$\mathrm{Ni}$

$\mathrm{Ni}$

$\mathrm{Ni}$

$\mathrm{Ni}$

$\mathrm{Ni}$

$\mathrm{Ni}$

$\mathrm{Ni}$

$\mathrm{Ni}$

$\mathrm{Ni}$

$\mathrm{Ni}$

$\mathrm{Ni}$

$\mathrm{Ni}$

$\mathrm{Ni}$

$\mathrm{Ni}$

$\mathrm{Ni}$
5.27663017

3. 51775344

3. 51775344

5. 33024944

5. 38721782

3.63936387

1.81298879

5.27663017

7.03550689

5.27663017

5.27663017

7.02976878

6.89004596

4.67657014

3.22720555

7.03550689

8.79438361

7.03550689

7.03550689

8.72266899

8.67157103

6.94376503

5.98822025

4.77520446

4.74340205

3.06294137

8.84276462

6.24074437

3.99066803

2.19687826

7.19171304

5.22849847

1.45629022

6.28687489
Fri Apr 16 14:51:55 2021

5.50615508

3.93296791

6.29274866

4.70778206

7.15539286

5.50735415

3.14637433

1.57318717

0.00000000

2.35978075

0.78294770

3.10728745

1.43376395

1.95763236

7.07934224

5.50615508

3.93296791

6.29274866

4.66563223

7.04129866

5.50062425

3.59046998

7.66543058

2.84280527

2.83711474

5.25027650

0.28825640

0.67407425

3. 40871487

4.82665293

6.02124926

1.08517788

2.56157094
4.03465190
8.78659358

9.57318717

10.35978075

11.09611782

11.91731265

12.71167988

14.53019918

8.00000000

8.78659358

9.57318717

10.35978075

11.29095848

12.08841678

13.40311178

15.86845875

8.00000000

8.78659358

9.57318717

10.35978075

11.18841819

11.94188617

13.13892554

15.35457176

15.28606219

16.24584304

13.58281929

13.04983034

12.99737415

14.90237226

16.10841767

14.90454827

13.34953395

15.89290832

13.86873758
8.00000000

8.78659358

9.57318717

10.35978075

11.14153480

12.04242168

12.71867073

13.44754154

8.00000000

8.78659358

9.57318717

10.35978075

11.13654898

11.87514663

12.68917292

13.45162522

8.00000000

8.78659358

9.57318717

10.35978075

11.13654898

11.87514663

12.68917292

13.45162522

8.00000000

8.78659358

9.57318717

10.35978075

11.14153480

12.04242168

1.53316443

5.50615508

3.93296791

6.29274866

4.71997817

7.06318253

$\begin{array}{llllll}7.86593582756403 & 0.0 & 0.0 & 0.0 & 21.50615\end{array}$ 
$\mathrm{Ni}$

7.02126353

$\mathrm{Ni}$

O

0

7.02125907
Fri Apr 16 14:51:55 2021

5.58068105

3.90395658

0.47967246

4. 41264037
12.71867073

13.44754154

14.18465794

14.18465794

35

Lattice $=" 7.035506886833694 \quad 0.0 \quad 0.0 \quad 3.5177534434168454 \quad 7.86593582756403 \quad 0.0 \quad 0.0 \quad 0.021 .50615$ 507929482" Properties=species:S:1:pos:R:3 pbc="T T T" $\mathrm{Ni} \quad 1.75887672 \quad 3.14637433 \quad 8.00000000$

$\begin{array}{llll}\mathrm{Ni} & 3.51775344 & 1.57318717 & 8.78659358\end{array}$

$\begin{array}{llll}\mathrm{Ni} & 1.75887672 & 0.00000000 & 9.57318717\end{array}$

$\begin{array}{llll}\mathrm{Ni} & 1.75887672 & 2.35978075 & 10.35978075\end{array}$

$\begin{array}{llll}\mathrm{Ni} & 3.53441187 & 0.77854208 & 11.13390078\end{array}$

$\mathrm{Ni} \quad 3.52757009 \quad 3.11306220 \quad 12.00591755$

$\mathrm{Ni} \quad 1.72665403 \quad 1.62112482 \quad 12.69799121$

$\mathrm{Ni} \quad 0.09959692 \quad 0.00606007 \quad 13.44448986$

$\mathrm{Ni} \quad 3.51775344 \quad 7.07934224$

$\mathrm{Ni} \quad 5.27663017 \quad 5.50615508$

$\mathrm{Ni} \quad 3.51775344 \quad 3.93296791$

$\mathrm{Ni} \quad 3.51775344 \quad 6.29274866$

$\mathrm{Ni} \quad 5.28070635 \quad 4.70932356$

$\mathrm{Ni} \quad 5.27469519 \quad 7.03608865$

$\mathrm{Ni} \quad 3.52258179 \quad 5.54048201$

$\mathrm{Ni} \quad 1.68184573 \quad 3.97593528$

$\mathrm{Ni} \quad 5.27663017 \quad 3.14637433$

$\mathrm{Ni} \quad 7.03550689 \quad 1.57318717$

$\mathrm{Ni} \quad 5.27663017 \quad 0.00000000$

$\mathrm{Ni} \quad 5.27663017 \quad 2.35978075$

$\mathrm{Ni} \quad 7.01934210 \quad 0.76691200$

$\mathrm{Ni} \quad 7.02547803 \quad 3.11337533$

$\mathrm{Ni} \quad 5.30296263 \quad 1.50432896$

$\mathrm{Ni} \quad 3.41137595 \quad 0.04025789$

$\begin{array}{lll}\mathrm{Ni} & 7.03550689 & 7.07934224\end{array}$

$\mathrm{Ni} \quad 8.79438361 \quad 5.50615508$

$\mathrm{Ni} \quad 7.03550689 \quad 3.93296791$

$\mathrm{Ni} \quad 7.03550689 \quad 6.29274866$

$\mathrm{Ni} \quad 8.78781230 \quad 4.72789870$

$\mathrm{Ni} \quad 8.79327186 \quad 7.06153435$

$\mathrm{Ni} \quad 7.02234714 \quad 5.55075867$

$\begin{array}{lll}\mathrm{Ni} & 5.35583543 & 3.87929752\end{array}$

O $\quad 1.75389879 \quad 0.46137453$

O $\quad 7.02411925 \quad 4.32815947$

o $3.52088439 \quad 3.96136470$

. /oxygen/Ni/210/iter_4/struct.xyz

36

Lattice $=" 7.035506886833694 \quad 0.0 \quad 0.0 \quad 3.5177534434168454$

$507929482 "$ Properties=species:S:1:pos:R:3 pbc="T T T"

$\mathrm{Ni} \quad 1.75887672 \quad 3.14637433$

$\mathrm{Ni} \quad 3.51775344 \quad 1.57318717$

$\mathrm{Ni} \quad 1.75887672$

0.00000000

8.00000000

2.35978075

8.78659358

9.57318717

1. 75887672

0.77964569

3.51249014
3.52106232

0.77964569
3.10107794

10.35978075

11.12222828

1.72635481

1.57010354

0.08126118

3. 51775344

$-0.02555464$

7.07934224

5.50615508

3.93296791

3.51775344

6.29274866

3.51775344

5. 28236352

5.27473287

4.70169893

7.03918836

3. 55255582

5.49621054

3. 94116274

3.14637433

5.27663017

1.57318717

0.00000000

2.35978075

12.00467061

12.69790157

13.60353542

8.00000000

8.78659358

9.57318717

10.35978075

11.11236475

11.91219789

12.69703799

13.58912764

8.00000000

8.78659358

9.57318717

10.35978075

0.76873102

11.11236475

5.27663017

$\mathrm{Ni}$

7. 04124024 $\begin{array}{llllll}7.86593582756403 & 0.0 & 0.0 & 0.0 & 21.50615\end{array}$ 
$\mathrm{Ni}$

$\mathrm{Ni}$

$\mathrm{Ni}$

$\mathrm{Ni}$

$\mathrm{Ni}$

$\mathrm{Ni}$

$\mathrm{Ni}$

$\mathrm{Ni}$

$\mathrm{Ni}$

$\mathrm{Ni}$

$\mathrm{Ni}$

O

0

0

0
7.03360959

5.31143254

3.43827487

7.03550689

8.79438361

7.03550689

7.03550689

8.78912030

8.79769248

7.00298497

5.35789135

1.75982364

7.03645380

3.51643249

8.79306265
Fri Apr 16 14:51:55 2021

3.10622045

1.56324263

0.00819483

7.07934224

5.50615508

3.93296791

6.29274866

4.71261360

7.03404585

5.50307145

3.90741327

0.38017180

4.31313972

3.93113164

7.86409956
11.91219789

12.69703799

13.58912764

8.00000000

8.78659358

9.57318717

10.35978075

11.12222828

12.00467061

12.69790157

13.60353542

14.16722164

14.16722164

13.82182143

13.82182143

37

Lattice $=" 7.035506886833694 \quad 0.0 \quad 0.0 \quad 3.5177534434168454$

$\begin{array}{llll}\mathrm{Ni} & 1.75887672 & 3.14637433 & 8.00000000\end{array}$

$\mathrm{Ni} \quad 3.51775344 \quad 1.57318717$

$\begin{array}{ll}1.75887672 & 0.00000000\end{array}$

$\begin{array}{ll}1.75887672 & 2.35978075\end{array}$

$\begin{array}{ll}3.52747182 & 0.78499759\end{array}$

$3.52815903 \quad 3.13172139$

$1.79242200 \quad 1.49983288$

$\begin{array}{ll}10.47208928 & 7.81819685\end{array}$

$\begin{array}{ll}3.51775344 & 7.07934224\end{array}$

$\begin{array}{ll}5.27663017 & 5.50615508\end{array}$

$\begin{array}{ll}3.51775344 & 3.93296791\end{array}$

$\begin{array}{ll}3.51775344 & 6.29274866\end{array}$

$5.28391090 \quad 4.68853653$

$\begin{array}{ll}5.26165039 & 7.02437183\end{array}$

$3.43395549 \quad 5.52191947$

$8.59898509 \quad 4.23609373$

$\begin{array}{ll}5.27663017 & 3.14637433\end{array}$

$\begin{array}{ll}7.03550689 & 1.57318717\end{array}$

$\begin{array}{ll}5.27663017 & 0.00000000\end{array}$

$\begin{array}{ll}5.27663017 & 2.35978075\end{array}$

$\begin{array}{ll}7.01947985 & 0.77157617\end{array}$

$\begin{array}{ll}7.03908116 & 3.11516237\end{array}$

$5.26714769 \quad 1.59431403$

$\begin{array}{ll}3.59654440 & 0.01544727\end{array}$

$\begin{array}{ll}7.03550689 & 7.07934224\end{array}$

$8.79438361 \quad 5.50615508$

$\begin{array}{ll}7.03550689 & 3.93296791\end{array}$

$\begin{array}{ll}7.03550689 & 6.29274866\end{array}$

$8.77989556 \quad 4.70187674$

$\begin{array}{ll}8.79348979 & 7.06215495\end{array}$

$\begin{array}{ll}7.06228407 & 5.56400584\end{array}$

$5.32293321 \quad 4.16756360$

$5.28182850 \quad 7.70037320$

$6.89075485 \quad 4.88377424$

$3.44537534 \quad 4.00646012$

$\begin{array}{ll}5.28274069 & 0.40069883\end{array}$

8.78659358

9.57318717

10.35978075

11.12505920

12.02956853

12.69090944

13.61435044

8.00000000

8.78659358

9.57318717

10.35978075

11.13417302

11.96696077

12.68901941

13.97884213

8.00000000

8.78659358

9.57318717

10.35978075

11.10914901

12.01754546

12.71283719

13.61750412

8.00000000

8.78659358

9.57318717

10.35978075

11.11967631

11.89706574

12.64591354

13.67492892

13.80477896

14.33847852

13.81540727

14.16746845

7.80782765

2.61375306

13.69732781

. /oxygen/Ni/210/iter_6/struct.xyz

38

Lattice $=" 7.035506886833694 \quad 0.0 \quad 0.0 \quad 3.5177534434168454 \quad 7.86593582756403 \quad 0.0 \quad 0.0 \quad 0.0 \quad 21.50615$

$507929482 "$ Properties=species:S:1:pos:R:3 pbc="T T T"

8.00000000

$\mathrm{N}$

1.75887672

3.51775344

3.14637433

8.00000000

1.75887672

1.57318717

9.57318717

1.75887672

0.00000000

2.35978075

3.53684787

0.76071766

3. 52328035

3.09632637

10.35978075

11.14946454

11.98230402

1.73869244

1. 53379452

12.71524417

$\mathrm{Ni}$

6.84092329

0.04921945

13.87367138

$7.86593582756403 \quad 0.0 \quad 0.0 \quad 0.0 \quad 21.50615$

$\mathrm{Ni}$ 
$\mathrm{Ni}$

$\mathrm{Ni}$

$\mathrm{Ni}$

$\mathrm{Ni}$

$\mathrm{Ni}$

$\mathrm{Ni}$

$\mathrm{Ni}$

$\mathrm{Ni}$

$\mathrm{Ni}$

$\mathrm{Ni}$

$\mathrm{Ni}$

$\mathrm{Ni}$

$\mathrm{Ni}$

$\mathrm{Ni}$

$\mathrm{Ni}$

$\mathrm{Ni}$

$\mathrm{Ni}$

$\mathrm{Ni}$

$\mathrm{Ni}$

$\mathrm{Ni}$

$\mathrm{Ni}$

$\mathrm{Ni}$

$\mathrm{Ni}$

$\mathrm{Ni}$

O

O

0

0

0

0

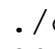

. / oxygen/Ni/210/iter_7/struct.xyz

Lattice $=" 7.035506886833694 \quad 0.0 \quad 0.0 \quad 3.5177534434168454$

507929482" Properties=species:S:1:pos:R:3 pbc="T T T"

$\mathrm{Ni}$

1.75887672

3.51775344

1.75887672

1.75887672

3.50285917

3.50505125

1.71532278

6.96239223

3.51775344

5.27663017

3.51775344

3.51775344

5.25548453

5.45520271

3.37553772

1.78521968

5.27663017

7.03550689

5.27663017

5.27663017

7.04678983

7.04241377

5.33369715

3.58310147

7.03550689

8.79438361

7.03550689

7.03550689

8.81219495

8.73268455

7.41898777

5.06106679

4.51045369

6.90782619
Fri Apr 16 14:51:55 2021

200

7.07934224

5.50615508

3. 93296791

4.68487984

7.08551048

5.44711096

4.45206589

3.14637433

1.57318717

0.00000000

2.35978075

0.77321702

3.14191347

1. 61865457

0.19378037

5.50615508

3. 93296791

6.29274866

4.69758834

7.02135865

5.58748161

4.16145436

7.78348638

4.98200218

3. 81519812

6.24701513

2.80011749

0.84443078

8.00000000

8.78659358

10.35978075

11.11841046

11.97758153

12.62186059

13.96146878

8.00000000

8.78659358

9.57318717

10.35978075

11.12553274

12.03308993

12.68680869

13.71909418

8.78659358

9.57318717

10.35978075

11.11000205

12.00712632

12.63623684

13.74748019

13.80967645

14.36002886

13.76642310

13.77274901

13.70586362

14.32800561
6.29274866

7.07934224

9.57318717

8.00000000

8.00000000

3.14637433

8.78659358

9.57318717

0.00000000

2.35978075

0.79127481

3.14333704

1.53818885

0.16393043

7.07934224

5.50615508

3.93296791

6.29274866

4.69842025

6.95049511

5.44550453

3. 26342821

3.14637433

1.57318717

0.00000000

2.35978075

0.73494153

3.19755664

1.59094110

1.10424281

7.07934224

5.50615508

3.93296791

6.29274866

4.72091234

7.11513860

10.35978075

11.08025390

11.93028642

12.55143408

13.74414000

8.00000000

8.78659358

9.57318717

10.35978075

11.17804867

12.18795767

12.90733492

14.29332000

8.00000000

8.78659358

9.57318717

10.35978075

11.11541432

11.95964288

12.56765231

14.18897907

8.00000000

8.78659358

9.57318717

10.35978075

11.12394931

11.93810605

13.17584664

13.97006914

3.50844050

13.76552948

6.56578463

13.78816942

3.75362748

$\begin{array}{llllll}7.86593582756403 & 0.0 & 0.0 & 0.0 & 21.50615\end{array}$ 


\section{0}

O

0

0

0
3.55808519

9.04194694

5.49529130

7.15132264

1.65406204
Fri Apr 16 14:51:55 2021

2.90822443

5.06028821

1.11964329

7.29242180

1.36811055
14.78140749

14.10358618

14.35010862

13.20853575

14.38244324

. /oxygen/Ni/210/iter_8/struct.xyz 40

Lattice $=" 7.035506886833694 \quad 0.0 \quad 0.0 \quad 3.5177534434168454 \quad 7.86593582756403 \quad 0.0 \quad 0.0 \quad 0.021 .50615$ $507929482 "$ Properties=species:S:1:pos:R:3 pbc="T T T"

$\begin{array}{lllr}\mathrm{Ni} & 1.75887672 & 3.14637433 & 8.00000000 \\ \mathrm{Ni} & 3.51775344 & 1.57318717 & 8.78659358 \\ \mathrm{Ni} & 1.75887672 & 0.0000000 & 9.57318717 \\ \mathrm{Ni} & 1.75887672 & 2.35978075 & 10.35978075 \\ \mathrm{Ni} & 3.46839397 & 0.74568395 & 11.15606898 \\ \mathrm{Ni} & 3.55244030 & 3.11060444 & 11.82801814 \\ \mathrm{Ni} & 1.83515245 & 1.71748000 & 12.86773933 \\ \mathrm{Ni} & 6.96996173 & 0.17132527 & 13.61804319 \\ \mathrm{Ni} & 3.51775344 & 7.07934224 & 8.00000000 \\ \mathrm{Ni} & 5.27663017 & 5.50615508 & 8.78659358 \\ \mathrm{Ni} & 3.51775344 & 3.93296791 & 9.57318717 \\ \mathrm{Ni} & 3.51775344 & 6.29274866 & 10.35978075 \\ \mathrm{Ni} & 5.27783507 & 4.71022061 & 11.12918731 \\ \mathrm{Ni} & 5.28140959 & 7.06824197 & 11.96831743 \\ \mathrm{Ni} & 4.05685602 & 5.00435181 & 13.31504898 \\ \mathrm{Ni} & 2.82237253 & 3.10119706 & 15.29251111 \\ \mathrm{Ni} & 5.27663017 & 3.14637433 & 8.00000000 \\ \mathrm{Ni} & 7.03550689 & 1.57318717 & 8.78659358 \\ \mathrm{Ni} & 5.27663017 & 0.00000000 & 9.57318717 \\ \mathrm{Ni} & 5.27663017 & 2.35978075 & 10.35978075 \\ \mathrm{Ni} & 7.06514200 & 0.77373330 & 11.14191806 \\ \mathrm{Ni} & 7.00095778 & 3.18304464 & 11.88027776 \\ \mathrm{Ni} & 5.20853353 & 1.53787161 & 12.57496703 \\ \mathrm{Ni} & 4.09102874 & 0.22907750 & 14.43336749 \\ \mathrm{Ni} & 7.03550689 & 7.07934224 & 8.00000000 \\ \mathrm{Ni} & 8.79438361 & 5.50615508 & 8.78659358 \\ \mathrm{Ni} & 7.03550689 & 3.93296791 & 9.57318717 \\ \mathrm{Ni} & 7.03550689 & 6.29274866 & 10.35978075 \\ \mathrm{Ni} & 8.81262884 & 4.70208351 & 11.20516009 \\ \mathrm{Ni} & 8.77886735 & 7.03720666 & 11.97521305 \\ \mathrm{Ni} & 8.09508059 & 4.83617612 & 14.48984652 \\ \mathrm{Ni} & 5.90638865 & 3.25911905 & 14.19424912 \\ \mathrm{O} & 4.67977821 & 6.74179086 & 13.65418721 \\ \mathrm{O} & 4.34578493 & 3.88484483 & 14.78297961 \\ \mathrm{O} & 2.80903324 & 1.50984568 & 14.46149235 \\ \mathrm{O} & 9.13466478 & 5.42979888 & 12.92360139 \\ \mathrm{O} & 5.68509587 & 1.28623057 & 14.37614974 \\ \mathrm{O} & 7.65919351 & 6.46189749 & 14.92558759 \\ \mathrm{O} & 8.52889264 & 3.93301168 & 15.98928264 \\ \mathrm{O} & 7.66042763 & 3.17224532 & 13.61104857\end{array}$

./oxygen/Ni/210/iter_9/struct.xyz

41

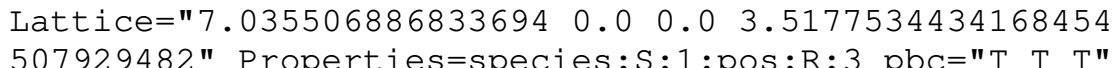

$\begin{array}{lrrr}\mathrm{Ni} & 1.75887672 & 3.14637433 & 8.00000000 \\ \mathrm{Ni} & 3.51775344 & 1.57318717 & 8.78659358 \\ \mathrm{Ni} & 1.75887672 & 0.00000000 & 9.57318717 \\ \mathrm{Ni} & 1.75887672 & 2.35978075 & 10.35978075 \\ \mathrm{Ni} & 3.53244295 & 0.76096513 & 11.12702653 \\ \mathrm{Ni} & 3.50650542 & 3.11000222 & 11.98625101 \\ \mathrm{Ni} & 1.65178280 & 1.64211307 & 12.66684352 \\ \mathrm{Ni} & 6.70261931 & -0.07926768 & 13.87344936 \\ \mathrm{Ni} & 3.51775344 & 7.07934224 & 8.0000000 \\ \mathrm{Ni} & 5.27663017 & 5.50615508 & 8.78659358 \\ \mathrm{Ni} & 3.51775344 & 3.93296791 & 9.57318717 \\ \mathrm{Ni} & 3.51775344 & 6.29274866 & 10.35978075 \\ \mathrm{Ni} & 5.27785488 & 4.69499942 & 11.11676422 \\ \mathrm{Ni} & 5.27510251 & 7.16920968 & 12.07300653 \\ \mathrm{Ni} & 3.58238544 & 5.46334081 & 13.02521481\end{array}$


$\mathrm{Ni}$

$\mathrm{Ni}$

$\mathrm{Ni}$

$\mathrm{Ni}$

$\mathrm{Ni}$

$\mathrm{Ni}$

$\mathrm{Ni}$

$\mathrm{Ni}$

$\mathrm{Ni}$

$\mathrm{Ni}$

$\mathrm{Ni}$

$\mathrm{Ni}$

$\mathrm{Ni}$

$\mathrm{Ni}$

$\mathrm{Ni}$

$\mathrm{Ni}$

$\mathrm{Ni}$

0

0

0

0

O

O

0

O

0
2.61455483

5.27663017

7.03550689

5.27663017

5.27663017

7.01320803

7.06476879

5.31048376

3.49350687

7.03550689

8.79438361

7.03550689

7.03550689

8.83346843

8.75263906

7.72065823

5.61665697

1.51476466

4.04353933

3.03628152

9.03910139

5.28097331

6.96020700

8.48912590

7.36740837

5.44088044
Fri Apr 16 14:51:55 2021

3.53908143

3.14637433

1.57318717

0.00000000

2.35978075

0.79128114

3.22282868

1.62271056

0.53100606

7.07934224

5.50615508

3.93296791

6.29274866

4.69382197

7.03189979

5.35760145

3. 92275613

0.19453118

4.53001786

2. 31612248

6.24282509

1.02360359

6.84788122

4.34376683

3.72630676

5.43363479
15.05085765

8.00000000

8.78659358

9.57318717

10.35978075

11.13033653

11.93691715

12.68291949

14.37103058

8.00000000

8.78659358

9.57318717

10.35978075

11.09950770

11.88399865

14.73907340

14.04342360

13.84306778

14.67236565

13.76967001

13.55105400

14.42627481

15.15984164

15.99618722

13.68228198

12.79432874

33

Lattice $=" 4.974854628764761 \quad 0.0 \quad 0.0 \quad 0.0 \quad 12.1858553849967 \quad 0.0 \quad 0.0 \quad 0.021 .026408903419032 "$ Pro perties=species:S:1:pos:R:3 pbc="T T T"

$\begin{array}{lrrr}\mathrm{Ni} & 1.24371366 & 4.06195179 & 8.00000000 \\ \mathrm{Ni} & 0.00000000 & 0.00000000 & 8.71805841 \\ \mathrm{Ni} & 1.24371366 & 2.03097590 & 9.43611683 \\ \mathrm{Ni} & 0.00000000 & 4.06195179 & 10.15417524 \\ \mathrm{Ni} & 1.24371366 & 0.03301764 & 10.83466442 \\ \mathrm{Ni} & 0.00487565 & 2.04096313 & 11.60820497 \\ \mathrm{Ni} & 1.24371366 & 4.02577211 & 12.35936693 \\ \mathrm{Ni} & 0.00113171 & -0.04924535 & 12.88177666 \\ \mathrm{Ni} & 1.24371366 & 10.15487949 & 8.00000000 \\ \mathrm{Ni} & 0.00000000 & 6.09292769 & 8.71805841 \\ \mathrm{Ni} & 1.24371366 & 8.12390359 & 9.43611683 \\ \mathrm{Ni} & 0.00000000 & 10.15487949 & 10.15417524 \\ \mathrm{Ni} & 1.24371366 & 6.13705612 & 10.80847904 \\ \mathrm{Ni} & -0.00550543 & 8.14081496 & 11.61854785 \\ \mathrm{Ni} & 1.24371366 & 10.14790466 & 12.26253878 \\ \mathrm{Ni} & -0.04428660 & 6.09981040 & 12.94242054 \\ \mathrm{Ni} & 3.73114097 & 4.06195179 & 8.00000000 \\ \mathrm{Ni} & 2.48742731 & 0.00000000 & 8.71805841 \\ \mathrm{Ni} & 3.73114097 & 2.03097590 & 9.43611683 \\ \mathrm{Ni} & 2.48742731 & 4.06195179 & 10.15417524 \\ \mathrm{Ni} & 3.73114097 & 0.03742154 & 10.83600481 \\ \mathrm{Ni} & 2.48255167 & 2.04096313 & 11.60820497 \\ \mathrm{Ni} & 3.73114097 & 4.04112847 & 12.21800192 \\ \mathrm{Ni} & 2.48629560 & -0.04924535 & 12.88177666 \\ \mathrm{Ni} & 3.73114097 & 10.15487949 & 8.00000000 \\ \mathrm{Ni} & 2.48742731 & 6.09292769 & 8.71805841 \\ \mathrm{Ni} & 3.73114097 & 8.12390359 & 9.43611683 \\ \mathrm{Ni} & 2.48742731 & 10.15487949 & 10.15417524 \\ \mathrm{Ni} & 3.73114097 & 6.13061202 & 10.84661369 \\ \mathrm{Ni} & 2.49293274 & 8.14081496 & 11.61854785 \\ \mathrm{Ni} & 3.73114097 & 10.15539055 & 12.26655503 \\ \mathrm{Ni} & 2.53171392 & 6.09981040 & 12.94242054 \\ \mathrm{O} & 1.24371366 & 5.18228829 & 13.84417641 \\ . / 0 \times y g e n & \mathrm{Ni} & & \end{array}$

. /oxygen/Ni/211/iter_10/struct.xyz

42

Lattice $=" 4.974854628764761 \quad 0.0 \quad 0.0 \quad 0.0 \quad 12.1858553849967 \quad 0.0 \quad 0.0 \quad 0.0 \quad 21.026408903419032 " \quad$ Pro perties=species:S:1:pos:R:3 pbc="T T T"

$\mathrm{Ni}$

1.24371366

4.06195179

8.00000000 
$\mathrm{Ni}$

$\mathrm{Ni}$

0.00000000

1.24371366

0.00000000

1.25765366

0.03871705

1.30965224

4.77963981

1.24371366

0.00000000

1.24371366

0.00000000

1.24920383

$-0.05424299$

0.58382677

4.38846731

3.73114097

2.48742731

3.73114097

2.48742731

3.78738616

2.55585240

3.77183837

2. 30702728

3.73114097

2.48742731

3.73114097

2.48742731

3.71575267

2.40951847

3.52699287

1.67021815

0.08659890

3.08143010

3.13785371

1.12475962

0.24379761

3.63133522

1.11850904

3.77437865

1. 41489222

2. 30005512
Fri Apr 16 14:51:55 2021

203

0.00000000

8.71805841

2.03097590

4.06195179

0.02140395

1.97109127

4.01277571

$-0.26836756$

10.15487949

6.09292769

8.12390359

10.15487949

6.06027926

8.18917593

10.52989291

6.54961298

4.06195179

0.00000000

2.03097590

4.06195179

$-0.04059200$

2.08779490

4.26528576

0.78651107

10.15487949

6.09292769

8.12390359

10.15487949

6.11160302

8.12414157

10.00448885

5.68422154

4.75938090

11.46408907

6.19875655

0.76393326

10.27994957

0.87562313

6.94486181

8.24189711

2.22889057

10.07066001
9.43611683

10.15417524

10.76239675

11.73602414

12.31701225

15.04144560

8.00000000

8.71805841

9.43611683

10.15417524

10.90709220

11.53709202

12.49279359

13.57307083

8.00000000

8.71805841

9.43611683

10.15417524

10.90647690

11.52987724

12.31931537

13.94383946

8.00000000

8.71805841

9.43611683

10.15417524

10.78038177

11.64811172

13.59048857

14.08772075

13.63954568

14.60530388

14.76948257

15.24463057

14.32096539

12.55402655

12.62729450

13.05385894

13.05698793

12.15075244

. /oxygen/Ni/211/iter_11/struct.xyz 43

Lattice $=" 4.974854628764761 \quad 0.0 \quad 0.0 \quad 0.0 \quad 12.1858553849967 \quad 0.0 \quad 0.0 \quad 0.021 .026408903419032 "$ Pro perties=species:S:1:pos:R:3 pbc="T T T"

$\mathrm{Ni} \quad 1.24371366 \quad$ pos:R:3 pbc=0 T

$\mathrm{Ni} \quad 0.00000000 \quad 0.00000000$

$\mathrm{Ni} \quad 1.24371366 \quad 2.03097590$

$\mathrm{Ni} \quad 0.00000000 \quad 4.06195179$

$1.26613985 \quad 0.01310101$

$0.05889696 \quad 2.11976952$

$1.24043558 \quad 4.14454623$

$0.30525644 \quad-0.32391262$

$1.24371366 \quad 10.15487949$

$0.00000000 \quad 6.09292769$

$1.24371366 \quad 8.12390359$

$0.00000000 \quad 10.15487949$

$1.21763431 \quad 6.04377650$

$-0.08409424$

6.04377650

1.19790615

$-0.18268695$

10.25317933

6.26181488

3. 73114097

4.06195179

2. 48742731

3.73114097

0.00000000

2.03097590

4.06195179

2.48742731

3. 76023684

2.56867118

$-0.01883672$

2.17165005

8.00000000

8.71805841

9.43611683

10.15417524

10.84527792

11.68410221

12.60673398

14.72197408

8.00000000

8.71805841

9.43611683

10.15417524

10.91561914

11.58263328

12.36546005

13.68706000

8.00000000

8.71805841

9.43611683

10.15417524

10.85548806

11.67941404

4.31028290

12.32261728 
$\mathrm{Ni}$

$\mathrm{Ni} \quad 3.73114097$

$\mathrm{Ni} \quad 2.48742731$

$\mathrm{Ni} \quad 3.73114097$

$\mathrm{Ni} \quad 2.48742731$

$\mathrm{Ni} \quad 3.70843368$

$\mathrm{Ni} \quad 2.49323858$

$\mathrm{Ni} \quad 3.69604986$

$\mathrm{Ni} \quad 2.38481439$

$0 \quad 1.09861574$

$0 \quad 3.74521183$

O $\quad 3.60416958$

1.34280273

$-0.02132998$

3.78326075

1.14867271

3.61151451

1.31625946

2.43941220

2.69361211
Fri Apr 16 14:51:55 2021

0.58141855

10.15487949

6.09292769

8.12390359

10.15487949

6.11697637

8.15835425

10.20073926

6.33302578

5.09823297

0.19597276

5.72775496

0.92905922

10.39801280

0.88458065

7.07828538

7.71924578

0.94160494

10.82540000 3.23286749
13.99274063

8.00000000

8.71805841

9.43611683

10.15417524

10.76708787

11.58047102

12.41032714

13.77267882

14.18110522

15.29299099

14.85950306

15.26158029

13.70050886

12.49558029

12.54059805

13.19016866

12.49015780

13.51376689

13.22475500

. /oxygen/Ni/211/iter_12/struct.xyz 44

Lattice $=" 4.974854628764761 \quad 0.0 \quad 0.0 \quad 0.0 \quad 12.1858553849967 \quad 0.0 \quad 0.0 \quad 0.021 .026408903419032 "$ Pro perties=species:S:1:pos:R:3 pbc="T T T"

4371366

0.00000000

0.00000000

1.24371366

2.03097590

0.00000000

4.06195179

1.25503453

0.00319614

$-0.00438367$

2.06735265

4.16672160

.13691093

0.22682377

$-0.17021457$

1.24371366

0.00000000

10.15487949

6.09292769

8.12390359

.00000000

10.15487949

6.07406966

8.08795783

1.26196768

0.04700448

10.28624575

1. 21386251

0.17367742

5.75679001

4.06195179

0.00000000

2. 48742731

3. 73114097

2.03097590

4.06195179

$-0.04078188$

2.18190622

4.27165367

0.61070633

51171848

3.73309593

2.49685193

3.73114097

2. 48742731

3. 73114097

2.48742731

3. 74916552

2. 48329011

3. 72097547

2.73744973

1.83764814

3. 65200692

3. 97116278

1.29180158

0.02438408

3.72731553

1.39281701

3. 67483208

1. 26860151

2. 46264616

2. 46729504

$-0.06111859$

10.15487949

6.09292769

8.12390359

10.15487949

6.09296435

8.15562558

10.12303037

7.01659589

5.95562994

0.29212375

6.92353998

1.06449466

10.55254026

0.89681592

6.99020177

8.21825484

0.93896167

10.90770602

3.31552983

8.00000000

8.71805841

9.43611683

10.15417524

10.83752937

11.63289096

12.39642152

14.76937586

8.00000000

8.71805841

9.43611683

10.15417524

10.90521056

11.68137639

12.38261082

14.95031866

8.00000000

8.71805841

9.43611683

10.15417524

10.88573883

11.68521373

12.38081582

13.99654223

8.00000000

8.71805841

9.43611683

10.15417524

10.76262805

11.50449495

12.50261207

14.04160352

15.11538872

15.32666931

15.32287061

15.27509628

13.72939998

12.51200343

12.59224759

12.98620176

12.48297190

13.49776142

13.24743963

4.46897765

13.74623702 
. /oxygen/Ni/211/iter_13/struct.xyz

45

Lattice $=" 4.974854628764761 \quad 0.0 \quad 0.0 \quad 0.0 \quad 12.1858553849967 \quad 0.0 \quad 0.0 \quad 0.021 .026408903419032 "$ Pro perties $=$ species:S:1:pos:R:3 pbc="T T T"

$\mathrm{Ni} \quad 0.00000000 \quad 0.00000000$

$\mathrm{Ni} \quad 1.24371366 \quad 2.03097590$

$\mathrm{Ni} \quad 0.00000000 \quad 4.06195179$

$\mathrm{Ni} \quad 1.26180184 \quad 0.02488159$

$\mathrm{Ni} \quad-0.00546301 \quad 2.08606227$

$\mathrm{Ni} \quad 1.97745177$

4.62265902

$\mathrm{Ni} \quad 0.29432178$

12.03536421

1. 24371366

10.15487949

0.00000000

6.09292769

1. 24371366

8.12390359

0.00000000

1. 20965553

10.15487949

6.00734013

$-0.03482720$

8.07295219

1.17340158

10.30863741

4.81413883

6.49092030

3. 73114097

4. 06195179

2. 48742731

0.00000000

3. 73114097

2.03097590

2. 48742731

4.06195179

3. 73438511

2. 46363842

12.14372329

2. 28707534

4.00227798

4.20914585

0.71287942

2.56104982

3. 73114097

10.15487949

2. 48742731

6.09292769

8.12390359

3.73114097

10.15487949

3. 71365948

6.07345659

8.13163139

2.41588353

3. 66438675

10.12686960

2. 42741628

7.27157025

6.05629153

0.37224389

3.73916563

7.59460898

1.09135992

3. 53093726

10.53331141

4.96571671

3. 72565869

0.92572334

6.99509783

1. 30326226

8. 24458427

1.02207951

1. 27279476

2. 40680192

10.94774988

3. 30392158

4.86369803

4.20278027

4.33925047

8.00000000

8.71805841

9.43611683

10.15417524

10.85509660

11.58847350

14.25758001

14.69382148

8.00000000

8.71805841

9.43611683

10.15417524

10.97416230

11.69534511

12.38020196

15.52009703

8.00000000

8.71805841

9.43611683

10.15417524

10.86788159

11.69368371

12.34698647

13.97290440

8.00000000

8.71805841

9.43611683

10.15417524

10.76380481

11.49985760

12.52458288

14.17988524

15.25095699

15.27664334

15.52139350

15.25148046

13.73894893

12.46074460

12.60415438

13.00044916

12.48563287

13.51908013

13.30389244

14.96348386

13.88166255

. /oxygen/Ni/211/iter_2/struct.xyz

34

Lattice $=" 4.974854628764761 \quad 0.0 \quad 0.0 \quad 0.0 \quad 12.1858553849967 \quad 0.0 \quad 0.0 \quad 0.021 .026408903419032 "$ Pro perties=species:S:1:pos:R:3 pbc="T T T"

$\begin{array}{lrrr}\mathrm{Ni} & 1.24371366 & 4.06195179 & 8.00000000 \\ \mathrm{Ni} & 0.00000000 & 0.00000000 & 8.71805841 \\ \mathrm{Ni} & 1.24371366 & 2.03097590 & 9.43611683 \\ \mathrm{Ni} & 0.00000000 & 4.06195179 & 10.15417524 \\ \mathrm{Ni} & 1.24371366 & 0.03111162 & 10.84835517 \\ \mathrm{Ni} & 0.00979873 & 2.03392961 & 11.60230160 \\ \mathrm{Ni} & 1.24371366 & 4.01877923 & 12.36035300 \\ \mathrm{Ni} & 0.04580074 & -0.00024483 & 12.94661635 \\ \mathrm{Ni} & 1.24371366 & 10.15487949 & 8.00000000 \\ \mathrm{Ni} & 0.00000000 & 6.09292769 & 8.71805841 \\ \mathrm{Ni} & 1.24371366 & 8.12390359 & 9.43611683 \\ \mathrm{Ni} & 0.00000000 & 10.15487949 & 10.15417524 \\ \mathrm{Ni} & 1.24371366 & 6.13351199 & 10.81346567 \\ \mathrm{Ni} & -0.00979873 & 8.12685731 & 11.60230160 \\ \mathrm{Ni} & 1.24371366 & 10.12439640 & 12.21443690\end{array}$




$\begin{array}{lr}\mathrm{Ni} & -0.04580074 \\ \mathrm{Ni} & 3.73114097 \\ \mathrm{Ni} & 2.48742731 \\ \mathrm{Ni} & 3.73114097 \\ \mathrm{Ni} & 2.48742731 \\ \mathrm{Ni} & 3.73114097 \\ \mathrm{Ni} & 2.47762859 \\ \mathrm{Ni} & 3.73114097 \\ \mathrm{Ni} & 2.44162658 \\ \mathrm{Ni} & 3.73114097 \\ \mathrm{Ni} & 2.48742731 \\ \mathrm{Ni} & 3.73114097 \\ \mathrm{Ni} & 2.48742731 \\ \mathrm{Ni} & 3.73114097 \\ \mathrm{Ni} & 2.49722604 \\ \mathrm{Ni} & 3.73114097 \\ \mathrm{Ni} & 2.53322805 \\ \mathrm{O} & 1.24371366 \\ \mathrm{O} & 3.73114097\end{array}$

$.10 \times 120$

35

Lattice $=" 4.974854628764761 \quad 0.0 \quad 0.0 \quad 0.0 \quad 12.1858553849967 \quad 0.0 \quad 0.0 \quad 0.021 .026408903419032 "$ Pro perties=species:S:1:pos:R:3 pbc="T T T"

$\mathrm{N}$

$\mathrm{Ni}$

$\mathrm{Ni}$

$\mathrm{Ni}$

$\mathrm{Ni}$

$\mathrm{Ni}$

$\mathrm{Ni}$

$\mathrm{Ni}$

$\mathrm{Ni}$

$\mathrm{Ni}$

$\mathrm{Ni}$

$\mathrm{Ni}$

$\mathrm{Ni}$

$\mathrm{Ni}$

$\mathrm{Ni}$

$\mathrm{Ni}$

$\mathrm{Ni}$

$\mathrm{Ni}$

$\mathrm{Ni}$

$\mathrm{Ni}$

$\mathrm{Ni}$

$\mathrm{Ni}$

$\mathrm{Ni}$

$\mathrm{Ni}$

$\mathrm{Ni}$

$\mathrm{Ni}$

$\mathrm{Ni}$

$\mathrm{Ni}$

$\mathrm{Ni}$

$\mathrm{Ni}$

$\mathrm{Ni}$

$\mathrm{Ni}$

O

0

0

Fri Apr 16 14:51:55 2021
6.09268286

4.06195179

0.00000000

2.03097590

4.06195179

0.04058430

2.03392961

4.03146871

$-0.00024483$

10.15487949

6.09292769

8.12390359

10.15487949

6.12403931

8.12685731

10.11170692

6.09268286

5.17577296

11.26870065
206
12.94661635

8.00000000

8.71805841

9.43611683

10.15417524

10.81346567

11.60230160

12.21443690

12.94661635

8.00000000

8.71805841

9.43611683

10.15417524

10.84835517

11.60230160

12.36035300

12.94661635

13.85649489

13.85649489
8.00000000
8.71805841

9.43611683

10.15417524

10.84906234

11.59724457

12.30232217

12.96251919

8.71805841

9.43611683

10.15417524

10.84471434

11.64316511

12.21977838

13.14244410

8.00000000

8.71805841

9.43611683

10.15417524

10.81068232

11.59724457

12.22683326

12.96251919

8.00000000

8.71805841

9.43611683

10.15417524

10.82053676

11.64316511

12.35207614

13.14244410

13.84895241

13.88849029

13.06491901
8.00000000

36

Lattice $=" 4.974854628764761 \quad 0.0 \quad 0.0 \quad 0.0 \quad 12.1858553849967 \quad 0.0 \quad 0.0 \quad 0.021 .026408903419032 "$ Pro perties=species:S:1:pos:R:3 pbc="T T T"

$\mathrm{Ni} \quad 1.24371366 \quad 4.06195179$

$\mathrm{Ni} \quad 0.00000000 \quad 0.00000000$

$\mathrm{Ni} \quad 1.24371366 \quad 2.03097590$

$\mathrm{Ni} \quad 0.00000000 \quad 4.06195179$

$\mathrm{Ni} \quad 1.24371366 \quad 0.02566932$

$\mathrm{Ni} \quad-0.00661704 \quad 2.08202944$

8.00000000

8.71805841

9.43611683

10.15417524

10.81753597

11.63533295 
$\mathrm{Ni} \quad 0.00829036$

$\mathrm{Ni} \quad 1.24371366$

$\mathrm{Ni} \quad 0.00000000$

$\mathrm{Ni} \quad 1.24371366$

$\mathrm{Ni} \quad 0.00000000$

$\mathrm{Ni} \quad 1.24371366$

$\mathrm{Ni} \quad 0.00661704$

$\mathrm{Ni} \quad 1.24371366$

$\mathrm{Ni} \quad-0.00829036$

$\mathrm{Ni} \quad 3.73114097$

$\mathrm{Ni} \quad 2.48742731$

$\mathrm{Ni} \quad 3.73114097$

$\mathrm{Ni} \quad 2.48742731$

$\mathrm{Ni} \quad 3.73114097$

$\mathrm{Ni} \quad 2.49404436$

$\mathrm{Ni} \quad 3.73114097$

$\mathrm{Ni} \quad 2.47913695$

$\mathrm{Ni} \quad 3.73114097$

$\mathrm{Ni} \quad 2.48742731$

$\mathrm{Ni} \quad 3.73114097$

$\mathrm{Ni} \quad 2.48742731$

$\mathrm{Ni} \quad 3.73114097$

$\mathrm{Ni} \quad 2.48081027$

$\mathrm{Ni} \quad 3.73114097$

$\mathrm{Ni} \quad 2.49571768$

O $\quad 1.24371366$

3.73114097

3.73114097

1.24371366
Fri Apr 16 14:51:55 2021

207

4.14092226

0.07660754

10.15487949

6.09292769

8.12390359

10.15487949

6.14980915

8.17495713

10.22693944

6.16953523

4.06195179

0.00000000

2.03097590

4.06195179

0.05688146

2.08202944

4.13401174

0.07660754

10.15487949

6.09292769

8.12390359

10.15487949

6.11859701

8.17495713

10.23384996

6.16953523

5.06652687

11.15945456

7.55288718

1.45995948
12.28766608

13.16811252

8.00000000

8.71805841

9.43611683

10.15417524

10.83866022

11.63533295

12.22856028

13.16811252

8.00000000

8.71805841

9.43611683

10.15417524

10.83866022

11.63533295

12.22856028

13.16811252

8.00000000

8.71805841

9.43611683

10.15417524

10.81753597

11.63533295

12.28766608

13.16811252

13.87656300

13.87656300

13.07859541

13.07859541

. /oxygen/Ni/211/iter_5/struct.xyz

37

Lattice $=" 4.974854628764761 \quad 0.0 \quad 0.0 \quad 0.0 \quad 12.1858553849967 \quad 0.0 \quad 0.0 \quad 0.021 .026408903419032 "$ Pro perties $=$ species:S:1:pos:R: 3 pbc $=" T$ T T"

$\mathrm{N}$

4.06195179

8.00000000

0.00000000

0.00000000

8.71805841

1.24371366

2.03097590

0.00000000

4.06195179

1.23266812

0.01787122

9.43611683

$\mathrm{Ni}$

0.01787122

10.15417524

$-0.01708229$

4.14242502

10.82507447

1.27094821

11.57548451

4. 92330346

0.22391742

1.24371366

0.00000000

10.15487949

12.27945037

13.19532299

8.00000000

6.09292769

8.12390359

1.24371366

0.00000000

1.25363649

10.15487949

6.11449600

8.05611966

0.01521488

1. 28073421

3.73828930

10.28141836

6.33799283

4.06195179

0.00000000

2.48742731

2.03097590

4.06195179

0.03922578

2.10638290

4.12004189

0.19923826

10.15487949

6.09292769

8.12390359

10.15487949

6.11942099

8.14055642

10.20784140

6.35447954

4.96571895

8.71805841

9.43611683

10.15417524

10.79863902

11.66878716

12.30602735

13.26447007

8.00000000

8.71805841

9.43611683

10.15417524

10.86329432

11.59758500

12.27819194

13.16948941

8.00000000

8.71805841

9.43611683

10.15417524

10.79839443

11.64869413

12.43905228

13.26890886

13.50900559

13.90905617 


$\begin{array}{llll}0 & 2.49896899 & 7.60815771 & 13.44655393 \\ 0 & 1.20455297 & 1.59069486 & 13.07757089 \\ 0 & 0.10391756 & 9.16385955 & 13.12100992\end{array}$

. /oxygen/Ni/211/iter_6/struct.xyz

38

Lattice $=" 4.974854628764761 \quad 0.0 \quad 0.0 \quad 0.0 \quad 12.1858553849967 \quad 0.0 \quad 0.0 \quad 0.0 \quad 21.026408903419032 "$ Pro perties=species:S:1:pos:R:3 pbc="T T T"

$\mathrm{Ni}$

$\mathrm{Ni}$

$\mathrm{Ni}$

$\mathrm{Ni}$

$\mathrm{Ni}$

$\mathrm{Ni}$

$\mathrm{Ni}$

$\mathrm{Ni}$

$\mathrm{Ni}$

$\mathrm{Ni}$

$\mathrm{Ni}$

$\mathrm{Ni}$

$\mathrm{Ni}$

$\mathrm{Ni}$

$\mathrm{Ni}$

$\mathrm{Ni}$

$\mathrm{Ni}$

$\mathrm{Ni}$

$\mathrm{Ni}$

$\mathrm{Ni}$

$\mathrm{Ni}$

$\mathrm{Ni}$

$\mathrm{Ni}$

$\mathrm{Ni}$

$\mathrm{Ni}$

$\mathrm{Ni}$

$\mathrm{Ni}$

$\mathrm{Ni}$

$\mathrm{Ni}$

$\mathrm{Ni}$

$\mathrm{Ni}$

$\mathrm{Ni}$

O

O

0

O

O

0
1.24371366

0.00000000

1.24371366

0.00000000

1.22163321

$-0.03754218$

1.09272842

$-0.01154007$

1. 24371366

0.00000000

1.24371366

0.00000000

1.28694373

0.11259686

1. 33525828

4. 23711617

3. 73114097

2.48742731

3. 73114097

2.48742731

3.70896937

2.41813230

3. 52219655

2.44043950

3.73114097

2. 48742731

3.73114097

2.48742731

3. 78096190

2.58080496

3.77858055

1.75937466

0.46839701

3. 67708072

2. 92087131

1. 20432811

0.22469160

3. 66995691
4.06195179

0.00000000

2.03097590

4.06195179

0.02159360

2.08599279

4.20570296

0.05969544

10.15487949

6.09292769

8.12390359

10.15487949

6.11324246

8.07917745

10.24319613

6.41787908

4.06195179

0.00000000

2.03097590

4.06195179

0.00089274

2.10233583

4. 31144185

0.06415205

10.15487949

6.09292769

8.12390359

10.15487949

6.13013809

8.12802317

10.13554637

6.30420677

5.13096138

11.24945652

7. 64202643

1.40455958

9.10231882

2.60993021
8.00000000

8.71805841

9.43611683

10.15417524

10.81604642

11.65958299

12.27767465

13.20768294

8.00000000

8.71805841

9.43611683

10.15417524

10.79661528

11.62101416

12.28608762

13.32348983

8.00000000

8.71805841

9.43611683

10.15417524

10.86016432

11.63858272

12.36288987

13.17229489

8.00000000

8.71805841

9.43611683

10.15417524

10.82543869

11.62356986

12.46212426

13.27108995

13.75364822

13.95924956

13.40480979

13.12115958

13.13630788

12.93868651

39

Lattice $=" 4.974854628764761 \quad 0.0 \quad 0.0 \quad 0.0 \quad 12.1858553849967$ perties=species:S:1:pos:R:3 pbc="T T T"

$\mathrm{N}$

$\mathrm{Ni}$

$\mathrm{Ni}$

$\mathrm{Ni}$

$\mathrm{Ni}$

$\mathrm{Ni}$

$\mathrm{Ni}$

$\mathrm{Ni}$

$\mathrm{Ni}$

$\mathrm{Ni}$

$\mathrm{Ni}$

$\mathrm{Ni}$

$\mathrm{Ni}$

$\mathrm{Ni}$

$\mathrm{Ni}$

$\mathrm{Ni}$

$\mathrm{Ni}$

$\mathrm{Ni}$

$\mathrm{Ni}$
1.24371366

0.00000000

1. 24371366

0.00000000

1. 25377150

0.05296739

1. 20967743

4. 13141513

1. 24371366

0.00000000

1. 24371366

0.00000000

1. 22589063

4.87853746

1. 34355102

4. 92065268

3. 73114097

2. 48742731

3. 73114097
4.06195179

0.00000000

2.03097590

4.06195179

0.03121588

2.03100888

4.04468674

0.58772983

10.15487949

6.09292769

8.12390359

10.15487949

6.11617054

8.20683817

10.42245679

6.04063920

4.06195179

0.00000000

2.03097590
8.00000000

8.71805841

9.43611683

10.15417524

10.85629295

11.56681056

12.31911272

13.51407364

8.00000000

8.71805841

9.43611683

10.15417524

10.83760033

11.69506095

12.64393468

13.14923069

8.00000000

8.71805841

9.43611683 
$\mathrm{Ni}$

$\mathrm{Ni}$

2.48742731

3.76291738

2.49107030

3.71996141

1.52734613

3.73114097

2.48742731

3.73114097

2.48742731

3.71584639

2. 44828327

3.74119002

2.47959002

1.22104761

2.55227234

3.69805498

0.27671289

4.80857275

3.26580944

1.17681741
Fri Apr 16 14:51:55 2021

209

4.06195179

0.05014048

2.07981387

3.99517801

1.99241888

10.15487949

6.09292769

8.12390359

10.15487949

6.07301179

8.19674953

10.31962227

6.04895418

5.01261914

11.90924706

7.40134610

1.29960813

11.03834819

2.30184018

8.65270433
10.15417524

10.79914753

11.51348936

12.32765230

13.77353909

8.00000000

8.71805841

9.43611683

10.15417524

10.84250875

11.67319823

12.27904046

13.13266045

13.90369097

12.42914972

13.09899568

14.68583281

13.57298842

13.28172713

12.93676395

. /oxygen/Ni/211/iter_8/struct.xyz

40

Lattice $=" 4.974854628764761 \quad 0.0 \quad 0.0 \quad 0.0 \quad 12.1858553849967 \quad 0.0 \quad 0.0 \quad 0.021 .026408903419032 "$ Pro perties=species:S:1:pos:R:3 pbc="T T T"

$\mathrm{Ni} \quad 0.00000000 \quad 0.00000000$

$\mathrm{Ni} \quad 1.24371366 \quad 2.03097590$

$\mathrm{Ni} \quad 0.00000000 \quad 4.06195179$

$\mathrm{Ni} \quad 1.24303027 \quad 0.00720482$

$\mathrm{Ni} \quad 0.02432021 \quad 1.95374225$

4.06339520

1.21708592

0.40514180

$-0.23349417$

1.24371366

0.00000000

10.15487949

6.09292769

8.12390359

1.24371366

10.15487949

0.00000000

1.25508556

6.08115986

$-0.02733377$

8.20688512

1.37336093

$-0.56281816$

10.24848347

6.52106087

3.73114097

4.06195179

0.00000000

2.03097590

3.73114097

4.06195179

2.48742731

$-0.03863178$

2.08982685

2. 44876654

4.21625345

0.64903922

.67451890

2.50904117
3.73114097

10.15487949
6.09292769

8.12390359

3. 73114097

2. 48742731

10.15487949

3.73048256

6.08395614

8.16884258

2. 44726450

3. 68786571

1. 90943047

10.00817229

5.69081654

4.80132821

0.23841406

3. 58599338

11.68536234

3. 23101992

0.89016529

6.23905127

1.47501553

0.53876559

10.14161604

3. 51170143

2. 36248140

6.99831438

8.00000000

8.71805841

9.43611683

10.15417524

10.84469122

11.80446986

12.23779898

13.66362259

8.00000000

8.71805841

9.43611683

10.15417524

10.87836023

11.53793662

12.28868869

13.53192843

8.00000000

8.71805841

9.43611683

10.15417524

10.88909898

11.44355237

12.50811876

13.32221448

8.00000000

8.71805841

9.43611683

10.15417524

10.81472276

11.61476751

13.40823188

13.90417334

13.64798129

14.12197017

14.83714663

13.43178037

13.89212055

12.92311681

12.58924337

13.01095122

. /oxygen/Ni/211/iter_9/struct. xyz

41

Lattice $=" 4.974854628764761 \quad 0.0 \quad 0.0 \quad 0.0 \quad 12.1858553849967 \quad 0.0 \quad 0.0 \quad 0.021 .026408903419032 "$ Pro perties=species:S:1:pos:R:3 pbc="T T T" 
$\mathrm{Ni}$

$\mathrm{Ni} \quad 0.00000000$

$\mathrm{Ni} \quad 1.24371366$

$\mathrm{Ni} \quad 0.00000000$

$\mathrm{Ni} \quad 1.22381489$

$\mathrm{Ni} \quad 4.87966767$

$\mathrm{Ni} \quad 1.25015996$

$\mathrm{Ni} \quad 4.38514128$

$\mathrm{Ni} \quad 1.24371366$

$\mathrm{Ni} \quad 0.00000000$

$\mathrm{Ni} \quad 1.24371366$

$\mathrm{Ni} \quad 0.00000000$

$\mathrm{Ni} \quad 1.24543194$

$\mathrm{Ni} \quad 0.01578678$

$\mathrm{Ni} \quad 1.33633239$

$\mathrm{Ni} \quad 0.01296539$

$\mathrm{Ni} \quad 3.73114097$

$\mathrm{Ni} \quad 2.48742731$

$\mathrm{Ni} \quad 3.73114097$

$\mathrm{Ni} \quad 2.48742731$

$\mathrm{Ni} \quad 3.72186325$

$\mathrm{Ni} \quad 2.48238982$

$\mathrm{Ni} \quad 3.72718917$

$\mathrm{Ni} \quad 1.93013825$

$\mathrm{Ni} \quad 3.73114097$

$\mathrm{Ni} \quad 2.48742731$

$\mathrm{Ni} \quad 3.73114097$

$\mathrm{Ni} \quad 2.48742731$

$\mathrm{Ni} \quad 3.73501677$

$\mathrm{Ni} \quad 2.48743808$

$\mathrm{Ni} \quad 3.76930398$

$\mathrm{Ni} \quad 2.49479975$

1.25261110

2.61576967

3. 74251134

0.63640250

0.02139073

3.62761467

1.25875503

3.73681360

1.23260929
Fri Apr 16 14:51:55 2021

210

4.06195179

0.00000000

2.03097590

4.06195179

0.01434399

1.99221385

4.21644312

12.07889985

10.15487949

6.09292769

8.12390359

10.15487949

6.09541509

8.17005250

10.42257486

6.37881979

4.06195179

0.00000000

2.03097590

4.06195179

0.00142407

2. 04329204

4.19481906

0.84848233

10.15487949

6.09292769

8.12390359

10.15487949

6.10168273

8.16805451

10.26070066

6.37684582

5.18870817

11.24353786

5.12222359

0.56123826

10.35560142

1.52147435

7.77818388

7.75248325

2.44790567
8.00000000

8.71805841

9.43611683

10.15417524

10.81056000

11.67917049

12.40125846

13.79332406

8.00000000

8.71805841

9.43611683

10.15417524

10.82486404

11.59329773

12.42184698

13.43822494

8.00000000

8.71805841

9.43611683

10.15417524

10.77947030

11.56002795

12.28075177

13.71257827

8.00000000

8.71805841

9.43611683

10.15417524

10.80046111

11.59299288

12.32096792

13.43429728

13.95788669

13.56577335

13.84325847

14.83451570

13.68350070

13.15254596

13.12327504

13.09742226

12.94199640

. /oxygen/Ni/221/iter_1/struct.xyz

41

Lattice $=" 4.974854628764762 \quad 0.0 \quad 0.0 \quad 0.0 \quad 14.9245638862942820 .0 \quad 0.0 \quad 0.0 \quad 21.27663016512527 "$ Pr operties=species:S:1:pos:R:3 pbc="T T T"

$\begin{array}{lrrr}\mathrm{Ni} & 1.24371366 & 3.73114097 & 8.00000000 \\ \mathrm{Ni} & 0.00000000 & 1.65828488 & 8.58629224 \\ \mathrm{Ni} & 1.24371366 & 7.04771072 & 9.17258448 \\ \mathrm{Ni} & 0.00000000 & 4.97485463 & 9.75887672 \\ \mathrm{Ni} & 1.24371366 & 2.90199853 & 10.34516896 \\ \mathrm{Ni} & 0.00085863 & 0.77706607 & 10.89464526 \\ \mathrm{Ni} & 1.23867183 & 6.14061210 & 11.53910028 \\ \mathrm{Ni} & 0.00074522 & 4.10423858 & 12.09128359 \\ \mathrm{Ni} & 1.23666983 & 2.08616978 & 12.71922328 \\ \mathrm{Ni} & -0.04504861 & -0.00367854 & 13.20304580 \\ \mathrm{Ni} & 1.24371366 & 11.19342291 & 8.00000000 \\ \mathrm{Ni} & 0.00000000 & 9.12056682 & 8.58629224 \\ \mathrm{Ni} & 1.24371366 & 14.50999267 & 9.17258448 \\ \mathrm{Ni} & 0.00000000 & 12.43713657 & 9.75887672 \\ \mathrm{Ni} & 1.24371366 & 10.36428048 & 10.34516896 \\ \mathrm{Ni} & -0.00387431 & 8.24287513 & 10.89533381 \\ \mathrm{Ni} & 1.24061041 & 13.59537664 & 11.52110800 \\ \mathrm{Ni} & -0.00624149 & 11.55596542 & 12.10509830 \\ \mathrm{Ni} & 1.23618711 & 9.49826661 & 12.62458799 \\ \mathrm{Ni} & -0.00879025 & 7.47020982 & 13.14451770 \\ \mathrm{Ni} & 3.73114097 & 3.73114097 & 8.00000000 \\ \mathrm{Ni} & 2.48742731 & 1.65828488 & 8.58629224 \\ \mathrm{Ni} & 3.73114097 & 7.04771072 & 9.17258448\end{array}$


$\mathrm{Ni}$

$\mathrm{Ni} \quad 3.73114097$

$\mathrm{Ni} \quad 2.48418553$

$\mathrm{Ni} \quad 3.72655010$

$\mathrm{Ni} \quad 2.47864464$

$\mathrm{Ni} \quad 3.72453223$

$\mathrm{Ni} \quad 2.51262680$

$\mathrm{Ni} \quad 3.73114097$

$\mathrm{Ni} \quad 2.48742731$

$\mathrm{Ni} \quad 3.73114097$

$\mathrm{Ni} \quad 2.48742731$

$\mathrm{Ni} \quad 3.73114097$

$\mathrm{Ni} \quad 2.48466451$

$\mathrm{Ni} \quad 3.72826874$

$\mathrm{Ni} \quad 2.48616834$

$\mathrm{Ni} \quad 3.72410590$

$\mathrm{Ni} \quad 2.47783673$

O 1.23329269
Fri Apr 16 14:51:55 2021

4.97485463

2.90199853

0.77681671

6.13453271

4.10632579

2.08192397

$-0.00355481$

11.19342291

9.12056682

14.50999267

12.43713657

10.36428048

8.24290449

13.60292286

11.55583951

9.49744026

7.47112362

0.89370136
9.75887672

10.34516896

10.89584967

11.53920625

12.09169130

12.58746682

13.20586382

8.00000000

8.58629224

9.17258448

9.75887672

10.34516896

10.89503388

11.54857530

12.10522640

12.62612138

13.14464593

14.15733298

oxygen/Ni/221/iter_10/struct.xyz

50

Lattice $=" 4.974854628764762 \quad 0.0 \quad 0.0 \quad 0.0 \quad 14.9245638862942820 .0 \quad 0.0 \quad 0.0 \quad 21.27663016512527 "$ Pr operties=species:S:1:pos:R:3 pbc="T T T"

$\mathrm{Ni}$

$\mathrm{Ni}$

1.24371366

3.73114097

0.00000000

1.65828488

1.24371366

7.04771072

0.00000000

4.97485463

1.24371366

2.90199853

0.00837560

0.82642168

1.23562691

6.20277170

4. 94141856

1.25841393

4. 12407164

2.05351295

0.02225440

1. 24371366

0.00000000

1. 24371366

0.00000000

1.24371366

4. 97031504

1. 24063714

0.00483448

1. 24778072

0.02400154

3. 73114097

2. 48742731

3. 73114097

2. 48742731

3. 73114097

2. 47711071

3. 74067800

2. 48526723

3. 69288850

2. 48004132

3. 73114097

2. 48742731

3. 73114097

2. 48742731

3. 73114097

2. 49805029

3. 72976618

2. 49737827

3. 75701535

14.92390881

11.19342291

9.12056682

14.50999267

12.43713657

10.36428048

8.27079632

13.71631923

11.56332949

9.46035896

7.47032814

3.73114097

1. 65828488

7.04771072

4.97485463

2.90199853

0.82200961

6.20870318

4.80718391

1.99146673

0.01143846

11.19342291

9.12056682

14.50999267

12.43713657

10.36428048

8.27430696

13.69814272

11.56488351

9.47515563

7.55979939

1.03804025

8.75926989

1. 25846265

1. 24855789

3. 73886796

3. 72723845

3. 73928341

3. 72298687

13.70535456

6.21387634

11.14934437

3. 54268469

8.00000000

8.58629224

9.17258448

9.75887672

10.34516896

10.90946505

11.45482590

12.13009058

12.71466486

13.50686523

8.00000000

8.58629224

9.17258448

9.75887672

10.34516896

10.89877921

11.52777300

12.15987677

12.61126085

13.97918983

8.00000000

8.58629224

9.17258448

9.75887672

10.34516896

10.87246620

11.49546720

13.43624287

12.61074575

13.45811853

8.00000000

8.58629224

9.17258448

9.75887672

10.34516896

10.89776453

11.49105756

12.16093201

12.76320319

13.85981961

14.26322635

14.30751784

13.36710311

13.42166558

13.41837282

13.49749861 


\section{0}

O

0

0
1.25051552

1.29454308

3.76602250

1.15507218
Fri Apr 16 14:51:55 2021

212
12.60387068

6.26929447

8.67324089

3.68040483
13.00772761

13.34851135

14.44664003

13.49280143

51

Lattice $=" 4.974854628764762 \quad 0.0 \quad 0.0 \quad 0.0 \quad 14.924563886294282 \quad 0.0 \quad 0.0 \quad 0.0 \quad 21.27663016512527 "$ Pr operties=species:S:1:pos:R:3 pbc="T T T"

$\mathrm{Ni}$

$\mathrm{Ni} \quad 0.00000000$

$\mathrm{Ni} \quad 1.24371366$

$\mathrm{Ni} \quad 0.00000000$

1.24371366

$\mathrm{Ni} \quad-0.00235738$

$\mathrm{Ni} \quad \begin{array}{rr}\mathrm{Ni} & -0.00615136\end{array}$

$\mathrm{Ni} \quad 1.25434436$

$\mathrm{Ni} \quad 0.00347980$

$\mathrm{Ni} \quad 1.24371366$

$\mathrm{Ni} \quad 0.00000000$

$\mathrm{Ni} \quad 1.24371366$

0.00000000

1.24371366

0.00233514

1.24231034

0.05766096

1.24576846

0.00708849

3.73114097

3.73114097

1.65828488

7.04771072

4.97485463

2.90199853

0.83209957

6.19864582

4.10612986

1.95539357

14.70020749

11.19342291

9.12056682

14.50999267

12.43713657

10.36428048

8.26788955

13.70361082

11.64717690

9.52047625

7.46729968

3.73114097

1. 65828488

7.04771072

3.73114097

4.97485463

2.90199853

3.73114097

0.83294983

6.20554365

.48080281

4.78797028

1.96938728

2.48006821

3.69991068

2. 43489124

3. 73114097

2. 48742731

3. 73114097

14.68322341

11.19342291

9.12056682

14.50999267

12.43713657

10.36428048

8.27079465

3.73114097

2. 49349767

3.72690471

13.70779897

2. 46978991

3. 75357200

11.63953987

9.46417384

7.55642665

0.94741979

8.76026258

1.22244018

1.22976130

3.70785637

3. 70098284

13.51934856

6.21975901

3. 74435112

3. 77750213

1.21567938

11.12335041

3.60429642

13.30216328

6.24366116

8.67102535

3.60311670

0.93969160

3.74610704

1.18484755

3.70307243

. /oxygen/Ni/221/iter_12/struct.xyz

8.00000000

8.58629224

9.17258448

9.75887672

10.34516896

10.91918670

11.46003000

12.10701026

12.74000856

13.71714094

8.00000000

8.58629224

9.17258448

9.75887672

10.34516896

10.90163999

11.43397445

12.17055067

12.61821478

13.96181765

8.00000000

8.58629224

9.17258448

9.75887672

10.34516896

10.88023800

11.49155869

13.39932413

12.71907084

13.74348427

8.00000000

8.58629224

9.17258448

9.75887672

10.34516896

10.90240129

11.42611801

12.15267113

12.72823878

13.84009956

14.30679197

14.28755346

13.27297372

13.40866786

13.36881026

13.47455256

13.27052364

13.35510405

14.41378304

13.47128758

14.25395871

52

Lattice $=" 4.974854628764762 \quad 0.0 \quad 0.0 \quad 0.0 \quad 14.9245638862942820 .0 \quad 0.0 \quad 0.021 .27663016512527 "$ Pr operties=species:S:1:pos:R:3 pbc="T T T"

$\begin{array}{lllr}\mathrm{Ni} & 1.24371366 & 3.73114097 & 8.00000000 \\ \mathrm{Ni} & 0.00000000 & 1.65828488 & 8.58629224 \\ \mathrm{Ni} & 1.24371366 & 7.04771072 & 9.17258448 \\ \mathrm{Ni} & 0.00000000 & 4.97485463 & 9.75887672 \\ \mathrm{Ni} & 1.24371366 & 2.90199853 & 10.34516896\end{array}$


$\mathrm{Ni}$

$\mathrm{Ni}$

$\mathrm{Ni}$

$\mathrm{Ni}$

$\mathrm{Ni}$

$\mathrm{Ni}$

$\mathrm{Ni}$

$\mathrm{Ni}$

$\mathrm{Ni}$

$\mathrm{Ni}$

$\mathrm{Ni}$

$\mathrm{Ni}$

$\mathrm{Ni}$

$\mathrm{Ni}$

$\mathrm{Ni}$

$\mathrm{Ni}$

$\mathrm{Ni}$

$\mathrm{Ni}$

$\mathrm{Ni}$

$\mathrm{Ni}$

$\mathrm{Ni}$

$\mathrm{Ni}$

$\mathrm{Ni}$

$\mathrm{Ni}$

$\mathrm{Ni}$

$\mathrm{Ni}$

$\mathrm{Ni}$

$\mathrm{Ni}$

$\mathrm{Ni}$

$\mathrm{Ni}$

$\mathrm{Ni}$

$\mathrm{Ni}$

$\mathrm{Ni}$

$\mathrm{Ni}$

$\mathrm{Ni}$

0

0

0

0

O

O

0

0

O

0

0

0
0.00284972

1.23721649

0.00489066

1.28462602

0.00599316

1.24371366

0.00000000

1.24371366

0.00000000

1.24371366

0.00267144

1. 25731420

0.00402259

1.21407873

0.00577681

3. 73114097

2. 48742731

3. 73114097

2. 48742731

3. 73114097

2. 49009876

3.74474151

2. 49144990

3. 70150604

2. 49320412

3. 73114097

2.48742731

3.73114097

3. 73114097

2. 49027703

3.72464380

2.49231797

3.77205334

2.49342048

1.26731131

1.23201309

3.76348670

3.70901791

3.67606638

3.79561135

1.22159060

1.27605938

3.75473863

1.18863906

3.71944040

1.30818404
2.48742731
Fri Apr 16 14:51:55 2021

0.82647966

6.22734434

4.14464412

2.02921105

0.09302478

11.19342291

9.12056682

14.50999267

12.43713657

10.36428048

8.28546369

13.68977132

12.29153480

9.49322893

7.44901699

3.73114097

1. 65828488

7.04771072

4.97485463

0.82318175

6.22748938

4.82925286

2.03094698

14.91129894

11.19342291

9.12056682

14.50999267

12.43713657

10.36428048

8.28876160

11.60692607

9.49149299

7.55530673

1.21585509

8.67866682

13.71299384

6.24960394

11.12938312

3.66844555

13.71188589

6.25071190

8.67813703

3.66710118

1.21638488

11.13072750
2.90199853

13.68962628
10.92403096

11.45582975

12.07399986

12.69733915

13.83762639

8.00000000

8.58629224

9.75887672

10.34516896

10.89049188

11.45615838

13.37269500

12.69883391

13.98056243

8.00000000

8.58629224

9.17258448

9.75887672

10.34516896

10.89049188

11.45615838

13.37269500

12.69883391

13.98056243

8.00000000

8.58629224

9.17258448

9.75887672

10.34516896

10.92403096

11.45582975

12.07399986

12.69733915

13.83762639

14.39234324

14.39239212

13.35601600

13.35566971

13.44665680

13.44856737

13.35566971

13.35601600

14.39234324

13.44665680

14.39239212

13.44856737
9.17258448

213

53

Lattice $=" 4.974854628764762 \quad 0.0 \quad 0.0 \quad 0.0 \quad 14.9245638862942820 .0 \quad 0.0 \quad 0.0 \quad 21.27663016512527 "$ Pr operties=species:S:1:pos:R:3 pbc="T T T"

$\begin{array}{llrr}\mathrm{Ni} & 1.24371366 & 3.73114097 & 8.00000000 \\ \mathrm{Ni} & 0.00000000 & 1.65828488 & 8.58629224 \\ \mathrm{Ni} & 1.24371366 & 7.04771072 & 9.17258448 \\ \mathrm{Ni} & 0.00000000 & 4.97485463 & 9.75887672 \\ \mathrm{Ni} & 1.24371366 & 2.90199853 & 10.34516896 \\ \mathrm{Ni} & 0.01696411 & 0.81497547 & 10.91914393 \\ \mathrm{Ni} & 1.22354375 & 6.23351854 & 11.47532130 \\ \mathrm{Ni} & 0.04462384 & 4.15121148 & 12.09357143 \\ \mathrm{Ni} & 1.31757880 & 1.96188069 & 12.69271637 \\ \mathrm{Ni} & 0.08600875 & 14.79628421 & 13.70836525 \\ \mathrm{Ni} & 1.24371366 & 11.19342291 & 8.00000000 \\ \mathrm{Ni} & 0.00000000 & 9.12056682 & 8.58629224 \\ \mathrm{Ni} & 1.24371366 & 14.50999267 & 9.17258448 \\ \mathrm{Ni} & 0.00000000 & 12.43713657 & 9.75887672 \\ \mathrm{Ni} & 1.24371366 & 10.36428048 & 10.34516896 \\ \mathrm{Ni} & 4.96632542 & 8.29807877 & 10.84888470 \\ \mathrm{Ni} & 1.24319481 & 13.65227276 & 11.47730994\end{array}$


$\mathrm{Ni}$

$\mathrm{Ni}$

4.81452288

0.99314254

4.95745089

3.73114097

2.48742731

3.73114097

2.48742731

3.73114097

2.50204228

3.74893199

2.53144231

3. 74988275

2.52314131

3.73114097

2.48742731

3.73114097

2. 48742731

3.73114097

2.50900852

3.74146437

2.45486496

3.84663845

2.40484893

1.31831559

1.25630242

3. 82794888

3. 67885238

3.25837575

3. 84152797

1.27488301

1.23428855

3.80740197

1.25307635

3.77313433

0.50862592

2.47114306
Fri Apr 16 14:51:55 2021

214

12.00266676

9.67760182

6.88906619

3.73114097

1. 65828488

7.04771072

4. 97485463

2.90199853

0.81426046

6.23519590

4.81656073

1.99931694

14.68830326

11.19342291

9.12056682

14.50999267

12.43713657

10.36428048

8.34936492

13.65622475

11.58715367

9.46898138

7.26675886

1.00101989

7.23878776

13.52295013

6.32299989

11.09603631

3. 64244009

13.30753829

6.16044090

8.11030582

3.60269585

1.00212070

10.40352246

8.64364673
13.61227512

12.64717313

14.57380480

8.00000000

8.58629224

9.17258448

9.75887672

10.34516896

10.88193525

11.46427089

13.30358650

12.67713774

13.86469306

8.00000000

8.58629224

9.17258448

9.75887672

10.34516896

11.10391826

11.41982621

11.99948862

14.00393821

14.41249122

14.29829182

15.72683936

13.26707239

13.32555135

13.53357192

13.43777874

13.35929447

13.36175085

15.25675284

13.41977750

14.23302561

14.24062170

12.98764744

. /oxygen/Ni/221/iter_14/struct.xyz

54

Lattice $=" 4.974854628764762 \quad 0.0 \quad 0.0 \quad 0.0 \quad 14.9245638862942820 .0 \quad 0.0 \quad 0.0 \quad 21.27663016512527 "$ Pr operties=species:S:1:pos:R:3 pbc="T T T"

$\mathrm{Ni}$

$\mathrm{Ni}$

$\mathrm{Ni}$

$\mathrm{Ni}$

$\mathrm{Ni}$

$\mathrm{Ni}$

$\mathrm{Ni}$

$\mathrm{Ni}$

$\mathrm{Ni}$

$\mathrm{Ni}$

$\mathrm{Ni}$

$\mathrm{Ni}$

$\mathrm{Ni}$

$\mathrm{Ni}$

$\mathrm{Ni}$

$\mathrm{Ni}$

$\mathrm{Ni}$

$\mathrm{Ni}$

$\mathrm{Ni}$

$\mathrm{Ni}$

$\mathrm{Ni}$

$\mathrm{Ni}$

$\mathrm{Ni}$

$\mathrm{Ni}$

$\mathrm{Ni}$

$\mathrm{Ni}$

$\mathrm{Ni}$

$\mathrm{Ni}$

\subsection{6}

0.00000000

1. 24371366

0.00000000

1. 24371366

0.04151685

1. 26821528

4. 89876129

1. 44406112

0.17527572

1. 24371366

0.00000000

1. 24371366

0.00000000

1.24371366

0.00682528

1. 27239686

4.90703467

1. 12481407

0.14435452

3. 73114097

2. 48742731

3. 73114097

2. 48742731

3. 73114097

2. 49425259

3. 75982418

2. 41960735
3.73114097

1. 65828488

7.04771072

4.97485463

2. 90199853

0.82927251

6.18422342

4.12931263

1. 77157207

14.33030077

11.19342291

9.12056682

14.50999267

12.43713657

10.36428048

8.28001983

13.66271954

11.78950662

9.53358288

6.60388534

3.73114097

1.65828488

7. 04771072

4.97485463

2. 90199853

0.81773789

6.20043760

4. 32722468
8.00000000

8.58629224

9.17258448

9.75887672

10.34516896

11.02316732

11.42404444

12.05491306

13.91238514

14.30534833

8.00000000

8.58629224

9.17258448

9.75887672

10.34516896

10.86387765

11.45729792

13.51781701

12.63736733

14.42546239

8.00000000

8.58629224

9.17258448

9.75887672

10.34516896

10.86387765

11.45729792

13.51781701 
$\mathrm{Ni}$

$\mathrm{Ni} \quad 2.63178183$

$\mathrm{Ni} \quad 3.73114097$

$\mathrm{Ni} \quad 2.48742731$

$\mathrm{Ni} \quad 3.73114097$

$\mathrm{Ni} \quad 2.48742731$

$\mathrm{Ni} \quad 3.73114097$

$\mathrm{Ni} \quad 2.52894417$

$\mathrm{Ni} \quad 3.75564260$

$\mathrm{Ni} \quad 2.41133398$

$\mathrm{Ni} \quad 3.93148843$

$\mathrm{Ni} \quad 2.66270304$

o $\quad 1.49237757$

0 $\quad 1.41361570$

O $\quad 3.92804511$

O $\quad 3.89422068$

O $\quad 3.34167551$

O $\quad 3.04937855$

o $\quad 1.40679337$

O $\quad 1.44061779$

O $\quad 3.97980489$

$0 \quad 0.85424820$

$0 \quad 3.90104302$

O $\quad 0.56195124$

o $\quad 2.55772945$ 0 $\quad 0.07030213$

55

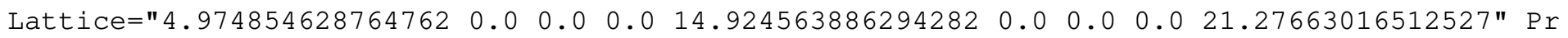
operties=species:S:1:pos:R:3 pbc="T T T"

\begin{tabular}{|c|c|c|c|}
\hline $\mathrm{Ni}$ & 1.24371366 & 3.73114097 & 8.00000000 \\
\hline $\mathrm{Ni}$ & 0.00000000 & 1.65828488 & 8.58629224 \\
\hline $\mathrm{Ni}$ & 1.24371366 & 7.04771072 & 9.17258448 \\
\hline $\mathrm{Ni}$ & 0.00000000 & 4.97485463 & 9.75887672 \\
\hline $\mathrm{Ni}$ & 1.24371366 & 2.90199853 & 10.34516896 \\
\hline $\mathrm{Ni}$ & 0.08562647 & 0.82873799 & 11.10710530 \\
\hline $\mathrm{Ni}$ & 1.23249083 & 6.20244229 & 11.44321047 \\
\hline $\mathrm{Ni}$ & -0.04796652 & 4.12682594 & 11.98247791 \\
\hline $\mathrm{Ni}$ & 1.58163838 & 2.00751698 & 13.97111864 \\
\hline $\mathrm{Ni}$ & -0.24822919 & -0.32226728 & 14.54069080 \\
\hline $\mathrm{Ni}$ & 1.24371366 & 11.19342291 & 8.00000000 \\
\hline $\mathrm{Ni}$ & 0.00000000 & 9.12056682 & 8.58629224 \\
\hline $\mathrm{Ni}$ & 1.24371366 & 14.50999267 & 9.17258448 \\
\hline $\mathrm{Ni}$ & 0.00000000 & 12.43713657 & 9.75887672 \\
\hline $\mathrm{Ni}$ & 1.24371366 & 10.36428048 & 10.34516896 \\
\hline $\mathrm{Ni}$ & -0.00846130 & 8.27908870 & 10.85358087 \\
\hline $\mathrm{Ni}$ & 1.26164999 & 13.67291842 & 11.44905649 \\
\hline $\mathrm{Ni}$ & -0.14904523 & 11.89218652 & 13.49956476 \\
\hline $\mathrm{Ni}$ & 1.06047981 & 9.56948056 & 12.61981166 \\
\hline $\mathrm{Ni}$ & 0.01841233 & 6.74867132 & 14.52906746 \\
\hline $\mathrm{Ni}$ & 3.73114097 & 3.73114097 & 8.00000000 \\
\hline $\mathrm{Ni}$ & 2.48742731 & 1.65828488 & 8.58629224 \\
\hline $\mathrm{Ni}$ & 3.73114097 & 7.04771072 & 9.17258448 \\
\hline $\mathrm{Ni}$ & 2.48742731 & 4.97485463 & 9.75887672 \\
\hline $\mathrm{Ni}$ & 3.73114097 & 2.90199853 & 10.34516896 \\
\hline $\mathrm{Ni}$ & 2.52626346 & 0.83916319 & 10.88203509 \\
\hline $\mathrm{Ni}$ & 3.73925156 & 6.19075089 & 11.46435854 \\
\hline $\mathrm{Ni}$ & 2.37904243 & 4.58719762 & 13.47013433 \\
\hline $\mathrm{Ni}$ & 3.71976300 & 2.08333294 & 12.65683911 \\
\hline $\mathrm{Ni}$ & 2.49318408 & -1.42738396 & 14.55339747 \\
\hline $\mathrm{Ni}$ & 3.73114097 & 11.19342291 & 8.00000000 \\
\hline & 2.48742731 & 9.12056682 & 8.58629224 \\
\hline $\mathrm{Ni}$ & 3.73114097 & 14.50999267 & 9.17258448 \\
\hline $\mathrm{Ni}$ & 2.48742731 & 12.43713657 & 9.75887672 \\
\hline & 3.73114097 & 10.36428048 & 10.34516896 \\
\hline $\mathrm{Ni}$ & 2.51943528 & 8.30296008 & 11.05192782 \\
\hline & 3.74030144 & 13.68655539 & 11.41862222 \\
\hline & & & \\
\hline
\end{tabular}

$2.07130094 \quad 12.63736733$

$14.06616728 \quad 14.42546239$

$11.19342291 \quad 8.00000000$

$9.12056682 \quad 8.58629224$

$14.50999267 \quad 9.17258448$

$12.43713657 \quad 9.75887672$

$10.36428048 \quad 10.34516896$

$8.29155445 \quad 11.02316732$

$13.64650536 \quad 11.42404444$

$11.59159458 \quad 12.05491306$

$9.23385401 \quad 13.91238514$

$6.86801882 \quad 14.30534833$

$0.35344664 \quad 15.11033382$

$7.11054091 \quad 15.51412001$

$13.33862387 \quad 13.26210306$

$5.57701687 \quad 13.27939440$

$10.88037888 \quad 13.46536676$

$2.72442191 \quad 14.21862940$

$13.03929881 \quad 13.27939440$

$5.87634192 \quad 13.26210306$

$7.81572858 \quad 15.11033382$

$3.41809693 \quad 13.46536676$

$14.57282285 \quad 15.51412001$

$10.18670385 \quad 14.21862940$

$8.41452255 \quad 12.92382479$

$0.95224061 \quad 12.92382479$
215 
$\mathrm{Ni}$

$\mathrm{Ni}$

O

0

0

O

0

0

0

0

0

O

O

O

O

O

0
3.88541256

2.50074096

2.13638022

1.30032546

3.87974281

3.74031927

3.24625760

3.14433675

1.39719605

1.29435478

3.86283288

0.87790147

3.83970903

0.50695026

2.52760164

0.18988712

1.31984119
Fri Apr 16 14:51:55 2021

13.94500806

14.36415755

15.28184810

15.62866884

13.24211642

13.34330607

13.55838358

14.09780271

13.17082358

13.31228189

15.20535218

13.41010857

15.70090863

14.18568233

12.95628058

13.26218265

15.51845319

. /oxygen/Ni/221/iter_2/struct.xyz

42

Lattice $=" 4.974854628764762 \quad 0.0 \quad 0.0 \quad 0.0 \quad 14.9245638862942820 .0 \quad 0.0 \quad 0.021 .27663016512527 "$ Pr operties=species:S:1:pos:R:3 pbc="T T T"

$\begin{array}{lrrr}\mathrm{Ni} & 1.24371366 & 3.73114097 & 8.00000000 \\ \mathrm{Ni} & 0.00000000 & 1.65828488 & 8.58629224 \\ \mathrm{Ni} & 1.24371366 & 7.04771072 & 9.17258448 \\ \mathrm{Ni} & 0.00000000 & 4.97485463 & 9.75887672 \\ \mathrm{Ni} & 1.24371366 & 2.90199853 & 10.34516896 \\ \mathrm{Ni} & 0.00101919 & 0.78182152 & 10.89216439 \\ \mathrm{Ni} & 1.24371366 & 6.14496323 & 11.51136102 \\ \mathrm{Ni} & 0.00310409 & 4.11338151 & 12.09209053 \\ \mathrm{Ni} & 1.24371366 & 2.08719091 & 12.71531394 \\ \mathrm{Ni} & 4.93656831 & 14.91448599 & 13.19643831 \\ \mathrm{Ni} & 1.24371366 & 11.19342291 & 8.00000000 \\ \mathrm{Ni} & 0.00000000 & 9.12056682 & 8.58629224 \\ \mathrm{Ni} & 1.24371366 & 14.50999267 & 9.17258448 \\ \mathrm{Ni} & 0.00000000 & 12.43713657 & 9.75887672 \\ \mathrm{Ni} & 1.24371366 & 10.36428048 & 10.34516896 \\ \mathrm{Ni} & 0.00092391 & 8.24506126 & 10.89200054 \\ \mathrm{Ni} & 1.24371366 & 13.60729913 & 11.51453509 \\ \mathrm{Ni} & 0.00373273 & 11.57360394 & 12.09287871 \\ \mathrm{Ni} & 1.24371366 & 9.54710974 & 12.71736382 \\ \mathrm{Ni} & 4.93655346 & 7.44888156 & 13.19471915 \\ \mathrm{Ni} & 3.73114097 & 3.73114097 & 8.00000000 \\ \mathrm{Ni} & 2.48742731 & 1.65828488 & 8.58629224 \\ \mathrm{Ni} & 3.73114097 & 7.04771072 & 9.17258448 \\ \mathrm{Ni} & 2.48742731 & 4.97485463 & 9.75887672 \\ \mathrm{Ni} & 3.73114097 & 2.90199853 & 10.34516896 \\ \mathrm{Ni} & 2.48640813 & 0.78182152 & 10.89216439 \\ \mathrm{Ni} & 3.73114097 & 6.14615037 & 11.53557528 \\ \mathrm{Ni} & 2.48432322 & 4.11338151 & 12.09209053 \\ \mathrm{Ni} & 3.73114097 & 2.08446701 & 12.58857436 \\ \mathrm{Ni} & 2.52571363 & 14.91448599 & 13.19643831 \\ \mathrm{Ni} & 3.73114097 & 11.19342291 & 8.00000000 \\ \mathrm{Ni} & 2.48742731 & 9.12056682 & 8.58629224 \\ \mathrm{Ni} & 3.73114097 & 14.50999267 & 9.17258448 \\ \mathrm{Ni} & 2.48742731 & 12.43713657 & 9.75887672 \\ \mathrm{Ni} & 3.73114097 & 10.36428048 & 10.34516896 \\ \mathrm{Ni} & 2.48650341 & 8.24506126 & 10.89200054 \\ \mathrm{Ni} & 3.73114097 & 13.60776324 & 11.53847684 \\ \mathrm{Ni} & 2.48369459 & 11.57360394 & 12.09287871 \\ \mathrm{Ni} & 3.73114097 & 9.54578109 & 12.58964444 \\ \mathrm{Ni} & 2.52572849 & 7.44888156 & 13.19471915 \\ \mathrm{O} & 1.24371366 & 0.87735301 & 14.14919326 \\ \mathrm{O} & 1.24371366 & 8.33423389 & 14.14920532 \\ \mathrm{~N} & / \mathrm{N} \times 1 / 221 / 169 & & \end{array}$

. /oxygen/Ni/221/iter_3/struct.xyz

43

Lattice $=" 4.974854628764762 \quad 0.0 \quad 0.0 \quad 0.0 \quad 14.9245638862942820 .0 \quad 0.0 \quad 0.021 .27663016512527 "$ Pr operties=species:S:1:pos:R:3 pbc="T T T" $\mathrm{Ni}$

$1.24371366 \quad 3.73114097$

8.00000000 
$\mathrm{Ni}$

$\mathrm{Ni}$

$\mathrm{Ni}$

$\mathrm{Ni}$

$\mathrm{Ni}$

$\mathrm{Ni}$

$\mathrm{Ni}$

$\mathrm{Ni}$

$\mathrm{Ni}$

$\mathrm{Ni}$

$\mathrm{Ni}$

$\mathrm{Ni}$

$\mathrm{Ni}$

$\mathrm{Ni}$

$\mathrm{Ni}$

$\mathrm{Ni}$

$\mathrm{Ni}$

$\mathrm{Ni}$

$\mathrm{Ni}$

$\mathrm{Ni}$

$\mathrm{Ni}$

$\mathrm{Ni}$

$\mathrm{Ni}$

$\mathrm{Ni}$

$\mathrm{Ni}$

$\mathrm{N}$

$\mathrm{Ni}$

$\mathrm{Ni}$

$\mathrm{Ni}$

$\mathrm{Ni}$

$\mathrm{Ni}$

$\mathrm{Ni}$

$\mathrm{Ni}$

$\mathrm{Ni}$

$\mathrm{Ni}$

$\mathrm{Ni}$

$\mathrm{Ni}$

$\mathrm{Ni}$

$\mathrm{Ni}$

0

0

0
0.00000000

1.24371366

0.00000000

1.24371366

0.00461280

1.24371366

4.97196248

1.24371366

0.00217678

1.24371366

0.00000000

1.24371366

0.00000000

1.24371366

0.00201543

1. 24371366

0.02021797

1.24371366

4.93640295

3. 73114097

2. 48742731

3. 73114097

2.48742731

3. 73114097

2. 48281452

3.73114097

2.49031946

3. 73114097

2. 48525054

3. 73114097

2. 48742731

3.73114097

2. 48742731

3. 73114097

2.48541189

3.73114097

2.46720935

3.73114097

2.52587899

1.24371366

1.24371366

3.73114097
Fri Apr 16 14:51:55 2021

217

1.65828488

7.04771072

4.97485463

2.90199853

0.78859176

6.14282635

4.11913099

2.09479302

14.89646134

11.19342291

9.12056682

14.50999267

12.43713657

10.36428048

8.24135066

13.64119324

11.54394708

9.53400375

7.43789543

3.73114097

1. 65828488

7.04771072

4.97485463

2.90199853

0.78859176

6.14856024

4.11913099

2.07222055

14.89646134

11.19342291

9.12056682

14.50999267

12.43713657

10.36428048

8.24135066

13.61649798

11.54394708

9.52548929

7.43789543

1.06231268

8.32405006

13.56779131
8.58629224

9.17258448

9.75887672

10.34516896

10.90134076

11.51440690

12.08822588

12.66051871

13.42206244

8.00000000

8.58629224

9.17258448

9.75887672

10.34516896

10.89127384

11.49895331

12.08599608

12.72399448

13.20038577

8.00000000

8.58629224

9.17258448

9.75887672

10.34516896

10.90134076

11.53288308

12.08822588

12.58762947

13.42206244

8.00000000

8.58629224

9.17258448

9.75887672

10.34516896

11.52984814

12.08599608

12.58808813

13.20038577

14.16710115

14.15144906

13.40747108
10.89127384

44

Lattice $=" 4.974854628764762 \quad 0.0 \quad 0.0 \quad 0.0 \quad 14.9245638862942820 .0 \quad 0.0 \quad 0.0 \quad 21.27663016512527 "$ Pr operties=species:S:1:pos:R:3 pbc="T T T"

$\mathrm{Ni}$

$\mathrm{Ni}$

$\mathrm{Ni}$

$\mathrm{Ni}$

$\mathrm{Ni}$

$\mathrm{Ni}$

$\mathrm{Ni}$

$\mathrm{Ni}$

$\mathrm{Ni}$

$\mathrm{Ni}$

$\mathrm{Ni}$

$\mathrm{Ni}$

$\mathrm{Ni}$

$\mathrm{Ni}$

$\mathrm{Ni}$

$\mathrm{Ni}$

$\mathrm{Ni}$

$\mathrm{Ni}$

$\mathrm{Ni}$

$\mathrm{Ni}$

$\mathrm{Ni}$

$\mathrm{Ni}$
1. 24371366

0.00000000

1. 24371366

0.00000000

1. 24371366

0.00478361

1. 24371366

0.01352531

1.24371366

0.00274398

1. 24371366

0.00000000

1. 24371366

0.00000000

1.24371366

0.00480781

1. 24371366

0.01393533

1. 24371366

0.00160304

3.73114097

2.48742731
3. 73114097

1. 65828488

7.04771072

4.97485463

2.90199853

0.78238748

6.17208032

4.08420329

2.07234956

$-0.05843965$

11.19342291

9.12056682

14.50999267

12.43713657

10.36428048

8.24483868

13.63294576

11.54082527

9.52726454

7.38534198

3.73114097

1. 65828488
8.00000000

8.58629224

9.17258448

9.75887672

10.34516896

10.89893696

11.49495101

12.08630784

12.66944412

13.42059704

8.00000000

8.58629224

9.17258448

9.75887672

10.34516896

10.89826593

11.49780944

12.08551939

12.66962523

13.41922388

8.00000000

8.58629224 
$\mathrm{Ni}$

$\mathrm{Ni}$

$\mathrm{Ni}$

$\mathrm{Ni}$

$\mathrm{Ni}$

$\mathrm{Ni}$

$\mathrm{Ni}$

$\mathrm{Ni}$

$\mathrm{Ni}$

$\mathrm{Ni}$

$\mathrm{Ni}$

$\mathrm{Ni}$

$\mathrm{Ni}$

$\mathrm{Ni}$

$\mathrm{Ni}$

$\mathrm{Ni}$

$\mathrm{Ni}$

$\mathrm{Ni}$

o

0

0

0

3. 73114097

3.73114097

2.48742731

3.73114097

2.48264371

3.73114097

2.47390201

3.73114097

2.48468333

3.73114097

2.48742731

3.73114097

2.48742731

3.73114097

2.48261950

3.73114097

2.47349198

3.73114097

2.48582428

1.24371366

1.24371366

3.73114097
Fri Apr 16 14:51:55 2021

7.04771072

4.97485463

2.90199853

0.78238748

6.15598973

4.08420329

2.03967502

$-0.05843965$

11.19342291

9.12056682

14.50999267

12.43713657

10.36428048

8.24483868

13.61490133

11.54082527

9.49506976

7.38534198

1.03188283

8.47619177

13.53253970

6.04853495
9.17258448

9.75887672

10.34516896

10.89893696

11.51450897

12.08630784

12.58664617

13.42059704

8.00000000

8.58629224

9.17258448

9.75887672

10.34516896

10.89826593

11.52524427

12.08551939

12.58844722

13.41922388

14.16707144

14.16164435

13.40225623

13.39096042

45

Lattice $=" 4.974854628764762 \quad 0.0 \quad 0.0 \quad 0.0 \quad 14.924563886294282 \quad 0.0 \quad 0.0 \quad 0.021 .27663016512527 "$ Pr operties=species:S:1:pos:R:3 pbc="T T T"

$\mathrm{Ni} \quad 1.24371366 \quad 3.73114097$

$\mathrm{Ni} \quad 0.00000000 \quad 1.65828488$

$\mathrm{Ni} \quad 1.24371366$

7.04771072

0.00000000

4.97485463

1.24371366

0.00723849

2.90199853

1.24371366

0.00142037

0.78657069

6.19606423

4.17051045

1.24371366

2.16000332

0.01325643

0.07711841

1.24371366

0.00000000

1.24371366

0.00000000

1.24371366

0.00359076

1.24371366

0.03417448

1.24371366

$-0.00667007$

3.73114097

2. 48742731

3.73114097

2. 48742731

3.73114097

2. 48018883

3. 73114097

2. 48600694

3. 73114097

2. 47417089

3.73114097

2. 48742731

3.73114097

2. 48742731

3.73114097

2. 48383655

3.73114097

2. 45325284

3.73114097

2. 49409738

1.24371366

11.19342291

9.12056682

14.50999267

12.43713657

10.36428048

8.25967576

13.61150822

11.49852834

9.33377794

7.09798790

3.73114097

1.65828488

7.04771072

4.97485463

2.90199853

0.78657069

6.21407304

4.17051045

2.10155751

0.07711841

11.19342291

9.12056682

14.50999267

12.43713657

10.36428048

8.25967576

13.62265910

11.49852834

9.24993285

7.09798790

8.00000000

8.58629224

9.17258448

9.75887672

10.34516896

10.90412275

11.44102749

12.13017354

12.65337912

13.42063975

8.00000000

8.58629224

9.17258448

9.75887672

10.34516896

10.87872365

11.52363494

12.16767930

12.67304477

13.41924013

8.00000000

8.58629224

9.17258448

9.75887672

10.34516896

10.90412275

11.42379455

12.13017354

12.57600951

13.42063975

8.00000000

8.58629224

9.17258448

9.75887672

10.34516896

10.87872365

11.55346142

12.16767930

12.70891458

13.41924013

1.17312474

14.18073255

14.13245470 


$\begin{array}{rrrr}0 & 3.73114097 & 13.72190211 & 13.42640893 \\ 0 & 3.73114097 & 5.67128073 & 13.26452822 \\ 0 & 3.73114097 & 10.94285270 & 13.38369582\end{array}$

. /oxygen/Ni/221/iter_6/struct.xyz

46

Lattice $=" 4.974854628764762 \quad 0.0 \quad 0.0 \quad 0.0 \quad 14.9245638862942820 .0 \quad 0.0 \quad 0.021 .27663016512527 "$ Pr operties $=$ species:S:1:pos:R:3 pbc="T T T"

$\mathrm{Ni}$

1.24371366

3.73114097

0.00000000

1.65828488

1.24371366

7.04771072

0.00000000

4.97485463

1.24371366

2.90199853

0.00188068

0.81722951

1. 26096494

6.21257341

$-0.04527413$

4.20677901

1. 16439348

$-0.08916137$

2.14628535

1.24371366

0.00000000

1. 24371366

0.00000000

$-0.00295131$

11.19342291

9. 12056682

14.50999267

12.43713657

1. 24371366

0.01882755

10.36428048

8.26759814

1.22595775

13.61250217

0.04143763

1. 27638121

11.52202152

9.39494945

7.25068532

3. 73114097

3.73114097

1. 65828488

7.04771072

2.48742731

4.97485463

2. 48742731

2.90199853

0.79541703

2. 47236359

6.22407601

3.74247568

4.19841613

2.09953551

3. 70019793

2. 37225611

3. 73114097

2. 48742731

3. 73114097

$-0.05464856$

11.19342291

9.12056682

14.50999267

2. 48742731

12.43713657

3. 73114097

2. 49648827

10.36428048

8.26030026

3. 71263549

13.62819149

2. 46439300

3. 75551212

11.52595889

9.29922068

7.17277820

0.91305733

1. 11200305

8.30363744

1.38512459

3. 65299102

13.62030405

5.84704165

8.00000000

8.58629224

9.17258448

9.75887672

10.34516896

10.92358316

11.45209936

12.11772877

12.83742399

13.29187075

8.00000000

8. 58629224

9.17258448

9.75887672

10.34516896

10.87815793

11.48679871

12.16329715

12.65306571

13.41015824

8.00000000

8.58629224

9.17258448

9.75887672

10.34516896

10.90811474

11.45090105

12.09699707

12.65744292

13.37918547

8.00000000

8.58629224

9.17258448

9.75887672

10.34516896

10.87731441

11.53189439

12.17034151

12.68162998

13.45470248

14.23581485

14.14355522

13.41658276

13.31330701

10.96602574

13.39157611

3.74632086

2. 53995512

3.16597640

13.59229553

. /oxygen/Ni/221/iter_7/struct.xyz

47

Lattice $=" 4.974854628764762 \quad 0.0 \quad 0.0 \quad 0.0 \quad 14.9245638862942820 .0 \quad 0.0 \quad 0.021 .27663016512527 "$ Pr operties=species:S:1:pos:R:3 pbc="T T T"

$\begin{array}{lrrr}\mathrm{Ni} & 1.24371366 & 3.73114097 & 8.00000000 \\ \mathrm{Ni} & 0.00000000 & 1.65828488 & 8.58629224 \\ \mathrm{Ni} & 1.24371366 & 7.04771072 & 9.17258448 \\ \mathrm{Ni} & 0.00000000 & 4.97485463 & 9.75887672 \\ \mathrm{Ni} & 1.24371366 & 2.90199853 & 10.34516896 \\ \mathrm{Ni} & -0.00394903 & 0.83415410 & 10.92472038 \\ \mathrm{Ni} & 1.26432871 & 6.23071263 & 11.44108742 \\ \mathrm{Ni} & 4.91556598 & 4.24691500 & 12.14223313 \\ \mathrm{Ni} & 1.15032056 & 2.20197694 & 12.81592379 \\ \mathrm{Ni} & 4.86608370 & 0.07244292 & 13.35475348 \\ \mathrm{Ni} & 1.24371366 & 11.19342291 & 8.00000000\end{array}$


$\mathrm{Ni}$

$\mathrm{Ni}$

0.00000000

1.24371366

0.00000000

1.24371366

0.02464422

1.22876289

4.95965128

1.28530270

0.13430141

3.73114097

2.48742731

3.73114097

2.48742731

3.73114097

2.46992762

3.74523336

2. 41477911

3. 67583733

2.37936165

3.73114097

2.48742731

3.73114097

2. 48742731

3.73114097

2.50005228

3. 71720524

2.50296360

3.76012629

2. 62928232

1.09897983

1.40607095

3. 65379657

3.95743292

3.73677792

2.52113659

1.22675044
Fri Apr 16 14:51:55 2021

220

9.12056682

14.50999267

12.43713657

10.36428048

8.27340222

13.64446633

11.42949879

9.27937977

7.19086339

3.73114097

1.65828488

7.04771072

4.97485463

2. 90199853

0.80719378

6.24902848

4.22597858

2.12656757

0.01124774

11.19342291

9.12056682

14.50999267

12.43713657

10.36428048

8.26514530

13.64513490

11.44548285

9. 23309629

7.10123658

1.02638568

8.21912926

13.69758834

5.77351334

10.82297730

3. 21246706

12.52184816
8. 58629224

9.17258448

9.75887672

10.34516896

10.86001402

11.54774421

12.26867405

12.66293421

13.42947569

8.00000000

8.58629224

9.17258448

9.75887672

10.34516896

10.91111487

11.43753783

12.07890562

12.66113282

13.48975978

8.00000000

8.58629224

9.17258448

9.75887672

10.34516896

11.50420975

12.28469117

12.67259250

13.47031871

14.24065932

14.17734305

13.36381816

13.29543924

13.49699832

13.58224827

13.00520638
10.85595174

. /oxygen/Ni/221/iter_8/struct.xyz

48

Lattice $=4.974854628764762 \quad 0.0 \quad 0.0 \quad 0.0 \quad 14.9245638862942820 .0 \quad 0.0 \quad 0.021 .27663016512527 "$ Pr operties=species:S:1:pos:R:3 pbc="T T T"

$\mathrm{Ni}$

$\mathrm{Ni}$

$\mathrm{Ni}$

$\mathrm{Ni}$

$\mathrm{Ni}$

$\mathrm{Ni}$

$\mathrm{Ni}$

$\mathrm{Ni}$

$\mathrm{Ni}$

$\mathrm{Ni}$

$\mathrm{Ni}$

$\mathrm{Ni}$

$\mathrm{Ni}$

$\mathrm{Ni}$

$\mathrm{Ni}$

$\mathrm{Ni}$

$\mathrm{Ni}$

$\mathrm{Ni}$

$\mathrm{Ni}$

$\mathrm{Ni}$

$\mathrm{Ni}$

$\mathrm{Ni}$

$\mathrm{Ni}$

$\mathrm{Ni}$

$\mathrm{Ni}$

$\mathrm{Ni}$

$\mathrm{Ni}$

$\mathrm{Ni}$
1.24371366

0.00000000

1. 24371366

0.00000000

1.24371366

0.02524299

1. 23607233

4. 96338688

1. 24344085

4. 91595938

1. 24371366

0.00000000

1. 24371366

0.00000000

1. 24371366

$-0.00469108$

1. 24157170

4. 95360894

1. 23525151

4. 96280412

3. 73114097

2. 48742731

3. 73114097

2. 48742731

3. 73114097

2. 48340004

3.72839967

2. 53792544
3.73114097

1. 65828488

7.04771072

4. 97485463

2.90199853

0.83183354

6.26490871

4. 09118668

2.09673067

14.92167617

11.19342291

9.12056682

14.50999267

12.43713657

10.36428048

8.27105405

13.68456535

11.53080470

9.42363239

7. 45075837

3.73114097

1.65828488

7. 04771072

4.97485463

2.90199853

0.81634090

6.23334259

4.16645133
8.00000000

8.58629224

9.17258448

9.75887672

10.34516896

10.92294641

11.51135959

12.09969486

12.84477162

13.42557499

8.00000000

8.58629224

9. 17258448

9.75887672

10.34516896

10.86334484

11.52909110

12.24467891

12.60566200

13.48299516

8.00000000

8.58629224

9.17258448

9.75887672

10.34516896

10.89268980

11.52831667

12.19539448 
$\mathrm{Ni}$

$\mathrm{Ni}$

3.77506405

2.41085488

3.73114097

2.48742731

3.73114097

2.48742731

3.73114097

2.48275885

3.72621246

2.49632557

3.72469927

2.48034073

1.13644779

1.22834765

3.68342266

3.71051874

3.72670859

2.67836189

1.23363244

1.17605057
Fri Apr 16 14:51:55 2021

1.99260922

14.86046270

11.19342291

9.12056682

14.50999267

12.43713657

10.36428048

8.26714783

13.67891013

11.53081864

9.39503816

7.46529058

0.97218036

8.53102470

13.65963959

6.16662169

10.94919604

3.01907016

12.60075977

5.14662609
12.63355309

13.58493434

8.00000000

8.58629224

9.17258448

9.75887672

10.34516896

10.86358166

11.48320096

12.25435081

12.61144338

13.48705182

14.30082513

14.21981929

13.34592297

13.41936533

13.48936792

13.59286853

13.01938629

12.97148892

. /oxygen/Ni/221/iter_9/struct.xyz

49

Lattice $=" 4.974854628764762 \quad 0.0 \quad 0.0 \quad 0.0 \quad 14.9245638862942820 .0 \quad 0.0 \quad 0.021 .27663016512527 "$ Pr operties=species:S:1:pos:R:3 pbc="T T T"$$
\mathrm{Ni}
$$

$\mathrm{Ni}$

$\mathrm{Ni}$

$\mathrm{Ni}$

$\mathrm{Ni}$

$\mathrm{Ni}$

$\mathrm{Ni}$

$\mathrm{Ni}$

$\mathrm{Ni}$

$\mathrm{Ni}$

$\mathrm{Ni}$

$\mathrm{Ni}$

$\mathrm{Ni}$

$\mathrm{Ni}$

$\mathrm{Ni}$

$\mathrm{Ni}$

$\mathrm{Ni}$

$\mathrm{Ni}$

$\mathrm{Ni}$

$\mathrm{Ni}$

$\mathrm{Ni}$

$\mathrm{Ni}$

$\mathrm{Ni}$

$\mathrm{Ni}$

$\mathrm{Ni}$

$\mathrm{Ni}$

$\mathrm{Ni}$

$\mathrm{Ni}$

$\mathrm{Ni}$

$\mathrm{Ni}$

$\mathrm{Ni}$

$\mathrm{Ni}$

$\mathrm{Ni}$

$\mathrm{Ni}$

$\mathrm{Ni}$

$\mathrm{Ni}$

$\mathrm{Ni}$

$\mathrm{Ni}$

$\mathrm{Ni}$

$\mathrm{Ni}$

O

O

O

O

\begin{abstract}
1.24371366
\end{abstract}
0.00000000

1.24371366

0.00000000

1. 24371366

0.00666785

1. 24805856

4.97334288

1. 19433868

4.87466351

1. 24371366

0.00000000

1. 24371366

0.00000000

1.24371366

$-0.00011770$

1. 22936569

4. 94669000

1. 24873569

0.00958127

3. 73114097

2. 48742731

3. 73114097

2. 48742731

3. 73114097

2. 47669585

3. 73400733

2. 49150452

3. 72212320

2. 37565058

3. 73114097

2. 48742731

3. 73114097

2. 48742731

3. 73114097

2. 49154129

3. 71597741

2. 49214028

3. 73214848

2. 49689536

1. 09625602

1. 25638578

3. 64994859

3. 75572704
3.73114097

4097

1. 65828488

7.04771072

4. 97485463

2. 90199853

0.82259312

6.24691867

4.19047900

2.13010727

0.02709456

11.19342291

9.12056682

14.50999267

12.43713657

10.36428048

8.29039826

13.65303219

11.44789514

9.30865820

7.01184667

3.73114097

1. 65828488

7.04771072

4. 97485463

2. 90199853

0.79962488

6.26258735

4.20407790

2.06311090

14.89382593

11.19342291

9.12056682

14.50999267

12.43713657

10.36428048

8.29122109

13.65275329

11.45529748

9.28044333

6.99500383

0.99882049

8.24572105

13.67436254

5.65484102
8.00000000

8.58629224

9.17258448

9.75887672

10.34516896

10.91799113

11.41461780

12.12232807

12.79605221

13.38135590

8.00000000

8.58629224

9.17258448

9.75887672

10.34516896

10.87243122

11.55165222

12.21384972

12.66297469

13.70155239

8.00000000

8.58629224

9.17258448

9.75887672

10.34516896

10.90081256

11.43409739

12.14838398

12.64368172

13.53092198

8.00000000

8.58629224

9.17258448

9.75887672

10.34516896

10.86580688

11.49804701

12.22786730

12.79200787

13.72223515

14.25639943

14.14609231

13.35851390

13.24494171 


\section{0}

O

0

0

0

\begin{abstract}
3.72441967
2.56881962

1.22130672

1.24300051

3.73731062
\end{abstract}

Fri Apr 16 14:51:55 2021

222
13.47487729

13.59815006

13.00177579

13.22753468

14.27433314

. / oxygen/Ni/311/iter_1/struct.xyz

37

Lattice="7.462281943147142 $0.0 \quad 0.0 \quad 2.4874273143823786 \quad 8.24986309508776 \quad 0.0 \quad 0.0 \quad 0.021 .30321$ 2853088358" Properties=species:S:1:pos:R:3 pbc="T T T"

$\mathrm{Ni}$

$\mathrm{Ni}$

$\mathrm{Ni}$

$\mathrm{Ni}$

$\mathrm{Ni}$

$\mathrm{Ni}$

$\mathrm{Ni}$

$\mathrm{Ni}$

$\mathrm{Ni}$

$\mathrm{Ni}$

$\mathrm{Ni}$

$\mathrm{Ni}$

$\mathrm{Ni}$

$\mathrm{Ni}$

$\mathrm{Ni}$

$\mathrm{Ni}$

$\mathrm{Ni}$

$\mathrm{Ni}$

$\mathrm{Ni}$

$\mathrm{Ni}$

$\mathrm{Ni}$

$\mathrm{Ni}$

$\mathrm{Ni}$

$\mathrm{Ni}$

$\mathrm{Ni}$

$\mathrm{Ni}$

$\mathrm{Ni}$

$\mathrm{Ni}$

$\mathrm{Ni}$

$\mathrm{Ni}$

$\mathrm{Ni}$

$\mathrm{Ni}$

$\mathrm{Ni}$

$\mathrm{Ni}$

$\mathrm{Ni}$

$\mathrm{Ni}$
2.48742731

2.48742731

1.24371366

1.25179691

2. 48693071

$-0.00104495$

3. 73114097

3.73114097

2.48742731

2.48571758

3. 72991058

1.17808607

4.97485463

4.97485463

3.73114097

3.71949563

5.00299254

2.48791653

6.21856829

6.21856829

4.97485463

4.97114731

6.22549390

3.79281836

7. 46228194

7.46228194

6.21856829

6.21867259

7.43616921

4.97051465

8.70599560

8.70599560

7.46228194

7.46559486

8.70359110

6.21618571

2. 48173257
2.99995022

0.74998755

2.62495644

0.39504243

2.19276028

$-0.02275980$

7.12488176

4.87491910

6.74988799

4.52127432

6.35600533

4.14678008

2.99995022

0.74998755

2.62495644

0.39441680

2.23361477

$-0.08978098$

7.12488176

4.87491910

6.74988799

4.51269800

6.34326850

4.13829574

2.99995022

0.74998755

2.62495644

0.38238364

2.23778360

$-0.02339004$

7.12488176

4.87491910

6.74988799

4.51689190

6.36659518

4.11236390

3. 17223612
8.00000000

9.06064257

10.12128514

11.17559382

12.39696010

13.17328662

8.00000000

9.06064257

10.12128514

11.14952998

12.25447692

13.24227727

8.00000000

9.06064257

10.12128514

11.17461552

12.23609779

13.19408812

8.00000000

9.06064257

10.12128514

11.16650453

12.27636172

13.24125140

8.00000000

9.06064257

10.12128514

11.16243489

12.23510931

13.17278824

8.00000000

9.06064257

10.12128514

11.16852158

12.26077624

13.18132685

14.03258188

46

Lattice $=" 7.462281943147142 \quad 0.0 \quad 0.0 \quad 2.4874273143823786 \quad 8.24986309508776 \quad 0.0 \quad 0.0 \quad 0.021 .30321$

$2853088358 "$ Properties=species:S:1:pos:R:3 pbc="T T T"

$\mathrm{Ni} \quad 2.48742731 \quad 2.99995022 \quad 8.00000000$

$\begin{array}{lll}\mathrm{Ni} & 2.48742731 & 0.74998755\end{array}$

$\begin{array}{ll}2.48742731 & 0.74998755 \\ 1.24371366 & 2.62495644\end{array}$

9.06064257

$1.26577102 \quad 0.33065035$

2.49238405

2.27515739

7. 43564508

0.69565765

3. 73114097

7.12488176

3.73114097

4.87491910

2. 48742731

6.74988799

2. 44310649

4.52042020

6.29830107

3.69225562

4.86013537

4.97485463

2.99995022

0.74998755

4.97485463

10.12128514

11.20605796

12.13640587

13.91489182

8.00000000

9.06064257

10.12128514

11.22583288

12.25644945

14.40283722

8.00000000

9.06064257

10.12128514

0.35093030

11.15620617

3. 74897528

2.10969888

12.21050261

4.97444849

0.74362887

13.97130872 
$\mathrm{Ni}$

$\mathrm{Ni}$

6.21856829

6.21856829

4.97485463

4.97917258

6.23472687

3.42625487

7.46228194

7.46228194

6.21856829

6.21176828

7.48139014

4.96561045

8.70599560

8.70599560

7.46228194

7.43449951

8.66184333

6.57766508

2.29782889

3.74639111

7.21056946

6.31920266

6.13944313

1.18384467

4.93302085

2.67487296

8.72926301

3.63279221
7.12488176

4.87491910

6.74988799

4.47612256

6.53388290

3.50082399

2.99995022

0.74998755

2.62495644

0.39336985

2.26980000

0.61539281

7.12488176

4.87491910

6.74988799

4.59599375

6.65001593

3. 38264588

4.15290291

7.96419958

4.95419921

1.77484826

7.61718681

2.17485682

3.68335300

4.94234180

7.55880709

1.75676992
8.00000000

9.06064257

10.12128514

11.29349040

12.44070477

14.29117265

8.00000000

9.06064257

10.12128514

11.11539133

12.07708969

14.39722976

8.00000000

9.06064257

10.12128514

11.28378776

12.32889497

14.02567346

15.51819161

13.05801202

13.22755903

14.89440550

14.00052503

13.65008251

13.22694084

13.10748405

13.99872443

14.97513458

. /oxygen/Ni/311/iter_11/struct.xyz

47

Lattice $=" 7.462281943147142 \quad 0.0 \quad 0.0 \quad 2.4874273143823786 \quad 8.24986309508776 \quad 0.0 \quad 0.0 \quad 0.021 .30321$

2853088358" Properties=species:S:1:pos:R:3 pbc="T T T"

$\mathrm{Ni} \quad 2.48742731 \quad 2.99995022 \quad 8.00000000$

$\mathrm{Ni} \quad 2.48742731$

0.74998755

8.00000000

1.24371366

2.62495644

10.12128514

1.25424549

0.33873128

2.49078242

2.27834703

7.43528812

11.20745481

0.77011467

3.73114097

7.12488176

12.12084431

13.92447292

8.00000000

9.06064257

3. 73114097

4.87491910

2.48742731

6.74988799

2. 45000527

4.53137614

3.69846409

6.32651632

8.69360825

4.74686410

4.97485463

2.99995022

0.74998755

4.97485463

2.62495644

.73114097

0.35085280

2.07759406

4.98095338

0.76660767

7.12488176

6.21856829

6.21856829

4.87491910

6.74988799

4.48678955

4.98281749

6.22668359

6.55124439

3. 49661519

3.54715250

7. 46228194

2.99995022

0.74998755

7. 46228194

2.62495644

0.37910544

6.22388341

7. 46923406

2.31561969

0.65572608

7.12488176

4.87491910

6.74988799

4.58908900

10.12128514

11.24264393

12.26037097

14.47999263

8.00000000

9.06064257

10.12128514

11.14354760

12.18817512

13.94771903

8.00000000

9.06064257

10.12128514

11.27711153

12.42695281

14.25044269

8.00000000

9.06064257

10.12128514

11.12267936

12.11346317

14.35282755

8.00000000

9.06064257

10.12128514

11.26102778

$\begin{array}{ll}6.62337246 & 12.36405238 \\ 3.56853471 & 14.25528284\end{array}$

$\begin{array}{ll}6.62337246 & 12.36405238 \\ 3.56853471 & 14.25528284\end{array}$ 


\section{0}

O

0

0

O

0

0

O

0

0

0
2.53411509

3.72630422

7.27368043

6.27791937

6.13696731

1.23173015

4.96845792

2.66220912

8.73658656

3.63916537

7.38975969
4.23820951

8.00060600

5.01901220

1.79440124

7.63162652

2.19806324

3. 67858050

4.97098285

7.63788669

1.78958881

4.25542371
15.54304311

13.06638986

13.14503209

14.91194667

13.97962125

13.63464521

13.11688587

13.14139272

13.96661619

14.93618212

15.54932195

48

Lattice="7.462281943147142 $0.0 \quad 0.0 \quad 2.4874273143823786 \quad 8.24986309508776 \quad 0.0 \quad 0.0 \quad 0.021 .30321$ 2853088358" Properties=species:S:1:pos:R:3 pbc="T T T"

$\begin{array}{lllr}\mathrm{Ni} & 2.48742731 & 2.99995022 & 8.00000000 \\ \mathrm{Ni} & 2.48742731 & 0.74998755 & 9.06064257 \\ \mathrm{Ni} & 1.24371366 & 2.62495644 & 10.12128514 \\ \mathrm{Ni} & 1.24547397 & 0.38328878 & 11.24915924 \\ \mathrm{Ni} & 2.49082097 & 2.29765013 & 12.21089427 \\ \mathrm{Ni} & 2.48563385 & 7.91095649 & 13.77397011 \\ \mathrm{Ni} & 3.73114097 & 7.12488176 & 8.0000000 \\ \mathrm{Ni} & 3.73114097 & 4.87491910 & 9.06064257 \\ \mathrm{Ni} & 2.48742731 & 6.74988799 & 10.12128514 \\ \mathrm{Ni} & 2.48588905 & 4.52649837 & 11.25220802 \\ \mathrm{Ni} & 3.73134572 & 6.37755278 & 12.17786543 \\ \mathrm{Ni} & 1.23640168 & 4.14098880 & 14.52614874 \\ \mathrm{Ni} & 4.97485463 & 2.99995022 & 8.00000000 \\ \mathrm{Ni} & 4.97485463 & 0.74998755 & 9.06064257 \\ \mathrm{Ni} & 3.73114097 & 2.62495644 & 10.12128514 \\ \mathrm{Ni} & 3.73322979 & 0.38355693 & 11.24845517 \\ \mathrm{Ni} & 4.97528240 & 2.29953747 & 12.21298203 \\ \mathrm{Ni} & 4.97561002 & 7.91184108 & 13.77535343 \\ \mathrm{Ni} & 6.21856829 & 7.12488176 & 8.0000000 \\ \mathrm{Ni} & 6.21856829 & 4.87491910 & 9.06064257 \\ \mathrm{Ni} & 4.97485463 & 6.74988799 & 10.12128514 \\ \mathrm{Ni} & 4.97534691 & 4.52660321 & 11.25252024 \\ \mathrm{Ni} & 6.21792563 & 6.37913528 & 12.17580541 \\ \mathrm{Ni} & 3.72564381 & 4.16212909 & 14.52630567 \\ \mathrm{Ni} & 7.46228194 & 2.99995022 & 8.0000000 \\ \mathrm{Ni} & 7.46228194 & 0.74998755 & 9.06064257 \\ \mathrm{Ni} & 6.21856829 & 2.62495644 & 10.12128514 \\ \mathrm{Ni} & 6.22020424 & 0.38478910 & 11.24907114 \\ \mathrm{Ni} & 7.46602507 & 2.29422713 & 12.20862927 \\ \mathrm{Ni} & 7.46199547 & 7.91022637 & 13.77462419 \\ \mathrm{Ni} & 8.70599560 & 7.12488176 & 8.00000000 \\ \mathrm{Ni} & 8.70599560 & 4.87491910 & 9.06064257 \\ \mathrm{Ni} & 7.46228194 & 6.74988799 & 10.12128514 \\ \mathrm{Ni} & 7.46305851 & 4.52562968 & 11.25679181 \\ \mathrm{Ni} & 8.70759747 & 6.37775483 & 12.17680799 \\ \mathrm{Ni} & 6.21186320 & 4.14556671 & 14.52817733 \\ \mathrm{O} & 2.48093359 & 4.09888979 & 15.74804638 \\ \mathrm{O} & 3.73079106 & 6.64027719 & 14.06811089 \\ \mathrm{O} & 7.45546939 & 3.94974414 & 13.12683262 \\ \mathrm{O} & 6.21727384 & 0.97142689 & 13.18750406 \\ \mathrm{O} & 6.21937174 & 6.63793490 & 14.06623494 \\ \mathrm{O} & 1.24317885 & 0.97015364 & 13.18834978 \\ \mathrm{O} & 4.96923175 & 3.96165429 & 13.12508876 \\ \mathrm{O} & 2.48195393 & 3.95875813 & 13.12514268 \\ \mathrm{O} & 8.70507315 & 6.63743062 & 14.06613731 \\ \mathrm{O} & 3.73106976 & 0.97295516 & 13.18712304 \\ \mathrm{O} & 7.45642486 & 4.06385713 & 15.74864051 \\ \mathrm{O} & 4.96784786 & 4.11318954 & 15.75116854\end{array}$

./oxygen/Ni/311/iter_2/struct.xyz 38

Lattice="7.462281943147142 $0.0 \quad 0.0 \quad 2.4874273143823786 \quad 8.24986309508776 \quad 0.0 \quad 0.0 \quad 0.021 .30321$ 2853088358" Properties=species:S:1:pos:R:3 pbc="T T T" $\mathrm{Ni}$ 
$\mathrm{Ni}$

$\mathrm{Ni}$

$\mathrm{Ni}$

$\mathrm{Ni}$

$\mathrm{Ni}$

$\mathrm{Ni}$

$\mathrm{Ni}$

$\mathrm{Ni}$

$\mathrm{Ni}$

$\mathrm{Ni}$

$\mathrm{Ni}$

$\mathrm{Ni}$

$\mathrm{Ni}$

$\mathrm{Ni}$

$\mathrm{Ni}$

$\mathrm{Ni}$

$\mathrm{Ni}$

$\mathrm{Ni}$

$\mathrm{Ni}$

$\mathrm{Ni}$

$\mathrm{Ni}$

$\mathrm{Ni}$

$\mathrm{Ni}$

$\mathrm{Ni}$

$\mathrm{Ni}$

$\mathrm{Ni}$

$\mathrm{Ni}$

$\mathrm{Ni}$

$\mathrm{Ni}$

$\mathrm{Ni}$

$\mathrm{Ni}$

$\mathrm{Ni}$

$\mathrm{Ni}$

$\mathrm{Ni}$

$\mathrm{Ni}$

O

0

3.68621038

39

oxgen/Ni/311/iter_3/struct.xyz
Fri Apr 16 14:51:55 2021

225

0.74998755

2.62495644

0.39974930

2. 18634968

0.02013481

7.12488176

4.87491910

6.74988799

4.52468084

6. 31128123

4.14506636

2.99995022

0.74998755

2.62495644

0.38914080

2. 21806887

$-0.06567787$

7.12488176

4.87491910

6.74988799

4.51407235

6.34300042

4.05925368

2. 99995022

0.74998755

2. 62495644

0.38048833

2. 24042455

$-0.01137781$

7.12488176

4.87491910

6.74988799

4.50541988

6.36535610

4.11355374

3.14996228

7.27489383
9.06064257

10.12128514

11.16632667

12.38583262

13.24186039

8.00000000

9.06064257

10.12128514

11.16632667

12.38583262

13.24186039

8.00000000

9.06064257

10.12128514

11.17899720

12.23592512

13.26960899

8.00000000

9.06064257

10.12128514

11.17899720

12.23592512

13.26960899

8.00000000

9.06064257

10.12128514

11.16096528

12.22819128

13.17516266

8.00000000

9.06064257

10.12128514

11.16096528

12.22819128

13.17516266

14.06315929

14.06315929

Lattice $=" 7.462281943147142 \quad 0.0 \quad 0.0 \quad 2.4874273143823786 \quad 8.24986309508776 \quad 0.0 \quad 0.0 \quad 0.021 .30321$ 2853088358" Properties=species:S:1:pos:R:3 pbc="T T T"

$\mathrm{Ni} \quad 2.48742731 \quad 2.99995022 \quad 8.00000000$

$\begin{array}{llll}\mathrm{Ni} & 2.48742731 & 0.74998755 & 9.06064257\end{array}$

$\mathrm{Ni} \quad 1.24371366 \quad 2.62495644$

$\mathrm{Ni} \quad 1.24475695 \quad 0.39942310$

2.21153721

2.48461025

$-0.06554469$

3.73114097

0.05825095

7. 12488176

3.73114097

4.87491910

2.48742731

6.74988799

2.48712425

4.52656013

3.72302745

6.33669431

1.21070964

4.14915769

4.97485463

2.99995022

0.74998755

4. 97485463

2.62495644

3. 73114097

0.37653871

2. 24109931

5.01923475

2.52644136

6.21856829

6.21856829

$-0.00345899$

7.12488176

4.87491910

6.74988799

4.97485463

4.51509898

10.12128514

11.15261183

12.33455448

13.25180615

8.00000000

9.06064257

10.12128514

11.18313784

12.36593934

13.42217315

8.00000000

9.06064257

10.12128514

11.17077793

12.21702898

13.26289292

8.00000000

9.06064257

10.12128514

11.17294197

12.26538307

6.23636490

6.37699010

4.06393627

2.99995022

13.25856786

8.00000000

9.06064257

0.74998755

10.12128514 
$\mathrm{Ni}$

$\mathrm{Ni}$

$\mathrm{Ni}$

$\mathrm{Ni}$

$\mathrm{Ni}$

$\mathrm{Ni}$

$\mathrm{Ni}$

$\mathrm{Ni}$

$\mathrm{Ni}$

0

0

0
6.22283492

$\begin{array}{llr}6.22283492 & 0.37453954 & 11.16842336 \\ 7.44828339 & 2.24020827 & 12.22747505 \\ 4.96150235 & 0.09307415 & 13.19843742 \\ 8.70599560 & 7.12488176 & 8.00000000 \\ 8.70599560 & 4.87491910 & 9.06064257 \\ 7.46228194 & 6.74988799 & 10.12128514 \\ 7.47917003 & 4.48161900 & 11.12426292 \\ 8.66154248 & 6.37658428 & 12.27484866 \\ 6.20278799 & 4.12076535 & 13.19402103 \\ 2.50494338 & 3.04596935 & 14.03053561 \\ 3.68519783 & 7.28382090 & 14.04995913 \\ 7.42098118 & 5.50797423 & 13.67113409\end{array}$

0.37453954

2.24020827

.09307415

ruct. $x y z$

40

Lattice="7.462281943147142 $0.0 \quad 0.0 \quad 2.4874273143823786 \quad 8.24986309508776 \quad 0.0 \quad 0.0 \quad 0.021 .30321$

2853088358" Properties=species:S:1:pos:R:3 pbc="T T T"

Nitis

2.48742731

2.99995022

2.48742731

0.74998755

1.24371366

2.62495644

1.23719940

0.40351751

2.47901525

2.23812576

$-0.03105162$

3.73114097

3.73114097

0.07060463

7.12488176

4.87491910

2. 48742731

6.74988799

2. 48091305

4.52844906

3.72272891

6.36305731

1.21266204

4.19553617

4.97485463

2.99995022

4.97485463

0.74998755

3.73114097

2.62495644

3.71126761

0.38465198

5.00466394

2.27789239

2. 52378719

0.00819725

6.21856829

7.12488176

6.21856829

4.87491910

4.97485463

6.74988799

4.95498126

4.50958352

6.24837760

6.40282393

3.76750085

4.13312880

7.46228194

2.99995022

7.46228194

0.74998755

2.62495644

6.21856829

0.35250609

2.25585380

7.43927913

0.08210813

4.93853501

7.12488176

4.87491910

6.74988799

7.46228194

4.47743764

7.47636277

6.38078534

4.20703968

6.18224867

3.06705555

7.19198710

5.56135440

8.00000000

9.06064257

10.12128514

11.16908456

12.32412994

13.44293814

8.00000000

9.06064257

10.12128514

11.16908456

12.32412994

13.44293814

8.00000000

9.06064257

10.12128514

11.16782892

12.25098807

13.25580407

8.00000000

9.06064257

10.12128514

11.16782892

12.25098807

13.25580407

8.00000000

9.06064257

10.12128514

11.13547374

12.26652859

13.21672658

8.00000000

9.06064257

10.12128514

11.13547374

12.26652859

13.21672658

14.02178753

14.02178753

13.67785637

7.43010711

1.43642285

13.67785637

. /oxygen/Ni/311/iter_5/struct.xyz

41

Lattice $=" 7.462281943147142 \quad 0.0 \quad 0.0 \quad 2.4874273143823786 \quad 8.24986309508776 \quad 0.0 \quad 0.0 \quad 0.021 .30321$ 2853088358" Properties=species:S:1:pos:R:3 pbc="T T T"

$\mathrm{Ni}$

$\mathrm{Ni}$

$\mathrm{Ni}$

$\mathrm{Ni}$

$\mathrm{Ni}$

$\mathrm{Ni}$

$\mathrm{Ni}$

$\mathrm{Ni}$
2.48742731

2. 48742731

1. 24371366

1. 25078596

2. 58174974

$-0.19997853$

3.73114097

3.73114097
2.99995022

0.74998755

2. 62495644

0.39596860

2.19178940

0.14577270

7.12488176

4.87491910
8.00000000

9.06064257

10.12128514

11.17831256

12.31771479

13.45561315

8.00000000

9.06064257 
$\mathrm{Ni}$

$\mathrm{Ni}$

$\mathrm{Ni}$

$\mathrm{Ni}$

$\mathrm{Ni}$

$\mathrm{Ni}$

$\mathrm{Ni}$

$\mathrm{Ni}$

$\mathrm{Ni}$

$\mathrm{Ni}$

$\mathrm{Ni}$

$\mathrm{Ni}$

$\mathrm{Ni}$

$\mathrm{Ni}$

$\mathrm{Ni}$

$\mathrm{Ni}$

$\mathrm{Ni}$

$\mathrm{Ni}$

$\mathrm{Ni}$

$\mathrm{Ni}$

$\mathrm{Ni}$

$\mathrm{Ni}$

$\mathrm{Ni}$

$\mathrm{Ni}$

$\mathrm{Ni}$

$\mathrm{Ni}$

$\mathrm{Ni}$

$\mathrm{Ni}$

o

0

0

0

0

$. / 0 \times$

./oxygen/Ni/311/iter_6/struct.xyz
2.48742731

2. 47566682

3. 60453144

1. 44227768

4.97485463

4.97485463

3.73114097

3. 73377666

5.09160076

2.22805386

6.21856829

6.21856829

4.97485463

4.98488915

6.09467816

3. 91468123

7. 46228194

7.46228194

6.21856829

6.22066558

7.52726294

4.81874990

8.70599560

8.70599560

7.46228194

7.47024704

8.54718633

6.37055477

2. 80302818

3. 39062167

7.78938485

6.11776458

6.13886064
Fri Apr 16 14:51:55 2021

6.74988799

4.51248606

6.36671106

3.99554954

2.99995022

0.74998755

2.62495644

0.39897219

2.34006588

0.03750405

7.12488176

4.87491910

6.74988799

4.54532690

6.46842040

4.16944210

2.99995022

0.74998755

2.62495644

0.32072191

2.26941583

0.25130396

7.12488176

4.87491910

6.74988799

4.50158293

6.33833613

4.29537258

2.93023644

7.10789318

5.31802498

1.56960725

7.31265211
ruct. xyz
10.12128514

11.18473132

12.28283019

13.48898820

8.00000000

9.06064257

10.12128514

11.15364813

12.19672376

13.45259133

8.00000000

9.06064257

10.12128514

11.16242134

12.27775480

13.25358953

8.00000000

9.06064257

10.12128514

11.17119350

12.22758117

13.42067348

8.00000000

9.06064257

10.12128514

11.13113417

12.38146171

13.24692797

14.02537103

13.97901290

13.85349418

13.64162976

13.97893416

2853088358" Properties=species:S:1:pos:R:3 pbc="T T T"

$\mathrm{Ni} \quad 2.48742731 \quad 2.99995022$

$\begin{array}{lll}\mathrm{Ni} & 2.48742731 & 0.74998755\end{array}$

2.62495644

1.24371366

0.39697418

8.00000000

9.06064257

1.25208316

2.51465874

2.17953144

6.29407397

0.32962376

3.73114097

7.12488176

3.73114097

4.87491910

2.48742731

6.74988799

2.47660854

4.51932824

3.70967193

6.40351093

1.23504546

4.20051874

4.97485463

2.99995022

4.97485463

0.74998755

3.73114097

2.62495644

3. 73410383

0.37085505

5.01581021

2. 25380137

1.38161637

0.24278714

7.12488176

6.21856829

4.87491910

6.74988799

4.97485463

4.50871535

10.12128514

11.13501218

12.32733527

13.70872058

8.00000000

9.06064257

10.12128514

11.17605043

12.29798417

13.45703646

8.00000000

9.06064257

10.12128514

11.13539304

12.26387371

13.62708850

8.00000000

9.06064257

10.12128514

11.14612968

12.26686974

13.22181324

8.00000000

9.06064257

10.12128514

11.15843982

12.26140347

13.58516247

8.00000000 
$\mathrm{Ni}$

$\mathrm{Ni}$

$\mathrm{Ni}$

$\mathrm{Ni}$

$\mathrm{Ni}$

0

0

0

0

0

0
8.70599560

7.46228194

7.47798413

8.64722673

6.20339649

2.56529051

2.42386255

7.40483413

5.01730504

5.14396923

7.67400186
Fri Apr 16 14:51:55 2021

4.87491910

6.74988799

4.50302825

6.42641455

4.14635149

3.13068986

7.07076528

5.45191522

1.56863020

7.19155156

1.56589607
9.06064257

10.12128514

11.13012472

12.32450139

13.20190323

13.98111099

13.64289863

13.71960228

13.99792903

13.67270825

13.98251555

. /oxygen/Ni/311/iter_7/struct.xyz

43

Lattice $=" 7.462281943147142 \quad 0.0 \quad 0.0 \quad 2.4874273143823786 \quad 8.24986309508776 \quad 0.0 \quad 0.0 \quad 0.021 .30321$ 2853088358" Properties=species:S:1:pos:R:3 pbc="T T T"

\begin{tabular}{|c|c|c|c|}
\hline $\mathrm{Ni}$ & 2.48742731 & 2.99995022 & 8.00000000 \\
\hline $\mathrm{Ni}$ & 2.48742731 & 0.74998755 & 9.06064257 \\
\hline $\mathrm{Ni}$ & 1.24371366 & 2.62495644 & 10.12128514 \\
\hline $\mathrm{Ni}$ & 1.23978020 & 0.38910420 & 11.12907469 \\
\hline $\mathrm{Ni}$ & 2.49231609 & 2.18229947 & 12.29378431 \\
\hline $\mathrm{Ni}$ & 6.23257126 & 0.30108566 & 13.70066119 \\
\hline $\mathrm{Ni}$ & 3.73114097 & 7.12488176 & 8.00000000 \\
\hline $\mathrm{Ni}$ & 3.73114097 & 4.87491910 & 9.06064257 \\
\hline $\mathrm{Ni}$ & 2.48742731 & 6.74988799 & 10.12128514 \\
\hline $\mathrm{Ni}$ & 2.49347547 & 4.50166644 & 11.19468304 \\
\hline $\mathrm{Ni}$ & 3.70918150 & 6.37663820 & 12.29679661 \\
\hline $\mathrm{Ni}$ & 1.23410602 & 4.19185455 & 13.40635615 \\
\hline $\mathrm{Ni}$ & 4.97485463 & 2.99995022 & 8.00000000 \\
\hline $\mathrm{Ni}$ & 4.97485463 & 0.74998755 & 9.06064257 \\
\hline $\mathrm{Ni}$ & 3.73114097 & 2.62495644 & 10.12128514 \\
\hline $\mathrm{Ni}$ & 3.73789956 & 0.38783356 & 11.12276025 \\
\hline $\mathrm{Ni}$ & 5.00647074 & 2.26729796 & 12.26439041 \\
\hline $\mathrm{Ni}$ & 1.32748478 & 0.24626701 & 13.60990677 \\
\hline $\mathrm{Ni}$ & 6.21856829 & 7.12488176 & 8.00000000 \\
\hline $\mathrm{Ni}$ & 6.21856829 & 4.87491910 & 9.06064257 \\
\hline $\mathrm{Ni}$ & 4.97485463 & 6.74988799 & 10.12128514 \\
\hline $\mathrm{Ni}$ & 4.95401593 & 4.49741329 & 11.18696317 \\
\hline $\mathrm{Ni}$ & 6.22460224 & 6.40976645 & 12.21392835 \\
\hline $\mathrm{Ni}$ & 3.77666132 & 4.08500912 & 13.46353974 \\
\hline $\mathrm{Ni}$ & 7.46228194 & 2.99995022 & 8.00000000 \\
\hline $\mathrm{Ni}$ & 7.46228194 & 0.74998755 & 9.06064257 \\
\hline $\mathrm{Ni}$ & 6.21856829 & 2.62495644 & 10.12128514 \\
\hline $\mathrm{Ni}$ & 6.21551851 & 0.38714141 & 11.15324395 \\
\hline $\mathrm{Ni}$ & 7.46245753 & 2.28378109 & 12.27738837 \\
\hline $\mathrm{Ni}$ & 3.78097005 & 0.28284169 & 13.55784140 \\
\hline $\mathrm{Ni}$ & 8.70599560 & 7.12488176 & 8.00000000 \\
\hline $\mathrm{Ni}$ & 8.70599560 & 4.87491910 & 9.06064257 \\
\hline $\mathrm{Ni}$ & 7.46228194 & 6.74988799 & 10.12128514 \\
\hline $\mathrm{Ni}$ & 7.45724412 & 4.48995023 & 11.14854687 \\
\hline $\mathrm{Ni}$ & 8.66075020 & 6.42956909 & 12.28845753 \\
\hline $\mathrm{Ni}$ & 6.21965424 & 4.25784054 & 13.37787135 \\
\hline O & 2.42350232 & 2.99553919 & 13.96752069 \\
\hline O & 2.41362515 & 7.08874733 & 13.63039018 \\
\hline O & 7.54198619 & 5.56263523 & 13.65795903 \\
\hline O & 4.95818230 & 1.52798176 & 13.97926971 \\
\hline O & 5.09010174 & 7.18168759 & 13.59609765 \\
\hline O & 7.60005050 & 1.54680105 & 13.97252745 \\
\hline O & 5.00975054 & 4.43353462 & 14.62524461 \\
\hline
\end{tabular}

. /oxygen/Ni/311/iter_8/struct.xyz

44

Lattice $=" 7.462281943147142 \quad 0.0 \quad 0.0 \quad 2.4874273143823786 \quad 8.24986309508776 \quad 0.0 \quad 0.0 \quad 0.021 .30321$

2853088358" Properties=species:S:1:pos:R:3 pbc="T T T"

$\begin{array}{llll}\mathrm{Ni} & 2.48742731 & 2.99995022 & 8.00000000\end{array}$

$\mathrm{Ni} \quad 2.48742731 \quad 0.74998755 \quad 9.06064257$

$\mathrm{Ni} \quad 1.24371366 \quad 2.62495644 \quad 10.12128514$

$\mathrm{Ni} \quad 1.24524290 \quad 0.30646769 \quad 11.22328113$

$\mathrm{Ni} \quad 2.48971314 \quad 2.19251597 \quad 12.12649999$

$\begin{array}{llll}\mathrm{Ni} & 6.20425730 & 0.87623586 & 13.96151982\end{array}$ 
$\mathrm{Ni}$

$\mathrm{Ni}$

3.73114097

3.73114097

2.48742731

2.49778366

2.49799509

1.10829541

4.97485463

4.97485463

3.73114097

3.73107352

4.99810831

1.36940533

6.21856829

6.21856829

4.97485463

4.96575311

4.98029629

3.82138938

7.46228194

7.46228194

6.21856829

6.22269155

7.46077491

3.88701449

8.70599560

8.70599560

7.46228194

7.48412482

7.47484232

6.23017209

2.39484989

2.48663842

7.54024584

4.83670377

5.09615296

7.51965889

4.89134581

2.50741364
7.12488176

4.87491910

6.74988799

4.59857968

6.87360685

3.94973491

2. 99995022

0.74998755

2.62495644

0.27956151

2.28930413

0.87593740

7.12488176

4.87491910

6.74988799

4.56372798

6.73038565

3.82384606

2.99995022

0.74998755

2.62495644

0.32956408

2. 30987160

0.55070116

7.12488176

4.87491910

6.74988799

4.53752410

6.75700615

4.37243110

2. 61128680

7.87011955

5.43950218

2.07382659

7. 82221602

2.25974592

5.24600269

5.03559857
8.00000000

9.06064257

10.12128514

11.29147436

12.65595546

13.77367213

9.06064257

10.12128514

11.16541661

12.14677940

14.09816205

8.00000000

9.06064257

10.12128514

11.12386142

12.75138222

13.77295503

8.00000000

9.06064257

10.12128514

11.25498270

12.19443684

13.55683646

8.00000000

10.12128514

11.10857214

12.54494763

13.15499265

13.98814032

14.19670700

13.82346080

14.02753816

14.26546418

14.13357015

13.93226220

13.14143437
8.00000000

9.06064257

. /oxygen/Ni/311/iter_9/struct.xyz

45

Lattice $=" 7.462281943147142 \quad 0.0 \quad 0.0 \quad 2.4874273143823786 \quad 8.24986309508776 \quad 0.0 \quad 0.0 \quad 0.021 .30321$ 2853088358" Properties=species:S:1:pos:R:3 pbc="T T T"

$\begin{array}{lllr}\mathrm{Ni} & 2.48742731 & 2.99995022 & 8.00000000 \\ \mathrm{Ni} & 2.48742731 & 0.74998755 & 9.06064257 \\ \mathrm{Ni} & 1.24371366 & 2.62495644 & 10.12128514 \\ \mathrm{Ni} & 1.24579047 & 0.30919864 & 11.19514252 \\ \mathrm{Ni} & 2.48915124 & 2.17057877 & 12.12693314 \\ \mathrm{Ni} & 6.22219431 & 0.81428505 & 14.07129779 \\ \mathrm{Ni} & 3.73114097 & 7.12488176 & 8.00000000 \\ \mathrm{Ni} & 3.73114097 & 4.87491910 & 9.06064257 \\ \mathrm{Ni} & 2.48742731 & 6.74988799 & 10.12128514 \\ \mathrm{Ni} & 2.49053322 & 4.58820198 & 11.25525316 \\ \mathrm{Ni} & 2.49046148 & 6.87101311 & 12.59661132 \\ \mathrm{Ni} & 1.24679661 & 3.81434350 & 13.66557934 \\ \mathrm{Ni} & 4.97485463 & 2.99995022 & 8.00000000 \\ \mathrm{Ni} & 4.97485463 & 0.74998755 & 9.06064257 \\ \mathrm{Ni} & 3.73114097 & 2.62495644 & 10.12128514 \\ \mathrm{Ni} & 3.73466404 & 0.30933124 & 11.19534006 \\ \mathrm{Ni} & 4.97808419 & 2.17095649 & 12.12684488 \\ \mathrm{Ni} & 1.24659516 & 0.81491352 & 14.07517144 \\ \mathrm{Ni} & 6.21856829 & 7.12488176 & 8.00000000 \\ \mathrm{Ni} & 6.21856829 & 4.87491910 & 9.06064257 \\ \mathrm{Ni} & 4.97485463 & 6.74988799 & 10.12128514 \\ \mathrm{Ni} & 4.97659619 & 4.58888662 & 11.25546893 \\ \mathrm{Ni} & 4.97759575 & 6.87195904 & 12.59719353 \\ \mathrm{Ni} & 3.73537561 & 3.81416272 & 13.66514138 \\ \mathrm{Ni} & 7.46228194 & 2.99995022 & 8.00000000 \\ \mathrm{Ni} & 7.46228194 & 0.74998755 & 9.06064257\end{array}$


$\mathrm{Ni}$

$\mathrm{Ni}$

6.21856829

6.22024993

7.46484169

3.73385250

8.70599560

8.70599560

7.46228194

7.46480527

7.46609432

6.22275106

2.49160938

2.49070580

7.46596816

4.97938246

4.97727063

7.46598330

4.97784498

2.49118631

7.46456326

./all_structures.xyz

./amd_subs/geo_opt/struct.xyz

10

Lattice="15.0 $0.0 \quad 0.0 \quad 0.015 .0 \quad 0.0 \quad 0.0 \quad 0.015 .0$ " Properties=species:S:1:pos:R:3 pbc="T T T"

$\begin{array}{lll}\mathrm{H} & 14.92902563 & 2.00610918 \\ \mathrm{H} & 13.44880413 & 1.20689151\end{array}$

$-0.09142288 \quad 13.06340551$

$1.97247198 \quad 13.98876594$

$2.04026204 \quad 0.54791169$

$1.82804319 \quad 0.51908999$

$0.05884755 \quad-0.03917839$

$1.56015692 \quad 0.00297399$

$14.46321475 \quad 1.20206282$

$14.32807668 \quad 13.90558776$

/atoms/Cu_atom/struct.xyz

1

Lattice $=" 14.5 \quad 0.0 \quad 0.0 \quad 0.0 \quad 15.0 \quad 0.0 \quad 0.0 \quad 0.0 \quad 15.5 "$ Properties=species:S:1:pos:R:3 pbc="T T T" $\mathrm{Cu} \quad 0.00000000 \quad 0.00000000$

. /atoms/C_atom/struct.xyz

1

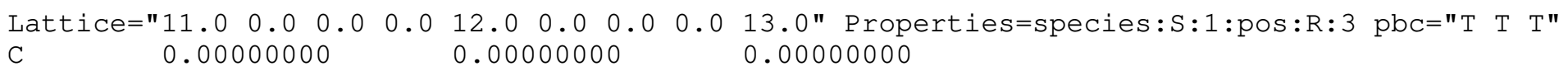

. /atoms/H_atom/struct.xyz

1

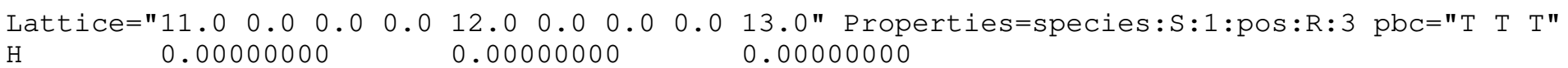

. /atoms/Ni_atom/struct.xyz

1

Lattice $=" 14.5 \quad 0.0 \quad 0.0 \quad 0.0 \quad 15.0 \quad 0.0 \quad 0.0 \quad 0.0 \quad 15.5 "$ Properties=species:S:1:pos:R:3 pbc="T T T" $\mathrm{Ni} \quad 0.00000000 \quad 0.00000000$

. /atoms/N_atom/struct.xyz

1

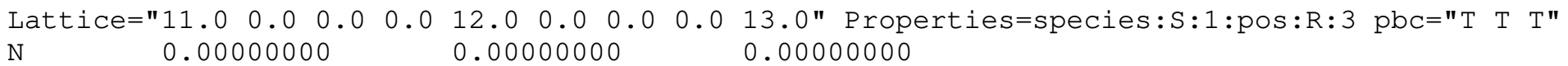

. /atoms/o_atom/struct.xyz

1

Lattice $=" 11.0 \quad 0.0 \quad 0.0 \quad 0.012 .0 \quad 0.0 \quad 0.0 \quad 0.013 .0 "$ Properties=species:S:1:pos:R:3 pbc="T T T" $00.00000000 \quad 0.00000000 \quad 0.00000000$

. /cu2fa2/geo_opt/struct.xyz

10

Lattice $=" 14.19990 .00 .00 .016 .7575$ $\mathrm{C}=" \mathrm{~T}$ T $\mathrm{T} "$

$\begin{array}{ll}\mathrm{C} & -3.27040000 \\ \mathrm{C} & -0.89530000 \\ \mathrm{O} & -4.18240000 \\ \mathrm{O} & -1.72700000 \\ \mathrm{O} & -2.43780000 \\ \mathrm{O} & 0.01750000 \\ \mathrm{H} & -3.78290000\end{array}$

$\mathrm{H}$
2.05450000

$-2.66630000$

0.80980000

2.04500000

$-2.65720000$

$-1.42200000$

3. 07280000
$0.00 .00 .010 .0964 "$ Properties=species:S:1:pos:R:3 pb

$$
\begin{array}{r}
0.02220000 \\
0.00460000 \\
0.06410000 \\
0.04780000 \\
0.05860000 \\
0.03320000 \\
-0.00340000
\end{array}
$$


$\mathrm{H}$

$-3.50720000$

$\mathrm{Cu} \quad-0.65830000$

.$/$

14

Lattice $=" 20.0 \quad 0.0 \quad 0.0 \quad 0.0 \quad 20.0 \quad 0.0 \quad 0.0 \quad 0.0$ $\mathrm{H} \quad 1.71715853$

$\mathrm{H} \quad 18.31898687$

$\mathrm{H} \quad 4.21871313$

$\mathrm{H} \quad 0.82054147$

$\mathrm{H} \quad 19.47690782$

$\mathrm{H} \quad 3.06079218$

C $\quad 0.02742104$

C $\quad 2.51027896$

$\mathrm{Cu} \quad 2.38920882$

$\mathrm{Cu} \quad 0.14849118$

$\mathrm{N} \quad 1.35481214$

$\mathrm{N} \quad 19.32508796$

3.21261204

$\mathrm{N} \quad 1.18288786$
19.05048017

1.01219159

3.38322841

5.34494983

5.30147804

0.04755873

4.34787127

1.55096369

2.84446631

0.00301204

1.17474747

3.22067253

4.39241796
19.09395196
Fri Apr 16 14:51:55 2021

231

$$
\begin{aligned}
-3.68470000 & -0.03230000 \\
-1.02300000 & -0.00030000 \\
0.41040000 & -0.02420000
\end{aligned}
$$

20.0" Properties=species: $\mathrm{S}: 1:$ pos:R: 3 pbc="T T T" 0.00000000

0.00000000

0.00000000

0.00000000

0.00000000

0.00000000

0.00000000

0.00000000

0.00000000

0.00000000

0.00000000

0.00000000

0.00000000

0.00000000

. /cu2gnd2/geo_opt/struct.xyz

18

Lattice $=" 20.0 \quad 0.0 \quad 0.0 \quad 0.0 \quad 20.0 \quad 0.0 \quad 0.0 \quad 0.0$

C

19.50244483

1.66408690

19.60830460

16.39223367

18.21995324

0.95522613

0.81132985

18.29953591

0.89199229

19.47278703

19.63627418

17.34948257

1.65500233

17.45661344

1.76371327

18.55989185

0.36372582

18.74370534

0.54844134

0.98370693

17.07265598

17.10153926

3.17376260

14.88255756

1.57848327

1.62552502

16.43070983

16.47919899

3. 71758120

3.73945482

14.31749467

14.33832006

19.01172927

18.05774164

19.04477387

0 20.0" Properties=species:S:1:pos:R:3 pbc="T T T" 0.25550402

0.38623728

0.74027734

19.80140409

0.84093773

19.90502905

0.22278844

0.41622597

1.03324775

19.47154532

1.17147479

19.61386311

0.54382809

19.87905129

0.75695333

0.09497095

0.86309159

19.78088189

. /fa/geo_opt/struct.xyz

5

Lattice $=" 12.2717999815940860 .0 \quad 0.0 \quad 0.011 .9135000705719030 .00 .00 .010 .0 "$ Properties=spe cies:S:1:pos:R:3 pbc="T T T"

$\begin{array}{rrrr}\mathrm{O} & 0.97112972 & 0.17908872 & 0.00000000 \\ \mathrm{O} & 11.24350624 & 1.21145687 & 0.00000000 \\ \mathrm{C} & 11.88060053 & 0.18698755 & 0.00000000 \\ \mathrm{H} & 11.43051643 & 11.08155848 & 0.00000000 \\ \mathrm{H} & 1.28954706 & 11.16780855 & 9.97000000\end{array}$

. /famd/geo_opt/struct.xyz

7

Lattice="15.0 0.0 0.0 0.0 15.0 $0.00 .0 \quad 0.015 .0 "$ Properties=species:S:1:pos:R:3 pbc="T T T" $\mathrm{H} \quad 1.10678109$

14.97593391

0.03021694

2.03337357

13.50639629

14.95604409

0.04748865

14.50853359

14.37149238

1.23716972

13.06660674

14.96670773

1.18956918

13.87997612

0.25465957

14.95744622

14.48826539

0.05198445

14.94809894

14.65158313

14.83416230

. /gnd/geo_opt/struct.xyz

9

Lattice $=" 20.0 \quad 0.0 \quad 0.0 \quad 0.0$

C

18.24028598

16.89386397

19.50523425

18.32155367

16.83380596

$20.0 \quad 0.0 \quad 0$.

1.30467729

0.62364312

0.48147648

2.80895089

19.51852352

$0.020 .0 "$ Properties=species: $\mathrm{S}: 1: \mathrm{pos}: \mathrm{R}: 3 \mathrm{pbc}=" \mathrm{~T} \mathrm{~T} \mathrm{~T} "$ 0.02610869

19.97379058

19.97820576

0.12867112

19.88810324 
all_structures.xyz

$\mathrm{H}$

$\mathrm{H}$

$\mathrm{H}$

./h2/geo_opt/struct.xyz

2

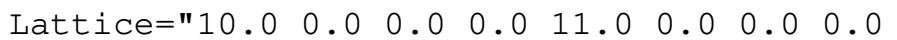

$\mathrm{H} \quad 5.00000000 \quad 5.50000000$

$\mathrm{H} \quad 5.00000000 \quad 5.50000000$

1.22774685

19.37653498

0.98179419

3.41373199
232

\author{
0.02179424 \\ 19.89318021 \\ 0.02914216 \\ 0.16860764
}

./h2o/geo_opt/struct.xyz

3

Lattice $=" 10.0 \quad 0.0 \quad 0.0 \quad 0.0 \quad 11.0 \quad 0.0 \quad 0.0 \quad 0.0 \quad 12.0 "$ Properties=species:S: $1: p o s: R: 3$ pbc="T T T"

$\begin{array}{llll}\mathrm{O} & 5.00000000 & 5.50000000 & 6.29879953 \\ \mathrm{H} & 5.00000000 & 6.26789362 & 5.70152298 \\ \mathrm{H} & 5.00000000 & 4.73210638 & 5.68652298\end{array}$

./n2/geo_opt/struct.xyz

2

Lattice $=" 10.0 \quad 0.0 \quad 0.0 \quad 0.0 \quad 11.0 \quad 0.0 \quad 0.0 \quad 0.0 \quad 12.0 "$ Properties=species:S:1:pos:R:3 pbc="T T T" $\begin{array}{lrll}\mathrm{N} & -0.00776380 & 0.00000000 & 0.00000000 \\ \mathrm{~N} & 1.10526380 & 0.00000000 & 0.00000000\end{array}$

./nh3/geo_opt/struct.xyz

4

Lattice $=" \begin{array}{llllllllll}6.0 & 0.0 & 0.0 & 0.0 & 7.0 & 0.0 & 0.0 & 0.0\end{array}$

$\begin{array}{llll}\mathrm{H} & 5.64827249 & 6.46351621 & 0.38481161 \\ \mathrm{H} & 0.20139471 & 1.00304247 & 0.29915468 \\ \mathrm{H} & 0.71646026 & 6.88861030 & 7.21960060 \\ \mathrm{~N} & 5.88957253 & 0.21203102 & 7.71363311\end{array}$

./ni2fa4/geo_opt/output.xyz

18

Properties=species: $\mathrm{S}: 1: \mathrm{pos}: \mathrm{R}: 3 \mathrm{pbc}=" \mathrm{~T} \mathrm{~T} \mathrm{~T}$

$\begin{array}{lrrr}\mathrm{C} & -0.03744617 & -1.31128383 & -2.12905183 \\ \mathrm{C} & 0.03441651 & 1.31257431 & 2.12873118 \\ \mathrm{C} & 0.00118962 & -2.12536738 & 1.31830716 \\ \mathrm{C} & 0.00124616 & 2.12444761 & -1.31909424 \\ \mathrm{H} & 0.04764679 & 1.89060009 & 3.07097114 \\ \mathrm{H} & -0.05297865 & -1.88780682 & -3.07225541 \\ \mathrm{H} & 0.00078798 & -3.06351970 & 1.90327089 \\ \mathrm{H} & 0.00116477 & 3.06167977 & -1.90540787 \\ \mathrm{O} & 1.11199488 & -1.02757569 & -1.66467610 \\ \mathrm{O} & -1.17199897 & -1.01062259 & -1.63863589 \\ \mathrm{O} & -1.11470701 & 1.02733303 & 1.66340519 \\ \mathrm{O} & 1.16935874 & 1.01199764 & 1.63996929 \\ \mathrm{O} & -1.14087396 & -1.64285769 & 1.03363158 \\ \mathrm{O} & -1.14098372 & 1.65697885 & -1.01090596 \\ \mathrm{O} & 1.14316369 & -1.65766882 & 1.01107148 \\ \mathrm{O} & 1.14304495 & 1.64239536 & -1.03410674 \\ \mathrm{Ni} & -1.15988942 & 0.00800903 & 0.01269187 \\ \mathrm{Ni} & 1.16034195 & -0.00809584 & -0.01233905\end{array}$

./ni2fa4/geo_opt/struct.xyz

18

Lattice $=" 20.0 \quad 0.0 \quad 0.00 .0$

$\mathrm{C}$
$\mathrm{C}$
$\mathrm{C}$
$\mathrm{C}$
$\mathrm{H}$
$\mathrm{H}$
$\mathrm{H}$
$\mathrm{H}$
$\mathrm{O}$
$\mathrm{O}$
$\mathrm{O}$
$\mathrm{O}$

$-5.19010000$

20.00 .00 .0

2.57100000

5.47660000

1.66880000

$-5.14410000$

6.37790000

6.07310000

1.98210000

0.70350000

7.34150000

2.93350000

6.36858300

6.36858300
5.63141700

$-5.14450000$

$-5.20350000$

$-5.14500000$

$-5.14450000$

$-3.81320000$

2. 95150000

$-6.54870000$

5.11170000

5.09490000

2.28380000

$-6.51140000$

5.77870000

2.26750000

5.76290000

$-3.77710000$

4.03220000

0.0

$20.0 "$ Prop
0.46540000
5.18910000

4.29240000

1. 36410000

6.16230000

$-0.50450000$

4.89440000

0.75780000

1.06710000

1.09260000

4.58990000

4.56460000

3.92210000

1.75830000

3.89780000

1.73450000

-3.77710000
-6.65570000

2.84230000 
$\mathrm{Ni} \quad-3.63700000$

. /ni2fa4/vib/output. xyz

18

Properties $=$ species: $\mathrm{S}: 1:$ pos: $\mathrm{R}: 3 \mathrm{pbc}=" \mathrm{~T} \mathrm{~T} \mathrm{~T}$ "

$\mathrm{C}$

C

$-0.03744617$

0.03441651

0.00118962

0.00124616

0.04764679

$-0.05297865$

0.00078798

0.00116477

1.11199488

$-1.17199897$

$-1.11470701$

1.16935874

$-1.14087396$

$-1.14098372$

1.14316369

1.14304495

$-1.15988942$

1.16034195
Fri Apr 16 14:51:55 2021
$4.01390000 \quad 2.81430000$

$$
\begin{array}{rr}
-1.31128383 & -2.12905183 \\
1.31257431 & 2.12873118 \\
-2.12536738 & 1.31830716 \\
2.12444761 & -1.31909424 \\
1.89060009 & 3.07097114 \\
-1.88780682 & -3.07225541 \\
-3.06351970 & 1.90327089 \\
3.06167977 & -1.90540787 \\
-1.02757569 & -1.66467610 \\
-1.01062259 & -1.63863589 \\
1.02733303 & 1.66340519 \\
1.01199764 & 1.63996929 \\
-1.64285769 & 1.03363158 \\
1.65697885 & -1.01090596 \\
-1.65766882 & 1.01107148 \\
1.64239536 & -1.03410674 \\
0.00800903 & 0.01269187 \\
-0.00809584 & -0.01233905
\end{array}
$$

/ni2famd4/geo_opt/output.xyz

26

Properties=species: $\mathrm{S}: 1:$ pos: $\mathrm{R}: 3$ pbc $=" \mathrm{~T}$ T T"

$\begin{array}{lrrr}\mathrm{C} & 0.04880471 & -1.31865126 & -2.14694121 \\ \mathrm{C} & -0.05011484 & 1.32055690 & 2.14561805 \\ \mathrm{C} & -0.01089443 & -2.14541476 & 1.32221019 \\ \mathrm{C} & 0.01208336 & 2.14520821 & -1.32177198 \\ \mathrm{H} & -0.07121891 & 1.89814969 & 3.08406203 \\ \mathrm{H} & 0.06966044 & -1.89295223 & -3.08740546 \\ \mathrm{H} & -0.01530160 & -3.08282964 & 1.90187908 \\ \mathrm{H} & 0.01679125 & 3.08279579 & -1.90105591 \\ \mathrm{H} & 2.02818151 & -1.28522350 & -2.11292091 \\ \mathrm{H} & -1.93080138 & -1.34071872 & -2.16695953 \\ \mathrm{H} & -2.02954248 & 1.31424866 & 2.09418207 \\ \mathrm{H} & 1.92929836 & 1.31633637 & 2.18242384 \\ \mathrm{H} & -1.96694835 & 2.11809415 & -1.36486835 \\ \mathrm{H} & 1.99150729 & 2.15706252 & -1.26918271 \\ \mathrm{H} & -1.99072122 & -2.14177605 & 1.29606488 \\ \mathrm{H} & 1.96882037 & -2.13403152 & 1.33883168 \\ \mathrm{~N} & 1.17632552 & 1.62611917 & -0.96744275 \\ \mathrm{~N} & -1.15732065 & 1.60437398 & -1.02141931 \\ \mathrm{~N} & 1.15868176 & -1.61177176 & 1.00994525 \\ \mathrm{~N} & -1.17552745 & -1.61953034 & 0.97942553 \\ \mathrm{~N} & -1.12990170 & -1.00781130 & -1.63292604 \\ \mathrm{~N} & -1.20506637 & 0.99221471 & 1.59026582 \\ \mathrm{~N} & 1.12877822 & 0.99623301 & 1.64013375 \\ \mathrm{~N} & 1.20404679 & -0.98043580 & -1.59789405 \\ \mathrm{Ni} & -1.27449453 & -0.00844062 & -0.02317873 \\ \mathrm{Ni} & 1.27452021 & 0.00808085 & 0.02342378\end{array}$

./ni2famd4/geo_opt/struct.xyz

26

Lattice $=" 20.0 \quad 0.0 \quad 0.0 \quad 0.0 \quad 20.0 \quad 0.0 \quad 0.0$

$\begin{array}{ll}\mathrm{C} & -5.08580000 \\ \mathrm{C} & -5.19520000 \\ \mathrm{C} & -5.15120000 \\ \mathrm{C} & -5.12510000 \\ \mathrm{H} & -5.21680000 \\ \mathrm{H} & -5.06430000 \\ \mathrm{H} & -5.15570000 \\ \mathrm{H} & -5.12030000 \\ \mathrm{H} & -2.78720000 \\ \mathrm{H} & -7.38780000 \\ \mathrm{H} & -7.49310000 \\ \mathrm{H} & -2.89430000 \\ \mathrm{H} & -7.42470000 \\ \mathrm{H} & -2.82650000\end{array}$

2.56860000

5.46400000

1.65900000

6.36740000

6.06310000

1.97600000

0.69130000

7. 33680000

2. 65160000

2.58400000

5.40510000

5.40520000

6.25750000

1.32221019

$-1.32177198$

3.08406203

$-3.08740546$

1.90187908

$-1.90105591$

$-2.11292091$

$-2.16695953$

2.09418207

2.18242384

1. 29606488

1. 33883168

$-0.96744275$

$-1.02141931$

1.00994525

0.97942553

1. 63292604

1. 59026582

1. 64013375

$-0.02317873$

.02342378

$0.020 .0 "$ Properties=species:S:1:pos:R:3 pbc="T T T" 0.47340000

5.18860000

4.29050000

1.38010000

6.15890000

$-0.49980000$

4.89120000

0.77850000

0.59750000

0.53660000

5.04950000

5.15260000

1. 38420000

1.49650000 


$\begin{array}{ll}\mathrm{H} & -7.44990000 \\ \mathrm{H} & -2.85040000 \\ \mathrm{~N} & -3.75810000 \\ \mathrm{~N} & -6.49880000 \\ \mathrm{~N} & -3.77790000 \\ \mathrm{~N} & -6.51930000 \\ \mathrm{~N} & -6.47020000 \\ \mathrm{~N} & -6.55190000 \\ \mathrm{~N} & -3.81090000 \\ \mathrm{~N} & -3.72860000 \\ \mathrm{Ni} & -6.64570000 \\ \mathrm{Ni} & -3.63350000\end{array}$

./ni2 famd4/geo_opt/vasp_opt. xyz

1.74000000

1.74690000

5.77610000

5.74940000

2.26730000

2.25860000

2. 92010000

5.08720000

5.09120000

2. 95450000

4.00280000

4.02290000
4.20160000
4.24870000
1.78870000
1.72480000
3.92810000
3.89460000
1.06340000
4.55390000
4.61230000
1.10310000
2.80660000
2.86070000

26

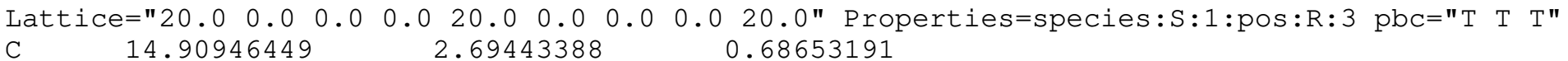

14.81054493

14.84976535

14.87274313

14.78944087

14.93032021

14.84535818

14.87745103

16.88884128

12.92985840

12.83111730

16.78995813

12.89371142

16.85216707

12.86993855

16.82948014

16.03698530

13.70333912

16.01934153

13.68513232

13.73075808

13.65559340

15.98943800

16.06470656

13.58616524

16.13517998
5. 33364204

1.86767038

6.15829334

5.91123482

2. 12013290

0.93025549

7.09588093

2.72786163

2.67236641

5.32733380

5.32942150

6.13117929

6.17014765

1. 87130908

1.87905361

5.63920431

5.61745911

2.40131337

2.39355480

3. 00527383

5.00529984

5.00931814

3.03264933

4.00464452

4.02116599
0.68653191

4.97909117

4.15568330

1.51170113

5.91753514

19.74606766

4.73535219

0.93241721

0.72055220

0.66651359

4.92765519

5.01589696

1.46860476

1. 56429041

4.12953799

4.17230479

1.86603036

1.81205381

3.84341837

3.81289865

1.20054707

4.42373894

4.47360687

1.23557906

2.81029438

2.85689690

. /ni2famd4/vib/output.xyz

26

Properties=species: $\mathrm{S}: 1: \mathrm{pos}: \mathrm{R}: 3 \mathrm{pbc}=" \mathrm{~T} \mathrm{~T} \mathrm{~T}$ "

$\begin{array}{lrrr}\mathrm{C} & 0.04880471 & -1.31865126 & -2.14694121 \\ \mathrm{C} & -0.05011484 & 1.32055690 & 2.14561805 \\ \mathrm{C} & -0.01089443 & -2.14541476 & 1.32221019 \\ \mathrm{C} & 0.01208336 & 2.14520821 & -1.32177198 \\ \mathrm{H} & -0.07121891 & 1.89814969 & 3.08406203 \\ \mathrm{H} & 0.06966044 & -1.89295223 & -3.08740546 \\ \mathrm{H} & -0.01530160 & -3.08282964 & 1.90187908 \\ \mathrm{H} & 0.01679125 & 3.08279579 & -1.90105591 \\ \mathrm{H} & 2.02818151 & -1.28522350 & -2.11292091 \\ \mathrm{H} & -1.93080138 & -1.34071872 & -2.16695953 \\ \mathrm{H} & -2.02954248 & 1.31424866 & 2.09418207 \\ \mathrm{H} & 1.92929836 & 1.31633637 & 2.18242384 \\ \mathrm{H} & -1.96694835 & 2.11809415 & -1.36486835 \\ \mathrm{H} & 1.99150729 & 2.15706252 & -1.26918271 \\ \mathrm{H} & -1.99072122 & -2.14177605 & 1.29606488 \\ \mathrm{H} & 1.96882037 & -2.13403152 & 1.33883168 \\ \mathrm{~N} & 1.17632552 & 1.62611917 & -0.96744275 \\ \mathrm{~N} & -1.15732065 & 1.60437398 & -1.02141931 \\ \mathrm{~N} & 1.15868176 & -1.61177176 & 1.00994525 \\ \mathrm{~N} & -1.17552745 & -1.61953034 & 0.97942553 \\ \mathrm{~N} & -1.12990170 & -1.00781130 & -1.63292604 \\ \mathrm{~N} & -1.20506637 & 0.99221471 & 1.59026582 \\ \mathrm{~N} & 1.12877822 & 0.99623301 & 1.64013375 \\ \mathrm{~N} & 1.20404679 & -0.98043580 & -1.59789405\end{array}$




$\begin{array}{rrrr}\mathrm{Ni} & -1.27449453 & -0.00844062 & -0.02317873 \\ \mathrm{Ni} & 1.27452021 & 0.00808085 & 0.02342378\end{array}$

./ni2famd4/vib/vasp_opt.xyz

26

$\begin{array}{ccccccccccc}\text { Lattice }=" 20.0 & 0.0 & 0.0 & 0.0 & 20.0 & 0.0 & 0.0 & 0.0 & 20.0 " & \text { Properties=species:S:1:pos:R:3 pbc="T T T" } \\ \mathrm{C} & 14.90946449 & 2.69443388 & 0.68653191\end{array}$

14.81054493

5.33364204

4.97909117

14.84976535

1. 86767038

4.15568330

14.87274313

6.15829334

1. 51170113

14.78944087

5.91123482

14.93032021

2.12013290

14.84535818

0.93025549

5.91753514

14.87745103

7.09588093

19.74606766

4.73535219

0.93241721

16.88884128

2.72786163

0.72055220

12.92985840

2. 67236641

0.66651359

12.83111730

5. 32733380

5.32942150

4.92765519

16.78995813

6.13117929

5.01589696

12.89371142

6.17014765

1.46860476

1. 56429041

12.86993855

1.87130908

4.12953799

16.82948014

1.87905361

4.17230479

5.63920431

13.70333912

5.61745911

1.86603036

1.81205381

16.01934153

2. 40131337

13.68513232

2.39355480

3. 00527383

3. 84341837

3. 81289865

1. 20054707

13.65559340

5.00529984

5.00931814

15.98943800

3.03264933

4.00464452

4.42373894

4.47360687

1.23557906

2.81029438

13.58616524

4.02116599

2.85689690

./ni3fa6/geo_opt/struct.xyz

27

Lattice $=" 20.0 \quad 0.0 \quad 0.0 \quad 0.020 .0 \quad 0.0 \quad 0.0 \quad 0.020 .0 "$ Properties=species:S:1:pos:R:3 pbc="T T T" $\mathrm{Ni} \quad 12.63928423$

$\mathrm{Ni} \quad 15.52987529$

1.94163146

14.62522063

3.07800590

1.53553235

0.21103116

14.40639465

13.21176506

15.82300211

3.12289235

2. 29351457

3.29919064

19.19079652

19.67017366

1.07158618

4.97166290

2.15146643

4.55916557

0.64202294

2. 55935321

11.87141343

0.77372439

3. 92014181

2.07991472

18.30739912

0.43988220

12.99744093

16.18722289

2. 39719154

5.98767700

14.56577008

3. 42353061

12.67623416

5.52353152

0.42336689

0.35130934

18.11637822

3. 72806332

12.34307164

1. 47781285

2.44928945

0.21186710

13.35234913

12.50079867

19.02886087

1.05141005

15.19919881

0.02205145

18.61643565

2.85160773

4.63136656

1.75642236

2.02078398

3. 00988894

19.88112612

0.82712265

1.92901995

4.87473600

4.13868843

$-0.02522563$

2.71679187

1.75491141

1.98478511

0.48018527

4.08093723

3. 01335243

2.81654662

. /ni3famd6/geo_opt/struct.xyz

40

Lattice $=" 20.0 \quad 0.0 \quad 0.0 \quad 0$.

$\mathrm{Ni} \quad 8.26453403$

$\mathrm{Ni} \quad 11.40023403$

$\mathrm{Ni} \quad 10.35723403$

$\mathrm{N} \quad 10.83403403$

$\begin{array}{rr}0 & 20.5 \quad 0.0 \\ 10.36729271\end{array}$

11.84899271

8.55539271

12.43639271

$21.0 "$ Prop
9.31249636

10.49779636

11.70859636

8.59529636 
$\mathrm{N}$

$\mathrm{N}$

$\mathrm{N}$

$\mathrm{N}$

$\mathrm{N}$

$\mathrm{N}$

$\mathrm{N}$

$\mathrm{N}$

$\mathrm{N}$

$\mathrm{N}$

$\mathrm{N}$

C

C

C

C

C

C

$\mathrm{H}$

$\mathrm{H}$

$\mathrm{H}$

$\mathrm{H}$

$\mathrm{H}$

$\mathrm{H}$

$\mathrm{H}$

$\mathrm{H}$

$\mathrm{H}$

$\mathrm{H}$

$\mathrm{H}$

$\mathrm{H}$

$\mathrm{H}$

H

$\mathrm{H}$

$\mathrm{H}$

$\mathrm{H}$

$\mathrm{H}$

$\mathrm{H}$

.$/$ nit

36

Lattice $=" 20.0 \quad 0.0 \quad 0.0 \quad 0.0$ $\mathrm{Ni}$

10.27073610

$\mathrm{Ni} \quad 7.38452381$

$\mathrm{Ni} \quad 9.74980376$

C $\quad 10.58205928$

C $\quad 13.06922851$

8.59677930

6.73866206

7.49327804

11.72300578

12.70113277

9.08752980

10.41991993

5.87771205

13.83407486

8.36420673

6.86535633

9.20549340

13.42699659

11.99523063

7.88086984

6.44346249

13.38585621

12.00300807

6.91257719

8.69028931

13.20359595

11.48301969

7.87145119
Fri Apr 16 14:51:55 2021

13.05639271

10.57779271

11.38389271

10.38129271

8.30749271

12.43669271

10.34929271

7.42419271

8.84129271

8.13959271

9.74949271

7.38479271

9.30539271

11.45829271

9.20879271

13.24729271

10.92799271

9.08209271

14.10559271

11.41549271

8.74129271

6.56039271

11.61449271

9.48749271

11.65879271

13.62259271

12.97199271

11.14329271

7.08349271

7.33159271

7.87569271

6.78249271

10.85539271

8.45689271

9.64019271

13.50589271

. xyz

020.50 .00 .0

9.74742990

12.50026372

10.69181413

8.05587216

7.83760680

11.92345656

10.29812061

8.01543185

13.51495590

12.16263982

7.54569844

10.70139339

7.19688591

7.36407468

12.29680046

9.79950507

14.42464461

11.24938984

6.91674143

12.75708551

7.55970204

9.11766075

10.88036375

12.60389328

12.50167391

13.62923241

8.33331565

7. 37104415

9.94960114
11.30419636

9.69229636

12.39839636

7.64889636

9.18579636

9.34819636

11.02199636

10.00459636

11.65429636

13.36849636

9.04349636

10.41719636

7.48249636

12.00169636

10.57939636

13.52129636

12.80459636

10.89389636

14.54669636

10.09559636

8.25709636

6.45479636

14.18729636

12.65739636

12.23629636

8.48899636

11.19429636

12.02269636

7.65899636

9.02509636

8.75699636

12.50749636

6.83949636

8.32649636
11.85509636

9.82509636
236 10.80945158

11.87665883

10.28877700

9.02500787

11.87330212

12.82827080

12.97280391

9.08190332

11.32985484

9.30620700

8.75941026

7.84820981

12.76549743

8.82379152

13.54090996

13.93729228

11.43140547

6.88979860

8.20295840

8. 40871890

8.77840750

9.63171141

12.18329733

12.76397383

10.83989349

11.72824915

9.61459957

8.45924176

11.99340746

$.021 .0 "$ Properties=species:S:1:pos:R:3 pbc="T T T" 
0

0

O

O

0

0

0
9.56578248

9.81698202

11.48834405

10.83131640

12.32871622

8.28206072

9.80007036
Fri Apr 16 14:51:55 2021

11.11215684

7.60348759

8.71556170

12.67647831

11.05765684

11.22186970

9.65955219
13.00442759

10.89227697

11.99436653

10.04638834

9.42770377

8.67407885

7.96740514 ./ni4fa8_2/geo_opt/struct.xyz

36

Lattice="20.0 0.0 0.0 0.0 20.5 0.0 $0.0 \quad 0.021 .0$ " Properties=species:S:1:pos:R:3 pbc="T T T" $\mathrm{Ni} \quad 12.69273015$

10.01279995

10.53796293

12.49923351

10.96567018

7.30270222

9.46530954

10.47378661

8.00996382

11.98700388

10.05637356

8.99814701

13.43616060

12.37032609

12.60930138

8.09056449

7.53751055

8.45514503

13.07917218

11.68833306

12.46889659

9.17852965

8.35106385

12.21149490

7.43803695

10.67846356

8.28100264

9.83060925

7.55791484

5.70548915

14.27049352

6.84096404

12.87686019

13.88216938

9. 44888445

11.29427960

6.64647114

13.16943678

11.82986465

10.49414096

8.22703976

7.68578242

6.24886424

13.73683565

12.30865669

7.00790426

8.74374546

12.99045059

11.26667035

10.89243493

12.32543488

8.33385894

9.77218417

10.20711347

11.62528113

9.13238603

7.71277939

12.73305545

9.21815206

7.75693408

7.53953392

9.16903943

11.66909528

9.30047304

9.30288513

7.80758900

11.70114737

13.18650834

7.97045754

13.13642591

11.99382345

6.92541000

8.95935482

8.33171483

14.06523262

12.67087818

8.33923238

9.05129877

11.29252567

12.94638610

12.14101092

12.01213174

12.68437017

11.00479090

13.23702063

8.35320125

7.29156646

12.76635424

11.12561736

11.19241559

9.56382045

10.95429174

9.30454064

7.72729404

9.36809287

12.01861351

10.00461712

8.93513810

10.11484970

9.43849924

8.59911060

7.90515943

13.08663790

12.40059073

10.87250269

11.57666895

/ni4famd8_1/geo_opt/struct.xyz

52

Lattice $=" 20.0 \quad 0.00 .00$.
$\mathrm{Ni}$

$\mathrm{Ni}$

$\mathrm{Ni}$

$\mathrm{Ni}$

$\mathrm{N}$

$\mathrm{N}$

$\mathrm{N}$

$\mathrm{N}$

$\mathrm{N}$

$\mathrm{N}$

$\mathrm{N}$

$\mathrm{N}$

$\mathrm{N}$

$\mathrm{N}$

$\mathrm{N}$

$\mathrm{N}$

$\mathrm{N}$

$\mathrm{N}$

N
10.23006961

8.17811706

9.77929235

13.21536421

11.40800767

9.68460417

10.78037611

8.94825936

12.20387443

12.94224503

7. 94162705

8.42980706

8.09860853

9. 68206058

11.15557605
11.81386182

11.83625782

6.68113190

7.12399029 $\begin{array}{lll}20.5 & 0.0 & 0.0 \\ 9.88128188\end{array}$

11.84603486

10.59387707

8.67957890

10.79212125

8.77484362

12.55994582

11.01492436

12.66273989

13.37344140

10.98480644

8.44363374

9.87793677

9.41268211

11.31304163

12.24277698

7.75479145

7.68746538

7.42143435
0.021 .0 " Properties=species:S:1:pos:R:3 pbc="T T T" 10.68465256

11.42832078

10.35903416

9.52760817

11.81080555

12.15078823

12.41174270

13.02453166

9.82500897

11.72003490

9.21238899

9.83704597

12.08307410

9.70753177

8.63908050

10.83568551

8.91362665

11.12287861

8.76383331 
9.87105346 10.51289243 13.01727462

8.62559829

6.89695777

7.65497634

11.71712045

12.43084294

9.14372519

10.43919953

12.01738630

9.00322250

6.03207964

5.71306384

8.11658876

13.87323538

14.19170043

11.85037195

10.18237053

7.13896232

8.32597861

6.99029812

9.19824859

6.10656081

9.15506945

10.41411459

13.10344305

13.92637422

10.89665400

10.42958092

12.07626193

7.89154000

12.92008115
9.68496760

7.81093714

11.96803986

10.24371435

8.28831748

13.27624415

12.19353218

7.46718119

10.75124103

7.11889416

8.83222522

6.93080886

7.76112912

9.73322189

6.91867880

12.47043701

10.49521315

13.48877984

11.26066495

9.27060325

9.87791363

14.10406214

14.19659408

12.28033713

11.19473604

9.29784712

6.66251054

8.31112244

6.57433136

13.59790017

12.80605917

12.14056896

10.66688066
7.94024447

12.13558740

12.38105205

13.14549696

9.04713909

11.46515456

9.02978694

9.10812885

7.68735652

12.99086144

12.96373269

11.16577373

8.60224484

9.66872989

8.32879349

12.86982713

11.80923327

12.83412645

13.87735827

12.23122112

14.14140730

11.77628499

12.26904224

10.76569416

6.67851420

7.17192214

8.75591231

10.07205454

8.25711184

9.63163899

8.18668486

8.39233440

8.56305879 /nifa2/geo_opt/struct.xyz 9

Lattice $=" 12.8777 \quad 0.0 \quad 0.0 \quad 0.0 \quad 12.9907$ $\mathrm{C}=$ "T $\mathrm{T}$ T"
13

I $\mathrm{Ni}$

C

C

$\mathrm{H}$

$\mathrm{H}$

$\mathrm{H}$

$\mathrm{H}$

$\mathrm{H}$

$\mathrm{H}$

$\mathrm{N}$

$\mathrm{N}$

$\mathrm{N}$

$\mathrm{N}$

O $\quad 5.00000000$

5.00000000

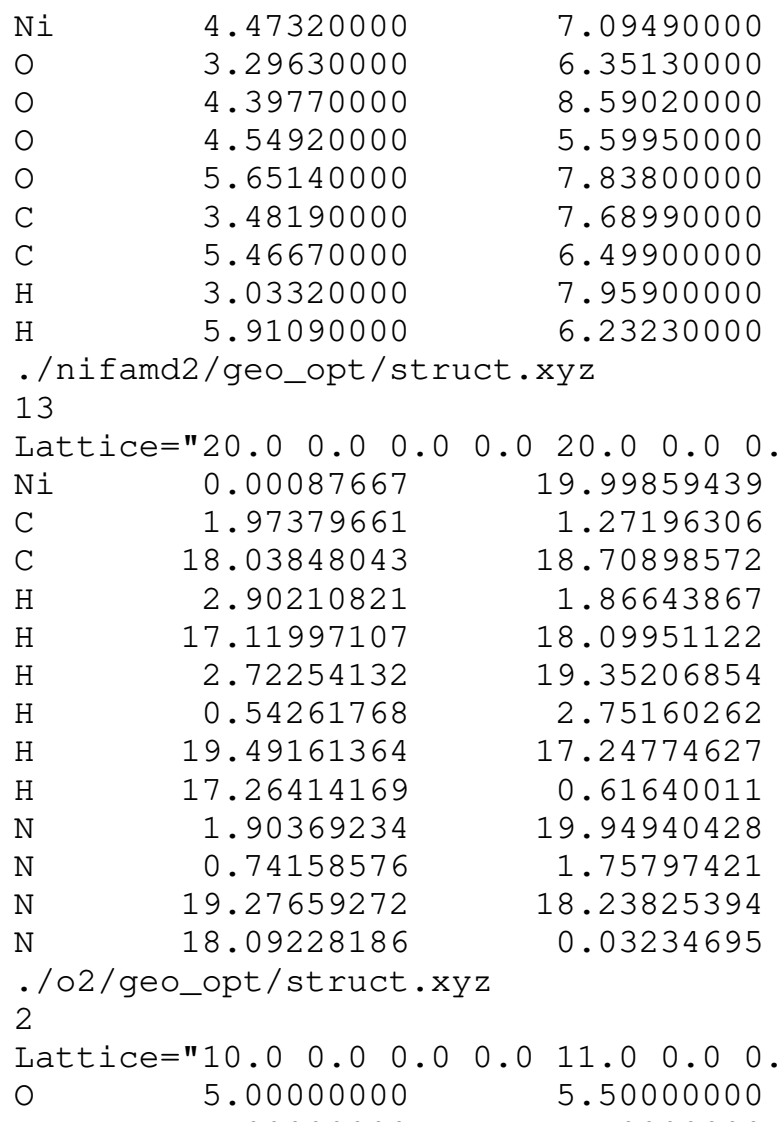

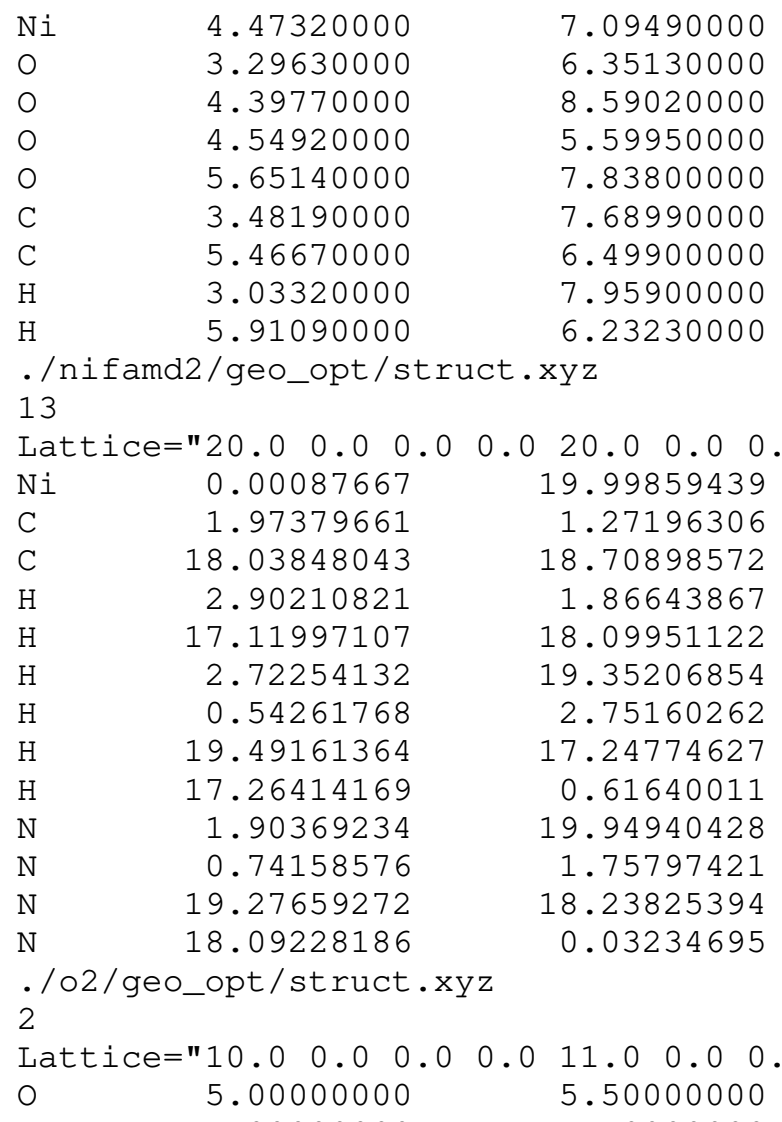

/OTHERS/cu2amd2_subs/Cu2Amd2_c1/geo_opt/struct.xyz

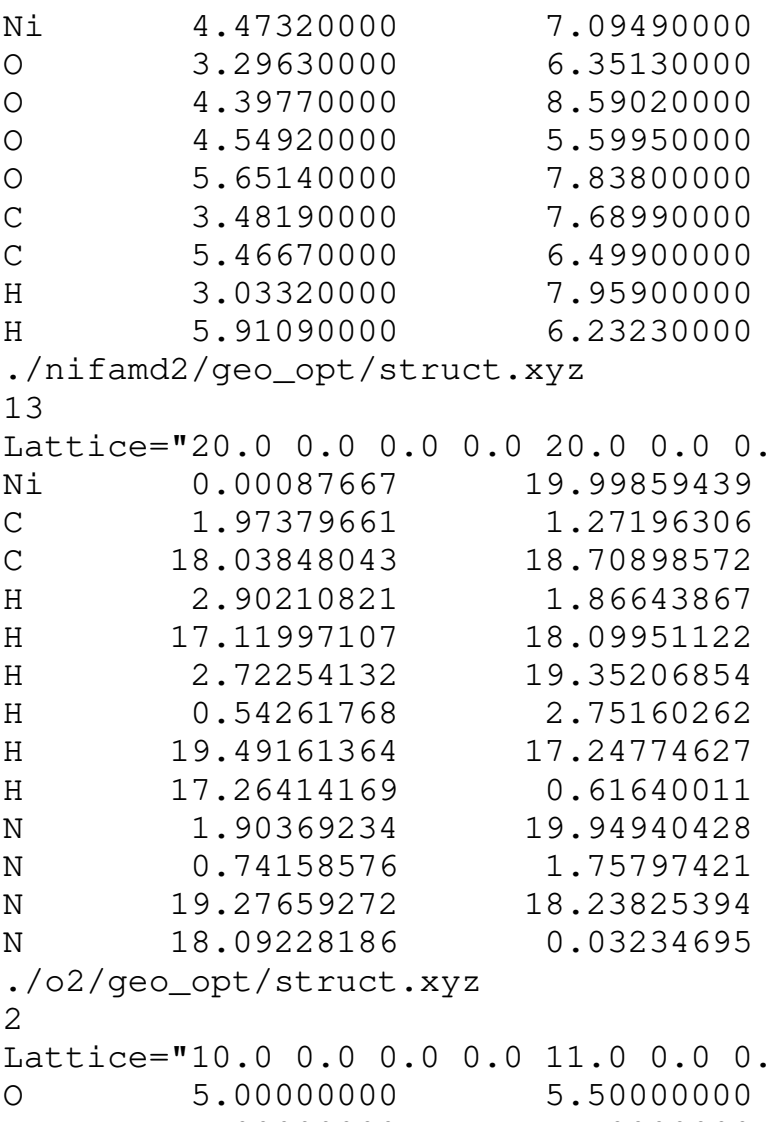

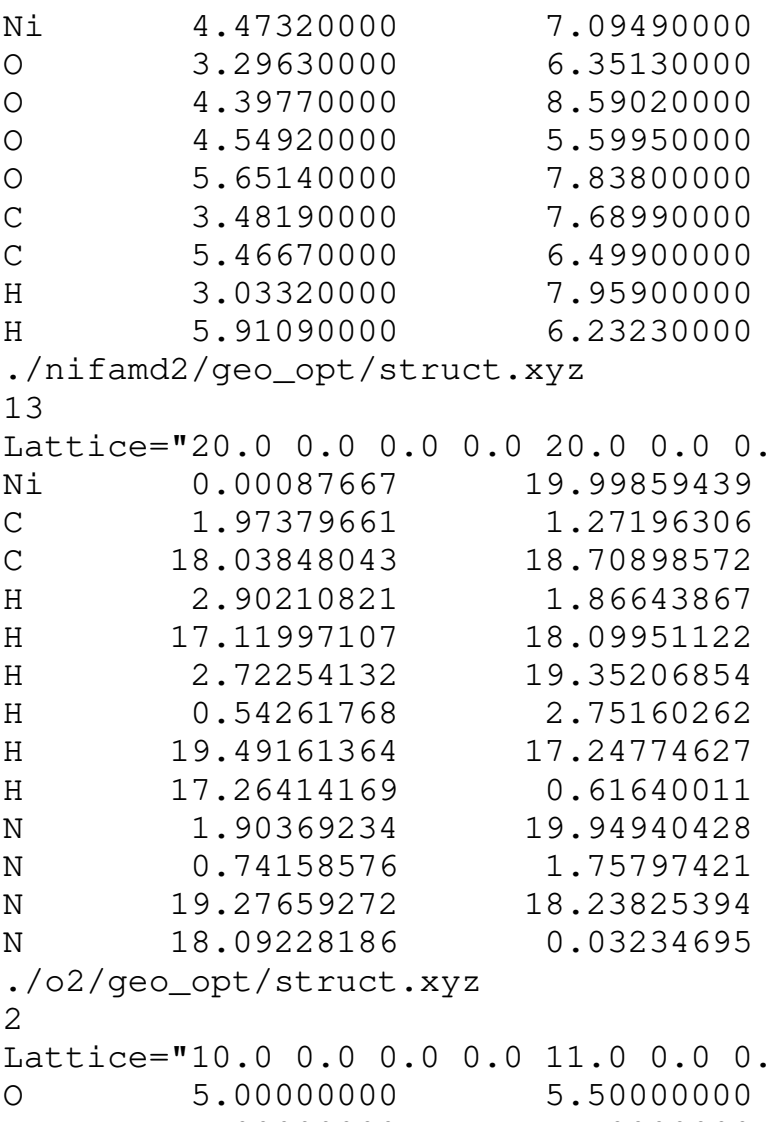

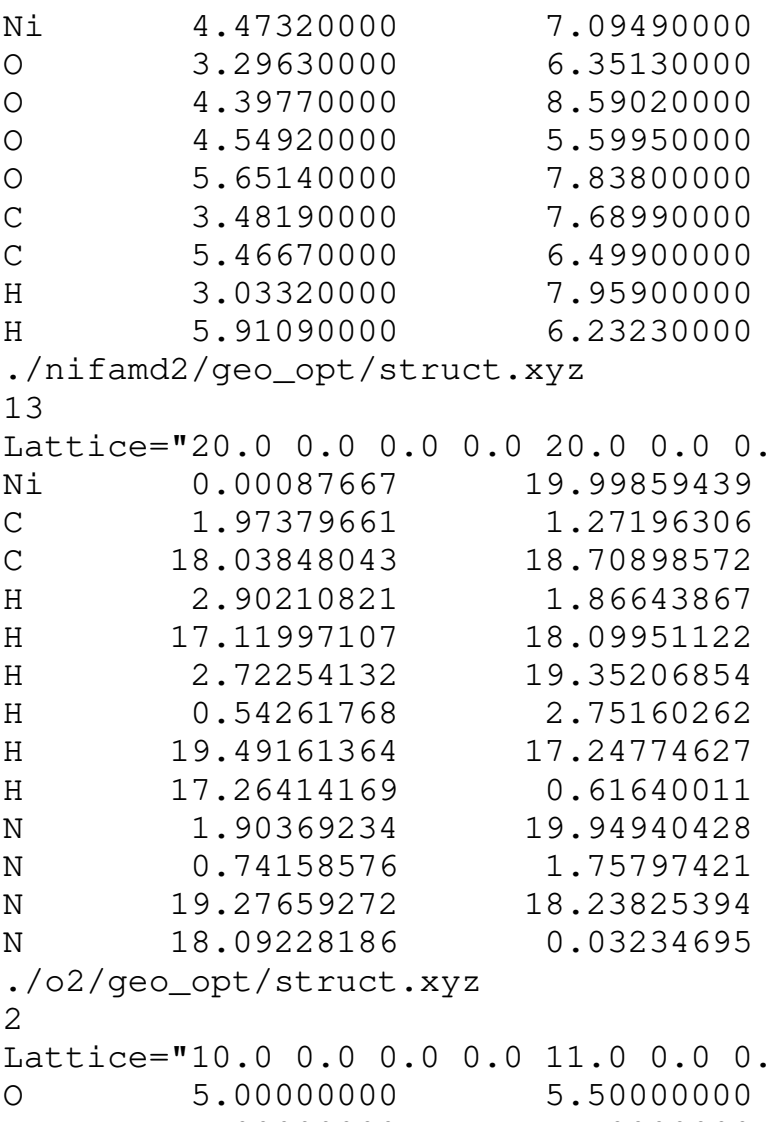

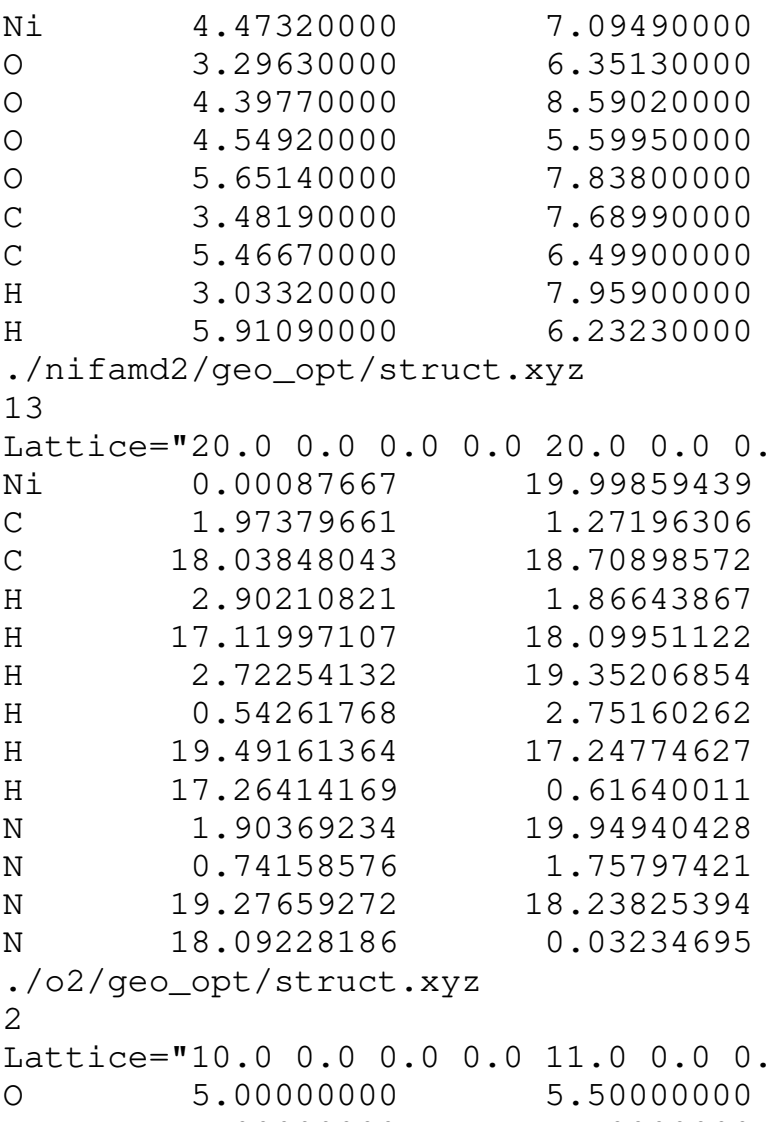

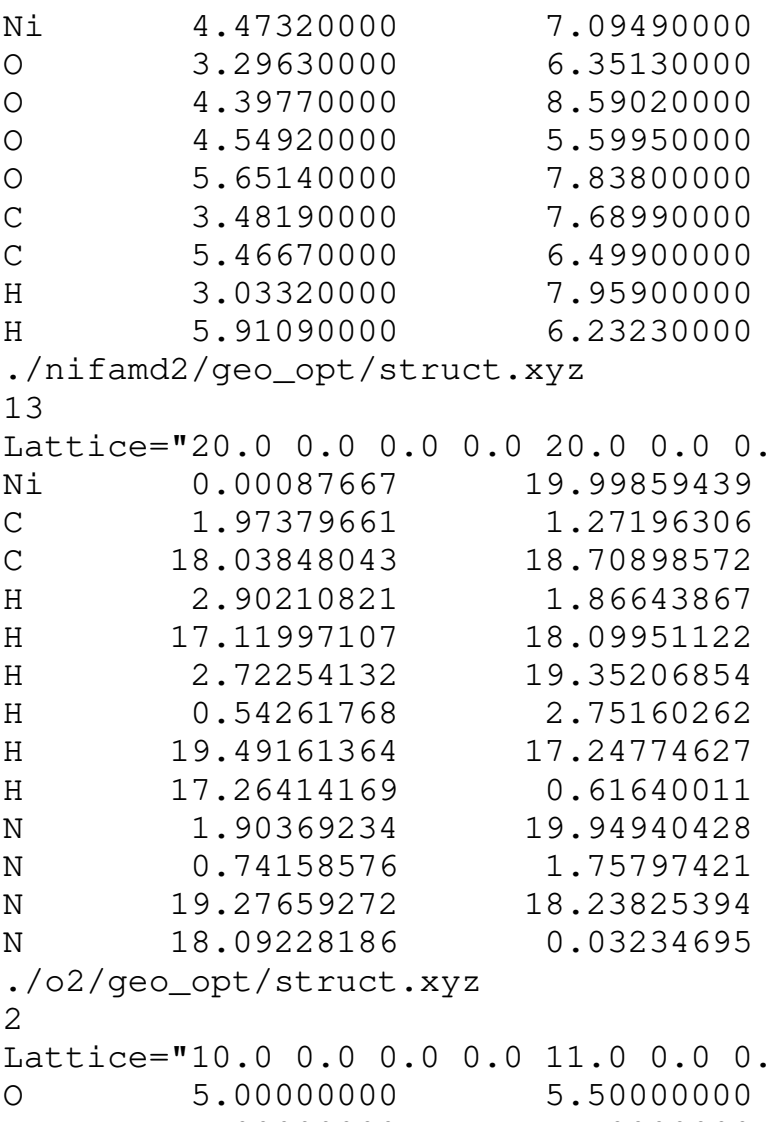

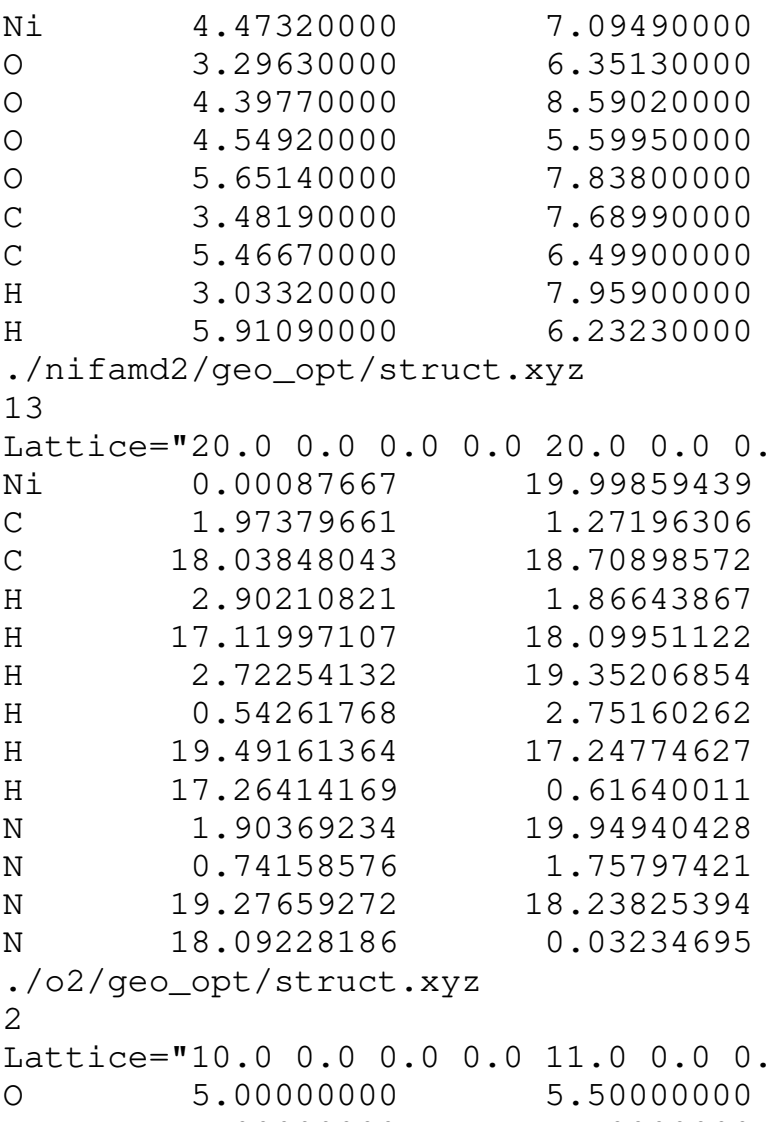

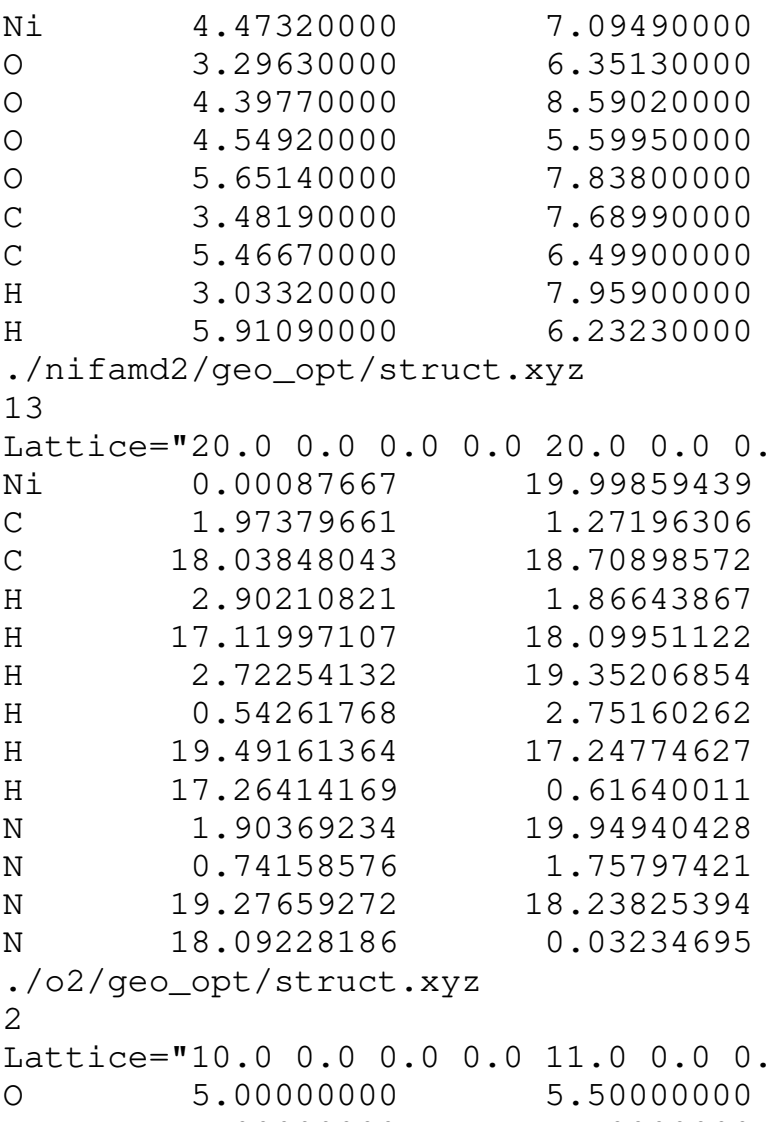

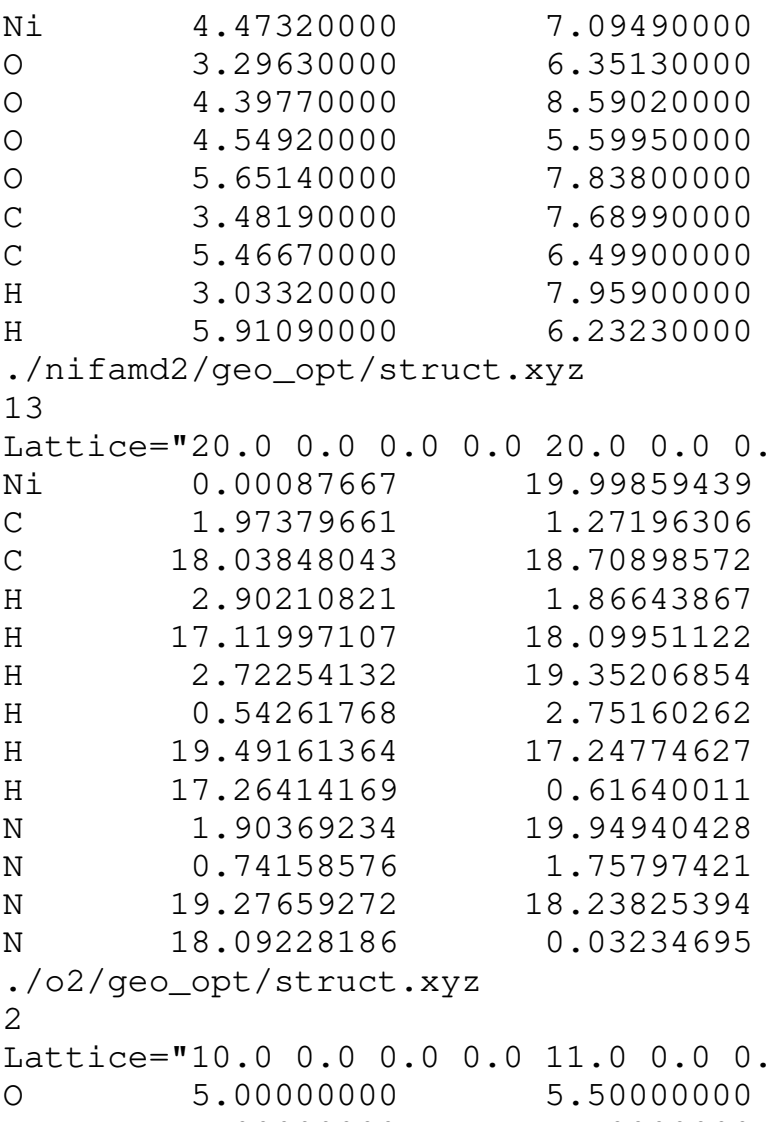

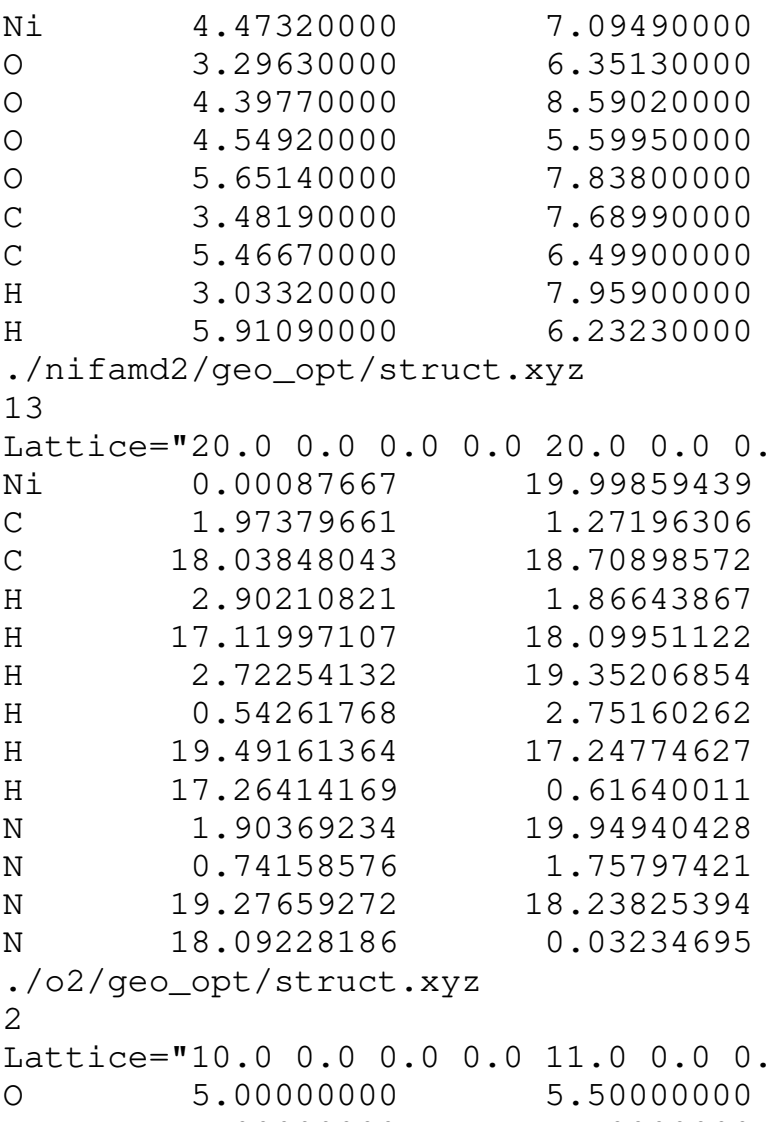

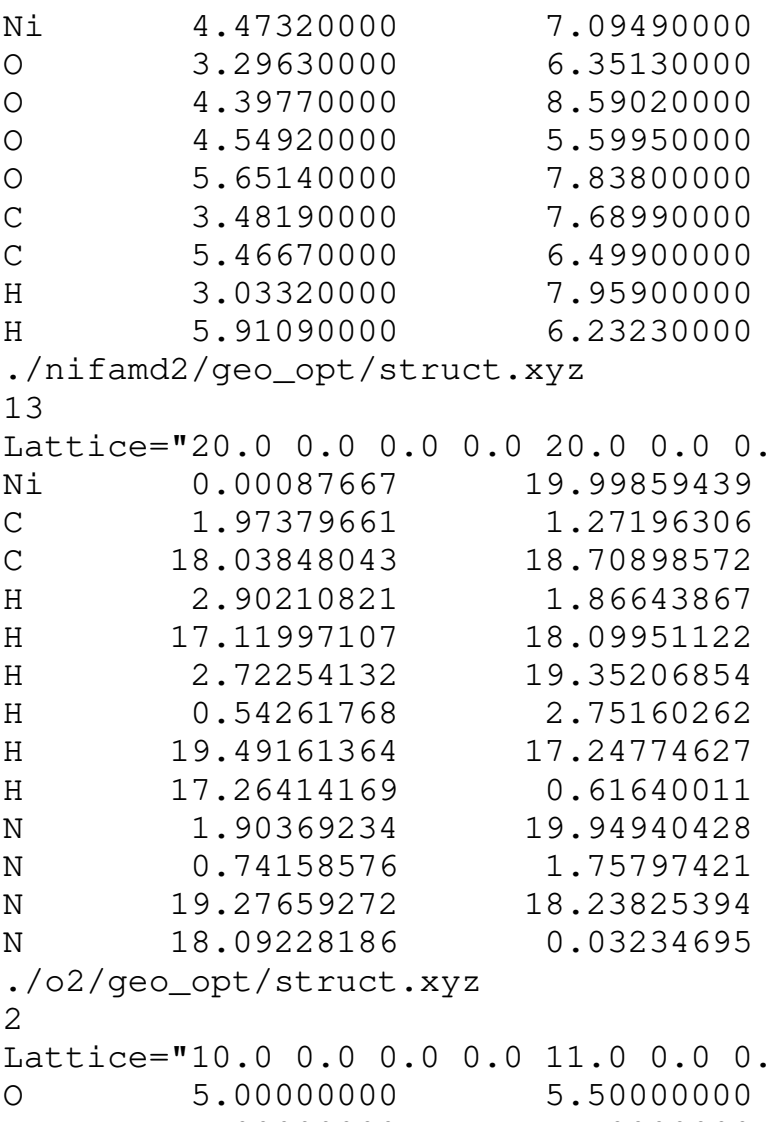

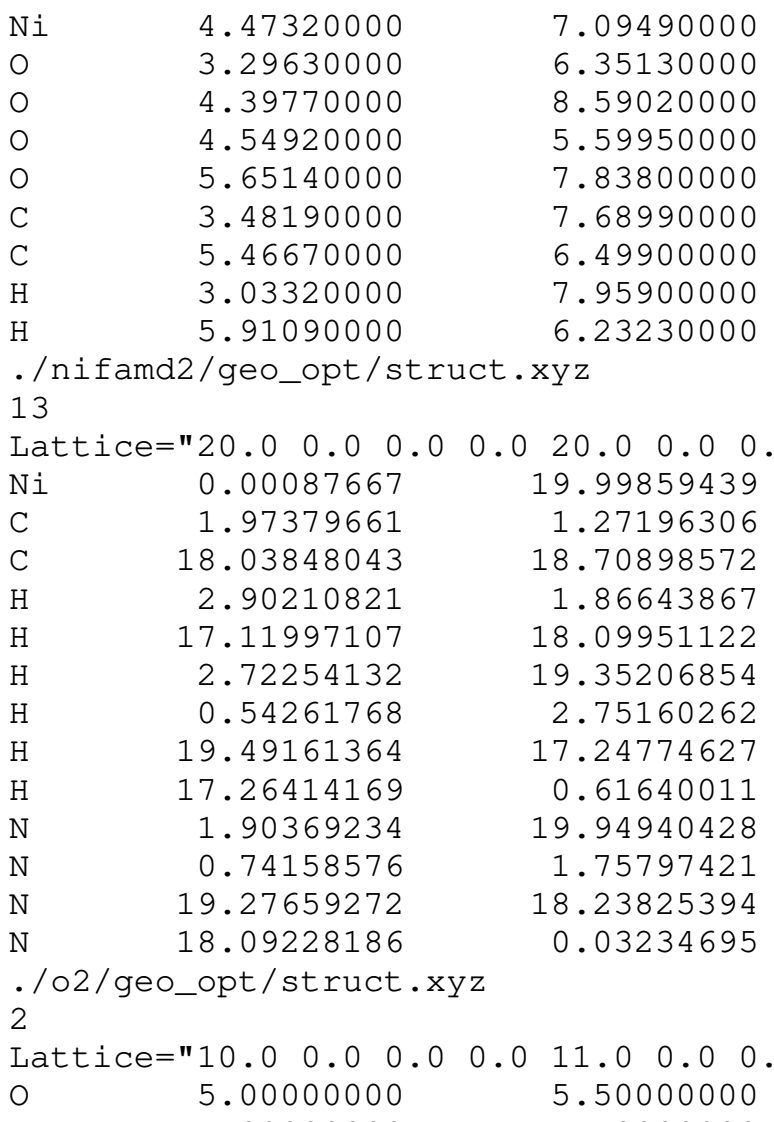

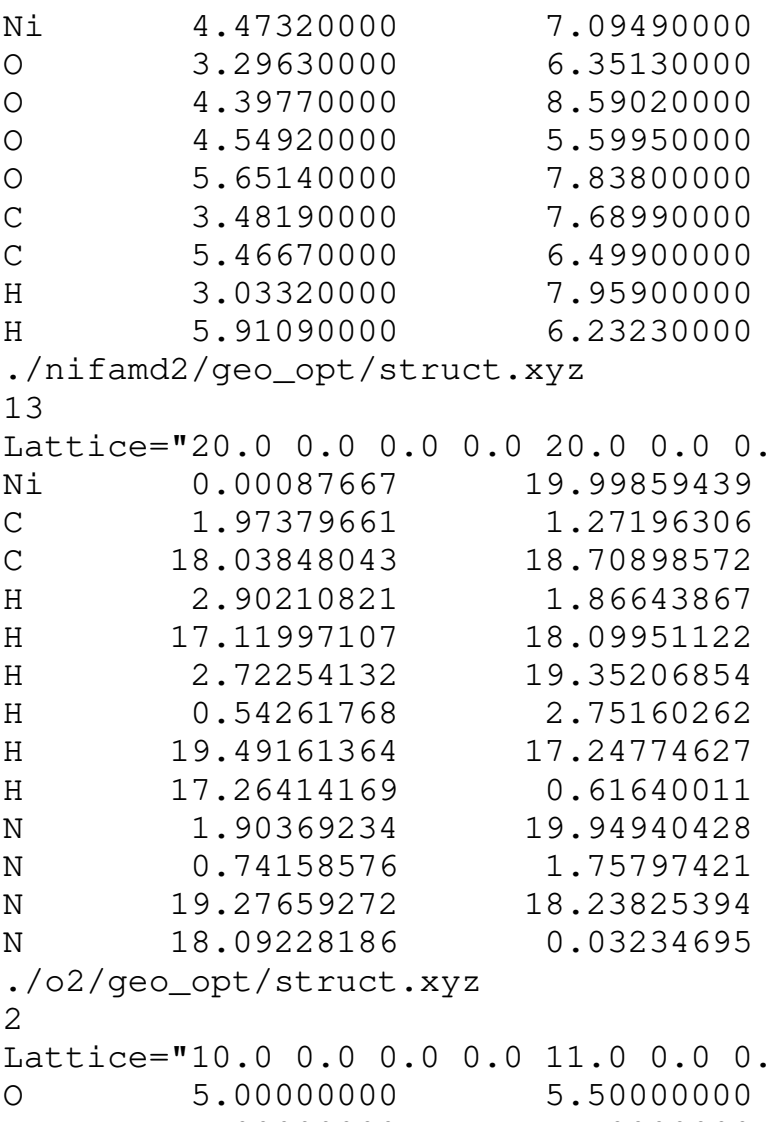

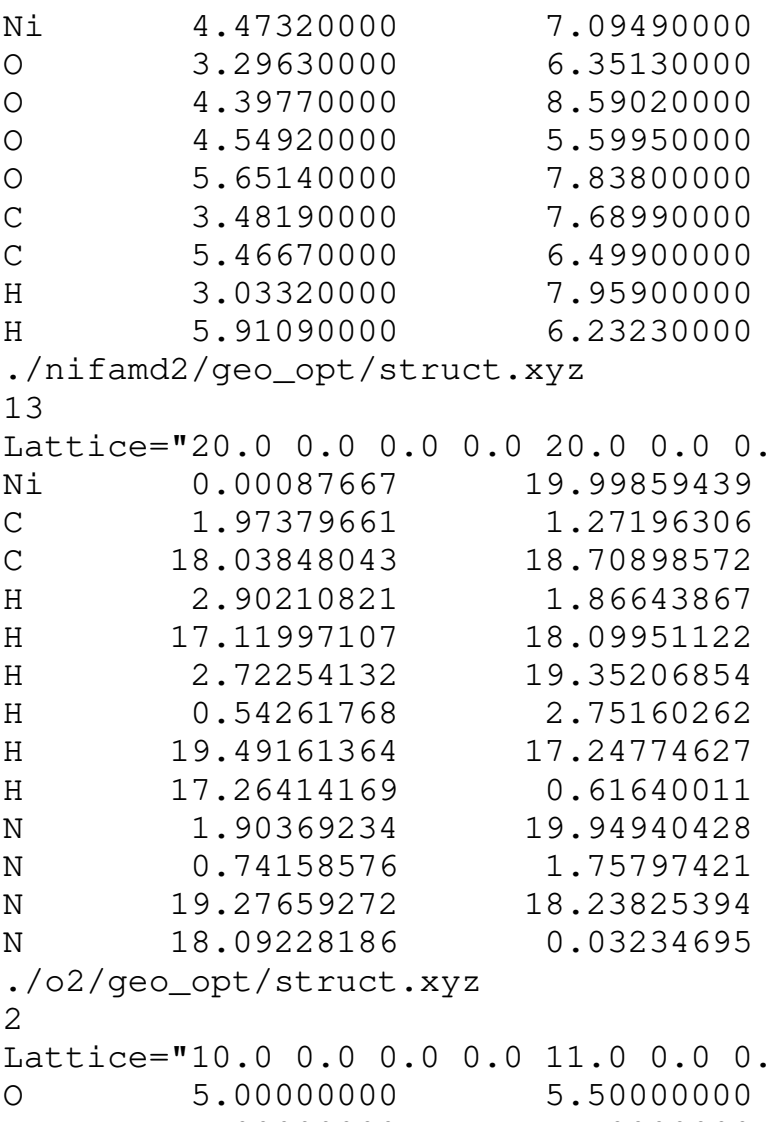

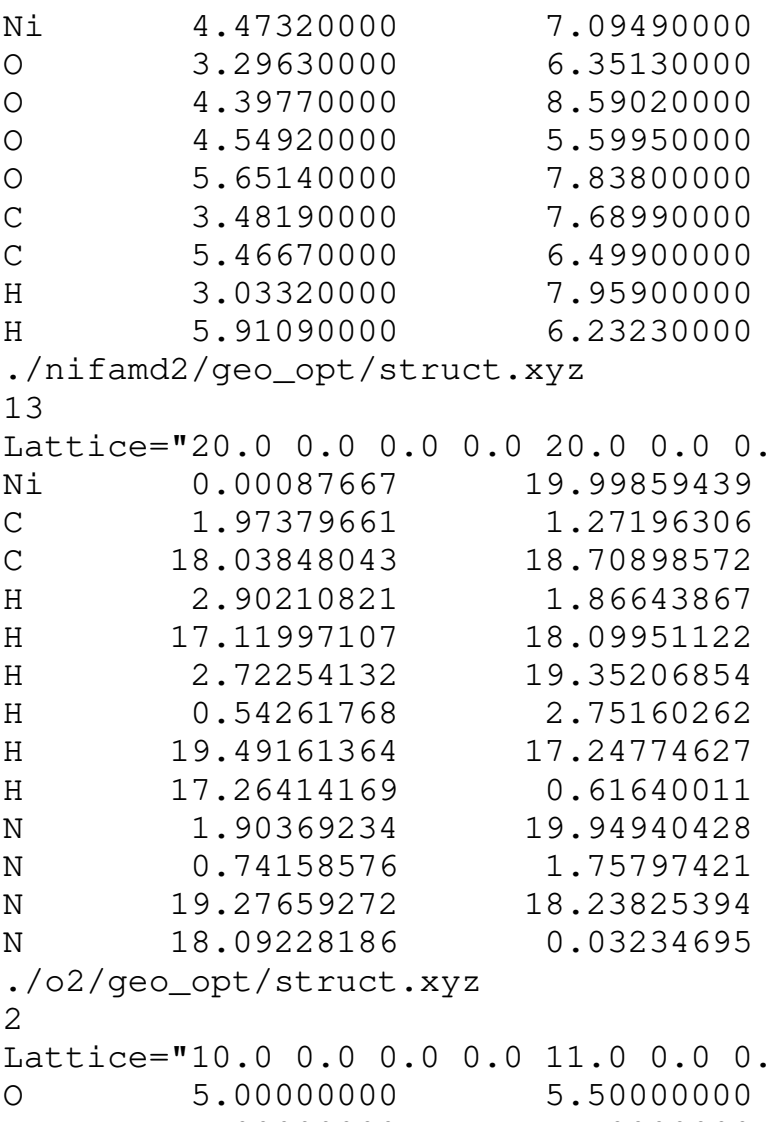

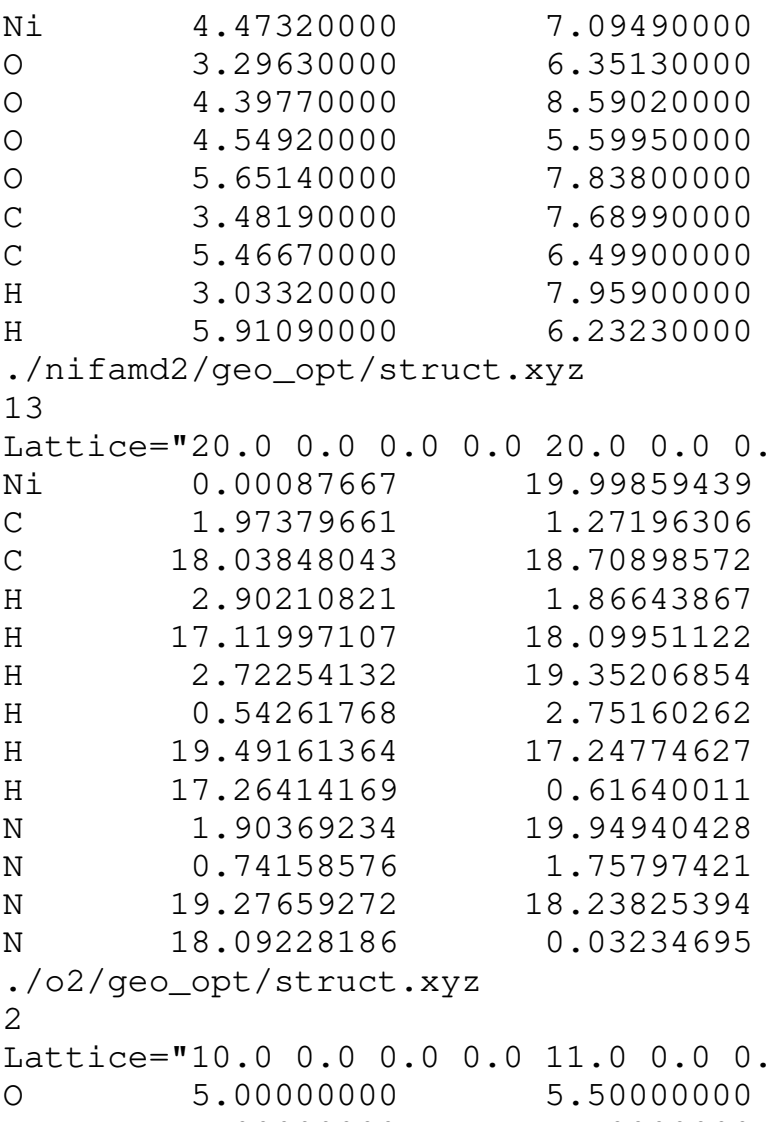

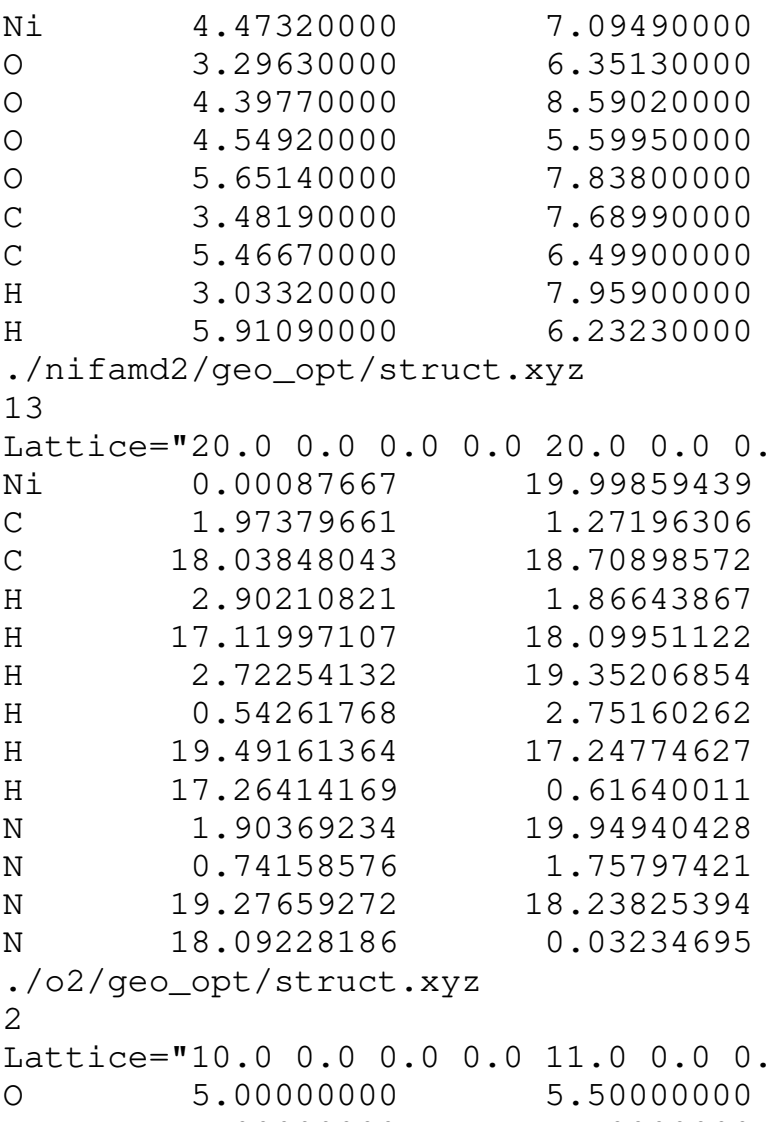

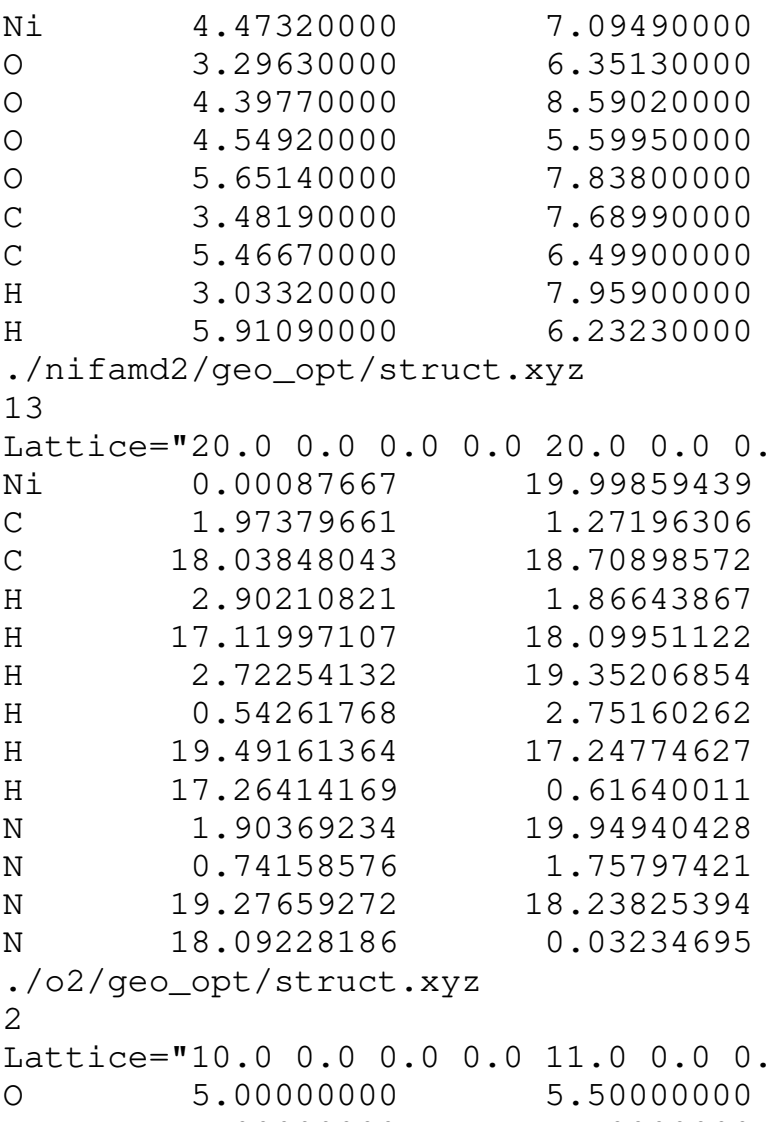

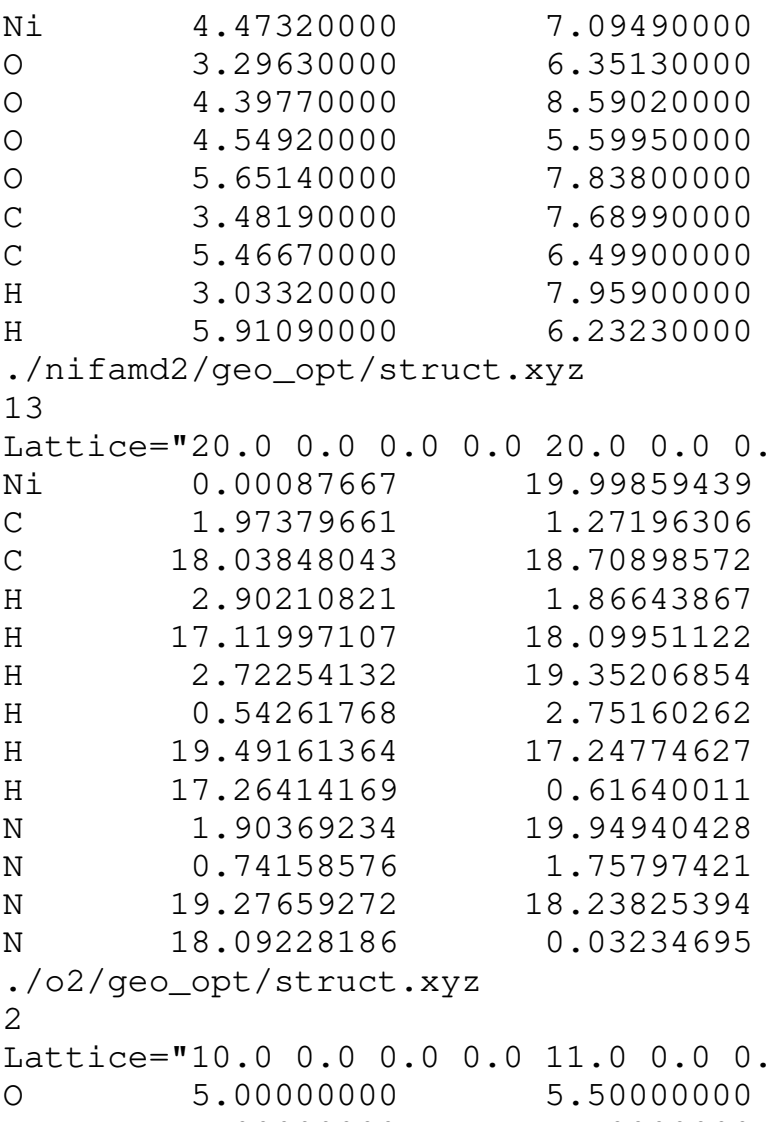

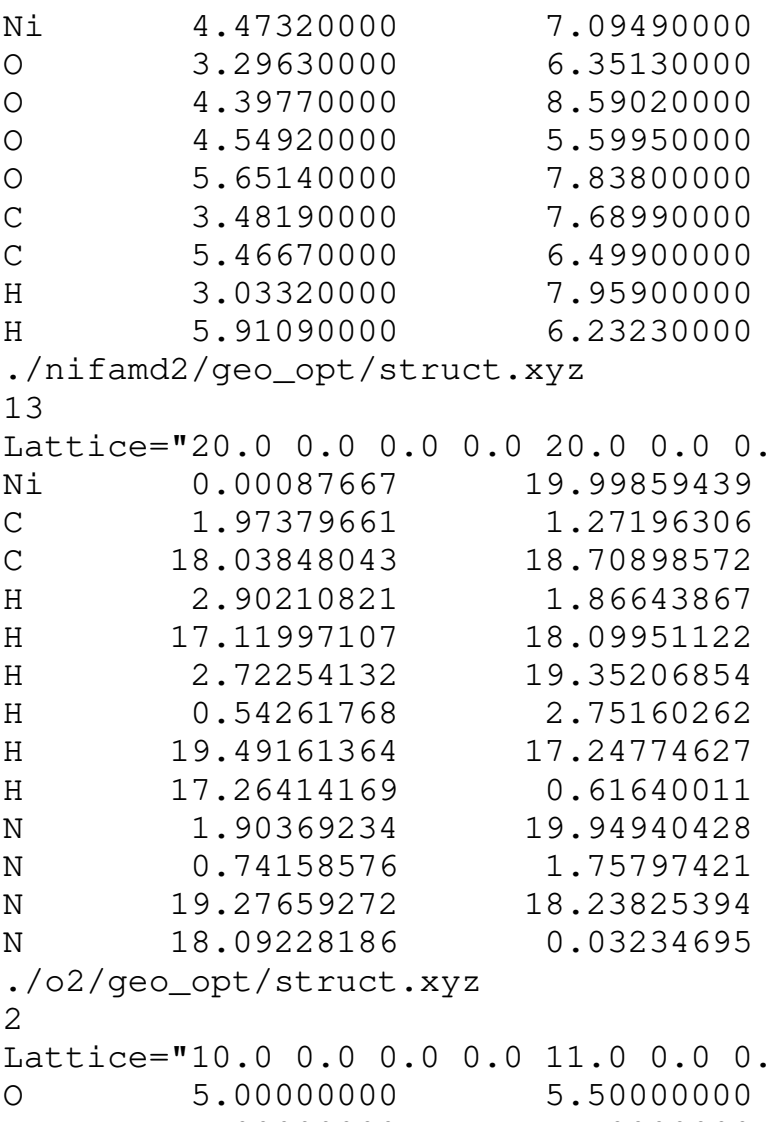

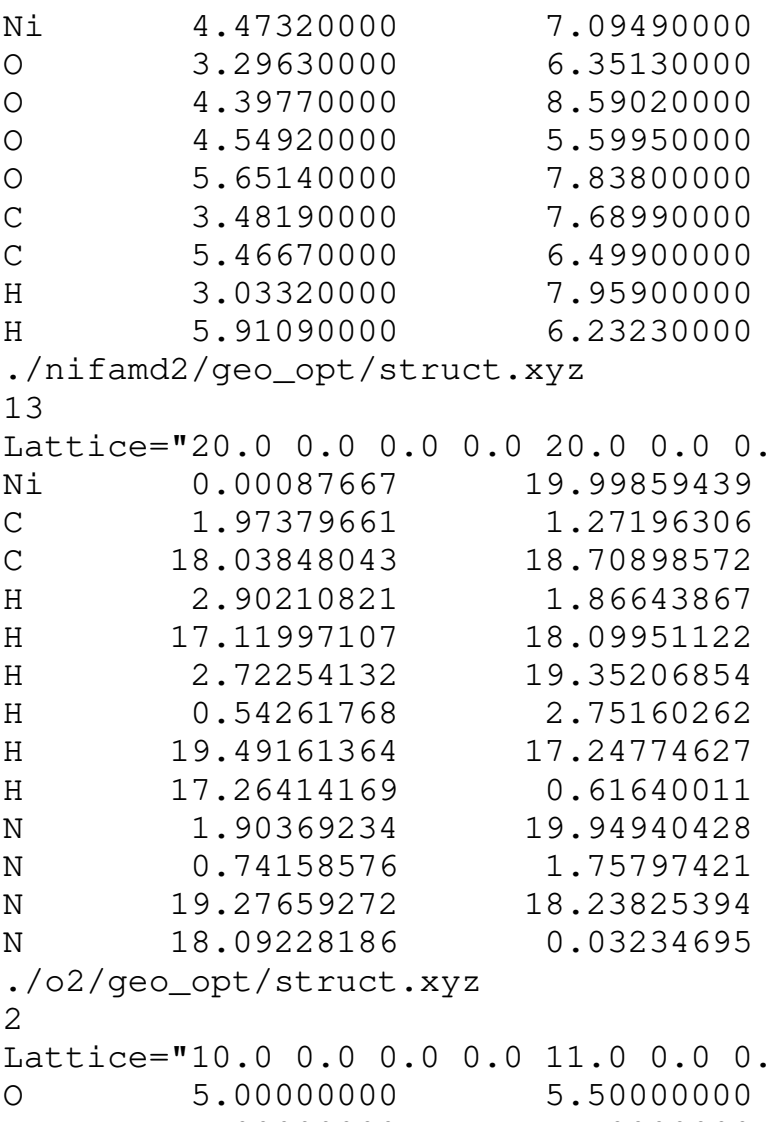

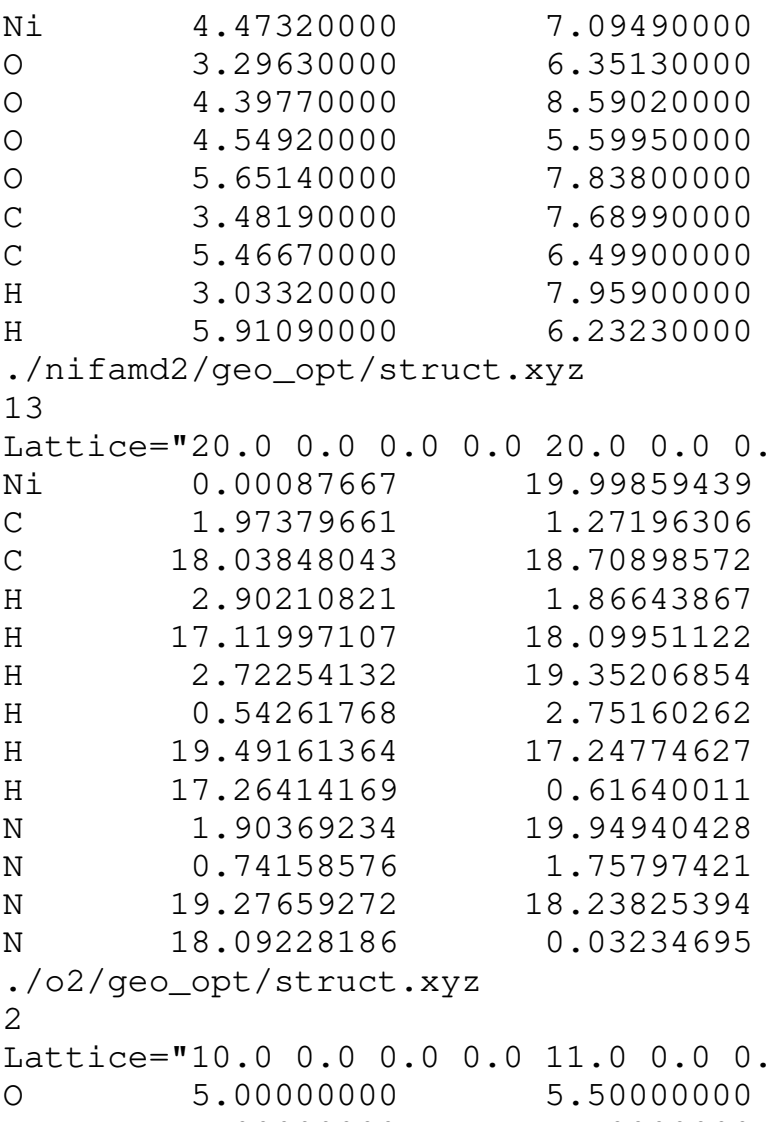

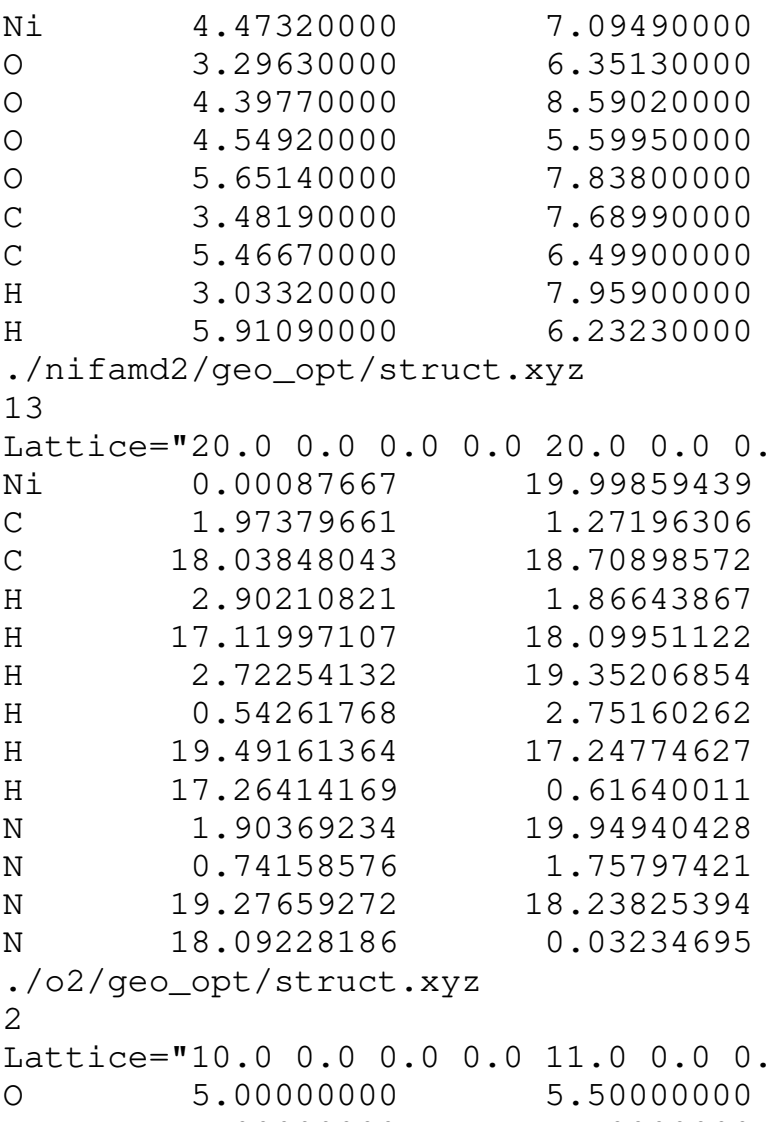

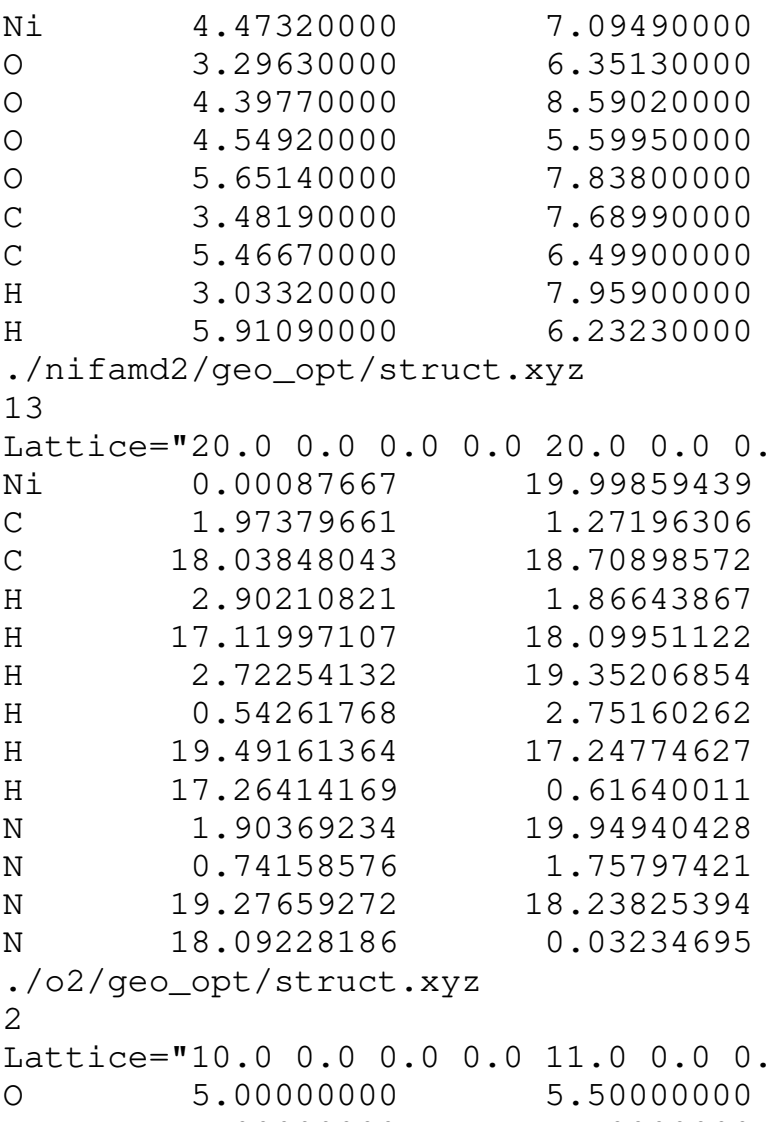

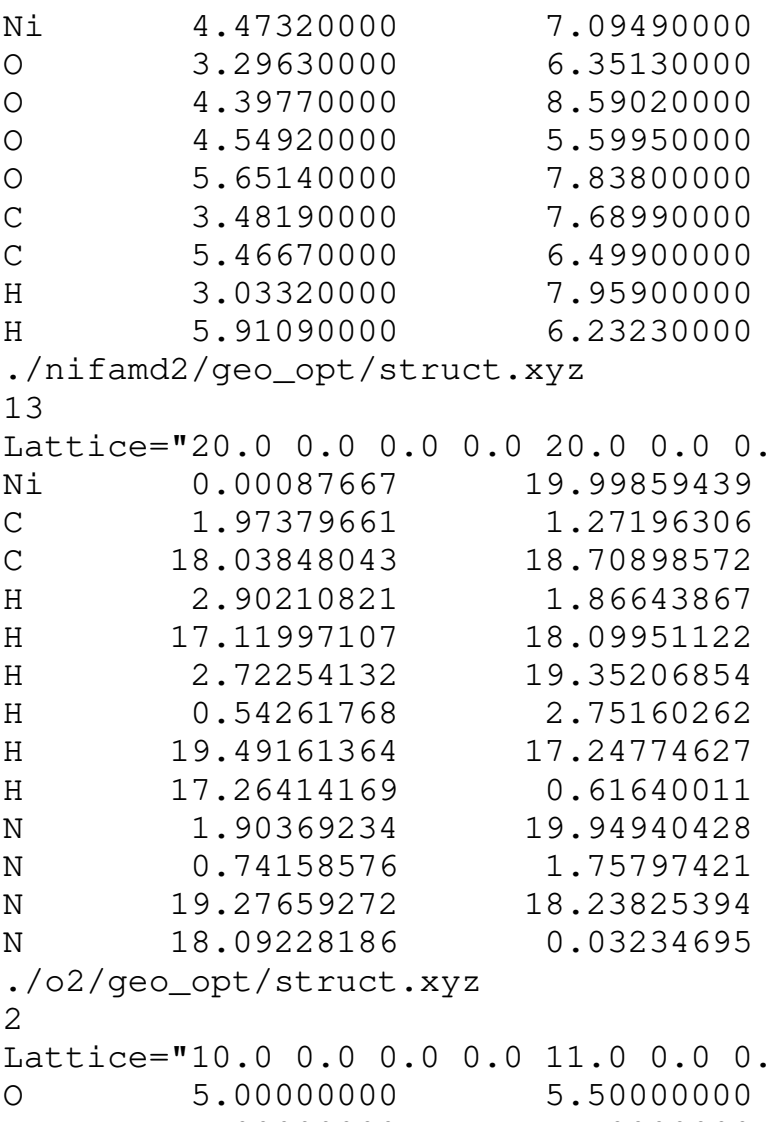
4.13580000
2.66460000
2.77140000
5.50020000
5.60640000
1.88970000
6.38120000
0.87630000
7.39650000

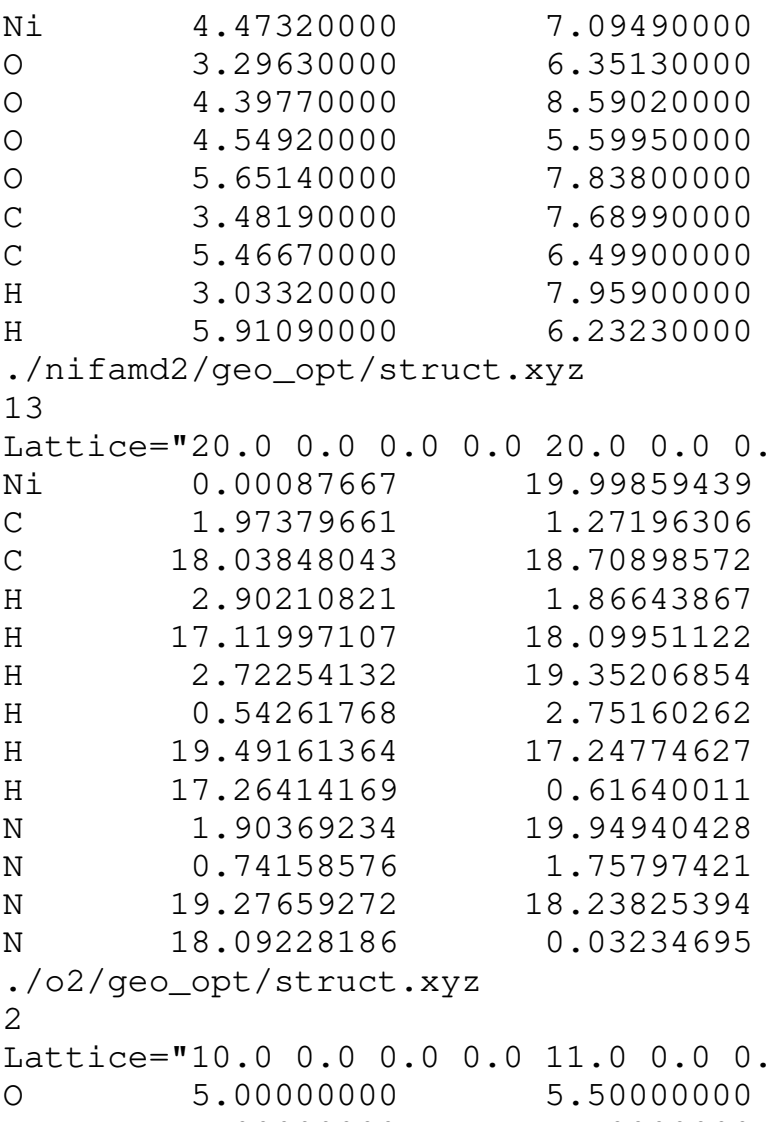

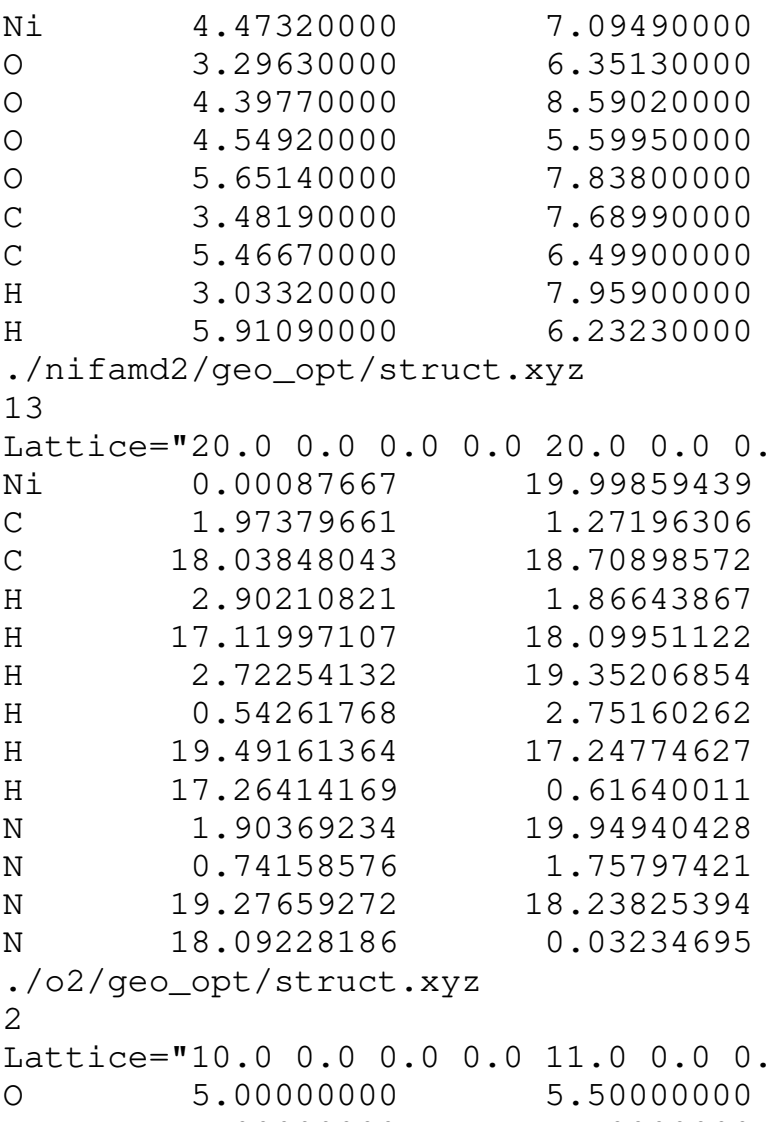

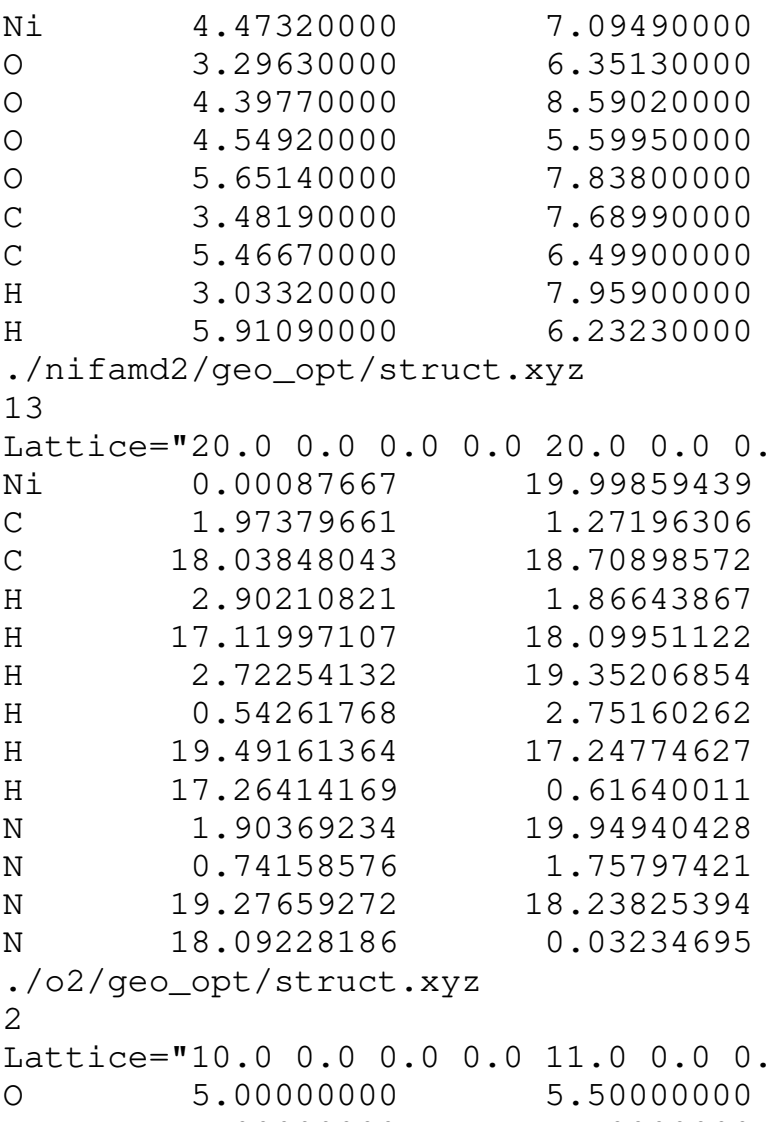

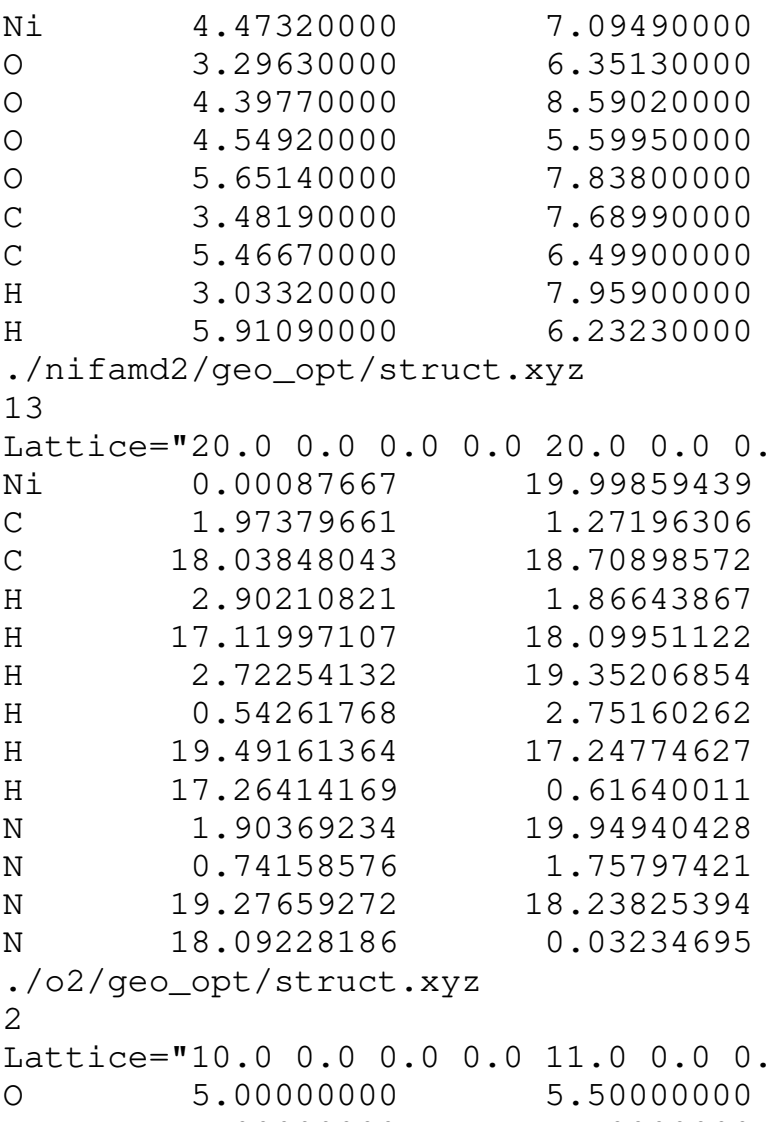

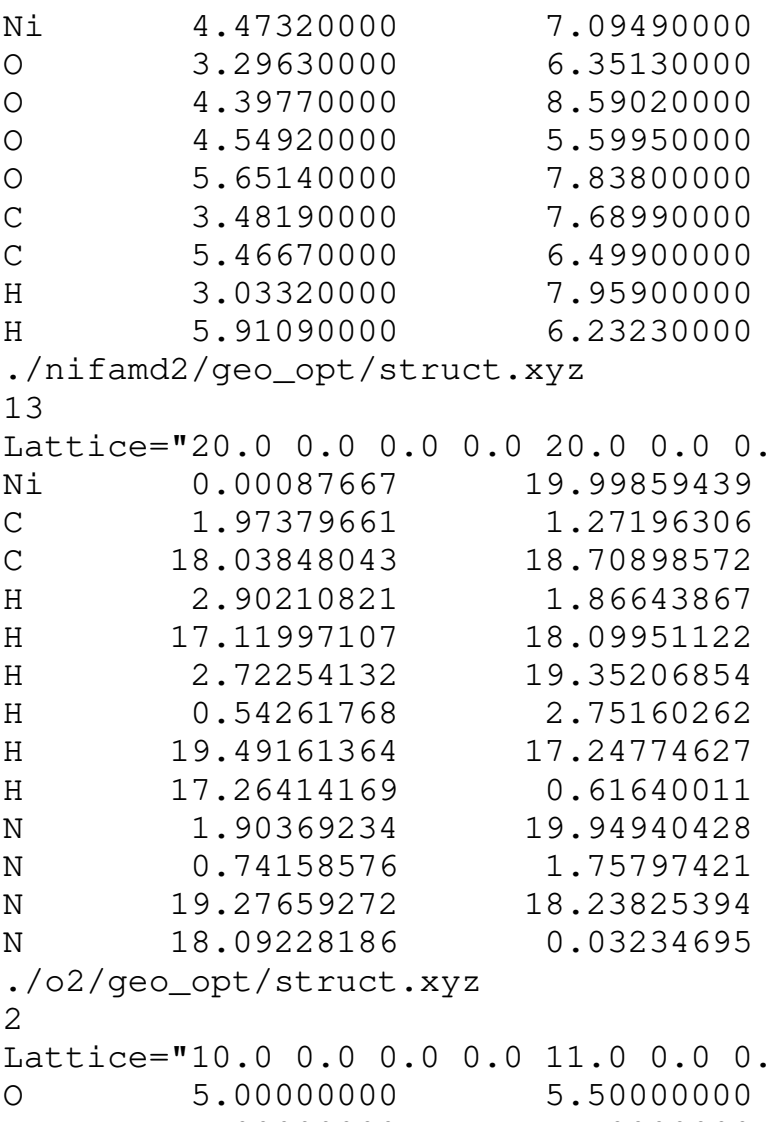

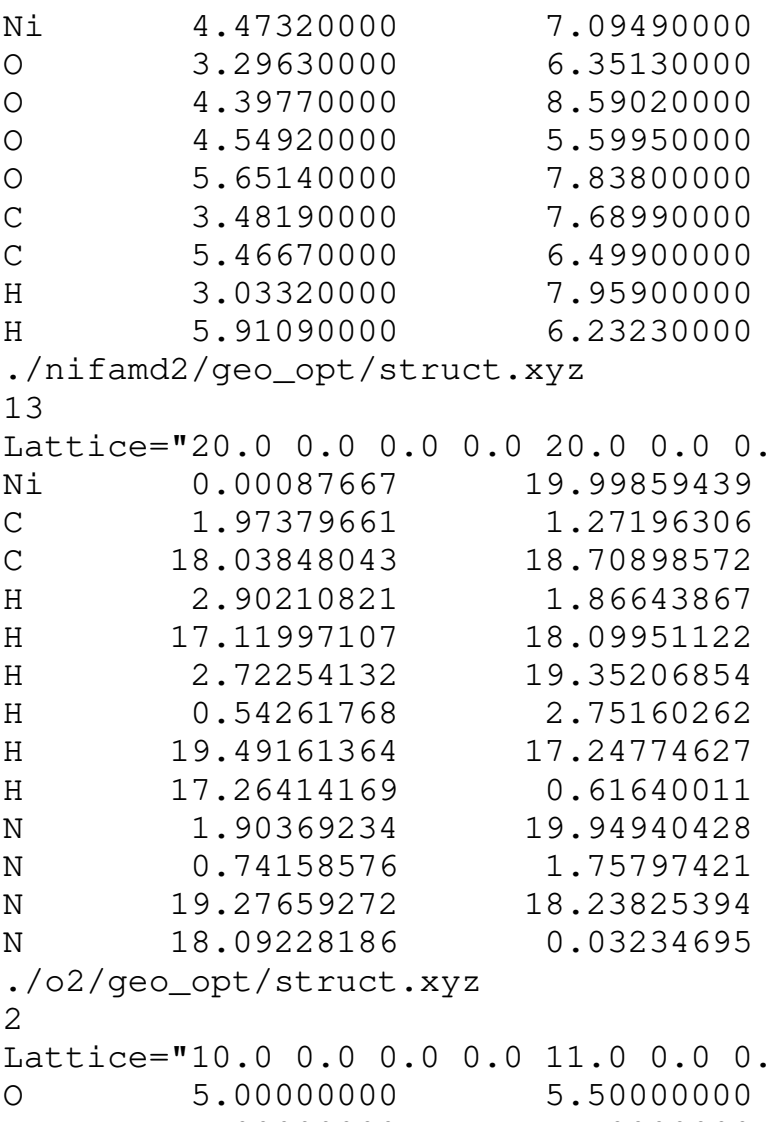

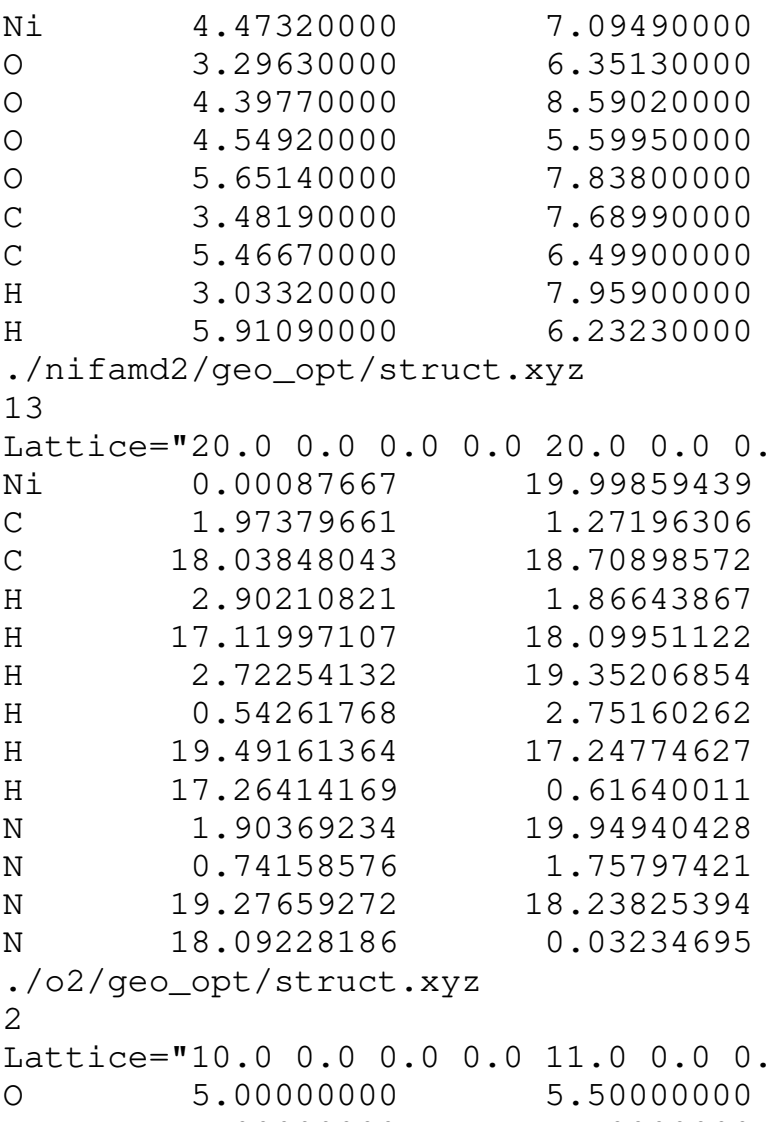

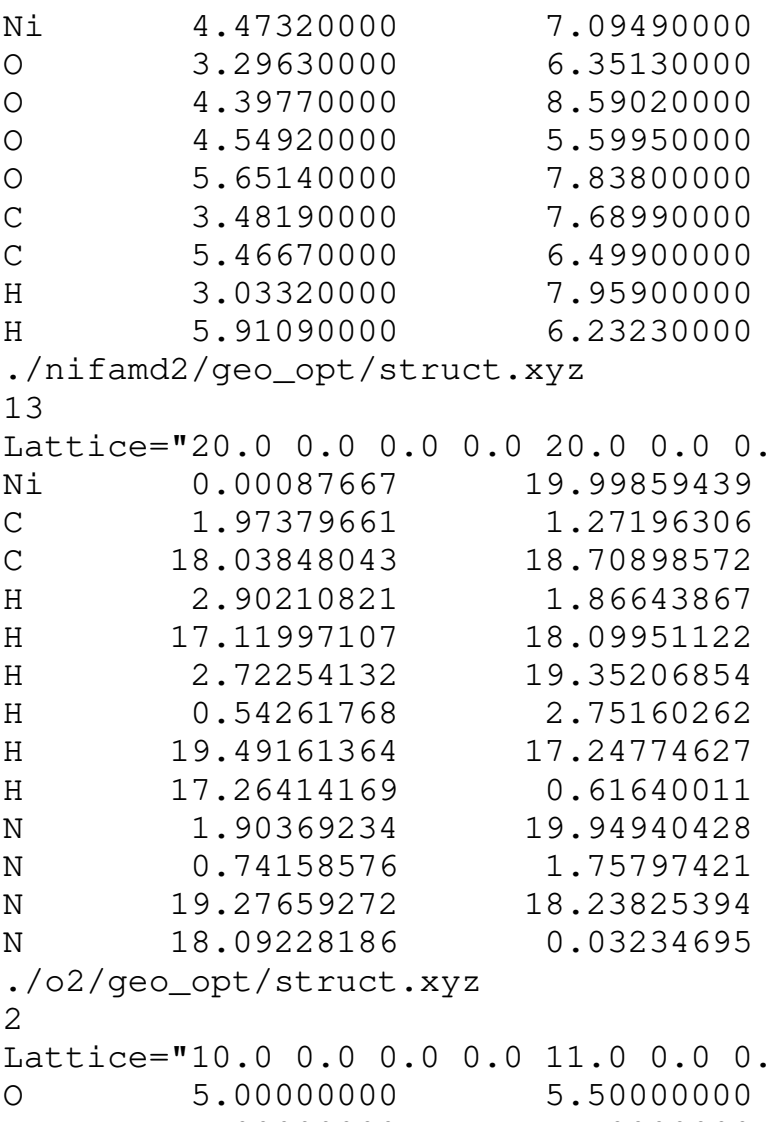

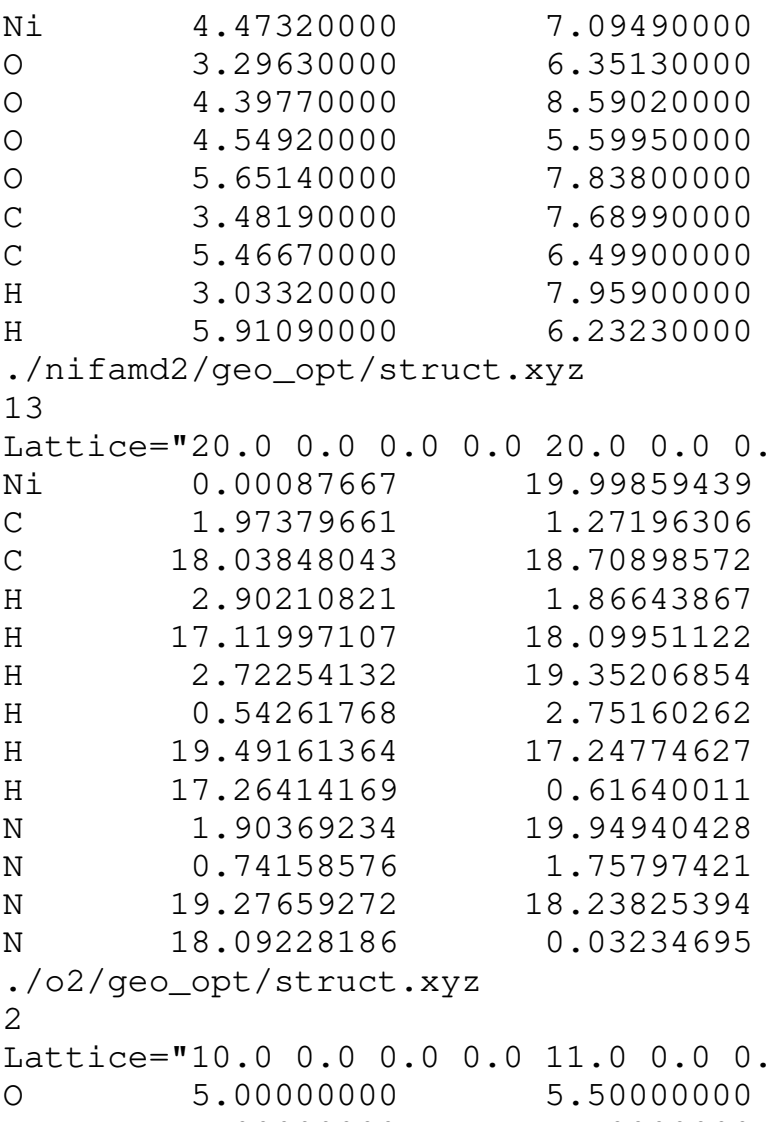

20.0" Properties=species:S:1:pos:R:3 pbc="T T T" 0.00000000

0.00000000

0.00000000

0.00000000

0.00000000

0.00000000

0.00000000

0.00000000

0.00000000

0.00000000

0.00000000

0.00000000

0.00000000

2 " Properties=species:S:1:pos:R:3 pb 
14

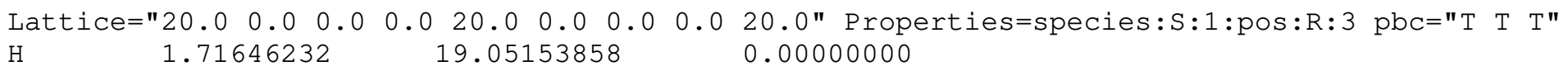

18.32023624

1.01211591

4.21746376

3.38330409

0.82123768

5.34389142

19.47739145

3.06030855

0.02753502

19.09479604

0.00000000

0.00000000

5.30063396

0.04776483

0.00000000

0.00000000

0.00000000

2. 51016498

4.34766517

0.00000000

1. 55103355

2.84439645

0.00000000

0.14855675

0.00197580

0.00000000

1. 35447917

1.17389301

0.00000000

19.32435060

0.00000000

3.21334940

0.00000000

1.18322083

3.22152699

0.00000000

. /OTHERS/cu2amd2_subs/Cu2Amd2_c2/geo_opt/struct.xyz

20

Lattice $=" 20.0 \quad 0.0 \quad 0.0 \quad 0.020 .0 \quad 0.0 \quad 0.0 \quad 0.020 .0 "$ Properties=species:S:1:pos:R:3 pbc="T T T"

$\mathrm{H}$

1.75630799

19.02889890

0.00000000

4.25474253

3.35991079

0.00000000

19.51669991

3.10082070

19.06990723

5.28024894

0.00000000

1. 96615574

0.00000000

17.41367852

0.43719399

.53451555

0.43719399

0.00000000

17.53451555

0.89496371

19.53954516

5.65210308

19.10503629

6.31361946

0.92305846

6.31361946

0.06621181

0.02707160

2. 54598164

4.32618449

0.97840160

5.73416201

0.63353138

1. 52245055

2. 80135172

0.00000000

19.10481583

0.89518417

0.00000000

0.00000000

0.00000000

0.00000000

0.00000000

0.00000000

$-0.01768781$

0.00000000

1.14023393

0.00000000

3.19540522

0.00000000

3. 24908463

4. 39296512

0.00000000

/ OTHERS / cu2amd2_subs/Cu2Amd2_c3/geo_opt/struct.xyz 20

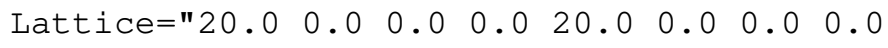

1. 77972871

0.75797129

19.09524630

5.30018370

19.54723473

2.99046527

19.05701685

5.33841315

17.34412471

17.51481615

17.51481615

1.88192991

0.36091984

0.36091984

5.19357529

2.51350009

4.03450016

4.03450016

5.02288385

0.03311654

4.36231346

0.91197387

3.48345613

1. 59715531

2.79827469

0.03436969

1.11999585

3.27542415

4.36106031

20.0" Properties=species: $\mathrm{S}: 1: \mathrm{pos}: \mathrm{R}: 3$ pbc="T T T" 0.00000000

0.00000000

0.00000000

0.00000000

0.00000000

0.89425180

19.10574820

0.00000000

0.89425180

19.10574820

0.00000000

0.00000000

0.00000000

0.00000000

0.00000000

0.00000000

0.00000000

0.00000000

0.00000000

3.23452443

1.14695243

0.00000000

. /OTHERS/cu2amd2_subs/Cu2Amd2_c4/geo_opt/struct.xyz 20

Lattice $=20.0 \quad 0.0 \quad 0.0 \quad 0.020 .0 \quad 0.0 \quad 0.0 \quad 0.020 .0 "$ Properties=species:S:1:pos:R:3 pbc="T T T"
$\mathrm{H}$
1.73084486
19.06541444
0.02619583
18.32495783
1.03160729
0.02599534
4.21274217
3.36381271
$-0.02599534$ 
$\mathrm{H}$

$\mathrm{H}$

$\mathrm{H}$

$\mathrm{H}$

$\mathrm{H}$

$\mathrm{H}$

$\mathrm{H}$

C

C

C

C

$\mathrm{Cu}$

$\mathrm{Cu}$

$\mathrm{N}$

$\mathrm{N}$

$\mathrm{N}$

$\mathrm{N}$
0.80685514

19.82500636

18.28301709

19.07761891

2.71269364

4.25468291

3.46008109

0.01426655

2.52343345

19.25733436

3.28036564

2.37793151

0.15976849

1.34784178

19.33233917

3.20536083

1.18985822

Fri Apr 16 14:51:55 2021

240

$\begin{array}{rr}5.33001556 & -0.02619583 \\ 17.91644393 & 0.49699168 \\ 18.80668550 & 0.49708856 \\ 18.40267623 & 18.95814440 \\ 6.47897607 & 19.50300832 \\ 5.58873450 & 19.50291144 \\ 5.99274377 & 1.04185560 \\ 0.02466177 & 0.02416474 \\ 4.37076823 & -0.02416474 \\ 18.71376627 & -0.00125674 \\ 5.68166373 & 0.00125674 \\ 1.55730086 & 0.00004590 \\ 2.83812914 & -0.00004590 \\ 0.00745751 & 0.01857389 \\ 1.17086634 & 0.01855998 \\ 3.22455366 & -0.01855998 \\ 4.38797249 & -0.01857389\end{array}$

5.33001556

17.91644393

18.80668550

8.40267623

5.99274377

0.02466177

4.37076823

8.71376627

5.68166373

1.55730086

.83812914

4.38797249

13

Lattice="20.0 0.0 0.0 $0.020 .00 .00 .00 .020 .0 "$ Properties=species:S:1:pos:R:3 pbc="T T T" $\mathrm{Ni}$

0.00022863

1.97676823

18.03594953

2.90436803

17.11536088

2.72266142

0.55044887

19.49243928

17.25784097

1.90619925

0.74563758

19.27387185

18.08852549

$-0.00046631$

1.27386625

18.70641712

0.00000000

0.00000000

0.00000000

1.86952735

0.00000000

18.09996365

0.00000000

19.35101944

0.00000000

2.75679214

0.00000000

17.24552543

0.00000000

0.61074028

0.00000000

$-0.04868482$

0.00000000

1.76203117

0.00000000

18.23500078

0.00000000

0.02955752

0.00000000

./OTHERS/niamd2_subs/NiAmd2_c2/geo_opt/struct.xyz

19

Lattice $=" \begin{array}{lllllllll}20.0 & 0.0 & 0.0 & 0.0 & 20.0 & 0.0 & 0.0 & 0.0\end{array}$

0.03002855

1.96674002

18.09104975

3.20607359

16.84821105

2.70602966

0.54312969

19.50747877

17.35912290

3.95669435

2.97392775

3.65281423

17.07490045

16.10599013

19.95415600

1.22014056

18.69319673

2.03026215

17.88651395

19.34312977

2.64697858

17.25790983

0.57047101

1.44934828

2.94395027

2. 33052445

16.96190841

18.46376912

16.39161171

1.91246046

0.72853377

17.60474810

19.89329137

1.70336051

18.20639917

0.02103176

18.14913951

0 20.0" Properties=species:S:1:pos:R:3 pbc="T T T" $-0.00295475$

19.79904055

0.20044222

0.04822358

19.95410839

$-0.01876747$

$-0.00359697$

0.01452761

0.00445267

0.60178373

0.61213611

19.08729710

19.40562756

19.38614844

0.91588523

19.65442902

19.65805425

0.34207056

0.34109215

./OTHERS/niamd2_subs/NiAmd2_c3/geo_opt/struct.xyz

19

Lattice $=20.0 \quad 0.00 .0$

$\mathrm{Ni} \quad 0.02911824$

C $\quad 1.95848573$

18.09665256

3.05709270

19.63379550

2.86073746

17.20895076

0.53785239

17.37907619

2.88583582

$20.0 \quad 0.0 \quad 0.0$
19.95938307

1.21736882

18.70553563

19.04424855

16.81786270

1.78618343

18.11164775

2.63117281

0.57756447

18.41580160
0 20.0" Properties=species:S:1:pos:R:3 pbc="T T T" 19.99851050

19.96187292

0.04032900

0.02509628

19.97145073

0.25502746

19.75251369

0.21382033

19.79300893

0.91605792 


\section{$\mathrm{H}$}

$\mathrm{H}$

$\mathrm{H}$

$\mathrm{H}$

$\mathrm{H}$

$\mathrm{N}$

$\mathrm{N}$

$\mathrm{N}$

$\mathrm{N}$
Fri Apr 16 14:51:55 2021

241

$\begin{array}{rr}19.62607229 & 0.18990284 \\ 18.36670677 & 19.17143315 \\ 16.71628264 & 19.08115731 \\ 16.21124973 & 19.80634477 \\ 16.38950225 & 0.82576934 \\ 19.91130931 & 19.77414873 \\ 1.72962587 & 19.77987586 \\ 18.19503320 & 0.22097871 \\ 0.03103912 & 0.22270154\end{array}$

19.62607229

3. 22313727

0.27803369

18.72460671

0.18354726

1.93664683

0.73554100

19.30005030

18.12508787
0.18990284
19.17143315
19.08115731
19.80634477
0.82576934
19.77414873
19.77987586
0.22097871
0.22270154

19

Lattice $=" 20.0 \quad 0.0 \quad 0.0 \quad 0.020 .0 \quad 0.0 \quad 0.0 \quad 0.020 .0 "$ Properties=species:S:1:pos:R:3 pbc="T T T" 0.00407209

1.94158571

18.07729725

3.00099944

17.00332603

2. 86622295

17.17027322

0.56305102

19.46929036

3.07233630

3. 95496510

2. 88142202

17.19687078

16.06774859

16.84581600

1.89423191

0.71533314

19.29903882

18.10556927

0.00264028

1.24750040

18.75536404

19.05540788

0.93414446

1. 80846821

18.19985311

2.69140175

17.32557635

18.29030988

19.61223645

18.52864746

1. 51217395

0.36355255

1.65578236

$-0.05970717$

1.77198545

18.23013661

0.05676598

$-0.03359746$

0.10638319

$-0.10486532$

0.03344485

$-0.03006046$

$-0.12413741$

0.19897155

$-0.20092893$

0.18721039

0.82271838

0.00348338

19.07049081

0.89010887

0.11467571

19.15289212

0.28592811

0.21309572

$-0.25301437$

$-0.33279911$

. /OTHERS/nifa2_others/bidentate_tetra/geo_opt/struct.xyz

9

Lattice $=" 13.00980 .0 \quad 0.0 \quad 0.012 .4613000000000010 .0 \quad 0.00 .016 .467 "$ Properties=species:S:1: pos:R:3 pbc="T T T"

$\begin{array}{llll}\mathrm{Ni} & 4.47320000 & 7.09490000 & 4.13580000 \\ \mathrm{O} & 2.90110000 & 7.97790000 & 3.33460000 \\ \mathrm{O} & 4.85450000 & 7.04460000 & 2.20050000 \\ \mathrm{O} & 4.54920000 & 5.59950000 & 5.50020000 \\ \mathrm{O} & 5.65140000 & 7.83800000 & 5.60640000 \\ \mathrm{C} & 3.51530000 & 7.76300000 & 1.93600000 \\ \mathrm{C} & 5.46670000 & 6.49900000 & 6.38120000 \\ \mathrm{H} & 3.07330000 & 8.06080000 & 0.92950000 \\ \mathrm{H} & 5.91090000 & 6.23230000 & 7.39650000\end{array}$

. /OTHERS/nifa2_others/monodentate/geo_opt/struct.xyz 9

Lattice $=" 12.81780 .0 \quad 0.0 \quad 0.014 .11820 .00 .00 .017 .0896 "$ Properties=species:S:1:pos:R:3 pb $\mathrm{C}=$ " $\mathrm{T}$ T $\mathrm{T} "$

$\begin{array}{ll}\mathrm{Ni} & 4.40476961 \\ \mathrm{O} & 3.07719596 \\ \mathrm{O} & 3.72533937 \\ \mathrm{O} & 5.17498697 \\ \mathrm{O} & 5.81344575 \\ \mathrm{C} & 3.90497088 \\ \mathrm{C} & 5.24415877 \\ \mathrm{H} & 4.90885964 \\ \mathrm{H} & 4.71507306\end{array}$

7.17119457
8.75019546
7.64977628
6.62242378
5.58870197
8.51887075
5.57163880
8.99792784
4.68037054

4.05219248

0.66921783

2. 53603689

5.49834917

7.40730793

1.52077946

6.33991703

1. 55574140

5. 93475782 